\title{
Copy number variation in fetal alcohol spectrum disorder
}

\begin{tabular}{|c|c|}
\hline Journal: & Biochemistry and Cell Biology \\
\hline Manuscript ID & bcb-2017-0241.R1 \\
\hline Manuscript Type: & Article \\
\hline Date Submitted by the Author: & 09-Nov-2017 \\
\hline Complete List of Authors: & $\begin{array}{l}\text { Zarrei, Mehdi; The Centre for Applied Genomics } \\
\text { Hicks, Geoffrey G.; University of Manitoba College of Medicine, } \\
\text { Regenerative Medicine } \\
\text { Reynolds, James N.; Queen's University School of Medicine, Biomedical and } \\
\text { Molecular Sciences } \\
\text { Thiruvahindrapuram, Bhooma; The Centre for Applied Genomics } \\
\text { Engchuan, Worrawat; Hospital for Sick Children SickKids Learning Institute } \\
\text { Pind, Molly; University of Manitoba College of Medicine, Regenerative } \\
\text { Medicine } \\
\text { Lamoureux, Sylvia; The Centre for Applied Genomics } \\
\text { Wei, John; The Centre for Applied Genomics } \\
\text { Wang, Zhouzhi; The Centre for Applied Genomics } \\
\text { Marshall, Christian R.; The Centre for Applied Genomics } \\
\text { Wintle, Richard; The Centre for Applied Genomics } \\
\text { Chudley, Albert; University of Manitoba } \\
\text { Scherer, Stephen W.; The Centre for Applied Genomics }\end{array}$ \\
\hline $\begin{array}{r}\text { Is the invited manuscript for } \\
\text { consideration in a Special } \\
\text { Issue? : }\end{array}$ & Fetal Alcohol Spectrum Disorder \\
\hline Keyword: & Fetal alcohol spectrum disorder, FASD, copy number variations, CNV \\
\hline
\end{tabular}

\section{SCHOLARONE}




\section{Copy number variation in fetal alcohol spectrum disorder}

2 Mehdi Zarrei, ${ }^{a}$ Geoffrey G. Hicks, ${ }^{b}$ James N. Reynolds, ${ }^{c, d}$ Bhooma Thiruvahindrapuram, ${ }^{a}$

3 Worrawat Engchuan, ${ }^{\mathrm{a}}$ Molly Pind, ${ }^{\mathrm{b}}$ Sylvia Lamoureux, ${ }^{\mathrm{a}}$ John Wei, ${ }^{\mathrm{a}}$ Zhouzhi Wang, ${ }^{\mathrm{a}}$ Christian R.

4 Marshall, ${ }^{a}$ Richard F. Wintle, ${ }^{a}$ Albert E. Chudley, ${ }^{e, f}$ and Stephen W. Scherer, ${ }^{\text {a,g }}$

$5 \quad{ }^{\mathrm{a}}$ The Centre for Applied Genomics and Program in Genetics and Genome Biology, The Hospital

6 for Sick Children, Toronto, Ontario, Canada

$7 \quad{ }^{b}$ Regenerative Medicine Program, University of Manitoba, Winnipeg, Canada

$8{ }^{\mathrm{c}}$ Centre for Neuroscience Studies, Queen's University, Kingston, Ontario, Canada.

$9{ }^{\mathrm{d}}$ Department of Biomedical and Molecular Sciences, Queen's University, Kingston, Ontario,

10 Canada

$11{ }^{\mathrm{e}}$ Department of Pediatrics and Child Health, Max Rady College of Medicine, Rady Faculty of

12 Health Sciences, University of Manitoba, Winnipeg, Canada.

13 f Department of Biochemistry and Medical Genetics, Max Rady College of Medicine, Rady

14 Faculty of Health Sciences, University of Manitoba, Winnipeg, Canada

$15{ }^{\mathrm{g}}$ Department of Molecular Genetics and McLaughlin Centre, University of Toronto, Toronto, 16 Ontario, Canada

17 Corresponding authors: Stephen W. Scherer (stephen.scherer@sickkids.ca) and Albert E. 18 Chudley (abchudley@gmail.com) 
20 Abstract: Fetal alcohol spectrum disorder (FASD) is characterized by a combination of

21 neurological, developmental, and congenital defects that may occur as a consequence of prenatal

22 alcohol exposure. Earlier reports showed that large chromosomal anomalies may link to FASD.

23 Here, we examined the prevalence and types of copy number variations (CNVs) in FASD cases

24 previously diagnosed by a multidisciplinary FASD team in sites across Canada. We genotyped

2595 children with FASD and 87 age-matched, typically developing controls on the Illumina

26 Human Omni2.5 SNP array platform. We compared their CNVs to those of 10,851 population

27 controls, in order to identify rare $\mathrm{CNVs}(<0.1 \%$ frequency), which may include large unbalanced

28 chromosomal abnormalities, that might be relevant to FASD. In 12/95 (13\%) of the FASD cases,

29 rare CNVs were found that impact potentially clinically-relevant developmental genes, including

30 the CACNA1H involved in epilepsy and autism, the $3 \mathrm{q} 29$ deletion disorder, and others. Our

31 results show that a subset of children diagnosed with FASD have chromosomal deletions and

32 duplications that may co-occur or explain the neurodevelopmental impairments in a diagnosed

33 cohort of FASD individuals. Children suspected to have FASD with or without sentinel facial

34 features of fetal alcohol syndrome and neurodevelopmental delays should potentially be

35 evaluated by a clinical geneticist and possibly have genetic investigations as appropriate to

36 exclude other etiologies.

37 Key words: Fetal alcohol spectrum disorder, FASD, copy number variations, CNV.

38 Résumé: [will be translated into French by the journal translator]

39 Mots-clés: Fetal alcohol spectrum disorder, FASD, copy number variations, CNV. 


\section{Introduction}

41 Fetal alcohol spectrum disorder (FASD) is an umbrella term for a group of alcohol-related

42 disorders, characterizing the full range of damage from prenatal alcohol exposure (Chudley et al.

43 2005). It comprises fetal alcohol syndrome (FAS), partial FAS, alcohol-related

44 neurodevelopmental disorders, and alcohol-related birth defects, which are congenital anomalies

45 that can include growth deficits, facial dysmorphism, and/or malformations in multiple

physiological systems (Abdelmalik et al. 2013; Chudley et al. 2005; Douzgou et al. 2012;

47 Popova et al. 2016). FASD is also associated with numerous co-morbid neurodevelopmental disorders, including intellectual disabilities, microcephaly, autism spectrum disorder (ASD), and

49 attention deficit hyperactivity disorder (Abdelmalik et al. 2013; Douzgou et al. 2012; Weyrauch 50 et al. 2017).

51 The reported incidence of FASD is 0.2 to 3.0 per 1,000 live births for most countries (Douzgou

52 et al. 2012). However, an incidence of up to 89.2 per 1,000 births was reported in regions of

53 South Africa where wine production is abundant (May et al. 2007). A recent review of the

54 global prevalence of FASD in children and youth reported a prevalence of 7.7 per 1000

55 population (95\% CI, 4.9-11.7 per 1000 population). The highest rate was reported by the World

56 Health Organization European region with 19.8 per 1000 population, (95\% CI, 14.1-28 per 1000

57 population)(Lange et al. 2017).

58 It is well established in the medical community that not all children exposed to alcohol

59 prenatally develop FASD (Abel 1995; Stoler 1999). This indicates that factors other than

60 prenatal alcohol exposure also contribute to FASD aetiology. The impact of the genetic

61 variations to the aetiology of FASD has been studied in animal models (reviewed in (Eberhart

62 and Parnell 2016; McCarthy and Eberhart 2014)). However, genetic studies on human are 
63 restricted to case reports with large chromosomal anomalies (Stoler 1999) and twin studies

64 (Chasnoff 1985). For example, association of trisomy 21 or ring chromosome 6 with FASD has

65 been reported (Bingol et al. 1987; Muller et al. 1993; Romke et al. 1987). Large chromosomal

66 anomalies such as 22q11.2 microdeletion and microduplication, and 1q21 microduplications,

67 among others, were reported in 9\% (7/90) of cases with FASD in a UK cohort (Douzgou et al.

68 2012). A retrospective study of younger and older siblings of patients with FAS clearly indicated

69 a greatly increased risk of FAS in younger siblings if an older sibling had been diagnosed with

70 FAS (Abel 1988). The contribution of CNVs to the aetiology of neurodevelopmental disorders

71 (Gazzellone et al. 2016; Lionel et al. 2011; Oskoui et al. 2015; Pinto et al. 2014; Pinto et al.

72 2010; Zarrei et al. 2017a), including those that may be comorbid with FASD (Popova et al.

73 2016), has been well documented. However, until the current study, a genome-wide CNV

74 analysis in FASD cases had not been performed.

75 Our objective was to assess the role of CNVs and chromosomal abnormalities, at a genome-

76 wide level, in a clinically well-defined collection of individuals with FASD, to further define

77 genomic risk contribution in this common disorder.

\section{Materials and methods}

\section{Participants}

80 Ninety-five children diagnosed with FASD and eighty-seven aged-matched, typically

81 developing controls as internal control were recruited through the NeuroDevNet Canadian FASD

82 study cohort (Portales-Casamar et al. 2016; Reynolds et al. 2011) and the majority of clinical

83 centers used the Canadian FASD Guidelines for diagnosis (Chudley et al. 2005). Siblings of

84 some probands, if consented, were also recruited for this study (Table S1). Our FASD cases have 
85 a sex ratio of 0.73 (male: 40; female: 55; Table S1; 0.85 in internal controls). Research Ethics

86 Board approval was obtained from each participating centre, and all caregivers and participants

87 provided written informed consent. Saliva samples were collected from the children. Details of

88 saliva collection and storage is provided elsewhere (Portales-Casamar et al. 2016).

\section{Genotyping, $\mathrm{CNV}$ calling, and detection of rare variants}

DNA extracted from saliva using standard protocols was genotyped on two Illumina Human

Omni2.5 SNP array platforms (Omni2.5m-Quad; $\mathrm{n}=132$ and Omni2.5m-8; $\mathrm{n}=50$ ) at The Centre

92 for Applied Genomics in Toronto according to the manufacturer's protocol. Relevant microarray data are deposited in the Gene Expression Omnibus (https://www.ncbi.nlm.nih.gov/geo/;

94 GSE102650). Quality control and ancestry assessment procedures were as described previously

95 (Gazzellone et al. 2016; Oskoui et al. 2015). Ancestry was determined using PLINK v1.07

96 (Purcell et al. 2007).

$\mathrm{CNV}$ calling was performed as previously described with slight modifications (Oskoui et al. 2015). In brief, three CNV calling algorithms were used for both cases and internal controls: i.e. iPattern (Pinto et al. 2011), PennCNV (Wang et al. 2007), and QuantiSNP (Colella et al. 2007). We then analyzed "stringent" CNVs, those detected by at least two methods. Detecting large CNVs particularly sex chromosomal aneuploidies is challenging (Oskoui et al. 2015). Hence,

102 these large CNVs are sometimes fragmented. If found, they were merged and their identity

103 confirmed by examining the probe density and B allele frequencies in the region.

We identified rare CNVs as those at not more than $0.1 \%$ frequency among 10,851 population 105 control samples (explained in detail in (Zarrei et al. 2017a)), using a 50\% reciprocal overlap strategy (Conrad et al. 2010; Redon et al. 2006). This list was further restricted with those that 
107 108

are not present in the study internal controls, which are age-matched to the cases. We additionally removed those with less than $75 \%$ overlap with copy number stable regions, according to our stringent CNV map of the human genome (Zarrei et al. 2015). We investigated CNVs $>20 \mathrm{~kb}$ and covered by $\geq 5$ consecutive probes (Mosca et al. 2016). Validation of relevant CNVs to FASD was performed by TaqMan Copy Number Assays. The resulting list of all CNVs is shown in Table S1. The genomic coordinates used are based on Human Genome Build GRCh37/hg19.

\section{Searching for CNVs deemed relevant to FASD}

We investigated CNVs impacting coding sequence of our FASD genes or those implicated in other neurodevelopmental disorders (Yuen et al. 2017; Yuen et al. 2016; Yuen et al. 2015). We also examined OMIM genes with neurological function or other functions that may be congruent with FASD physiology. Our FASD gene list comprises the top 30 genes differentially methylated in children with FASD (Portales-Casamar et al. 2016), top-ranked genes identified using binary matrix filtering (Lombard et al. 2007), and genes involved in alcohol metabolism in humans and human homologues of those with established interactions with alcohol in animal models (Eberhart and Parnell 2016). CNVs in regions associated with known genomic disorders including DECIPHER syndrome loci (Firth et al. 2009) and ClinGen loci (Rehm et al. 2015), were also analyzed. All CNV data was also compared to population control data in the Database of Genomic Variants (MacDonald et al. 2014).

\section{Gene-set burden enrichment analysis and burden of pLI scores}

Gene-sets with an a priori association to neuropsychiatric disorders were compiled following methods described elsewhere (Marshall et al. 2017; Merico et al. 2015) (Table S2). We also 
129 included a protein-expression module obtained from our previous publication (Uddin et al.

130 2016). The latter gene-set, which was called the "blue module", is comprised of 2,484 genes.

131 These are highly associated with pathways involved in brain development and functions

132 including synaptic transmission, neuron projection, cell signaling, nervous system development,

133 and axon guidance. A logistic regression model was fitted for each gene-set using the glm

134 function and additional in-house scripts in R (Team 2017). Population stratification based on 135 principal component analysis (PCA) of SNP data extracted using PLINK (Purcell et al. 2007)

136 and sex were used as covariates. The model was corrected by the number of genes impacted by

137 rare $\mathrm{CNVs}$ that larger multigene CNVs did not contribute to the signal more than smaller CNVs.

138 The multiple test correction by Benjamini Hochberg False Discovery Rate (BH-FDR) was done

139 with R function, p.adjust with option method = "BH". Since many of our subjects were related,

140 we analyzed only unrelated subjects (51 cases, 41 controls). Only rare CNVs that perturbed

141 coding sequences of protein coding genes were considered in this analysis.

142 We obtained the probability of truncating loss-of-function intolerance (pLI) (Lek et al. 2016)

143 for genes impacted by deletions in our cases, compared with all 10,851 population controls at the

144 individual level. We also analyzed deletions in our unrelated cases compared with 2,884

145 platform-matched control samples genotyped on Illumina Omni 2.5 platforms obtained from

146 KORA (Cooperative Research in the Region of Augsburg) (Verhoeven et al. 2013) and

147 COGEND (Collaborative Genetic Study of Nicotine Dependence) (Bierut et al. 2007). We

148 compared cases to controls using an unpaired $t$-test between sums of pLI scores in cases

149 compared with controls to evaluate significance in genetic burden as described in (Zarrei et al.

150 2017a; Zarrei et al. 2017b).

\section{Results}


Our study cohort comprises 103 individuals of European ancestry, and 79 from other

153 backgrounds. We detected 225 rare stringent CNVs in proband DNAs (2.4 rare CNVs per

154 individual; Table S1). These range from $20 \mathrm{~kb}$ to a $1.6 \mathrm{Mb}$ deletion in the $3 \mathrm{q} 29$ genomic

155 syndrome locus. Rare deletions were slightly more frequent than rare duplications (56\%

156 deletions, 44\% duplications). Of all rare CNVs, 87 (39\%) impact coding sequences, whereas

$15761 \%$ involved noncoding regions of the genome. CNVs from internal controls are presented in

158 Table S1. These CNVs from internal controls are used as an additional filter besides external

159 controls in identifying rare CNVs in FASD cases; therefore, we are unable to discuss variants in

160 these individuals similar to the FASD cases. The inheritance patterns of the relevant CNVs are

161 unknown since parental samples were not obtained in this study. The reason is that most of the

162 children with FASD were in foster or adoptive homes, and biological parents therefore not

163 available to us. We discovered multiple discrepancies between self-reported sibling-relationships

164 and those inferred from genotypes. We reported here the latter type.

165 Fourteen CNVs with potential relevance to FASD were identified in a total of 12 individuals

166 (Table 1). These CNVs either impact a gene with an established role associated with brain

167 function, are involved in ASD or other neurodevelopmental disorders, or are in regions

168 associated with known genomic disorders (Citta et al. 2013). We describe five cases with notable

169 CNV findings below.

Case 184346 has a $1.6 \mathrm{Mb}$ deletion, which is congruent with $3 \mathrm{q} 29$ microdeletion syndrome.

171 Intellectual disability, cognitive behavioral problems, and mild morphological anomalies have

172 been reported in patients with this syndrome (Citta et al. 2013).

174 (Table 1). The first two genes code for subunits of a voltage-dependent calcium channel 
175 complex. GWAS studies indicate that SNPs in CACNA1C are associated with psychiatric 176 disorders, particularly bipolar disorder (Jakobsson et al. 2016). Variations in CACNA1C have 177 also been reported in Timothy syndrome (OMIM: 601005). This syndrome is characterized by 178 multi-organ dysfunction, including cognitive abnormalities and autism (Splawski et al. 2004). 179 This CNV partially duplicates all known isoforms of both calcium-channel genes. Additionally, 180 two sisters both diagnosed with FASD (187242 and 187243) each carry identical $88.2 \mathrm{~kb}$ 181 duplications impacting the first two exons of $C A C N A 1 H$, another member of the calcium channel 182 complex (Fig. 1). Mutations in this gene have been found to be associated with idiopathic 183 epilepsy (Eckle et al. 2014). In previous studies, we found six duplications impacting these genes in cases of ASD (Pinto 2010; Pinto et al. 2014). This $88.2 \mathrm{~kb}$ duplication also impacts SSTR5, 185 which is a somatostatin receptor. Polymorphisms in this gene were linked to acromegaly 186 (Ciganoka et al. 2011).

Cases 204530 and 204538, both sisters, inferred from genotype data using PLINK and diagnosed with FASD, have identical $181.5 \mathrm{~kb}$ deletions impacting two exons of NCKAP5

189 (Table 1). A GWAS study found a SNP in this gene to be associated with bipolar disorder (Smith 190 et al. 2009). The first sister also carries an $89.8 \mathrm{~kb}$ duplication impacting $S 100 B$ and PRMT2.

191 Variations in $S 100 B$ (S100 calcium binding protein B), which is highly expressed in brain and fat 192 tissues, have been linked with several neurological disorders, including epilepsy (Chen et al. 193 2015).

194 We found eight gene-sets with statistically significant enrichment in either cases or controls $195(\mathrm{p}<0.05$; Table S2). However, only one gene-set reached our multiple test correction threshold of $196 \quad 0.25$. This gene-set is comprised of genes moderately expressed in brain, but with a negative 197 coefficient $(\mathrm{p}=0.0002, \mathrm{FDR}=0.0057$, coefficient $=-2.68)$. The negative coefficient translates to 
198 199

more variants found in controls impacting members of the set compared with those found in cases (eleven CNVs in controls and only one in cases). These variants are shown in Table S2.

Our results show that there is a higher mean for the pLI scores in cases of FASD compared with platform matched controls ( 0.096 vs $0.058 ; p=0.32)$. The difference between these two scores was not significant when we compared our cases with controls genotyped on different platforms $(0.096$ vs $0.103 ; p=0.86)$.

\section{Discussion}

This paper is the first publication of a relatively large diagnosed cohort of FASD individuals (and some prenatal alcohol exposure (PAE)) with CNV analyses, unlike previous studies that are from cohorts of patients referred to a genetics unit with a query diagnosis of FAS or PAE. Our results suggest that FASD can be associated with rare CNVs often impacting important neurodevelopmental genes. Some of the CNV findings are clinically important, and point to other pathologies that contribute to the developmental problems in a few individuals. On this basis alone, our study should be of interest to clinicians hoping to thoroughly assess a diagnosis of FASD and exclude other etiologies.

In particular, we found rare CNVs, both genic and intergenic, in 85/95 (90\%) of cases with FASD. In 48/95 (50\%) of cases, rare CNVs impact coding sequences were found, and in 12/95 (13\%) of cases they carried genic CNVs that might possibly contribute to the aetiology of FASD. These range in size from $20 \mathrm{~kb}$ to a $1.6 \mathrm{Mb}$ deletion affecting the $3 \mathrm{q} 29$ genomic syndrome locus, which was known to be causative for neurodevelopmental disorders (Citta et al. 2013). We also found multiple rare CNVs impacting coding sequences of genes with neurological functions, particularly genes associated with neuropsychiatric disorders, including deletions and 
220 duplications impacting $C A C N A 1 C, C A C N A 2 D 4, C A C N A 1 H$, and $S 100 B$ (Chen et al. 2015; Eckle

221 et al. 2014; Pinto et al. 2014; Pinto et al. 2010; Splawski et al. 2004). Although it is difficult to

222 fully interpret our gene-set enrichment findings because of the negative coefficient finding, a

223 larger cohort may help to resolve specific genetic pathways in FASD. FASD is a multifactorial

224 disorder with numerous mitigating factors influencing development during the pregnancy and

225 effects on mental development in early childhood. Risk factors include older mothers, lower

226 income families, nutritional deficiencies, lower education, lower employment, family discord

227 and experience with abuse or violence, minority groups especially those that have been

228 marginalized, oppressed and/or colonized, a diagnosis later in life and genetic and epigenetic

229 factors (Liyanage et al. 2017). Earlier studies suggested that only a small percentage (4.3\%) of

230 children with a history of heavy prenatal alcohol exposure develop the full Fetal Alcohol

231 Syndrome (FAS) (Abel 1995). However, this figure is based on FAS, which makes up only 10-

$23215 \%$ of cases of FASD, suggesting that the number of children adversely affected by prenatal

233 alcohol exposure may be higher.

234 Our objective was to describe CNV findings in this cohort. Given the nature of the study 235 design, which did not allow for the collection of parental samples, we could not determine the 236 mode of inheritance of the CNVs making the interpretation of the data more challenging. For

237 example, in the clinical setting, de novo CNVs of significant size affecting critical

238 neurodevelopmental genes are often assigned as 'pathogenic' for the ascertained phenotype

239 (Buchanan and Scherer 2008; Firth et al. 2009; Kearney et al. 2011; Lee and Scherer 2010;

240 Rehm et al. 2015).

241 Here, we can only make a descriptive association of CNV to comorbidities, but we postulate

242 that some of the CNV findings in this study may reflect underlying neurodevelopmental 
243 pathology that is in some cases masquerading as FASD. By including family based analysis of

244 CNVs, in our own research and that of others (Oskoui et al. 2015; Segel et al. 2015; Zarrei et al.

245 2017a; Zarrei et al. 2017b), we could begin to resolve the role of CNVs in cerebral palsy (CP),

246 which is another heterogeneous disorder previously thought to arise almost entirely by

247 environmental (non-genetic) events. Resolving the complex nature and often shared features

248 between CP, ASD and other neurodevelopmental disorders, may also help to resolve the genetic

249 susceptibility factors in FASD (Lionel et al. 2011; McGrath et al. 2014). Moreover, microarray

250 platforms have a resolution limitation, $20 \mathrm{~kb}$ in this study, and therefore cannot detect small

251 variants and point mutations (Zarrei et al. 2015). Therefore, whole-genome sequencing, which

252 has potential of detecting CNVs and complex structural variations including smaller

253 chromosomal rearrangements, should be considered for future studies (Yuen et al. 2017).

The British Medical Association has recommended a referral to a clinical geneticist in 255 children with suspected FASD, to consider appropriate genetic testing that might include a 256 microarray analysis (Douzgou et al. 2012). For at least the 12 (of 95, or 13\%) of the participants

257 CNV data was found that would be reported back in the clinical setting, most as variants of 258 unknown significance to FASD. Notwithstanding this ambiguity in reporting language it is quite 259 likely many of these CNVs are contributing to the natural history of general disease in these 260 individuals, and may need to be reported back in genetic counseling.

\section{Acknowledgments}

262 The authors thank the staff at The Centre for Applied Genomics for technical assistance and 263 helpful discussions. This project was supported by grants from the Kids Brain Health Network 264 (formerly NeuroDevNet), the Ontario Brain Institute (funding in part by the Government of 265 Ontario), Genome Canada, Canada Foundation for Innovation, Canadian Institutes of Health 
266 Research (CIHR), Canadian Institute for Advanced Research (CIFAR), the Government of 267 Ontario, Canadian Institutes of Health Research, The Hospital for Sick Children and the 268 University of Toronto McLaughlin Centre, and Manitoba Liquor and Lotteries. SWS holds the 269 GlaxoSmithKline-CIHR Chair in Genome Sciences at The Hospital for Sick Children and the 270 University of Toronto. 


\section{References}

272 Abdelmalik, N., van Haelst, M., Mancini, G., Schrander-Stumpel, C., Marcus-Soekarman, D.,

273 Hennekam, R., et al. 2013. Diagnostic outcomes of 27 children referred by pediatricians to a

274 genetics clinic in the Netherlands with suspicion of fetal alcohol spectrum disorders. Am J Med

275 Genet Part A 161A(2): 254-260. doi: 10.1002/ajmg.a.35672.

276 Abel, E.L. 1988. Fetal alcohol syndrome in families. Neurotoxicol Teratol 10(1): 1-2.

277 Abel, E.L. 1995. An update on incidence of FAS: FAS is not an equal opportunity birth defect.

278 Neurotoxicol Teratol 17(4): 437-443.

279 Bierut, L.J., Madden, P.A., Breslau, N., Johnson, E.O., Hatsukami, D., Pomerleau, O.F., et al.

280 2007. Novel genes identified in a high-density genome wide association study for nicotine

281 dependence. Human molecular genetics 16(1): 24-35. doi: 10.1093/hmg/ddl441.

282 Bingol, N., Fuchs, M., Iosub, S., Kumar, S., Stone, R.K., and Gromisch, D.S. 1987. Fetal alcohol

283 syndrome associated with trisomy 21. Alcohol Clin Exp Res 11(1): 42-44.

284 Buchanan, J.A., and Scherer, S.W. 2008. Contemplating effects of genomic structural variation.

285 Genetics in medicine : official journal of the American College of Medical Genetics 10(9): 639-

286 647. doi: 10.1097GIM.0b013e318183f848.

287 Chasnoff, I.J. 1985. Fetal alcohol syndrome in twin pregnancy. Acta Genet Med Gemellol

288 (Roma) 34(3-4): 229-232.

289 Chen, W., Tan, Y., Ge, Y., Chen, Y., and Liu, X. 2015. The Effects of Levetiracetam on

290 Cerebrospinal Fluid and Plasma NPY and GAL, and on the Components of Stress Response

291 System, hs-CRP, and S100B Protein in Serum of Patients with Refractory Epilepsy. Cell

292 biochemistry and biophysics 73(2): 489-494. doi: 10.1007/s12013-015-0683-8. 
293 Chudley, A.E., Conry, J., Cook, J.L., Loock, C., Rosales, T., LeBlanc, N., et al. 2005. Fetal 294 alcohol spectrum disorder: Canadian guidelines for diagnosis. CMAJ 172(5 Suppl): S1-S21. doi: $295 \quad 10.1503 /$ cmaj.1040302.

296 Ciganoka, D., Balcere, I., Kapa, I., Peculis, R., Valtere, A., Nikitina-Zake, L., et al. 2011.

297 Identification of somatostatin receptor type 5 gene polymorphisms associated with acromegaly. 298 European journal of endocrinology / European Federation of Endocrine Societies 165(4): 517299 525. doi: 10.1530/EJE-11-0416.

300 Citta, S., Buono, S., Greco, D., Barone, C., Alfei, E., Bulgheroni, S., et al. 2013. 3q29

301 microdeletion syndrome: Cognitive and behavioral phenotype in four patients. Am J Med Genet 302 Part A 161A(12): 3018-3022. doi: 10.1002/ajmg.a.36142.

303 Colella, S., Yau, C., Taylor, J.M., Mirza, G., Butler, H., Clouston, P., et al. 2007. QuantiSNP: an 304 Objective Bayes Hidden-Markov Model to detect and accurately map copy number variation 305 using SNP genotyping data. Nucleic acids research 35(6): 2013-2025. doi: 10.1093/nar/gkm076. 306 Conrad, D.F., Pinto, D., Redon, R., Feuk, L., Gokcumen, O., Zhang, Y., et al. 2010. Origins and 307 functional impact of copy number variation in the human genome. Nature 464(7289): 704-712. 308 doi: $10.1038 /$ nature 08516.

309 Douzgou, S., Breen, C., Crow, Y.J., Chandler, K., Metcalfe, K., Jones, E., et al. 2012.

310 Diagnosing fetal alcohol syndrome: new insights from newer genetic technologies. Archives of 311 disease in childhood 97(9): 812-817. doi: 10.1136/archdischild-2012-302125.

312 Eberhart, J.K., and Parnell, S.E. 2016. The Genetics of Fetal Alcohol Spectrum Disorders.

313 Alcohol Clin Exp Res 40(6): 1154-1165. doi: 10.1111/acer.13066. 
314 Eckle, V.S., Shcheglovitov, A., Vitko, I., Dey, D., Yap, C.C., Winckler, B., et al. 2014.

315 Mechanisms by which a CACNA1H mutation in epilepsy patients increases seizure

316 susceptibility. The Journal of physiology 592(4): 795-809. doi: 10.1113/jphysiol.2013.264176.

317 Firth, H.V., Richards, S.M., Bevan, A.P., Clayton, S., Corpas, M., Rajan, D., et al. 2009.

318 DECIPHER: Database of Chromosomal Imbalance and Phenotype in Humans Using Ensembl

319 Resources. American journal of human genetics 84(4): 524-533. doi:

320 10.1016/j.ajhg.2009.03.010.

321 Gazzellone, M.J., Zarrei, M., Burton, C.L., Walker, S., Uddin, M., Shaheen, S.M., et al. 2016.

322 Uncovering obsessive-compulsive disorder risk genes in a pediatric cohort by high-resolution

323 analysis of copy number variation. Journal of neurodevelopmental disorders 8: 36 . doi:

324 10.1186/s11689-016-9170-9.

325 Jakobsson, J., Palsson, E., Sellgren, C., Rydberg, F., Ekman, A., Zetterberg, H., et al. 2016.

326 CACNA1C polymorphism and altered phosphorylation of tau in bipolar disorder. The British

327 journal of psychiatry : the journal of mental science 208(2): 195-196. doi:

328 10.1192/bjp.bp.114.159806.

329 Kearney, H.M., South, S.T., Wolff, D.J., Lamb, A., Hamosh, A., Rao, K.W., et al. 2011.

330 American College of Medical Genetics recommendations for the design and performance

331 expectations for clinical genomic copy number microarrays intended for use in the postnatal

332 setting for detection of constitutional abnormalities. Genetics in medicine : official journal of the

333 American College of Medical Genetics 13(7): 676-679. doi: 10.1097/GIM.0b013e31822272ac.

334 Lange, S., Probst, C., Gmel, G., Rehm, J., Burd, L., and Popova, S. 2017. Global prevalence of

335 fetal alcohol spectrum disorder among children and youth: A systematic review and meta-

336 analysis. JAMA pediatrics. doi: 10.1001/jamapediatrics.2017.1919. 
337 Lee, C., and Scherer, S.W. 2010. The clinical context of copy number variation in the human

338 genome. Expert reviews in molecular medicine 12: e8. doi: 10.1017/S1462399410001390.

339 Lek, M., Karczewski, K.J., Minikel, E.V., Samocha, K.E., Banks, E., Fennell, T., et al. 2016.

340 Analysis of protein-coding genetic variation in 60,706 humans. Nature 536(7616): 285-291. doi:

$341 \quad 10.1038 /$ nature19057.

342 Lionel, A.C., Crosbie, J., Barbosa, N., Goodale, T., Thiruvahindrapuram, B., Rickaby, J., et al.

343 2011. Rare copy number variation discovery and cross-disorder comparisons identify risk genes

344 for ADHD. Science translational medicine 3(95): 95ra75. doi: 10.1126/scitranslmed.3002464.

345 Liyanage, V.R., Curtis, K., Zachariah, R.M., Chudley, A.E., and Rastegar, M. 2017. Overview of 346 the Genetic Basis and Epigenetic Mechanisms that Contribute to FASD Pathobiology. Curr Top

347 Med Chem 17(7): 808-828.

348 Lombard, Z., Tiffin, N., Hofmann, O., Bajic, V.B., Hide, W., and Ramsay, M. 2007.

349 Computational selection and prioritization of candidate genes for fetal alcohol syndrome. BMC 350 genomics 8: 389. doi: 10.1186/1471-2164-8-389.

351 MacDonald, J.R., Ziman, R., Yuen, R.K., Feuk, L., and Scherer, S.W. 2014. The Database of 352 Genomic Variants: a curated collection of structural variation in the human genome. Nucleic 353 acids research 42(Database issue): D986-992. doi: 10.1093/nar/gkt958.

354 Marshall, C.R., Howrigan, D.P., Merico, D., Thiruvahindrapuram, B., Wu, W., Greer, D.S., et al. 355 2017. Contribution of copy number variants to schizophrenia from a genome-wide study of 356 41,321 subjects. Nature genetics 49(1): 27-35. doi: 10.1038/ng.3725.

357 May, P.A., Gossage, J.P., Marais, A.S., Adnams, C.M., Hoyme, H.E., Jones, K.L., et al. 2007.

358 The epidemiology of fetal alcohol syndrome and partial FAS in a South African community.

359 Drug Alcohol Depend 88(2-3): 259-271. doi: 10.1016/j.drugalcdep.2006.11.007. 
360 McCarthy, N., and Eberhart, J.K. 2014. Gene-ethanol interactions underlying fetal alcohol

361 spectrum disorders. Cell Mol Life Sci 71(14): 2699-2706. doi: 10.1007/s00018-014-1578-3.

362 McGrath, L.M., Yu, D., Marshall, C., Davis, L.K., Thiruvahindrapuram, B., Li, B., et al. 2014.

363 Copy number variation in obsessive-compulsive disorder and tourette syndrome: a cross-disorder 364 study. Journal of the American Academy of Child and Adolescent Psychiatry 53(8): 910-919.

365 doi: 10.1016/j.jaac.2014.04.022.

366 Merico, D., Zarrei, M., Costain, G., Ogura, L., Alipanahi, B., Gazzellone, M.J., et al. 2015.

367 Whole-Genome Sequencing Suggests Schizophrenia Risk Mechanisms in Humans with 22q11.2

368 Deletion Syndrome. G3 5(11): 2453-2461. doi: 10.1534/g3.115.021345.

369 Mosca, S.J., Langevin, L.M., Dewey, D., Innes, A.M., Lionel, A.C., Marshall, C.C., et al. 2016.

370 Copy-number variations are enriched for neurodevelopmental genes in children with

371 developmental coordination disorder. Journal of medical genetics 53(12): 812-819. doi:

$372 \quad 10.1136 /$ jmedgenet-2016-103818.

373 Muller, J., Cobet, G., Laske, G., Degen, B., Grauel, C., and Lehmann, K. 1993. [Partial

374 monosomy 21 or fetal alcohol embryopathy in a retarded boy?]. Padiatr Grenzgeb 31(5): 313-

375319.

376 Oskoui, M., Gazzellone, M.J., Thiruvahindrapuram, B., Zarrei, M., Andersen, J., Wei, J., et al.

377 2015. Clinically relevant copy number variations detected in cerebral palsy. Nature

378 communications 6: 7949. doi: 10.1038/ncomms8949.

379 Pinto, D. 2010. Functional impact of global rare copy number variation in autism spectrum 380 disorders. Nature 466: 368-372. 
381 Pinto, D., Darvishi, K., Shi, X., Rajan, D., Rigler, D., Fitzgerald, T., et al. 2011. Comprehensive 382 assessment of array-based platforms and calling algorithms for detection of copy number 383 variants. Nature biotechnology 29(6): 512-520. doi: 10.1038/nbt.1852.

384 Pinto, D., Delaby, E., Merico, D., Barbosa, M., Merikangas, A., Klei, L., et al. 2014.

385 Convergence of genes and cellular pathways dysregulated in autism spectrum disorders.

386 American journal of human genetics 94(5): 677-694. doi: 10.1016/j.ajhg.2014.03.018.

387 Pinto, D., Pagnamenta, A.T., Klei, L., Anney, R., Merico, D., Regan, R., et al. 2010. Functional 388 impact of global rare copy number variation in autism spectrum disorders. Nature 466(7304):

389 368-372. doi: 10.1038/nature09146.

390 Popova, S., Lange, S., Shield, K., Mihic, A., Chudley, A.E., Mukherjee, R.A., et al. 2016.

391 Comorbidity of fetal alcohol spectrum disorder: a systematic review and meta-analysis. Lancet 392 387(10022): 978-987. doi: 10.1016/S0140-6736(15)01345-8.

393 Portales-Casamar, E., Lussier, A.A., Jones, M.J., MacIsaac, J.L., Edgar, R.D., Mah, S.M., et al. 394 2016. DNA methylation signature of human fetal alcohol spectrum disorder. Epigenetics 395 Chromatin 9: 25. doi: 10.1186/s13072-016-0074-4.

396 Purcell, S., Neale, B., Todd-Brown, K., Thomas, L., Ferreira, M.A., Bender, D., et al. 2007.

397 PLINK: a tool set for whole-genome association and population-based linkage analyses.

398 American journal of human genetics 81(3): 559-575. doi: 10.1086/519795.

399 Redon, R., Ishikawa, S., Fitch, K.R., Feuk, L., Perry, G.H., Andrews, T.D., et al. 2006. Global

400 variation in copy number in the human genome. Nature 444(7118): 444-454. doi:

$401 \quad 10.1038 /$ nature05329. 
402 Rehm, H.L., Berg, J.S., Brooks, L.D., Bustamante, C.D., Evans, J.P., Landrum, M.J., et al. 2015.

403 ClinGen--the Clinical Genome Resource. N Engl J Med 372(23): 2235-2242. doi:

404 10.1056/NEJMsr1406261.

405 Reynolds, J.N., Weinberg, J., Clarren, S., Beaulieu, C., Rasmussen, C., Kobor, M., et al. 2011.

406 Fetal alcohol spectrum disorders: gene-environment interactions, predictive biomarkers, and the

407 relationship between structural alterations in the brain and functional outcomes. Seminars in

408 pediatric neurology 18(1): 49-55. doi: 10.1016/j.spen.2011.02.006.

409 Romke, C., Heyne, K., Stewens, J., and Schwinger, E. 1987. Erroneous diagnosis of fetal alcohol

410 syndrome in a patient with ring chromosome 6. European journal of pediatrics 146(4): 443.

411 Segel, R., Ben-Pazi, H., Zeligson, S., Fatal-Valevski, A., Aran, A., Gross-Tsur, V., et al. 2015.

412 Copy number variations in cryptogenic cerebral palsy. Neurology 84(16): 1660-1668. doi:

413 10.1212/WNL.0000000000001494.

414 Smith, E.N., Bloss, C.S., Badner, J.A., Barrett, T., Belmonte, P.L., Berrettini, W., et al. 2009.

415 Genome-wide association study of bipolar disorder in European American and African American

416 individuals. Molecular psychiatry 14(8): 755-763. doi: 10.1038/mp.2009.43.

417 Splawski, I., Timothy, K.W., Sharpe, L.M., Decher, N., Kumar, P., Bloise, R., et al. 2004.

$418 \mathrm{Ca}(\mathrm{V}) 1.2$ calcium channel dysfunction causes a multisystem disorder including arrhythmia and

419 autism. Cell 119(1): 19-31. doi: 10.1016/j.cell.2004.09.011.

420 Stoler, J.M. 1999. Reassessment of patients with the diagnosis of fetal alcohol syndrome.

421 Pediatrics 103(6 Pt 1): 1313-1315.

422 Team, R.D.C. 2017. R: A language and environment for statistical computing. R Foundation for

423 Statistical Computing, Vienna, Austria. 
424 Uddin, M., Pellecchia, G., Thiruvahindrapuram, B., D'Abate, L., Merico, D., Chan, A., et al. 425 2016. Indexing Effects of Copy Number Variation on Genes Involved in Developmental Delay. 426 Scientific reports 6: 28663. doi: 10.1038/srep28663.

427 Verhoeven, V.J., Hysi, P.G., Wojciechowski, R., Fan, Q., Guggenheim, J.A., Hohn, R., et al. 428 2013. Genome-wide meta-analyses of multiancestry cohorts identify multiple new susceptibility 429 loci for refractive error and myopia. Nature genetics 45(3): 314-318. doi: 10.1038/ng.2554.

430 Wang, K., Li, M., Hadley, D., Liu, R., Glessner, J., Grant, S.F., et al. 2007. PennCNV: an

431 integrated hidden Markov model designed for high-resolution copy number variation detection in 432 whole-genome SNP genotyping data. Genome research 17(11): 1665-1674. doi:

$43310.1101 /$ gr.6861907.

434 Weyrauch, D., Schwartz, M., Hart, B., Klug, M.G., and Burd, L. 2017. Comorbid Mental 435 Disorders in Fetal Alcohol Spectrum Disorders: A Systematic Review. Journal of developmental 436 and behavioral pediatrics : JDBP 38(4): 283-291. doi: 10.1097/DBP.0000000000000440.

437 Yuen, R., Merico, D., Bookman, M., J, L.H., Thiruvahindrapuram, B., Patel, R.V., et al. 2017. 438 Whole genome sequencing resource identifies 18 new candidate genes for autism spectrum 439 disorder. Nature neuroscience 20(4): 602-611. doi: 10.1038/nn.4524.

440 Yuen, R.K., Merico, D., Cao, H., Pellecchia, G., Alipanahi, B., Thiruvahindrapuram, B., et al. 441 2016. Genome-wide characteristics of de novo mutations in autism. NPJ Genom Med 1: 160271442 1602710. doi: 10.1038/npjgenmed.2016.27.

443 Yuen, R.K., Thiruvahindrapuram, B., Merico, D., Walker, S., Tammimies, K., Hoang, N., et al.

444 2015. Whole-genome sequencing of quartet families with autism spectrum disorder. Nature 445 medicine 21(2): 185-191. doi: 10.1038/nm.3792. 
446 Zarrei, M., Fehlings, D.L., Mawjee, K., Switzer, L., Thiruvahindrapuram, B., Walker, S., et al.

447 2017a. De novo and rare inherited copy-number variations in the hemiplegic form of cerebral

448 palsy. Genetics in medicine : official journal of the American College of Medical Genetics. doi:

$449 \quad 10.1038 /$ gim.2017.83.

450 Zarrei, M., MacDonald, J.R., Merico, D., and Scherer, S.W. 2015. A copy number variation map

451 of the human genome. Nature reviews. Genetics 16(3): 172-183. doi: 10.1038/nrg3871.

452 Zarrei, M., Merico, D., Kellam, B., Engchuan, W., Scriver, T., Jokhan, R., et al. 2017b. A de

453 novo deletion in a boy with cerebral palsy suggests a refined critical region for the $4 \mathrm{q} 21.22$

454 microdeletion syndrome. Am J Med Genet Part A 173(5): 1287-1293. doi:

$455 \quad$ 10.1002/ajmg.a.38176. 


\section{Figure legend:}

$458 \quad$ Fig 1: Schematic of chromosome 16 with two duplications (blue bars) in two sisters both 459 diagnosed with FASD (187242 and 187243). Other blue bars represent duplications in ASD 460 cases from our previous publications. The red bars for CNV map indicate deletions found in the 461 general population (Zarrei et al. 2015).

462 Supplementary online material:

463 Table S1: Subject information and stringent CNVs in FASD cases and age-matched study 464 controls.

465 Table S2: Gene-set burden enrichment analysis results for case/control analysis in FASD. 


\section{Figure 1}

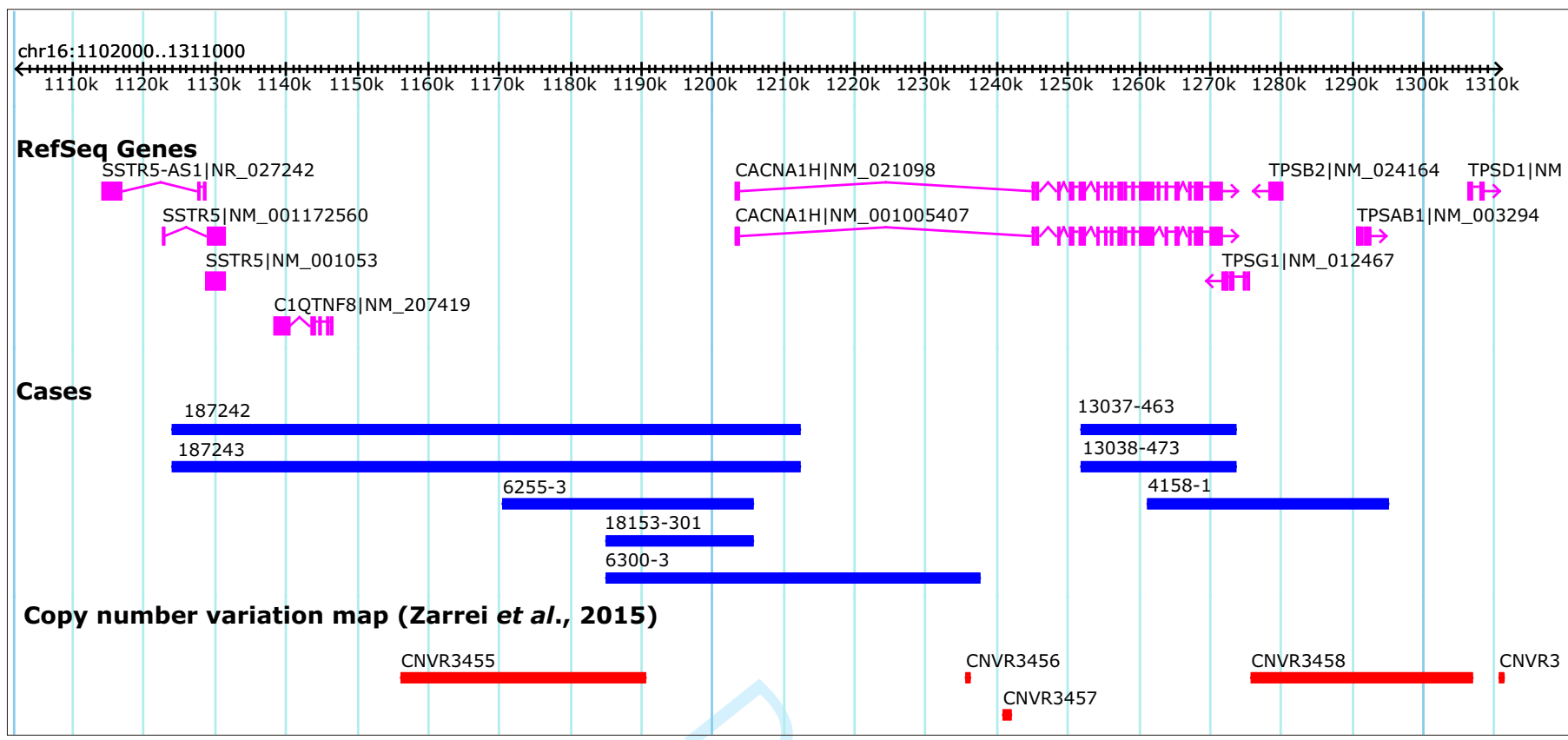


Table 1: Copy number variants of potential significance in 12 children diagnosed with FASD

\begin{tabular}{|c|c|c|c|c|c|c|c|c|}
\hline Case & $\operatorname{sex}$ & chr & start & end & cytoband & type* & size (bp) & genes \\
\hline 184346 & $\mathrm{~F}$ & 3 & $195,725,336$ & $197,346,971$ & $3 q 29$ & del & $1,621,636$ & $\begin{array}{l}\text { PCYT1A, FBXO45, SMCO1, } \\
\text { CEP19, WDR53, NCBP2, DLG1, } \\
\text { SENP5, RNF168, ZDHHC19, } \\
\text { SLC51A, TFRC, NRROS, } \\
\text { TCTEX1D2, PIGX, TM4SF19, } \\
\text { PIGZ, UBXN7, MELTF, PAK2, } \\
\text { BDH1 }\end{array}$ \\
\hline \multirow[t]{2}{*}{184275} & M & 12 & $2,018,149$ & $2,674,893$ & $12 \mathrm{p} 13.33$ & dup & 656,745 & $C A C N A 1 C, D C P 1 B, C A C N A 2 D 4$ \\
\hline & M & 6 & $162,329,287$ & $162,438,621$ & $6 q 26$ & dup & 109,335 & $P R K N$ \\
\hline 184339 & $\mathrm{~F}$ & 15 & $31,963,721$ & $32,515,849$ & $15 q 13.3$ & del & 552,129 & CHRNA7 \\
\hline 204537 & M & 22 & $24,516,291$ & $24,999,249$ & $22 \mathrm{q} 11.23$ & dup & 482,959 & $\begin{array}{l}\text { ADORA2A, SNRPD3, CABIN1, } \\
\text { SPECC1L, LRRC75B, GUCD1, } \\
\text { SUSD2, GGT5, UPB1 }\end{array}$ \\
\hline 184273 & $\mathrm{~F}$ & 2 & $172,239,759$ & $172,512,646$ & $2 \mathrm{q} 31.1$ & dup & 272,888 & METTL8, DCAF17, CYBRD1 \\
\hline $204538^{\wedge}$ & $\mathrm{F}$ & 2 & $133,924,454$ & $134,105,917$ & $2 \mathrm{q} 21.2$ & del & 181,464 & NCKAP5 \\
\hline \multirow[t]{2}{*}{$204530^{\wedge}$} & $\mathrm{F}$ & 2 & $133,924,454$ & $134,105,917$ & $2 \mathrm{q} 21.2$ & del & 181,464 & NCKAP5 \\
\hline & $\mathrm{F}$ & 21 & $48,006,209$ & $48,095,982$ & $21 \mathrm{q} 22.3$ & dup & 89,774 & PRMT2, S100B \\
\hline 187239 & M & 18 & $31,117,401$ & $31,290,424$ & $18 \mathrm{q} 12.1$ & dup & 173,024 & $A S X L 3$ \\
\hline 184341 & $\mathrm{~F}$ & 12 & $80,849,328$ & $80,997,556$ & $12 \mathrm{q} 21.31$ & del & 148,229 & $P T P R Q$ \\
\hline $187242^{\$}$ & $\mathrm{~F}$ & 16 & $1,124,086$ & $1,212,279$ & $16 \mathrm{p} 13.3$ & dup & 88,194 & CACNA1H, CIQTNF8, SSTR5 \\
\hline $187243^{\$}$ & $\mathrm{~F}$ & 16 & $1,124,086$ & $1,212,279$ & $16 \mathrm{p} 13.3$ & dup & 88,194 & CACNA1H, CIQTNF8, SSTR5 \\
\hline 184338 & $\mathrm{~F}$ & 6 & $33,115,024$ & $33,138,955$ & $6 \mathrm{p} 21.32$ & dup & 23,932 & COL11A2 \\
\hline
\end{tabular}

F: female; M: male; del: deletion; dup: duplication; CNV: copy number variant; FASD: fetal alcohol spectrum disorder

*Each CNV validated by TaqMan assay; ^: siblings; ${ }^{\$}$ : siblings 


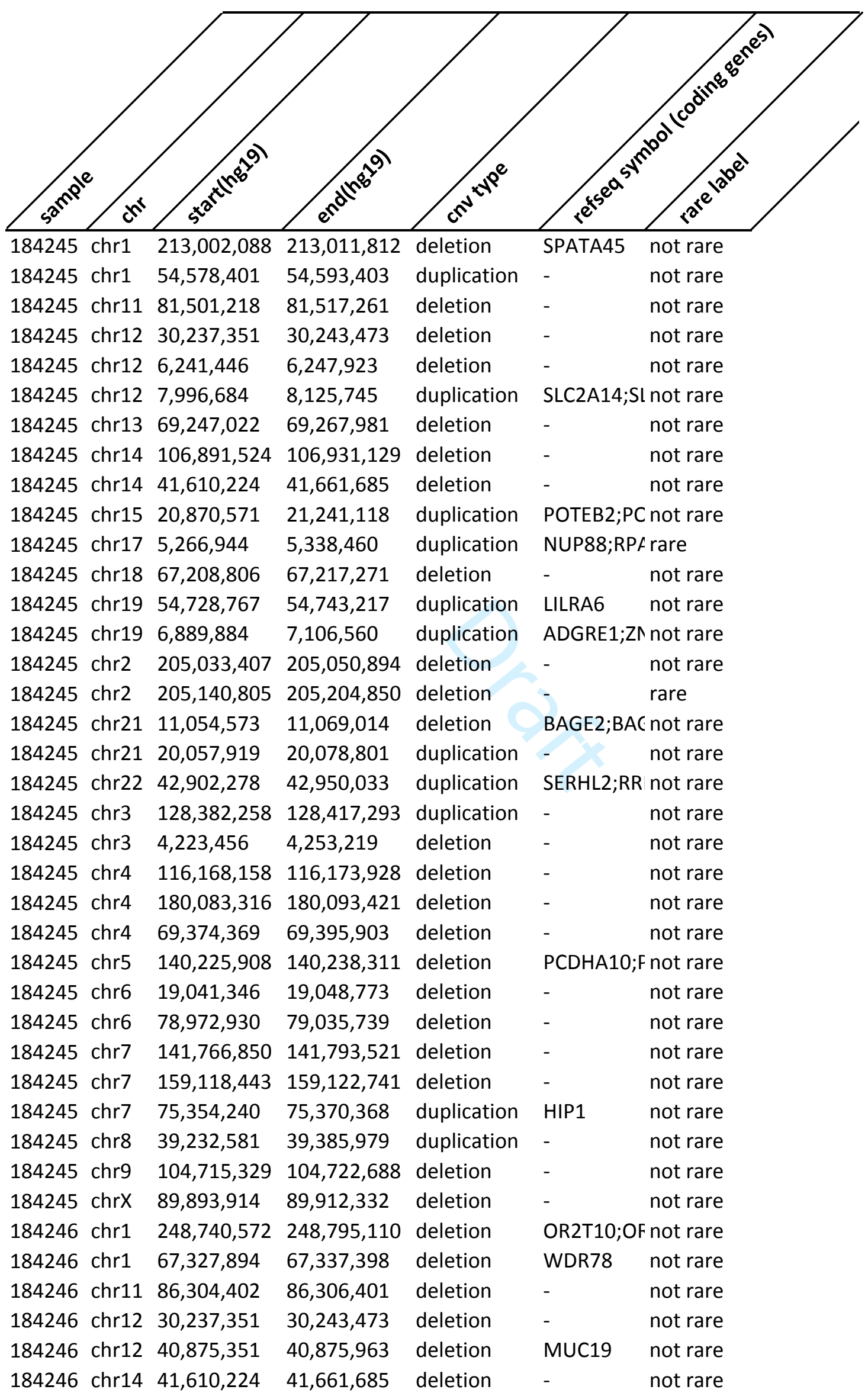




\begin{tabular}{|c|c|c|c|c|c|}
\hline 184246 chr17 & $10,890,859$ & $10,893,799$ & deletion & - & not rare \\
\hline 184246 chr17 & $54,160,465$ & $54,172,668$ & deletion & - & not rare \\
\hline 184246 chr18 & $1,724,943$ & $1,839,339$ & deletion & - & not rare \\
\hline 184246 chr18 & $36,946,222$ & $37,031,162$ & deletion & - & rare \\
\hline $184246 \mathrm{chr} 18$ & $41,976,831$ & $41,982,102$ & deletion & - & not rare \\
\hline 184246 chr18 & $63,889,832$ & $63,910,708$ & deletion & - & rare \\
\hline 184246 chr19 & $43,703,965$ & $43,759,382$ & deletion & \multicolumn{2}{|c|}{ PSG4;PSG9 not rare } \\
\hline $184246 \mathrm{chr} 2$ & $184,085,551$ & $184,088,256$ & deletion & - & not rare \\
\hline $184246 \mathrm{chr} 2$ & $195,979,780$ & $195,982,450$ & deletion & - & not rare \\
\hline $184246 \mathrm{chr} 2$ & $41,239,457$ & $41,248,468$ & deletion & - & not rare \\
\hline 184246 chr2 & $89,540,620$ & $90,275,455$ & duplication & - & not rare \\
\hline 184246 chr3 & $69,858,081$ & $69,870,775$ & deletion & - & not rare \\
\hline $184246 \mathrm{chr} 4$ & $64,136,609$ & $64,154,477$ & deletion & - & not rare \\
\hline 184246 chr5 & $117,388,410$ & $117,393,058$ & deletion & - & t rare \\
\hline 184246 chr5 & $70,305,696$ & $70,308,251$ & deletion & NAIP & not rare \\
\hline 184246 chr6 & $124,432,882$ & $124,470,796$ & duplication & NKAIN2 & not rare \\
\hline 184246 chr6 & $151,507,355$ & $151,509,176$ & deletion & - & not rare \\
\hline 184246 chr6 & $29,855,945$ & $29,899,189$ & deletion & - & not rare \\
\hline 184246 chr6 & $31,338,528$ & $31,341,357$ & deletion & - & are \\
\hline 184246 chr6 & $78,972,930$ & $79,035,739$ & deletion & - & not rare \\
\hline $184246 \mathrm{chr} 7$ & $141,766,850$ & $141,793,521$ & deletion & - & not rare \\
\hline 184246 chr7 & $156,387,296$ & $156,394,307$ & del & - & not rare \\
\hline 184246 chr7 & 41,421 & 68,920 & del & & rare \\
\hline 184246 chr8 & $40,184,872$ & $40,189,688$ & dele & & not rare \\
\hline 184246 chr9 & $24,502,737$ & $24,518,795$ & deletion & & not rare \\
\hline 184248 chr1 & $106,685,410$ & $106,750,803$ & duplication & - & rare \\
\hline 184248 chr10 & $38,792,512$ & $38,903,858$ & deletion & - & not rare \\
\hline 184248 chr10 & $56,449,714$ & $56,468,486$ & duplication & - & not rare \\
\hline 184248 chr11 & $81,501,218$ & $81,517,261$ & deletion & - & not rare \\
\hline 184248 chr14 & $106,891,524$ & $106,913,843$ & deletion & - & not rare \\
\hline 184248 chr14 & $41,610,224$ & $41,661,685$ & deletion & - & not rare \\
\hline 184248 chr16 & $19,945,540$ & $19,962,636$ & deletion & - & not rare \\
\hline 184248 chr16 & $28,614,734$ & $28,620,752$ & deletion & $1 \mathrm{~A} 1$ & not rare \\
\hline 184248 chr18 & $66,746,208$ & $66,755,736$ & deletion & - & not rare \\
\hline 184248 & $67,208,806$ & $67,217,271$ & deletion & - & not rare \\
\hline 184248 chr19 & $43,703,965$ & $43,759,382$ & deletion & \multicolumn{2}{|c|}{ PSG4;PSG9 not rare } \\
\hline $184248 \mathrm{chr} 2$ & $123,477,465$ & $123,482,301$ & deletion & - & not rare \\
\hline 184248 chr2 & $150,747,039$ & $150,756,064$ & deletion & - & not rare \\
\hline 184248 chr2 & $238,554,352$ & $238,558,478$ & deletion & - & not rare \\
\hline 184248 chr2 & $242,953,337$ & $243,068,869$ & deletion & - & not rare \\
\hline 184248 chr2 & $38,956,947$ & $38,971,623$ & duplication & GALM & not rare \\
\hline 184248 chr2 & $51,926,599$ & $51,926,904$ & deletion & - & not rare \\
\hline 184248 chr2 & $87,405,001$ & $87,821,567$ & duplication & - & not rare \\
\hline 184248 chr21 & $20,057,919$ & $20,078,801$ & duplication & - & not rare \\
\hline 184248 chr3 & $129,769,827$ & $129,806,304$ & deletion & ALG1L2 & not rare \\
\hline $184248 \mathrm{chr} 3$ & $191,062,751$ & $191,070,300$ & deletion & - & not rare \\
\hline 184248 chr3 & $195,449,715$ & $195,475,799$ & duplication & \multicolumn{2}{|c|}{ MUC20;MI not rare } \\
\hline
\end{tabular}




\begin{tabular}{|c|c|c|c|c|c|}
\hline $184248 \mathrm{chr} 3$ & $197,844,320$ & $197,846,134$ & deletion & - & not rare \\
\hline 184248 chr3 & $53,032,639$ & $53,038,786$ & deletion & - & not rare \\
\hline 84248 chr4 & $138,092,298$ & $138,098,601$ & deletion & - & not rare \\
\hline $184248 \mathrm{chr} 4$ & $152,991,094$ & $152,993,620$ & deletion & - & not rare \\
\hline $184248 \mathrm{chr} 4$ & $22,048,637$ & $22,070,701$ & deletion & - & not rare \\
\hline $184248 \mathrm{chr} 4$ & $34,785,955$ & $34,818,502$ & deletion & - & ot rare \\
\hline $184248 \mathrm{chr} 4$ & $64,136,609$ & $64,154,477$ & deletion & - & not rare \\
\hline $184248 \mathrm{chr} 4$ & $69,374,369$ & $69,489,473$ & deletion & UGT2B17 & not rare \\
\hline 184248 chr5 & $180,175,886$ & $180,195,526$ & duplication & - & not rare \\
\hline 184248 chr6 & $139,603,393$ & $139,606,731$ & deletion & - & not rare \\
\hline 184248 chr6 & $168,334,239$ & $168,597,816$ & duplication & \multicolumn{2}{|c|}{ MLLT4;KIFð_ not rare } \\
\hline 184248 chr7 & $13,022,810$ & $13,028,215$ & deletion & - & not rare \\
\hline 184248 chr7 & $75,354,240$ & $75,370,368$ & duplication & HIP1 & not rare \\
\hline 184248 chr8 & $115,634,278$ & $115,650,409$ & deletion & - & not rare \\
\hline 184248 chr8 & $39,232,581$ & $39,385,979$ & duplication & - & not rare \\
\hline 184248 chr9 & $24,146,203$ & $24,150,034$ & deletion & - & not rare \\
\hline 184248 chr9 & $6,701,130$ & $6,707,794$ & deletion & - & not rare \\
\hline 184248 chr9 & $69,836,485$ & $69,992,206$ & duplication & - & not rare \\
\hline $184250 \mathrm{chr} 1$ & $149,032,299$ & $149,233,182$ & del & - & are \\
\hline $184250 \mathrm{chr} 1$ & $245,636,900$ & $245,647,809$ & deletion & - & not rare \\
\hline $184250 \mathrm{chr} 1$ & $25,598,276$ & $25,659,509$ & deletion & RHD & not rare \\
\hline 184250 chr10 & $81,788,104$ & $81,791,683$ & deletion & - & not rare \\
\hline 184250 chr11 & $18,949,220$ & $18,961,743$ & duplication & MRGPRX1 & not rare \\
\hline 184250 chr11 & $90,193,796$ & $90,195,742$ & deletion & 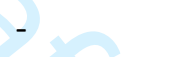 & not rare \\
\hline 184250 chr12 & $11,220,827$ & $11,244,117$ & deletion & TAS2R43 & not rare \\
\hline 184250 chr12 & $7,996,684$ & $8,125,745$ & duplication & \multicolumn{2}{|c|}{ SLC2A14;SI not rare } \\
\hline 184250 & $69,247,022$ & $69,267,981$ & deletion & - & are \\
\hline 184250 chr14 & $41,610,224$ & $41,661,685$ & deletion & - & not rare \\
\hline 184250 chr14 & $44,040,854$ & $44,088,427$ & deletion & - & rare \\
\hline 184250 chr14 & $86,457,952$ & $86,489,093$ & deletion & - & not rare \\
\hline 184250 chr15 & $97,815,182$ & $97,831,832$ & deletion & - & not rare \\
\hline 184250 chr16 & $34,482,158$ & $34,756,258$ & duplication & 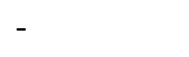 & not rare \\
\hline 184250 chr17 & $33,681,335$ & $33,768,284$ & deletion & \multicolumn{2}{|c|}{ SLFN11;SLF not rare } \\
\hline 184250 chr17 & $34,438,753$ & $34,483,342$ & duplication & - & not rare \\
\hline 184250 chr17 & $54,160,465$ & $54,172,668$ & deletion & - & not rare \\
\hline 184250 chr17 & $7,264,717$ & $7,265,681$ & deletion & - & not rare \\
\hline 184250 chr19 & $54,728,767$ & $54,743,217$ & duplication & LILRA6 & not rare \\
\hline $184250 \mathrm{chr} 2$ & $38,956,947$ & $38,971,623$ & duplication & GALM & not rare \\
\hline $184250 \mathrm{chr} 2$ & $51,925,954$ & $51,926,904$ & deletion & - & not rare \\
\hline $184250 \mathrm{chr} 2$ & $81,518,453$ & $81,558,942$ & deletion & - & not rare \\
\hline 184250 chr22 & $42,902,278$ & $42,950,033$ & duplication & \multicolumn{2}{|c|}{ SERHL2;RR not rare } \\
\hline $184250 \mathrm{chr} 3$ & $131,711,896$ & $131,712,898$ & deletion & - & not rare \\
\hline $184250 \mathrm{chr} 3$ & $175,887,314$ & $175,909,890$ & deletion & - & not rare \\
\hline 184250 chr3 & $192,776,867$ & $192,782,294$ & deletion & - & not rare \\
\hline $184250 \mathrm{chr} 3$ & $75,428,675$ & $75,639,184$ & deletion & - & not rare \\
\hline $184250 \mathrm{chr} 4$ & $152,790,580$ & $152,793,891$ & deletion & - & not rare \\
\hline $184250 \mathrm{chr} 4$ & $190,194,200$ & $190,200,463$ & deletion & - & not rare \\
\hline
\end{tabular}




\begin{tabular}{|c|c|c|c|c|c|}
\hline $184250 \mathrm{chr} 4$ & $29,927,916$ & $29,938,283$ & deletion & - & not rare \\
\hline $184250 \mathrm{chr} 5$ & $140,225,908$ & $140,238,311$ & deletion & \multicolumn{2}{|c|}{ PCDHA10; F not rare } \\
\hline 84250 chr6 & $110,396,540$ & $110,412,756$ & deletion & - & not rare \\
\hline 184250 chr6 & $150,512,533$ & $150,517,701$ & deletion & - & not rare \\
\hline 184250 chr6 & $26,344,333$ & $26,351,349$ & deletion & - & not rare \\
\hline 184250 chr6 & $55,471,178$ & $55,474,701$ & deletion & - & not rare \\
\hline 184250 chr6 & $69,231,883$ & $69,241,954$ & duplication & - & not rare \\
\hline 184250 chr6 & $78,985,226$ & $79,009,742$ & duplication & - & not rare \\
\hline 184250 chr7 & $3,611,608$ & $3,618,143$ & deletion & - & not rare \\
\hline 184250 chr7 & $97,396,709$ & $97,401,677$ & deletion & - & not rare \\
\hline 184250 chr8 & $39,232,581$ & $39,385,979$ & deletion & - & not rare \\
\hline $184250 \mathrm{chr} 8$ & $83,270,172$ & $83,293,872$ & deletion & - & not rare \\
\hline 184250 chr9 & $104,715,329$ & $104,722,688$ & deletion & - & not rare \\
\hline 184250 chr9 & $135,947,165$ & $135,957,452$ & duplication & - & not rare \\
\hline 184250 chr9 & $6,701,130$ & $6,707,794$ & deletion & - & not rare \\
\hline 184250 chr9 & $78,004,378$ & $78,011,616$ & deletion & - & not rare \\
\hline 184251 chr1 & $12,908,591$ & $12,919,928$ & deletion & PRAMEF2 & not rare \\
\hline $184251 \mathrm{chr} 1$ & $17,022,921$ & $17,252,392$ & duplication & \multicolumn{2}{|c|}{ FAM231A; f not rare } \\
\hline $184251 \mathrm{chr} 1$ & $213,002,088$ & $213,011,812$ & deletion & SPATA45 & not rare \\
\hline 184251 chr10 & $47,032,813$ & $47,703,946$ & duplication & \multicolumn{2}{|c|}{ ANTXRL;NF not rare } \\
\hline 184251 chr11 & $125,075,915$ & $125,084,742$ & deletion & - & not rare \\
\hline 184251 chr11 & $85,263,724$ & $85,271,049$ & dele & - & not rare \\
\hline 184251 chr11 & $90,193,427$ & $90,195,742$ & del & & not rare \\
\hline 184251 chr12 & $43,021,431$ & $43,027,453$ & & & rare \\
\hline 184251 chr12 & $59,935,926$ & $59,942,122$ & dele & & not rare \\
\hline 184251 chr12 & $96,285,847$ & $96,293,058$ & deletion & CCDC38 & not rare \\
\hline 184251 chr13 & $50,590,260$ & $50,594,836$ & deletion & \multicolumn{2}{|c|}{ TRIM13;KC not rare } \\
\hline 184251 chr13 & $69,247,022$ & $69,267,981$ & deletion & - & not rare \\
\hline 184251 chr13 & $98,530,124$ & $98,532,757$ & deletion & - & not rare \\
\hline 184251 chr15 & $20,196,661$ & $21,241,118$ & duplication & \multicolumn{2}{|c|}{ GOLGA6L6; not rare } \\
\hline 184251 chr15 & $97,815,182$ & $97,831,832$ & deletion & - & not rare \\
\hline 184251 chr17 & $65,438,501$ & $65,442,685$ & deletion & - & not rare \\
\hline 184251 chr18 & $63,200,484$ & $63,206,335$ & duplication & - & not rare \\
\hline 184251 chr18 & $76,157,694$ & $76,160,278$ & deletion & - & not rare \\
\hline 184251 chr19 & $53,731,394$ & $53,733,101$ & ion & - & not rare \\
\hline 184251 chr19 & $54,728,767$ & $54,743,217$ & duplication & LIL & not rare \\
\hline 184251 chr2 & $123,477,465$ & $123,482,301$ & deletion & - & not rare \\
\hline $184251 \mathrm{chr} 2$ & $181,942,795$ & $182,076,305$ & deletion & - & rare \\
\hline $184251 \mathrm{chr} 2$ & $202,739,991$ & $202,742,455$ & duplication & - & not rare \\
\hline 184251 chr2 & $233,214,406$ & $233,219,891$ & deletion & - & not rare \\
\hline $184251 \mathrm{chr} 2$ & $41,239,457$ & $41,248,468$ & deletion & - & not rare \\
\hline 184251 chr2 & $4,212,725$ & $4,222,144$ & deletion & - & not rare \\
\hline 184251 chr20 & $14,677,564$ & $14,765,094$ & deletion & - & not rare \\
\hline 184251 chr3 & $37,979,882$ & $37,986,734$ & deletion & - & not rare \\
\hline $184251 \mathrm{chr} 3$ & $46,798,133$ & $46,845,618$ & deletion & - & not rare \\
\hline 184251 chr3 & $53,032,639$ & $53,038,786$ & deletion & - & not rare \\
\hline 184251 chr3 & $89,954,229$ & $90,153,262$ & duplication & - & rare \\
\hline
\end{tabular}




\begin{tabular}{|c|c|c|c|c|c|}
\hline $184251 \mathrm{chr} 4$ & $122,285,263$ & $122,289,863$ & deletion & - & not rare \\
\hline $184251 \mathrm{chr} 4$ & $69,374,369$ & $69,467,200$ & deletion & UGT2B17 & not rare \\
\hline 84251 chr4 & $70,137,897$ & $70,229,260$ & deletion & UGT2B28 & not rare \\
\hline $84251 \mathrm{chr} 5$ & $111,937,859$ & $111,944,746$ & deletion & - & not rare \\
\hline 184251 chr5 & $114,726,913$ & $114,729,143$ & deletion & - & not rare \\
\hline 84251 chr6 & $78,972,930$ & $79,035,739$ & deletion & - & ot rare \\
\hline 84251 chr7 & $141,766,850$ & $141,793,521$ & deletion & - & not rare \\
\hline $184251 \mathrm{chr} 7$ & 149,765 & 159,207 & deletion & - & not rare \\
\hline 184251 chr7 & $62,149,006$ & $62,162,385$ & deletion & - & not rare \\
\hline $184251 \mathrm{chr} 8$ & $10,303,265$ & $10,309,373$ & deletion & - & not rare \\
\hline 184251 chr8 & $36,070,428$ & $36,076,949$ & deletion & - & not rare \\
\hline 184251 chr8 & $39,232,581$ & $39,385,979$ & duplication & - & not rare \\
\hline 184251 chr9 & $44,779,633$ & $44,834,316$ & deletion & - & not rare \\
\hline 184251 chr9 & $8,009,428$ & $8,015,596$ & deletion & - & not rare \\
\hline $184251 \mathrm{chrX}$ & $47,881,362$ & $47,976,174$ & deletion & ZNF630 & not rare \\
\hline 184252 chr1 & $104,153,766$ & $104,211,046$ & deletion & \multicolumn{2}{|c|}{ AMY1A;AN not rare } \\
\hline $184252 \mathrm{chr} 1$ & $12,908,591$ & $12,919,928$ & deletion & PRAMEF2 & not rare \\
\hline 184252 chr1 & $155,630,752$ & $155,735,012$ & deletion & \multicolumn{2}{|c|}{ DAP3;YY1A rare } \\
\hline 184252 chr1 & $16,095,104$ & $16,116,197$ & duplication & & rare \\
\hline 184252 chr10 & $4,528,276$ & $4,553,643$ & deletion & - & rare \\
\hline 184252 chr11 & $115,844,365$ & $115,872,716$ & deletion & - & rare \\
\hline 184252 chr11 & $55,365,761$ & $55,424,207$ & deletion & \multicolumn{2}{|c|}{$\mathrm{OR} 4 \mathrm{P} 4 ; \mathrm{OR} \angle$ not rare } \\
\hline 184252 chr11 & $81,501,218$ & $81,517,261$ & del & & not rare \\
\hline 184252 chr11 & $93,020,527$ & $93,028,940$ & & 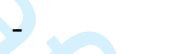 & not rare \\
\hline 184252 chr12 & $27,647,382$ & $27,655,163$ & deletion & SMCO2 & not rare \\
\hline 184252 chr12 & $6,241,446$ & $6,247,923$ & deletion & - & not rare \\
\hline 184252 & 4,315 & $100,008,773$ & & - & not rare \\
\hline 184252 chr16 & $1,111,302$ & $1,147,810$ & deletion & \multicolumn{2}{|c|}{ C1QTNF8;S rare } \\
\hline 184252 chr16 & $19,945,540$ & $19,962,636$ & deletion & - & not rare \\
\hline 184252 chr16 & $24,467,771$ & $24,479,830$ & duplication & - & not rare \\
\hline 184252 chr16 & $70,183,624$ & $70,196,139$ & duplication & PDPR & not rare \\
\hline 184252 chr16 & $72,246,084$ & $72,252,752$ & deletion & 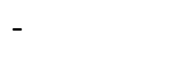 & not rare \\
\hline 184252 chr17 & $34,438,753$ & $34,534,918$ & duplication & \multicolumn{2}{|c|}{ CCL3L3;CCI not rare } \\
\hline 184252 chr19 & $43,214,714$ & $43,378,265$ & duplication & \multicolumn{2}{|c|}{ PSG3;PSG8 not rare } \\
\hline 184252 chr19 & $53,518,747$ & $53,548,950$ & duplication & & not rare \\
\hline 184252 chr2 & $195,742,629$ & $195,748,022$ & deletion & - & not rare \\
\hline 184252 chr2 & $41,239,457$ & $41,248,468$ & deletion & - & not rare \\
\hline 184252 chr2 & $52,759,890$ & $52,784,952$ & deletion & - & not rare \\
\hline 184252 chr2 & $79,752,148$ & $79,765,482$ & deletion & - & not rare \\
\hline 184252 chr2 & $87,392,157$ & $87,997,476$ & deletion & - & not rare \\
\hline 184252 chr22 & $39,359,885$ & $39,385,663$ & deletion & \multicolumn{2}{|c|}{ APOBEC3B not rare } \\
\hline $184252 \mathrm{chr} 3$ & $119,352,669$ & $119,353,893$ & deletion & - & not rare \\
\hline 184252 chr3 & $131,711,896$ & $131,712,898$ & deletion & - & not rare \\
\hline 184252 chr3 & $195,433,728$ & $195,468,690$ & duplication & MUC20 & not rare \\
\hline 184252 chr3 & $197,844,320$ & $197,846,134$ & deletion & - & not rare \\
\hline 184252 chr3 & $65,117,947$ & $65,118,762$ & deletion & - & not rare \\
\hline $184252 \mathrm{chr} 4$ & $190,194,200$ & $190,200,463$ & deletion & - & not rare \\
\hline
\end{tabular}




\begin{tabular}{|c|c|c|c|c|c|}
\hline $184252 \mathrm{chr} 4$ & $60,325,010$ & $60,330,880$ & duplication & - & not rare \\
\hline $184252 \mathrm{chr} 4$ & $70,137,897$ & $70,229,260$ & deletion & UGT2B28 & not rare \\
\hline 84252 chr4 & $7,182,916$ & $7,187,397$ & deletion & - & not rare \\
\hline 184252 chr5 & $103,443,017$ & $103,452,929$ & deletion & - & not rare \\
\hline 184252 chr5 & $155,477,866$ & $155,488,917$ & deletion & - & not rare \\
\hline 184252 chr6 & $78,972,930$ & $79,035,739$ & deletion & - & ot rare \\
\hline 184252 chr7 & $141,766,850$ & $141,793,521$ & deletion & - & not rare \\
\hline $184252 \mathrm{chr} 7$ & $38,388,889$ & $38,397,322$ & deletion & - & not rare \\
\hline 184252 chr7 & 41,421 & 73,060 & duplication & - & not rare \\
\hline 184252 chr8 & $115,632,593$ & $115,651,344$ & deletion & - & not rare \\
\hline 184252 chr8 & $144,942,305$ & $144,948,861$ & deletion & EPPK1 & not rare \\
\hline 184252 chr8 & $145,001,031$ & $145,018,354$ & deletion & PLEC & not rare \\
\hline 184252 chr9 & $13,798,727$ & $13,804,612$ & deletion & - & not rare \\
\hline 184252 chr9 & $68,307,449$ & $68,431,416$ & duplication & - & not rare \\
\hline 184252 chr9 & $73,766,471$ & $73,771,616$ & deletion & - & not rare \\
\hline 184254 chr1 & $104,153,766$ & $104,161,227$ & deletion & AMY2A & not rare \\
\hline $184254 \mathrm{chr} 1$ & $12,908,591$ & $12,919,928$ & deletion & PRAMEF2 & not rare \\
\hline 184254 chr1 & $72,754,314$ & $72,763,324$ & deletion & - & not rare \\
\hline 184254 chr11 & $134,602,333$ & $134,607,484$ & & - & are \\
\hline 184254 chr11 & $18,949,220$ & $18,961,743$ & duplication & MRGPRX1 & not rare \\
\hline 184254 chr11 & $55,365,761$ & $55,427,700$ & deletion & OR4P4;OR< & rare \\
\hline 184254 chr11 & $86,304,402$ & $86,306,401$ & deletion & - & not rare \\
\hline 184254 chr11 & $90,193,796$ & $90,196,843$ & del & & not rare \\
\hline 184254 chr12 & $16,537,026$ & $16,540,305$ & & - & not rare \\
\hline 184254 chr12 & $27,648,411$ & $27,657,025$ & deletion & SMC & not rare \\
\hline 184254 chr12 & $43,021,431$ & $43,027,453$ & deletion & - & not rare \\
\hline 184254 & 38,0 & 38,085 & del & - & \\
\hline 184254 chr16 & $19,945,540$ & $19,962,636$ & deletion & - & not rare \\
\hline 184254 chr16 & $22,552,780$ & $22,703,741$ & duplication & - & not rare \\
\hline 184254 chr16 & $24,467,771$ & $24,479,830$ & duplication & - & not rare \\
\hline 184254 chr16 & $5,109,651$ & $5,110,738$ & deletion & C16orf89 & not rare \\
\hline 184254 chr16 & $70,183,624$ & $70,196,139$ & duplication & PDPR & not rare \\
\hline 184254 chr17 & $34,438,753$ & $34,534,918$ & duplication & CCL3L3;CCl I & not rare \\
\hline 184254 chr2 & $129,638,609$ & $129,645,321$ & deletion & - & not rare \\
\hline 184254 chr2 & $14,704,369$ & $14,709,611$ & deletion & - & not rare \\
\hline 184254 chr2 & $195,742,629$ & $195,748,022$ & deletion & - & not rare \\
\hline 184254 chr2 & $195,979,780$ & $195,982,450$ & deletion & - & not rare \\
\hline 184254 chr2 & $41,239,457$ & $41,248,468$ & deletion & - & not rare \\
\hline 184254 chr2 & $52,759,890$ & $52,784,952$ & deletion & - & not rare \\
\hline 184254 chr2 & $79,752,148$ & $79,761,222$ & deletion & - & not rare \\
\hline 184254 chr22 & $39,359,885$ & $39,385,663$ & deletion & АРОВЕСЗВ & not rare \\
\hline $184254 \mathrm{chr} 3$ & $119,352,669$ & $119,353,893$ & deletion & - & not rare \\
\hline 184254 chr3 & $195,457,853$ & $195,468,690$ & duplication & $\mathrm{Ml}$ & not rare \\
\hline 184254 chr4 & $152,989,386$ & $152,993,620$ & deletion & - & not rare \\
\hline 184254 chr4 & $60,325,010$ & $60,330,880$ & duplication & - & not rare \\
\hline 184254 & $70,137,897$ & $70,229,260$ & deletion & UGT2B28 & not rare \\
\hline 184254 chr5 & $103,443,017$ & $103,452,929$ & deletion & - & not rare \\
\hline
\end{tabular}




\begin{tabular}{|c|c|c|c|c|c|}
\hline 184254 chr6 & $29,856,693$ & $29,899,189$ & deletion & - & not rare \\
\hline 184254 chr6 & $31,338,528$ & $31,341,357$ & deletion & - & not rare \\
\hline 84254 chr6 & $78,972,930$ & $79,035,739$ & deletion & - & ot rare \\
\hline 184254 chr7 & $16,877,244$ & $16,882,780$ & deletion & - & not rare \\
\hline 184254 chr8 & $115,633,704$ & $115,642,459$ & deletion & - & not rare \\
\hline 184254 chr8 & $39,232,581$ & $39,385,979$ & duplication & - & are \\
\hline 184254 chr8 & $5,595,511$ & $5,605,706$ & deletion & - & not rare \\
\hline 184254 chr8 & $72,214,753$ & $72,217,690$ & deletion & - & not rare \\
\hline 184255 chr1 & $112,693,423$ & $112,704,581$ & deletion & - & not rare \\
\hline 184255 chr1 & $12,850,282$ & $12,998,343$ & duplication & HNRNPCL1 & not rare \\
\hline 184255 chr1 & $149,022,110$ & $149,432,892$ & deletion & - & not rare \\
\hline 184255 chr1 & $248,740,572$ & $248,795,110$ & deletion & OR2T10;OF & not rare \\
\hline 184255 chr10 & $20,790,926$ & $20,814,583$ & duplication & - & not rare \\
\hline 184255 chr12 & $52,688,766$ & $52,782,141$ & duplication & $\mathrm{KR}$ & not rare \\
\hline 184255 chr13 & $64,223,907$ & $64,236,288$ & deletion & - & not rare \\
\hline 184255 chr14 & $31,430,261$ & $31,458,519$ & deletion & - & rare \\
\hline 184255 chr14 & $73,527,253$ & $73,530,678$ & deletion & - & not rare \\
\hline 184255 chr15 & $21,903,815$ & $22,753,733$ & duplication & A6L2: & not rare \\
\hline 184255 chr15 & $24,361,858$ & $24,496,990$ & ation & - & rare \\
\hline 184255 chr16 & $19,945,540$ & $19,962,636$ & deletion & - & not rare \\
\hline 184255 chr16 & $33,519,862$ & $33,590,959$ & deletion & - & not rare \\
\hline 184255 chr16 & $70,183,624$ & $70,196,139$ & duplication & & not rare \\
\hline 184255 chr17 & $34,438,753$ & $34,534,918$ & duplication & $\mathrm{L} 3 ; \mathrm{CCl}$ & not rare \\
\hline 184255 chr17 & $44,177,365$ & $44,213,925$ & duplication & & not rare \\
\hline 184255 chr17 & $77,365,534$ & $77,392,062$ & dup & & not rare \\
\hline 184255 chr2 & $208,351,315$ & $208,357,838$ & deletion & 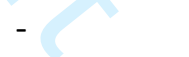 & not rare \\
\hline 184255 chr2 & $224,804,043$ & $224,814,186$ & & & \\
\hline 184255 chr22 & $37,745,101$ & $37,749,946$ & deletion & - & not rare \\
\hline 184255 chr22 & $42,906,877$ & $42,950,033$ & duplication & IL2;RR & not rare \\
\hline 184255 chr3 & $129,769,827$ & $129,806,304$ & deletion & ALG1L2 & not rare \\
\hline 184255 chr3 & $195,457,853$ & $195,468,690$ & duplication & MUC20 & not rare \\
\hline $184255 \mathrm{chr} 4$ & $34,785,955$ & $34,818,502$ & deletion & - & not rare \\
\hline 184255 chr5 & $112,254,377$ & $112,289,462$ & deletion & RE & not rare \\
\hline 184255 chr5 & $177,390,271$ & $177,400,704$ & deletion & - & not rare \\
\hline 184255 chr5 & $58,237,234$ & $58,242,966$ & deletion & - & not rare \\
\hline 184255 chr6 & $124,432,882$ & $124,470,796$ & duplication & NK & not rare \\
\hline 184255 chr6 & $29,916,391$ & $29,922,720$ & deletion & - & not rare \\
\hline 184255 chr6 & $78,972,930$ & $79,035,739$ & deletion & - & not rare \\
\hline 184255 chr7 & $141,766,850$ & $141,793,521$ & deletion & - & not rare \\
\hline 184255 chr7 & $142,476,094$ & $142,484,042$ & deletion & - & not rare \\
\hline 184255 chr7 & $62,149,006$ & $62,162,385$ & deletion & - & not rare \\
\hline 184255 chr7 & $70,321,643$ & $70,364,102$ & duplication & - & not rare \\
\hline 184255 chr7 & $80,635,149$ & $80,680,149$ & deletion & - & rare \\
\hline 184255 chr8 & $104,367,673$ & $104,876,026$ & duplication & \multicolumn{2}{|c|}{ DCAF13;CT rare } \\
\hline 184255 chr8 & $39,322,364$ & $39,357,332$ & deletion & - & not rare \\
\hline 184255 chr9 & $11,936,751$ & $12,001,203$ & deletion & - & not rare \\
\hline 184255 chr9 & $94,395,522$ & $94,402,923$ & deletion & - & not rare \\
\hline
\end{tabular}




\begin{tabular}{|c|c|c|c|c|c|}
\hline 184256 chr10 & $71,282,981$ & $71,289,190$ & deletion & - & not rare \\
\hline 184256 chr12 & $29,944,159$ & $29,946,546$ & duplication & - & not rare \\
\hline 84256 chr12 & $30,237,351$ & $30,243,473$ & deletion & - & not rare \\
\hline 184256 chr12 & $6,255,862$ & $6,259,552$ & deletion & - & not rare \\
\hline $184256 \mathrm{chr} 13$ & $38,076,308$ & $38,085,537$ & deletion & - & not rare \\
\hline 184256 chr13 & $69,247,022$ & $69,267,981$ & deletion & - & ot rare \\
\hline 184256 chr14 & $41,610,224$ & $41,661,685$ & deletion & - & not rare \\
\hline 184256 chr14 & $79,160,530$ & $79,165,608$ & deletion & - & not rare \\
\hline 184256 chr15 & $34,717,697$ & $34,814,869$ & deletion & - & not rare \\
\hline 184256 chr16 & $32,363,472$ & $32,833,893$ & deletion & 3TG3B; & not rare \\
\hline 184256 chr16 & $33,548,357$ & $33,590,959$ & deletion & - & not rare \\
\hline 184256 chr16 & $35,185,903$ & $35,197,323$ & deletion & - & not rare \\
\hline $184256 \mathrm{chr} 16$ & $78,372,894$ & $78,383,509$ & deletion & - & not rare \\
\hline 184256 chr17 & $14,997,297$ & $15,010,250$ & deletion & - & not rare \\
\hline 184256 chr17 & $15,044,804$ & $15,058,236$ & deletion & - & not rare \\
\hline 184256 chr17 & $34,438,753$ & $34,483,342$ & duplication & - & not rare \\
\hline 184256 chr18 & $63,200,484$ & $63,206,335$ & duplication & - & not rare \\
\hline 184256 chr18 & $67,208,806$ & $67,217,271$ & deletion & - & not rare \\
\hline 184256 chr19 & $53,518,747$ & $53,552,296$ & ation & & rare \\
\hline 184256 chr2 & $14,704,369$ & $14,709,611$ & deletion & - & not rare \\
\hline $184256 \mathrm{chr} 2$ & $184,795,725$ & $184,803,456$ & deletion & - & not rare \\
\hline $184256 \mathrm{chr} 2$ & $208,351,315$ & $208,357,838$ & deletion & - & not rare \\
\hline 184256 chr2 & $227,343,513$ & $227,347,453$ & del & & not rare \\
\hline $184256 \mathrm{chr} 2$ & $66,188,209$ & $66,196,726$ & & & not rare \\
\hline 184256 chr22 & $37,745,101$ & $37,749,946$ & dele & & not rare \\
\hline 184256 chr3 & $110,793,050$ & $110,796,218$ & deletion & . & not rare \\
\hline 184256 chr3 & $193,136,358$ & $193,140,348$ & & - & are \\
\hline $184256 \mathrm{chr} 3$ & $195,457,853$ & $195,468,690$ & duplication & ML & not rare \\
\hline $184256 \mathrm{chr} 3$ & $22,279,685$ & $22,286,262$ & deletion & - & not rare \\
\hline $184256 \mathrm{chr} 4$ & $167,162,342$ & $167,186,484$ & deletion & - & not rare \\
\hline $184256 \mathrm{chr} 4$ & $34,785,955$ & $34,818,502$ & deletion & - & not rare \\
\hline 184256 chr5 & $155,477,866$ & $155,488,917$ & deletion & - & not rare \\
\hline 184256 chr5 & $82,156,492$ & $82,170,556$ & deletion & - & not rare \\
\hline 184256 chr6 & $30,994,010$ & $30,994,787$ & deletion & MUC22 & not rare \\
\hline 184256 chr6 & $31,785,453$ & $31,795,550$ & deletion & $A 1 A$ & not rare \\
\hline 184256 chr6 & $32,486,060$ & $32,509,357$ & deletion & HLA-DRB5 & not rare \\
\hline 184256 chr6 & $78,985,226$ & $79,012,428$ & duplication & - & not rare \\
\hline 184256 chr7 & $62,043,993$ & $62,713,168$ & duplication & - & not rare \\
\hline 184256 chr7 & $91,033,074$ & $91,041,618$ & deletion & - & not rare \\
\hline 184256 chr8 & $39,232,581$ & $39,385,979$ & deletion & - & not rare \\
\hline 184256 chr9 & $119,688,600$ & $119,706,183$ & deletion & - & not rare \\
\hline 184256 chr9 & $44,739,805$ & $44,834,316$ & deletion & - & not rare \\
\hline 184256 chr9 & $6,701,130$ & $6,707,794$ & deletion & - & not rare \\
\hline 184256 chr9 & $90,817,188$ & $90,821,421$ & deletion & - & not rare \\
\hline 184257 chr1 & $104,153,766$ & $104,161,227$ & deletion & AMY2A & not rare \\
\hline 184257 chr1 & $149,032,299$ & $149,432,892$ & deletion & - & not rare \\
\hline 184257 chr1 & $161,563,386$ & $161,619,741$ & duplication & \multicolumn{2}{|c|}{ FCGR2C;FC not rare } \\
\hline
\end{tabular}




\begin{tabular}{|c|c|c|c|c|c|c|}
\hline 184257 & chr1 & $17,009,576$ & $17,261,738$ & duplication & \multicolumn{2}{|c|}{ FAM231A; not rare } \\
\hline 184257 & chr1 & $8,360,487$ & $8,363,575$ & deletion & - & not rare \\
\hline 84257 & chr10 & $71,278,805$ & $71,289,190$ & deletion & - & not rare \\
\hline 84257 & chr11 & $25,750,699$ & $25,753,430$ & deletion & - & not rare \\
\hline 84257 & chr11 & $55,365,761$ & $55,427,700$ & deletion & \multicolumn{2}{|c|}{$\mathrm{OR} 4 \mathrm{P} 4 ; \mathrm{OR} \angle$ not rare } \\
\hline 184257 & chr12 & $30,237,351$ & $30,243,473$ & deletion & - & not rare \\
\hline 184257 & $\operatorname{chr} 13$ & $69,247,022$ & $69,267,981$ & deletion & - & not rare \\
\hline 184257 & chr14 & $106,062,675$ & $106,178,945$ & duplication & - & not rare \\
\hline 184257 & chr14 & $20,197,311$ & $20,424,184$ & duplication & \multicolumn{2}{|c|}{ OR4K5;OR $\angle$ not rare } \\
\hline 184257 & chr14 & $39,129,744$ & $39,140,536$ & deletion & - & not rare \\
\hline 184257 & chr14 & $41,610,224$ & $41,661,685$ & deletion & - & not rare \\
\hline 184257 & chr14 & $46,508,465$ & $46,603,680$ & deletion & - & rare \\
\hline 184257 & chr16 & $32,528,023$ & $32,833,893$ & deletion & \multicolumn{2}{|c|}{ TP53TG3B; not rare } \\
\hline 184257 & chr16 & $57,610,423$ & $57,611,856$ & deletion & - & not rare \\
\hline 184257 & chr17 & $10,890,859$ & $10,893,799$ & deletion & - & not rare \\
\hline 184257 & chr2 & $123,477,263$ & $123,482,301$ & deletion & - & not rare \\
\hline 184257 & chr2 & $195,979,780$ & $195,982,450$ & deletion & - & not rare \\
\hline 184257 & chr2 & $212,770,611$ & $212,776,546$ & deletion & - & care \\
\hline 184257 & chr2 & $38,301,735$ & $38,305,451$ & deletion & BB1 & not rare \\
\hline 184257 & chr2 & $41,239,457$ & $41,248,468$ & deletion & - & rare \\
\hline 184257 & chr20 & $62,194,128$ & $62,198,819$ & & & care \\
\hline 184257 & chr21 & $20,057,919$ & $20,081,051$ & duplication & - & not rare \\
\hline 184257 & $\operatorname{chr} 21$ & $28,985,549$ & $28,993,938$ & deletion & 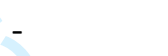 & not rare \\
\hline 184257 & chr22 & $25,663,994$ & $25,919,492$ & deletion & \multirow[t]{3}{*}{ LRP5L } & not rare \\
\hline 184257 & chr3 & $191,935,121$ & $191,996,202$ & cation & & rare \\
\hline 184257 & chr4 & $138,092,298$ & $138,098,601$ & dele & & not rare \\
\hline 184257 & chr4 & $34,785,955$ & $34,818,502$ & deletion & - & not rare \\
\hline 184257 & chr4 & $64,694,117$ & $64,709,753$ & deletion & - & not rare \\
\hline 184257 & chr4 & $69,374,369$ & $69,489,473$ & dele & 2B17 & are \\
\hline 184257 & chr5 & $5,694,586$ & $5,713,766$ & duplication & - & not rare \\
\hline 184257 & chr5 & $97,933,671$ & $97,939,616$ & deletion & - & not rare \\
\hline 184257 & chr6 & $29,855,945$ & $29,899,189$ & deletion & - & not rare \\
\hline 184257 & chr6 & $31,338,528$ & $31,341,357$ & deletion & - & not rare \\
\hline 184257 & chr6 & $44,577,322$ & $44,591,850$ & deletion & - & not rare \\
\hline 184257 & chr6 & $65,110,673$ & $65,136,490$ & deletion & - & rare \\
\hline 184257 & chr7 & $110,582,600$ & $110,585,307$ & deletion & - & not rare \\
\hline 184257 & chr7 & $110,977,136$ & $111,159,896$ & deletion & $|\mathrm{M}|$ & not rare \\
\hline 184257 & chr7 & $91,033,074$ & $91,048,548$ & deletion & - & not rare \\
\hline 184257 & chr8 & $115,634,278$ & $115,651,344$ & deletion & - & not rare \\
\hline 184257 & chr8 & $2,150,949$ & $2,161,040$ & deletion & - & not rare \\
\hline 184257 & chr8 & $33,506,910$ & $33,530,099$ & deletion & - & not rare \\
\hline 184257 & chr8 & $39,232,581$ & $39,385,979$ & deletion & - & not rare \\
\hline 184257 & chr9 & $4,526,780$ & $4,529,671$ & deletion & - & not rare \\
\hline 184257 & chr9 & $6,664,282$ & $6,673,957$ & deletion & - & not rare \\
\hline 184258 & chr1 & $104,153,766$ & $104,211,046$ & deletion & \multicolumn{2}{|c|}{ AMY1A;AN not rare } \\
\hline 184258 & chr1 & $112,693,423$ & $112,704,581$ & deletion & - & not rare \\
\hline 184258 & $\mathrm{hr} 1$ & $149,024,218$ & $149,432,892$ & deletion & - & not rare \\
\hline
\end{tabular}




\begin{tabular}{|c|c|c|c|c|c|}
\hline $184258 \mathrm{chr} 1$ & $161,563,386$ & 161, & del & \multicolumn{2}{|c|}{ FCGR2C;FC not rare } \\
\hline $184258 \mathrm{chr} 1$ & $174,794,144$ & $174,801,639$ & deletion & - & not rare \\
\hline 84258 chr10 & $71,280,107$ & $71,289,190$ & deletion & - & not rare \\
\hline 84258 chr11 & $25,750,699$ & $25,753,430$ & deletion & - & not rare \\
\hline 184258 chr12 & $2,245,636$ & $2,254,219$ & deletion & - & not rare \\
\hline 184258 chr12 & $30,237,351$ & $30,243,473$ & deletion & - & not rare \\
\hline 184258 chr12 & $59,935,926$ & $59,942,122$ & deletion & - & not rare \\
\hline 184258 chr13 & $23,545,717$ & $23,553,518$ & deletion & - & not rare \\
\hline 184258 chr13 & $84,102,440$ & $84,157,927$ & deletion & - & not rare \\
\hline 184258 chr14 & $106,067,375$ & $106,178,945$ & duplication & - & not rare \\
\hline 184258 chr14 & $41,610,224$ & $41,661,685$ & deletion & - & not rare \\
\hline 184258 chr14 & $74,240,176$ & $74,245,607$ & deletion & - & not rare \\
\hline 184258 chr15 & $55,342,771$ & $55,356,202$ & duplication & - & not rare \\
\hline 184258 chr16 & $55,800,009$ & $55,819,443$ & deletion & - & not rare \\
\hline 184258 chr16 & $60,082,237$ & $60,098,544$ & deletion & - & not rare \\
\hline 184258 chr19 & $35,661,787$ & $35,665,515$ & deletion & - & not rare \\
\hline 184258 chr19 & $41,355,999$ & $41,379,321$ & deletion & CYP2A6 & not rare \\
\hline 184258 chr19 & $43,703,965$ & $43,759,382$ & deletion & \multicolumn{2}{|c|}{ PSG4;PSG9 not rare } \\
\hline 184258 chr19 & $53,518,747$ & $53,552,296$ & duplication & & not rare \\
\hline 184258 chr19 & $55,298,309$ & $55,339,383$ & duplication & \multicolumn{2}{|c|}{ KIR3DL1;KI not rare } \\
\hline $184258 \mathrm{chr} 2$ & $123,477,465$ & $123,482,301$ & deletion & - & not rare \\
\hline $184258 \mathrm{chr} 2$ & $129,638,609$ & $129,645,321$ & deletion & - & not rare \\
\hline 184258 chr2 & $14,704,369$ & $14,709,611$ & del & & not rare \\
\hline $184258 \mathrm{chr} 2$ & $177,374,056$ & $177,377,367$ & del & & rare \\
\hline $184258 \mathrm{chr} 2$ & $195,979,780$ & $195,982,450$ & dele & & not rare \\
\hline $184258 \mathrm{chr} 2$ & $212,771,670$ & $212,773,893$ & deletion & - & not rare \\
\hline 184258 chr2 & $34,697,895$ & $34,726,904$ & ation & - & are \\
\hline $184258 \mathrm{chr} 2$ & $51,926,599$ & $51,926,904$ & deletion & - & not rare \\
\hline $184258 \mathrm{chr} 2$ & $79,752,148$ & $79,761,222$ & deletion & - & not rare \\
\hline 184258 chr2 & $89,540,620$ & $90,256,530$ & deletion & - & not rare \\
\hline $184258 \mathrm{chr} 3$ & $195,418,291$ & $195,468,690$ & duplication & MUC20 & not rare \\
\hline $184258 \mathrm{chr} 3$ & $20,990,739$ & $20,996,035$ & deletion & - & not rare \\
\hline $184258 \mathrm{chr} 4$ & $115,073,729$ & $115,089,046$ & deletion & - & not rare \\
\hline $184258 \mathrm{chr} 4$ & $138,092,298$ & $138,098,601$ & deletion & - & not rare \\
\hline $184258 \mathrm{chr} 4$ & $31,464,058$ & $31,469,190$ & deletion & - & not rare \\
\hline $184258 \mathrm{chr} 4$ & $34,785,955$ & $34,805,860$ & duplication & - & not rare \\
\hline $184258 \mathrm{chr} 4$ & $54,596,025$ & $54,603,082$ & deletion & - & not rare \\
\hline $184258 \mathrm{chr} 4$ & $58,256,666$ & $58,261,728$ & deletion & - & not rare \\
\hline $184258 \mathrm{chr} 4$ & $60,325,010$ & $60,330,880$ & duplication & - & not rare \\
\hline $184258 \mathrm{chr} 4$ & $69,374,369$ & $69,489,473$ & deletion & UGT2B17 & not rare \\
\hline 184258 chr5 & $62,781,249$ & $62,823,933$ & deletion & - & rare \\
\hline 184258 chr5 & 717,675 & 824,025 & duplication & ZDHHC11 & not rare \\
\hline 184258 chr6 & $151,507,355$ & $151,509,176$ & deletion & - & not rare \\
\hline 184258 chr6 & $29,855,945$ & $29,899,189$ & deletion & - & not rare \\
\hline 184258 chr6 & $31,338,528$ & $31,341,357$ & deletion & - & not rare \\
\hline 184258 chr7 & $99,562,258$ & $99,625,041$ & duplication & \multicolumn{2}{|c|}{ AZGP1;ZKS rare } \\
\hline $184258 \mathrm{chr} 8$ & $135,059,968$ & $135,065,947$ & deletion & - & not rare \\
\hline
\end{tabular}




\begin{tabular}{|c|c|c|c|c|c|}
\hline $184258 \mathrm{chr} 8$ & $2,150,949$ & $2,161,040$ & deletion & - & not rare \\
\hline 184258 chr8 & $39,322,364$ & $39,372,087$ & deletion & - & not rare \\
\hline 184258 chr8 & $51,029,371$ & $51,038,149$ & deletion & - & ot rare \\
\hline $184258 \mathrm{chr} 8$ & $51,226,087$ & $51,227,777$ & deletion & - & not rare \\
\hline 184258 chr9 & $23,363,526$ & $23,376,817$ & deletion & - & not rare \\
\hline $184260 \mathrm{chr} 1$ & $104,153,766$ & $104,161,227$ & deletion & AMY2A & not rare \\
\hline $184260 \mathrm{chr} 1$ & $1,651,355$ & $1,680,951$ & duplication & \multicolumn{2}{|c|}{ SLC35E2;C[ not rare } \\
\hline $184260 \mathrm{chr} 1$ & $187,716,107$ & $187,722,032$ & deletion & - & not rare \\
\hline 184260 chr10 & $90,941,441$ & $90,949,605$ & deletion & - & not rare \\
\hline 184260 chr11 & $106,396,192$ & $106,404,608$ & deletion & - & not rare \\
\hline 184260 chr12 & $21,519,708$ & $21,547,875$ & deletion & IAPP & rare \\
\hline 184260 chr12 & $43,021,431$ & $43,027,453$ & deletion & - & not rare \\
\hline 184260 chr15 & $24,494,024$ & $24,710,031$ & duplication & - & not rare \\
\hline 184260 chr16 & $58,945,839$ & $58,948,100$ & deletion & - & not rare \\
\hline 184260 chr17 & $44,166,604$ & $44,356,058$ & duplication & KANSL1 & not rare \\
\hline 184260 chr17 & $50,680,882$ & $50,740,695$ & deletion & - & rare \\
\hline 184260 chr18 & $41,976,831$ & $41,982,102$ & deletion & - & not rare \\
\hline 184260 chr18 & $7,730,117$ & $7,761,056$ & deletion & - & not rare \\
\hline 184260 & $54,728,767$ & 54,74 & tion & & \\
\hline 184260 chr19 & $55,233,850$ & $55,332,601$ & duplication & \multicolumn{2}{|c|}{ KIR2DL3;KI not rare } \\
\hline $184260 \mathrm{chr} 2$ & $110,852,875$ & $110,982,530$ & duplication & \multicolumn{2}{|c|}{ MALL;NPHI not rare } \\
\hline $184260 \mathrm{chr} 2$ & $14,702,635$ & $14,709,611$ & deletion & - & not rare \\
\hline $184260 \mathrm{chr} 2$ & $213,187,034$ & $213,191,389$ & deletion & & not rare \\
\hline $184260 \mathrm{chr} 2$ & $228,241,621$ & $228,258,288$ & duplication & TM4SF20 & not rare \\
\hline 184260 chr2 & $41,239,457$ & $41,248,468$ & deletion & & not rare \\
\hline $184260 \mathrm{chr} 2$ & $4,212,452$ & $4,222,144$ & deletion & . & not rare \\
\hline 184260 chr20 & $59,568,187$ & $59,590,280$ & duplication & - & are \\
\hline 184260 chr21 & $10,766,457$ & $10,858,651$ & deletion & - & not rare \\
\hline 184260 chr21 & $23,655,900$ & $23,665,470$ & deletion & - & not rare \\
\hline 184260 chr22 & $33,463,222$ & $33,474,789$ & duplication & - & not rare \\
\hline $184260 \mathrm{chr} 3$ & $11,411,823$ & $11,414,339$ & deletion & - & not rare \\
\hline $184260 \mathrm{chr} 3$ & $151,514,590$ & $151,549,959$ & deletion & AA & not rare \\
\hline $184260 \mathrm{chr} 3$ & $191,065,392$ & $191,070,300$ & deletion & - & not rare \\
\hline 184260 chr3 & $195,457,853$ & $195,468,690$ & duplication & MUC20 & not rare \\
\hline $184260 \mathrm{chr} 3$ & $98,945,571$ & $98,947,547$ & deletion & - & not rare \\
\hline $184260 \mathrm{chr} 4$ & $122,285,263$ & $122,289,863$ & deletion & - & not rare \\
\hline $184260 \mathrm{chr} 4$ & $138,092,298$ & $138,098,601$ & deletion & - & not rare \\
\hline $184260 \mathrm{chr} 4$ & $152,790,580$ & $152,793,891$ & deletion & - & not rare \\
\hline $184260 \mathrm{chr} 4$ & $25,557,047$ & $25,578,269$ & duplication & - & not rare \\
\hline $184260 \mathrm{chr} 4$ & $64,697,704$ & $64,709,753$ & deletion & - & not rare \\
\hline 184260 chr5 & $155,477,866$ & $155,488,917$ & deletion & - & not rare \\
\hline 184260 chr5 & $8,258,458$ & $8,260,630$ & deletion & - & not rare \\
\hline 184260 chr6 & $32,458,168$ & $32,509,357$ & deletion & RB5 & not rare \\
\hline 184260 chr6 & $78,972,930$ & $79,035,739$ & deletion & - & not rare \\
\hline 184260 chr7 & 151,829 & 159,207 & deletion & - & not rare \\
\hline $184260 \mathrm{chr} 7$ & $156,387,296$ & $156,394,307$ & deletion & - & not rare \\
\hline $184260 \mathrm{chr} 8$ & $12,761,080$ & $12,790,944$ & deletion & - & rare \\
\hline
\end{tabular}




\begin{tabular}{|c|c|c|c|c|c|}
\hline $184260 \mathrm{chr} 8$ & $39,232,581$ & $39,385,979$ & duplication & - & not rare \\
\hline 184260 chr9 & $78,004,378$ & $78,011,616$ & deletion & - & not rare \\
\hline 84261 chr1 & $104,104,990$ & $104,211,046$ & duplication & \multicolumn{2}{|c|}{ AMY1C;AN not rare } \\
\hline 84261 chr1 & $12,846,082$ & $12,944,989$ & deletion & \multicolumn{2}{|c|}{ HNRNPCL1 not rare } \\
\hline $184261 \mathrm{chr} 1$ & $152,526,812$ & $152,552,461$ & deletion & \multicolumn{2}{|c|}{ LCE3D;LCE: not rare } \\
\hline $184261 \mathrm{c}$ & $245,636,900$ & $245,647,809$ & deletion & - & not rare \\
\hline $184261 \mathrm{chr} 1$ & $248,740,572$ & $248,795,110$ & deletion & \multicolumn{2}{|c|}{ OR2T10;OF not rare } \\
\hline 184261 chr10 & $58,845,646$ & $58,849,853$ & deletion & - & not rare \\
\hline 184261 chr10 & $71,282,981$ & $71,289,190$ & deletion & - & not rare \\
\hline 184261 chr11 & $18,949,220$ & $18,961,743$ & deletion & MRGPRX1 & not rare \\
\hline 184261 chr11 & $55,365,761$ & $55,443,269$ & duplication & \multicolumn{2}{|c|}{$\mathrm{OR} 4 \mathrm{P} 4 ; \mathrm{OR} \angle$ not rare } \\
\hline 184261 chr12 & $30,237,351$ & $30,243,473$ & deletion & - & not rare \\
\hline 184261 chr13 & $69,247,022$ & $69,267,981$ & deletion & - & not rare \\
\hline 184261 & $88,294,496$ & $88,301,122$ & deletion & - & not rare \\
\hline 184261 chr15 & $24,648,891$ & $24,808,488$ & duplication & - & not rare \\
\hline 184261 chr15 & $34,717,697$ & $34,859,791$ & deletion & GOLGA8B & not rare \\
\hline 184261 chr16 & $28,614,734$ & $28,620,752$ & duplication & SULT1A1 & not rare \\
\hline 184261 chr17 & $10,890,859$ & $10,893,799$ & deletion & - & not rare \\
\hline 184261 & 77,3 & $77,392,062$ & tion & - & \\
\hline 184261 chr19 & $24,460,170$ & $24,462,326$ & deletion & - & not rare \\
\hline 184261 chr19 & $43,703,965$ & $43,759,382$ & deletion & \multicolumn{2}{|c|}{ PSG4;PSG9 not rare } \\
\hline 184261 chr19 & $55,293,760$ & $55,338,833$ & duplication & \multicolumn{2}{|c|}{ KIR3DL1;KI not rare } \\
\hline 184261 chr2 & $214,989,439$ & $214,992,692$ & deletion & & not rare \\
\hline $184261 \mathrm{chr} 2$ & $54,479,117$ & $54,481,636$ & & & care \\
\hline 184261 chr21 & $10,766,457$ & $10,811,787$ & dele & & not rare \\
\hline 184261 chr22 & $45,134,981$ & $45,136,558$ & dele & . & not rare \\
\hline 184261 chr3 & $162,130,691$ & $162,145,531$ & & - & are \\
\hline $184261 \mathrm{chr} 3$ & $46,798,133$ & $46,845,618$ & deletion & - & not rare \\
\hline $184261 \mathrm{chr} 3$ & $98,945,571$ & $98,947,547$ & deletion & - & not rare \\
\hline $184261 \mathrm{chr} 4$ & $69,374,369$ & $69,489,473$ & duplication & 17 & are \\
\hline $184261 \mathrm{chr} 4$ & $7,182,916$ & $7,187,397$ & deletion & - & not rare \\
\hline 184261 chr5 & $155,477,866$ & $155,488,917$ & deletion & - & not rare \\
\hline 184261 chr5 & $171,043,230$ & $171,049,024$ & deletion & - & not rare \\
\hline 184261 chr5 & $18,529,962$ & $18,572,605$ & deletion & - & rare \\
\hline 184261 chr5 & $45,858,842$ & $45,879,274$ & deletion & - & not rare \\
\hline 184261 chr6 & $151,507,355$ & $151,509,176$ & deletion & - & not rare \\
\hline 184261 chr6 & $30,994,010$ & $30,994,787$ & deletion & ML & not rare \\
\hline 184261 chr6 & $78,985,226$ & $79,012,428$ & duplication & - & not rare \\
\hline 184261 chr7 & $111,020,216$ & $111,054,249$ & deletion & - & not rare \\
\hline 184261 chr7 & $141,766,850$ & $141,793,521$ & deletion & - & not rare \\
\hline 184261 chr7 & 41,421 & 73,060 & duplication & - & not rare \\
\hline 184261 chr7 & $9,934,923$ & $9,940,421$ & deletion & - & not rare \\
\hline 184261 chr8 & $3,786,543$ & $3,790,254$ & deletion & - & not rare \\
\hline 184261 chr8 & $39,232,581$ & $39,385,979$ & duplication & - & not rare \\
\hline 184261 chr8 & $51,013,729$ & $51,038,149$ & deletion & - & not rare \\
\hline 184261 chr8 & $5,417,839$ & $5,435,083$ & deletion & - & not rare \\
\hline 184261 chr9 & $1,446,522$ & $1,450,465$ & deletion & - & not rare \\
\hline
\end{tabular}




\begin{tabular}{|c|c|c|c|c|c|}
\hline 184261 chr9 & $78,004,378$ & $78,011,616$ & deletion & - & not rare \\
\hline 184262 chr 1 & $152,526,812$ & $152,568,230$ & deletion & LCE3D;LCE: I & not rare \\
\hline 84262 chr1 & $165,644,865$ & $165,649,715$ & deletion & ALDH9A1 & not rare \\
\hline 84262 chr1 & $174,797,867$ & $174,802,731$ & deletion & - & not rare \\
\hline 184262 chr11 & $18,949,220$ & $18,961,743$ & deletion & MRGPRX1 & not rare \\
\hline 84262 chr11 & $55,365,761$ & $55,432,933$ & deletion & OR4P4;OR । & rare \\
\hline 184262 chr12 & $132,131,087$ & $132,136,919$ & deletion & - & not rare \\
\hline 184262 chr13 & $34,134,809$ & $34,143,545$ & deletion & - & not rare \\
\hline 184262 chr14 & $41,610,224$ & $41,661,685$ & deletion & - & not rare \\
\hline 84262 chr14 & $48,230,056$ & $48,277,472$ & deletion & - & not rare \\
\hline 184262 & $34,717,697$ & $34,814,869$ & deletion & - & rare \\
\hline 184262 chr16 & $32,531,934$ & $32,833,893$ & deletion & TP53TG3B; & not rare \\
\hline 184262 chr17 & $10,890,859$ & $10,893,799$ & deletion & - & not rare \\
\hline 184262 chr17 & $44,166,604$ & $44,225,181$ & duplication & KANSL1 & not rare \\
\hline 184262 chr19 & $43,703,965$ & $43,759,382$ & deletion & PSG4;PSG9 & not rare \\
\hline 184262 chr19 & $53,518,747$ & $53,552,296$ & duplication & ERVV-1 & not rare \\
\hline 184262 chr19 & $54,728,767$ & $54,743,217$ & duplication & LILRA6 & not rare \\
\hline 184262 chr2 & $54,479,117$ & $54,481,636$ & deletion & - & rare \\
\hline 184262 chr2 & $66,188,209$ & $66,196,726$ & dele & - & \\
\hline 184262 chr21 & $10,813,582$ & $10,873,592$ & duplication & - & not rare \\
\hline 184262 chr22 & $45,134,981$ & $45,136,558$ & deletion & - & not rare \\
\hline 184262 chr3 & $29,835,228$ & $29,850,829$ & dele & - & not rare \\
\hline 184262 chr3 & $37,979,882$ & $37,986,734$ & del & - & not rare \\
\hline $184262 \mathrm{chr} 4$ & $69,374,369$ & $69,489,473$ & ation & T2B17 & not rare \\
\hline 184262 chr5 & $18,529,962$ & $18,572,605$ & deletion & & rare \\
\hline 184262 chr5 & $74,182,586$ & $74,186,901$ & dele & - & not rare \\
\hline 184262 chr5 & $97,048,466$ & $97,096,042$ & & - & \\
\hline 184262 chr6 & $151,506,254$ & $151,509,176$ & deletion & - & not rare \\
\hline 184262 chr6 & $3,958,054$ & $3,961,288$ & deletion & - & not rare \\
\hline 184262 chr7 & $111,020,216$ & $111,054,249$ & deletion & - & not rare \\
\hline 184262 chr7 & $141,766,850$ & $141,793,521$ & deletion & - & not rare \\
\hline 184262 chr7 & 41,421 & 68,920 & duplication & - & not rare \\
\hline 184262 chr7 & $62,285,495$ & $62,306,851$ & duplication & - & not rare \\
\hline 184262 chr8 & $3,786,543$ & $3,790,254$ & deletion & - & not rare \\
\hline 184262 chr8 & $40,184,872$ & $40,189,688$ & deletion & - & are \\
\hline 184262 chr8 & $5,417,415$ & $5,435,083$ & deletion & - & not rare \\
\hline 184262 chr9 & $12,907,731$ & $12,908,447$ & deletion & - & not rare \\
\hline 184262 chr9 & $78,004,378$ & $78,011,616$ & deletion & - & not rare \\
\hline 184262 chr9 & $79,150,527$ & $79,160,759$ & deletion & - & not rare \\
\hline 184263 chr 1 & $104,104,990$ & $104,211,046$ & duplication & AMY1C;AN & not rare \\
\hline 184263 chr1 & $12,156,236$ & $12,169,295$ & duplication & TNFRSF8 & not rare \\
\hline 184263 chr1 & $12,206,652$ & $12,316,432$ & duplication & VPS13D;TN & rare \\
\hline 184263 chr1 & $152,526,812$ & $152,552,461$ & deletion & LCE3D;LCE: I & not rare \\
\hline 184263 chr 1 & $25,598,276$ & $25,659,509$ & deletion & RHD & not rare \\
\hline 184263 chr10 & $58,516,470$ & $58,526,153$ & deletion & - & not rare \\
\hline 184263 chr11 & $18,941,894$ & $18,961,743$ & deletion & MRGPRX1 & not rare \\
\hline 184263 chr13 & $19,342,790$ & $19,361,026$ & deletion & - & not rare \\
\hline
\end{tabular}




\begin{tabular}{|c|c|c|c|c|c|}
\hline 184263 chr14 & 35,60 & $35,613,500$ & deletion & - & not rare \\
\hline 184263 chr14 & $41,610,224$ & $41,661,685$ & deletion & - & not rare \\
\hline 84263 chr14 & $48,230,056$ & $48,278,934$ & deletion & - & not rare \\
\hline 184263 chr15 & $24,648,891$ & $24,742,652$ & duplication & - & not rare \\
\hline 184263 chr15 & $34,717,697$ & $34,814,869$ & deletion & - & not rare \\
\hline 184263 chr16 & $72,598,884$ & $72,614,366$ & deletion & - & not rare \\
\hline 184263 chr17 & $6,300,290$ & $6,312,899$ & duplication & - & not rare \\
\hline 184263 chr19 & $35,852,103$ & $35,861,695$ & deletion & - & not rare \\
\hline 184263 chr2 & $208,351,315$ & $208,357,838$ & deletion & - & not rare \\
\hline 184263 chr2 & $214,989,439$ & $214,992,692$ & deletion & - & not rare \\
\hline 184263 chr20 & $16,663,069$ & $16,666,053$ & deletion & - & not rare \\
\hline 184263 chr22 & $45,134,981$ & $45,136,558$ & deletion & - & not rare \\
\hline 184263 chr3 & $162,130,691$ & $162,142,475$ & deletion & - & not rare \\
\hline 184263 chr3 & $29,835,228$ & $29,850,829$ & deletion & - & not rare \\
\hline $184263 \mathrm{chr} 3$ & $37,979,882$ & $37,986,734$ & deletion & - & not rare \\
\hline 184263 chr3 & $46,798,133$ & $46,845,618$ & deletion & - & not rare \\
\hline $184263 \mathrm{chr} 4$ & $138,092,298$ & $138,098,601$ & deletion & - & not rare \\
\hline $184263 \mathrm{chr} 4$ & $69,374,369$ & $69,489,473$ & duplication & B17 & not rare \\
\hline 184263 chr5 & $155,477,866$ & $155,488,917$ & deletion & - & not rare \\
\hline 184263 chr5 & $171,043,230$ & $171,049,024$ & deletion & - & not rare \\
\hline 184263 chr5 & $18,529,962$ & $18,572,605$ & deletion & - & rare \\
\hline 184263 chr5 & $45,858,842$ & $45,879,274$ & dele & - & not rare \\
\hline 184263 chr6 & $30,994,010$ & $30,994,787$ & deletion & & not rare \\
\hline 184263 chr6 & $31,281,682$ & $31,286,577$ & delet & & not rare \\
\hline 184263 chr6 & $3,958,054$ & $3,961,288$ & dele & & not rare \\
\hline 184263 chr7 & 151,829 & 159,207 & deletion & - & not rare \\
\hline 184263 chr7 & $156,387,296$ & $156,394,307$ & deletion & - & not rare \\
\hline 184263 chr8 & $39,232,581$ & $39,385,979$ & duplication & - & not rare \\
\hline $184263 \mathrm{chr} 8$ & $40,184,872$ & $40,189,688$ & deletion & - & not rare \\
\hline 184263 chr9 & $12,907,731$ & $12,908,447$ & deletion & - & not rare \\
\hline 184263 chr9 & $73,906,136$ & $73,909,871$ & duplication & - & not rare \\
\hline 184265 chr1 & $119,073,435$ & $119,085,749$ & deletion & - & not rare \\
\hline 184265 chr1 & $149,032,299$ & $149,237,009$ & deletion & - & not rare \\
\hline 184265 chr1 & $152,526,812$ & $152,568,230$ & deletion & \multicolumn{2}{|c|}{ LCE3D;LCE: not rare } \\
\hline 184265 chr1 & $161,559,327$ & $161,617,673$ & duplication & \multicolumn{2}{|c|}{ FCGR2C;FC not rare } \\
\hline 184265 chr1 & $182,084,486$ & $182,087,284$ & deletion & - & not rare \\
\hline 184265 chr1 & $187,716,107$ & $187,722,032$ & deletion & - & not rare \\
\hline 184265 chr10 & $39,058,671$ & $39,154,535$ & duplication & - & not rare \\
\hline 184265 chr10 & $71,279,367$ & $71,289,190$ & deletion & - & not rare \\
\hline 184265 chr11 & $24,083,684$ & $24,140,697$ & deletion & - & not rare \\
\hline 184265 chr11 & $55,365,761$ & $55,424,207$ & deletion & \multicolumn{2}{|c|}{ OR4P4;OR $\angle$ not rare } \\
\hline 184265 chr11 & $90,193,796$ & $90,196,843$ & deletion & - & not rare \\
\hline 184265 chr12 & $119,986,882$ & $119,991,777$ & deletion & - & not rare \\
\hline 184265 chr12 & $43,021,431$ & $43,027,453$ & deletion & - & not rare \\
\hline 184265 chr13 & $34,134,809$ & $34,143,545$ & deletion & - & not rare \\
\hline 184265 chr14 & $41,610,224$ & $41,661,685$ & deletion & - & not rare \\
\hline 184265 chr15 & $32,509,090$ & $32,515,849$ & duplication & - & not rare \\
\hline
\end{tabular}




\begin{tabular}{|c|c|c|c|c|c|}
\hline 184265 chr15 & $56,789,979$ & $56,800,635$ & deletion & - & not rare \\
\hline 184265 chr16 & $32,559,149$ & $32,603,033$ & duplication & - & not rare \\
\hline 84265 chr16 & $55,798,095$ & $55,819,443$ & deletion & - & ot rare \\
\hline 184265 chr17 & $14,994,610$ & $15,010,250$ & deletion & - & not rare \\
\hline 184265 chr17 & $15,044,804$ & $15,058,236$ & deletion & - & not rare \\
\hline 184265 chr19 & $1,671,549$ & $1,677,696$ & deletion & - & ot rare \\
\hline 184265 chr19 & $35,852,103$ & $35,861,695$ & deletion & - & not rare \\
\hline 184265 chr19 & $43,703,965$ & $43,759,382$ & deletion & \multicolumn{2}{|c|}{ PSG4;PSG9 not rare } \\
\hline 184265 chr2 & $184,085,551$ & $184,088,256$ & deletion & - & not rare \\
\hline 184265 chr2 & $34,697,895$ & $34,726,904$ & deletion & - & not rare \\
\hline 184265 chr2 & $41,239,457$ & $41,248,468$ & deletion & - & not rare \\
\hline 184265 chr2 & $4,212,725$ & $4,222,144$ & deletion & - & not rare \\
\hline 184265 chr2 & $48,851,541$ & $48,856,770$ & deletion & - & not rare \\
\hline 184265 chr2 & $52,032,381$ & $52,077,000$ & deletion & - & rare \\
\hline 184265 chr2 & $54,479,117$ & $54,481,636$ & deletion & - & not rare \\
\hline 184265 chr20 & $29,454,201$ & $29,500,566$ & deletion & - & not rare \\
\hline 184265 chr3 & $18,235,914$ & $18,244,588$ & deletion & - & not rare \\
\hline 184265 chr3 & $191,065,392$ & $191,070,300$ & deletion & - & not rare \\
\hline 184265 & $193,136,358$ & $193,140,348$ & on & - & \\
\hline 184265 chr3 & $37,979,882$ & $37,990,299$ & deletion & CTDSPL & not rare \\
\hline $184265 \mathrm{chr} 3$ & $65,117,947$ & $65,118,762$ & deletion & - & not rare \\
\hline $184265 \mathrm{chr} 4$ & $70,137,897$ & $70,229,260$ & deletion & UGT2B28 & not rare \\
\hline 184265 chr5 & $180,176,005$ & $180,195,526$ & duplicatio & & not rare \\
\hline 184265 chr5 & $97,933,671$ & $97,939,616$ & deletion & 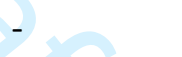 & not rare \\
\hline 184265 chr6 & $30,994,010$ & $30,994,787$ & deletion & MUC22 & not rare \\
\hline 184265 chr7 & $142,479,577$ & $142,484,042$ & deletion & - & not rare \\
\hline 184265 & $148,670,881$ & $148,675,815$ & del & - & are \\
\hline 184265 chr7 & $86,238,045$ & $86,251,244$ & deletion & - & not rare \\
\hline 184265 chr8 & $39,236,582$ & $39,385,979$ & deletion & - & not rare \\
\hline 184265 chr9 & $106,792,558$ & $106,806,206$ & deletion & - & not rare \\
\hline 184265 chr9 & $6,664,282$ & $6,673,465$ & deletion & - & not rare \\
\hline 184266 chr1 & $12,910,449$ & $12,998,343$ & duplication & \multicolumn{2}{|c|}{ PRAMEF4;F not rare } \\
\hline 184266 chr1 & $142,603,938$ & $143,978,807$ & duplication & \multicolumn{2}{|c|}{ FAM72D;F/ not rare } \\
\hline 184266 chr1 & $1,846,582$ & $1,879,954$ & deletion & \multicolumn{2}{|c|}{ CFAP74;TN rare } \\
\hline 184266 chr11 & $134,602,333$ & $134,607,484$ & deletion & - & not rare \\
\hline 184266 chr12 & $119,988,733$ & $119,991,777$ & deletion & - & not rare \\
\hline 184266 chr12 & $126,994,335$ & $127,004,296$ & deletion & - & not rare \\
\hline 184266 chr12 & $129,228,378$ & $129,233,013$ & deletion & - & not rare \\
\hline 184266 chr12 & $43,021,431$ & $43,027,453$ & deletion & - & not rare \\
\hline 184266 chr13 & $69,247,022$ & $69,267,981$ & deletion & - & not rare \\
\hline 184266 chr14 & $20,258,645$ & $20,420,338$ & duplication & \multicolumn{2}{|c|}{ OR4N2;OR` not rare } \\
\hline 184266 chr14 & $41,610,224$ & $41,661,685$ & deletion & - & not rare \\
\hline 184266 chr14 & $50,373,817$ & $50,385,124$ & duplication & - & not rare \\
\hline 184266 chr16 & $32,531,934$ & $32,833,893$ & deletion & \multicolumn{2}{|c|}{ TP53TG3B; not rare } \\
\hline 184266 chr17 & $34,438,753$ & $34,534,918$ & duplication & \multicolumn{2}{|c|}{ CCL3L3;CCI not rare } \\
\hline 184266 chr19 & $33,693,005$ & $33,698,448$ & deletion & LRP3 & not rare \\
\hline 184266 chr19 & $35,852,103$ & $35,861,695$ & deletion & - & not rare \\
\hline
\end{tabular}




\begin{tabular}{|c|c|c|c|c|c|}
\hline 184266 chr19 & $43,261,897$ & 9,979 & duplication & PSG8 & not rare \\
\hline 184266 chr2 & $184,083,676$ & $184,088,256$ & deletion & - & not rare \\
\hline 84266 chr2 & $213,187,034$ & $213,191,389$ & deletion & - & ot rare \\
\hline 184266 chr2 & $228,243,310$ & $228,258,288$ & duplication & TM4SF20 & not rare \\
\hline $184266 \mathrm{chr} 2$ & $241,824,566$ & $241,876,606$ & deletion & C2orf54 & rare \\
\hline 184266 chr2 & $41,239,457$ & $41,248,468$ & deletion & - & not rare \\
\hline 184266 chr2 & $48,851,541$ & $48,856,770$ & deletion & - & not rare \\
\hline 184266 chr20 & $62,194,128$ & $62,200,576$ & deletion & HELZ2 & not rare \\
\hline 184266 chr21 & $24,020,223$ & $24,043,069$ & deletion & - & not rare \\
\hline 184266 chr21 & $47,387,112$ & $47,433,055$ & deletion & COL6A1 & not rare \\
\hline 184266 chr22 & $30,340,138$ & $30,362,719$ & deletion & - & not rare \\
\hline 184266 chr22 & $37,745,101$ & $37,749,946$ & deletion & - & not rare \\
\hline 184266 chr3 & $131,711,896$ & $131,712,898$ & deletion & - & not rare \\
\hline 184266 chr3 & $165,040,903$ & $165,074,650$ & deletion & - & not rare \\
\hline 184266 chr3 & $1,782,524$ & $1,787,581$ & deletion & - & not rare \\
\hline 184266 chr3 & $195,418,291$ & $195,468,690$ & duplication & MUC20 & not rare \\
\hline 184266 chr3 & $46,798,133$ & $46,845,618$ & deletion & - & not rare \\
\hline 184266 chr3 & $53,032,639$ & $53,042,099$ & deletion & - & not rare \\
\hline $184266 \mathrm{chr} 4$ & $16,413,099$ & $16,428,907$ & & - & \\
\hline $184266 \mathrm{chr} 4$ & $49,069,684$ & $49,651,131$ & duplication & - & not rare \\
\hline $184266 \mathrm{chr} 4$ & $92,076,288$ & $92,079,569$ & deletion & - & not rare \\
\hline 184266 chr5 & 701,026 & 840,717 & duplication & ZDHHC11 & not rare \\
\hline 184266 chr6 & $57,160,035$ & $57,165,948$ & deletion & & not rare \\
\hline $184266 \mathrm{chr} 8$ & $115,633,704$ & $115,642,459$ & & & not rare \\
\hline 184266 chr8 & $144,976,572$ & $145,032,302$ & deletion & PL & not rare \\
\hline 184266 chr8 & $39,232,581$ & $39,385,979$ & ation & 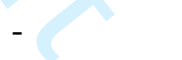 & not rare \\
\hline 184266 & $12,907,731$ & $12,908,447$ & & - & not rare \\
\hline 184266 chr9 & $6,619,776$ & $6,753,072$ & duplication & \multicolumn{2}{|c|}{ KDM4C;GLI not rare } \\
\hline 184266 chr9 & $68,380,409$ & $68,502,538$ & duplication & - & not rare \\
\hline 184266 chr9 & $73,906,136$ & $73,909,871$ & sation & - & not rare \\
\hline 184267 chr1 & $149,033,600$ & $149,432,892$ & duplication & - & not rare \\
\hline 184267 chr1 & $161,563,386$ & $161,619,741$ & duplication & \multicolumn{2}{|c|}{ FCGR2C;FC not rare } \\
\hline 184267 chr1 & $187,716,107$ & $187,722,032$ & deletion & - & not rare \\
\hline 184267 chr1 & $196,823,300$ & $196,901,753$ & duplication & CFHR4 & not rare \\
\hline 184267 chr1 & $248,740,572$ & $248,813,829$ & deletion & \multicolumn{2}{|c|}{ OR2T10;OF not rare } \\
\hline 184267 chr10 & $58,846,575$ & $58,849,853$ & deletion & - & not rare \\
\hline 184267 chr11 & $55,365,761$ & $55,424,207$ & deletion & \multicolumn{2}{|c|}{ OR4P4;OR $\angle$ not rare } \\
\hline 184267 chr11 & $90,193,556$ & $90,196,843$ & deletion & - & not rare \\
\hline 184267 chr12 & $30,237,351$ & $30,243,473$ & deletion & - & not rare \\
\hline 184267 chr12 & $43,021,431$ & $43,027,453$ & deletion & - & not rare \\
\hline 184267 chr12 & $59,935,926$ & $59,942,122$ & deletion & - & not rare \\
\hline 184267 chr13 & $34,134,809$ & $34,143,545$ & deletion & - & not rare \\
\hline 184267 chr13 & $58,589,893$ & $58,599,174$ & deletion & - & not rare \\
\hline 184267 chr13 & $69,247,022$ & $69,267,981$ & deletion & - & not rare \\
\hline 184267 chr14 & $41,610,224$ & $41,661,685$ & deletion & - & not rare \\
\hline 184267 chr16 & $70,183,624$ & $70,196,139$ & duplication & PD & not rare \\
\hline 184267 chr16 & $7,182,538$ & $7,204,523$ & deletion & - & rare \\
\hline
\end{tabular}




\begin{tabular}{|c|c|c|c|c|c|c|}
\hline 84267 & chr16 & 676 & $85,304,326$ & deletion & - & not rare \\
\hline 184267 & chr19 & $49,304,215$ & $49,351,040$ & duplication & \multicolumn{2}{|c|}{ PLEKHA4;B rare } \\
\hline 84267 & chr2 & $195,677,877$ & $195,703,010$ & deletion & - & not rare \\
\hline 84267 & chr2 & $212,771,670$ & $212,773,893$ & deletion & - & not rare \\
\hline 84267 & chr2 & $242,854,089$ & $243,034,519$ & deletion & - & not rare \\
\hline 184267 & chr2 & $38,956,947$ & $38,971,623$ & duplication & GA & not rare \\
\hline 184267 & chr20 & $52,647,631$ & $52,656,535$ & deletion & - & not rare \\
\hline 184267 & chr22 & $18,844,632$ & $19,016,663$ & duplication & \multicolumn{2}{|c|}{ PRODH;DG not rare } \\
\hline 184267 & chr4 & $152,789,786$ & $152,795,802$ & deletion & - & not rare \\
\hline 184267 & chr4 & $34,785,955$ & $34,818,502$ & deletion & - & not rare \\
\hline 184267 & chr4 & $69,374,369$ & $69,489,473$ & deletion & 2B17 & not rare \\
\hline 184267 & chr5 & $155,477,866$ & $155,495,107$ & deletion & - & not rare \\
\hline 184267 & chr5 & 762,435 & 825,338 & deletion & -C11 & not rare \\
\hline 184267 & chr6 & $19,040,857$ & $19,048,773$ & deletion & - & are \\
\hline 184267 & chr6 & $30,994,010$ & $30,994,787$ & deletion & $\mathrm{M}$ & not rare \\
\hline 184267 & chr6 & $67,011,900$ & $67,048,885$ & deletion & - & not rare \\
\hline 184267 & chr6 & $77,439,769$ & $77,451,301$ & deletion & - & not rare \\
\hline 184267 & chr7 & $111,943,602$ & $111,960,972$ & deletion & & not rare \\
\hline 184267 & chr7 & $62,145,873$ & $62,162,385$ & & - & rare \\
\hline 184267 & chr7 & $97,396,709$ & $97,401,677$ & deletion & - & not rare \\
\hline 184267 & chr8 & $103,054,099$ & $103,056,645$ & deletion & - & not rare \\
\hline 184267 & chr8 & $115,633,704$ & $115,642,459$ & deletion & - & not rare \\
\hline 184267 & chr8 & $117,929,458$ & $117,938,722$ & duplicatio & & not rare \\
\hline 184267 & chr8 & $39,232,581$ & $39,385,979$ & & & not rare \\
\hline 184267 & chr8 & $87,172,818$ & $87,192,895$ & dele & & rare \\
\hline 184267 & chr9 & $23,363,526$ & $23,376,817$ & dup & . & not rare \\
\hline 184267 & chr9 & $6,701,130$ & $6,707,794$ & & - & are \\
\hline 184267 & chr9 & $69,838,649$ & $69,992,206$ & deletion & - & not rare \\
\hline 184267 & chr9 & $78,004,378$ & $78,011,616$ & deletion & - & not rare \\
\hline 184269 & chr1 & $104,103,057$ & $104,211,046$ & duplication & \multicolumn{2}{|c|}{ AMY1C;AN not rare } \\
\hline 184269 & chr1 & $149,032,299$ & $149,233,182$ & deletion & - & not rare \\
\hline 184269 & chr1 & $187,716,107$ & $187,722,032$ & deletion & - & not rare \\
\hline 184269 & chr10 & $57,249,829$ & $57,342,252$ & deletion & - & rare \\
\hline 184269 & chr10 & $71,278,805$ & $71,289,190$ & deletion & - & not rare \\
\hline 184269 & chr11 & $18,949,220$ & $18,961,743$ & duplication & MRGPRX1 & not rare \\
\hline 184269 & chr11 & $7,814,299$ & $7,827,879$ & deletion & OR5P2 & not rare \\
\hline 184269 & chr11 & $81,501,218$ & $81,517,261$ & deletion & - & not rare \\
\hline 184269 & $\operatorname{chr} 12$ & $2,245,636$ & $2,254,219$ & deletion & - & not rare \\
\hline 184269 & chr12 & $32,331,195$ & $32,334,813$ & deletion & - & not rare \\
\hline 184269 & chr12 & $43,021,431$ & $43,027,453$ & deletion & - & not rare \\
\hline 184269 & chr12 & $59,935,926$ & $59,942,122$ & deletion & - & not rare \\
\hline 184269 & $\operatorname{chr} 12$ & $98,313,235$ & $98,342,834$ & deletion & - & rare \\
\hline 184269 & chr13 & $114,545,317$ & $114,548,359$ & deletion & - & not rare \\
\hline 184269 & chr14 & $106,891,524$ & $106,931,129$ & deletion & - & not rare \\
\hline 184269 & chr14 & $20,197,311$ & $20,424,184$ & duplication & \multicolumn{2}{|c|}{ OR4K5;OR $\angle$ not rare } \\
\hline 184269 & chr14 & $24,447,203$ & $24,464,885$ & deletion & DHRS4L2 & not rare \\
\hline 184269 & chr14 & $75,663,348$ & $75,674,246$ & deletion & - & not rare \\
\hline
\end{tabular}




\begin{tabular}{|c|c|c|c|c|c|}
\hline 184269 chr15 & $20,870,571$ & $21,200,107$ & duplication & \multicolumn{2}{|c|}{ POTEB2;PC not rare } \\
\hline 184269 chr15 & $21,903,815$ & $22,753,733$ & duplication & \multicolumn{2}{|c|}{ GOLGA6L2: not rare } \\
\hline 84269 chr16 & $19,945,540$ & $19,962,636$ & deletion & - & not rare \\
\hline $184269 \mathrm{chr} 16$ & $70,016,280$ & $70,183,624$ & duplication & PD & not rare \\
\hline $184269 \mathrm{chr} 16$ & $78,372,894$ & $78,383,509$ & deletion & - & not rare \\
\hline 184269 chr17 & $73,334,770$ & $73,346,460$ & deletion & - & not rare \\
\hline 184269 chr18 & $14,549,799$ & $14,552,505$ & deletion & - & not rare \\
\hline 184269 chr19 & $53,518,747$ & $53,552,296$ & duplication & N-1 & not rare \\
\hline 184269 chr2 & $208,351,315$ & $208,357,838$ & deletion & - & not rare \\
\hline $184269 \mathrm{chr} 2$ & $29,098,459$ & $29,104,237$ & deletion & - & not rare \\
\hline $184269 \mathrm{chr} 2$ & $48,851,541$ & $48,856,770$ & deletion & - & not rare \\
\hline $184269 \mathrm{chr} 2$ & $51,926,599$ & $51,926,904$ & deletion & - & not rare \\
\hline 184269 chr2 & $52,759,890$ & $52,784,952$ & deletion & - & not rare \\
\hline $184269 \mathrm{chr} 2$ & $66,188,209$ & $66,196,726$ & deletion & - & t rare \\
\hline 184269 chr21 & $15,235,002$ & $15,270,083$ & duplication & - & not rare \\
\hline $184269 \mathrm{chr} 21$ & $23,655,900$ & $23,665,470$ & deletion & - & not rare \\
\hline $184269 \mathrm{chr} 3$ & $162,130,691$ & $162,142,475$ & deletion & - & not rare \\
\hline $184269 \mathrm{chr} 3$ & $195,433,728$ & $195,468,690$ & duplication & 20 & not rare \\
\hline 184269 chr3 & $6,651,929$ & $6,654,060$ & & - & \\
\hline 184269 chr3 & $89,402,409$ & $89,418,331$ & deletion & - & not rare \\
\hline $184269 \mathrm{chr} 4$ & $190,194,200$ & $190,200,463$ & deletion & - & not rare \\
\hline $184269 \mathrm{chr} 4$ & $34,785,955$ & $34,818,502$ & dele & - & not rare \\
\hline $184269 \mathrm{chr} 4$ & $70,137,897$ & $70,229,260$ & deletion & B28 & not rare \\
\hline 184269 chr5 & $174,436,246$ & $174,441,565$ & dele & & not rare \\
\hline 184269 chr6 & $29,855,945$ & $29,899,189$ & dele & & not rare \\
\hline 184269 chr6 & $69,687,698$ & $69,690,567$ & dele & - & not rare \\
\hline 184269 chr6 & $77,439,769$ & $77,451,301$ & & - & are \\
\hline 184269 chr7 & $38,388,889$ & $38,402,517$ & delet & - & not rare \\
\hline 184269 chr7 & $40,588,933$ & $40,602,105$ & deletion & - & not rare \\
\hline 184269 chr7 & $75,479,047$ & $75,489,038$ & & - & are \\
\hline 184269 chr7 & $97,396,709$ & $97,401,677$ & deletion & - & not rare \\
\hline 184269 chr8 & $115,633,704$ & $115,642,459$ & deletion & - & not rare \\
\hline 184269 chr8 & $16,262,222$ & $16,272,444$ & deletion & - & not rare \\
\hline 184269 chr8 & $39,232,581$ & $39,385,979$ & duplication & - & not rare \\
\hline 184269 chr8 & $51,029,371$ & $51,038,149$ & deletion & - & not rare \\
\hline 184269 chr8 & $51,226,087$ & $51,227,777$ & delet & - & not rare \\
\hline 184269 chr8 & $5,595,511$ & $5,605,706$ & deletion & - & not rare \\
\hline 184269 chr9 & $104,715,329$ & $104,722,688$ & deletion & - & not rare \\
\hline $184270 \mathrm{chr} 1$ & $149,032,299$ & $149,233,182$ & deletion & - & not rare \\
\hline 184270 chr1 & $213,002,088$ & $213,020,221$ & deletion & SPATA45 & not rare \\
\hline 184270 chr1 & $248,740,572$ & $248,795,110$ & deletion & \multicolumn{2}{|c|}{ OR2T10;OF not rare } \\
\hline 184270 chr1 & $62,113,597$ & $62,119,136$ & deletion & - & not rare \\
\hline 184270 chr10 & $38,789,617$ & $38,877,428$ & duplication & - & not rare \\
\hline 184270 chr11 & $55,370,325$ & $55,419,073$ & deletion & \multicolumn{2}{|c|}{$\mathrm{OR} 4 \mathrm{P} 4 ; \mathrm{OR} \angle$ not rare } \\
\hline 184270 chr12 & $129,528,229$ & $129,539,946$ & deletion & - & not rare \\
\hline 184270 chr13 & $38,076,308$ & $38,085,537$ & deletion & - & not rare \\
\hline 184270 chr14 & $19,457,976$ & $20,424,184$ & deletion & \multicolumn{2}{|c|}{ OR4K5;OR $\angle$ not rare } \\
\hline
\end{tabular}




\begin{tabular}{|c|c|c|c|c|c|}
\hline 184270 chr15 & 97,8 & $97,831,832$ & deletion & - & not rare \\
\hline 184270 chr16 & $34,471,298$ & $34,756,258$ & duplication & - & not rare \\
\hline 84270 chr17 & $34,438,753$ & $34,483,342$ & duplication & - & ot rare \\
\hline 84270 chr17 & $44,166,604$ & $44,225,181$ & duplication & KANSL1 & not rare \\
\hline L4270 chr17 & $75,266,251$ & $75,271,787$ & deletion & - & not rare \\
\hline $184270 \mathrm{chr} 18$ & $67,208,806$ & $67,217,271$ & deletion & - & not rare \\
\hline 84270 chr19 & $43,647,130$ & $43,744,060$ & deletion & PSG4;PSG5 & not rare \\
\hline 184270 chr19 & $54,728,767$ & $54,743,217$ & duplication & LILRA6 & not rare \\
\hline 184270 chr19 & $55,233,850$ & $55,339,383$ & duplication & KIR2DL3;KI & not rare \\
\hline 184270 chr2 & $184,795,725$ & $184,803,456$ & deletion & - & not rare \\
\hline 184270 chr2 & $212,770,611$ & $212,773,893$ & deletion & - & not rare \\
\hline 184270 chr2 & $213,187,034$ & $213,191,389$ & deletion & - & not rare \\
\hline 184270 chr21 & $47,387,112$ & $47,433,055$ & deletion & COL6A1 & not rare \\
\hline 184270 chr22 & $37,745,101$ & $37,749,946$ & deletion & - & are \\
\hline $184270 \mathrm{chr} 3$ & $162,130,691$ & $162,142,475$ & deletion & - & not rare \\
\hline 184270 chr3 & 82,010 & 130,397 & duplication & - & not rare \\
\hline $184270 \mathrm{chr} 4$ & $171,267,562$ & $171,273,360$ & deletion & - & not rare \\
\hline $184270 \mathrm{chr} 4$ & $34,785,955$ & $34,818,502$ & deletion & - & not rare \\
\hline $184270 \mathrm{chr} 4$ & $69,374,369$ & $69,489,473$ & tion & 17 & are \\
\hline 184270 chr5 & $140,225,908$ & $140,238,311$ & deletion & PCDHA10;F & F not rare \\
\hline 184270 chr5 & $161,111,588$ & $161,132,478$ & deletion & GABRA6 & rare \\
\hline 184270 chr5 & $7,178,644$ & $7,191,074$ & dele & - & not rare \\
\hline 184270 chr5 & $8,258,458$ & $8,260,630$ & 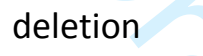 & & not rare \\
\hline 184270 chr6 & $139,603,393$ & $139,606,731$ & & & are \\
\hline 184270 chr6 & $29,855,945$ & $29,899,189$ & dele & & not \\
\hline 184270 chr7 & $7,292,458$ & $7,324,800$ & deletion & 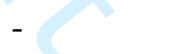 & rare \\
\hline 184270 chr8 & $115,634,278$ & $115,642,459$ & & - & \\
\hline 184270 chr8 & $24,972,808$ & $24,990,418$ & duplication & - & not rare \\
\hline 184270 chr8 & $39,232,581$ & $39,385,979$ & deletion & - & not rare \\
\hline 184270 chr8 & 47,526,999 & $47,539,088$ & duplication & - & not rare \\
\hline 184270 chr9 & $135,947,165$ & $135,957,452$ & duplication & - & not rare \\
\hline 184270 chr9 & $23,363,526$ & $23,376,817$ & duplication & - & not rare \\
\hline 184270 chr9 & $78,004,378$ & $78,011,616$ & deletion & - & not rare \\
\hline 184270 chr9 & $85,373,362$ & $85,383,934$ & deletion & - & not rare \\
\hline 184271 chr1 & $16,367,339$ & $16,380,869$ & duplication & CLCNKB & not rare \\
\hline 184271 chr1 & $213,002,088$ & $213,011,812$ & deletion & SPATA45 & not rare \\
\hline 184271 chr10 & $132,635,459$ & $132,638,422$ & deletion & - & not rare \\
\hline 184271 chr10 & $35,927,293$ & $35,936,753$ & deletion & FZD8 & not rare \\
\hline 184271 chr10 & $71,282,981$ & $71,289,190$ & deletion & - & not rare \\
\hline 184271 chr11 & $121,739,802$ & $121,740,944$ & deletion & - & not rare \\
\hline 184271 chr11 & $134,602,333$ & $134,607,484$ & deletion & - & not rare \\
\hline 184271 chr11 & $55,365,761$ & $55,427,700$ & deletion & \multicolumn{2}{|c|}{$\mathrm{OR} 4 \mathrm{P} 4 ; \mathrm{OR} \angle$ not rare } \\
\hline 184271 chr12 & $11,511,132$ & $11,543,084$ & duplication & - & not rare \\
\hline 184271 chr12 & $33,300,995$ & $33,305,855$ & deletion & - & not rare \\
\hline 184271 chr12 & $43,021,431$ & $43,027,453$ & deletion & - & not rare \\
\hline 184271 chr14 & $106,891,524$ & $106,931,129$ & deletion & - & not rare \\
\hline 184271 chr14 & $38,058,924$ & $38,065,813$ & deletion & FOXA1 & not rare \\
\hline
\end{tabular}




\begin{tabular}{|c|c|c|c|c|c|}
\hline 84271 chr14 & 44 & $44,718,622$ & deletion & - & not rare \\
\hline 84271 chr14 & $65,710,580$ & $65,712,420$ & deletion & - & not rare \\
\hline 84271 chr15 & $20,434,155$ & $21,241,118$ & duplication & \multicolumn{2}{|c|}{ GOLGA6L6; not rare } \\
\hline 84271 chr15 & $22,425,916$ & $22,514,794$ & duplication & - & not rare \\
\hline 84271 chr16 & $19,945,540$ & $19,962,636$ & deletion & - & not rare \\
\hline 184271 chr16 & $27,337,036$ & $27,350,687$ & deletion & - & t rare \\
\hline 84271 chr16 & $32,562,332$ & $32,609,440$ & duplication & - & not rare \\
\hline 184271 chr16 & $70,155,033$ & $70,189,705$ & deletion & PDPR & not rare \\
\hline 184271 chr16 & $85,302,676$ & $85,304,326$ & deletion & - & not rare \\
\hline 184271 chr17 & $44,166,604$ & $44,213,925$ & duplication & KANSL1 & not rare \\
\hline 184271 chr19 & $11,777,889$ & $11,781,456$ & deletion & - & not rare \\
\hline 184271 chr19 & $24,460,170$ & $24,462,326$ & deletion & - & not rare \\
\hline 184271 chr19 & $35,852,103$ & $35,861,695$ & deletion & - & not rare \\
\hline 184271 chr19 & $54,728,767$ & $54,743,217$ & duplication & & not rare \\
\hline $184271 \mathrm{chr} 2$ & $129,638,609$ & $129,645,321$ & deletion & - & not rare \\
\hline 184271 chr2 & $184,085,551$ & $184,088,256$ & deletion & - & not rare \\
\hline 184271 chr2 & $208,351,315$ & $208,357,838$ & deletion & - & not rare \\
\hline 184271 chr2 & $212,771,670$ & $212,773,893$ & deletion & - & not rare \\
\hline 184271 chr2 & $213,187,034$ & $213,191,389$ & & - & \\
\hline 184271 chr2 & $51,926,599$ & $51,926,904$ & deletion & - & not rare \\
\hline 184271 chr21 & $10,803,242$ & $10,833,351$ & cation & - & not rare \\
\hline 184271 chr21 & $34,601,021$ & $34,602,321$ & del & - & not rare \\
\hline 184271 chr22 & $37,745,101$ & $37,749,946$ & de & & not rare \\
\hline 184271 chr22 & $39,359,885$ & $39,385,663$ & dele & \multicolumn{2}{|l|}{ АРОВЕСЗВ } \\
\hline 184271 chr3 & $162,130,691$ & $162,145,531$ & dele & & not rare \\
\hline $184271 \mathrm{chr} 4$ & $63,645,262$ & $63,681,840$ & dupl & 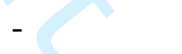 & not rare \\
\hline $184271 \mathrm{chr} 4$ & $64,136,609$ & $64,154,477$ & & - & \\
\hline $184271 \mathrm{chr} 4$ & $64,697,704$ & $64,709,753$ & deletion & - & not rare \\
\hline $184271 \mathrm{chr} 4$ & $70,137,897$ & $70,229,260$ & deletion & 328 & not rare \\
\hline 184271 chr5 & $155,477,866$ & $155,488,917$ & deletion & - & not rare \\
\hline 184271 chr5 & $32,106,628$ & $32,164,826$ & duplication & \multicolumn{2}{|c|}{ GOLPH3;P[ not rare } \\
\hline 184271 chr5 & $45,939,294$ & $45,958,465$ & deletion & - & not rare \\
\hline 184271 chr5 & $80,501,981$ & $80,600,006$ & duplication & \multicolumn{2}{|c|}{ CKMT2;RA!rare } \\
\hline 184271 chr5 & $99,198,267$ & $99,231,572$ & duplication & - & not rare \\
\hline 184271 chr6 & $139,601,136$ & $139,606,731$ & deletion & - & not rare \\
\hline 184271 chr6 & $29,856,693$ & $29,899,189$ & deletion & - & not rare \\
\hline 184271 chr7 & $134,131,341$ & $134,156,417$ & deletion & 1B1 & rare \\
\hline 184271 chr7 & $75,664,539$ & $75,667,743$ & deletion & - & not rare \\
\hline 184271 chr8 & $15,401,929$ & $15,411,867$ & deletion & - & not rare \\
\hline 184271 chr8 & $39,236,582$ & $39,385,262$ & deletion & - & not rare \\
\hline 184271 chr8 & $85,261,157$ & $85,268,951$ & deletion & - & not rare \\
\hline 184271 chr9 & $121,689,662$ & $121,756,868$ & duplication & - & rare \\
\hline 184271 chr9 & $23,363,526$ & $23,376,817$ & duplication & - & not rare \\
\hline 184271 chr9 & $85,373,362$ & $85,383,934$ & deletion & - & not rare \\
\hline 184273 chr 1 & $12,846,082$ & $12,920,067$ & duplication & \multicolumn{2}{|c|}{ HNRNPCL1 not rare } \\
\hline 184273 chr1 & $161,559,327$ & $161,619,741$ & duplication & \multicolumn{2}{|c|}{ FCGR2C;FC not rare } \\
\hline 184273 chr1 & $187,716,107$ & $187,722,032$ & deletion & - & not rare \\
\hline
\end{tabular}




\begin{tabular}{|c|c|c|c|c|c|}
\hline 184273 chr1 & $213,002,088$ & $213,011,812$ & deletion & SPATA45 & not rare \\
\hline 84273 chr1 & $22,316,110$ & $22,336,305$ & deletion & CELA3A & not rare \\
\hline 34273 chr1 & $248,151,999$ & $248,215,316$ & deletion & \multicolumn{2}{|c|}{ OR2L5;OR2 not rare } \\
\hline 84273 chr1 & $248,740,572$ & $248,795,110$ & deletion & \multicolumn{2}{|c|}{ OR2T10;OF not rare } \\
\hline 84273 chr1 & $73,011,443$ & $73,090,527$ & deletion & _ & not rare \\
\hline 184273 chr10 & $135,242,873$ & $135,381,927$ & duplication & \multicolumn{2}{|c|}{ SYCE1;CYP; not rare } \\
\hline 184273 chr10 & $39,058,671$ & $39,154,535$ & duplication & - & not rare \\
\hline 184273 chr10 & $46,949,421$ & $47,940,417$ & duplication & \multicolumn{2}{|c|}{ ANTXRL;NF not rare } \\
\hline 184273 chr10 & $76,372,037$ & $76,374,526$ & deletion & - & not rare \\
\hline 184273 chr11 & $134,602,333$ & $134,607,484$ & deletion & - & not rare \\
\hline 184273 chr11 & $67,511,694$ & $67,731,956$ & deletion & - & not rare \\
\hline 184273 chr11 & $90,193,427$ & $90,195,742$ & deletion & - & not rare \\
\hline 184273 chr11 & $93,020,527$ & $93,028,940$ & deletion & - & not rare \\
\hline 184273 & $93,683,680$ & $93,688,541$ & deletion & - & not rare \\
\hline 184273 chr12 & $2,245,636$ & $2,254,219$ & deletion & - & not rare \\
\hline 184273 chr12 & $31,907,259$ & $31,909,635$ & deletion & - & not rare \\
\hline 184273 chr12 & $59,935,926$ & $59,942,122$ & deletion & - & not \\
\hline 184273 chr13 & $24,783,504$ & $24,794,329$ & deletion & - & are \\
\hline 184273 chr13 & $95,984,005$ & $95,999,229$ & deletion & - & not rare \\
\hline 184273 chr14 & $20,197,311$ & $20,424,184$ & duplication & \multicolumn{2}{|c|}{ OR4K5;OR $\angle$ not rare } \\
\hline 184273 & $21,903,815$ & $22,753,733$ & duplication & \multicolumn{2}{|c|}{ GOLGA6L2: not rare } \\
\hline 184273 chr15 & $24,486,234$ & $24,714,849$ & deletion & - & not rare \\
\hline 184273 chr16 & $19,945,540$ & $19,962,636$ & & & not rare \\
\hline 184273 chr16 & $27,336,427$ & $27,350,687$ & dele & & rare \\
\hline 184273 chr16 & $35,185,903$ & $35,197,323$ & del & & not rare \\
\hline 184273 chr16 & $55,800,009$ & $55,819,443$ & del & & \\
\hline 184273 chr16 & $55,843,252$ & $55,865,127$ & duplication & CES1 & not rare \\
\hline 184273 chr16 & $85,502,428$ & $85,509,167$ & deletion & - & not rare \\
\hline 184273 & 48,3 & $48,318,328$ & on & - & are \\
\hline 184273 chr18 & $69,428,556$ & $69,441,154$ & deletion & - & not rare \\
\hline $184273 \mathrm{chr} 2$ & $172,239,759$ & $172,512,646$ & duplication & \multicolumn{2}{|c|}{ METTL8;DC rare } \\
\hline $184273 \mathrm{chr} 2$ & $195,364,534$ & $195,366,958$ & deletion & - & not rare \\
\hline $184273 \mathrm{chr} 2$ & $212,873,497$ & $212,938,165$ & deletion & - & rare \\
\hline $184273 \mathrm{chr} 2$ & $34,697,895$ & $34,726,904$ & duplication & - & not rare \\
\hline 184273 chr2 & $48,851,541$ & $48,856,770$ & deletion & - & not rare \\
\hline $184273 \mathrm{chr} 2$ & $57,203,475$ & $57,213,161$ & deletion & - & not rare \\
\hline 184273 & $78,707,767$ & $78,711,422$ & deletion & - & are \\
\hline 184273 chr3 & $195,433,728$ & $195,468,690$ & duplication & $\mathrm{MI}$ & not rare \\
\hline 184273 chr3 & $50,854,125$ & $50,878,734$ & deletion & - & rare \\
\hline 184273 chr3 & $65,189,366$ & $65,214,685$ & deletion & - & not rare \\
\hline $184273 \mathrm{chr} 4$ & $25,679,209$ & $25,682,826$ & deletion & - & not rare \\
\hline 184273 chr4 & $34,785,955$ & $34,818,502$ & deletion & - & not rare \\
\hline $184273 \mathrm{chr} 4$ & $39,705,484$ & $39,713,134$ & deletion & - & not rare \\
\hline $184273 \mathrm{chr} 4$ & $92,280,583$ & $92,284,935$ & deletion & - & not rare \\
\hline 184273 chr5 & $151,514,956$ & $151,518,615$ & deletion & - & not rare \\
\hline 184273 & $17,345,282$ & $17,356,536$ & deletion & - & not rare \\
\hline 184273 & $180,176,005$ & $180,195,526$ & duplicatic & 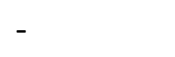 & not rar \\
\hline
\end{tabular}




\begin{tabular}{|c|c|c|c|c|c|}
\hline $184273 \mathrm{chr} 5$ & $7,178,644$ & $7,191,074$ & deletion & - & not rare \\
\hline 84273 chr6 & $78,972,930$ & $79,035,739$ & deletion & - & not rare \\
\hline 184273 chr7 & $141,766,850$ & $141,793,521$ & deletion & - & not rare \\
\hline 34273 chr7 & $62,149,006$ & $62,162,385$ & deletion & - & not rare \\
\hline 184273 chr8 & $115,633,704$ & $115,650,409$ & deletion & - & not rare \\
\hline 184273 chr8 & $24,972,808$ & $24,990,418$ & duplication & - & not rare \\
\hline 184273 chr8 & $39,236,582$ & $39,385,979$ & deletion & - & not rare \\
\hline 184273 chr8 & $5,595,511$ & $5,605,706$ & deletion & - & not rare \\
\hline 184273 chr9 & $23,363,526$ & $23,376,817$ & deletion & - & not rare \\
\hline $184273 \mathrm{chrX}$ & $138,863,360$ & $139,290,872$ & duplication & \multicolumn{2}{|c|}{ LOC389895 not rare } \\
\hline $184273 \mathrm{chrX}$ & $6,648,590$ & $7,103,283$ & duplication & PUDP & not rare \\
\hline 184275 chr1 & $149,032,299$ & $149,215,927$ & duplication & - & not rare \\
\hline 184275 chr10 & $47,141,927$ & $47,756,065$ & duplication & \multicolumn{2}{|c|}{ ANXA8;AN; not rare } \\
\hline 184275 chr10 & $58,516,470$ & $58,526,153$ & deletion & - & not rare \\
\hline 184275 chr11 & $18,949,220$ & $18,961,743$ & duplication & MRGPRX1 & not rare \\
\hline 184275 chr11 & $90,193,796$ & $90,195,742$ & deletion & - & not rare \\
\hline 184275 chr12 & $12,532,984$ & $12,544,338$ & deletion & - & not rare \\
\hline 184275 chr12 & $129,230,513$ & $129,233,013$ & deletion & - & not rare \\
\hline 184275 chr12 & $1,422,253$ & $1,549,146$ & duplication & ERC1 & rare \\
\hline 184275 chr12 & $2,018,149$ & $2,674,893$ & duplication & \multicolumn{2}{|c|}{ CACNA1C; [ rare } \\
\hline 184275 chr13 & $34,134,809$ & $34,143,545$ & deletion & - & not rare \\
\hline 184275 chr13 & $61,500,155$ & $61,508,150$ & & - & \\
\hline 184275 chr13 & $83,788,742$ & $83,791,615$ & dele & & not rare \\
\hline 184275 chr14 & $106,891,524$ & $106,931,129$ & dele & & not rare \\
\hline 184275 chr14 & 41,472 & $41,483,267$ & delet & & not rare \\
\hline 184275 chr15 & $63,267,778$ & $63,269,100$ & deletion & - & not rare \\
\hline 184275 chr16 & $78,702,508$ & $78,761,364$ & deletion & - & rare \\
\hline 184275 chr17 & $34,438,753$ & $34,483,342$ & duplication & - & not rare \\
\hline 184275 chr17 & $7,264,717$ & $7,265,681$ & deletion & - & not rare \\
\hline 184275 & $41,976,831$ & $41,982,102$ & dele & - & not rare \\
\hline 184275 chr19 & $54,728,767$ & $54,743,217$ & deletion & LIL & not rare \\
\hline 184275 chr2 & $184,085,551$ & $184,088,256$ & deletion & - & not rare \\
\hline 184275 chr22 & $44,321,641$ & $44,377,557$ & duplication & \multicolumn{2}{|c|}{ PNPLA3;SA rare } \\
\hline 184275 chr3 & $11,411,823$ & $11,414,339$ & deletion & - & not rare \\
\hline 184275 chr3 & $53,032,639$ & $53,042,099$ & deletion & - & not rare \\
\hline 184275 chr3 & $90,467,441$ & $90,477,597$ & deletion & - & not rare \\
\hline 184275 chr4 & $168,808,600$ & $168,992,519$ & deletion & - & not rare \\
\hline 184275 chr4 & $34,785,955$ & $34,818,502$ & deletion & - & not rare \\
\hline $184275 \mathrm{chr} 4$ & $69,374,369$ & $69,489,473$ & duplication & UGT2B17 & not rare \\
\hline 184275 chr4 & $9,370,814$ & $9,504,845$ & duplication & DEFB131 & not rare \\
\hline 184275 chr5 & $151,514,956$ & $151,518,615$ & deletion & - & not rare \\
\hline 184275 chr5 & $17,623,605$ & $17,642,516$ & deletion & - & not rare \\
\hline 184275 chr6 & $124,432,882$ & $124,470,796$ & duplication & NKAIN2 & not rare \\
\hline 184275 chr6 & $162,329,287$ & $162,438,621$ & duplication & PARK2 & rare \\
\hline 184275 & $31,338,528$ & $31,341,357$ & deletion & - & not rare \\
\hline 184275 chr6 & $57,140,831$ & $57,206,230$ & deletion & & rare \\
\hline 184275 chr6 & $78,972,930$ & $79,035,739$ & deletion & - & not rare \\
\hline
\end{tabular}




\begin{tabular}{|c|c|c|c|c|c|}
\hline 184275 chr8 & $39,232,581$ & $39,385,979$ & duplication & - & not rare \\
\hline 184275 chr8 & $81,990,903$ & $81,993,964$ & deletion & - & not rare \\
\hline 84275 chr8 & $87,180,635$ & $87,331,746$ & duplication & SLC7A13 & not rare \\
\hline 84275 chr8 & $9,108,843$ & $9,116,575$ & deletion & - & not rare \\
\hline 184275 chr9 & $16,978,385$ & $17,127,664$ & deletion & - & rare \\
\hline 184275 chr9 & $26,141,480$ & $26,770,758$ & duplication & - & rare \\
\hline 184275 chr9 & $78,004,378$ & $78,011,616$ & deletion & - & not rare \\
\hline $184275 \mathrm{chrX}$ & $69,439,260$ & $69,445,176$ & deletion & - & not rare \\
\hline 184277 chr1 & $104,104,990$ & $104,211,046$ & duplication & \multicolumn{2}{|c|}{ AMY1C;AN not rare } \\
\hline 184277 chr1 & $58,726,579$ & $58,733,753$ & duplication & - & not rare \\
\hline 184277 chr1 & 723,918 & 759,036 & duplication & - & rare \\
\hline 184277 chr1 & 776,546 & 849,998 & duplication & - & not rare \\
\hline 184277 chr1 & $8,359,110$ & $8,363,575$ & deletion & - & not rare \\
\hline 184277 chr10 & $135,242,873$ & $135,381,927$ & duplication & \multicolumn{2}{|c|}{ SYCE1;CYP; not rare } \\
\hline 184277 chr10 & $39,119,741$ & $39,154,535$ & duplication & - & not rare \\
\hline 184277 chr10 & $58,901,976$ & $58,936,939$ & deletion & - & not rare \\
\hline 184277 chr11 & $3,239,077$ & $3,243,665$ & deletion & MRGPRG & not rare \\
\hline 184277 chr11 & $55,365,761$ & $55,443,269$ & deletion & \multicolumn{2}{|c|}{$\mathrm{OR} 4 \mathrm{P} 4 ; \mathrm{OR} \angle$ not rare } \\
\hline 184277 & $81,501,218$ & $81,517,261$ & dele & - & not rare \\
\hline 184277 chr11 & $90,193,427$ & $90,195,742$ & deletion & - & not rare \\
\hline 184277 chr11 & $93,699,768$ & $93,705,299$ & deletion & - & not rare \\
\hline 184277 chr12 & $129,230,513$ & $129,233,013$ & deletion & - & not rare \\
\hline 184277 chr12 & $30,237,351$ & $30,243,631$ & dele & & not rare \\
\hline 184277 chr12 & $70,874,037$ & $70,879,131$ & & & not rare \\
\hline 184277 chr12 & $99,994,315$ & $100,008,773$ & dele & & not rare \\
\hline 184277 chr14 & $20,361,945$ & $20,422,799$ & duplication & \multicolumn{2}{|c|}{ OR4K5;OR $\angle$ not rare } \\
\hline 184277 & $26,751,068$ & $26,752,373$ & deletion & - & are \\
\hline 184277 chr17 & $10,890,859$ & $10,893,799$ & deletion & - & not rare \\
\hline 184277 chr17 & $47,983,805$ & $47,985,668$ & deletion & - & not rare \\
\hline 184277 chr17 & $70,562,063$ & $70,565,828$ & deletion & - & not rare \\
\hline 184277 chr2 & $110,852,875$ & $110,982,530$ & duplication & \multicolumn{2}{|c|}{ MALL;NPHI not rare } \\
\hline 184277 chr2 & $123,477,465$ & $123,482,301$ & deletion & - & not rare \\
\hline 184277 chr2 & $242,854,089$ & $243,034,519$ & deletion & - & not rare \\
\hline 184277 chr2 & $4,212,725$ & $4,222,144$ & deletion & - & not rare \\
\hline 184277 chr2 & $89,540,620$ & $90,256,530$ & deletion & - & not rare \\
\hline 184277 chr21 & $10,818,766$ & $10,858,651$ & duplication & - & not rare \\
\hline 184277 chr21 & $23,655,900$ & $23,665,470$ & deletion & - & not rare \\
\hline 184277 chr3 & $104,778,910$ & $104,786,447$ & deletion & - & not rare \\
\hline 184277 chr3 & $128,382,258$ & $128,412,024$ & duplication & - & not rare \\
\hline 184277 chr3 & $131,711,896$ & $131,712,898$ & deletion & - & not rare \\
\hline 184277 chr3 & $192,777,710$ & $192,782,294$ & deletion & - & not rare \\
\hline 184277 chr3 & $20,990,739$ & $20,996,035$ & deletion & - & not rare \\
\hline 184277 chr3 & $37,979,882$ & $37,986,734$ & deletion & - & not rare \\
\hline 184277 chr3 & $65,117,947$ & $65,118,762$ & deletion & - & not rare \\
\hline 184277 chr4 & $145,199,920$ & $145,211,387$ & deletion & - & not rare \\
\hline 184277 chr4 & $176,491,850$ & $176,496,877$ & deletion & - & not rare \\
\hline 184277 chr4 & $69,374,369$ & $69,467,200$ & deletion & UGT2B17 & not rare \\
\hline
\end{tabular}




\begin{tabular}{|c|c|c|c|c|c|}
\hline 84277 chr5 & 106, & 106 & & - & not rare \\
\hline 184277 chr5 & $115,926,603$ & $116,282,454$ & deletion & - & rare \\
\hline 84277 chr5 & $120,054,967$ & $120,067,140$ & duplication & - & not rare \\
\hline 84277 chr5 & $32,106,628$ & $32,164,826$ & duplication & \multicolumn{2}{|c|}{ GOLPH3;P[ not rare } \\
\hline 184277 chr5 & 762,435 & 825,338 & duplication & ZDHHC11 & not rare \\
\hline $184277 \mathrm{c}$ & $21,826,729$ & $21,834,059$ & deletion & - & not rare \\
\hline 84277 chr6 & $29,855,945$ & $29,899,189$ & deletion & - & not rare \\
\hline 184277 chr6 & $32,458,168$ & $32,520,879$ & deletion & HLA-DRB5 & not rare \\
\hline 184277 chr6 & $76,829,307$ & $76,847,454$ & duplication & - & not rare \\
\hline 184277 chr7 & $111,042,920$ & $111,127,053$ & deletion & - & not rare \\
\hline 184277 chr7 & $141,766,850$ & $141,793,521$ & deletion & - & not rare \\
\hline 184277 chr7 & 151,829 & 159,207 & deletion & - & not rare \\
\hline 184277 chr7 & $29,688,702$ & $29,690,262$ & deletion & - & not rare \\
\hline 184277 chr7 & $38,388,889$ & $38,402,517$ & deletion & - & not rare \\
\hline 184277 chr7 & $91,033,074$ & $91,041,618$ & deletion & - & not rare \\
\hline 184277 chr8 & $15,947,559$ & $16,023,673$ & deletion & MSR1 & not rare \\
\hline $184277 \mathrm{chrX}$ & $16,346,245$ & $16,353,797$ & deletion & - & not rare \\
\hline 184279 chr1 & $104,103,057$ & $104,211,046$ & duplication & \multicolumn{2}{|c|}{ AMY1C;AN not rare } \\
\hline 184279 & 12,9 & 12,9 & & PRAMEF2 & \\
\hline 184279 chr1 & $1,585,642$ & $1,628,197$ & duplication & SLC35E2B & not rare \\
\hline 184279 chr1 & $236,139,894$ & $236,157,433$ & duplication & NID1 & not rare \\
\hline 184279 chr1 & $25,598,276$ & $25,659,509$ & deletion & RHD & not rare \\
\hline 184279 chr1 & $45,635,175$ & $45,645,069$ & dele & & not rare \\
\hline 184279 chr10 & $38,789,617$ & $38,877,428$ & dup & - & not rare \\
\hline 184279 chr11 & $3,239,077$ & $3,243,665$ & deletion & & not rare \\
\hline 184279 chr11 & $90,947,633$ & $90,949,496$ & duplication & - & not rare \\
\hline 184279 & 91,2 & 91,23 & dup & - & are \\
\hline 184279 chr12 & $59,935,926$ & $59,942,122$ & deletion & - & not rare \\
\hline 184279 chr13 & $111,681,665$ & $111,700,050$ & deletion & - & not rare \\
\hline 184279 chr13 & $38,076,308$ & $38,085,537$ & deletion & - & not rare \\
\hline 184279 chr15 & $22,299,434$ & $22,477,128$ & duplication & \multicolumn{2}{|c|}{ OR4M2;OR not rare } \\
\hline 184279 chr16 & $55,800,009$ & $55,819,443$ & deletion & - & not rare \\
\hline 184279 chr17 & $10,890,859$ & $10,893,799$ & deletion & - & not rare \\
\hline 184279 chr18 & $41,976,831$ & $41,982,102$ & deletion & - & not rare \\
\hline 184279 chr19 & $20,601,006$ & $20,717,536$ & deletion & - & not rare \\
\hline 184279 chr2 & $123,891,492$ & $124,011,120$ & duplication & - & rare \\
\hline 184279 chr20 & $52,647,631$ & $52,656,535$ & deletion & - & not rare \\
\hline 184279 chr21 & $23,655,900$ & $23,665,470$ & deletion & - & not rare \\
\hline 184279 chr21 & $35,724,739$ & $35,906,068$ & duplication & \multicolumn{2}{|c|}{$\mathrm{KCNE} 2 ; \mathrm{KCA}$ not rare } \\
\hline 184279 chr3 & $172,396,155$ & $172,399,955$ & duplication & - & not rare \\
\hline 184279 chr3 & $37,979,882$ & $37,986,734$ & deletion & - & not rare \\
\hline 184279 chr3 & $65,117,947$ & $65,118,762$ & deletion & - & not rare \\
\hline $184279 \mathrm{chr} 4$ & $122,285,263$ & $122,289,863$ & deletion & - & not rare \\
\hline $184279 \mathrm{chr} 4$ & 123,872 & 240,079 & duplication & $\mathrm{ZN}$ & rare \\
\hline $184279 \mathrm{chr} 4$ & $138,091,446$ & $138,098,601$ & deletion & - & not rare \\
\hline 184279 chr4 & $152,790,580$ & $152,793,891$ & deletion & - & not rare \\
\hline $184279 \mathrm{chr} 4$ & $69,374,369$ & $69,489,473$ & duplication & UGT2B17 & not rare \\
\hline
\end{tabular}




\begin{tabular}{|c|c|c|c|c|c|}
\hline 84279 chr5 & $110,417,428$ & 110, & ion & WI & not rare \\
\hline 184279 chr5 & $113,327,484$ & $113,335,230$ & deletion & - & not rare \\
\hline 84279 chr5 & $115,926,603$ & $116,282,454$ & deletion & - & rare \\
\hline 84279 chr5 & $32,106,628$ & $32,164,826$ & duplication & \multicolumn{2}{|c|}{ GOLPH3;P[ not rare } \\
\hline $184279 \mathrm{chr} 7$ & $38,388,889$ & $38,402,517$ & deletion & - & not rare \\
\hline 184279 chr8 & $16,262,222$ & $16,272,444$ & deletion & - & ot rare \\
\hline 84279 chr8 & $2,588,813$ & $2,591,021$ & deletion & - & not rare \\
\hline 184279 chr8 & $72,214,753$ & $72,217,690$ & deletion & - & not rare \\
\hline 184279 chr9 & $104,715,329$ & $104,722,688$ & deletion & - & not rare \\
\hline $184298 \mathrm{chr} 1$ & $161,559,327$ & $161,619,741$ & duplication & \multicolumn{2}{|c|}{ FCGR2C;FC not rare } \\
\hline 184298 chr10 & $47,141,927$ & $47,703,946$ & duplication & \multicolumn{2}{|c|}{ FAM25C;Al not rare } \\
\hline 184298 chr11 & $134,602,333$ & $134,607,484$ & deletion & - & not rare \\
\hline 184298 chr11 & $18,949,220$ & $18,961,743$ & duplication & MRGPRX1 & not rare \\
\hline 184298 chr11 & $3,239,077$ & $3,243,435$ & deletion & RG & not rare \\
\hline 184298 chr11 & $49,715,154$ & $49,757,690$ & deletion & - & not rare \\
\hline 184298 & $81,501,218$ & $81,517,261$ & deletion & - & rare \\
\hline 184298 chr12 & $12,532,984$ & $12,541,869$ & deletion & - & not rare \\
\hline 184298 chr12 & $30,237,351$ & $30,243,473$ & deletion & - & rare \\
\hline 184298 & $59,935,926$ & $59,942,122$ & & - & are \\
\hline 184298 chr12 & $70,874,037$ & $70,879,131$ & deletion & - & not rare \\
\hline 184298 chr13 & $34,134,809$ & $34,143,545$ & dele & - & not rare \\
\hline 184298 chr14 & $41,610,224$ & $41,661,685$ & dele & - & not rare \\
\hline 184298 chr14 & $86,285,493$ & $86,312,888$ & del & & not rare \\
\hline 184298 chr15 & $54,263,271$ & $54,270,291$ & & & ot rare \\
\hline 184298 chr15 & $60,086,249$ & $60,103,464$ & dele & & not rare \\
\hline 184298 chr16 & $32,562,332$ & $32,609,440$ & ation & - & not rare \\
\hline 184298 & $32,642,384$ & $33,827,904$ & duplication & \multicolumn{2}{|c|}{ TP53TG3B; not rare } \\
\hline 184298 chr16 & $55,800,009$ & $55,819,443$ & deletion & - & not rare \\
\hline 184298 chr16 & $71,356,102$ & $71,360,594$ & deletion & - & not rare \\
\hline 184298 chr2 & $123,477,465$ & $123,482,301$ & deletion & - & not rare \\
\hline 184298 chr2 & $227,343,513$ & $227,347,453$ & deletion & - & not rare \\
\hline 184298 chr2 & $32,630,551$ & $33,332,938$ & duplication & \multicolumn{2}{|c|}{ BIRC6;TTC2 not rare } \\
\hline 184298 chr2 & $34,697,895$ & $34,726,904$ & deletion & - & not rare \\
\hline 184298 chr2 & $97,738,917$ & $98,024,837$ & deletion & \multicolumn{2}{|c|}{ FAHD2B;Ar not rare } \\
\hline 184298 chr21 & $20,057,919$ & $20,078,801$ & duplication & - & not rare \\
\hline 184298 chr3 & $173,239,453$ & $173,290,024$ & duplication & - & not rare \\
\hline $184298 \mathrm{chr} 4$ & $190,194,200$ & $190,200,463$ & deletion & - & not rare \\
\hline $184298 \mathrm{chr} 4$ & $69,374,369$ & $69,489,473$ & duplication & 17 & not rare \\
\hline 184298 chr5 & $117,388,410$ & $117,393,058$ & deletion & - & not rare \\
\hline 184298 chr5 & $128,046,998$ & $128,072,042$ & duplication & - & rare \\
\hline 184298 chr5 & $155,477,866$ & $155,488,917$ & deletion & - & not rare \\
\hline 184298 chr6 & $19,041,346$ & $19,048,773$ & deletion & - & not rare \\
\hline 184298 chr6 & $29,855,945$ & $29,899,189$ & deletion & - & not rare \\
\hline 184298 chr6 & $30,994,010$ & $30,994,787$ & deletion & ML & not rare \\
\hline 184298 chr6 & $66,074,421$ & $66,080,908$ & deletion & - & not rare \\
\hline 184298 & $142,476,094$ & $142,484,042$ & deletion & - & not rare \\
\hline $184298 \mathrm{chr} 7$ & $156,387,296$ & $156,394,307$ & deletion & - & not rare \\
\hline
\end{tabular}




\begin{tabular}{|c|c|c|c|c|c|}
\hline $184298 \mathrm{chr} 8$ & $115,634,278$ & $115,642,459$ & deletion & - & not rare \\
\hline $84298 \mathrm{chr} 8$ & $2,256,926$ & $2,263,719$ & deletion & - & not rare \\
\hline 184298 chr8 & $24,972,808$ & $24,990,418$ & duplication & - & ot rare \\
\hline 84298 chr8 & $5,595,511$ & $5,605,706$ & deletion & - & not rare \\
\hline 184298 chr9 & $107,799,020$ & $107,803,370$ & deletion & - & not rare \\
\hline 184298 chr9 & $26,567,999$ & $26,572,537$ & deletion & - & ot rare \\
\hline $184298 \mathrm{chrX}$ & $51,741,943$ & $51,760,413$ & deletion & - & not rare \\
\hline 184301 chr1 & $161,563,386$ & $161,619,741$ & duplication & \multicolumn{2}{|c|}{ FCGR2C;FC not rare } \\
\hline 184301 chr1 & $243,116,818$ & $243,338,216$ & duplication & CEP170 & rare \\
\hline 184301 chr10 & $58,846,575$ & $58,849,853$ & deletion & - & not rare \\
\hline 184301 chr11 & $25,750,699$ & $25,753,430$ & deletion & - & not rare \\
\hline 184301 chr11 & $86,304,402$ & $86,306,401$ & deletion & - & not rare \\
\hline 184301 chr11 & $90,193,796$ & $90,195,742$ & deletion & - & not rare \\
\hline 184301 & $93,027,130$ & $93,033,807$ & deletion & - & not rare \\
\hline 184301 & $32,785,284$ & $32,831,091$ & duplication & FGD4 & rare \\
\hline 184301 chr13 & $69,247,022$ & $69,267,981$ & deletion & - & not rare \\
\hline 184301 chr14 & $20,295,510$ & $20,422,799$ & duplication & \multicolumn{2}{|c|}{ OR4N2;OR، not rare } \\
\hline 184301 chr14 & $41,610,224$ & $41,661,685$ & deletion & - & not rare \\
\hline 184301 chr14 & $44,504,048$ & $44,531,417$ & deletion & - & not rare \\
\hline 184301 chr14 & $47,247,998$ & $47,255,362$ & deletion & - & not rare \\
\hline 184301 chr16 & $78,372,894$ & $78,383,509$ & deletion & - & not rare \\
\hline 184301 chr2 & $212,771,670$ & $212,776,546$ & & - & \\
\hline 184301 chr2 & $48,851,541$ & $48,856,770$ & dele & & not rare \\
\hline 184301 chr20 & $24,887,251$ & $24,893,947$ & del & & not rare \\
\hline $184301 \mathrm{chr} 3$ & 191,9 & 191,9 & & & \\
\hline 184301 chr3 & $37,979,882$ & $37,986,734$ & deletion & - & not rare \\
\hline $184301 \mathrm{chr} 4$ & $136,532,271$ & $136,606,270$ & deletion & - & not rare \\
\hline $184301 \mathrm{chr} 4$ & $60,325,010$ & $60,330,880$ & duplication & - & not rare \\
\hline 184301 chr4 & $69,374,369$ & $69,489,473$ & duplication & UGT2B17 & not rare \\
\hline $184301 \mathrm{chr} 4$ & $70,137,897$ & $70,229,260$ & deletion & UGT2B28 & not rare \\
\hline 184301 chr5 & $73,993,871$ & $74,073,542$ & duplication & \multicolumn{2}{|c|}{ NSA2;GFM rare } \\
\hline 184301 chr6 & $29,855,945$ & $29,899,189$ & deletion & - & not rare \\
\hline 184301 chr6 & $32,458,168$ & $32,556,530$ & deletion & \multicolumn{2}{|c|}{ HLA-DRB1; not rare } \\
\hline 184301 chr7 & $110,582,600$ & $110,585,307$ & deletion & - & not rare \\
\hline 184301 chr7 & $155,883,507$ & $155,885,143$ & deletion & - & not rare \\
\hline 184301 chr8 & $135,059,968$ & $135,065,947$ & deletion & - & not rare \\
\hline 184301 chr8 & $39,236,582$ & $39,382,903$ & deletion & - & not rare \\
\hline 184301 chr9 & $138,195,113$ & $138,195,756$ & deletion & - & not rare \\
\hline 184301 chr9 & $69,494,085$ & $69,992,206$ & deletion & - & not rare \\
\hline 184302 chr1 & $12,908,591$ & $12,919,928$ & deletion & PRAMEF2 & not rare \\
\hline 184302 chr1 & $55,114,469$ & $55,120,469$ & deletion & MROH7 & not rare \\
\hline 184302 chr10 & $38,774,749$ & $38,877,428$ & duplication & - & not rare \\
\hline 184302 chr10 & $58,846,575$ & $58,849,853$ & deletion & - & not rare \\
\hline 184302 chr11 & $18,949,220$ & $18,961,743$ & duplication & MRGPRX1 & not rare \\
\hline 184302 chr11 & $24,083,684$ & $24,140,697$ & deletion & - & not rare \\
\hline 184302 chr11 & $25,750,699$ & $25,753,430$ & deletion & - & not rare \\
\hline 184302 chr11 & $86,304,402$ & $86,306,401$ & deletion & - & not rare \\
\hline
\end{tabular}




\begin{tabular}{|c|c|c|c|c|c|}
\hline 184302 chr11 & 90 & $90,196,843$ & deletion & - & not rare \\
\hline 184302 chr12 & $11,220,827$ & $11,244,117$ & deletion & TAS2R43 & not rare \\
\hline 84302 chr12 & $132,131,087$ & $132,136,919$ & deletion & - & not rare \\
\hline 34302 chr13 & $69,247,022$ & $69,267,981$ & deletion & - & not rare \\
\hline 84302 chr14 & $19,457,976$ & $20,424,184$ & deletion & \multicolumn{2}{|c|}{ OR4K5;OR $\angle$ not rare } \\
\hline 184302 chr14 & $41,610,224$ & $41,661,685$ & deletion & - & not rare \\
\hline 184302 chr14 & $44,504,048$ & $44,531,417$ & deletion & - & not rare \\
\hline 184302 chr14 & $47,247,998$ & $47,255,362$ & deletion & - & not rare \\
\hline 184302 chr14 & $47,885,499$ & $47,911,283$ & deletion & - & rare \\
\hline 184302 chr16 & $32,531,934$ & $32,833,893$ & deletion & \multicolumn{2}{|c|}{ TP53TG3B; not rare } \\
\hline 184302 chr16 & $55,800,009$ & $55,819,443$ & deletion & - & not rare \\
\hline 184302 chr16 & $58,945,599$ & $58,948,100$ & deletion & - & not rare \\
\hline 184302 chr17 & $10,890,859$ & $10,893,799$ & deletion & - & not rare \\
\hline 184302 chr19 & $53,518,747$ & $53,552,296$ & duplication & ERVV-1 & not rare \\
\hline 184302 chr19 & $54,728,767$ & $54,743,217$ & duplication & LILRA6 & not rare \\
\hline 184302 chr2 & $40,048,031$ & $40,051,216$ & deletion & - & not rare \\
\hline 184302 chr2 & $48,851,541$ & $48,856,770$ & deletion & - & not rare \\
\hline 184302 chr2 & $92,293,128$ & $92,304,211$ & deletion & - & not rare \\
\hline 184302 chr20 &, 251 & $24,893,947$ & & - & are \\
\hline 184302 chr20 & $29,804,016$ & $29,809,681$ & duplication & - & not rare \\
\hline 184302 chr20 & $52,288,724$ & $52,290,223$ & deletion & - & not rare \\
\hline 184302 chr3 & $143,133,504$ & $143,167,322$ & deletion & - & rare \\
\hline 184302 chr3 & $191,989,376$ & $191,996,202$ & duplicatio & & not rare \\
\hline $184302 \mathrm{chr} 3$ & $37,979,882$ & $37,986,734$ & & & are \\
\hline 184302 chr3 & $53,032,639$ & $53,038,786$ & dele & & not rare \\
\hline 184302 chr3 & $89,402,409$ & $89,418,331$ & dele & - & not rare \\
\hline $184302 \mathrm{chr} 4$ & $10,396,298$ & $10,400,156$ & & - & are \\
\hline $184302 \mathrm{chr} 4$ & $138,092,298$ & $138,098,601$ & deletion & - & not rare \\
\hline $184302 \mathrm{chr} 4$ & $34,785,955$ & $34,818,502$ & deletion & - & not rare \\
\hline 184302 chr4 & $69,374,369$ & $69,489,473$ & duplication & 17 & not rare \\
\hline 184302 chr5 & $151,514,956$ & $151,518,615$ & deletion & - & not rare \\
\hline 184302 chr5 & $175,512,089$ & $175,638,369$ & duplication & FAM153B & not rare \\
\hline 184302 chr5 & $32,106,628$ & $32,164,826$ & duplication & \multicolumn{2}{|c|}{ GOLPH3;P[ not rare } \\
\hline 184302 chr6 & $165,129,935$ & $165,194,584$ & duplication & - & not rare \\
\hline 184302 chr6 & $29,856,693$ & $29,899,189$ & deletion & - & not rare \\
\hline 184302 chr6 & $30,994,010$ & $30,994,787$ & deletion & & not rare \\
\hline 184302 chr6 & $69,231,883$ & $69,241,954$ & duplication & - & not rare \\
\hline 184302 chr7 & $156,387,296$ & $156,394,307$ & deletion & - & not rare \\
\hline 184302 chr7 & $86,940,652$ & $86,948,364$ & deletion & - & not rare \\
\hline 184302 chr8 & $115,634,278$ & $115,650,409$ & deletion & - & not rare \\
\hline 184302 chr8 & $12,551,156$ & $12,560,152$ & deletion & - & not rare \\
\hline 184304 chr10 & $103,083,978$ & $103,281,308$ & duplication & BTRC & rare \\
\hline 184304 chr10 & $20,850,624$ & $20,857,365$ & deletion & - & not rare \\
\hline 184304 chr10 & $87,953,705$ & $87,959,563$ & deletion & - & not rare \\
\hline 184304 chr11 & $24,446,609$ & $24,451,830$ & deletion & - & not rare \\
\hline 184304 chr11 & $55,365,761$ & $55,443,269$ & deletion & \multicolumn{2}{|c|}{$\mathrm{OR} 4 \mathrm{P} 4 ; \mathrm{OR} \angle$ not rare } \\
\hline 184304 chr12 & $11,511,132$ & $11,543,084$ & duplication & - & not rare \\
\hline
\end{tabular}




\begin{tabular}{|c|c|c|c|c|c|}
\hline 84304 chr12 & 35 & 127 & & - & 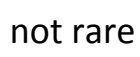 \\
\hline 84304 chr12 & $129,230,513$ & $129,233,013$ & deletion & - & not rare \\
\hline 34304 chr12 & $27,648,411$ & $27,657,025$ & deletion & SMCO2 & t rare \\
\hline 34304 chr12 & $63,942,707$ & $64,137,937$ & duplication & DPY19L2 & not rare \\
\hline 84304 chr14 & $20,341,645$ & $20,373,822$ & duplication & OR4K2 & not rare \\
\hline 84304 chr14 & $41,610,224$ & $41,661,685$ & deletion & - & ot rare \\
\hline 84304 chr15 & $24,680,494$ & $24,742,652$ & deletion & - & not rare \\
\hline 184304 chr17 & $33,681,335$ & $33,768,284$ & deletion & \multicolumn{2}{|c|}{ SLFN11;SLF not rare } \\
\hline 184304 chr17 & $44,166,604$ & $44,288,620$ & duplication & KANSL1 & not rare \\
\hline 184304 chr18 & $41,976,831$ & $41,982,102$ & deletion & - & not rare \\
\hline 184304 & $63,200,484$ & $63,206,335$ & duplication & - & are \\
\hline 184304 chr18 & $67,208,806$ & $67,217,271$ & deletion & - & not rare \\
\hline 184304 chr19 & $54,728,767$ & $54,743,217$ & duplication & LILRA6 & not rare \\
\hline 184304 chr2 & $123,477,465$ & $123,482,301$ & deletion & - & not rare \\
\hline 184304 chr2 & $53,682,501$ & $53,706,903$ & deletion & - & rare \\
\hline 184304 chr20 & $52,647,631$ & $52,656,535$ & dele & - & t rare \\
\hline 184304 chr3 & $162,130,691$ & $162,142,475$ & deletion & - & not rare \\
\hline 184304 chr3 & $175,887,314$ & $175,909,890$ & deletion & - & are \\
\hline 184304 chr3 & $191,065,392$ & $191,070,300$ & dele & - & \\
\hline 184304 chr3 & $6,651,929$ & $6,654,060$ & deletion & - & not rare \\
\hline $184304 \mathrm{chr} 4$ & $138,092,298$ & $138,098,601$ & dele & - & not rare \\
\hline 184304 chr4 & $171,269,504$ & $171,273,360$ & dele & - & not rare \\
\hline $184304 \mathrm{chr} 4$ & $34,785,955$ & $34,805,860$ & dupl & & not rare \\
\hline $184304 \mathrm{chr} 4$ & $64,697,704$ & $64,709,753$ & & & \\
\hline $184304 \mathrm{chr} 4$ & $69,374,369$ & $69,489,473$ & deletion & UGT2B17 & not rare \\
\hline 184304 chr5 & $117,388,410$ & $117,393,058$ & deletion & & not rare \\
\hline 184304 chr5 & $140,555,135$ & $140,558,618$ & ation & \multicolumn{2}{|c|}{ PCDHB8;PC not rare } \\
\hline 184304 chr5 & $3,315,071$ & $3,320,665$ & deletion & - & not rare \\
\hline 184304 chr5 & $75,431,699$ & $75,454,751$ & duplication & - & not rare \\
\hline 184304 chr6 & $124,432,882$ & $124,470,796$ & duplication & NKAIN2 & not rare \\
\hline 184304 chr6 & $151,862,142$ & $151,869,645$ & deletion & CCDC170 & not rare \\
\hline 184304 chr6 & $168,334,239$ & $168,597,816$ & duplication & \multicolumn{2}{|c|}{ MLLT4;KIFZ̈ not rare } \\
\hline 184304 chr6 & $31,276,513$ & $31,277,988$ & deletion & - & not rare \\
\hline 184304 chr6 & $78,972,930$ & $79,035,739$ & deletion & - & not rare \\
\hline 184304 chr7 & $97,396,709$ & $97,401,677$ & dele & - & are \\
\hline 184304 chr8 & $39,232,581$ & $39,385,979$ & deletion & - & not rare \\
\hline 184304 chr8 & $8,093,240$ & $8,148,289$ & duplication & - & not rare \\
\hline 184304 chr9 & $24,502,737$ & $24,518,795$ & deletion & - & not rare \\
\hline 184307 chr1 & $104,104,990$ & $104,211,046$ & duplication & \multicolumn{2}{|c|}{ AMY1C;AN not rare } \\
\hline 184307 chr1 & $11,656,684$ & $11,666,315$ & deletion & - & not rare \\
\hline 184307 chr1 & $152,651,840$ & $152,659,194$ & duplication & - & not rare \\
\hline 184307 chr1 & $15,921,774$ & $15,932,242$ & deletion & - & not rare \\
\hline 184307 chr1 & $161,559,327$ & $161,617,673$ & duplication & \multicolumn{2}{|c|}{ FCGR2C;FC not rare } \\
\hline 184307 chr1 & $17,053,565$ & $17,288,389$ & duplication & \multicolumn{2}{|c|}{ FAM231A; f not rare } \\
\hline 184307 chr1 & $189,772,005$ & $189,777,996$ & deletion & - & not rare \\
\hline 184307 chr1 & $8,182,535$ & $8,190,707$ & deletion & - & not rare \\
\hline 184307 chr10 & $122,769,975$ & $122,784,282$ & deletion & - & not rare \\
\hline
\end{tabular}




\begin{tabular}{|c|c|c|c|c|c|c|}
\hline 84307 & chr11 & 20 & $18,961,743$ & duplication & MRGPRX1 & \\
\hline 184307 & chr11 & $24,662,885$ & $24,664,320$ & deletion & - & not rare \\
\hline 84307 & chr11 & $55,365,761$ & $55,427,700$ & deletion & \multicolumn{2}{|c|}{$\mathrm{OR} 4 \mathrm{P} 4 ; \mathrm{OR} \angle$ not rare } \\
\hline 34307 & chr11 & $5,876,086$ & $5,884,123$ & deletion & OR52E8 & not rare \\
\hline 184307 & chr11 & $64,201,639$ & $64,202,814$ & deletion & - & not rare \\
\hline 84307 & chr11 & $91,215,706$ & $91,216,313$ & deletion & - & not rare \\
\hline 84307 & chr11 & $93,020,527$ & $93,028,940$ & deletion & - & not rare \\
\hline 184307 & $\operatorname{chr} 12$ & $10,275,072$ & $10,276,001$ & deletion & CLEC7A & not rare \\
\hline 184307 & chr12 & $11,504,864$ & $11,546,987$ & deletion & \multicolumn{2}{|c|}{ PRB1;PRB2 not rare } \\
\hline 184307 & $\operatorname{chr} 12$ & $27,648,411$ & $27,657,025$ & deletion & & not rare \\
\hline 184307 & chr13 & $114,545,317$ & $114,548,359$ & deletion & - & not rare \\
\hline 184307 & chr13 & $38,070,549$ & $38,120,275$ & deletion & - & not rare \\
\hline 184307 & chr13 & $57,867,569$ & $57,891,197$ & dele & - & not rare \\
\hline 184307 & chr13 & $66,193,989$ & $66,206,279$ & deletion & - & not rare \\
\hline 184307 & chr13 & $99,236,471$ & $99,238,580$ & deletion & - & not rare \\
\hline 184307 & chr14 & $106,891,524$ & $106,931,129$ & deletion & - & not rare \\
\hline 184307 & chr14 & $41,610,224$ & $41,661,685$ & deletion & - & not rare \\
\hline 184307 & chr15 & $20,196,661$ & $21,241,118$ & duplication & \multicolumn{2}{|c|}{ GOLGA6L6; not rare } \\
\hline 184307 & chr15 & $21,903,815$ & $22,753,733$ & duplication & \multicolumn{2}{|c|}{ GOLGA6L2: not rare } \\
\hline 184307 & chr16 & $19,945,540$ & $19,962,636$ & deletion & - & not rare \\
\hline 184307 & chr16 & $35,185,903$ & $35,197,323$ & dele & - & care \\
\hline 184307 & chr16 & $5,513,523$ & $5,527,809$ & duplication & - & not rare \\
\hline 184307 & chr17 & $15,641,642$ & $15,676,900$ & duplication & D26 & not rare \\
\hline 184307 & chr18 & $41,976,831$ & $41,982,102$ & delet & & are \\
\hline 184307 & chr19 & 566,738 & 568,189 & del & & are \\
\hline 184307 & chr2 & $38,871,161$ & $38,883,974$ & del & 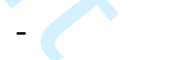 & not rare \\
\hline 184 & chr20 & $14,729,622$ & 14,7 & & - & \\
\hline 184307 & chr20 & $37,008,769$ & $37,014,876$ & deletion & - & not \\
\hline 184307 & $\operatorname{chr} 21$ & $10,766,457$ & $10,833,351$ & deletion & - & not rare \\
\hline 184307 & chr21 & $21,611,559$ & $21,620,786$ & dele & - & not rare \\
\hline 184307 & chr22 & $18,844,632$ & $18,940,271$ & duplication & \multicolumn{2}{|c|}{ PRODH;DG not rare } \\
\hline 184307 & chr22 & $18,959,684$ & $19,010,149$ & duplication & - & not rare \\
\hline 184307 & chr22 & $36,571,916$ & $36,574,767$ & deletion & - & not rare \\
\hline 184307 & chr22 & $39,359,885$ & $39,385,663$ & deletion & \multicolumn{2}{|c|}{ APOBEC $3 \mathrm{~B}$ not rare } \\
\hline 184307 & chr3 & $108,583,871$ & $108,586,312$ & deletion & - & not rare \\
\hline 184307 & chr3 & $191,065,392$ & $191,070,300$ & deletion & - & not rare \\
\hline 184307 & chr3 & $193,136,358$ & $193,140,348$ & deletion & - & not rare \\
\hline 184307 & chr3 & $46,798,133$ & $46,845,618$ & deletion & - & not rare \\
\hline 184307 & chr4 & $12,374,586$ & $12,395,247$ & deletion & - & rare \\
\hline 184307 & chr4 & $171,269,504$ & $171,273,360$ & deletion & - & not rare \\
\hline 184307 & chr4 & $70,137,897$ & $70,229,260$ & deletion & UG & not rare \\
\hline 184307 & chr5 & $115,586,404$ & $115,623,835$ & deletion & - & not rare \\
\hline 184307 & chr5 & $140,225,908$ & $140,238,311$ & deletion & \multicolumn{2}{|c|}{ PCDHA10; F not rare } \\
\hline 184307 & chr5 & $155,477,866$ & $155,488,917$ & deletion & - & not rare \\
\hline 184307 & chr5 & $45,954,604$ & $45,970,396$ & deletion & - & not rare \\
\hline 184307 & chr5 & 717,675 & 816,908 & duplication & ZDr & not rare \\
\hline 184307 & chr6 & $139,603,393$ & $139,606,731$ & deletion & - & not rare \\
\hline
\end{tabular}




\begin{tabular}{|c|c|c|c|c|c|}
\hline 184307 chr6 & $29,855,945$ & $29,899,189$ & deletion & - & not rare \\
\hline 184307 chr6 & $3,958,054$ & $3,961,288$ & deletion & - & not rare \\
\hline 84307 chr6 & $93,575,149$ & $93,578,359$ & deletion & - & ot rare \\
\hline 184307 chr7 & $141,766,850$ & $141,793,521$ & deletion & - & not rare \\
\hline 184307 chr7 & $47,283,974$ & $47,288,332$ & deletion & - & not rare \\
\hline 184307 chr7 & $61,831,893$ & $61,903,396$ & deletion & - & ot rare \\
\hline 184307 chr7 & $66,783,279$ & $66,865,643$ & deletion & - & rare \\
\hline 184307 chr8 & $115,634,278$ & $115,642,459$ & deletion & - & not rare \\
\hline 184307 chr8 & $13,600,024$ & $13,608,763$ & deletion & - & not rare \\
\hline $184307 \mathrm{chr} 8$ & $24,972,808$ & $24,990,418$ & duplication & - & not rare \\
\hline 184307 chr8 & $39,232,581$ & $39,385,979$ & duplication & - & not rare \\
\hline 184307 chr8 & $43,508,916$ & $43,535,592$ & deletion & - & rare \\
\hline 184307 chr9 & $23,363,526$ & $23,376,817$ & duplication & - & not rare \\
\hline 184308 chr1 & $104,153,766$ & $104,211,046$ & deletion & AMY1A;AI & not rare \\
\hline 184308 chr1 & $109,367,944$ & $109,371,874$ & deletion & AKNAD1 & not rare \\
\hline $184308 \mathrm{chr} 1$ & $12,846,082$ & $12,919,928$ & deletion & HNRNPCL & not rare \\
\hline $184308 \mathrm{chr} 1$ & $17,009,576$ & $17,261,738$ & duplication & FAM231A & not rare \\
\hline 184308 chr1 & $240,224,754$ & $240,229,161$ & deletion & - & not rare \\
\hline 184308 chr1 & $248,740,572$ & $248,795,110$ & deletion & $\mathrm{Ol}$ & \\
\hline 184308 chr1 & $72,754,314$ & $72,763,324$ & deletion & - & not rare \\
\hline $184308 \mathrm{chr} 1$ & $74,649,265$ & $74,659,043$ & duplication & LRRIQ3 & not rare \\
\hline 184308 chr10 & $60,090,140$ & $60,094,274$ & deletion & - & not rare \\
\hline 184308 chr10 & $71,280,107$ & $71,289,190$ & dele & & not rare \\
\hline 184308 chr10 & $90,940,221$ & $90,949,605$ & del & & not rare \\
\hline 184308 chr11 & $134,602,333$ & $134,607,484$ & deletion & & not rare \\
\hline 184308 chr11 & $55,365,761$ & $55,427,700$ & deletion & OR4P4;OR & not rare \\
\hline 184308 & $58,811,241$ & $58,850,926$ & duplication & - & are \\
\hline 184308 chr11 & $86,304,402$ & $86,306,401$ & deletion & - & not rare \\
\hline 184308 chr12 & $11,220,827$ & $11,244,117$ & deletion & TAS2R43 & not rare \\
\hline 184308 chr12 & $129,230,513$ & $129,233,013$ & deletion & - & not rare \\
\hline 184308 chr12 & $59,935,926$ & $59,942,122$ & deletion & - & not rare \\
\hline 184308 chr13 & $34,134,809$ & $34,143,545$ & deletion & - & not rare \\
\hline 184308 chr14 & $19,457,976$ & $20,424,184$ & deletion & OR4K5;C & not rare \\
\hline 184308 chr14 & $41,610,224$ & $41,661,685$ & deletion & - & not rare \\
\hline 184308 chr14 & $42,988,346$ & $42,992,844$ & deletion & - & not rare \\
\hline 184308 chr15 & $55,010,133$ & $55,015,367$ & deletion & - & not rare \\
\hline 184308 chr16 & $34,471,298$ & $34,756,258$ & duplication & - & not rare \\
\hline 184308 chr17 & $14,997,297$ & $15,010,250$ & deletion & - & not rare \\
\hline 184308 chr17 & $15,044,804$ & $15,058,236$ & deletion & - & not rare \\
\hline 184308 chr18 & $69,428,556$ & $69,441,154$ & deletion & - & not rare \\
\hline 184308 chr19 & $53,518,747$ & $53,552,296$ & duplication & ERVV-1 & not rare \\
\hline 184308 chr19 & $9,275,282$ & $9,287,189$ & deletion & - & not rare \\
\hline $184308 \mathrm{chr} 2$ & $208,351,315$ & $208,357,838$ & deletion & - & not rare \\
\hline $184308 \mathrm{chr} 2$ & $223,865,393$ & $223,869,820$ & deletion & - & not rare \\
\hline $184308 \mathrm{chr} 2$ & $4,212,725$ & $4,224,068$ & deletion & - & not rare \\
\hline $184308 \mathrm{chr} 2$ & $48,851,541$ & $48,856,770$ & deletion & - & not rare \\
\hline 184308 chr2 & $97,738,917$ & $98,024,837$ & deletion & \multicolumn{2}{|c|}{ FAHD2B;Ar not rare } \\
\hline
\end{tabular}




\begin{tabular}{|c|c|c|c|c|c|}
\hline 184308 chr20 & $24,887,251$ & $24,893,947$ & deletion & - & not rare \\
\hline 184308 chr20 & $37,008,769$ & $37,014,876$ & deletion & - & not rare \\
\hline 184308 chr20 & $52,645,166$ & $52,656,535$ & deletion & BCAS1 & ot rare \\
\hline 184308 chr21 & $15,235,002$ & $15,275,981$ & duplication & - & not rare \\
\hline $184308 \mathrm{chr} 3$ & $173,239,453$ & $173,290,024$ & duplication & - & not rare \\
\hline 184308 chr3 & $191,065,392$ & $191,070,300$ & deletion & - & ot rare \\
\hline 184308 chr3 & $195,457,853$ & $195,468,690$ & duplication & MUC20 & not rare \\
\hline 184308 chr3 & $37,979,882$ & $37,986,734$ & deletion & - & not rare \\
\hline 184308 chr3 & $63,578,618$ & $63,580,540$ & deletion & - & not rare \\
\hline 184308 chr3 & $89,402,409$ & $89,418,331$ & deletion & - & not rare \\
\hline $184308 \mathrm{chr} 4$ & $11,370,217$ & $11,380,361$ & deletion & - & not rare \\
\hline $184308 \mathrm{chr} 4$ & $188,927,822$ & $188,938,071$ & deletion & - & not rare \\
\hline $184308 \mathrm{chr} 4$ & $18,932,707$ & $18,993,753$ & deletion & - & not rare \\
\hline $184308 \mathrm{chr} 4$ & $59,980,062$ & $59,988,095$ & duplication & - & are \\
\hline $184308 \mathrm{chr} 4$ & $69,374,369$ & $69,431,280$ & deletion & UGT2B17 & not rare \\
\hline $184308 \mathrm{chr} 4$ & $70,137,897$ & $70,229,260$ & deletion & UGT2B28 & not rare \\
\hline 184308 chr5 & $140,555,135$ & $140,558,618$ & duplication & \multicolumn{2}{|c|}{ PCDHB8;PC not rare } \\
\hline 184308 chr5 & $151,514,956$ & $151,518,615$ & deletion & - & not rare \\
\hline 184308 chr5 & $17,599,857$ & $17,625,744$ & del & - & \\
\hline 184308 chr5 & $7,178,644$ & $7,191,074$ & deletion & - & not rare \\
\hline $184308 \mathrm{chr} 5$ & 744,005 & 836,608 & duplication & ZDHHC11 & not rare \\
\hline 184308 chr6 & $165,129,935$ & $165,194,584$ & duplication & - & not rare \\
\hline 184308 chr6 & $30,994,010$ & $30,994,787$ & deletion & MUC22 & not rare \\
\hline 184308 chr7 & $141,766,850$ & $141,793,521$ & dele & 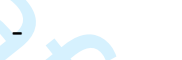 & not rare \\
\hline 184308 chr7 & $142,824,355$ & $142,890,868$ & deletion & \multicolumn{2}{|c|}{ PIP;TAS2R 3 not rare } \\
\hline 184308 chr7 & 41,421 & 68,920 & duplication & - & not rare \\
\hline 08 chr7 & $86,238,045$ & $86,244,535$ & dele & - & \\
\hline 184308 chr7 & $97,396,709$ & $97,401,677$ & deletion & - & not rare \\
\hline $184308 \mathrm{chr} 8$ & $51,029,371$ & $51,038,149$ & deletion & - & not rare \\
\hline 184308 chr8 & $92,116,351$ & $92,185,168$ & deletion & LR & not rare \\
\hline 184308 chr9 & $138,195,113$ & $138,195,756$ & deletion & - & not rare \\
\hline 184308 chr9 & $69,838,649$ & $69,992,206$ & deletion & - & not rare \\
\hline 184308 chr9 & $78,004,378$ & $78,011,616$ & deletion & - & not rare \\
\hline 184308 chr9 & $8,393,996$ & $8,398,021$ & deletion & - & not rare \\
\hline 184308 chr9 & $8,418,224$ & $8,421,388$ & ion & - & not rare \\
\hline $184308 \mathrm{chrX}$ & $94,343,494$ & $94,393,450$ & duplication & - & not rare \\
\hline 184317 chr1 & $104,103,057$ & $104,211,046$ & duplication & \multicolumn{2}{|c|}{ AMY1C;AN not rare } \\
\hline 184317 chr1 & $149,032,299$ & $149,233,182$ & deletion & - & not rare \\
\hline 184317 chr1 & $161,559,327$ & $161,617,673$ & deletion & \multicolumn{2}{|c|}{ FCGR2C;FC not rare } \\
\hline 184317 chr1 & $215,859,447$ & $215,861,792$ & deletion & - & not rare \\
\hline 184317 chr1 & $62,113,597$ & $62,119,136$ & deletion & - & not rare \\
\hline 184317 chr10 & $33,853,394$ & $33,865,876$ & duplication & - & not rare \\
\hline 184317 chr10 & $68,174,504$ & $68,208,752$ & deletion & - & rare \\
\hline 184317 chr11 & $134,602,333$ & $134,607,484$ & deletion & - & not rare \\
\hline 184317 chr11 & $24,446,609$ & $24,451,830$ & deletion & - & not rare \\
\hline 184317 chr12 & $11,220,827$ & $11,244,117$ & deletion & TA & not rare \\
\hline 184317 chr12 & $32,331,195$ & $32,334,813$ & deletion & - & not rare \\
\hline
\end{tabular}




\begin{tabular}{|c|c|c|c|c|c|c|}
\hline 84317 & $\operatorname{chr} 12$ & 21 & $33,734,935$ & deletion & - & not rare \\
\hline 184317 & $\operatorname{chr} 12$ & $59,935,926$ & $59,942,122$ & deletion & - & not rare \\
\hline 84317 & chr12 & $70,869,625$ & $70,879,131$ & deletion & - & ot rare \\
\hline 84317 & chr13 & $38,076,308$ & $38,085,537$ & deletion & - & not rare \\
\hline 184317 & chr14 & $74,240,176$ & $74,245,607$ & deletion & - & not rare \\
\hline 184317 & chr16 & $19,945,540$ & $19,962,636$ & deletion & - & t rare \\
\hline 184317 & chr16 & $34,482,158$ & $34,756,258$ & duplication & - & not rare \\
\hline 184317 & chr16 & $55,800,009$ & $55,819,443$ & deletion & - & not rare \\
\hline 184317 & chr16 & $78,372,894$ & $78,383,509$ & deletion & - & not rare \\
\hline 184317 & chr17 & $44,166,604$ & $44,285,531$ & duplication & KANSL1 & not rare \\
\hline 184317 & chr17 & $54,160,465$ & $54,172,668$ & deletion & - & not rare \\
\hline 184317 & chr19 & $3,283,789$ & $3,294,359$ & duplication & CELF5 & not rare \\
\hline 184317 & chr19 & $35,852,103$ & $35,861,695$ & deletion & - & not rare \\
\hline 184317 & chr19 & $43,263,508$ & $43,359,979$ & duplication & PS & not rare \\
\hline 184317 & chr2 & $123,477,465$ & $123,482,301$ & deletion & - & not rare \\
\hline 184317 & chr2 & $34,697,895$ & $34,726,904$ & deletion & - & not rare \\
\hline 184317 & chr3 & $65,189,366$ & $65,214,685$ & deletion & - & not rare \\
\hline 184317 & chr4 & $108,064,023$ & $108,076,199$ & deletion & - & not rare \\
\hline 184317 & chr4 & $185,753,007$ & $185,760,834$ & on & - & \\
\hline 184317 & chr4 & $64,136,609$ & $64,154,477$ & deletion & - & not rare \\
\hline 184317 & chr4 & $69,374,369$ & $69,467,200$ & deletion & 2B17 & not rare \\
\hline 184317 & chr4 & $70,137,897$ & $70,229,260$ & dele & 28 & not rare \\
\hline 184317 & chr5 & $151,514,956$ & $151,518,615$ & del & & not rare \\
\hline 184317 & chr5 & $17,609,458$ & $17,642,516$ & & & not rare \\
\hline 184317 & chr5 & $59,713,726$ & $59,769,702$ & dup & & not rare \\
\hline 184317 & chr6 & $132,709,027$ & $132,711,200$ & deletion & - & not rare \\
\hline 184317 & chr6 & $19,040,857$ & $19,048,773$ & & - & are \\
\hline 184317 & chr6 & $30,994,010$ & $30,994,787$ & deletion & ML & not rare \\
\hline 184317 & chr6 & $81,284,205$ & $81,293,326$ & deletion & - & not rare \\
\hline 184317 & chr7 & $12,542,866$ & $12,550,763$ & deletion & - & not rare \\
\hline 184317 & chr7 & $141,766,850$ & $141,793,521$ & deletion & - & not rare \\
\hline 184317 & chr7 & $97,396,709$ & $97,401,677$ & deletion & - & not rare \\
\hline 184317 & chr8 & $115,634,278$ & $115,642,459$ & deletion & - & not rare \\
\hline 184317 & chr8 & $17,580,791$ & $17,581,700$ & deletion & MTUS1 & not rare \\
\hline 184317 & chr8 & $39,232,581$ & $39,385,979$ & duplication & - & not rare \\
\hline 184317 & chr9 & $16,902,524$ & $16,913,174$ & deletion & - & not rare \\
\hline 184317 & chr9 & $78,004,378$ & $78,011,616$ & deletion & - & not rare \\
\hline 184320 & chr1 & $104,153,766$ & $104,211,046$ & deletion & \multicolumn{2}{|c|}{ AMY1A;AN not rare } \\
\hline 184320 & chr1 & $248,730,623$ & $248,795,110$ & deletion & \multicolumn{2}{|c|}{ OR2T10;OF not rare } \\
\hline 184320 & chr10 & $46,965,834$ & $47,541,708$ & deletion & \multicolumn{2}{|c|}{ NPY4R;GPF not rare } \\
\hline 184320 & chr12 & $2,245,636$ & $2,254,219$ & deletion & - & not rare \\
\hline 184320 & $\operatorname{chr} 12$ & $38,315,433$ & $38,328,711$ & deletion & - & not rare \\
\hline 184320 & chr13 & $34,131,102$ & $34,143,545$ & deletion & - & not rare \\
\hline 184320 & chr14 & $20,295,510$ & $20,404,736$ & duplication & \multicolumn{2}{|c|}{ OR4N2;OR، not rare } \\
\hline 184320 & chr14 & $73,527,253$ & $73,530,678$ & deletion & - & not rare \\
\hline 184320 & chr15 & $20,005,287$ & $20,173,102$ & duplication & - & not rare \\
\hline 184320 & chr15 & $22,368,738$ & $22,585,470$ & duplication & \multicolumn{2}{|c|}{ OR4M2;OR not rare } \\
\hline
\end{tabular}




\begin{tabular}{|c|c|c|c|c|c|}
\hline 184320 chr16 & 934 & $32,642,384$ & deletion & - & not rare \\
\hline 184320 chr16 & $4,135,350$ & $4,140,459$ & deletion & - & not rare \\
\hline 184320 chr17 & $25,264,289$ & $25,309,973$ & duplication & - & ot rare \\
\hline 184320 chr18 & $1,724,943$ & $1,839,339$ & deletion & - & not rare \\
\hline 184320 chr18 & $63,200,484$ & $63,206,335$ & duplication & - & not rare \\
\hline 184320 chr18 & $76,157,694$ & $76,160,278$ & deletion & - & not rare \\
\hline 184320 chr19 & $35,852,103$ & $35,861,695$ & deletion & - & not rare \\
\hline 184320 chr19 & $54,556,755$ & $54,560,078$ & deletion & - & not rare \\
\hline 184320 chr19 & $54,728,767$ & $54,743,217$ & duplication & LILRA6 & not rare \\
\hline $184320 \mathrm{chr} 2$ & $228,241,621$ & $228,258,288$ & duplication & TM4SF20 & not rare \\
\hline 184320 chr2 & $49,038,154$ & $49,055,023$ & deletion & - & not rare \\
\hline 184320 chr20 & $29,530,268$ & $29,839,864$ & duplication & - & not rare \\
\hline 184320 chr3 & $195,457,853$ & $195,468,690$ & duplication & MUC20 & not rare \\
\hline 184320 chr3 & $46,798,133$ & $46,845,618$ & deletion & - & not rare \\
\hline $184320 \mathrm{chr} 3$ & $53,032,639$ & $53,042,099$ & deletion & - & not rare \\
\hline 184320 chr3 & $65,189,366$ & $65,214,685$ & deletion & - & not rare \\
\hline $184320 \mathrm{chr} 4$ & $108,064,023$ & $108,074,123$ & deletion & - & not rare \\
\hline $184320 \mathrm{chr} 4$ & $116,168,158$ & $116,173,928$ & deletion & - & not rare \\
\hline $184320 \mathrm{chr} 4$ & $156,957,485$ & $156,974,697$ & deletion & - & not rare \\
\hline $184320 \mathrm{chr} 4$ & $171,269,504$ & $171,273,360$ & deletion & - & not rare \\
\hline $184320 \mathrm{chr} 4$ & $49,069,684$ & $49,651,131$ & duplication & - & not rare \\
\hline $184320 \mathrm{chr} 4$ & $69,374,369$ & $69,489,473$ & duplication & UGT2B17 & not rare \\
\hline 184320 chr5 & $117,388,410$ & $117,393,058$ & deletion & . & not rare \\
\hline 184320 chr5 & $140,225,908$ & $140,238,311$ & deletion & $\mathrm{PC}$ & not rare \\
\hline 184320 chr6 & $139,603,393$ & $139,606,731$ & deletion & & not rare \\
\hline 184320 chr6 & $15,092,665$ & $15,130,064$ & deletion & - & rare \\
\hline 184320 chr6 & $40,362,751$ & $40,368,860$ & & - & not rare \\
\hline 184320 chr6 & $69,687,698$ & $69,690,567$ & deletion & - & not rare \\
\hline 184320 chr6 & $78,972,930$ & $79,035,739$ & deletion & - & not rare \\
\hline 184320 chr7 & $142,476,094$ & $142,484,042$ & deletion & - & not rare \\
\hline 184320 chr7 & $17,388,039$ & $17,629,654$ & duplication & - & rare \\
\hline 184320 chr7 & $53,281,291$ & $53,284,220$ & deletion & - & not rare \\
\hline 184320 chr7 & $62,149,006$ & $62,162,385$ & deletion & - & not rare \\
\hline 184320 chr8 & $100,213,925$ & $100,228,085$ & duplication & $3 B$ & not rare \\
\hline 184320 chr8 & $26,334,167$ & $26,343,814$ & duplication & - & not rare \\
\hline 184320 chr8 & $39,232,581$ & $39,385,979$ & deletion & - & not rare \\
\hline 184320 chr8 & $5,595,511$ & $5,605,706$ & deletion & - & not rare \\
\hline 184320 chr9 & $135,624,197$ & $135,627,634$ & deletion & - & not rare \\
\hline 184320 chr9 & $6,117,562$ & $6,129,017$ & duplication & - & not rare \\
\hline 184320 chr9 & $6,701,130$ & $6,707,794$ & deletion & - & not rare \\
\hline 184320 chr9 & $78,004,378$ & $78,011,616$ & deletion & - & not rare \\
\hline 184325 chr1 & $62,113,597$ & $62,119,136$ & deletion & - & not rare \\
\hline 184325 chr10 & $9,951,063$ & $9,979,063$ & duplication & - & rare \\
\hline 184325 chr11 & $106,398,020$ & $106,404,608$ & deletion & - & not rare \\
\hline 184325 chr11 & $93,683,680$ & $93,688,541$ & deletion & - & not rare \\
\hline 184325 chr12 & $11,504,864$ & $11,546,987$ & deletion & \multicolumn{2}{|c|}{ PRB1;PRB2 not rare } \\
\hline 184325 chr12 & $6,241,446$ & $6,259,552$ & deletion & - & not rare \\
\hline
\end{tabular}




\begin{tabular}{|c|c|c|c|c|c|}
\hline 184325 chr13 & 34, & $34,143,545$ & deletion & - & not rare \\
\hline 184325 chr13 & $96,020,342$ & $96,023,960$ & deletion & - & not rare \\
\hline 84325 chr14 & $106,891,524$ & $106,931,129$ & deletion & - & ot rare \\
\hline 84325 chr14 & $20,197,311$ & $20,402,122$ & duplication & \multicolumn{2}{|c|}{ OR4Q3;OR، not rare } \\
\hline 184325 chr14 & $22,028,579$ & $22,036,009$ & deletion & - & not rare \\
\hline 184325 & $42,988,346$ & $42,992,844$ & deletion & - & t rare \\
\hline 84325 chr15 & $56,789,979$ & $56,800,635$ & deletion & - & not rare \\
\hline 184325 chr15 & $69,503,967$ & $69,520,428$ & duplication & - & not rare \\
\hline 184325 chr16 & $55,800,009$ & $55,819,443$ & deletion & - & not rare \\
\hline 184325 chr17 & $77,367,139$ & $77,392,062$ & duplication & - & not rare \\
\hline 184325 chr18 & $40,880,729$ & $40,902,067$ & deletion & - & not rare \\
\hline 184325 chr19 & $41,355,999$ & $41,379,321$ & deletion & CYP2A6 & not rare \\
\hline 184325 chr19 & $7,769,188$ & $7,775,654$ & deletion & - & not rare \\
\hline 184325 chr2 & $184,085,551$ & $184,088,256$ & deletion & - & no \\
\hline 184325 chr2 & $228,241,621$ & $228,258,288$ & duplication & SF20 & not rare \\
\hline 184325 chr2 & $242,854,089$ & $243,034,519$ & deletion & - & not rare \\
\hline 184325 chr22 & $18,874,965$ & $19,010,149$ & duplication & \multicolumn{2}{|c|}{ PRODH;DG not rare } \\
\hline 184325 chr3 & $112,878,536$ & $112,893,640$ & duplication & - & not rare \\
\hline 184325 chr3 & $4,155,831$ & $4,234,405$ & & - & \\
\hline 184325 chr3 & $5,536,686$ & $5,539,065$ & deletion & - & not rare \\
\hline $184325 \mathrm{chr} 3$ & $65,189,366$ & $65,214,685$ & deletion & - & not rare \\
\hline 184325 chr3 & $75,149,932$ & $75,181,452$ & duplication & - & \\
\hline $184325 \mathrm{chr} 4$ & $104,740,495$ & $104,761,084$ & deletion & & not rare \\
\hline $184325 \mathrm{chr} 4$ & $122,285,263$ & $122,289,863$ & & & rare \\
\hline $184325 \mathrm{chr} 4$ & $171,269,504$ & $171,273,360$ & dele & & not rare \\
\hline 184325 chr4 & $64,697,704$ & $64,709,753$ & deletion & - & not rare \\
\hline $184325 \mathrm{chr} 4$ & $69,374,369$ & $69,489,473$ & ation & UG & not \\
\hline 184325 chr5 & $117,388,410$ & $117,393,058$ & deletion & - & not rare \\
\hline 184325 chr5 & $155,477,866$ & $155,488,917$ & deletion & - & not rare \\
\hline 184325 chr5 & $45,858,842$ & $45,879,274$ & deletion & - & not rare \\
\hline 184325 chr5 & $7,178,644$ & $7,191,074$ & deletion & - & not rare \\
\hline 184325 chr6 & $29,855,945$ & $29,899,189$ & deletion & - & not rare \\
\hline 184325 chr6 & $78,972,930$ & $79,035,739$ & deletion & - & not rare \\
\hline 184325 chr7 & $11,920,873$ & $11,922,312$ & deletion & - & not rare \\
\hline 184325 chr7 & $38,291,851$ & $38,305,404$ & duplication & TA & not rare \\
\hline 184325 chr8 & $16,262,222$ & $16,272,444$ & deletion & - & not rare \\
\hline 184325 chr8 & $24,972,808$ & $24,990,418$ & deletion & - & not rare \\
\hline 184325 chr8 & $39,322,364$ & $39,335,392$ & deletion & - & not rare \\
\hline 184325 chr8 & $51,013,729$ & $51,038,149$ & deletion & - & not rare \\
\hline 184325 chr9 & $23,363,526$ & $23,376,817$ & duplication & - & not rare \\
\hline 184325 chr9 & $2,904,655$ & $2,932,710$ & deletion & - & not rare \\
\hline 184325 chr9 & $94,396,059$ & $94,402,923$ & deletion & - & not rare \\
\hline 184327 chr1 & $104,153,766$ & $104,211,046$ & deletion & \multicolumn{2}{|c|}{ AMY1A;AN not rare } \\
\hline 184327 chr1 & $153,674,704$ & $153,690,013$ & duplication & - & not rare \\
\hline 184327 chr1 & $161,559,327$ & $161,619,741$ & duplication & \multicolumn{2}{|c|}{ FCGR2C;FC not rare } \\
\hline 184327 chr1 & $174,797,867$ & $174,801,639$ & deletion & - & not rare \\
\hline 184327 chr1 & $213,002,088$ & $213,011,812$ & deletion & SPATA45 & not rare \\
\hline
\end{tabular}




\begin{tabular}{|c|c|c|c|c|c|c|}
\hline 184327 & chr1 & $248,740,572$ & $248,795,110$ & deletion & \multicolumn{2}{|c|}{ OR2T10;OF not rare } \\
\hline 184327 & chr1 & $30,480,091$ & $30,488,320$ & deletion & - & not rare \\
\hline 84327 & chr1 & $67,744,601$ & $67,769,006$ & duplication & - & rare \\
\hline 184327 & chr10 & $20,850,624$ & $20,857,365$ & deletion & - & not rare \\
\hline 184327 & chr10 & $38,792,512$ & $38,903,858$ & deletion & - & not rare \\
\hline 184327 & chr10 & $86,522,877$ & $86,531,815$ & deletion & - & not rare \\
\hline 184327 & chr11 & $104,366,089$ & $104,372,318$ & deletion & - & not rare \\
\hline 184327 & chr11 & $107,653,650$ & $107,671,155$ & duplication & SLC35F2 & not rare \\
\hline 184327 & chr11 & $131,925,050$ & $131,929,835$ & duplication & - & not rare \\
\hline 184327 & chr11 & $18,949,220$ & $18,961,743$ & duplication & MRGPRX1 & not rare \\
\hline 184327 & chr11 & $81,501,218$ & $81,517,261$ & deletion & - & not rare \\
\hline 184327 & $\operatorname{chr} 12$ & $11,511,132$ & $11,543,084$ & duplication & - & not rare \\
\hline 184327 & chr12 & $31,907,259$ & $31,909,635$ & deletion & - & not rare \\
\hline 184327 & chr12 & $32,331,195$ & $32,334,813$ & deletion & - & rare \\
\hline 184327 & chr13 & $114,545,317$ & $114,548,359$ & deletion & - & not rare \\
\hline 184327 & chr13 & $64,225,427$ & $64,236,288$ & deletion & - & not rare \\
\hline 184327 & chr14 & $106,891,524$ & $106,931,129$ & deletion & - & not rare \\
\hline 184327 & chr14 & $20,341,645$ & $20,373,822$ & duplication & & not rare \\
\hline 184327 & chr14 & $41,610,224$ & $41,661,685$ & deletion & - & are \\
\hline 184327 & chr14 & $47,708,397$ & $47,715,702$ & deletion & - & not rare \\
\hline 184327 & chr16 & $19,945,540$ & $19,962,636$ & deletion & - & not rare \\
\hline 184327 & chr16 & $34,471,298$ & $34,756,258$ & duplication & - & ot rare \\
\hline 184327 & chr16 & $55,800,009$ & $55,819,443$ & deletion & & not rare \\
\hline 184327 & chr16 & $85,302,707$ & $85,304,326$ & dele & & not rare \\
\hline 184327 & chr2 & $129,638,609$ & $129,645,321$ & deletion & & not rare \\
\hline 184327 & chr2 & $227,343,513$ & $227,347,453$ & deletion & . & not rare \\
\hline 184327 & chr2 & $41,239,457$ & $41,248,468$ & deletion & - & rare \\
\hline 184327 & chr21 & $10,815,443$ & $10,858,651$ & duplication & - & not rare \\
\hline 184327 & $\operatorname{chr} 22$ & $42,918,711$ & $42,950,033$ & duplication & SERHL2 & not rare \\
\hline 184327 & chr3 & $195,457,853$ & $195,468,690$ & duplication & MUC20 & not rare \\
\hline 184327 & chr3 & $37,979,882$ & $37,986,734$ & deletion & - & not rare \\
\hline 184327 & chr4 & $11,372,956$ & $11,380,361$ & deletion & - & not rare \\
\hline 184327 & chr4 & $34,785,955$ & $34,818,502$ & deletion & - & not rare \\
\hline 184327 & chr4 & $59,980,062$ & $59,988,095$ & duplication & - & not rare \\
\hline 184327 & chr5 & $117,388,410$ & $117,393,058$ & deletion & - & not rare \\
\hline 184327 & chr5 & $17,345,282$ & $17,356,536$ & deletion & - & not rare \\
\hline 184327 & chr5 & $17,499,492$ & $17,515,026$ & deletion & - & not rare \\
\hline 184327 & chr6 & $139,603,393$ & $139,606,731$ & deletion & - & not rare \\
\hline 184327 & chr6 & $19,041,346$ & $19,048,773$ & deletion & - & not rare \\
\hline 184327 & chr6 & $29,855,945$ & $29,899,189$ & deletion & - & not rare \\
\hline 184327 & chr6 & $77,439,769$ & $77,451,301$ & deletion & - & not rare \\
\hline 184327 & chr7 & 151,829 & 159,207 & deletion & - & not rare \\
\hline 184327 & chr7 & $156,387,296$ & $156,394,307$ & deletion & - & not rare \\
\hline 184327 & chr7 & $38,291,851$ & $38,305,404$ & duplication & TARP & not rare \\
\hline 184327 & chr7 & 41,421 & 73,060 & duplication & - & not rare \\
\hline 184327 & chr8 & $115,634,278$ & $115,642,459$ & deletion & - & not rare \\
\hline 184327 & chr8 & $138,822,798$ & $138,824,695$ & deletion & - & not rare \\
\hline
\end{tabular}




\begin{tabular}{|c|c|c|c|c|c|}
\hline $184327 \mathrm{chr} 8$ & $39,290,901$ & $39,385,979$ & deletion & - & not rare \\
\hline 184327 chr8 & $40,184,872$ & $40,189,688$ & deletion & - & not rare \\
\hline 84327 chr8 & $8,583,109$ & $8,588,552$ & deletion & - & t rare \\
\hline 84327 chr9 & $23,363,526$ & $23,376,817$ & duplication & - & not rare \\
\hline 184327 chr9 & $6,701,130$ & $6,707,794$ & deletion & - & not rare \\
\hline 84329 chr1 & $145,625,979$ & $146,089,254$ & duplication & \multicolumn{2}{|c|}{ CD160;RNF not rare } \\
\hline 184329 chr 1 & $161,489,467$ & $161,619,741$ & duplication & \multicolumn{2}{|c|}{ FCGR3B;FC not rare } \\
\hline 184329 chr 1 & $248,740,572$ & $248,795,110$ & deletion & \multicolumn{2}{|c|}{ OR2T10;OF not rare } \\
\hline 184329 chr10 & $39,127,750$ & $39,154,535$ & duplication & - & not rare \\
\hline 184329 chr10 & $58,901,976$ & $58,936,939$ & deletion & - & not rare \\
\hline 184329 & $71,279,367$ & $71,289,190$ & deletion & - & are \\
\hline 184329 & $90,193,796$ & $90,195,742$ & deletion & - & not rare \\
\hline 184329 chr12 & $11,220,827$ & $11,258,011$ & deletion & TAS2R43 & not rare \\
\hline 184329 chr12 & $33,300,995$ & $33,305,855$ & deletion & - & not rare \\
\hline 184329 chr12 & $94,197,679$ & $94,202,652$ & deletion & - & not rare \\
\hline 184329 chr14 & $19,457,976$ & $20,424,184$ & deletion & \multicolumn{2}{|c|}{ OR4K5;OR $\angle$ not rare } \\
\hline 184329 chr14 & $41,610,224$ & $41,661,685$ & deletion & - & not rare \\
\hline 184329 chr15 & $23,090,818$ & $23,116,232$ & deletion & - & not rare \\
\hline 184329 & $32,528,023$ & $32,833,893$ & deletion & \multicolumn{2}{|c|}{ TP53TG3B; not rare } \\
\hline 184329 chr16 & $33,516,067$ & $33,590,959$ & deletion & - & not rare \\
\hline 184329 chr16 & $70,016,280$ & $70,155,033$ & duplication & & not rare \\
\hline 184329 chr17 & $7,262,498$ & $7,265,681$ & deletion & - & not rare \\
\hline 184329 chr17 & $78,601,169$ & $78,612,034$ & duplication & 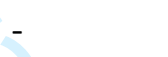 & not rare \\
\hline 184329 chr19 & $17,030,021$ & $17,054,492$ & deletion & \multirow[t]{2}{*}{ CPAMD8 } & rare \\
\hline 184329 chr19 & $53,152,453$ & $53,159,177$ & deletion & & not rare \\
\hline 184329 chr2 & $208,351,315$ & $208,357,838$ & dele & - & not rare \\
\hline 184329 chr2 & $212,771,670$ & $212,773,893$ & & - & \\
\hline 184329 chr2 & $216,039,339$ & $216,039,701$ & deletion & - & not rare \\
\hline 184329 chr2 & $41,239,457$ & $41,248,468$ & deletion & - & not rare \\
\hline 184329 chr2 & $48,851,541$ & $48,856,770$ & dele & - & not rare \\
\hline 184329 chr20 & $59,568,187$ & $59,590,280$ & duplication & - & not rare \\
\hline 184329 chr20 & $61,372,822$ & $61,420,032$ & deletion & & rare \\
\hline 184329 chr21 & $10,833,209$ & $10,858,651$ & duplication & - & not rare \\
\hline 184329 chr3 & $127,637,136$ & $127,652,712$ & duplication & BD12 & not rare \\
\hline $184329 \mathrm{chr} 3$ & $187,587,002$ & $187,607,826$ & ation & - & Tale \\
\hline 184329 chr3 & $193,136,358$ & $193,140,348$ & deletion & - & not rare \\
\hline 184329 chr3 & $195,457,853$ & $195,468,690$ & duplication & MUC20 & not rare \\
\hline $184329 \mathrm{chr} 4$ & $152,790,580$ & $152,793,891$ & deletion & - & not rare \\
\hline $184329 \mathrm{chr} 4$ & $60,852,284$ & $60,861,990$ & deletion & - & not rare \\
\hline $184329 \mathrm{chr} 4$ & $69,374,369$ & $69,489,473$ & deletion & UGT2B17 & not rare \\
\hline $184329 \mathrm{chr} 4$ & $70,137,897$ & $70,229,260$ & deletion & UGT2B28 & not rare \\
\hline 184329 chr5 & $151,514,956$ & $151,518,615$ & deletion & - & not rare \\
\hline 184329 chr5 & $84,990,560$ & $85,023,326$ & deletion & - & rare \\
\hline 184329 chr6 & $29,855,945$ & $29,899,189$ & deletion & - & not rare \\
\hline 184329 chr6 & $30,994,010$ & $30,994,787$ & deletion & MUC22 & not rare \\
\hline 184329 chr6 & $31,219,869$ & $31,229,552$ & deletion & - & not rare \\
\hline 184329 chr6 & $37,963,308$ & $37,983,593$ & duplication & - & not rare \\
\hline
\end{tabular}




\begin{tabular}{|c|c|c|c|c|c|}
\hline 184329 chr6 & $49,780,993$ & $49,806,196$ & duplication & CRISP1 & rare \\
\hline 184329 chr6 & $81,284,205$ & $81,293,326$ & deletion & - & not rare \\
\hline 84329 chr6 & 979,803 & 988,245 & deletion & - & not rare \\
\hline 184329 chr8 & $115,634,278$ & $115,642,459$ & deletion & - & not rare \\
\hline $184329 \mathrm{chr} 8$ & $24,972,808$ & $24,990,418$ & duplication & - & not rare \\
\hline 184329 chr8 & $39,322,364$ & $39,385,979$ & deletion & - & ot rare \\
\hline 184329 chr9 & $133,253,482$ & $133,271,928$ & deletion & HMCN2 & not rare \\
\hline 184332 chr1 & $111,380,720$ & $111,387,723$ & deletion & - & not rare \\
\hline 184332 chr1 & $12,900,831$ & $12,944,989$ & deletion & \multicolumn{2}{|c|}{ HNRNPCL1 not rare } \\
\hline 184332 chr1 & $149,032,299$ & $149,432,892$ & deletion & - & not rare \\
\hline 184332 chr1 & $1,585,642$ & $1,680,951$ & duplication & \multicolumn{2}{|c|}{ SLC35E2;C[ not rare } \\
\hline 184332 chr1 & $203,907,840$ & $203,921,875$ & deletion & - & not rare \\
\hline 184332 chr1 & $25,598,276$ & $25,659,509$ & deletion & RHD & not rare \\
\hline 184332 chr1 & 785,100 & 919,501 & duplication & \multicolumn{2}{|c|}{ KLHL17;NO rare } \\
\hline 184332 chr11 & $24,446,609$ & $24,451,830$ & deletion & - & not rare \\
\hline 184332 chr11 & $90,193,796$ & $90,196,843$ & deletion & - & not rare \\
\hline 184332 chr13 & $45,949,677$ & $45,995,982$ & duplication & SLC25A30 & rare \\
\hline 184332 chr13 & $64,225,427$ & $64,236,288$ & deletion & - & not rare \\
\hline 184332 & $64,343,579$ & $64,387,767$ & duplication & - & are \\
\hline 184332 chr13 & $84,102,440$ & $84,157,927$ & deletion & - & not rare \\
\hline 184332 chr14 & $41,610,224$ & $41,661,685$ & deletion & - & not rare \\
\hline 184332 & $55,010,133$ & $55,015,367$ & de & - & ot rare \\
\hline 184332 chr16 & $55,800,009$ & $55,819,443$ & del & & not rare \\
\hline 184332 chr17 & $71,099,671$ & $71,102,042$ & & - & not rare \\
\hline 184332 chr19 & $43,266,647$ & $43,322,065$ & duplicatio & PSC & not rare \\
\hline 184332 chr2 & $183,760,854$ & $183,766,917$ & deletion & - & not rare \\
\hline 184332 chr2 & $97,738,917$ & $98,024,837$ & deletion & \multicolumn{2}{|c|}{ FAHD2B;Ar not rare } \\
\hline $184332 \mathrm{chr} 3$ & $131,711,896$ & $131,712,898$ & deletion & - & not rare \\
\hline $184332 \mathrm{chr} 3$ & $193,136,358$ & $193,140,348$ & deletion & - & not rare \\
\hline $184332 \mathrm{chr} 4$ & $156,959,568$ & $156,973,205$ & deletion & - & not rare \\
\hline $184332 \mathrm{chr} 4$ & $18,932,707$ & $18,993,753$ & deletion & - & not rare \\
\hline $184332 \mathrm{chr} 4$ & $60,325,010$ & $60,330,880$ & duplication & - & not rare \\
\hline $184332 \mathrm{chr} 4$ & $70,137,897$ & $70,229,260$ & deletion & UGT2B28 & not rare \\
\hline 184332 chr4 & $7,182,916$ & $7,187,397$ & deletion & - & not rare \\
\hline 184332 chr5 & $117,388,410$ & $117,393,058$ & deletion & - & not rare \\
\hline 184332 chr5 & $7,178,644$ & $7,191,074$ & deletion & - & not rare \\
\hline 184332 chr6 & $30,994,010$ & $30,994,787$ & deletion & MUC22 & not rare \\
\hline 184332 chr6 & $31,360,255$ & $31,453,640$ & deletion & MICA & not rare \\
\hline 184332 chr6 & $66,074,421$ & $66,080,908$ & deletion & - & not rare \\
\hline 184332 chr6 & 979,803 & 988,245 & deletion & - & not rare \\
\hline 184332 chr7 & $141,766,850$ & $141,793,521$ & deletion & - & not rare \\
\hline 184332 chr7 & $86,940,652$ & $86,948,364$ & deletion & - & not rare \\
\hline 184332 chr7 & $97,396,709$ & $97,401,677$ & deletion & - & not rare \\
\hline 184332 chr8 & $115,633,704$ & $115,642,459$ & deletion & - & not rare \\
\hline 184332 chr8 & $2,588,813$ & $2,591,021$ & deletion & - & not rare \\
\hline 184332 chr8 & $39,232,581$ & $39,382,903$ & deletion & - & not rare \\
\hline 184332 chr9 & 311,600 & 331,490 & deletion & DOCK8 & not rare \\
\hline
\end{tabular}




\begin{tabular}{|c|c|c|c|c|c|}
\hline 84334 chr1 & ,766 & 104 & & AMY2A & not rare \\
\hline 84334 chr1 & $109,367,944$ & $109,371,874$ & deletion & AKNAD1 & not rare \\
\hline 184334 chr1 & $161,489,467$ & $161,619,741$ & duplication & \multicolumn{2}{|c|}{ FCGR3B;FC not rare } \\
\hline 84334 chr1 & $16,363,132$ & $16,382,966$ & duplication & CLCNKB & not rare \\
\hline 34334 chr10 & $23,154,715$ & $23,157,251$ & deletion & - & not rare \\
\hline $184334 c$ & $71,282,981$ & $71,289,190$ & deletion & - & not rare \\
\hline 84334 chr11 & $18,949,220$ & $18,961,743$ & duplication & MRGPRX1 & not rare \\
\hline 184334 chr11 & $55,365,761$ & $55,427,700$ & deletion & \multicolumn{2}{|c|}{$\mathrm{OR} 4 \mathrm{P} 4 ; \mathrm{OR} \angle$ not rare } \\
\hline 184334 chr12 & $30,237,351$ & $30,243,473$ & deletion & - & not rare \\
\hline 184334 chr12 & $31,288,884$ & $31,407,303$ & duplication & - & not rare \\
\hline 184334 chr12 & $43,021,431$ & $43,027,453$ & deletion & - & not rare \\
\hline 184334 chr12 & $94,197,679$ & $94,202,652$ & deletion & - & not rare \\
\hline 184334 chr13 & $34,134,809$ & $34,143,545$ & deletion & - & not rare \\
\hline 184334 & $106,062,675$ & $106,178,679$ & duplication & - & care \\
\hline 184334 chr17 & $10,890,859$ & $10,893,799$ & deletion & - & not rare \\
\hline 184334 chr17 & $7,264,717$ & $7,265,681$ & dele & - & care \\
\hline 184334 chr19 & $35,852,103$ & $35,861,695$ & deletion & - & not rare \\
\hline 184334 chr19 & $9,275,282$ & $9,287,189$ & deletion & - & not rare \\
\hline 184334 chr2 & $242,854,089$ & $243,034,519$ & deletion & - & rare \\
\hline 184334 chr2 & $48,851,541$ & $48,856,770$ & deletion & - & not rare \\
\hline 184334 chr20 & $53,407,575$ & $53,419,685$ & deletion & - & not rare \\
\hline 184334 chr21 & 10,6 & 10,8 & duplication & - & \\
\hline 184334 chr22 & $39,359,885$ & $39,385,663$ & deletion & \multicolumn{2}{|c|}{ APOBEC3B not rare } \\
\hline $184334 \mathrm{chr} 3$ & $129,769,827$ & $129,806,304$ & deletion & & not rare \\
\hline 184334 chr3 & 193, & 193 & del & & \\
\hline $184334 \mathrm{chr} 4$ & $31,464,058$ & $31,469,190$ & dele & - & not rare \\
\hline $184334 \mathrm{chr} 4$ & $58,256,666$ & $58,261,728$ & deletion & - & not rare \\
\hline $184334 \mathrm{chr} 4$ & $60,325,010$ & $60,330,880$ & duplication & - & not rare \\
\hline 184334 chr5 & $140,225,908$ & $140,238,311$ & deletion & \multicolumn{2}{|c|}{ PCDHA10;F not rare } \\
\hline 184334 chr5 & $144,679,911$ & $144,713,515$ & duplication & - & \\
\hline 184334 chr5 & $151,514,956$ & $151,518,615$ & deletion & - & not rare \\
\hline 184334 chr6 & $30,994,010$ & $30,994,787$ & deletion & MUC22 & not rare \\
\hline 184334 chr6 & $78,972,930$ & $79,009,742$ & sation & - & not rare \\
\hline 184334 chr7 & $15,154,324$ & $15,159,059$ & deletion & - & not rare \\
\hline 184334 chr7 & $154,431,180$ & $154,459,674$ & deletion & - & rare \\
\hline 184334 chr7 & $38,291,851$ & $38,305,404$ & duplication & TARP & not rare \\
\hline 184334 chr7 & $91,033,074$ & $91,041,618$ & deletion & - & not rare \\
\hline 184334 chr8 & $135,059,968$ & $135,065,947$ & deletion & - & not rare \\
\hline 184334 chr8 & $39,322,364$ & $39,345,423$ & deletion & - & not rare \\
\hline 184334 chr8 & $51,029,371$ & $51,038,149$ & deletion & - & not rare \\
\hline 184334 chr8 & $51,226,087$ & $51,227,777$ & deletion & - & not rare \\
\hline 184334 chr9 & $138,195,113$ & $138,195,756$ & deletion & - & not rare \\
\hline $184334 \mathrm{chrX}$ & $95,446,396$ & $95,480,629$ & deletion & - & not rare \\
\hline 184335 chr1 & $1,385,211$ & $1,430,707$ & deletion & \multicolumn{2}{|c|}{ ATAD3C;AT not rare } \\
\hline 184335 chr1 & $152,526,812$ & $152,568,230$ & deletion & \multicolumn{2}{|c|}{ LCE3D;LCE: not rare } \\
\hline 184335 chr1 & $161,563,386$ & $161,619,741$ & duplication & \multicolumn{2}{|c|}{ FCGR2C;FC not rare } \\
\hline 184335 chr1 & $55,114,469$ & $55,120,469$ & deletion & MROH7 & not rare \\
\hline
\end{tabular}




\begin{tabular}{|c|c|c|c|c|c|}
\hline 184335 chr1 & $62,113,597$ & $62,119,136$ & deletion & - & not rare \\
\hline 184335 chr10 & $121,545,750$ & $121,577,062$ & deletion & INPP5F & rare \\
\hline 84335 chr10 & $58,516,470$ & $58,526,153$ & deletion & - & not rare \\
\hline 184335 chr10 & $71,282,981$ & $71,289,190$ & deletion & - & not rare \\
\hline 184335 chr11 & $42,968,875$ & $42,973,253$ & duplication & - & not rare \\
\hline 184335 chr11 & $90,193,796$ & $90,196,843$ & deletion & - & ot rare \\
\hline 184335 chr11 & $93,020,527$ & $93,028,940$ & deletion & - & not rare \\
\hline 184335 chr12 & $32,785,284$ & $32,843,021$ & duplication & \multicolumn{2}{|c|}{ FGD4;DNM rare } \\
\hline 184335 chr12 & $33,300,995$ & $33,305,855$ & deletion & - & not rare \\
\hline 184335 chr12 & $59,935,926$ & $59,942,122$ & deletion & - & not rare \\
\hline 184335 chr13 & $34,134,809$ & $34,143,545$ & deletion & - & not rare \\
\hline 184335 chr13 & $69,247,022$ & $69,267,981$ & deletion & - & not rare \\
\hline 184335 chr13 & $84,102,440$ & $84,157,927$ & deletion & - & not rare \\
\hline 184335 chr14 & $20,197,311$ & $20,424,184$ & duplication & \multicolumn{2}{|c|}{ OR4K5;OR $\angle$ not rare } \\
\hline 184335 chr15 & $20,196,661$ & $21,241,118$ & deletion & \multicolumn{2}{|c|}{ GOLGA6L6; not rare } \\
\hline 184335 chr15 & $29,901,216$ & $29,902,919$ & deletion & - & not rare \\
\hline 184335 chr15 & $43,888,976$ & $43,895,643$ & deletion & \multicolumn{2}{|c|}{ STRC;CKM1 not rare } \\
\hline 184335 chr16 & $32,511,914$ & $32,603,033$ & duplication & - & not rare \\
\hline 184335 chr16 & $88,638,854$ & $88,640,165$ & & - & rare \\
\hline 184335 chr17 & $34,438,753$ & $34,534,918$ & duplication & \multicolumn{2}{|c|}{ CCL3L3;CCI not rare } \\
\hline 184335 chr17 & $44,166,604$ & $44,225,181$ & duplication & KANSL1 & not rare \\
\hline 184335 chr19 & $53,518,747$ & $53,552,296$ & duplication & & not rare \\
\hline 184335 chr2 & $123,477,465$ & $123,482,301$ & deletion & & not rare \\
\hline 184335 chr2 & $196,421,568$ & $196,429,252$ & del & & not rare \\
\hline 184335 chr2 & $52,032,381$ & $52,077,000$ & dele & & \\
\hline 184335 chr2 & $54,479,117$ & $54,481,636$ & deletion & - & not rare \\
\hline 184335 chr2 & $97,738,917$ & $98,024,837$ & deletion & \multicolumn{2}{|c|}{ FAHD2B;Ar not rare } \\
\hline 184335 chr20 & $29,454,201$ & $29,500,566$ & deletion & - & not rare \\
\hline 184335 chr20 & $53,407,575$ & $53,419,685$ & deletion & - & not rare \\
\hline 184335 chr20 & $59,568,187$ & $59,590,280$ & duplication & - & not rare \\
\hline 184335 chr21 & $10,699,687$ & $10,888,876$ & duplication & - & not rare \\
\hline 184335 chr22 & $25,660,776$ & $25,909,677$ & duplication & LRP5L & not rare \\
\hline 184335 chr22 & $37,745,101$ & $37,749,946$ & deletion & - & not rare \\
\hline 184335 chr22 & $39,359,885$ & $39,385,663$ & deletion & \multicolumn{2}{|c|}{ APOBEC $3 \mathrm{~B}$ not rare } \\
\hline 184335 chr3 & $37,979,882$ & $37,986,734$ & deletion & - & not rare \\
\hline 184335 chr3 & $41,092,547$ & $41,144,695$ & deletion & - & not rare \\
\hline 184335 chr3 & $53,032,639$ & $53,038,786$ & deletion & - & not rare \\
\hline 184335 chr3 & $6,651,929$ & $6,654,060$ & deletion & - & not rare \\
\hline $184335 \mathrm{chr} 4$ & $138,092,298$ & $138,098,601$ & deletion & - & not rare \\
\hline $184335 \mathrm{chr} 4$ & $161,068,421$ & $161,179,694$ & deletion & - & rare \\
\hline $184335 \mathrm{chr} 4$ & $34,785,955$ & $34,818,502$ & deletion & - & not rare \\
\hline $184335 \mathrm{chr} 4$ & $39,705,484$ & $39,713,134$ & deletion & - & not rare \\
\hline $184335 \mathrm{chr} 4$ & $64,697,704$ & $64,709,753$ & deletion & - & not rare \\
\hline $184335 \mathrm{chr} 4$ & $7,182,916$ & 7,187,397 & deletion & - & not rare \\
\hline 184335 chr5 & $103,443,017$ & $103,452,929$ & deletion & - & not rare \\
\hline 184335 chr5 & $151,514,956$ & $151,518,615$ & deletion & - & not rare \\
\hline 184335 chr5 & $17,599,857$ & $17,625,744$ & deletion & - & not rare \\
\hline
\end{tabular}




\begin{tabular}{|c|c|c|c|c|c|}
\hline 184335 chr5 & $70,305,696$ & $70,308,251$ & duplication & NAIP & not rare \\
\hline 184335 chr5 & 770,153 & 825,338 & duplication & ZDHHC11 & not rare \\
\hline 184335 chr5 & $97,933,671$ & $97,939,616$ & deletion & - & not rare \\
\hline 184335 chr6 & $132,114,944$ & $132,118,192$ & deletion & - & rare \\
\hline 184335 chr6 & $139,603,393$ & $139,606,731$ & deletion & - & not rare \\
\hline 184335 chr6 & $29,855,945$ & $29,899,189$ & deletion & - & not rare \\
\hline 184335 chr6 & 665,448 & 670,182 & deletion & - & not rare \\
\hline 184335 chr6 & $77,439,769$ & $77,451,301$ & deletion & - & not rare \\
\hline 184335 chr6 & $78,979,398$ & $79,012,428$ & duplication & - & not rare \\
\hline 184335 chr7 & $75,664,539$ & $75,667,743$ & deletion & - & not rare \\
\hline 184335 chr7 & $86,940,652$ & $86,948,364$ & deletion & - & not rare \\
\hline 184335 chr7 & $8,827,959$ & $8,866,383$ & deletion & - & are \\
\hline 184335 & $17,580,791$ & $17,581,700$ & deletion & MTL & not rare \\
\hline 184335 chr8 & $24,972,808$ & $24,990,418$ & duplication & - & not rare \\
\hline 184335 chr8 & $39,232,581$ & $39,385,979$ & duplication & - & not rare \\
\hline 184335 chr9 & $139,668,443$ & $139,676,734$ & deletion & - & not rare \\
\hline 184335 chr9 & $23,363,526$ & $23,376,817$ & duplication & - & not rare \\
\hline $184335 \mathrm{chrX}$ & $47,881,362$ & $47,976,174$ & deletion & ZNF630 & not rare \\
\hline $184335 \mathrm{chr} X$ & $51,741,943$ & $51,760,413$ & dele & - & not rare \\
\hline 184336 chr1 & $104,153,766$ & $104,211,046$ & deletion & \multicolumn{2}{|c|}{ AMY1A;AN not rare } \\
\hline 184336 chr1 & $161,563,386$ & $161,619,741$ & duplication & \multicolumn{2}{|c|}{ FCGR2C;FC not rare } \\
\hline 184336 chr1 & $47,509,513$ & $47,524,454$ & duplication & CYP4X1 & not rare \\
\hline 184336 chr10 & $38,774,749$ & $38,877,428$ & duplication & & not rare \\
\hline 184336 chr10 & $42,791,910$ & $42,811,180$ & dup & - & not rare \\
\hline 184336 chr11 & $55,365,761$ & $55,443,269$ & dele & \multicolumn{2}{|c|}{$\mathrm{OR} 4 \mathrm{P} 4 ; \mathrm{OR} \angle$ not rare } \\
\hline 184336 chr11 & $91,228,448$ & $91,235,067$ & duplication & - & not rare \\
\hline 184336 chr12 & $43,021,431$ & $43,027,453$ & deletion & - & not rare \\
\hline 184336 chr13 & $34,134,809$ & $34,143,545$ & deletion & - & not rare \\
\hline 184336 chr13 & $69,247,022$ & $69,267,981$ & deletion & - & not rare \\
\hline 184336 chr14 & $106,178,679$ & $106,199,579$ & duplication & - & not rare \\
\hline 184336 chr14 & $20,361,945$ & $20,404,736$ & duplication & \multicolumn{2}{|c|}{ OR4K5;OR $\angle$ not rare } \\
\hline 184336 chr14 & $41,610,224$ & $41,661,685$ & deletion & - & not rare \\
\hline 184336 & $46,476,963$ & $46,492,062$ & dele & - & not rare \\
\hline 184336 chr16 & $55,800,009$ & $55,819,443$ & dele & - & not rare \\
\hline 184336 chr16 & $6,504,661$ & $6,508,784$ & deletion & - & not rare \\
\hline 184336 chr17 & $14,997,297$ & $15,010,250$ & deletion & - & not rare \\
\hline 184336 chr17 & $15,044,804$ & $15,058,236$ & deletion & - & not rare \\
\hline 184336 chr19 & $43,703,965$ & $43,759,382$ & deletion & \multicolumn{2}{|c|}{ PSG4;PSG9 not rare } \\
\hline 184336 chr19 & $53,518,747$ & $53,552,296$ & duplication & ERVV-1 & not rare \\
\hline $184336 \mathrm{chr} 2$ & $214,989,439$ & $214,992,692$ & deletion & - & not rare \\
\hline 184336 chr2 & $4,212,725$ & $4,222,144$ & deletion & - & not rare \\
\hline 184336 chr21 & $10,749,786$ & $10,858,651$ & duplication & - & not rare \\
\hline 184336 chr21 & $23,655,900$ & $23,665,470$ & deletion & - & not rare \\
\hline 184336 chr3 & $195,457,853$ & $195,468,690$ & duplication & MUC20 & not rare \\
\hline 184336 chr3 & $37,979,882$ & $37,986,734$ & deletion & - & not rare \\
\hline 184336 & $190,194,200$ & $190,199,993$ & deletion & - & not rare \\
\hline $184336 \mathrm{chr} 4$ & $60,325,010$ & $60,330,880$ & duplication & - & not rare \\
\hline
\end{tabular}




\begin{tabular}{|c|c|c|c|c|c|}
\hline $184336 \mathrm{chr} 4$ & $64,232,579$ & $64,241,398$ & deletion & - & not rare \\
\hline 84336 chr4 & $69,374,369$ & $69,392,193$ & deletion & - & not rare \\
\hline $34336 \mathrm{chr} 4$ & $70,137,897$ & $70,229,260$ & deletion & UGT2B28 & not rare \\
\hline 84336 chr5 & $17,609,458$ & $17,642,516$ & deletion & - & not rare \\
\hline 184336 chr5 & $70,305,696$ & $70,308,251$ & deletion & NAIP & not rare \\
\hline 84336 chr5 & 744,005 & 825,338 & duplication & ZDHHC11 & not rare \\
\hline $184336 \mathrm{chr} 5$ & $97,933,671$ & $97,939,616$ & deletion & - & not rare \\
\hline 184336 chr6 & $30,994,010$ & $30,994,787$ & deletion & MUC22 & not rare \\
\hline 184336 chr6 & $32,486,060$ & $32,549,192$ & deletion & HLA-DRB1; & not rare \\
\hline 184336 chr6 & $68,819,790$ & $68,835,697$ & duplication & - & not rare \\
\hline 184336 chr6 & $93,713,622$ & $93,724,029$ & deletion & - & not rare \\
\hline 184336 chr7 & $142,476,094$ & $142,484,042$ & deletion & - & not rare \\
\hline 184336 chr7 & $57,991,743$ & $57,996,333$ & duplication & - & not rare \\
\hline 184336 chr8 & $115,633,704$ & $115,642,459$ & deletion & - & not rare \\
\hline $184336 \mathrm{chr} 8$ & $24,972,808$ & $24,990,418$ & duplication & - & not rare \\
\hline $184336 \mathrm{chr} 8$ & $39,232,581$ & $39,385,979$ & duplication & - & not rare \\
\hline $184336 \mathrm{chrX}$ & $39,645,132$ & $39,662,452$ & duplication & - & not \\
\hline 184337 chr1 & $104,153,766$ & $104,211,046$ & deletion & AMY1A;AN & are \\
\hline 184337 chr1 & $149,024,218$ & $149,233,182$ & deletion & - & not rare \\
\hline 184337 chr1 & $161,563,386$ & $161,619,741$ & duplication & FCGR2C;FC & no \\
\hline 184337 chr11 & 18,9 & $18,961,743$ & duplication & MRGPI & \\
\hline 184337 chr11 & $55,365,761$ & $55,443,269$ & deletion & OR4P4;OR & $\angle$ not rare \\
\hline 184337 chr12 & $43,021,431$ & $43,027,453$ & & & rare \\
\hline 184337 chr13 & $34,134,809$ & $34,143,545$ & dele & & not rare \\
\hline 184337 chr13 & $78,806,521$ & $78,808,628$ & del & & not rare \\
\hline 184337 chr14 & $20,331,117$ & $20,422,182$ & duplication & OR4K5;C & $\angle$ not \\
\hline 184337 chr16 & $55,798,095$ & $55,819,443$ & deletion & - & not rare \\
\hline 184337 chr16 & $6,504,661$ & $6,508,784$ & deletion & - & not rare \\
\hline 184337 chr17 & 14,9 & 15,0 & del & - & are \\
\hline 184337 chr17 & $15,044,804$ & $15,058,236$ & deletion & - & not rare \\
\hline 184337 chr19 & $55,028,894$ & $55,153,092$ & deletion & LILRA2;L & rare \\
\hline 184337 chr2 & $195,677,877$ & $195,703,010$ & deletion & - & not rare \\
\hline 184337 chr2 & $208,351,315$ & $208,357,838$ & deletion & - & not rare \\
\hline 184337 chr2 & $48,851,541$ & $48,856,770$ & deletion & - & not rare \\
\hline 184337 chr21 & $10,699,687$ & $10,888,876$ & duplication & - & not rare \\
\hline 184337 chr21 & $23,655,900$ & $23,665,470$ & deletion & - & not rare \\
\hline 184337 chr22 & $31,258,967$ & $31,262,905$ & deletion & - & are \\
\hline 184337 chr3 & $37,979,882$ & $37,986,734$ & deletion & - & not rare \\
\hline 184337 chr3 & $6,651,929$ & $6,654,060$ & deletion & - & not rare \\
\hline 184337 chr4 & $190,194,200$ & $190,200,463$ & deletion & - & not rare \\
\hline $184337 \mathrm{chr} 4$ & $34,785,955$ & $34,818,502$ & deletion & - & not rare \\
\hline 184337 chr4 & $64,136,609$ & $64,154,477$ & deletion & - & not rare \\
\hline $184337 \mathrm{chr} 4$ & $69,374,369$ & $69,489,473$ & duplication & UGT2B17 & not rare \\
\hline $184337 \mathrm{chr} 5$ & $17,609,458$ & $17,642,516$ & deletion & - & not rare \\
\hline 184337 chr5 & $180,377,470$ & $180,430,540$ & deletion & \multicolumn{2}{|c|}{ BTNL8;BTN not rare } \\
\hline 184337 chr5 & $70,305,696$ & $70,308,251$ & deletion & NAIP & not rare \\
\hline 184337 & 733,565 & 825,338 & duplicatic & ZDHHC11 & not rar \\
\hline
\end{tabular}




\begin{tabular}{|c|c|c|c|c|c|}
\hline 184337 chr5 & $97,933,671$ & $97,939,616$ & deletion & - & not rare \\
\hline 184337 chr6 & $30,994,010$ & $30,994,787$ & deletion & MUC22 & not rare \\
\hline 84337 chr6 & $31,338,528$ & $31,341,357$ & deletion & - & ot rare \\
\hline 84337 chr6 & $68,819,790$ & $68,836,838$ & duplication & - & not rare \\
\hline 184337 chr6 & $78,972,930$ & $79,012,428$ & duplication & - & ot rare \\
\hline $184337 c$ & $142,476,094$ & $142,484,042$ & deletion & - & t rare \\
\hline 184337 chr7 & 41,421 & 73,060 & duplication & - & not rare \\
\hline 184337 chr7 & $57,991,743$ & $57,996,333$ & duplication & - & not rare \\
\hline 184337 chr8 & $39,232,581$ & $39,385,979$ & duplication & - & not rare \\
\hline 184337 chr8 & $51,226,087$ & $51,228,442$ & deletion & - & not rare \\
\hline 184337 chr9 & $6,662,216$ & $6,670,263$ & deletion & - & not rare \\
\hline $184337 \mathrm{chr} X$ & $39,645,132$ & $39,664,301$ & duplication & - & not rare \\
\hline 184338 chr1 & $104,153,766$ & $104,211,046$ & deletion & \multicolumn{2}{|c|}{ AMY1A;AN not rare } \\
\hline 184338 chr1 & $152,526,812$ & $152,568,230$ & deletion & \multicolumn{2}{|c|}{ LCE3D;LCE: not rare } \\
\hline $184338 \mathrm{chr} 1$ & $161,489,467$ & $161,536,131$ & duplication & \multicolumn{2}{|c|}{ FCGR3A;HS not rare } \\
\hline 184338 chr1 & $161,563,386$ & $161,619,741$ & deletion & \multicolumn{2}{|c|}{ FCGR2C;FC not rare } \\
\hline 184338 chr10 & $38,774,749$ & $38,877,428$ & duplication & - & not rare \\
\hline 184338 chr10 & $39,078,767$ & $39,093,434$ & duplication & - & not rare \\
\hline 184338 & 4,204 & 2,738 & deletion & - & \\
\hline 184338 chr11 & $55,365,761$ & $55,443,269$ & deletion & \multicolumn{2}{|c|}{$\mathrm{OR} 4 \mathrm{P} 4 ; \mathrm{OR} \angle$ not rare } \\
\hline 184338 chr12 & $70,874,037$ & $70,879,131$ & deletion & - & not rare \\
\hline 184338 & $21,728,134$ & $21,746,637$ & ation & SK & not rare \\
\hline 184338 chr13 & $32,532,761$ & $32,537,768$ & dele & & not rare \\
\hline 184338 chr13 & $34,134,809$ & $34,143,545$ & & & care \\
\hline 184338 chr13 & $38,076,308$ & $38,085,537$ & dele & & not rare \\
\hline 184338 chr13 & $84,102,440$ & $84,157,927$ & dele & - & not rare \\
\hline 184338 & $93,951,505$ & $94,003,438$ & dele & - & \\
\hline 184338 chr16 & $12,418,582$ & $12,427,729$ & deletion & - & not rare \\
\hline 184338 chr16 & $18,253,329$ & $18,607,586$ & deletion & \multicolumn{2}{|c|}{ NOMO2;NF not rare } \\
\hline 184338 chr16 & $34,471,298$ & $34,756,258$ & duplication & - & are \\
\hline 184338 chr16 & $55,798,095$ & $55,819,443$ & deletion & - & not rare \\
\hline 184338 chr18 & $66,536,333$ & $66,538,301$ & deletion & - & not rare \\
\hline 184338 chr19 & $35,852,103$ & $35,861,695$ & deletion & - & not rare \\
\hline 184338 chr2 & $123,477,465$ & $123,482,301$ & deletion & - & not rare \\
\hline 184338 chr2 & $195,979,780$ & $195,982,450$ & deletion & - & not rare \\
\hline 184338 chr20 & $59,568,187$ & $59,590,280$ & duplication & - & not rare \\
\hline 184338 chr21 & $10,749,786$ & $10,808,192$ & duplication & - & not rare \\
\hline 184338 chr21 & $10,815,443$ & $10,858,651$ & duplication & - & not rare \\
\hline 184338 chr22 & $39,359,885$ & $39,385,663$ & deletion & \multicolumn{2}{|c|}{ APOBEC3B not rare } \\
\hline $184338 \mathrm{chr} 3$ & $131,711,896$ & $131,712,898$ & deletion & - & not rare \\
\hline 184338 chr3 & $18,235,914$ & $18,244,588$ & deletion & - & not rare \\
\hline $184338 \mathrm{chr} 4$ & $34,785,955$ & $34,818,502$ & deletion & - & not rare \\
\hline $184338 \mathrm{chr} 4$ & $7,182,916$ & $7,187,397$ & deletion & - & not rare \\
\hline 184338 chr5 & $103,443,017$ & $103,452,929$ & deletion & - & not rare \\
\hline 184338 chr5 & $126,232,069$ & $126,234,277$ & deletion & - & not rare \\
\hline 184338 & $140,225,908$ & $140,238,311$ & deletion & \multicolumn{2}{|c|}{ PCDHA10; F not rare } \\
\hline 184338 chr5 & $159,212,767$ & $159,218,574$ & deletion & - & not rare \\
\hline
\end{tabular}




\begin{tabular}{|c|c|c|c|c|c|}
\hline 184338 chr5 & $97,933,671$ & $97,939,616$ & deletion & - & not rare \\
\hline $184338 \mathrm{chr} 6$ & $31,338,528$ & $31,341,357$ & deletion & - & not rare \\
\hline 184338 chr6 & $32,652,359$ & $32,654,454$ & deletion & - & not rare \\
\hline 184338 chr6 & $33,115,024$ & $33,138,955$ & duplication & COL11A2 & rare \\
\hline 184338 chr6 & $66,074,421$ & $66,080,908$ & deletion & - & not rare \\
\hline 184338 chr6 & $78,972,930$ & $79,012,428$ & duplication & - & not rare \\
\hline 184338 chr8 & $115,633,704$ & $115,650,409$ & deletion & - & not rare \\
\hline $184338 \mathrm{chr} 8$ & $24,972,808$ & $24,990,418$ & duplication & - & not rare \\
\hline 184338 chr8 & $39,232,581$ & $39,385,979$ & duplication & - & not rare \\
\hline 184338 chr8 & $3,974,190$ & $3,974,736$ & deletion & - & not rare \\
\hline 184338 chr8 & $51,029,371$ & $51,038,149$ & deletion & - & not rare \\
\hline $184338 \mathrm{chr} 8$ & $51,226,087$ & $51,227,777$ & deletion & - & not rare \\
\hline 184338 chr9 & $113,047,790$ & $113,051,296$ & deletion & - & not rare \\
\hline 184338 chr9 & $23,362,379$ & $23,376,817$ & duplication & - & not rare \\
\hline 184338 chr9 & $37,461,064$ & $37,478,497$ & deletion & - & not rare \\
\hline 184338 chr9 & $44,739,805$ & $44,834,316$ & deletion & - & not rare \\
\hline 184338 chr9 & $6,662,822$ & $6,670,263$ & deletion & - & not rare \\
\hline $184338 \mathrm{chrX}$ & $94,343,494$ & $94,393,450$ & duplication & - & not rare \\
\hline $184339 \mathrm{chr} 1$ & $12,846,082$ & $12,919,928$ & deletion & HNRNPCL1 & not rare \\
\hline 184339 chr1 & $161,559,327$ & $161,619,741$ & duplication & FCGR2C;FC & t rare \\
\hline 184339 chr1 & $248,740,572$ & $248,795,110$ & deletion & OR2T10 & are \\
\hline $184339 \mathrm{chr} 1$ & $62,113,597$ & $62,119,136$ & deletion & - & not rare \\
\hline 184339 chr10 & $23,154,715$ & $23,157,251$ & & & not rare \\
\hline 184339 chr10 & $71,282,981$ & $71,289,190$ & deletion & - & not rare \\
\hline 184339 chr11 & $18,949,220$ & $18,961,743$ & deletion & MRGPRX1 & not rare \\
\hline $184339 \mathrm{chr} 11$ & $55,370,325$ & $55,427,700$ & deletion & OR4P4;ORL & $\angle$ not rare \\
\hline 184339 chr11 & $90,193,796$ & $90,195,742$ & deletion & - & not rare \\
\hline $184339 \mathrm{chr} 12$ & $27,647,382$ & $27,655,163$ & deletion & SMCO2 & not rare \\
\hline $184339 \mathrm{chr} 12$ & 835,074 & 839,011 & ion & - & not rare \\
\hline 184339 chr13 & $23,545,717$ & $23,553,518$ & deletion & - & not rare \\
\hline 184339 chr13 & $34,134,809$ & $34,143,545$ & deletion & - & not rare \\
\hline 184339 chr15 & $30,934,220$ & $31,024,804$ & duplication & - & not rare \\
\hline 184339 chr15 & $31,963,721$ & $32,515,849$ & deletion & CHRNA7 & rare \\
\hline 184339 chr16 & $32,531,934$ & $32,833,893$ & deletion & TP53TG3B; & ; not rare \\
\hline 184339 chr16 & $55,800,009$ & $55,819,443$ & deletion & - & not rare \\
\hline $184339 \mathrm{chr} 16$ & $60,082,237$ & $60,098,544$ & deletion & - & not rare \\
\hline 184339 chr16 & $78,372,894$ & $78,383,509$ & duplication & - & not rare \\
\hline 184339 chr17 & $10,890,859$ & $10,893,799$ & deletion & - & not rare \\
\hline $184339 \mathrm{chr} 18$ & $63,200,484$ & $63,206,335$ & duplication & - & not rare \\
\hline $184339 \mathrm{chr} 18$ & $63,907,596$ & $63,910,708$ & deletion & - & not rare \\
\hline 184339 chr19 & $53,518,747$ & $53,552,296$ & duplication & ERVV-1 & not rare \\
\hline $184339 \mathrm{chr} 2$ & $123,477,465$ & $123,482,301$ & deletion & - & not rare \\
\hline $184339 \mathrm{chr} 2$ & $208,351,315$ & $208,357,838$ & deletion & - & not rare \\
\hline $184339 \mathrm{chr} 2$ & $223,865,393$ & $223,869,820$ & deletion & - & not rare \\
\hline $184339 \mathrm{chr} 2$ & $48,851,541$ & $48,856,770$ & deletion & - & not rare \\
\hline $184339 \mathrm{chr} 2$ & $51,926,599$ & $51,926,904$ & deletion & - & not rare \\
\hline 184339 & $14,573,513$ & $14,576,448$ & deletion & - & not rare \\
\hline
\end{tabular}




\begin{tabular}{|c|c|c|c|c|c|}
\hline 184339 chr20 & $53,407,575$ & $53,419,685$ & deletion & - & not rare \\
\hline 184339 chr20 & $53,764,306$ & $54,122,219$ & deletion & - & rare \\
\hline 84339 chr21 & $23,655,900$ & $23,665,470$ & deletion & - & not rare \\
\hline $184339 \mathrm{chr} 22$ & $29,758,178$ & $29,814,381$ & duplication & AP1B1 & rare \\
\hline $184339 \mathrm{chr} 3$ & $10,766,629$ & $10,774,759$ & deletion & - & not rare \\
\hline $184339 \mathrm{chr} 4$ & $138,092,298$ & $138,098,601$ & deletion & - & not rare \\
\hline $184339 \mathrm{chr} 4$ & $156,959,568$ & $156,974,697$ & deletion & - & not rare \\
\hline $184339 \mathrm{chr} 4$ & $59,980,062$ & $59,988,095$ & duplication & - & not rare \\
\hline $184339 \mathrm{chr} 4$ & $64,697,704$ & $64,709,753$ & deletion & - & not rare \\
\hline $184339 \mathrm{chr} 4$ & $69,374,369$ & $69,489,473$ & deletion & UGT2B17 & not rare \\
\hline $184339 \mathrm{chr} 4$ & $70,130,965$ & $70,229,260$ & deletion & UGT2B28 & not rare \\
\hline 184339 chr5 & $151,514,956$ & $151,518,615$ & deletion & - & not rare \\
\hline 184339 chr5 & $180,377,470$ & $180,430,540$ & deletion & \multicolumn{2}{|c|}{ BTNL8;BTN not rare } \\
\hline 184339 chr5 & $97,933,671$ & $97,939,616$ & deletion & - & not rare \\
\hline 184339 chr6 & $31,219,869$ & $31,229,552$ & deletion & - & not rare \\
\hline 184339 chr6 & $31,276,513$ & $31,286,577$ & deletion & - & not rare \\
\hline 184339 chr6 & $78,999,898$ & $79,012,428$ & duplication & - & not rare \\
\hline 184339 chr7 & 41,421 & 73,060 & duplication & - & not rare \\
\hline 184339 chr7 & $66,783,279$ & $66,787,661$ & deletion & - & ot rare \\
\hline 184339 chr7 & $91,033,074$ & $91,041,618$ & deletion & - & not rare \\
\hline $184339 \mathrm{chr} 8$ & $115,633,704$ & $115,642,459$ & deletion & - & not rare \\
\hline 184339 chr8 & $39,232,581$ & $39,385,979$ & del & - & not rare \\
\hline 184339 chr8 & $3,973,859$ & $3,974,736$ & del & & not rare \\
\hline 184339 chr9 & $1,446,522$ & $1,450,465$ & del & & not rare \\
\hline 184339 chr9 & $21,211,979$ & $21,227,459$ & deletion & IFNA16 & not rare \\
\hline 184339 chr9 & $23,363,526$ & $23,376,817$ & deletion & - & not rare \\
\hline 184339 chr9 & $24,502,737$ & $24,518,795$ & del & - & not rare \\
\hline 184339 chr9 & $78,002,560$ & $78,011,616$ & deletion & - & not rare \\
\hline $184339 \mathrm{chrX}$ & $51,741,943$ & $51,760,413$ & deletion & - & not rare \\
\hline 184340 chr1 & $104,153,766$ & $104,161,227$ & deletion & AMY2A & not rare \\
\hline $184340 \mathrm{chr} 1$ & $248,740,572$ & $248,795,110$ & deletion & \multicolumn{2}{|c|}{ OR2T10;OF not rare } \\
\hline 184340 chr1 & $62,113,597$ & $62,119,136$ & deletion & - & not rare \\
\hline 184340 chr10 & $38,677,023$ & $38,972,666$ & duplication & - & not rare \\
\hline 184340 chr10 & $39,058,671$ & $39,154,535$ & duplication & - & not rare \\
\hline 184340 chr11 & $55,370,325$ & $55,427,700$ & deletion & \multicolumn{2}{|c|}{ OR4P4;OR $\angle$ not rare } \\
\hline 184340 chr11 & $90,193,796$ & $90,196,843$ & deletion & - & not rare \\
\hline 184340 chr12 & $30,237,351$ & $30,243,473$ & deletion & - & not rare \\
\hline 184340 chr12 & $31,283,913$ & $31,407,303$ & duplication & - & not rare \\
\hline 184340 chr12 & $43,021,431$ & $43,027,453$ & deletion & - & not rare \\
\hline 184340 chr12 & $59,935,926$ & $59,942,122$ & deletion & - & not rare \\
\hline 184340 chr13 & $34,134,809$ & $34,143,545$ & deletion & - & not rare \\
\hline 184340 chr14 & $20,361,945$ & $20,375,610$ & duplication & - & not rare \\
\hline 184340 chr14 & $35,604,892$ & $35,613,500$ & deletion & - & not rare \\
\hline 184340 chr14 & $41,610,224$ & $41,661,685$ & deletion & - & not rare \\
\hline 184340 chr15 & $20,120,214$ & $20,133,383$ & deletion & - & not rare \\
\hline 184340 chr15 & $60,086,249$ & $60,103,464$ & deletion & - & not rare \\
\hline 184340 chr16 & $5,109,651$ & $5,110,738$ & deletion & C16orf89 & not rare \\
\hline
\end{tabular}




\begin{tabular}{|c|c|c|c|c|c|}
\hline 184340 chr16 &, 009 & $55,819,443$ & deletion & - & not rare \\
\hline $184340 \mathrm{chr} 16$ & $55,843,252$ & $55,865,127$ & duplication & CES1 & not rare \\
\hline 84340 chr16 & $58,945,599$ & $58,948,100$ & deletion & - & ot rare \\
\hline 34340 chr17 & $34,438,753$ & $34,534,918$ & duplication & \multicolumn{2}{|c|}{ CCL3L3;CCI not rare } \\
\hline $84340 \mathrm{chr} 18$ & $63,907,596$ & $63,910,708$ & deletion & - & not rare \\
\hline 184340 chr2 & $184,085,551$ & $184,088,256$ & deletion & - & ot rare \\
\hline $84340 \mathrm{chr} 2$ & $208,351,315$ & $208,357,838$ & deletion & - & not rare \\
\hline $184340 \mathrm{chr} 2$ & $213,187,034$ & $213,191,389$ & deletion & - & not rare \\
\hline 184340 chr2 & $34,697,895$ & $34,726,904$ & duplication & - & not rare \\
\hline $184340 \mathrm{chr} 2$ & $51,926,599$ & $51,926,904$ & deletion & - & not rare \\
\hline 184340 chr20 & $52,647,631$ & $52,656,535$ & deletion & - & not rare \\
\hline 184340 chr21 & $10,699,687$ & $10,888,876$ & duplication & - & not rare \\
\hline 184340 chr22 & $42,522,613$ & $42,531,210$ & deletion & CYP2D6 & not rare \\
\hline 184340 chr3 & $193,136,358$ & $193,140,348$ & deletion & - & not rare \\
\hline $184340 \mathrm{chr} 3$ & $37,979,882$ & $37,990,299$ & deletion & $\mathrm{CT}$ & not rare \\
\hline 184340 chr3 & $53,032,639$ & $53,042,099$ & deletion & - & rare \\
\hline $184340 \mathrm{chr} 4$ & $138,092,298$ & $138,098,601$ & deletion & - & not rare \\
\hline $184340 \mathrm{chr} 4$ & $152,789,786$ & $152,793,891$ & deletion & - & not rare \\
\hline 184340 chr5 & 0,864 & $119,509,546$ & tion & - & \\
\hline 184340 chr5 & $32,106,628$ & $32,164,826$ & duplication & \multicolumn{2}{|c|}{ GOLPH3;P[ not rare } \\
\hline $184340 \mathrm{chr} 5$ & $7,178,644$ & $7,191,074$ & deletion & - & not rare \\
\hline 184340 chr5 & 760,502 & 825,338 & duplication & HC11 & not rare \\
\hline 184340 chr6 & $29,855,945$ & $29,899,189$ & dele & & not rare \\
\hline 184340 chr6 & $31,281,682$ & $31,289,316$ & & & not rare \\
\hline 184340 chr6 & $32,486,060$ & $32,555,728$ & deletion & \multicolumn{2}{|c|}{ HLA-DRB1; not rare } \\
\hline 184340 chr7 & $159,118,443$ & $159,122,741$ & deletion & - & not rare \\
\hline 184340 chr7 & $62,285,495$ & $62,306,851$ & ation & - & \\
\hline 184340 chr7 & $75,354,240$ & $75,370,368$ & duplication & HIP1 & not rare \\
\hline $184340 \mathrm{chr} 8$ & $12,551,156$ & $12,564,733$ & deletion & - & not rare \\
\hline 184340 chr8 & $135,059,968$ & $135,065,947$ & delet & - & are \\
\hline 184340 chr8 & $24,972,808$ & $24,990,418$ & duplication & - & not rare \\
\hline 184340 chr8 & $39,232,581$ & $39,382,903$ & deletion & - & not rare \\
\hline 184340 chr9 & $138,195,113$ & $138,195,756$ & deletion & - & not rare \\
\hline $184340 \mathrm{chrX}$ & $95,446,396$ & $95,480,629$ & deletion & - & not rare \\
\hline $184341 \mathrm{chr} 1$ & $104,153,766$ & $104,161,227$ & deletion & AMY2A & not rare \\
\hline 184341 chr1 & $109,367,944$ & $109,371,874$ & deletion & AKNAD1 & not rare \\
\hline 184341 chr1 & $161,489,467$ & $161,619,741$ & duplication & \multicolumn{2}{|c|}{ FCGR3B;FC not rare } \\
\hline 184341 chr1 & $1,851,185$ & $1,854,579$ & deletion & CFAP74 & not rare \\
\hline 184341 chr10 & $71,278,805$ & $71,289,190$ & deletion & - & not rare \\
\hline 184341 chr11 & $18,949,220$ & $18,961,743$ & duplication & MRGPRX1 & not rare \\
\hline 184341 chr11 & $55,365,761$ & $55,427,700$ & deletion & OR4P4;OR & not rare \\
\hline 184341 chr12 & $27,648,411$ & $27,657,025$ & deletion & SMCO2 & not rare \\
\hline 184341 chr12 & $30,237,351$ & $30,243,631$ & deletion & - & not rare \\
\hline 184341 chr12 & $31,285,101$ & $31,407,303$ & duplication & - & not rare \\
\hline 184341 chr12 & $43,021,431$ & $43,027,453$ & deletion & - & not rare \\
\hline 184341 chr12 & $80,849,328$ & $80,997,556$ & deletion & PTPRQ & rare \\
\hline 184341 chr14 & $20,197,311$ & $20,422,799$ & duplication & \multicolumn{2}{|c|}{ OR4K5;OR $\angle$ not rare } \\
\hline
\end{tabular}




\begin{tabular}{|c|c|c|c|c|c|}
\hline 84341 chr15 & 20,1 & $21,241,118$ & deletion & \multicolumn{2}{|c|}{ GOLGA6L6; not rare } \\
\hline 184341 chr16 & $5,106,184$ & $5,112,590$ & deletion & C16orf89 & not rare \\
\hline 34341 chr17 & $10,890,859$ & $10,893,799$ & deletion & - & rare \\
\hline 34341 chr17 & $46,673,539$ & $46,676,460$ & deletion & $\mathrm{HC}$ & not rare \\
\hline L84341 chr19 & $9,275,282$ & $9,282,176$ & deletion & - & not rare \\
\hline $84341 \mathrm{chr} 2$ & $242,854,089$ & $243,036,003$ & deletion & - & not rare \\
\hline $84341 \mathrm{chr} 2$ & $89,540,620$ & $90,256,530$ & deletion & - & not rare \\
\hline 184341 chr20 & $52,647,631$ & $52,656,535$ & deletion & - & not rare \\
\hline 184341 chr21 & $10,813,582$ & $10,863,251$ & duplication & - & not rare \\
\hline 184341 chr22 & $39,355,109$ & $39,385,663$ & deletion & \multicolumn{2}{|c|}{ APOBEC $3 \mathrm{~A}$ not rare } \\
\hline 184341 chr3 & $129,769,827$ & $129,806,304$ & deletion & ALG1L2 & not rare \\
\hline $184341 \mathrm{chr} 3$ & $193,136,358$ & $193,140,348$ & deletion & - & care \\
\hline $184341 \mathrm{chr} 4$ & $138,092,298$ & $138,098,601$ & deletion & - & not rare \\
\hline $184341 \mathrm{chr} 4$ & $49,588,812$ & $49,651,131$ & deletion & - & not rare \\
\hline $184341 \mathrm{chr} 4$ & $60,325,010$ & $60,330,880$ & ation & - & are \\
\hline $184341 \mathrm{chr} 4$ & $69,374,369$ & $69,467,200$ & dele & $2 \mathrm{~B} 17$ & not rare \\
\hline $184341 \mathrm{chr} 5$ & $32,106,628$ & $32,164,826$ & duplication & \multicolumn{2}{|c|}{ GOLPH3;P[ not rare } \\
\hline 184341 chr5 & 762,435 & 825,338 & duplication & HC11 & not rare \\
\hline 184341 chr7 & 149,765 & 159 & & - & \\
\hline 184341 chr7 & $15,154,324$ & $15,159,059$ & deletion & - & not rare \\
\hline $184341 \mathrm{chr} 7$ & $159,118,443$ & $159,122,741$ & dele & - & not rare \\
\hline 184341 chr7 & $91,033,074$ & $91,041,618$ & dele & - & not rare \\
\hline $184341 \mathrm{chr} 8$ & $135,059,968$ & $135,065,947$ & dele & & not rare \\
\hline 184341 chr8 & $39,232,581$ & $39,385,979$ & & & not rare \\
\hline 184341 chr8 & $4,313,576$ & $4,335,682$ & del & & not rare \\
\hline 184341 chr9 & $138,195,113$ & $138,195,756$ & dele & & not rare \\
\hline 184341 chr9 & $139,668,443$ & 139 & & - & \\
\hline 184341 chr9 & $23,363,526$ & $23,376,817$ & deletion & - & not \\
\hline 184342 chr1 & $112,693,423$ & $112,704,581$ & deletion & - & not rare \\
\hline 184342 chr1 & $12,846,082$ & $12,919,928$ & dele & \multicolumn{2}{|c|}{ HNRNPCL1 not rare } \\
\hline 184342 chr10 & $47,541,708$ & $47,756,065$ & duplication & \multicolumn{2}{|c|}{ ANTXRL;A^ not rare } \\
\hline 184342 chr11 & $24,083,684$ & $24,140,697$ & deletion & - & not rare \\
\hline 184342 chr11 & $55,365,761$ & $55,443,269$ & deletion & \multicolumn{2}{|c|}{ OR4P4;OR $\angle$ not rare } \\
\hline 184342 chr11 & $90,193,796$ & $90,195,742$ & deletion & - & not rare \\
\hline 184342 chr12 & $43,021,431$ & $43,027,453$ & on & - & are \\
\hline 184342 chr12 & $59,935,926$ & $59,942,122$ & dele & - & not rare \\
\hline 184342 chr12 & $70,871,858$ & $70,879,131$ & deletion & - & not rare \\
\hline 184342 chr13 & $34,134,809$ & $34,143,545$ & deletion & - & not rare \\
\hline 184342 chr13 & $69,247,022$ & $69,267,981$ & deletion & - & not rare \\
\hline 184342 chr14 & $106,082,680$ & $106,199,579$ & duplication & - & not rare \\
\hline 184342 chr14 & $20,197,311$ & $20,403,643$ & duplication & \multicolumn{2}{|c|}{ OR4Q3;OR، not rare } \\
\hline 184342 chr14 & $41,610,224$ & $41,661,685$ & deletion & - & not rare \\
\hline 184342 chr14 & $44,504,048$ & $44,531,417$ & deletion & - & not rare \\
\hline 184342 chr14 & $47,247,998$ & $47,255,362$ & deletion & - & not rare \\
\hline 184342 chr15 & $20,196,661$ & $21,241,118$ & deletion & \multicolumn{2}{|c|}{ GOLGA6L6; not rare } \\
\hline 184342 chr16 & $18,253,329$ & $18,607,586$ & duplication & \multicolumn{2}{|c|}{ NOMO2; NF not rare } \\
\hline 184342 chr16 & $55,800,009$ & $55,819,443$ & deletion & - & not rare \\
\hline
\end{tabular}




\begin{tabular}{|c|c|c|c|c|c|}
\hline 184342 chr17 & 97 & 15,0 & deletion & - & not rare \\
\hline 184342 chr17 & $15,044,804$ & $15,058,236$ & deletion & - & not rare \\
\hline 84342 chr17 & $34,438,753$ & $34,534,918$ & duplication & \multicolumn{2}{|c|}{ CCL3L3;CCI not rare } \\
\hline 84342 chr18 & $41,976,831$ & $41,982,102$ & deletion & - & not rare \\
\hline 184342 chr18 & $56,463,861$ & $56,467,150$ & deletion & - & not rare \\
\hline 184342 chr2 & $177,374,056$ & $177,377,367$ & deletion & - & not rare \\
\hline 184342 chr2 & $92,293,128$ & $92,304,211$ & deletion & - & not rare \\
\hline 184342 chr2 & $97,738,917$ & $98,024,837$ & deletion & \multicolumn{2}{|c|}{ FAHD2B;Ar not rare } \\
\hline 184342 chr20 & $29,804,016$ & $30,232,673$ & duplication & \multicolumn{2}{|c|}{ DEFB116;D rare } \\
\hline 184342 chr20 & $37,008,769$ & $37,014,876$ & deletion & - & not rare \\
\hline 184342 chr20 & $3,880,128$ & $3,885,100$ & deletion & - & not rare \\
\hline 184342 chr20 & $53,407,575$ & $53,419,685$ & deletion & - & not rare \\
\hline 184342 chr22 & $39,359,885$ & $39,385,663$ & deletion & \multicolumn{2}{|c|}{ APOBEC3B not rare } \\
\hline 184342 chr3 & $143,133,504$ & $143,167,322$ & deletion & - & rare \\
\hline 184342 chr3 & $65,117,947$ & $65,118,762$ & deletion & - & not rare \\
\hline $184342 \mathrm{chr} 4$ & $34,785,955$ & $34,805,860$ & duplication & - & not rare \\
\hline $184342 \mathrm{chr} 4$ & $69,374,369$ & $69,402,799$ & deletion & - & not rare \\
\hline $184342 \mathrm{chr} 4$ & $70,137,897$ & $70,229,260$ & deletion & B28 & not rare \\
\hline $184342 \mathrm{chr} 4$ & 7,18 & $7,187,397$ & & - & \\
\hline 184342 chr5 & $103,443,017$ & $103,452,929$ & deletion & - & not rare \\
\hline 184342 chr5 & $140,225,908$ & $140,238,311$ & deletion & \multicolumn{2}{|c|}{ PCDHA10; F not rare } \\
\hline 184342 chr6 & $20,884,080$ & $20,914,617$ & duplication & - & rare \\
\hline 184342 chr6 & $29,855,945$ & $29,899,189$ & deletion & & not rare \\
\hline 184342 chr6 & $31,276,513$ & $31,277,988$ & & - & not rare \\
\hline 184342 chr6 & $31,360,255$ & $31,453,640$ & deletion & M & not rare \\
\hline 184342 chr6 & $66,074,421$ & $66,080,908$ & deletion & - & not rare \\
\hline 184342 chr6 & $67,014,958$ & $67,017,722$ & & - & \\
\hline 184342 chr6 & $78,972,930$ & $79,035,739$ & deletion & - & not rare \\
\hline 184342 chr7 & $15,154,324$ & $15,159,059$ & deletion & - & not rare \\
\hline 184342 chr7 & $38,388,889$ & $38,402,517$ & deletion & - & not rare \\
\hline 184342 chr7 & 41,421 & 73,060 & duplication & - & not rare \\
\hline 184342 chr7 & $86,238,045$ & $86,244,535$ & deletion & - & not rare \\
\hline 184342 chr7 & $86,940,652$ & $86,948,364$ & deletion & - & not rare \\
\hline 184342 chr8 & $103,054,099$ & $103,056,645$ & deletion & - & not rare \\
\hline 184342 chr8 & $117,928,825$ & $117,938,722$ & duplication & - & not rare \\
\hline 184342 chr8 & $39,232,581$ & $39,385,979$ & deletion & - & not rare \\
\hline 184342 chr8 & $40,184,872$ & $40,189,688$ & deletion & - & not rare \\
\hline 184342 chr8 & $4,691,734$ & $4,711,912$ & deletion & - & rare \\
\hline 184342 chr9 & $23,363,526$ & $23,376,817$ & duplication & - & not rare \\
\hline 184342 chr9 & $5,304,710$ & $5,337,760$ & duplication & RLN1 & not rare \\
\hline $184342 \mathrm{chrX}$ & $95,446,396$ & $95,480,629$ & deletion & - & not rare \\
\hline 184343 chr1 & $149,024,218$ & $149,233,182$ & deletion & - & not rare \\
\hline 184343 chr1 & $1,585,642$ & $1,668,298$ & duplication & \multicolumn{2}{|c|}{ SLC35E2;C[ not rare } \\
\hline 184343 chr10 & $38,774,749$ & $38,877,428$ & duplication & - & not rare \\
\hline 184343 chr10 & $47,374,006$ & $47,756,065$ & duplication & \multicolumn{2}{|c|}{ ANTXRL;A^ not rare } \\
\hline 184343 chr10 & $58,516,470$ & $58,526,153$ & deletion & - & not rare \\
\hline 184343 chr13 & $58,589,893$ & $58,599,174$ & deletion & - & not rare \\
\hline
\end{tabular}




\begin{tabular}{|c|c|c|c|c|c|}
\hline 4343 chr14 & 50 & & & & \\
\hline 184343 chr14 & $41,610,224$ & $41,661,685$ & deletion & - & not rare \\
\hline 84343 chr16 & $32,531,934$ & $32,833,893$ & deletion & \multicolumn{2}{|c|}{ TP53TG3B; not rare } \\
\hline 34343 chr16 & $33,543,313$ & $33,590,959$ & deletion & - & not rare \\
\hline 84343 chr17 & $10,890,859$ & $10,893,799$ & deletion & - & not rare \\
\hline 84343 chr17 & $44,166,604$ & $44,225,181$ & duplication & KANSL1 & not rare \\
\hline 184343 chr18 & $41,976,831$ & $41,982,102$ & deletion & - & not rare \\
\hline 184343 chr19 & $20,828,777$ & $20,989,664$ & duplication & ZNF626 & not rare \\
\hline 184343 chr19 & $4,322,990$ & $4,385,562$ & duplication & \multicolumn{2}{|c|}{ FSD1;STAP: rare } \\
\hline 184343 chr19 & $54,728,767$ & $54,743,217$ & duplication & LILRA6 & not rare \\
\hline 184343 & $52,759,890$ & $52,784,952$ & deletion & - & are \\
\hline 184343 chr20 & $59,112,918$ & $59,122,693$ & deletion & - & not rare \\
\hline 184343 chr21 & $10,776,405$ & $10,828,683$ & deletion & - & not rare \\
\hline 184343 chr21 & $10,892,752$ & $11,063,273$ & deletion & \multicolumn{2}{|c|}{ TPTE;BAGE not rare } \\
\hline 184343 chr21 & $23,655,900$ & $23,665,470$ & deletion & - & not rare \\
\hline 184343 chr22 & $42,918,711$ & $42,950,033$ & duplication & SERHL2 & not rare \\
\hline $184343 \mathrm{chr} 3$ & $195,433,728$ & $195,468,690$ & duplication & MUC20 & not rare \\
\hline 184343 chr3 & $65,117,947$ & $65,118,762$ & deletion & - & not rare \\
\hline 184343 chr3 & $87,657,432$ & $87,662,325$ & dele & - & \\
\hline $184343 \mathrm{chr} 4$ & $122,285,263$ & $122,289,863$ & deletion & - & not rare \\
\hline $184343 \mathrm{chr} 4$ & $152,790,580$ & $152,793,891$ & deletion & - & not rare \\
\hline $184343 \mathrm{chr} 4$ & $25,557,519$ & $25,577,184$ & duplication & - & not rare \\
\hline $184343 \mathrm{chr} 4$ & $34,785,955$ & $34,818,502$ & dele & - & not rare \\
\hline 184343 chr5 & $140,225,908$ & $140,238,311$ & dele & \multicolumn{2}{|c|}{ PCDHA10;F not rare } \\
\hline 184343 chr5 & $17,345,282$ & $17,356,536$ & deletion & & not rare \\
\hline 184343 chr5 & $180,377,470$ & $180,430,540$ & deletion & \multicolumn{2}{|c|}{ BTNL8;BTN not rare } \\
\hline 184343 chr5 & $97,048,466$ & $97,096,042$ & & - & \\
\hline 184343 chr6 & $132,113,933$ & $132,118,192$ & deletion & - & not rare \\
\hline 184343 chr6 & $30,994,010$ & $30,994,787$ & deletion & MUC22 & not rare \\
\hline 184343 chr7 & $57,950,641$ & $58,008,189$ & sation & - & not rare \\
\hline 184343 chr8 & $12,551,156$ & $12,560,152$ & deletion & - & not rare \\
\hline 184343 chr8 & $138,822,798$ & $138,824,695$ & deletion & - & not rare \\
\hline 184343 chr8 & $24,972,808$ & $24,990,418$ & duplication & - & not rare \\
\hline 184343 chr8 & $40,184,872$ & $40,189,688$ & deletion & - & not rare \\
\hline 184343 chr8 & $57,041,268$ & $57,098,250$ & ation & PLAG1 & not rare \\
\hline 184343 chr9 & $66,820,824$ & $66,862,785$ & dele & - & rare \\
\hline 184343 chr9 & $6,683,293$ & $6,693,059$ & duplication & - & not rare \\
\hline 184343 chr9 & $78,004,378$ & $78,011,616$ & deletion & - & not rare \\
\hline 184343 chrX & $51,741,943$ & $51,760,413$ & deletion & - & not rare \\
\hline 184344 chr1 & $152,526,812$ & $152,568,230$ & deletion & \multicolumn{2}{|c|}{ LCE3D;LCE: not rare } \\
\hline 184344 chr1 & $72,754,314$ & $72,763,324$ & deletion & - & not rare \\
\hline 184344 chr10 & $105,716,540$ & $105,720,104$ & deletion & - & not rare \\
\hline 184344 chr11 & $55,365,761$ & $55,427,700$ & deletion & \multicolumn{2}{|c|}{ OR4P4;OR $\angle$ not rare } \\
\hline 184344 chr11 & $90,193,796$ & $90,195,742$ & deletion & - & not rare \\
\hline 184344 chr14 & $106,067,375$ & $106,178,679$ & deletion & - & not rare \\
\hline 184344 chr14 & $106,724,166$ & $106,734,169$ & duplication & - & not rare \\
\hline 184344 chr14 & $41,610,224$ & $41,661,685$ & deletion & - & not rare \\
\hline
\end{tabular}




\begin{tabular}{|c|c|c|c|c|c|}
\hline 84344 chr15 & 749 & 102 & on & \multicolumn{2}{|c|}{ TM2D3;TAI rare } \\
\hline 184344 chr15 & $56,789,979$ & $56,800,635$ & deletion & - & not rare \\
\hline 84344 chr16 & $60,082,237$ & $60,098,544$ & deletion & - & not rare \\
\hline 84344 chr17 & $66,559,123$ & $66,562,824$ & deletion & - & not rare \\
\hline 184344 chr18 & $63,907,596$ & $63,910,708$ & deletion & - & not rare \\
\hline 184344 chr19 & $43,703,965$ & $43,759,382$ & deletion & \multicolumn{2}{|c|}{ PSG4;PSG9 not rare } \\
\hline 184344 chr2 & $159,726,920$ & $159,738,206$ & duplication & - & not rare \\
\hline 184344 chr2 & $214,989,439$ & $214,992,692$ & deletion & - & not rare \\
\hline 184344 chr22 & $42,918,711$ & $42,950,033$ & duplication & SERHL2 & not rare \\
\hline 184344 chr3 & $128,382,258$ & $128,412,024$ & duplication & - & not rare \\
\hline 184344 chr3 & $191,065,392$ & $191,070,300$ & deletion & - & not rare \\
\hline 184344 chr3 & $193,136,358$ & $193,140,348$ & deletion & - & not rare \\
\hline 184344 chr3 & $37,979,882$ & $37,986,734$ & deletion & - & not rare \\
\hline $184344 \mathrm{chr} 4$ & $138,091,446$ & $138,098,601$ & deletion & - & not rare \\
\hline $184344 \mathrm{chr} 4$ & $152,991,094$ & $152,993,620$ & deletion & - & not rare \\
\hline 184344 chr4 & $64,697,704$ & $64,709,753$ & deletion & - & not rare \\
\hline $184344 \mathrm{chr} 4$ & $69,374,369$ & $69,431,280$ & deletion & UGT2B17 & not rare \\
\hline $184344 \mathrm{chr} 4$ & $70,137,897$ & $70,229,260$ & deletion & $2 \mathrm{~B} 28$ & not rare \\
\hline $184344 \mathrm{chr} 4$ & $7,182,916$ & 7,187,397 & & - & rare \\
\hline 184344 chr5 & $111,937,859$ & $111,944,746$ & deletion & - & not rare \\
\hline 184344 chr5 & $13,564,351$ & $13,583,651$ & dele & - & not rare \\
\hline 184344 chr5 & $155,477,866$ & $155,488,917$ & de & - & not rare \\
\hline 184344 chr6 & $19,041,346$ & $19,048,773$ & del & & not rare \\
\hline 184344 chr6 & $32,486,060$ & $32,520,879$ & del & HLA-DRB5 & not rare \\
\hline 184344 chr6 & $77,439,769$ & $77,451,301$ & dele & & not rare \\
\hline 184344 chr6 & $78,972,930$ & $79,035,739$ & dele & - & not rare \\
\hline 184344 chr7 & $155,882,711$ & $155,885,143$ & & - & rare \\
\hline 184344 chr7 & $86,940,652$ & $86,948,364$ & deletion & - & not rare \\
\hline 184344 chr7 & $91,033,074$ & $91,041,618$ & deletion & - & not rare \\
\hline 184344 chr7 & $97,396,709$ & $97,401,677$ & deletion & - & not rare \\
\hline 184344 chr8 & $135,059,968$ & $135,065,947$ & deletion & - & not rare \\
\hline 184344 chr8 & $2,289,839$ & $2,324,246$ & duplication & - & not rare \\
\hline 184344 chr8 & $39,232,581$ & $39,385,979$ & duplication & - & not rare \\
\hline 184344 chr9 & $1,446,522$ & $1,450,465$ & deletion & - & not rare \\
\hline 184345 chr1 & $104,153,766$ & $104,211,046$ & deletion & \multicolumn{2}{|c|}{ AMY1A;AN not rare } \\
\hline 184345 chr1 & $152,526,812$ & $152,568,230$ & deletion & \multicolumn{2}{|c|}{ LCE3D;LCE not rare } \\
\hline 184345 chr1 & $17,009,576$ & $17,261,738$ & duplication & \multicolumn{2}{|c|}{ FAM231A; f not rare } \\
\hline 184345 chr1 & $55,114,469$ & $55,120,469$ & deletion & MROH7 & not rare \\
\hline 184345 chr1 & $9,847,290$ & $9,855,529$ & deletion & - & not rare \\
\hline 184345 chr10 & $38,677,023$ & $38,972,666$ & duplication & - & not rare \\
\hline 184345 chr10 & $89,068,877$ & $89,112,638$ & deletion & - & not rare \\
\hline 184345 chr11 & $18,949,220$ & $18,961,743$ & duplication & MRGPRX1 & not rare \\
\hline 184345 chr11 & $25,750,699$ & $25,753,430$ & deletion & - & not rare \\
\hline 184345 chr11 & $3,239,077$ & $3,243,665$ & deletion & MRGPRG & not rare \\
\hline 184345 chr11 & $55,365,761$ & $55,443,269$ & deletion & \multicolumn{2}{|c|}{ OR4P4;OR $\angle$ not rare } \\
\hline 184345 chr11 & $90,193,796$ & $90,196,843$ & deletion & - & not rare \\
\hline 184345 chr12 & $11,220,827$ & $11,244,117$ & deletion & TAS2R43 & not rare \\
\hline
\end{tabular}




\begin{tabular}{|c|c|c|c|c|c|}
\hline L84345 chr12 & 84 & $32,843,021$ & duplication & \multicolumn{2}{|c|}{ FGD4;DNM rare } \\
\hline 184345 chr12 & $40,875,351$ & $40,875,963$ & deletion & MUC19 & not rare \\
\hline 84345 chr12 & $70,869,625$ & $70,879,131$ & deletion & - & not rare \\
\hline 34345 chr12 & $94,197,679$ & $94,202,652$ & deletion & - & not rare \\
\hline 184345 chr12 & $9,756,322$ & $9,762,917$ & deletion & & not rare \\
\hline 184345 chr13 & $106,229,594$ & $106,307,349$ & deletion & - & not rare \\
\hline 184345 chr13 & $21,728,134$ & $21,732,193$ & duplication & SK & not rare \\
\hline 184345 chr13 & $34,134,809$ & $34,143,545$ & deletion & - & not rare \\
\hline 184345 chr13 & $84,102,440$ & $84,157,927$ & deletion & - & not rare \\
\hline 184345 chr14 & $20,341,645$ & $20,422,799$ & duplication & \multicolumn{2}{|c|}{ OR4K5;OR $\angle$ not rare } \\
\hline 184345 chr14 & $41,610,224$ & $41,661,685$ & deletion & - & not rare \\
\hline 184345 chr16 & $55,800,009$ & $55,819,443$ & deletion & - & not rare \\
\hline 184345 chr16 & $55,843,252$ & $55,865,127$ & duplication & CES1 & not rare \\
\hline 184345 chr16 & $58,945,839$ & $58,948,100$ & deletion & - & not rare \\
\hline 184345 chr16 & $60,082,237$ & $60,098,544$ & deletion & - & not rare \\
\hline 184345 chr16 & $78,372,894$ & $78,383,509$ & deletion & - & not rare \\
\hline 184345 chr16 & $90,102,143$ & $90,170,495$ & duplication & \multicolumn{2}{|c|}{ GAS8;PRDA rare } \\
\hline 184345 chr18 & $63,907,596$ & $63,910,708$ & deletion & - & not rare \\
\hline 184345 & $35,852,103$ & $35,861,695$ & & - & \\
\hline 184345 chr19 & $41,350,895$ & $41,379,321$ & duplication & CYP2A6 & not rare \\
\hline 184345 chr19 & $53,518,747$ & $53,548,950$ & duplication & & rare \\
\hline 184345 chr19 & $9,275,282$ & $9,282,176$ & deletion & - & not rare \\
\hline 184345 chr2 & $10,885,901$ & $10,890,881$ & duplicatio & & not rare \\
\hline 184345 chr2 & $212,771,670$ & $212,773,893$ & & & rare \\
\hline 184345 chr2 & $233,214,406$ & $233,219,891$ & del & & not rare \\
\hline 184345 chr2 & $51,926,599$ & $51,926,904$ & del & . & not rare \\
\hline 184345 chr20 & $14,755,790$ & $14,827,620$ & & - & rare \\
\hline 184345 chr20 & $53,407,575$ & $53,419,685$ & deletion & - & not rare \\
\hline 184345 chr20 & $53,764,306$ & $54,122,219$ & deletion & - & rare \\
\hline 184345 chr21 & $10,699,687$ & $10,888,876$ & duplication & - & not rare \\
\hline 184345 chr22 & $39,359,885$ & $39,385,663$ & deletion & \multicolumn{2}{|c|}{ АРОВЕС $3 \mathrm{~B}$ not rare } \\
\hline 184345 chr22 & $45,129,262$ & $45,137,783$ & deletion & PRR5 & not rare \\
\hline 184345 chr3 & $131,711,896$ & $131,712,898$ & deletion & - & not rare \\
\hline 184345 chr3 & $18,235,914$ & $18,244,588$ & deletion & - & not rare \\
\hline 184345 chr3 & $191,065,392$ & $191,070,300$ & deletion & - & not rare \\
\hline $184345 \mathrm{chr} 4$ & $69,374,369$ & $69,447,592$ & duplication & B17 & not rare \\
\hline $184345 \mathrm{chr} 4$ & $7,182,916$ & $7,187,397$ & deletion & - & not rare \\
\hline 184345 chr5 & $18,043,852$ & $18,059,000$ & duplication & - & not rare \\
\hline 184345 chr5 & $32,106,628$ & $32,164,826$ & duplication & \multicolumn{2}{|c|}{ GOLPH3;P[ not rare } \\
\hline 184345 chr6 & $30,994,010$ & $30,994,787$ & deletion & MUC22 & not rare \\
\hline 184345 chr6 & $32,486,060$ & $32,520,879$ & deletion & HLA-DRB5 & not rare \\
\hline 184345 chr6 & $66,074,421$ & $66,080,908$ & deletion & - & not rare \\
\hline 184345 chr7 & $110,582,600$ & $110,585,307$ & deletion & - & not rare \\
\hline 184345 chr7 & $142,098,342$ & $142,108,878$ & deletion & - & not rare \\
\hline 184345 chr7 & $38,388,889$ & $38,402,517$ & deletion & - & not rare \\
\hline 184345 chr7 & $91,033,074$ & $91,041,618$ & deletion & - & not rare \\
\hline 184345 chr8 & $103,054,099$ & $103,056,645$ & deletion & - & not rare \\
\hline
\end{tabular}




\begin{tabular}{|c|c|c|c|c|c|}
\hline 184345 chr8 & $16,993,943$ & $16,997,863$ & deletion & - & not rare \\
\hline 184345 chr8 & $24,972,808$ & $24,990,418$ & duplication & - & not rare \\
\hline 84345 chr8 & $39,236,582$ & $39,385,979$ & deletion & - & trare \\
\hline 34345 chr8 & $51,029,371$ & $51,038,149$ & deletion & - & not rare \\
\hline 184345 chr9 & $138,195,113$ & $138,195,756$ & deletion & - & not rare \\
\hline $84345 \mathrm{c}$ & $23,290,141$ & $23,299,151$ & deletion & - & not rare \\
\hline 84345 chr9 & $43,395,008$ & $43,705,991$ & deletion & \multicolumn{2}{|c|}{ CNTNAP3B not rare } \\
\hline 184345 chr9 & $6,664,282$ & $6,670,263$ & deletion & - & not rare \\
\hline 184345 chr9 & $69,514,657$ & $69,881,429$ & deletion & - & not rare \\
\hline 184345 chr9 & $8,282,119$ & $8,289,073$ & deletion & - & not rare \\
\hline $184345 \mathrm{chr} X$ & $94,348,661$ & $94,392,523$ & duplication & - & not rare \\
\hline 184346 chr1 & $152,526,812$ & $152,568,230$ & deletion & \multicolumn{2}{|c|}{ LCE3D;LCE not rare } \\
\hline 184346 chr1 & $161,563,386$ & $161,619,741$ & duplication & \multicolumn{2}{|c|}{ FCGR2C;FC not rare } \\
\hline 184346 chr1 & $46,795,447$ & $46,806,480$ & deletion & - & not rare \\
\hline 184346 chr1 & $55,114,469$ & $55,120,469$ & deletion & M & not rare \\
\hline 184346 chr1 & $62,113,597$ & $62,119,136$ & deletion & - & not rare \\
\hline 184346 chr10 & $71,278,805$ & $71,289,190$ & deletion & - & not rare \\
\hline 184346 chr11 & $55,365,761$ & $55,427,700$ & deletion & \multicolumn{2}{|c|}{$\mathrm{OR} 4 \mathrm{P} 4 ; \mathrm{OR} \angle$ not rare } \\
\hline 184346 & 4,402 & $86,306,401$ & & - & \\
\hline 184346 chr11 & $90,193,796$ & $90,195,742$ & deletion & - & not rare \\
\hline 184346 chr12 & $132,987,231$ & $132,988,515$ & deletion & - & not rare \\
\hline 184346 chr12 & $32,785,284$ & $32,843,021$ & duplication & \multicolumn{2}{|c|}{ FGD4;DNM rare } \\
\hline 184346 chr12 & $70,874,037$ & $70,879,131$ & deletion & & not rare \\
\hline 184346 chr12 & $80,155,600$ & $80,160,230$ & & & are \\
\hline 184346 chr13 & $34,134,809$ & $34,143,545$ & dele & & not rare \\
\hline 184346 chr13 & $38,076,308$ & $38,085,537$ & dele & . & not rare \\
\hline 184346 & $106,062,675$ & $106,199,579$ & ation & - & not rare \\
\hline 184346 chr14 & $19,223,219$ & $19,267,042$ & deletion & - & rare \\
\hline 184346 chr14 & $41,610,224$ & $41,661,685$ & deletion & - & not rare \\
\hline 184346 chr16 & $55,800,009$ & $55,819,443$ & dele & - & not rare \\
\hline 184346 chr16 & $55,843,252$ & $55,865,127$ & duplication & CES1 & not rare \\
\hline 184346 chr16 & $90,102,143$ & $90,170,495$ & duplication & \multicolumn{2}{|c|}{ GAS8;PRDA rare } \\
\hline 184346 chr17 & $10,889,594$ & $10,893,799$ & deletion & - & not rare \\
\hline 184346 chr17 & $21,316,983$ & $21,534,405$ & duplication & \multicolumn{2}{|c|}{ C17orf51;K not rare } \\
\hline 184346 chr19 & $53,518,747$ & $53,548,950$ & duplication & & not rare \\
\hline 184346 chr2 & $212,770,611$ & $212,776,546$ & deletion & - & not rare \\
\hline 184346 chr2 & $41,239,457$ & $41,248,468$ & deletion & - & not rare \\
\hline 184346 chr2 & $51,925,954$ & $51,926,904$ & deletion & - & not rare \\
\hline 184346 chr22 & $39,359,885$ & $39,385,663$ & deletion & \multicolumn{2}{|c|}{ APOBEC3B not rare } \\
\hline 184346 chr3 & $195,725,336$ & $197,346,971$ & deletion & \multicolumn{2}{|c|}{ PCYT1A;FB' rare } \\
\hline $184346 \mathrm{chr} 4$ & $139,939,052$ & $139,941,594$ & deletion & - & not rare \\
\hline $184346 \mathrm{chr} 4$ & $60,325,010$ & $60,330,880$ & duplication & - & not rare \\
\hline $184346 \mathrm{chr} 4$ & $69,374,369$ & $69,467,200$ & deletion & UGT2B17 & not rare \\
\hline $184346 \mathrm{chr} 4$ & $70,137,897$ & $70,229,260$ & deletion & UGT2B28 & not rare \\
\hline $184346 \mathrm{chr} 4$ & $7,182,916$ & $7,187,397$ & deletion & - & not rare \\
\hline 184346 & $144,679,911$ & $144,713,515$ & duplication & - & rare \\
\hline 184346 chr5 & $151,514,956$ & $151,518,615$ & deletion & - & not rare \\
\hline
\end{tabular}




\begin{tabular}{|c|c|c|c|c|c|}
\hline 184346 chr5 & $18,043,852$ & $18,059,000$ & duplication & - & not rare \\
\hline 184346 chr5 & $97,555,945$ & $97,563,781$ & deletion & - & not rare \\
\hline 84346 chr6 & $29,855,945$ & $29,899,189$ & deletion & - & ot rare \\
\hline 84346 chr6 & $31,275,231$ & $31,277,988$ & deletion & - & not rare \\
\hline 184346 chr6 & $31,360,255$ & $31,453,640$ & deletion & MICA & not rare \\
\hline 184346 & $66,074,421$ & $66,080,908$ & deletion & - & ot rare \\
\hline 184346 chr6 & $78,985,226$ & $79,012,428$ & duplication & - & not rare \\
\hline $184346 \mathrm{chr} 7$ & $155,882,711$ & $155,885,143$ & deletion & - & not rare \\
\hline 184346 chr7 & $38,388,889$ & $38,402,517$ & deletion & - & not rare \\
\hline 184346 chr7 & 41,421 & 73,060 & duplication & - & not rare \\
\hline 184346 chr8 & $24,972,808$ & $24,990,418$ & duplication & - & not rare \\
\hline 184346 chr8 & $39,232,581$ & $39,385,979$ & duplication & - & not rare \\
\hline 184346 chr8 & $51,029,371$ & $51,038,149$ & deletion & - & not rare \\
\hline 184346 chr9 & $138,195,113$ & $138,195,756$ & deletion & - & not rare \\
\hline 184346 chr9 & $1,446,522$ & $1,450,465$ & deletion & - & not rare \\
\hline 184347 chr1 & $104,153,766$ & $104,211,046$ & deletion & \multicolumn{2}{|c|}{ AMY1A;AN not rare } \\
\hline 184347 chr1 & $12,846,082$ & $12,909,273$ & deletion & \multicolumn{2}{|c|}{ HNRNPCL1 not rare } \\
\hline 184347 chr1 & $17,009,576$ & $17,226,354$ & duplication & \multicolumn{2}{|c|}{ FAM231A; f not rare } \\
\hline 184347 chr1 & $248,621,384$ & $248,645,623$ & dupli & OR2T3 & not rare \\
\hline 184347 chr1 & $248,740,572$ & $248,795,110$ & deletion & \multicolumn{2}{|c|}{ OR2T10;OF not rare } \\
\hline 184347 chr1 & $55,354,335$ & $55,362,241$ & duplication & - & not rare \\
\hline 184347 chr10 & $126,128,109$ & $126,254,695$ & duplication & \multicolumn{2}{|c|}{ LHPP;NKX1 rare } \\
\hline 184347 chr10 & 601,031 & 641,961 & duplication & & not rare \\
\hline 184347 chr11 & $18,949,220$ & $18,961,743$ & duplication & MRGPRX1 & not rare \\
\hline 184347 chr11 & $25,750,699$ & $25,753,430$ & deletion & & not rare \\
\hline 184347 chr11 & $55,365,761$ & $55,427,700$ & deletion & \multicolumn{2}{|c|}{$\mathrm{OR} 4 \mathrm{P} 4 ; \mathrm{OR} \angle$ not rare } \\
\hline 184347 & $90,193,796$ & $90,195,742$ & & - & not \\
\hline 184347 chr12 & $11,220,827$ & $11,244,117$ & deletion & TAS2R43 & not rare \\
\hline 184347 chr12 & $2,245,636$ & $2,254,219$ & deletion & - & not rare \\
\hline 184347 chr12 & $40,875,351$ & $40,875,963$ & deletion & ML & not rare \\
\hline 184347 chr12 & $43,021,431$ & $43,027,453$ & deletion & - & not rare \\
\hline 184347 chr13 & $34,134,809$ & $34,143,545$ & deletion & - & not rare \\
\hline 184347 chr14 & $37,202,022$ & $37,204,131$ & deletion & SLC25A21 & not rare \\
\hline 184347 chr14 & $41,610,224$ & $41,661,685$ & deletion & - & not rare \\
\hline 184347 chr14 & $73,527,253$ & $73,530,678$ & deletion & - & not rare \\
\hline 184347 chr16 & $32,511,914$ & $32,603,033$ & duplication & - & not rare \\
\hline 184347 chr16 & $58,945,839$ & $58,948,100$ & deletion & - & not rare \\
\hline 184347 chr16 & $77,543,178$ & $77,556,200$ & deletion & - & not rare \\
\hline 184347 chr17 & $7,264,717$ & $7,265,681$ & deletion & - & not rare \\
\hline 184347 chr19 & $41,350,895$ & $41,379,321$ & duplication & CYP2A6 & not rare \\
\hline 184347 chr19 & $41,660,464$ & $41,664,145$ & deletion & - & not rare \\
\hline 184347 chr19 & $53,518,747$ & $53,552,296$ & duplication & ERVV-1 & not rare \\
\hline 184347 chr19 & $54,556,755$ & $54,560,078$ & deletion & - & not rare \\
\hline 184347 chr2 & $213,187,034$ & $213,191,389$ & deletion & - & not rare \\
\hline 184347 chr2 & $242,854,089$ & $243,034,519$ & deletion & - & not rare \\
\hline 184347 chr2 & $34,697,895$ & $34,726,904$ & deletion & - & not rare \\
\hline 184347 chr2 & $51,926,599$ & $51,926,904$ & deletion & - & not rare \\
\hline
\end{tabular}




\begin{tabular}{|c|c|c|c|c|c|}
\hline 184347 chr2 & $52,759,890$ & $52,784,952$ & deletion & - & not rare \\
\hline 184347 chr2 & $58,614,740$ & $58,624,757$ & deletion & - & not rare \\
\hline 84347 chr20 & $17,214,842$ & $17,272,251$ & deletion & PCSK2 & rare \\
\hline 84347 chr20 & $29,804,016$ & $29,828,235$ & duplication & - & not rare \\
\hline 184347 chr20 & $43,981,995$ & $43,996,189$ & deletion & SYS1 & not rare \\
\hline 184347 chr22 & $42,915,843$ & $42,950,033$ & duplication & SERHL2 & ot rare \\
\hline 184347 chr3 & $133,644,169$ & $133,652,671$ & deletion & C3orf36 & not rare \\
\hline 184347 chr3 & $18,235,914$ & $18,244,588$ & deletion & - & not rare \\
\hline 184347 chr3 & $37,979,882$ & $37,986,734$ & deletion & - & not rare \\
\hline 184347 chr4 & $122,285,263$ & $122,289,863$ & deletion & - & not rare \\
\hline 184347 chr4 & $70,137,897$ & $70,229,260$ & deletion & UGT2B28 & not rare \\
\hline 184347 chr5 & $155,477,866$ & $155,488,917$ & deletion & - & not rare \\
\hline 184347 chr5 & $175,475,525$ & $175,646,717$ & deletion & FAM153B & not rare \\
\hline 184347 chr5 & $177,117,072$ & $177,165,594$ & duplication & FAM153A & rare \\
\hline 184347 chr5 & $180,377,470$ & $180,430,540$ & deletion & BTNL8;BTN & are \\
\hline 184347 chr5 & $18,043,852$ & $18,059,000$ & duplication & - & not rare \\
\hline 184347 chr5 & $32,106,628$ & $32,164,826$ & duplication & GOLPH3;PL & not rare \\
\hline 184347 chr5 & 701,026 & 807,499 & duplication & ZDHHC11 & not rare \\
\hline 184347 chr5 & $70,305,696$ & $70,308,251$ & & & \\
\hline 184347 chr6 & $30,994,010$ & $30,995,187$ & deletion & MUC22 & not rare \\
\hline 184347 chr6 & $49,780,993$ & $49,806,196$ & ation & CRISP1 & \\
\hline 184347 chr6 & $78,972,930$ & $79,035,739$ & deletion & - & not rare \\
\hline 184347 chr7 & $156,139,940$ & $156,149,460$ & dele & & not rare \\
\hline 184347 chr8 & $115,634,278$ & $115,642,459$ & & & are \\
\hline 184347 chr8 & $24,972,808$ & $24,990,418$ & & & not rare \\
\hline 184347 chr8 & $39,232,581$ & $39,385,979$ & dele & - & not rare \\
\hline 184347 chr8 & $51,029,371$ & $51,038,149$ & & - & \\
\hline 184347 chr8 & $8,318,936$ & $8,324,686$ & deletion & - & not rare \\
\hline 184347 chr9 & $104,715,329$ & $104,722,688$ & deletion & - & not rare \\
\hline 184347 chr9 & $138,195,113$ & $138,195,756$ & deletion & - & not rare \\
\hline 184347 chr9 & $6,664,282$ & $6,670,263$ & deletion & - & not rare \\
\hline $184347 \mathrm{chrX}$ & $94,348,661$ & $94,392,523$ & duplication & - & not rare \\
\hline 184349 chr1 & $106,880,390$ & $106,961,455$ & deletion & - & rare \\
\hline 184349 chr1 & $12,846,082$ & $12,919,928$ & deletion & \multicolumn{2}{|c|}{ HNRNPCL1 not rare } \\
\hline 184349 chr1 & $161,559,327$ & $161,619,741$ & duplication & \multicolumn{2}{|c|}{ FCGR2C;FC not rare } \\
\hline 184349 chr1 & $46,058,525$ & $46,129,348$ & duplication & \multicolumn{2}{|c|}{ NASP;CCDC rare } \\
\hline 184349 chr10 & $71,280,107$ & $71,289,190$ & deletion & - & not rare \\
\hline 184349 chr11 & $25,750,699$ & $25,753,430$ & deletion & - & not rare \\
\hline 184349 chr11 & $44,223,836$ & $44,236,652$ & deletion & EXT2 & not rare \\
\hline 184349 chr11 & $55,365,761$ & $55,443,269$ & deletion & \multicolumn{2}{|c|}{ OR4P4;OR $\angle$ not rare } \\
\hline 184349 chr11 & $90,193,796$ & $90,196,843$ & deletion & - & not rare \\
\hline 184349 chr12 & $11,511,132$ & $11,543,084$ & duplication & - & not rare \\
\hline 184349 chr12 & $29,940,186$ & $29,949,915$ & duplication & - & not rare \\
\hline 184349 chr12 & $43,021,431$ & $43,027,453$ & deletion & - & not rare \\
\hline 184349 chr13 & $34,134,809$ & $34,143,545$ & deletion & - & not rare \\
\hline 184349 chr13 & $64,225,427$ & $64,236,288$ & deletion & - & not rare \\
\hline 184349 chr13 & $69,247,022$ & $69,267,981$ & deletion & - & not rare \\
\hline
\end{tabular}




\begin{tabular}{|c|c|c|c|c|c|}
\hline 184349 chr13 & $80,681,247$ & $80,685,663$ & deletion & - & not rare \\
\hline 184349 chr14 & $35,604,892$ & $35,613,500$ & deletion & - & not rare \\
\hline 84349 chr14 & $41,610,224$ & $41,661,685$ & deletion & - & ot rare \\
\hline 84349 chr16 & $34,471,298$ & $34,756,258$ & duplication & - & not rare \\
\hline 184349 chr16 & $85,302,676$ & $85,304,326$ & deletion & - & not rare \\
\hline 184349 chr17 & $10,890,859$ & $10,893,799$ & deletion & - & t rare \\
\hline 184349 chr17 & $34,438,753$ & $34,483,342$ & duplication & - & not rare \\
\hline 184349 chr18 & $64,890,340$ & $64,894,225$ & deletion & - & not rare \\
\hline 184349 chr19 & $43,298,958$ & $43,539,189$ & deletion & \multicolumn{2}{|c|}{ PSG6;PSG7 not rare } \\
\hline 184349 chr2 & $14,704,369$ & $14,709,611$ & deletion & - & not rare \\
\hline 184349 chr2 & $195,979,780$ & $195,982,450$ & deletion & - & not rare \\
\hline 184349 chr2 & $212,771,670$ & $212,773,893$ & deletion & - & not rare \\
\hline 184349 chr2 & $214,989,439$ & $214,992,692$ & deletion & - & not rare \\
\hline 184349 chr2 & $4,212,725$ & $4,222,144$ & deletion & - & not rare \\
\hline 184349 chr2 & $48,851,541$ & $48,856,770$ & deletion & - & not rare \\
\hline 184349 chr21 & $10,815,443$ & $10,863,251$ & duplication & - & not rare \\
\hline 184349 chr22 & $39,359,885$ & $39,385,663$ & deletion & \multicolumn{2}{|c|}{ APOBEC $3 \mathrm{~B}$ not rare } \\
\hline 184349 chr3 & $11,411,823$ & $11,414,339$ & deletion & - & not rare \\
\hline 184349 chr3 & $160,680,773$ & $160,685,788$ & del & - & \\
\hline 184349 chr3 & $173,239,453$ & $173,290,024$ & duplication & - & not rare \\
\hline $184349 \mathrm{chr} 3$ & $191,065,392$ & $191,070,300$ & deletion & - & not rare \\
\hline 184349 chr3 & $37,979,882$ & $37,986,734$ & deletion & - & not rare \\
\hline 184349 chr3 & $8,378,359$ & $8,431,954$ & duplicatio & & rare \\
\hline $184349 \mathrm{chr} 4$ & $138,092,298$ & $138,098,601$ & deletion & & not rare \\
\hline $184349 \mathrm{chr} 4$ & $168,307,924$ & $168,324,109$ & dele & & not rare \\
\hline $184349 \mathrm{chr} 4$ & $187,747,224$ & $187,758,032$ & deletion & 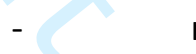 & not rare \\
\hline $184349 \mathrm{chr} 4$ & $34,785,955$ & $34,805,860$ & duplication & - & not rare \\
\hline $184349 \mathrm{chr} 4$ & $69,374,369$ & $69,489,473$ & deletion & UGT2B17 & not rare \\
\hline $184349 \mathrm{chr} 4$ & $70,137,897$ & $70,229,260$ & deletion & B28 & not rare \\
\hline 184349 chr5 & $120,054,967$ & $120,067,140$ & duplication & - & not rare \\
\hline 184349 chr5 & $139,311,848$ & $139,335,948$ & deletion & - & rare \\
\hline 184349 chr5 & $151,514,956$ & $151,518,615$ & deletion & - & not rare \\
\hline 184349 chr5 & $155,477,866$ & $155,488,917$ & deletion & - & not rare \\
\hline 184349 chr5 & $70,305,696$ & $70,308,251$ & deletion & NAIP & not rare \\
\hline 184349 chr6 & $124,432,882$ & $124,470,796$ & duplication & & not rare \\
\hline 184349 chr6 & $31,219,869$ & $31,229,552$ & deletion & - & not rare \\
\hline 184349 chr6 & $78,972,930$ & $79,012,428$ & duplication & - & not rare \\
\hline 184349 chr7 & $142,476,094$ & $142,484,042$ & deletion & - & not rare \\
\hline 184349 chr7 & $15,154,324$ & $15,159,059$ & deletion & - & not rare \\
\hline 184349 chr8 & $39,232,581$ & $39,385,979$ & deletion & - & not rare \\
\hline 184349 chr8 & $5,595,511$ & $5,605,706$ & deletion & - & not rare \\
\hline 184349 chr8 & $84,678,170$ & $84,684,004$ & deletion & - & not rare \\
\hline $184350 \mathrm{chr} 1$ & $152,526,812$ & $152,568,230$ & deletion & \multicolumn{2}{|c|}{ LCE3D;LCE: not rare } \\
\hline 184350 chr1 & $248,740,314$ & $248,795,110$ & deletion & \multicolumn{2}{|c|}{ OR2T10;OF not rare } \\
\hline 184350 chr10 & $58,846,575$ & $58,849,853$ & deletion & - & not rare \\
\hline 184350 chr11 & $18,949,220$ & $18,961,743$ & duplication & MRGPRX1 & not rare \\
\hline $184350 \mathrm{chr} 11$ & $54,821,812$ & $54,867,808$ & deletion & - & rare \\
\hline
\end{tabular}




\begin{tabular}{|c|c|c|c|c|c|}
\hline 184350 chr11 & 761 & $55,443,269$ & deletion & \multicolumn{2}{|c|}{$\mathrm{OR} 4 \mathrm{P} 4 ; \mathrm{OR} \angle$ not rare } \\
\hline 184350 chr11 & $90,193,796$ & $90,195,742$ & deletion & - & not rare \\
\hline 84350 chr12 & $59,935,926$ & $59,942,122$ & deletion & - & not rare \\
\hline 84350 chr12 & $6,255,862$ & $6,259,552$ & deletion & - & not rare \\
\hline 184350 chr12 & $70,513,571$ & $70,517,836$ & duplication & - & not rare \\
\hline 184350 chr13 & $34,134,809$ & $34,143,545$ & deletion & - & ot rare \\
\hline 184350 chr13 & $58,589,893$ & $58,599,174$ & deletion & - & not rare \\
\hline 184350 chr16 & $32,528,023$ & $32,833,893$ & deletion & \multicolumn{2}{|c|}{ TP53TG3B; not rare } \\
\hline 184350 chr16 & $33,551,243$ & $33,590,959$ & deletion & - & not rare \\
\hline 184350 chr16 & $78,372,894$ & $78,383,509$ & duplication & - & not rare \\
\hline 184350 chr17 & $10,890,859$ & $10,893,799$ & deletion & - & not rare \\
\hline 184350 chr17 & $14,997,297$ & $15,010,250$ & deletion & - & not rare \\
\hline 184350 chr17 & $15,044,804$ & $15,058,236$ & deletion & - & not rare \\
\hline 184350 chr18 & $63,907,596$ & $63,910,708$ & deletion & - & not rare \\
\hline 184350 chr19 & $35,852,103$ & $35,861,695$ & deletion & - & not rare \\
\hline 184350 chr19 & $53,152,453$ & $53,159,177$ & deletion & - & not rare \\
\hline 184350 chr19 & $53,520,702$ & $53,552,296$ & duplication & - & not rare \\
\hline 184350 chr19 & $9,275,282$ & $9,282,176$ & deletion & - & not rare \\
\hline $184350 \mathrm{chr} 2$ & $123,477,465$ & $123,482,301$ & on & - & rare \\
\hline 184350 chr2 & $196,421,568$ & $196,429,106$ & deletion & - & not rare \\
\hline $184350 \mathrm{chr} 2$ & $212,771,670$ & $212,776,546$ & deletion & - & not rare \\
\hline 184350 chr2 & $223,865,393$ & $223,869,820$ & deletion & - & not rare \\
\hline 184350 chr2 & $97,738,917$ & $98,024,837$ & deletion & \multicolumn{2}{|c|}{ FAHD2B;Ar not rare } \\
\hline 184350 chr21 & $33,392,441$ & $33,394,823$ & deletion & & not rare \\
\hline 184350 chr3 & $131,711,896$ & $131,712,898$ & dele & & not rare \\
\hline 184350 chr3 & $1,782,524$ & $1,787,581$ & deletion & - & not rare \\
\hline 184350 chr3 & $193,136,358$ & $193,140,348$ & deletion & - & not rare \\
\hline $184350 \mathrm{chr} 3$ & $24,546,267$ & $24,645,631$ & duplication & - & rare \\
\hline $184350 \mathrm{chr} 3$ & $65,189,366$ & $65,214,685$ & deletion & - & not rare \\
\hline $184350 \mathrm{chr} 4$ & $152,789,786$ & $152,793,891$ & deletion & - & not rare \\
\hline $184350 \mathrm{chr} 4$ & $187,747,224$ & $187,758,032$ & deletion & - & not rare \\
\hline $184350 \mathrm{chr} 4$ & $34,785,955$ & $34,818,502$ & deletion & - & not rare \\
\hline $184350 \mathrm{chr} 4$ & $69,374,369$ & $69,431,280$ & deletion & UG & not rare \\
\hline 184350 chr5 & $25,210,358$ & $25,224,844$ & deletion & - & not rare \\
\hline 184350 chr5 & $32,106,628$ & $32,164,826$ & duplication & \multicolumn{2}{|c|}{ GOLPH3;P[ not rare } \\
\hline 184350 chr5 & $46,192,689$ & $46,213,735$ & deletion & - & rare \\
\hline 184350 chr5 & 744,005 & 825,338 & duplication & ZDHHC11 & not rare \\
\hline 184350 chr6 & $30,994,010$ & $30,994,787$ & deletion & MUC22 & not rare \\
\hline 184350 chr6 & $31,276,513$ & $31,277,988$ & deletion & - & not rare \\
\hline 184350 chr7 & $141,766,850$ & $141,793,521$ & deletion & - & not rare \\
\hline 184350 chr7 & $142,476,094$ & $142,484,042$ & deletion & - & not rare \\
\hline 184350 chr7 & $153,794,636$ & $153,814,001$ & duplication & - & not rare \\
\hline $184350 \mathrm{chr} 8$ & $103,054,099$ & $103,056,645$ & deletion & - & not rare \\
\hline 184350 chr8 & $115,634,278$ & $115,642,459$ & deletion & - & not rare \\
\hline $184350 \mathrm{chr} 8$ & $39,232,581$ & $39,385,979$ & duplication & - & not rare \\
\hline 184350 chr9 & $138,195,113$ & $138,195,756$ & deletion & - & not rare \\
\hline 184350 chr9 & $139,668,443$ & $139,676,734$ & deletion & - & not rare \\
\hline
\end{tabular}




\begin{tabular}{|c|c|c|c|c|c|}
\hline 184350 chr9 & $5,304,710$ & $5,337,760$ & duplication & RLN1 & not rare \\
\hline 184350 chr9 & $6,701,130$ & $6,707,794$ & deletion & - & not rare \\
\hline 84351 chr1 & $104,153,766$ & $104,211,046$ & deletion & \multicolumn{2}{|c|}{ AMY1A;AN not rare } \\
\hline 34351 chr1 & $112,693,423$ & $112,704,581$ & deletion & - & not rare \\
\hline 84351 chr1 & $152,526,812$ & $152,568,230$ & deletion & \multicolumn{2}{|c|}{ LCE3D;LCE not rare } \\
\hline 184351 & $248,740,314$ & $248,795,110$ & deletion & \multicolumn{2}{|c|}{ OR2T10;OF not rare } \\
\hline 84351 chr10 & $58,846,575$ & $58,849,853$ & deletion & - & not rare \\
\hline 184351 chr11 & $18,949,220$ & $18,961,743$ & duplication & MRGPRX1 & not rare \\
\hline 184351 chr11 & $25,750,699$ & $25,753,430$ & deletion & - & not rare \\
\hline 184351 chr11 & $55,365,761$ & $55,443,269$ & deletion & \multicolumn{2}{|c|}{$\mathrm{OR} 4 \mathrm{P} 4 ; \mathrm{OR} \angle$ not rare } \\
\hline 184351 chr11 & $58,811,241$ & $58,844,734$ & duplication & - & not rare \\
\hline 184351 chr11 & $86,304,402$ & $86,306,401$ & deletion & - & not rare \\
\hline 184351 chr11 & $90,193,796$ & $90,195,742$ & deletion & - & not rare \\
\hline 184351 & $91,228,448$ & $91,235,067$ & duplication & - & not rare \\
\hline $184351 \mathrm{c}$ & $93,027,130$ & $93,033,807$ & deletion & - & not rare \\
\hline 184351 chr12 & $10,165,421$ & $10,169,041$ & deletion & CLEC12B & not rare \\
\hline 184351 chr12 & $43,021,431$ & $43,027,453$ & deletion & - & not rare \\
\hline 184351 chr12 & $59,935,926$ & $59,942,122$ & deletion & - & not rare \\
\hline 184351 chr12 & $6,255,862$ & $6,259,552$ & & - & \\
\hline 184351 chr12 & $94,197,679$ & $94,202,652$ & deletion & - & not rare \\
\hline 184351 chr13 & $34,134,809$ & $34,143,545$ & deletion & - & not rare \\
\hline 184351 chr13 & $69,247,022$ & $69,267,981$ & & - & not rare \\
\hline 184351 chr14 & $106,784,840$ & $106,816,856$ & del & & not rare \\
\hline 184351 chr16 & $32,531,934$ & $32,833,893$ & dele & \multicolumn{2}{|c|}{ TP53TG3B; not rare } \\
\hline 184351 chr16 & $33,548,357$ & $33,590,959$ & dele & & not rare \\
\hline 184351 chr17 & $44,166,604$ & $44,238,126$ & duplication & KANSL1 & not rare \\
\hline 184351 chr19 & $35,852,103$ & $35,861,695$ & del & - & are \\
\hline 184351 chr19 & $53,152,453$ & $53,159,177$ & deletion & - & not rare \\
\hline 184351 chr19 & $53,520,702$ & $53,552,296$ & duplication & - & not rare \\
\hline 184351 chr19 & $9,275,282$ & $9,282,176$ & deletion & - & not rare \\
\hline $184351 \mathrm{chr} 2$ & $123,477,465$ & $123,482,301$ & deletion & - & not rare \\
\hline 184351 chr2 & $196,421,568$ & $196,429,252$ & deletion & - & not rare \\
\hline $184351 \mathrm{chr} 2$ & $212,771,670$ & $212,773,893$ & deletion & - & not rare \\
\hline 184351 chr2 & $51,926,599$ & $51,926,904$ & deletion & - & not rare \\
\hline 184351 chr2 & $97,738,917$ & $98,024,837$ & deletion & \multicolumn{2}{|c|}{ FAHD2B;Ar not rare } \\
\hline 184351 chr20 & $24,887,251$ & $24,893,947$ & deletion & - & not rare \\
\hline 184351 chr20 & $59,568,187$ & $59,590,280$ & duplication & - & not rare \\
\hline 184351 chr22 & $39,359,885$ & $39,385,663$ & deletion & \multicolumn{2}{|c|}{ APOBEC3B not rare } \\
\hline $184351 \mathrm{chr} 3$ & $131,711,896$ & $131,712,898$ & deletion & - & not rare \\
\hline $184351 \mathrm{chr} 3$ & $191,061,214$ & $191,070,300$ & deletion & - & not rare \\
\hline 184351 chr3 & $192,777,710$ & $192,782,294$ & deletion & - & not rare \\
\hline 184351 chr3 & $193,136,358$ & $193,140,348$ & deletion & - & not rare \\
\hline 184351 chr3 & $24,546,267$ & $24,645,631$ & duplication & - & rare \\
\hline 184351 chr3 & $65,189,366$ & $65,214,685$ & deletion & - & not rare \\
\hline 184351 chr3 & $66,771,340$ & $66,781,466$ & deletion & - & not rare \\
\hline 184351 chr4 & $187,747,224$ & $187,758,032$ & deletion & - & not rare \\
\hline 184351 chr4 & $64,697,704$ & $64,709,753$ & deletion & - & not rare \\
\hline
\end{tabular}




\begin{tabular}{|c|c|c|c|c|c|}
\hline 184351 chr5 & $32,106,628$ & $32,164,826$ & duplication & \multicolumn{2}{|c|}{ GOLPH3;P[ not rare } \\
\hline 184351 chr5 & 730,567 & 825,338 & duplication & ZDHHC11 & not rare \\
\hline 84351 chr7 & $141,766,850$ & $141,793,521$ & deletion & - & not rare \\
\hline 84351 chr7 & $142,476,094$ & $142,484,042$ & deletion & - & not rare \\
\hline 184351 chr7 & $38,388,889$ & $38,402,517$ & deletion & - & not rare \\
\hline 184351 chr8 & $103,054,099$ & $103,056,645$ & deletion & - & not rare \\
\hline 184351 chr8 & $115,634,278$ & $115,642,459$ & deletion & - & not rare \\
\hline 184351 chr8 & $39,232,581$ & $39,385,979$ & duplication & - & not rare \\
\hline 184351 chr9 & $6,701,130$ & $6,707,794$ & deletion & - & not rare \\
\hline 184353 chr10 & $38,792,512$ & $38,903,858$ & deletion & - & not rare \\
\hline 184353 chr10 & $58,516,470$ & $58,526,153$ & deletion & - & not rare \\
\hline 184353 chr11 & $107,653,650$ & $107,671,155$ & duplication & SLC35F2 & not rare \\
\hline 184353 chr11 & $90,947,633$ & $90,948,703$ & duplication & - & not rare \\
\hline 184353 chr12 & $31,266,287$ & $31,407,303$ & duplication & - & not rare \\
\hline 184353 chr12 & $40,875,351$ & $40,875,963$ & deletion & MUC19 & not rare \\
\hline 184353 chr12 & $59,935,926$ & $59,942,122$ & deletion & - & not rare \\
\hline 184353 chr12 & $6,241,446$ & $6,247,923$ & deletion & - & not rare \\
\hline 184353 chr12 & $7,996,684$ & $8,123,306$ & duplication & \multicolumn{2}{|c|}{ SLC2A14;SI not rare } \\
\hline 184353 & 80,6 & $80,685,663$ & deletion & - & \\
\hline 184353 chr14 & $104,953,794$ & $104,985,486$ & duplication & - & rare \\
\hline 184353 chr14 & $106,624,360$ & $106,774,914$ & duplication & - & not rare \\
\hline 184353 chr14 & $35,604,892$ & $35,613,500$ & deletion & - & not rare \\
\hline 184353 chr14 & $44,820,190$ & $44,930,894$ & duplication & & not rare \\
\hline 184353 chr15 & $43,888,976$ & $43,939,642$ & deletion & \multicolumn{2}{|c|}{ STRC;CATSI not rare } \\
\hline 184353 chr15 & $97,815,182$ & $97,831,832$ & deletion & & not rare \\
\hline 184353 chr16 & $28,603,889$ & $28,643,773$ & deletion & \multicolumn{2}{|c|}{ SULT1A1;SI not rare } \\
\hline 184353 chr16 & $78,372,894$ & $78,383,509$ & deletion & - & are \\
\hline 184353 chr18 & $14,549,799$ & $14,550,375$ & deletion & - & not rare \\
\hline $184353 \mathrm{chr} 18$ & $63,200,484$ & $63,206,335$ & duplication & - & not rare \\
\hline 184353 chr18 & $71,131,189$ & $71,159,898$ & deletion & - & not rare \\
\hline 184353 chr19 & $41,355,999$ & $41,379,321$ & deletion & CYP2A6 & not rare \\
\hline 184353 chr19 & $53,664,448$ & $53,669,492$ & deletion & ZNF665 & not rare \\
\hline 184353 chr2 & $102,965,204$ & $102,968,362$ & deletion & IL1RL1 & not rare \\
\hline 184353 chr2 & $17,220,064$ & $17,239,502$ & deletion & - & not rare \\
\hline 184353 chr2 & $242,854,089$ & $243,034,519$ & deletion & - & not rare \\
\hline 184353 chr2 & $92,293,128$ & $92,304,211$ & deletion & - & not rare \\
\hline 184353 chr20 & $37,008,769$ & $37,014,876$ & deletion & - & not rare \\
\hline 184353 chr3 & $129,769,827$ & $129,806,304$ & deletion & $\mathrm{ALC}$ & not rare \\
\hline 184353 chr3 & $173,239,831$ & $173,290,024$ & duplication & - & not rare \\
\hline 184353 chr3 & $53,032,639$ & $53,038,786$ & deletion & - & not rare \\
\hline 184353 chr3 & $90,467,441$ & $90,477,597$ & deletion & - & not rare \\
\hline $184353 \mathrm{chr} 4$ & $108,064,023$ & $108,074,123$ & deletion & - & not rare \\
\hline $184353 \mathrm{chr} 4$ & $122,285,263$ & $122,289,863$ & deletion & - & not rare \\
\hline 184353 chr4 & $171,269,504$ & $171,273,360$ & deletion & - & not rare \\
\hline $184353 \mathrm{chr} 4$ & $29,927,916$ & $29,938,283$ & deletion & - & not rare \\
\hline 184353 chr4 & $34,785,955$ & $34,818,502$ & deletion & - & not rare \\
\hline 184353 chr4 & $39,705,484$ & $39,713,134$ & deletion & - & not rare \\
\hline
\end{tabular}




\begin{tabular}{|c|c|c|c|c|c|}
\hline 184353 chr6 & $50,483,579$ & $50,511,038$ & deletion & - & rare \\
\hline 184353 chr6 & $78,972,930$ & $79,035,739$ & deletion & - & not rare \\
\hline 184353 chr7 & $150,234,430$ & $150,247,798$ & deletion & - & not rare \\
\hline 184353 chr7 & $156,387,296$ & $156,394,307$ & deletion & - & not rare \\
\hline 184353 chr7 & $38,291,851$ & $38,305,404$ & duplication & TARP & not rare \\
\hline 184353 chr8 & $24,972,808$ & $24,990,418$ & deletion & - & ot rare \\
\hline 184353 chr8 & $39,332,194$ & $39,344,455$ & deletion & - & not rare \\
\hline 184353 chr8 & $51,029,371$ & $51,038,149$ & deletion & - & not rare \\
\hline 184353 chr9 & $135,947,165$ & $135,957,452$ & duplication & - & not rare \\
\hline 184353 chr9 & $23,363,526$ & $23,376,817$ & duplication & - & not rare \\
\hline 184353 chr9 & $44,739,805$ & $44,834,316$ & deletion & - & not rare \\
\hline 184354 chr1 & $248,740,572$ & $248,795,110$ & deletion & \multicolumn{2}{|c|}{ OR2T10;OF not rare } \\
\hline 184354 chr10 & $38,792,512$ & $38,903,858$ & deletion & - & not rare \\
\hline 184354 chr11 & $49,715,154$ & $49,757,690$ & deletion & - & not rare \\
\hline 184354 chr11 & $90,193,796$ & $90,196,843$ & deletion & - & not rare \\
\hline 184354 chr12 & $32,053,440$ & $32,058,066$ & deletion & - & not rare \\
\hline 184354 chr12 & $59,934,071$ & $59,942,122$ & deletion & - & not rare \\
\hline 184354 chr14 & $104,961,982$ & $104,985,486$ & duplication & - & rare \\
\hline 184354 & $106,575,614$ & $106,774,755$ & ation & - & are \\
\hline 184354 chr14 & $35,604,892$ & $35,613,500$ & deletion & - & not rare \\
\hline 184354 chr14 & $41,610,224$ & $41,661,685$ & deletion & - & not rare \\
\hline 184354 chr14 & $42,988,346$ & $42,992,844$ & deletion & - & not rare \\
\hline 184354 chr14 & $46,506,349$ & $47,125,523$ & duplication & RPL10L & rare \\
\hline 184354 chr14 & $73,527,253$ & $73,530,678$ & deletion & & not rare \\
\hline 184354 chr15 & $22,750,305$ & $22,986,435$ & duplication & \multicolumn{2}{|c|}{ CYFIP1;TUE not rare } \\
\hline 184354 chr15 & $23,001,299$ & $23,288,063$ & duplication & \multicolumn{2}{|c|}{ NIPA2;NIP/ not rare } \\
\hline 184354 chr15 & $78,010,069$ & $78,012,688$ & deletion & - & ot rare \\
\hline 184354 chr15 & $97,815,182$ & $97,831,832$ & deletion & - & not rare \\
\hline 184354 chr16 & $32,542,736$ & $32,609,440$ & duplication & - & not rare \\
\hline 184354 chr16 & $34,471,298$ & $34,756,258$ & duplication & - & not rare \\
\hline 184354 chr16 & $85,302,676$ & $85,304,326$ & deletion & - & not rare \\
\hline 184354 chr17 & $44,166,604$ & $44,280,724$ & duplication & KANSL1 & not rare \\
\hline 184354 chr18 & $14,549,799$ & $14,550,375$ & deletion & - & not rare \\
\hline 184354 chr18 & $67,208,806$ & $67,217,271$ & deletion & - & not rare \\
\hline 184354 chr19 & $35,661,787$ & $35,665,515$ & deletion & - & not rare \\
\hline 184354 chr19 & $35,852,103$ & $35,861,695$ & deletion & - & not rare \\
\hline 184354 chr19 & $43,703,965$ & $43,759,382$ & deletion & \multicolumn{2}{|c|}{ PSG4;PSG9 not rare } \\
\hline 184354 chr2 & $184,795,725$ & $184,803,456$ & deletion & - & not rare \\
\hline 184354 chr2 & $242,854,089$ & $243,034,519$ & deletion & - & not rare \\
\hline 184354 chr2 & $4,212,725$ & $4,222,144$ & deletion & - & not rare \\
\hline 184354 chr2 & $52,759,890$ & $52,784,952$ & duplication & - & not rare \\
\hline 184354 chr3 & $129,769,827$ & $129,806,304$ & deletion & ALG1L2 & not rare \\
\hline 184354 chr3 & $131,711,896$ & $131,712,898$ & deletion & - & not rare \\
\hline 184354 chr3 & $191,065,392$ & $191,070,300$ & deletion & - & not rare \\
\hline 184354 chr3 & $195,457,853$ & $195,468,690$ & duplication & MUC20 & not rare \\
\hline 184354 chr3 & $53,032,639$ & $53,038,786$ & deletion & - & not rare \\
\hline 184354 chr3 & $90,467,441$ & $90,477,597$ & deletion & - & not rare \\
\hline
\end{tabular}




\begin{tabular}{|c|c|c|c|c|c|}
\hline $84354 \mathrm{chr} 4$ & $138,092,298$ & $138,098,601$ & deletion & - & not rare \\
\hline $184354 \mathrm{chr} 4$ & $29,927,916$ & $29,930,433$ & deletion & - & not rare \\
\hline 84354 chr4 & $34,785,955$ & $34,818,502$ & deletion & - & not rare \\
\hline $184354 \mathrm{chr} 4$ & $39,705,484$ & $39,713,134$ & deletion & - & not rare \\
\hline 184354 chr4 & $42,697,553$ & $42,708,694$ & deletion & - & not rare \\
\hline $84354 \mathrm{chr} 4$ & $69,374,369$ & $69,489,473$ & deletion & UGT2B17 & not rare \\
\hline $184354 \mathrm{chr} 5$ & $113,327,484$ & $113,335,230$ & deletion & - & not rare \\
\hline $184354 \mathrm{chr} 5$ & $155,477,866$ & $155,488,917$ & deletion & - & not rare \\
\hline 184354 chr5 & $180,377,470$ & $180,430,540$ & deletion & \multicolumn{2}{|c|}{ BTNL8;BTN not rare } \\
\hline 184354 chr6 & $19,041,346$ & $19,048,773$ & deletion & - & not rare \\
\hline 184354 chr6 & $31,276,513$ & $31,286,577$ & deletion & - & not rare \\
\hline 184354 chr6 & $50,487,927$ & $50,511,038$ & deletion & - & rare \\
\hline 184354 chr6 & $78,972,930$ & $79,035,739$ & deletion & - & not rare \\
\hline 184354 chr7 & $156,387,296$ & $156,394,307$ & deletion & - & not rare \\
\hline $184354 \mathrm{chr} 7$ & $62,149,006$ & $62,162,385$ & deletion & - & not rare \\
\hline 184354 chr8 & $39,232,581$ & $39,385,979$ & deletion & - & not rare \\
\hline 184354 chr9 & $135,947,165$ & $135,957,452$ & duplication & - & not rare \\
\hline $184356 \mathrm{chr} 1$ & $104,153,766$ & $104,211,046$ & deletion & \multicolumn{2}{|c|}{ AMY1A;AN not rare } \\
\hline $184356 \mathrm{chr} 1$ & $152,526,812$ & $152,568,230$ & deletion & \multicolumn{2}{|c|}{ LCE3D;LCE: not rare } \\
\hline $184356 \mathrm{chr} 1$ & $161,559,327$ & $161,619,741$ & duplication & \multicolumn{2}{|c|}{ FCGR2C;FC not rare } \\
\hline 184356 chr1 & $248,740,572$ & $248,795,110$ & deletion & \multicolumn{2}{|c|}{ OR2T10;OF not rare } \\
\hline $184356 \mathrm{chr} 1$ & $55,114,469$ & $55,120,469$ & deletion & $\mathrm{MROH} 7$ & not rare \\
\hline 184356 chr10 & $23,154,715$ & $23,157,251$ & & & not rare \\
\hline 184356 chr10 & $36,414,157$ & $36,423,110$ & & & not rare \\
\hline 184356 chr10 & $38,774,749$ & $38,877,428$ & $\mathrm{du}$ & & not rare \\
\hline $184356 \mathrm{chr} 10$ & $58,846,575$ & $58,849,853$ & on & & not \\
\hline 184356 chr11 & $18,949,220$ & $18,961,743$ & deletion & MRGPRX1 & not rare \\
\hline 184356 chr11 & $49,715,154$ & $49,757,690$ & deletion & - & not rare \\
\hline 184356 & $67,511,694$ & $67,760,366$ & duplication & BB1 & no \\
\hline 184356 chr11 & $90,193,796$ & $90,195,742$ & deletion & - & not rare \\
\hline 184356 chr12 & $11,220,827$ & $11,244,117$ & deletion & TAS2R43 & not rare \\
\hline 184356 chr12 & $40,875,351$ & $40,875,963$ & deletion & MUC19 & not rare \\
\hline 184356 chr12 & $99,994,315$ & $100,008,773$ & deletion & - & not rare \\
\hline 184356 chr14 & $41,610,224$ & $41,661,685$ & deletion & - & not rare \\
\hline 184356 chr18 & $63,907,596$ & $63,910,708$ & deletion & - & not rare \\
\hline $184356 \mathrm{chr} 18$ & $66,746,208$ & $66,755,736$ & deletion & - & not rare \\
\hline 184356 chr19 & $9,275,282$ & $9,282,176$ & deletion & - & not rare \\
\hline $184356 \mathrm{chr} 2$ & $123,477,465$ & $123,482,301$ & deletion & - & not rare \\
\hline $184356 \mathrm{chr} 2$ & $48,851,541$ & $48,856,770$ & deletion & - & not rare \\
\hline $184356 \mathrm{chr} 2$ & $97,738,917$ & $98,024,837$ & deletion & \multicolumn{2}{|c|}{ FAHD2B;Ar not rare } \\
\hline 184356 chr22 & $39,359,885$ & $39,385,663$ & deletion & \multicolumn{2}{|c|}{ APOBEC3B not rare } \\
\hline $184356 \mathrm{chr} 3$ & $193,136,358$ & $193,140,348$ & deletion & - & not rare \\
\hline $184356 \mathrm{chr} 4$ & $185,762,881$ & $185,769,481$ & deletion & - & not rare \\
\hline $184356 \mathrm{chr} 4$ & $34,785,955$ & $34,805,860$ & duplication & - & not rare \\
\hline $184356 \mathrm{chr} 4$ & $69,374,369$ & $69,489,473$ & deletion & UGT2B17 & not rare \\
\hline 184356 & $70,137,897$ & $70,229,260$ & deletion & UGT2B28 & not rare \\
\hline 184356 & $165,129,935$ & $165,194,584$ & duplication & - & not rare \\
\hline
\end{tabular}




\begin{tabular}{|c|c|c|c|c|c|}
\hline 184356 chr6 & $29,855,945$ & $29,899,189$ & deletion & - & not rare \\
\hline 184356 chr6 & $31,219,869$ & $31,228,972$ & deletion & - & not rare \\
\hline 84356 chr6 & $31,275,231$ & $31,277,988$ & deletion & - & not rare \\
\hline 184356 chr6 & $66,074,421$ & $66,080,908$ & deletion & - & not rare \\
\hline 184356 chr6 & $69,229,759$ & $69,241,954$ & duplication & - & not rare \\
\hline 184356 chr6 & $78,972,930$ & $79,035,739$ & duplication & - & ot rare \\
\hline 184356 chr7 & $142,476,094$ & $142,484,042$ & deletion & - & not rare \\
\hline $184356 \mathrm{chr} 7$ & $38,388,889$ & $38,402,517$ & deletion & - & not rare \\
\hline 184356 chr7 & $91,033,074$ & $91,041,618$ & deletion & - & not rare \\
\hline 184356 chr7 & $97,396,709$ & $97,401,677$ & deletion & - & not rare \\
\hline 184356 chr8 & $123,383,935$ & $123,390,052$ & deletion & - & not rare \\
\hline 184356 chr8 & $39,232,581$ & $39,385,979$ & duplication & - & not rare \\
\hline 184356 chr8 & $51,029,371$ & $51,038,149$ & deletion & - & not rare \\
\hline 184356 chr9 & $44,739,805$ & $44,834,316$ & deletion & - & not rare \\
\hline 184356 chr9 & $68,838,523$ & $69,042,382$ & deletion & - & rare \\
\hline 184356 chr9 & $78,004,378$ & $78,011,616$ & deletion & - & not rare \\
\hline $184356 \mathrm{chrX}$ & $94,343,494$ & $94,393,450$ & duplication & - & not rare \\
\hline 184357 chr1 & $104,153,766$ & $104,211,046$ & deletion & AMY1A;AN & not rare \\
\hline 184357 chr1 & $12,908,591$ & $12,919,928$ & on & PRAMEF2 & rare \\
\hline 184357 chr 1 & $152,526,812$ & $152,568,230$ & deletion & LCE3D;LCE: & not rare \\
\hline 184357 chr1 & $248,621,384$ & $248,632,568$ & duplication & - & not rare \\
\hline 184357 chr1 & $248,740,572$ & $248,795,110$ & deletion & OR2T10;OF & not rare \\
\hline 184357 chr1 & $55,114,469$ & $55,120,469$ & deletion & MROH7 & not rare \\
\hline 184357 chr10 & $36,414,157$ & $36,423,110$ & deletion & & not rare \\
\hline 184357 chr10 & $55,232,434$ & $55,244,487$ & dup & & not rare \\
\hline 184357 chr10 & $58,846,575$ & $58,849,853$ & deletion & . & not rare \\
\hline 184357 chr11 & $18,949,220$ & $18,961,743$ & tion & $\mathrm{RX1}$ & rare \\
\hline 184357 chr11 & $55,370,325$ & $55,427,700$ & deletion & OR4P4;OR & not rare \\
\hline 184357 chr11 & $90,193,796$ & $90,195,742$ & deletion & - & not rare \\
\hline 184357 chr12 & $43,021,431$ & $43,027,453$ & deletion & - & not rare \\
\hline 184357 chr12 & $59,935,926$ & $59,942,122$ & deletion & - & not rare \\
\hline 184357 chr12 & $99,994,315$ & $100,008,773$ & deletion & - & not rare \\
\hline 184357 chr13 & $20,461,245$ & $20,469,457$ & duplication & - & not rare \\
\hline 184357 chr13 & $23,545,717$ & $23,553,518$ & deletion & - & not rare \\
\hline 184357 chr13 & $38,076,308$ & $38,085,537$ & deletion & - & not rare \\
\hline 184357 chr13 & $84,102,440$ & $84,157,927$ & deletion & - & not rare \\
\hline 184357 chr14 & $20,341,645$ & $20,422,182$ & duplication & OR4K5;OR & not rare \\
\hline 184357 chr14 & $41,610,224$ & $41,661,685$ & deletion & - & not rare \\
\hline 184357 chr14 & $48,708,134$ & $48,720,633$ & duplication & - & not rare \\
\hline 184357 chr15 & $26,747,139$ & $26,753,344$ & deletion & - & not rare \\
\hline 184357 chr15 & $34,717,697$ & $34,814,869$ & deletion & - & not rare \\
\hline 184357 chr17 & $76,419,882$ & $76,421,467$ & deletion & DNAH17 & not rare \\
\hline 184357 chr18 & $41,976,831$ & $41,982,102$ & deletion & - & not rare \\
\hline 184357 chr18 & $63,907,596$ & $63,910,708$ & deletion & - & not rare \\
\hline 184357 chr18 & $66,746,208$ & $66,755,736$ & deletion & - & not rare \\
\hline 184357 chr19 & $9,275,282$ & $9,282,176$ & deletion & - & not rare \\
\hline 184357 chr2 & $123,477,465$ & $123,482,301$ & deletion & - & not rare \\
\hline
\end{tabular}




\begin{tabular}{|c|c|c|c|c|c|}
\hline 184357 chr2 & $51,926,599$ & $51,926,904$ & deletion & - & not rare \\
\hline 184357 chr2 & $97,738,917$ & $98,024,837$ & deletion & \multicolumn{2}{|c|}{ FAHD2B;Ar not rare } \\
\hline 184357 chr22 & $39,359,885$ & $39,385,663$ & deletion & \multicolumn{2}{|c|}{ APOBEC3B not rare } \\
\hline 184357 chr3 & $193,136,358$ & $193,140,348$ & deletion & - & not rare \\
\hline 184357 chr4 & $187,747,224$ & $187,758,032$ & deletion & - & not rare \\
\hline $184357 \mathrm{chr} 4$ & $3,468,031$ & $3,475,406$ & duplication & DOK7 & not rare \\
\hline $184357 \mathrm{chr} 4$ & $69,374,369$ & $69,489,473$ & deletion & UGT2B17 & not rare \\
\hline $184357 \mathrm{chr} 4$ & $70,137,897$ & $70,229,260$ & deletion & UGT2B28 & not rare \\
\hline 184357 chr6 & $165,129,935$ & $165,194,584$ & duplication & - & not rare \\
\hline 184357 chr6 & $31,219,869$ & $31,229,552$ & deletion & - & not rare \\
\hline 184357 chr6 & $31,275,455$ & $31,277,988$ & deletion & - & not rare \\
\hline 184357 chr6 & $66,074,421$ & $66,080,908$ & deletion & - & not rare \\
\hline 184357 chr6 & $69,231,883$ & $69,241,954$ & duplication & - & not rare \\
\hline 184357 chr6 & $78,972,930$ & $79,012,428$ & duplication & - & rare \\
\hline 184357 chr7 & $142,476,094$ & $142,484,042$ & deletion & - & not rare \\
\hline 184357 chr7 & $91,033,074$ & $91,041,618$ & deletion & - & not rare \\
\hline 184357 chr7 & $97,396,709$ & $97,401,677$ & deletion & - & not rare \\
\hline 184357 chr8 & $115,633,704$ & $115,642,459$ & deletion & - & not rare \\
\hline 184357 chr8 & $123,383,935$ & $123,390,052$ & on & - & \\
\hline 184357 chr8 & $137,682,484$ & $137,862,080$ & deletion & - & not rare \\
\hline 184357 chr8 & $39,232,581$ & $39,385,979$ & duplication & - & not rare \\
\hline 184357 chr8 & $51,029,371$ & $51,038,149$ & dele & - & not rare \\
\hline 184357 chr9 & $12,581,270$ & $12,612,897$ & del & & rare \\
\hline 184357 chr9 & $78,004,378$ & $78,011,616$ & & & not rare \\
\hline 187232 chr1 & $161,563,386$ & $161,619,741$ & duplication & \multicolumn{2}{|c|}{ FCGR2C;FC not rare } \\
\hline 187232 chr1 & $62,113,597$ & $62,119,136$ & deletion & - & not rare \\
\hline 187232 chr1 & $71,240,621$ & $71,248,704$ & deletion & - & are \\
\hline 187232 chr1 & $8,359,110$ & $8,363,575$ & deletion & - & not rare \\
\hline 187232 chr10 & $125,162,193$ & $125,173,740$ & deletion & - & not rare \\
\hline 187232 chr11 & $55,370,325$ & $55,422,275$ & deletion & \multicolumn{2}{|c|}{$\mathrm{OR} 4 \mathrm{P} 4 ; \mathrm{OR}<$ not rare } \\
\hline 187232 chr11 & $86,304,402$ & $86,306,401$ & deletion & - & not rare \\
\hline 187232 chr12 & $2,245,636$ & $2,254,219$ & deletion & - & not rare \\
\hline 187232 chr12 & $33,300,995$ & $33,305,855$ & deletion & - & not rare \\
\hline 187232 chr12 & $40,875,351$ & $40,875,963$ & deletion & MUC19 & not rare \\
\hline 187232 chr12 & $43,021,431$ & $43,027,453$ & deletion & - & not rare \\
\hline 187232 chr12 & $59,935,926$ & $59,942,122$ & deletion & - & not rare \\
\hline 187232 chr13 & $32,532,761$ & $32,537,768$ & deletion & - & not rare \\
\hline 187232 chr13 & $34,134,809$ & $34,143,545$ & deletion & - & not rare \\
\hline 187232 chr13 & $69,247,022$ & $69,267,981$ & deletion & - & not rare \\
\hline 187232 chr13 & $83,282,387$ & $83,418,060$ & duplication & - & rare \\
\hline 187232 chr15 & $23,090,818$ & $23,116,232$ & deletion & - & not rare \\
\hline 187232 chr16 & $72,246,084$ & $72,252,752$ & deletion & - & not rare \\
\hline 187232 chr17 & $10,890,859$ & $10,893,799$ & deletion & - & not rare \\
\hline 187232 chr19 & $51,330,932$ & $51,333,442$ & deletion & KLK15 & not rare \\
\hline 187232 chr19 & $53,518,747$ & $53,552,296$ & duplication & ERVV-1 & not rare \\
\hline 187232 chr2 & $223,865,393$ & $223,869,820$ & deletion & - & not rare \\
\hline 187232 chr2 & $48,851,541$ & $48,856,770$ & deletion & - & not rare \\
\hline
\end{tabular}




\begin{tabular}{|c|c|c|c|c|c|}
\hline 187232 chr2 & $52,759,890$ & $52,784,952$ & deletion & - & not rare \\
\hline 187232 chr20 & $53,407,575$ & $53,419,685$ & deletion & - & not rare \\
\hline 187232 chr21 & $20,057,919$ & $20,078,801$ & duplication & - & not rare \\
\hline 187232 chr22 & $39,359,885$ & $39,385,663$ & deletion & АРОВЕСЗВ & not rare \\
\hline $187232 \mathrm{chr} 3$ & $11,411,823$ & $11,414,339$ & deletion & - & not rare \\
\hline 187232 chr3 & $153,679,375$ & $153,685,843$ & deletion & - & not rare \\
\hline 187232 chr3 & $193,136,358$ & $193,140,348$ & deletion & - & not rare \\
\hline 187232 chr3 & $84,887,157$ & $84,897,368$ & deletion & - & not rare \\
\hline 187232 chr4 & $156,959,568$ & $156,973,205$ & deletion & - & not rare \\
\hline $187232 \mathrm{chr} 4$ & $31,464,058$ & $31,469,190$ & deletion & - & not rare \\
\hline $187232 \mathrm{chr} 4$ & $34,785,955$ & $34,818,502$ & duplication & - & not rare \\
\hline $187232 \mathrm{chr} 4$ & $69,374,369$ & $69,431,280$ & deletion & UGT2B17 & not rare \\
\hline $187232 \mathrm{chr} 4$ & $7,182,916$ & $7,187,397$ & deletion & - & not rare \\
\hline 187232 chr5 & $117,385,681$ & $117,393,058$ & deletion & - & not rare \\
\hline 187232 chr6 & $29,856,693$ & $29,899,189$ & deletion & - & not rare \\
\hline 187232 chr6 & $31,276,513$ & $31,286,577$ & deletion & - & not rare \\
\hline 187232 chr6 & $32,458,168$ & $32,520,879$ & deletion & RB5 & not rare \\
\hline 187232 chr6 & $78,972,930$ & $79,035,739$ & deletion & - & not rare \\
\hline 187232 chr7 & $156,387,296$ & $156,394,307$ & on & - & are \\
\hline 187232 chr7 & $20,750,774$ & $20,753,528$ & deletion & - & not rare \\
\hline 187232 chr7 & $38,388,889$ & $38,402,517$ & deletion & - & not rare \\
\hline 187232 chr7 & $86,940,652$ & $86,948,364$ & de & - & not rare \\
\hline 187232 chr7 & $91,033,074$ & $91,041,618$ & del & & not rare \\
\hline 187232 chr8 & $109,406,155$ & $109,422,327$ & del & & not rare \\
\hline 187232 chr8 & $115,634,278$ & $115,651,344$ & dele & & not rare \\
\hline 187232 chr8 & $17,580,791$ & $17,581,700$ & deletion & MTUS1 & not rare \\
\hline 187232 chr8 & $39,232,581$ & $39,385,979$ & duplication & - & are \\
\hline 187232 chr8 & $51,226,087$ & $51,227,777$ & deletion & - & not rare \\
\hline 187232 chr8 & $58,126,687$ & $58,134,058$ & deletion & - & not rare \\
\hline 187232 chr8 & $6,977,269$ & $8,094,699$ & deletion & US & not rare \\
\hline 187232 chr9 & $23,290,141$ & $23,299,151$ & deletion & - & not rare \\
\hline $187232 \mathrm{chrX}$ & $16,346,245$ & $16,353,797$ & deletion & - & not rare \\
\hline $187233 \mathrm{chr} 1$ & $161,563,386$ & $161,619,741$ & duplication & $\mathrm{FC}$ & not rare \\
\hline 187233 chr10 & $71,788,176$ & $71,808,518$ & duplication & - & not rare \\
\hline 187233 & $18,949,220$ & $18,961,743$ & duplication & MRGPRX1 & not rare \\
\hline 187233 chr11 & $55,370,325$ & $55,427,700$ & deletion & OR4P4;OR & not rare \\
\hline 187233 chr11 & $86,304,402$ & $86,306,401$ & deletion & - & not rare \\
\hline 187233 chr12 & $2,245,636$ & $2,259,042$ & deletion & - & not rare \\
\hline 187233 chr12 & $33,300,995$ & $33,305,855$ & deletion & - & not rare \\
\hline 187233 chr12 & $43,021,431$ & $43,027,453$ & deletion & - & not rare \\
\hline 187233 chr13 & $32,532,541$ & $32,537,768$ & deletion & - & not rare \\
\hline 187233 chr13 & $34,134,809$ & $34,143,545$ & deletion & - & not rare \\
\hline 187233 chr13 & $69,247,022$ & $69,267,981$ & deletion & - & not rare \\
\hline 187233 chr13 & $83,282,387$ & $83,418,060$ & duplication & - & rare \\
\hline 187233 chr14 & $107,157,561$ & $107,181,152$ & duplication & - & not rare \\
\hline 187233 chr14 & $41,610,224$ & $41,661,685$ & deletion & - & not rare \\
\hline 187233 chr14 & $42,988,346$ & $42,992,844$ & deletion & - & not rare \\
\hline
\end{tabular}




\begin{tabular}{|c|c|c|c|c|c|}
\hline 187233 chr14 & 87,4 & $87,466,418$ & deletion & - & not rare \\
\hline 187233 chr16 & $22,635,867$ & $22,703,741$ & deletion & - & not rare \\
\hline 187233 chr16 & $33,433,576$ & $33,590,959$ & deletion & - & ot rare \\
\hline 187233 chr16 & $72,246,084$ & $72,252,752$ & deletion & - & not rare \\
\hline 187233 chr17 & $44,166,604$ & $44,356,058$ & duplication & KANSL1 & not rare \\
\hline 187233 & $65,847,099$ & $65,892,444$ & deletion & - & t rare \\
\hline 187233 chr18 & $67,208,806$ & $67,217,271$ & deletion & - & not rare \\
\hline 187233 chr19 & $51,330,932$ & $51,333,442$ & deletion & KLK15 & not rare \\
\hline 187233 chr19 & $53,518,747$ & $53,552,296$ & duplication & ERVV-1 & not rare \\
\hline 187233 chr2 & $212,771,670$ & $212,773,893$ & deletion & - & not rare \\
\hline 187233 chr2 & $228,244,107$ & $228,258,288$ & duplication & - & not rare \\
\hline 187233 chr2 & $52,759,890$ & $52,784,952$ & deletion & - & not rare \\
\hline 187233 chr2 & $89,540,620$ & $90,256,530$ & deletion & - & not rare \\
\hline 187233 & $53,407,575$ & $53,419,685$ & deletion & - & rare \\
\hline 187233 chr22 & $39,359,885$ & $39,385,663$ & deletion & АРОВЕСЗВ & not rare \\
\hline 187233 chr3 & $195,418,291$ & $195,468,690$ & duplication & MUC20 & not rare \\
\hline $187233 \mathrm{chr} 3$ & $65,189,366$ & $65,214,685$ & deletion & - & not rare \\
\hline 187233 chr3 & $84,887,157$ & $84,897,368$ & deletion & - & not rare \\
\hline 187233 chr3 & $89,402,409$ & $89,418,331$ & del & - & are \\
\hline $187233 \mathrm{chr} 4$ & $136,532,271$ & $136,601,944$ & deletion & - & not rare \\
\hline $187233 \mathrm{chr} 4$ & $156,959,568$ & $156,973,205$ & deletion & - & not rare \\
\hline $187233 \mathrm{chr} 4$ & $70,137,897$ & $70,229,260$ & & UGT2B28 & not rare \\
\hline $187233 \mathrm{chr} 4$ & $7,182,916$ & $7,187,397$ & de & & not rare \\
\hline 187233 chr5 & $117,388,410$ & $117,393,058$ & & & care \\
\hline 187233 chr6 & $29,855,945$ & $29,899,189$ & dele & & not rare \\
\hline 187233 chr6 & $32,458,168$ & $32,520,879$ & deletion & DRB5 & not rare \\
\hline 187233 & $38,388,889$ & $38,402,517$ & & - & are \\
\hline $187233 \mathrm{chr} 7$ & $86,940,652$ & $86,948,364$ & deletion & - & not rare \\
\hline 187233 chr7 & $91,033,074$ & $91,041,618$ & deletion & - & not rare \\
\hline 187233 chr8 & $39,279,418$ & $39,357,332$ & & - & are \\
\hline 187233 chr9 & $69,838,649$ & $69,992,206$ & deletion & - & not rare \\
\hline $187233 \mathrm{chr} X$ & $16,346,245$ & $16,353,797$ & deletion & - & not rare \\
\hline $187238 \mathrm{chr} 1$ & $104,153,766$ & $104,211,046$ & deletion & \multicolumn{2}{|c|}{ AMY1A;AN not rare } \\
\hline 187238 chr1 & $112,693,423$ & $112,704,581$ & deletion & - & not rare \\
\hline $187238 \mathrm{chr} 1$ & $161,489,467$ & $161,536,131$ & duplication & \multicolumn{2}{|c|}{ FCGR3A;HS not rare } \\
\hline 187238 chr1 & $248,740,572$ & $248,795,110$ & deletion & \multicolumn{2}{|c|}{ OR2T10;OF not rare } \\
\hline 187238 chr1 & $62,113,597$ & $62,119,136$ & deletion & - & not rare \\
\hline 187238 chr10 & $12,298,194$ & $12,307,963$ & deletion & - & not rare \\
\hline 187238 chr10 & $23,154,715$ & $23,157,251$ & deletion & - & not rare \\
\hline 187238 chr10 & $38,774,749$ & $38,785,636$ & duplication & - & not rare \\
\hline 187238 chr11 & $134,602,333$ & $134,607,484$ & deletion & - & not rare \\
\hline 187238 chr11 & $18,949,220$ & $18,961,743$ & duplication & MRGPRX1 & not rare \\
\hline 187238 chr11 & $3,239,077$ & $3,243,665$ & deletion & MRGPRG & not rare \\
\hline 187238 chr11 & $55,365,761$ & $55,427,700$ & deletion & \multicolumn{2}{|c|}{$\mathrm{OR} 4 \mathrm{P} 4 ; \mathrm{OR} \angle$ not rare } \\
\hline 187238 chr12 & $2,245,636$ & $2,254,219$ & deletion & - & not rare \\
\hline 187238 chr12 & $30,237,351$ & $30,243,473$ & deletion & - & not rare \\
\hline 187238 chr14 & $20,213,037$ & $20,375,610$ & duplication & \multicolumn{2}{|c|}{ OR4Q3;OR، not rare } \\
\hline
\end{tabular}




\begin{tabular}{|c|c|c|c|c|c|}
\hline 87238 chr14 & 41,6 & $41,661,685$ & deletion & - & not rare \\
\hline 187238 chr15 & $61,688,169$ & $61,698,179$ & deletion & - & not rare \\
\hline 187238 chr16 & $55,798,095$ & $55,819,443$ & deletion & - & ot rare \\
\hline 187238 chr16 & $72,246,084$ & $72,252,752$ & deletion & - & not rare \\
\hline 187238 chr17 & $10,890,859$ & $10,893,799$ & deletion & - & not rare \\
\hline 187238 chr17 & $14,997,297$ & $15,010,250$ & deletion & - & ot rare \\
\hline 187238 chr17 & $15,044,804$ & $15,058,236$ & deletion & - & not rare \\
\hline 187238 chr19 & $40,120,958$ & $40,123,536$ & deletion & - & not rare \\
\hline 187238 chr19 & $53,518,747$ & $53,552,296$ & duplication & ERVV-1 & not rare \\
\hline 187238 chr19 & $54,728,767$ & $54,743,217$ & duplication & LILRA6 & not rare \\
\hline 187238 chr2 & $184,795,725$ & $184,803,456$ & deletion & - & not rare \\
\hline 187238 chr2 & $212,771,670$ & $212,773,893$ & deletion & - & not rare \\
\hline 187238 chr2 & $48,851,541$ & $48,856,770$ & deletion & - & not rare \\
\hline 187238 chr2 & $51,926,599$ & $51,926,904$ & deletion & - & not rare \\
\hline 187238 chr2 & $81,518,838$ & $81,558,942$ & deletion & - & not rare \\
\hline 187238 chr21 & $23,655,900$ & $23,665,470$ & deletion & - & not rare \\
\hline 187238 chr22 & $39,359,885$ & $39,385,663$ & deletion & EC $3 B$ & not rare \\
\hline 187238 chr3 & $160,680,773$ & $160,685,788$ & deletion & - & not rare \\
\hline 187238 chr3 & $191,064,647$ & $191,070,300$ & on & - & \\
\hline 187238 chr3 & $63,578,618$ & $63,580,540$ & deletion & - & not rare \\
\hline $187238 \mathrm{chr} 4$ & $64,697,704$ & $64,709,753$ & deletion & - & not rare \\
\hline $187238 \mathrm{chr} 4$ & $69,374,369$ & $69,489,473$ & del & UGT2B17 & not rare \\
\hline $187238 \mathrm{chr} 4$ & $92,280,583$ & $92,284,935$ & del & & not rare \\
\hline 187238 chr5 & 685,748 & 776,691 & del & - & not rare \\
\hline 187238 chr6 & $124,432,882$ & $124,470,796$ & duplication & NKAIN2 & not rare \\
\hline 187238 chr6 & $162,966,431$ & $162,974,881$ & deletion & - & not rare \\
\hline 187238 chr6 & $29,856,693$ & $29,899,189$ & deletion & - & not rare \\
\hline 187238 chr6 & $30,994,010$ & $30,994,787$ & deletion & MUC22 & not rare \\
\hline 187238 chr6 & $32,494,038$ & $32,527,045$ & deletion & HLA-DRB5 & not rare \\
\hline 187238 chr6 & $78,972,930$ & $79,035,739$ & deletion & - & not rare \\
\hline 187238 chr7 & $159,118,443$ & $159,122,741$ & deletion & - & not rare \\
\hline 187238 chr7 & $75,354,240$ & $75,370,368$ & duplication & HIP1 & not rare \\
\hline 187238 chr7 & $86,940,652$ & $86,948,364$ & deletion & - & not rare \\
\hline 187238 chr8 & $39,232,581$ & $39,385,979$ & duplication & - & not rare \\
\hline 187238 chr8 & $51,013,729$ & $51,038,149$ & deletion & - & not rare \\
\hline 187239 chr1 & $149,032,299$ & $149,432,892$ & deletion & - & not rare \\
\hline 187239 chr1 & $187,716,107$ & $187,722,032$ & deletion & - & not rare \\
\hline 187239 chr10 & $135,242,873$ & $135,381,927$ & duplication & \multicolumn{2}{|c|}{ SYCE1;CYP; not rare } \\
\hline 187239 chr11 & $24,446,609$ & $24,451,830$ & deletion & - & not rare \\
\hline 187239 chr11 & $55,365,761$ & $55,443,269$ & duplication & \multicolumn{2}{|c|}{ OR4P4;OR $\angle$ not rare } \\
\hline 187239 chr11 & $90,193,796$ & $90,195,742$ & deletion & - & not rare \\
\hline 187239 chr12 & $11,220,827$ & $11,244,117$ & deletion & TAS2R43 & not rare \\
\hline 187239 chr12 & $119,988,733$ & $119,991,777$ & deletion & - & not rare \\
\hline 187239 chr12 & $31,266,287$ & $31,407,303$ & duplication & - & not rare \\
\hline 187239 chr13 & $64,225,427$ & $64,236,288$ & deletion & - & not rare \\
\hline 187239 chr15 & $34,717,697$ & $34,814,869$ & deletion & - & not rare \\
\hline 187239 chr15 & $34,929,955$ & $34,982,803$ & deletion & - & not rare \\
\hline
\end{tabular}




\begin{tabular}{|c|c|c|c|c|c|}
\hline 187239 chr16 & $78,944,055$ & $78,948,077$ & deletion & - & not rare \\
\hline 187239 chr17 & $67,268,689$ & $67,316,837$ & deletion & ABCA5 & not rare \\
\hline $87239 \mathrm{chr} 18$ & $31,117,401$ & $31,290,424$ & duplication & ASXL3 & rare \\
\hline 87239 chr19 & $41,355,999$ & $41,386,033$ & deletion & \multicolumn{2}{|c|}{$\mathrm{CYP} 2 \mathrm{~A} 7 ; \mathrm{CY}$ not rare } \\
\hline $187239 \mathrm{chr} 2$ & $10,885,901$ & $10,890,881$ & duplication & - & not rare \\
\hline $187239 \mathrm{chr} 2$ & $212,771,670$ & $212,773,893$ & deletion & - & not rare \\
\hline $187239 \mathrm{chr} 2$ & $34,697,895$ & $34,726,904$ & duplication & - & not rare \\
\hline $187239 \mathrm{chr} 2$ & $85,808,871$ & $85,821,204$ & deletion & 5 & not rare \\
\hline 187239 chr20 & $22,453,423$ & $22,465,098$ & deletion & - & not rare \\
\hline 187239 chr21 & $23,655,900$ & $23,665,470$ & deletion & - & not rare \\
\hline $187239 \mathrm{chr} 3$ & $131,711,896$ & $131,712,898$ & deletion & - & not rare \\
\hline $187239 \mathrm{chr} 3$ & $193,136,358$ & $193,140,348$ & deletion & - & not rare \\
\hline $187239 \mathrm{chr} 3$ & $68,793,418$ & $68,797,070$ & deletion & - & t rare \\
\hline $187239 \mathrm{chr} 4$ & $11,873,033$ & $11,880,161$ & duplication & - & not rare \\
\hline $187239 \mathrm{chr} 4$ & $138,092,298$ & $138,098,601$ & deletion & - & not rare \\
\hline 187239 chr5 & $140,225,908$ & $140,238,311$ & deletion & \multicolumn{2}{|c|}{ PCDHA10; F not rare } \\
\hline 187239 chr5 & $155,477,866$ & $155,488,917$ & deletion & - & not rare \\
\hline 187239 chr5 & $62,535,314$ & $62,678,398$ & deletion & - & \\
\hline 187239 chr5 & $7,178,644$ & $7,191,074$ & deletion & - & not rare \\
\hline 187239 chr5 & $97,048,466$ & $97,096,042$ & dele & - & not rare \\
\hline 187239 chr5 & $97,933,671$ & $97,939,616$ & & - & ot rare \\
\hline 187239 chr6 & $29,856,693$ & $29,899,189$ & deletion & - & not rare \\
\hline $187239 \mathrm{chr} 6$ & $30,994,010$ & $30,994,787$ & & & not rare \\
\hline $187239 \mathrm{chr} 6$ & $69,687,698$ & $69,690,567$ & del & & not rare \\
\hline 187239 chr7 & $3,371,291$ & $3,384,298$ & del & & not rare \\
\hline $187239 \mathrm{chr} 7$ & $62,149,006$ & $62,162,385$ & & & care \\
\hline $187239 \mathrm{chr} 8$ & $24,972,808$ & $24,990,418$ & duplication & - & not rare \\
\hline $187239 \mathrm{chr} 8$ & $51,013,729$ & $51,038,149$ & deletion & - & not rare \\
\hline $187239 \mathrm{chr} 8$ & $51,226,087$ & $51,227,777$ & del & - & are \\
\hline 187239 chr9 & $24,502,737$ & $24,518,795$ & deletion & - & not rare \\
\hline $187240 \mathrm{chr} 1$ & $153,674,704$ & $153,690,013$ & duplication & - & not rare \\
\hline $187240 \mathrm{chr} 1$ & $161,559,327$ & $161,619,741$ & duplication & \multicolumn{2}{|c|}{ FCGR2C;FC not rare } \\
\hline $187240 \mathrm{chr} 1$ & $248,740,572$ & $248,795,110$ & deletion & \multicolumn{2}{|c|}{ OR2T10;OF not rare } \\
\hline $187240 \mathrm{chr} 1$ & $25,598,276$ & $25,659,509$ & deletion & RHD & not rare \\
\hline 187240 chr1 & $62,113,597$ & $62,119,136$ & deletion & - & not rare \\
\hline 187240 chr11 & $55,370,325$ & $55,415,406$ & deletion & \multicolumn{2}{|c|}{$\mathrm{OR} 4 \mathrm{P} 4 ; \mathrm{OR} \angle$ not rare } \\
\hline 187240 chr12 & $11,220,827$ & $11,244,117$ & deletion & TAS2R43 & not rare \\
\hline 187240 chr12 & $40,875,351$ & $40,875,963$ & deletion & MUC19 & not rare \\
\hline 187240 chr12 & $43,021,431$ & $43,027,453$ & deletion & - & not rare \\
\hline 187240 chr13 & $38,076,308$ & $38,085,537$ & deletion & - & not rare \\
\hline 187240 chr14 & $106,103,358$ & $106,199,579$ & duplication & - & not rare \\
\hline 187240 chr14 & $106,575,614$ & $106,776,739$ & deletion & - & not rare \\
\hline 187240 chr15 & $24,521,050$ & $24,660,014$ & duplication & - & not rare \\
\hline 187240 chr15 & $25,420,978$ & $25,430,654$ & deletion & - & not rare \\
\hline 187240 chr15 & $32,509,090$ & $32,515,849$ & duplication & - & not rare \\
\hline 187240 chr15 & $34,717,697$ & $34,814,869$ & deletion & - & not rare \\
\hline 187240 & $32,511,914$ & $32,609,440$ & duplication & - & not rare \\
\hline
\end{tabular}




\begin{tabular}{|c|c|c|c|c|c|}
\hline 187240 chr16 & $55,800,009$ & $55,819,443$ & deletion & - & not rare \\
\hline 187240 chr17 & $34,438,753$ & $34,483,342$ & duplication & - & not rare \\
\hline 187240 chr17 & $44,166,604$ & $44,225,181$ & duplication & KANSL1 & not rare \\
\hline 187240 chr17 & $71,099,671$ & $71,102,042$ & deletion & - & not rare \\
\hline $187240 \mathrm{chr} 18$ & $63,907,596$ & $63,910,708$ & deletion & - & not rare \\
\hline 187240 chr19 & $24,460,170$ & $24,462,326$ & deletion & - & not rare \\
\hline 187240 chr19 & $43,703,965$ & $43,759,382$ & deletion & \multicolumn{2}{|c|}{ PSG4;PSG9 not rare } \\
\hline 187240 chr19 & $9,275,282$ & $9,282,176$ & deletion & - & not rare \\
\hline $187240 \mathrm{chr} 2$ & $123,477,465$ & $123,482,301$ & deletion & - & not rare \\
\hline $187240 \mathrm{chr} 2$ & $134,768,062$ & $134,777,220$ & deletion & - & not rare \\
\hline $187240 \mathrm{chr} 2$ & $184,085,551$ & $184,088,256$ & deletion & - & not rare \\
\hline 187240 chr2 & $242,854,089$ & $243,034,519$ & deletion & - & not rare \\
\hline $187240 \mathrm{chr} 2$ & $34,697,895$ & $34,726,904$ & deletion & - & not rare \\
\hline $187240 \mathrm{chr} 2$ & $41,239,457$ & $41,248,468$ & deletion & - & not rare \\
\hline $187240 \mathrm{chr} 2$ & $48,851,541$ & $48,856,770$ & deletion & - & not rare \\
\hline 187240 chr21 & $23,655,900$ & $23,665,470$ & deletion & - & not rare \\
\hline 187240 chr22 & $39,359,885$ & $39,385,663$ & deletion & \multicolumn{2}{|c|}{ APOBEC $3 \mathrm{~B}$ not rare } \\
\hline $187240 \mathrm{chr} 3$ & $129,769,827$ & $129,806,304$ & deletion & ALG1L2 & not rare \\
\hline $187240 \mathrm{chr} 3$ & $65,117,947$ & $65,118,762$ & on & - & are \\
\hline $187240 \mathrm{chr} 4$ & $138,092,298$ & $138,098,601$ & deletion & - & not rare \\
\hline $187240 \mathrm{chr} 4$ & $59,980,062$ & $59,988,095$ & duplication & - & not rare \\
\hline $187240 \mathrm{chr} 4$ & $60,325,010$ & $60,330,880$ & duplication & - & not rare \\
\hline $187240 \mathrm{chr} 4$ & $70,137,897$ & $70,229,260$ & deletion & B28 & not rare \\
\hline 187240 chr5 & $151,514,956$ & $151,518,615$ & deletion & & are \\
\hline 187240 chr6 & $132,114,944$ & $132,118,192$ & dele & & not rare \\
\hline 187240 chr6 & $139,603,393$ & $139,606,731$ & deletion & - & not rare \\
\hline 187240 chr6 & $29,855,945$ & $29,899,189$ & dele & - & are \\
\hline 187240 chr6 & $31,281,682$ & $31,297,908$ & duplication & - & not rare \\
\hline 187240 chr7 & $141,766,850$ & $141,793,521$ & deletion & - & not rare \\
\hline $187240 \mathrm{chr} 7$ & $97,396,709$ & $97,401,677$ & deletion & - & not rare \\
\hline 187240 chr8 & $40,184,872$ & $40,189,688$ & deletion & - & not rare \\
\hline 187240 chr9 & $138,195,113$ & $138,195,756$ & deletion & - & not rare \\
\hline $187240 \mathrm{chrX}$ & $94,343,494$ & $94,393,450$ & duplication & - & not rare \\
\hline 187242 chr1 & $195,546,330$ & $195,567,234$ & deletion & - & rare \\
\hline 187242 chr1 & $213,002,088$ & $213,011,812$ & deletion & SPATA45 & not rare \\
\hline 187242 chr1 & $248,740,572$ & $248,795,110$ & deletion & \multicolumn{2}{|c|}{ OR2T10;OF not rare } \\
\hline $187242 \mathrm{chr} 1$ & $43,447,091$ & $43,451,901$ & deletion & - & not rare \\
\hline 187242 chr1 & $62,113,597$ & $62,119,136$ & deletion & - & not rare \\
\hline 187242 chr10 & $58,516,470$ & $58,526,153$ & deletion & - & not rare \\
\hline 187242 chr10 & $71,280,107$ & $71,289,190$ & deletion & - & not rare \\
\hline 187242 chr10 & $76,372,037$ & $76,374,526$ & deletion & - & not rare \\
\hline 187242 chr11 & $90,193,796$ & $90,195,742$ & deletion & - & not rare \\
\hline 187242 chr12 & $33,300,995$ & $33,305,855$ & deletion & - & not rare \\
\hline 187242 chr13 & $34,134,809$ & $34,143,545$ & deletion & - & not rare \\
\hline 187242 chr13 & $91,024,205$ & $91,027,128$ & deletion & - & not rare \\
\hline 187242 chr14 & $106,891,524$ & $106,931,129$ & deletion & - & not rare \\
\hline 187242 chr15 & $22,299,434$ & $22,432,634$ & duplication & \multicolumn{2}{|c|}{ OR4M2;OR not rare } \\
\hline
\end{tabular}




\begin{tabular}{|c|c|c|c|c|c|}
\hline 87242 chr15 & 56 & $56,551,747$ & deletion & - & not rare \\
\hline 187242 chr16 & $1,124,086$ & $1,212,279$ & duplication & \multicolumn{2}{|c|}{ CACNA1H; (rare } \\
\hline 87242 chr16 & $55,800,009$ & $55,819,443$ & deletion & - & not rare \\
\hline 87242 chr16 & 961,054 & $1,063,457$ & duplication & \multicolumn{2}{|c|}{ LMF1;SOX\& rare } \\
\hline 187242 chr17 & $14,997,297$ & $15,010,250$ & deletion & - & not rare \\
\hline 187242 chr17 & $15,044,804$ & $15,058,236$ & deletion & - & not rare \\
\hline 87242 chr19 & $35,852,103$ & $35,861,695$ & deletion & - & not rare \\
\hline 187242 chr19 & $43,298,958$ & $43,539,189$ & deletion & \multicolumn{2}{|c|}{ PSG6;PSG7 not rare } \\
\hline 187242 chr19 & $53,518,747$ & $53,548,950$ & duplication & -1 & not rare \\
\hline 187242 chr2 & $130,242,413$ & $130,250,978$ & deletion & - & not rare \\
\hline 187242 chr2 & $214,989,439$ & $214,992,692$ & deletion & - & not rare \\
\hline 187242 chr2 & $4,212,725$ & $4,222,144$ & deletion & - & not rare \\
\hline 187242 chr2 & $48,851,541$ & $48,856,770$ & dele & - & not rare \\
\hline 187242 chr2 & $49,626,017$ & $49,662,921$ & deletion & - & rare \\
\hline 187242 chr2 & $57,398,769$ & $57,446,548$ & duplication & - & not rare \\
\hline 187242 chr3 & $110,793,050$ & $110,796,218$ & deletion & - & not rare \\
\hline 187242 chr3 & $128,382,258$ & $128,412,024$ & duplication & - & not rare \\
\hline 187242 chr3 & $195,433,728$ & $195,475,799$ & duplication & \multicolumn{2}{|c|}{ MUC20;Ml not rare } \\
\hline 187242 chr3 & $65,189,366$ & $65,214,685$ & & - & are \\
\hline $187242 \mathrm{chr} 4$ & $138,092,298$ & $138,098,601$ & deletion & - & not rare \\
\hline $187242 \mathrm{chr} 4$ & $64,697,704$ & $64,709,753$ & dele & - & not rare \\
\hline 187242 chr5 & 5,908 & $140,238,311$ & & \multicolumn{2}{|c|}{ PCDHA10; F not rare } \\
\hline 187242 chr5 & $142,859,606$ & $142,865,795$ & c & & not rare \\
\hline 187242 chr5 & $155,477,866$ & $155,488,917$ & & & not rare \\
\hline 187242 chr5 & $97,555,945$ & $97,563,781$ & del & & not rare \\
\hline 187242 chr6 & $78,972,930$ & $79,035,739$ & del & - & not rare \\
\hline 187242 chr7 & $141,766,850$ & $141,793,521$ & & - & not rare \\
\hline 187242 chr7 & $156,387,296$ & $156,394,307$ & deletion & - & not rare \\
\hline 187242 chr7 & $17,237,990$ & $17,267,538$ & deletion & - & rare \\
\hline 187242 chr7 & 86,9 & 8,364 & dele & - & are \\
\hline 187242 chr7 & $91,033,074$ & $91,041,618$ & deletion & - & not rare \\
\hline 187242 chr8 & $115,634,278$ & $115,642,459$ & deletion & - & not rare \\
\hline 187242 chr8 & $39,232,581$ & $39,385,979$ & duplication & - & not rare \\
\hline 187242 chr9 & $44,739,805$ & $44,834,316$ & deletion & - & not rare \\
\hline $187242 \mathrm{chrX}$ & $47,881,362$ & $48,003,920$ & duplication & \multicolumn{2}{|c|}{ SPACA5B;S not rare } \\
\hline $187242 \mathrm{chrX}$ & $51,741,943$ & $51,760,413$ & deletion & - & not rare \\
\hline 187243 chr1 & $104,153,766$ & $104,211,046$ & deletion & \multicolumn{2}{|c|}{ AMY1A;AN not rare } \\
\hline 187243 chr1 & $195,546,330$ & $195,567,234$ & deletion & - & rare \\
\hline 187243 chr1 & $248,740,572$ & $248,795,110$ & deletion & \multicolumn{2}{|c|}{ OR2T10;OF not rare } \\
\hline 187243 chr 1 & $91,093,909$ & $91,099,903$ & deletion & - & not rare \\
\hline 187243 chr10 & $58,516,470$ & $58,526,153$ & deletion & - & not rare \\
\hline 187243 chr10 & $71,282,981$ & $71,289,190$ & deletion & - & not rare \\
\hline 187243 chr10 & $76,372,037$ & $76,374,526$ & deletion & - & not rare \\
\hline 187243 chr10 & $83,883,951$ & $83,888,343$ & deletion & - & not rare \\
\hline 187243 chr11 & $18,949,220$ & $18,961,743$ & duplication & MRGPRX1 & not rare \\
\hline 187243 chr11 & $58,811,241$ & $58,844,734$ & duplication & - & not rare \\
\hline 187243 chr11 & $90,193,796$ & $90,195,742$ & deletion & - & not rare \\
\hline
\end{tabular}




\begin{tabular}{|c|c|c|c|c|c|}
\hline 87243 chr12 & 547 & 130 & & - & not rare \\
\hline 187243 chr13 & $34,134,809$ & $34,143,545$ & deletion & - & not rare \\
\hline 87243 chr13 & $38,076,308$ & $38,085,537$ & deletion & - & ot rare \\
\hline 87243 chr14 & $20,197,311$ & $20,422,182$ & duplication & \multicolumn{2}{|c|}{ OR4K5;OR $\angle$ not rare } \\
\hline 187243 chr14 & $41,610,224$ & $41,661,685$ & deletion & - & not rare \\
\hline 87243 & $42,988,346$ & $42,992,844$ & deletion & - & not rare \\
\hline 87243 chr15 & $102,356,497$ & $102,364,833$ & deletion & OR4F15 & not rare \\
\hline 187243 chr15 & $20,196,661$ & $21,241,118$ & duplication & \multicolumn{2}{|c|}{ GOLGA6L6; not rare } \\
\hline 187243 chr15 & $21,903,815$ & $22,753,733$ & duplication & \multicolumn{2}{|c|}{ GOLGA6L2: not rare } \\
\hline 187243 chr15 & $56,543,546$ & $56,551,747$ & deletion & - & not rare \\
\hline 187243 chr16 & $1,036,486$ & $1,063,457$ & duplication & - & rare \\
\hline 187243 chr16 & $1,124,086$ & $1,212,279$ & duplication & \multicolumn{2}{|c|}{ CACNA1H;( rare } \\
\hline 187243 chr16 & $55,800,009$ & $55,819,443$ & deletion & - & not rare \\
\hline 187243 chr16 & $85,301,988$ & $85,304,326$ & deletion & - & not rare \\
\hline 187243 chr16 & 961,054 & $1,028,819$ & duplication & LN & rare \\
\hline 187243 chr17 & $14,997,297$ & $15,010,250$ & deletion & - & not rare \\
\hline 187243 chr17 & $15,044,804$ & $15,058,236$ & deletion & - & not rare \\
\hline 187243 chr19 & $35,852,103$ & $35,861,695$ & deletion & - & not rare \\
\hline 187243 & $43,703,965$ & 9,382 & deletion & \multicolumn{2}{|c|}{ PSG4;PSG9 not rare } \\
\hline 187243 chr19 & $51,330,932$ & $51,333,442$ & deletion & KLK15 & not rare \\
\hline 187243 chr19 & $53,518,747$ & $53,552,296$ & duplication & ERVV-1 & not rare \\
\hline 187243 chr2 & $130,242,413$ & $130,250,978$ & delet & - & not rare \\
\hline 187243 chr2 & $213,187,034$ & $213,191,389$ & dele & & not rare \\
\hline 187243 chr2 & $231,415,013$ & $231,434,203$ & du & & not rare \\
\hline 187243 chr2 & $34,697,895$ & $34,724,199$ & dele & & not rare \\
\hline 187243 chr2 & $4,212,725$ & $4,222,144$ & dele & 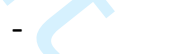 & not rare \\
\hline 187243 chr2 & $48,851,541$ & $48,856,770$ & dele & - & not rare \\
\hline 187243 chr2 & $49,626,017$ & $49,662,921$ & deletion & - & rare \\
\hline 187243 chr2 & $57,398,769$ & $57,446,548$ & duplication & - & not rare \\
\hline 187243 chr2 & $66,188,209$ & $66,196,726$ & deletion & - & are \\
\hline 187243 chr20 & $50,601,439$ & $50,606,738$ & deletion & - & not rare \\
\hline 187243 chr3 & $110,793,050$ & $110,796,218$ & deletion & - & not rare \\
\hline 187243 chr3 & $128,382,258$ & $128,412,024$ & duplication & - & not rare \\
\hline 187243 chr3 & $182,434,708$ & $182,508,807$ & duplication & - & rare \\
\hline 187243 chr3 & $37,979,882$ & $37,986,734$ & deletion & - & not rare \\
\hline $187243 \mathrm{chr} 4$ & $60,325,010$ & $60,330,880$ & duplication & - & not rare \\
\hline 187243 chr4 & $69,374,369$ & $69,489,473$ & deletion & UGT2B17 & not rare \\
\hline 187243 chr5 & $17,609,458$ & $17,642,516$ & deletion & - & not rare \\
\hline 187243 chr5 & 733,565 & 825,338 & duplication & ZDHHC11 & not rare \\
\hline 187243 chr5 & $97,555,945$ & $97,563,781$ & deletion & - & not rare \\
\hline 187243 chr5 & $97,933,671$ & $97,939,616$ & deletion & - & not rare \\
\hline 187243 chr6 & $132,114,944$ & $132,118,192$ & deletion & - & not rare \\
\hline 187243 chr6 & $139,603,393$ & $139,606,731$ & deletion & - & not rare \\
\hline 187243 chr6 & $29,856,693$ & $29,910,742$ & deletion & $\mathrm{HL}$ & not rare \\
\hline 187243 chr6 & $31,281,682$ & $31,286,577$ & deletion & - & not rare \\
\hline 187243 chr7 & $141,766,850$ & $141,793,521$ & deletion & - & not rare \\
\hline 187243 chr7 & $156,387,296$ & $156,394,307$ & deletion & - & not rare \\
\hline
\end{tabular}




\begin{tabular}{|c|c|c|c|c|c|}
\hline 187243 chr7 & $17,237,990$ & $17,267,538$ & deletion & - & rare \\
\hline 187243 chr7 & $86,940,652$ & $86,948,364$ & deletion & - & not rare \\
\hline 187243 chr7 & $91,033,074$ & $91,041,618$ & deletion & - & ot rare \\
\hline 187243 chr8 & $115,634,278$ & $115,642,459$ & deletion & - & not rare \\
\hline 187243 chr8 & $39,232,581$ & $39,385,979$ & deletion & - & not rare \\
\hline 187243 chr8 & $40,184,872$ & $40,189,688$ & deletion & - & not rare \\
\hline $187243 \mathrm{chrX}$ & $47,881,362$ & $48,003,920$ & duplication & \multicolumn{2}{|c|}{ SPACA5B;S not rare } \\
\hline 187243 chrX & $51,741,943$ & $51,760,413$ & deletion & - & not rare \\
\hline 187251 chr1 & $104,153,766$ & $104,211,046$ & deletion & \multicolumn{2}{|c|}{ AMY1A;AN not rare } \\
\hline $187251 \mathrm{chr} 1$ & $148,937,908$ & $149,432,892$ & duplication & - & not rare \\
\hline $187251 \mathrm{chr} 1$ & $87,028,669$ & $87,039,309$ & deletion & CLCA4 & not rare \\
\hline 187251 chr10 & $46,949,421$ & $47,756,065$ & duplication & \multicolumn{2}{|c|}{ ANTXRL;NF not rare } \\
\hline 187251 chr10 & $71,280,107$ & $71,289,190$ & deletion & - & not rare \\
\hline 187251 chr12 & $129,230,513$ & $129,233,013$ & deletion & - & not rare \\
\hline 187251 chr12 & $2,245,636$ & $2,254,219$ & deletion & - & not rare \\
\hline 187251 chr12 & $30,237,351$ & $30,243,473$ & deletion & - & not rare \\
\hline 187251 chr12 & $33,300,995$ & $33,305,855$ & deletion & - & not rare \\
\hline 187251 chr13 & $19,174,634$ & $19,263,735$ & duplication & - & not rare \\
\hline 187251 chr13 & $21,728,134$ & $21,732,193$ & dupli & & rare \\
\hline 187251 chr13 & $34,134,809$ & $34,143,545$ & deletion & - & not rare \\
\hline 187251 chr14 & $20,197,311$ & $20,424,184$ & duplication & \multicolumn{2}{|c|}{ OR4K5;OR $\angle$ not rare } \\
\hline 187251 chr14 & $41,610,224$ & $41,661,685$ & deletion & - & not rare \\
\hline 187251 chr15 & $34,717,697$ & $34,814,869$ & dele & & not rare \\
\hline 187251 chr16 & $19,945,540$ & $19,962,636$ & & & ot rare \\
\hline 187251 chr16 & $6,986,351$ & $6,991,478$ & dele & & not rare \\
\hline 187251 chr16 & $85,302,707$ & $85,304,326$ & dele & . & not rare \\
\hline 187251 chr18 & $41,976,831$ & $41,982,102$ & dele & - & rare \\
\hline 187251 chr19 & $24,460,170$ & $24,462,326$ & deletion & - & not rare \\
\hline 187251 chr19 & $43,703,965$ & $43,759,382$ & deletion & \multicolumn{2}{|c|}{ PSG4;PSG9 not rare } \\
\hline 187251 chr19 & $53,518,747$ & $53,548,950$ & duplication & ERI & not rare \\
\hline $187251 \mathrm{chr} 2$ & $165,850,456$ & $165,864,123$ & deletion & - & not rare \\
\hline $187251 \mathrm{chr} 2$ & $184,795,725$ & $184,803,456$ & deletion & - & not rare \\
\hline $187251 \mathrm{chr} 2$ & $213,187,034$ & $213,191,389$ & deletion & - & not rare \\
\hline 187251 chr2 & $48,851,541$ & $48,856,770$ & deletion & - & not rare \\
\hline 187251 chr21 & $14,478,785$ & $15,153,902$ & deletion & & not rare \\
\hline 187251 chr22 & $37,745,101$ & $37,749,946$ & deletion & - & not rare \\
\hline $187251 \mathrm{chr} 4$ & $138,092,298$ & $138,098,601$ & deletion & - & not rare \\
\hline $187251 \mathrm{chr} 4$ & $64,136,609$ & $64,154,477$ & deletion & - & not rare \\
\hline $187251 \mathrm{chr} 4$ & $69,374,369$ & $69,489,473$ & duplication & UGT2B17 & not rare \\
\hline 187251 chr5 & $111,937,859$ & $111,944,746$ & deletion & - & not rare \\
\hline 187251 chr5 & $151,514,956$ & $151,518,615$ & deletion & - & not rare \\
\hline 187251 chr5 & $155,477,866$ & $155,488,917$ & deletion & - & not rare \\
\hline 187251 chr5 & $25,210,358$ & $25,224,844$ & deletion & - & not rare \\
\hline 187251 chr6 & $69,231,883$ & $69,241,954$ & duplication & - & not rare \\
\hline 187251 chr6 & $77,439,769$ & $77,451,301$ & deletion & - & not rare \\
\hline 187251 chr7 & $155,882,711$ & $155,885,143$ & deletion & - & not rare \\
\hline $187251 \mathrm{chr} 7$ & $159,118,443$ & $159,122,741$ & deletion & - & not rare \\
\hline
\end{tabular}




\begin{tabular}{|c|c|c|c|c|c|}
\hline 187251 chr7 & $38,388,889$ & $38,402,517$ & deletion & - & not rare \\
\hline 187251 chr7 & 41,421 & 73,060 & duplication & - & not rare \\
\hline 187251 chr7 & $75,354,240$ & $75,370,368$ & duplication & HIP1 & not rare \\
\hline 87251 chr7 & $91,033,074$ & $91,041,618$ & deletion & - & not rare \\
\hline 187251 chr8 & $115,633,704$ & $115,642,459$ & deletion & - & not rare \\
\hline 187251 chr8 & $24,972,808$ & $24,990,418$ & duplication & - & not rare \\
\hline 87251 chr8 & $39,236,582$ & $39,290,901$ & deletion & - & not rare \\
\hline 187251 chr8 & $39,322,364$ & $39,382,903$ & deletion & - & not rare \\
\hline 187251 chr8 & $51,029,371$ & $51,038,149$ & deletion & - & not rare \\
\hline 187251 chr9 & $23,363,526$ & $23,376,817$ & deletion & - & not rare \\
\hline 187251 chr9 & $24,146,203$ & $24,150,034$ & deletion & - & not rare \\
\hline 187251 chr9 & $6,398,529$ & $6,408,429$ & deletion & - & not rare \\
\hline 198116 chr1 & $161,570,803$ & $161,601,370$ & duplication & FCGR3B & not rare \\
\hline 198116 chr1 & $1,651,355$ & $1,668,298$ & deletion & SLC35E2;CL & rare \\
\hline 198116 chr1 & $248,740,572$ & $248,795,110$ & deletion & OR2T10;OF & not rare \\
\hline 198116 chr1 & $62,113,597$ & $62,119,136$ & deletion & - & rare \\
\hline 198116 chr1 & $9,847,290$ & $9,855,529$ & deletion & - & not rare \\
\hline 198116 chr10 & $132,635,459$ & $132,638,422$ & deletion & - & not rare \\
\hline 198116 chr10 & $28,939,852$ & $28,945,223$ & & - & are \\
\hline 198116 chr10 & $30,876,076$ & $30,891,380$ & duplication & - & not rare \\
\hline 198116 chr10 & $71,282,981$ & $71,289,190$ & deletion & - & not rare \\
\hline 198116 chr11 & $134,601,963$ & $134,606,394$ & deletion & - & not rare \\
\hline 198116 chr11 & $18,951,137$ & $18,958,916$ & duplication & MRGPRX1 & not rare \\
\hline 198116 chr11 & $3,239,988$ & $3,243,665$ & deletion & MRGPRG & not rare \\
\hline 198116 chr11 & $55,365,761$ & $55,443,269$ & deletion & OR4P4;OR & not rare \\
\hline 198116 chr12 & $11,511,132$ & $11,543,002$ & duplication & - & not rare \\
\hline 198116 chr12 & $19,472,747$ & $19,578,107$ & dup & PLEKHA5 & \\
\hline 198116 chr12 & $31,266,287$ & $31,407,303$ & duplication & - & not rare \\
\hline 198116 chr12 & $43,021,431$ & $43,026,485$ & deletion & - & not rare \\
\hline 198116 chr12 & $6,241,446$ & $6,247,923$ & deletion & - & not rare \\
\hline 198116 chr13 & $21,728,134$ & $21,732,193$ & duplication & SKA3 & not rare \\
\hline 198116 chr13 & $38,076,308$ & $38,085,537$ & deletion & - & not rare \\
\hline 198116 chr13 & $88,294,496$ & $88,300,323$ & deletion & - & not rare \\
\hline 198116 chr14 & $24,447,448$ & $24,484,060$ & deletion & DHRS4L2 & not rare \\
\hline 198116 chr14 & $27,479,798$ & $27,493,119$ & ion & - & not rare \\
\hline 198116 chr14 & $42,988,346$ & $42,992,844$ & deletion & - & not rare \\
\hline 198116 chr15 & $32,509,090$ & $32,620,088$ & duplication & - & not rare \\
\hline 198116 chr15 & $41,682,094$ & $41,689,232$ & duplication & NDUFAF1 & not rare \\
\hline 198116 chr16 & $60,082,237$ & $60,098,544$ & deletion & - & not rare \\
\hline 198116 chr19 & $43,298,958$ & $43,539,189$ & deletion & \multicolumn{2}{|c|}{ PSG6;PSG7 not rare } \\
\hline 198116 chr19 & $50,079,451$ & $50,113,109$ & deletion & \multicolumn{2}{|c|}{ PRR12;PRR rare } \\
\hline 198116 chr19 & $53,518,747$ & $53,552,296$ & duplication & ERVV-1 & not rare \\
\hline 198116 chr19 & $54,730,202$ & $54,743,217$ & duplication & LILRA6 & not rare \\
\hline 198116 chr2 & $212,771,670$ & $212,773,893$ & deletion & - & not rare \\
\hline 198116 chr2 & $214,989,439$ & $214,992,692$ & deletion & - & not rare \\
\hline 198116 chr2 & $38,956,947$ & $38,971,623$ & duplication & GALM & not rare \\
\hline 198116 chr2 & $52,759,890$ & $52,784,952$ & duplication & - & not rare \\
\hline
\end{tabular}




\begin{tabular}{|c|c|c|c|c|c|}
\hline 198116 chr20 & 903 & $29,585,801$ & deletion & - & not rare \\
\hline 198116 chr20 & $59,567,371$ & $59,586,828$ & duplication & - & not rare \\
\hline 98116 chr21 & $10,766,457$ & $10,863,251$ & duplication & - & not rare \\
\hline 98116 chr22 & $20,133,378$ & $20,204,924$ & duplication & \multicolumn{2}{|c|}{ ZDHHC8;CC rare } \\
\hline 198116 chr22 & $25,663,994$ & $25,919,492$ & duplication & LRP5L & not rare \\
\hline 198116 chr3 & $193,136,358$ & $193,140,348$ & deletion & - & not rare \\
\hline $98116 \mathrm{chr} 3$ & $195,458,723$ & $195,463,099$ & duplication & C20 & not rare \\
\hline 198116 chr3 & $197,844,320$ & $197,846,134$ & deletion & - & not rare \\
\hline 198116 chr3 & $28,810,588$ & $28,817,583$ & deletion & - & not rare \\
\hline 198116 chr3 & $37,979,882$ & $37,986,734$ & deletion & - & not rare \\
\hline 198116 chr3 & $71,929,521$ & $71,932,634$ & deletion & - & not rare \\
\hline $198116 \mathrm{chr} 4$ & $138,092,298$ & $138,098,601$ & deletion & - & not rare \\
\hline $198116 \mathrm{chr} 4$ & $190,194,200$ & $190,199,993$ & deletion & - & not rare \\
\hline $198116 \mathrm{chr} 4$ & $31,464,058$ & $31,469,190$ & deletion & - & not rare \\
\hline $198116 \mathrm{chr} 4$ & $34,785,955$ & $34,818,502$ & deletion & - & not rare \\
\hline $198116 \mathrm{chr} 4$ & $59,167,915$ & $59,180,928$ & deletion & - & not rare \\
\hline $198116 \mathrm{chr} 4$ & $69,374,369$ & $69,489,473$ & deletion & 2B17 & not rare \\
\hline 198116 chr4 & $7,182,916$ & $7,187,397$ & deletion & - & not rare \\
\hline 198116 chr5 & $140,555,135$ & $140,558,618$ & duplication & \multicolumn{2}{|c|}{ PCDHB8;PC not rare } \\
\hline 198116 chr5 & $180,377,470$ & $180,424,820$ & deletion & \multicolumn{2}{|c|}{ BTNL8;BTN not rare } \\
\hline 198116 chr6 & $101,607,733$ & $101,632,702$ & deletion & - & rare \\
\hline 198116 chr6 & $29,859,135$ & $29,899,389$ & deletion & - & not rare \\
\hline 198116 chr6 & $31,281,682$ & $31,286,577$ & e & & not rare \\
\hline 198116 chr6 & $31,337,815$ & $31,343,632$ & & & \\
\hline 198116 chr6 & $66,074,421$ & $66,080,908$ & dele & & not rare \\
\hline 198116 chr6 & $81,284,205$ & $81,293,326$ & dele & - & not rare \\
\hline 198116 chr7 & $141,766,850$ & $141,793,419$ & & - & are \\
\hline 198116 chr7 & $142,098,342$ & $142,105,433$ & deletion & - & not rare \\
\hline 198116 chr7 & $156,387,296$ & $156,394,307$ & deletion & - & not rare \\
\hline 198116 chr7 & $20,750,774$ & $20,753,987$ & dele & - & not rare \\
\hline 198116 chr7 & 41,421 & 73,060 & duplication & - & not rare \\
\hline 198116 chr8 & $24,972,808$ & $24,990,418$ & duplication & - & not rare \\
\hline 198116 chr8 & $39,232,581$ & $39,335,392$ & deletion & - & not rare \\
\hline 198116 chr8 & $39,369,329$ & $39,385,979$ & deletion & - & not rare \\
\hline 198116 chr8 & $51,031,221$ & $51,038,149$ & on & - & are \\
\hline 198116 chr8 & $51,226,087$ & $51,227,777$ & deletion & - & not rare \\
\hline 198116 chr9 & $2,148,213$ & $2,151,397$ & deletion & - & not rare \\
\hline 198116 chr9 & $77,999,354$ & $78,011,616$ & deletion & - & not rare \\
\hline $198116 \mathrm{chr} X$ & $144,312,825$ & $144,343,312$ & deletion & SPANXN1 & rare \\
\hline 198117 chr1 & $108,734,695$ & $108,737,586$ & deletion & SLC25A24 & not rare \\
\hline 198117 chr1 & $112,693,423$ & $112,704,581$ & deletion & - & not rare \\
\hline 198117 chr1 & $149,022,110$ & $149,237,009$ & deletion & - & not rare \\
\hline 198117 chr1 & $1,646,574$ & $1,680,951$ & duplication & \multicolumn{2}{|c|}{ SLC35E2;C[ not rare } \\
\hline 198117 chr1 & $248,740,572$ & $248,795,110$ & deletion & \multicolumn{2}{|c|}{ OR2T10;OF not rare } \\
\hline 198117 chr1 & $32,075,036$ & $32,075,703$ & deletion & - & not rare \\
\hline 198117 chr1 & $62,113,597$ & $62,119,136$ & deletion & - & not rare \\
\hline 198117 chr10 & $56,448,886$ & $56,468,486$ & duplication & - & not rare \\
\hline
\end{tabular}




\begin{tabular}{|c|c|c|c|c|c|c|}
\hline 198117 & chr10 & $71,282,981$ & $71,289,190$ & deletion & - & not rare \\
\hline 198117 & chr11 & $55,365,761$ & $55,443,269$ & deletion & \multicolumn{2}{|c|}{$\mathrm{OR} 4 \mathrm{P} 4 ; \mathrm{OR} \angle$ not rare } \\
\hline 98117 & chr11 & $5,886,773$ & $5,934,737$ & duplication & OR52E4 & not rare \\
\hline 198117 & chr11 & $93,063,200$ & $93,068,949$ & deletion & CCDC67 & not rare \\
\hline 198117 & chr12 & $12,532,984$ & $12,541,869$ & deletion & - & not rare \\
\hline 198117 & chr12 & $43,021,431$ & $43,026,485$ & deletion & - & not rare \\
\hline 198117 & chr13 & $88,294,496$ & $88,300,323$ & deletion & - & not rare \\
\hline 198117 & chr14 & $27,479,798$ & $27,493,119$ & deletion & - & not rare \\
\hline 198117 & chr15 & $20,120,214$ & $20,144,522$ & deletion & - & not rare \\
\hline 198117 & chr15 & $41,682,094$ & $41,689,232$ & duplication & NDUFAF1 & not rare \\
\hline 198117 & chr15 & $56,789,979$ & $56,800,635$ & deletion & - & not rare \\
\hline 198117 & chr16 & $46,471,024$ & $46,492,062$ & deletion & - & not rare \\
\hline 198117 & chr16 & $70,141,115$ & $70,196,139$ & deletion & PDPR & not rare \\
\hline 198117 & chr17 & $54,160,465$ & $54,172,668$ & deletion & - & not rare \\
\hline 198117 & chr17 & $70,815,357$ & $70,820,207$ & deletion & - & not rare \\
\hline 198117 & chr2 & $129,638,609$ & $129,645,321$ & deletion & - & not rare \\
\hline 198117 & chr2 & $41,239,457$ & $41,248,468$ & deletion & - & not rare \\
\hline 198117 & chr2 & $48,851,541$ & $48,856,770$ & deletion & - & not rare \\
\hline 198117 & chr20 & $25,890,697$ & $25,896,760$ & ion & - & are \\
\hline 198117 & chr20 & $29,450,698$ & $29,483,606$ & deletion & - & not rare \\
\hline 198117 & $\operatorname{chr} 20$ & $29,566,903$ & $29,585,801$ & deletion & - & not rare \\
\hline 198117 & chr20 & $60,781,095$ & $60,785,802$ & deletion & - & not rare \\
\hline 198117 & chr21 & $11,055,462$ & $11,063,273$ & deletion & \multicolumn{2}{|c|}{ BAGE2;BAC not rare } \\
\hline 198117 & chr22 & $20,131,055$ & $20,207,328$ & duplication & \multicolumn{2}{|c|}{ ZDHHC8;CC rare } \\
\hline 198117 & chr22 & $25,663,994$ & $25,919,492$ & duplication & LRP5L & not rare \\
\hline 198117 & chr3 & $193,136,358$ & $193,140,348$ & deletion & - & not rare \\
\hline 198117 & chr3 & $197,844,320$ & $197,846,927$ & dele & - & \\
\hline 198117 & chr3 & $53,032,639$ & $53,038,786$ & deletion & - & not rare \\
\hline 198117 & chr4 & $138,092,298$ & $138,098,601$ & deletion & - & not rare \\
\hline 198117 & chr4 & $34,785,955$ & $34,818,502$ & duplication & - & not rare \\
\hline 198117 & chr4 & $3,494,600$ & $3,496,058$ & duplication & DOK7 & not rare \\
\hline 198117 & chr4 & $56,779,555$ & $56,800,102$ & deletion & - & rare \\
\hline 198117 & chr4 & $60,325,242$ & $60,330,880$ & duplication & - & not rare \\
\hline 198117 & chr4 & $69,374,369$ & $69,489,473$ & deletion & UGT2B17 & not rare \\
\hline 198117 & chr4 & $70,137,897$ & $70,229,260$ & deletion & $2 \mathrm{~B} 28$ & not rare \\
\hline 198117 & chr4 & $7,182,916$ & $7,187,397$ & deletion & - & not rare \\
\hline 198117 & chr5 & $113,476,277$ & $113,507,843$ & deletion & - & rare \\
\hline 198117 & chr5 & $140,225,908$ & $140,238,311$ & deletion & \multicolumn{2}{|c|}{ PCDHA10; F not rare } \\
\hline 198117 & chr5 & $155,477,866$ & $155,488,917$ & deletion & - & not rare \\
\hline 198117 & chr5 & $1,561,873$ & $1,566,307$ & duplication & - & not rare \\
\hline 198117 & chr6 & $101,607,733$ & $101,632,702$ & deletion & - & rare \\
\hline 198117 & chr6 & $110,396,540$ & $110,411,065$ & deletion & - & not rare \\
\hline 198117 & chr6 & $66,074,421$ & $66,080,908$ & deletion & - & not rare \\
\hline 198117 & chr6 & $78,972,930$ & $79,035,739$ & deletion & - & not rare \\
\hline 198117 & chr7 & $142,098,342$ & $142,105,433$ & deletion & - & not rare \\
\hline 198117 & chr7 & $38,372,869$ & $38,375,575$ & duplication & - & not rare \\
\hline 198117 & chr7 & $38,388,889$ & $38,402,517$ & deletion & - & not rare \\
\hline
\end{tabular}




\begin{tabular}{|c|c|c|c|c|c|}
\hline 198117 chr7 & 41,421 & 67,820 & duplication & - & not rare \\
\hline 198117 chr7 & $91,033,074$ & $91,041,618$ & deletion & - & not rare \\
\hline 198117 chr8 & $103,054,099$ & $103,056,645$ & deletion & - & not rare \\
\hline 198117 chr8 & $3,786,543$ & $3,790,254$ & deletion & - & not rare \\
\hline 198117 chr8 & $39,232,581$ & $39,385,979$ & deletion & - & not rare \\
\hline 198117 chr9 & $24,502,737$ & $24,518,795$ & deletion & - & not rare \\
\hline 198118 chr1 & $112,693,423$ & $112,704,581$ & deletion & - & not rare \\
\hline 198118 chr1 & $178,659,933$ & $178,676,113$ & deletion & - & not rare \\
\hline 198118 chr1 & $188,047,560$ & $188,136,382$ & deletion & - & rare \\
\hline 198118 chr1 & $213,003,428$ & $213,011,812$ & deletion & SPATA45 & not rare \\
\hline 198118 chr10 & $112,835,590$ & $112,840,171$ & deletion & ADRA2A & not rare \\
\hline 198118 chr10 & $47,541,708$ & $47,703,946$ & duplication & ANTXRL & not rare \\
\hline 198118 chr10 & $71,282,981$ & $71,289,190$ & deletion & - & not rare \\
\hline 198118 chr10 & $89,068,877$ & $89,112,638$ & deletion & - & not rare \\
\hline 198118 chr11 & $104,923,842$ & $104,930,873$ & deletion & - & not rare \\
\hline 198118 chr11 & $24,083,684$ & $24,140,697$ & deletion & - & not rare \\
\hline 198118 chr11 & $25,750,699$ & $25,753,430$ & deletion & - & not rare \\
\hline 198118 chr11 & $43,319,979$ & $43,323,407$ & deletion & - & not rare \\
\hline 198118 chr11 & $55,365,761$ & $55,443,269$ & deletion & \multicolumn{2}{|c|}{$\mathrm{OR} 4 \mathrm{P} 4 ; \mathrm{OR} \angle$ not rare } \\
\hline 198118 chr11 & $91,017,701$ & $91,026,649$ & duplication & - & not rare \\
\hline 198118 chr12 & $11,232,402$ & $11,243,274$ & duplication & - & not rare \\
\hline 198118 chr12 & $126,998,114$ & $127,013,007$ & deletion & - & not rare \\
\hline 198118 chr12 & $27,648,411$ & $27,654,998$ & deletion & SMCO2 & not rare \\
\hline 198118 chr13 & $21,728,134$ & $21,732,193$ & duplication & SKA3 & not rare \\
\hline 198118 chr14 & $41,610,224$ & $41,661,685$ & deletion & & not rare \\
\hline 198118 chr15 & $20,878,391$ & $21,200,107$ & deletion & \multicolumn{2}{|c|}{ POTEB2;PC not rare } \\
\hline 198118 & $24,055,488$ & $24,061,557$ & deletion & - & are \\
\hline 198118 chr15 & $97,815,182$ & $97,831,832$ & deletion & - & not rare \\
\hline 198118 chr16 & $46,471,024$ & $46,492,062$ & deletion & - & not rare \\
\hline 198118 chr16 & $55,800,009$ & $55,819,443$ & deletion & - & not rare \\
\hline 198118 chr16 & $58,945,839$ & $58,948,100$ & deletion & - & not rare \\
\hline 198118 chr16 & $79,631,697$ & $79,635,140$ & deletion & MAF & not rare \\
\hline 198118 chr17 & $10,891,369$ & $10,893,799$ & deletion & - & not rare \\
\hline 198118 chr18 & $63,907,596$ & $63,910,708$ & deletion & - & not rare \\
\hline 198118 chr19 & $54,730,202$ & $54,743,217$ & duplication & LILRA6 & not rare \\
\hline 198118 chr2 & $123,477,263$ & $123,482,301$ & deletion & - & not rare \\
\hline 198118 chr2 & $13,202,216$ & $13,279,945$ & duplication & - & not rare \\
\hline 198118 chr2 & $14,704,369$ & $14,709,611$ & deletion & - & not rare \\
\hline 198118 chr2 & $48,851,541$ & $48,856,770$ & deletion & - & not rare \\
\hline 198118 chr2 & $51,926,599$ & $51,926,904$ & deletion & - & not rare \\
\hline 198118 chr2 & $82,203,525$ & $82,315,179$ & duplication & - & not rare \\
\hline 198118 chr20 & $25,890,697$ & $25,896,449$ & deletion & - & not rare \\
\hline 198118 chr21 & $23,655,900$ & $23,667,066$ & deletion & - & not rare \\
\hline 198118 chr22 & $45,135,790$ & $45,136,558$ & deletion & - & not rare \\
\hline $198118 \mathrm{chr} 4$ & $69,374,369$ & $69,489,473$ & deletion & UGT2B17 & not rare \\
\hline 198118 chr5 & 701,026 & 776,691 & duplication & - & not rare \\
\hline 198118 chr6 & $26,344,333$ & $26,351,593$ & deletion & - & not rare \\
\hline
\end{tabular}




\begin{tabular}{|c|c|c|c|c|c|}
\hline $198118 \mathrm{chr} 6$ & $29,921,001$ & $29,922,138$ & deletion & - & not rare \\
\hline 198118 chr6 & $31,276,760$ & $31,278,472$ & deletion & - & not rare \\
\hline 198118 chr6 & $48,662,078$ & $48,685,078$ & duplication & - & rare \\
\hline 198118 chr6 & 665,448 & 670,182 & deletion & - & not rare \\
\hline 198118 chr6 & 979,803 & 985,196 & deletion & - & not rare \\
\hline 198118 chr7 & $141,766,850$ & $141,793,419$ & deletion & - & not rare \\
\hline 198118 chr7 & $155,882,711$ & $155,885,143$ & deletion & - & not rare \\
\hline 198118 chr7 & $97,396,709$ & $97,401,677$ & deletion & - & not rare \\
\hline 198118 chr8 & $115,634,278$ & $115,642,459$ & deletion & - & not rare \\
\hline 198118 chr8 & $39,232,581$ & $39,385,979$ & duplication & - & not rare \\
\hline 198119 chr1 & $112,693,423$ & $112,704,581$ & deletion & - & not rare \\
\hline 198119 chr1 & $12,908,591$ & $12,920,067$ & deletion & PRAMEF2 & not rare \\
\hline 198119 chr1 & $143,481,684$ & $143,504,049$ & deletion & - & not rare \\
\hline 198119 chr1 & $161,570,803$ & $161,619,741$ & deletion & FCGR3B & not rare \\
\hline 198119 chr1 & $174,797,867$ & $174,801,639$ & deletion & - & not rare \\
\hline 198119 chr1 & $188,047,560$ & $188,136,382$ & deletion & - & rare \\
\hline 198119 chr10 & $71,282,981$ & $71,289,190$ & deletion & - & not rare \\
\hline 198119 chr10 & 94,316 & 135,853 & duplication & TUBB8 & rare \\
\hline 198119 chr11 & $134,700,936$ & $134,777,616$ & deletion & - & rare \\
\hline 198119 chr11 & $24,083,684$ & $24,140,697$ & deletion & - & not rare \\
\hline 198119 chr11 & $25,750,699$ & $25,753,430$ & deletion & - & not rare \\
\hline 198119 & $43,319,979$ & $43,323,407$ & dele & - & ot rare \\
\hline 198119 chr11 & $92,178,278$ & $92,188,498$ & deletion & . & not rare \\
\hline 198119 chr11 & $95,979,334$ & $95,981,067$ & deletion & & not rare \\
\hline 198119 chr12 & $126,998,114$ & $127,013,007$ & deletion & & not rare \\
\hline 198119 chr12 & $27,648,411$ & $27,654,998$ & deletion & SMCO2 & not rare \\
\hline 198119 chr12 & $32,331,195$ & $32,334,813$ & deletion & - & not rare \\
\hline 198119 chr14 & $35,604,892$ & $35,613,500$ & deletion & - & not rare \\
\hline 198119 chr14 & $41,610,224$ & $41,661,685$ & deletion & - & not rare \\
\hline 198119 chr15 & $22,393,248$ & $22,512,696$ & deletion & - & not rare \\
\hline 198119 chr16 & $32,542,736$ & $32,642,384$ & deletion & - & not rare \\
\hline 198119 chr16 & $33,519,862$ & $33,555,310$ & deletion & - & not rare \\
\hline 198119 chr16 & $46,471,024$ & $46,492,062$ & deletion & - & not rare \\
\hline 198119 chr16 & $76,664,462$ & $76,670,907$ & deletion & - & not rare \\
\hline 198119 chr17 & $54,160,465$ & $54,172,668$ & deletion & - & not rare \\
\hline 198119 chr18 & $41,976,831$ & $41,982,102$ & deletion & - & not rare \\
\hline 198119 chr18 & $63,907,596$ & $63,910,708$ & deletion & - & not rare \\
\hline 198119 chr19 & $54,730,202$ & $54,743,217$ & deletion & & not rare \\
\hline 198119 chr2 & $14,704,369$ & $14,709,611$ & deletion & - & not rare \\
\hline 198119 chr2 & $214,989,439$ & $214,992,692$ & deletion & - & not rare \\
\hline 198119 chr2 & $233,214,566$ & $233,219,891$ & deletion & - & not rare \\
\hline 198119 chr20 & $25,890,697$ & $25,896,760$ & deletion & - & not rare \\
\hline 198119 chr20 & $29,566,903$ & $29,585,801$ & deletion & - & not rare \\
\hline 198119 chr20 & $40,809,970$ & $40,820,457$ & deletion & - & not rare \\
\hline 198119 chr21 & $23,655,900$ & $23,667,066$ & deletion & - & not rare \\
\hline 198119 chr3 & $160,680,773$ & $160,685,788$ & deletion & - & not rare \\
\hline $198119 \mathrm{chr} 4$ & $69,374,369$ & $69,489,473$ & deletion & UGT2B17 & not rare \\
\hline
\end{tabular}




\begin{tabular}{|c|c|c|c|c|c|}
\hline $198119 \mathrm{chr} 5$ & $140,225,908$ & 140, & deletion & \multicolumn{2}{|c|}{ PCDHA10; F not rare } \\
\hline 198119 chr5 & $177,390,271$ & $177,400,704$ & deletion & - & not rare \\
\hline 198119 chr5 & $180,285,913$ & $180,488,297$ & duplication & \multicolumn{2}{|c|}{ BTNL9;BTN rare } \\
\hline 198119 chr6 & $29,921,001$ & $29,921,927$ & deletion & - & not rare \\
\hline 198119 chr6 & $31,337,850$ & $31,341,379$ & deletion & - & not rare \\
\hline 198119 chr6 & $66,074,421$ & $66,080,908$ & deletion & - & not rare \\
\hline 198119 chr6 & 665,448 & 670,182 & deletion & - & not rare \\
\hline 198119 chr6 & 979,803 & 985,196 & deletion & - & not rare \\
\hline $198119 \mathrm{chr} 7$ & $11,920,873$ & $11,922,312$ & deletion & - & not rare \\
\hline $198119 \mathrm{chr} 7$ & $155,882,711$ & $155,885,143$ & deletion & - & not rare \\
\hline $198119 \mathrm{chr} 7$ & 41,421 & 68,920 & duplication & - & not rare \\
\hline 198119 chr7 & $97,396,709$ & $97,401,677$ & deletion & - & not rare \\
\hline 198119 chr8 & $115,634,278$ & $115,642,459$ & deletion & - & not rare \\
\hline 198119 chr8 & $39,232,581$ & $39,385,979$ & duplication & - & not rare \\
\hline 198119 chr8 & $40,184,872$ & $40,189,688$ & deletion & - & not rare \\
\hline 198119 chr8 & $56,379,772$ & $56,388,749$ & deletion & - & not rare \\
\hline $198119 \mathrm{chr} 8$ & $58,359,519$ & $58,366,958$ & deletion & - & not rare \\
\hline 198119 chr8 & $65,245,116$ & $65,256,049$ & deletion & - & not rare \\
\hline $198120 \mathrm{chr} 1$ & $108,734,695$ & $108,737,586$ & ion & $A 24$ & not rare \\
\hline 198120 chr1 & $112,693,423$ & $112,704,581$ & deletion & - & not rare \\
\hline $198120 \mathrm{chr} 1$ & $12,908,591$ & $12,919,928$ & deletion & PRAMEF2 & not rare \\
\hline $198120 \mathrm{chr} 1$ & $186,784,140$ & $186,788,264$ & dele & - & not rare \\
\hline 198120 chr1 & $188,047,560$ & $188,136,382$ & dele & & rare \\
\hline $198120 \mathrm{chr} 1$ & $8,360,487$ & $8,363,575$ & del & & not rare \\
\hline 198120 chr10 & $71,282,981$ & $71,289,190$ & dele & & not rare \\
\hline 198120 chr11 & $43,319,979$ & $43,323,407$ & deletion & - & not rare \\
\hline 198120 & $55,365,761$ & $55,443,269$ & deletion & \multicolumn{2}{|c|}{ OR4P4;OR $\angle$ not rare } \\
\hline 198120 chr11 & $92,178,278$ & $92,188,498$ & deletion & - & not rare \\
\hline 198120 chr11 & $95,979,334$ & $95,981,067$ & deletion & - & not rare \\
\hline 198120 chr12 & $32,331,195$ & $32,334,813$ & deletion & - & not rare \\
\hline 198120 chr12 & $70,875,808$ & $70,877,258$ & deletion & - & not rare \\
\hline 198120 chr13 & $69,247,022$ & $69,267,981$ & deletion & - & not rare \\
\hline 198120 chr14 & $28,669,164$ & $28,672,334$ & deletion & - & not rare \\
\hline 198120 chr14 & $35,116,289$ & $35,122,186$ & deletion & - & not rare \\
\hline 198120 & $20,120,214$ & $20,145,193$ & deletion & - & not rare \\
\hline 198120 chr16 & $46,471,024$ & $46,492,062$ & deletion & - & not rare \\
\hline $198120 \mathrm{chr} 16$ & $58,945,839$ & $58,948,100$ & deletion & - & not rare \\
\hline 198120 chr16 & $72,094,348$ & $72,108,093$ & duplication & HP & not rare \\
\hline 198120 chr17 & $34,438,753$ & $34,480,718$ & duplication & - & not rare \\
\hline 198120 chr18 & $63,907,596$ & $63,910,708$ & deletion & - & not rare \\
\hline 198120 chr18 & $66,746,208$ & $66,755,736$ & deletion & - & not rare \\
\hline 198120 chr19 & $43,703,965$ & $43,759,382$ & deletion & \multicolumn{2}{|c|}{ PSG4;PSG9 not rare } \\
\hline $198120 \mathrm{chr} 2$ & $13,202,216$ & $13,279,945$ & duplication & - & not rare \\
\hline $198120 \mathrm{chr} 2$ & $151,150,611$ & $151,172,764$ & deletion & - & rare \\
\hline $198120 \mathrm{chr} 2$ & $51,926,599$ & $51,926,904$ & deletion & - & not rare \\
\hline 198120 chr21 & $10,810,892$ & $10,813,820$ & deletion & - & not rare \\
\hline 198120 chr22 & $42,526,710$ & $42,537,623$ & duplication & CYP2D6 & not rare \\
\hline
\end{tabular}




\begin{tabular}{|c|c|c|c|c|c|}
\hline 198120 chr22 & $45,135,790$ & $45,136,558$ & deletion & - & not rare \\
\hline 198120 chr3 & $131,711,896$ & $131,712,898$ & deletion & - & not rare \\
\hline $198120 \mathrm{chr} 3$ & $160,680,773$ & $160,685,788$ & deletion & - & not rare \\
\hline 98120 chr3 & $1,917,909$ & $1,922,565$ & deletion & - & not rare \\
\hline 198120 chr3 & $46,798,133$ & $46,845,618$ & deletion & - & not rare \\
\hline 198120 chr3 & $89,402,409$ & $89,418,331$ & deletion & - & not rare \\
\hline 198120 chr3 & $9,637,451$ & $9,649,470$ & deletion & - & not rare \\
\hline $198120 \mathrm{chr} 4$ & $58,256,666$ & $58,261,728$ & deletion & - & not rare \\
\hline $198120 \mathrm{chr} 4$ & $60,325,242$ & $60,330,880$ & duplication & - & not rare \\
\hline $198120 \mathrm{chr} 4$ & $64,697,704$ & $64,709,753$ & duplication & - & not rare \\
\hline $198120 \mathrm{chr} 4$ & $69,374,369$ & $69,489,473$ & deletion & UGT2B17 & not rare \\
\hline 198120 chr5 & 682,606 & 776,691 & duplication & - & not rare \\
\hline 198120 chr6 & $29,921,001$ & $29,922,138$ & deletion & - & not rare \\
\hline 198120 chr6 & 665,448 & 670,182 & deletion & - & not rare \\
\hline 198120 chr6 & 979,803 & 985,196 & deletion & - & not rare \\
\hline 198120 chr7 & $61,796,377$ & $61,804,744$ & deletion & - & not rare \\
\hline 198120 chr7 & $62,285,495$ & $62,306,851$ & duplication & - & not rare \\
\hline 198120 chr8 & $18,612,008$ & $18,615,397$ & deletion & - & not rare \\
\hline 198120 chr8 & $39,232,581$ & $39,385,979$ & duplication & - & not rare \\
\hline 198120 chr8 & $67,186,387$ & $67,188,888$ & deletion & - & not rare \\
\hline 198120 chr9 & $23,290,141$ & $23,299,151$ & deletion & - & not rare \\
\hline $198121 \mathrm{chr} 1$ & $104,153,766$ & $104,211,046$ & & \multicolumn{2}{|c|}{ AMY1A;AN not rare } \\
\hline 198121 chr1 & $16,568,271$ & $16,593,404$ & duplication & FBX042 & not rare \\
\hline 198121 chr1 & $17,224,089$ & $17,229,007$ & dele & - & not rare \\
\hline 198121 chr1 & $248,740,314$ & $248,795,110$ & deletion & \multicolumn{2}{|c|}{ OR2T10;OF not rare } \\
\hline $198121 \mathrm{chr} 1$ & $9,847,290$ & $9,855,529$ & deletion & - & not rare \\
\hline 198121 chr10 & $39,119,741$ & $39,149,666$ & duplication & - & not rare \\
\hline 198121 chr10 & $58,845,646$ & $58,849,853$ & deletion & - & not rare \\
\hline 198121 chr11 & $55,365,761$ & $55,443,269$ & deletion & \multicolumn{2}{|c|}{ OR4P4;OR $\angle$ not rare } \\
\hline 198121 chr12 & $31,266,287$ & $31,407,303$ & duplication & - & not rare \\
\hline 198121 chr12 & $70,513,571$ & $70,517,836$ & duplication & - & not rare \\
\hline 198121 chr13 & $97,499,278$ & $97,513,234$ & deletion & - & not rare \\
\hline 198121 chr14 & $20,341,645$ & $20,383,323$ & duplication & OR4K2 & not rare \\
\hline 198121 chr15 & $20,120,214$ & $20,145,193$ & deletion & - & not rare \\
\hline 198121 chr15 & $24,055,488$ & $24,061,557$ & deletion & - & not rare \\
\hline 198121 chr15 & $34,730,758$ & $34,860,040$ & duplication & GOLGA8B & not rare \\
\hline 198121 chr15 & $60,086,249$ & $60,103,464$ & deletion & - & not rare \\
\hline 198121 chr16 & $34,178,980$ & $34,189,937$ & deletion & - & not rare \\
\hline 198121 chr16 & $46,471,024$ & $46,492,062$ & deletion & - & not rare \\
\hline 198121 chr16 & $60,082,237$ & $60,098,544$ & deletion & - & not rare \\
\hline 198121 chr16 & $70,016,280$ & $70,141,115$ & duplication & - & not rare \\
\hline 198121 chr19 & $43,456,765$ & $43,539,189$ & deletion & PSG11 & not rare \\
\hline 198121 chr19 & $43,703,965$ & $43,759,382$ & deletion & \multicolumn{2}{|c|}{ PSG4;PSG9 not rare } \\
\hline 198121 chr2 & $57,399,830$ & $57,446,412$ & duplication & - & not rare \\
\hline 198121 chr2 & $6,591,865$ & $6,596,646$ & deletion & - & not rare \\
\hline 198121 chr20 & $29,566,903$ & $29,585,801$ & deletion & - & not rare \\
\hline 198121 chr20 & $29,627,973$ & $29,629,342$ & deletion & - & not rare \\
\hline
\end{tabular}




\begin{tabular}{|c|c|c|c|c|c|}
\hline 198121 chr20 & $7,961,715$ & $7,965,595$ & deletion & TMX4 & not rare \\
\hline 198121 chr21 & $20,057,919$ & $20,078,801$ & duplication & - & not rare \\
\hline 98121 chr22 & $39,359,885$ & $39,385,663$ & deletion & АРОВЕСЗВ & rare \\
\hline 198121 chr3 & $22,279,685$ & $22,286,262$ & deletion & - & not rare \\
\hline $198121 \mathrm{chr} 4$ & $59,980,062$ & $59,988,095$ & duplication & - & not rare \\
\hline 198121 chr6 & $150,412,539$ & $150,426,272$ & deletion & - & ot rare \\
\hline 198121 chr6 & $29,859,135$ & $29,899,389$ & deletion & - & not rare \\
\hline 198121 chr7 & $156,387,296$ & $156,394,307$ & deletion & - & not rare \\
\hline 198121 chr8 & $39,232,581$ & $39,385,979$ & deletion & - & not rare \\
\hline 198121 chr8 & $92,110,936$ & $92,185,168$ & deletion & LRRC69 & not rare \\
\hline 198121 chr9 & $69,494,085$ & $69,881,429$ & deletion & - & not rare \\
\hline 198125 chr1 & $12,850,282$ & $12,891,503$ & deletion & PRAMEF1;F & rare \\
\hline 198125 chr1 & $152,526,812$ & $152,568,230$ & deletion & LCE3D;LCE: & not rare \\
\hline 198125 chr1 & $161,570,803$ & $161,619,741$ & deletion & FCGR3B & not rare \\
\hline 198125 chr1 & $187,717,153$ & $187,722,032$ & deletion & - & not rare \\
\hline 198125 chr1 & $8,360,487$ & $8,363,575$ & deletion & - & not rare \\
\hline 198125 chr10 & $107,125,649$ & $107,127,799$ & deletion & - & not rare \\
\hline 198125 chr10 & $54,930,114$ & $54,937,235$ & deletion & - & not rare \\
\hline 198125 chr10 & $58,327,709$ & $58,482,756$ & & - & are \\
\hline 198125 chr11 & $106,398,020$ & $106,404,608$ & deletion & - & not rare \\
\hline 198125 chr11 & $107,653,650$ & $107,671,155$ & duplication & SLC35F2 & not rare \\
\hline 198125 chr11 & $55,365,761$ & $55,443,269$ & deletion & OR4P4;O & not rare \\
\hline 198125 chr11 & $7,814,299$ & $7,827,879$ & deletion & & not rare \\
\hline 198125 chr12 & $30,237,351$ & $30,243,473$ & & & are \\
\hline 198125 chr12 & $31,907,259$ & $31,909,635$ & dele & & not rare \\
\hline 198125 chr12 & $94,197,679$ & $94,202,652$ & deletion & - & not rare \\
\hline 198125 chr13 & $21,728,134$ & $21,732,193$ & ation & $\mathrm{Sk}$ & are \\
\hline 198125 chr14 & $45,822,243$ & $46,008,210$ & deletion & - & not rare \\
\hline 198125 chr15 & $34,874,651$ & $34,884,471$ & duplication & - & not rare \\
\hline 198125 chr17 & $15,044,804$ & $15,058,236$ & deletion & - & not rare \\
\hline 198125 chr17 & $74,652,274$ & $74,658,084$ & deletion & - & not rare \\
\hline 198125 chr18 & $63,907,596$ & $63,910,708$ & deletion & - & not rare \\
\hline 198125 chr2 & $23,234,798$ & $23,238,601$ & deletion & - & not rare \\
\hline 198125 chr2 & $71,345,404$ & $71,347,759$ & deletion & - & not rare \\
\hline 198125 chr2 & $79,752,148$ & $79,761,222$ & deletion & - & not rare \\
\hline 198125 chr21 & $23,655,900$ & $23,667,066$ & deletion & - & not rare \\
\hline 198125 chr22 & $39,359,885$ & $39,385,663$ & deletion & АРОВЕСЗВ & not rare \\
\hline 198125 chr3 & $13,681,185$ & $13,682,137$ & deletion & - & not rare \\
\hline 198125 chr3 & $148,964,692$ & $148,969,220$ & deletion & - & not rare \\
\hline 198125 chr3 & $155,479,723$ & $155,505,597$ & duplication & $\mathrm{C} 3 \mathrm{c}$ & not rare \\
\hline 198125 chr3 & $173,239,453$ & $173,290,024$ & duplication & - & not rare \\
\hline 198125 chr3 & $191,989,376$ & $191,996,202$ & duplication & - & not rare \\
\hline 198125 chr3 & $195,458,723$ & $195,467,820$ & duplication & MUC20 & not rare \\
\hline 198125 chr5 & $70,305,696$ & $70,308,251$ & duplication & NAIP & not rare \\
\hline 198125 chr5 & $98,552,170$ & $98,560,100$ & duplication & - & not rare \\
\hline 198125 chr6 & $165,129,935$ & $165,194,584$ & duplication & - & not rare \\
\hline 198125 chr6 & $29,819,280$ & $29,819,741$ & deletion & - & not rare \\
\hline
\end{tabular}




\begin{tabular}{|c|c|c|c|c|c|}
\hline 198125 chr6 & $29,921,001$ & $29,921,927$ & deletion & - & not rare \\
\hline 198125 chr7 & $142,476,094$ & $142,487,836$ & deletion & - & not rare \\
\hline 198125 chr7 & $155,882,711$ & $155,885,143$ & deletion & - & not rare \\
\hline 98125 chr7 & $20,750,774$ & $20,753,528$ & deletion & - & not rare \\
\hline 198125 chr7 & $52,730,700$ & $52,744,420$ & duplication & - & not rare \\
\hline 198125 chr7 & $86,940,652$ & $86,948,364$ & deletion & - & not rare \\
\hline 198125 chr7 & $97,396,709$ & $97,401,677$ & duplication & - & not rare \\
\hline 198125 chr8 & $13,615,303$ & $13,650,690$ & deletion & - & not rare \\
\hline 198125 chr8 & $24,972,808$ & $24,990,418$ & duplication & - & not rare \\
\hline 198125 chr8 & $39,232,581$ & $39,385,979$ & deletion & - & not rare \\
\hline 198125 chr9 & $6,701,130$ & $6,707,794$ & deletion & - & not rare \\
\hline 198126 chr1 & $149,024,218$ & $149,237,009$ & deletion & - & not rare \\
\hline 198126 chr1 & $161,570,803$ & $161,619,741$ & deletion & FCGR3B & not rare \\
\hline 198126 chr1 & $248,507,806$ & $248,689,794$ & duplication & OR2G6;OR & \\
\hline 198126 chr1 & $248,796,402$ & $248,881,991$ & duplication & OR2T27;OF & rare \\
\hline 198126 chr10 & $82,937,618$ & $82,945,572$ & deletion & - & not rare \\
\hline 198126 chr11 & $18,949,220$ & $18,953,876$ & duplication & - & not rare \\
\hline 198126 chr12 & $11,220,827$ & $11,244,117$ & deletion & 243 & not rare \\
\hline 198126 chr12 & $24,099,479$ & $24,100,692$ & deletion & - & not rare \\
\hline 198126 chr12 & $32,004,170$ & $32,061,988$ & duplication & - & not rare \\
\hline 198126 chr12 & $53,086,532$ & $53,087,449$ & deletion & & not rare \\
\hline 198126 & $106,134,981$ & $106,198,034$ & & - & not rare \\
\hline 198126 chr14 & $86,285,493$ & $86,312,888$ & deletion & . & not rare \\
\hline 198126 chr15 & $56,789,979$ & $56,800,635$ & dele & & not rare \\
\hline 198126 chr16 & 78,3 & $78,383,509$ & deletion & & \\
\hline 198126 chr18 & $14,549,799$ & $14,550,375$ & deletion & 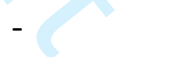 & not rare \\
\hline 198126 chr18 & $33,350,239$ & $33,361,604$ & deletion & - & not rare \\
\hline 198126 chr19 & $41,362,173$ & $41,387,154$ & duplication & C) & not rare \\
\hline 198126 chr2 & $34,697,895$ & $34,726,904$ & duplication & - & not rare \\
\hline 198126 chr2 & $38,956,947$ & $38,971,623$ & duplication & & not rare \\
\hline 198126 chr2 & $49,534,329$ & $49,538,143$ & deletion & - & not rare \\
\hline $198126 \mathrm{chr} 2$ & $90,065,400$ & $90,275,455$ & duplication & - & not rare \\
\hline 198126 chr20 & $14,781,684$ & $14,823,472$ & deletion & - & not rare \\
\hline 198126 chr20 & $52,647,631$ & $52,656,535$ & deletion & - & not rare \\
\hline 198126 chr21 & $23,655,900$ & $23,667,066$ & deletion & - & not rare \\
\hline 198126 chr3 & $152,347,482$ & $152,355,226$ & deletion & - & not rare \\
\hline 198126 chr3 & $186,386,674$ & $186,425,186$ & deletion & HRG & not rare \\
\hline 198126 chr3 & $46,798,133$ & $46,845,618$ & deletion & - & not rare \\
\hline $198126 \mathrm{chr} 3$ & $75,419,736$ & $75,703,113$ & deletion & - & not rare \\
\hline $198126 \mathrm{chr} 3$ & $98,945,571$ & $98,947,547$ & deletion & - & not rare \\
\hline $198126 \mathrm{chr} 4$ & $108,064,023$ & $108,074,123$ & deletion & - & not rare \\
\hline $198126 \mathrm{chr} 4$ & $122,285,263$ & $122,289,863$ & deletion & - & not rare \\
\hline $198126 \mathrm{chr} 4$ & $42,697,553$ & $42,708,694$ & deletion & - & not rare \\
\hline $198126 \mathrm{chr} 4$ & $60,325,242$ & $60,330,880$ & duplication & - & not rare \\
\hline $198126 \mathrm{chr} 4$ & $69,374,369$ & $69,489,473$ & deletion & UGT2B17 & not rare \\
\hline 198126 & $140,225,908$ & $140,238,311$ & deletion & \multicolumn{2}{|c|}{ PCDHA10; F not rare } \\
\hline 198126 chr5 & $70,305,696$ & $70,308,251$ & duplication & NAIP & not rare \\
\hline
\end{tabular}




\begin{tabular}{|c|c|c|c|c|c|}
\hline 198126 chr5 & 788,646 & 824,172 & deletion & C11 & not rare \\
\hline 198126 chr6 & $31,281,682$ & $31,286,577$ & deletion & - & not rare \\
\hline 98126 chr6 & $69,687,698$ & $69,690,567$ & deletion & - & not rare \\
\hline 98126 chr6 & $84,229,114$ & $84,231,999$ & deletion & - & not rare \\
\hline 198126 chr7 & $141,766,850$ & $141,793,419$ & deletion & - & not rare \\
\hline 198126 chr7 & $153,931,637$ & $153,946,793$ & deletion & - & not rare \\
\hline 198126 chr7 & $155,882,711$ & $155,885,143$ & deletion & - & not rare \\
\hline 198126 chr8 & $117,277,011$ & $117,305,398$ & deletion & - & rare \\
\hline 198126 chr8 & $39,232,581$ & $39,385,979$ & duplication & - & not rare \\
\hline 198126 chr9 & $135,949,324$ & $135,956,640$ & duplication & - & not rare \\
\hline 198126 chr9 & $2,148,213$ & $2,151,397$ & deletion & - & trare \\
\hline $198126 \mathrm{chr} X$ & $62,472,314$ & $62,497,551$ & duplication & - & not rare \\
\hline 198128 chr1 & $187,717,153$ & $187,722,032$ & deletion & - & not rare \\
\hline 198128 chr10 & $58,516,470$ & $58,526,153$ & deletion & - & not rare \\
\hline 198128 chr10 & $71,282,981$ & $71,289,190$ & deletion & - & not rare \\
\hline 198128 chr10 & $90,940,413$ & $90,945,998$ & deletion & - & not rare \\
\hline 198128 chr11 & $3,239,988$ & $3,243,665$ & deletion & RG & not rare \\
\hline 198128 chr11 & $49,715,154$ & $49,757,690$ & deletion & - & not rare \\
\hline 198128 & $55,365,761$ & $55,443,269$ & deletion & \multicolumn{2}{|c|}{$\mathrm{OR} 4 \mathrm{P} 4 ; \mathrm{OR} \angle$ not rare } \\
\hline 198128 chr11 & $81,501,218$ & $81,517,261$ & deletion & - & not rare \\
\hline 198128 chr12 & $11,220,827$ & $11,244,117$ & deletion & TAS2R43 & not rare \\
\hline 198128 chr12 & $43,021,431$ & $43,026,485$ & deletion & - & not rare \\
\hline 198128 chr12 & $99,994,315$ & $100,008,773$ & dele & & not rare \\
\hline 198128 chr13 & $78,806,521$ & $78,807,439$ & de & & not rare \\
\hline 198128 chr14 & $41,610,224$ & $41,661,685$ & deletion & & not rare \\
\hline 198128 chr15 & $87,607,311$ & $87,613,895$ & deletion & & not rare \\
\hline 198128 & $3,215,104$ & $3,245,872$ & & - & are \\
\hline 198128 chr16 & $34,471,298$ & $34,756,258$ & duplication & - & not rare \\
\hline 198128 chr16 & $89,314,821$ & $89,321,238$ & duplication & - & not rare \\
\hline 198128 chr17 & $34,438,753$ & $34,480,718$ & duplication & - & not rare \\
\hline 198128 chr17 & $7,264,717$ & $7,265,681$ & deletion & - & not rare \\
\hline 198128 chr18 & $1,905,525$ & $1,982,551$ & deletion & - & not rare \\
\hline 198128 chr19 & $24,460,170$ & $24,462,326$ & deletion & - & not rare \\
\hline 198128 chr19 & $43,647,130$ & $43,744,060$ & deletion & \multicolumn{2}{|c|}{ PSG4;PSG5 not rare } \\
\hline 198128 chr2 & $233,446,568$ & $233,482,060$ & duplication & & rare \\
\hline 198128 chr2 & $66,188,209$ & $66,196,726$ & deletion & - & not rare \\
\hline 198128 chr21 & $11,055,462$ & $11,063,273$ & deletion & \multicolumn{2}{|c|}{ BAGE2;BAC not rare } \\
\hline 198128 chr21 & $23,655,900$ & $23,667,066$ & deletion & - & not rare \\
\hline 198128 chr22 & $18,844,632$ & $19,023,133$ & duplication & \multicolumn{2}{|c|}{ PRODH;DG not rare } \\
\hline 198128 chr22 & $51,068,560$ & $51,078,251$ & duplication & - & not rare \\
\hline 198128 chr3 & $128,382,258$ & $128,412,024$ & duplication & - & not rare \\
\hline $198128 \mathrm{chr} 3$ & $129,769,827$ & $129,798,600$ & deletion & - & not rare \\
\hline $198128 \mathrm{chr} 4$ & $10,396,298$ & $10,400,156$ & deletion & - & not rare \\
\hline $198128 \mathrm{chr} 4$ & $123,003,141$ & $123,046,521$ & deletion & - & rare \\
\hline $198128 \mathrm{chr} 4$ & $54,596,025$ & $54,603,082$ & deletion & - & not rare \\
\hline $198128 \mathrm{chr} 4$ & $69,374,369$ & $69,489,473$ & deletion & 317 & not rare \\
\hline 198128 chr5 & $17,623,605$ & $17,642,516$ & deletion & - & not rare \\
\hline
\end{tabular}




\begin{tabular}{|c|c|c|c|c|c|}
\hline $198128 \mathrm{chr} 5$ & 788,646 & 824,172 & deletion & ZDHHC11 & not rare \\
\hline 198128 chr6 & $78,972,930$ & $79,035,739$ & deletion & - & not rare \\
\hline 98128 chr6 & $79,687,346$ & $79,776,936$ & duplication & PHIP & rare \\
\hline 98128 chr7 & $112,340,114$ & $112,379,258$ & deletion & - & rare \\
\hline $198128 \mathrm{chr} 7$ & $20,750,774$ & $20,753,528$ & deletion & - & not rare \\
\hline 198128 chr7 & $38,388,889$ & $38,402,517$ & deletion & - & not rare \\
\hline 198128 chr7 & $75,664,539$ & $75,667,743$ & deletion & - & not rare \\
\hline 198128 chr8 & $39,232,581$ & $39,385,979$ & duplication & - & not rare \\
\hline 198128 chr8 & $51,031,221$ & $51,038,149$ & deletion & - & not rare \\
\hline 198128 chr9 & $12,359,235$ & $12,367,988$ & deletion & - & not rare \\
\hline 198128 chr9 & $139,668,443$ & $139,676,734$ & deletion & - & t rare \\
\hline 203888 & $149,022,110$ & $149,237,009$ & deletion & - & not rare \\
\hline 203888 chr1 & $152,526,812$ & $152,552,461$ & deletion & \multicolumn{2}{|c|}{ LCE3D;LCE: not rare } \\
\hline 203888 chr1 & $62,113,597$ & $62,119,136$ & deletion & - & not rare \\
\hline 203888 chr1 & $9,847,290$ & $9,856,826$ & deletion & - & not rare \\
\hline 203888 chr10 & $71,282,981$ & $71,289,190$ & deletion & - & not rare \\
\hline 203888 chr11 & $55,365,761$ & $55,443,269$ & deletion & \multicolumn{2}{|c|}{$\mathrm{OR} 4 \mathrm{P} 4 ; \mathrm{OR} \angle$ not rare } \\
\hline 203888 chr11 & $86,304,402$ & $86,306,401$ & deletion & - & not rare \\
\hline 203888 & $12,532,984$ & $12,541,869$ & deletion & - & t rare \\
\hline 203888 chr12 & $129,230,513$ & $129,233,013$ & deletion & - & not rare \\
\hline 203888 chr13 & $112,720,332$ & $112,730,210$ & deletion & SOX1 & not rare \\
\hline 203888 chr13 & $34,134,809$ & $34,143,545$ & dele & - & not rare \\
\hline 203888 chr14 & $106,575,614$ & $106,776,739$ & $d \epsilon$ & & not rare \\
\hline 203888 chr15 & $20,120,214$ & $20,144,522$ & & & ot rare \\
\hline 203888 chr15 & $24,055,488$ & $24,061,557$ & dele & & not rare \\
\hline 203888 chr15 & $60,086,249$ & $60,103,464$ & dele & & not rare \\
\hline 203888 & $32,542,736$ & $32,642,384$ & & - & not rare \\
\hline 203888 chr16 & 576,495 & 630,902 & deletion & \multicolumn{2}{|c|}{ PIGQ;CAPN rare } \\
\hline 203888 chr16 & 681,071 & 783,865 & deletion & \multicolumn{2}{|c|}{ WDR90;WF rare } \\
\hline 203888 chr16 & $72,246,084$ & $72,252,752$ & deletion & - & not rare \\
\hline 203888 chr17 & $10,891,369$ & $10,893,799$ & deletion & - & not rare \\
\hline 203888 & $34,438,753$ & $34,481,492$ & duplication & - & not rare \\
\hline 203888 chr19 & $43,352,823$ & $43,520,965$ & duplication & \multicolumn{2}{|c|}{ PSG6;PSG7 not rare } \\
\hline 203888 chr19 & $9,275,282$ & $9,282,176$ & deletion & - & not rare \\
\hline 203888 chr2 & $214,989,439$ & $214,992,692$ & deletion & - & not rare \\
\hline 203888 chr2 & $52,032,381$ & $52,077,000$ & deletion & - & rare \\
\hline 203888 chr2 & $54,479,117$ & $54,481,636$ & deletion & - & not rare \\
\hline 203888 chr2 & $97,738,917$ & $98,024,837$ & deletion & \multicolumn{2}{|c|}{ FAHD2B;Ar not rare } \\
\hline 203888 chr20 & $52,647,631$ & $52,656,535$ & deletion & - & not rare \\
\hline 203888 chr22 & $25,663,994$ & $25,919,492$ & duplication & LRP5L & not rare \\
\hline $203888 \mathrm{chr} 4$ & $34,785,955$ & $34,815,308$ & deletion & - & not rare \\
\hline $203888 \mathrm{chr} 4$ & $64,697,704$ & $64,709,753$ & deletion & - & not rare \\
\hline $203888 \mathrm{chr} 4$ & $95,370,805$ & $95,386,465$ & deletion & PDLIM5 & not rare \\
\hline 203888 chr4 & 982,347 & 999,255 & deletion & \multicolumn{2}{|c|}{ SLC26A1; I[ not rare } \\
\hline 203888 chr5 & $155,875,529$ & $155,880,332$ & deletion & - & not rare \\
\hline 203888 chr5 & $179,227,865$ & $179,238,794$ & deletion & MGAT4B & not rare \\
\hline 203888 chr5 & $70,305,696$ & $70,308,251$ & duplication & NAIP & not rare \\
\hline
\end{tabular}




\begin{tabular}{|c|c|c|c|c|c|}
\hline 03888 chr6 & $142,133,656$ & $142,236,407$ & deletion & - & not rare \\
\hline 03888 chr6 & $29,859,135$ & $29,899,389$ & deletion & - & not rare \\
\hline 3888 chr6 & $30,994,010$ & $30,994,787$ & deletion & MUC22 & ot rare \\
\hline 3888 chr6 & $31,281,682$ & $31,286,577$ & deletion & - & not rare \\
\hline 03888 chr6 & $47,064,147$ & $47,070,766$ & deletion & - & ot rare \\
\hline 03888 chr6 & $66,071,980$ & $66,080,908$ & deletion & - & t rare \\
\hline 03888 chr6 & $77,439,769$ & $77,451,301$ & deletion & - & not rare \\
\hline 03888 chr7 & $15,154,324$ & $15,159,059$ & deletion & - & not rare \\
\hline $03888 \mathrm{chr} 7$ & $156,387,296$ & $156,394,307$ & deletion & - & not rare \\
\hline 3888 chr7 & $38,388,889$ & $38,402,517$ & deletion & - & not rare \\
\hline 03888 chr8 & $115,634,278$ & $115,642,459$ & deletion & - & not rare \\
\hline 03888 chr8 & $144,993,943$ & $144,997,356$ & deletion & PLEC & not rare \\
\hline $03888 \mathrm{chr} 8$ & $145,100,105$ & $145,120,635$ & deletion & \multicolumn{2}{|c|}{ SPATC1;OP rare } \\
\hline 203888 chr9 & $23,290,141$ & $23,299,151$ & deletion & - & not rare \\
\hline 203888 chr9 & $43,601,888$ & $44,087,860$ & deletion & \multicolumn{2}{|c|}{ CNTNAP3B not rare } \\
\hline 203888 chr9 & $69,494,085$ & $69,881,429$ & deletion & - & not rare \\
\hline 203889 chr1 & $104,153,766$ & $104,211,046$ & deletion & \multicolumn{2}{|c|}{ AMY1A;AN not rare } \\
\hline $203889 \mathrm{chr} 1$ & $248,740,572$ & $248,795,110$ & deletion & \multicolumn{2}{|c|}{ OR2T10;OF not rare } \\
\hline 203889 & $39,093,215$ & $39,127,750$ & deletion & - & \\
\hline 203889 chr11 & $42,288,274$ & $42,327,777$ & deletion & - & not rare \\
\hline 203889 chr11 & $7,814,299$ & $7,827,879$ & deletion & OR5P2 & not rare \\
\hline 203889 & 31,9 & $31,909,635$ & & - & not rare \\
\hline 203889 chr12 & $43,021,431$ & $43,026,485$ & del & & not rare \\
\hline 203889 chr12 & $7,688,753$ & $7,712,932$ & & & rare \\
\hline 203889 chr15 & $25,420,978$ & $25,430,654$ & dele & & not rare \\
\hline 203889 chr16 & $17,475,929$ & $17,478,212$ & dele & . & not rare \\
\hline 203889 & 19,9 & $19,962,636$ & & - & are \\
\hline 203889 chr16 & $33,863,814$ & $33,884,046$ & deletion & - & not rare \\
\hline 203889 chr17 & $34,438,753$ & $34,481,492$ & duplication & - & not rare \\
\hline 203889 chr19 & $43,555,291$ & $43,848,797$ & deletion & \multicolumn{2}{|c|}{ PSG4;PSG5 not rare } \\
\hline 203889 chr19 & $53,518,747$ & $53,552,296$ & duplication & ERVV-1 & not rare \\
\hline 203889 chr19 & $54,730,202$ & $54,743,217$ & duplication & LILRA6 & not rare \\
\hline 203889 chr2 & $41,239,457$ & $41,248,468$ & deletion & - & not rare \\
\hline 203889 chr2 & $59,949,129$ & $59,953,475$ & deletion & - & not rare \\
\hline 203889 chr21 & $10,818,766$ & $10,858,651$ & duplication & - & not rare \\
\hline 203889 chr22 & $25,663,994$ & $25,919,492$ & duplication & LR & not rare \\
\hline $203889 \mathrm{chr} 3$ & $175,887,314$ & $175,909,890$ & deletion & - & not rare \\
\hline 203889 chr3 & $195,433,728$ & $195,462,869$ & duplication & ML & not rare \\
\hline $203889 \mathrm{chr} 4$ & $122,285,263$ & $122,289,863$ & deletion & - & not rare \\
\hline 203889 chr4 & $34,785,955$ & $34,818,502$ & duplication & - & not rare \\
\hline $203889 \mathrm{chr} 4$ & $59,980,062$ & $59,988,095$ & duplication & - & not rare \\
\hline 203889 chr4 & $60,325,010$ & $60,330,880$ & duplication & - & not rare \\
\hline 203889 chr4 & $69,374,369$ & $69,489,473$ & deletion & UGT2B17 & not rare \\
\hline 203889 chr4 & $70,137,897$ & $70,229,260$ & deletion & UGT2B28 & not rare \\
\hline 203889 chr5 & $151,514,956$ & $151,518,615$ & deletion & - & not rare \\
\hline 203889 chr5 & $155,477,866$ & $155,488,917$ & deletion & - & not rare \\
\hline 203889 chr5 & 744,005 & 824,025 & duplication & ZDHHC11 & not rare \\
\hline
\end{tabular}




\begin{tabular}{|c|c|c|c|c|c|}
\hline $203889 \mathrm{chr} 6$ & $169,271,326$ & $169,273,635$ & deletion & - & not rare \\
\hline 203889 chr6 & $29,853,641$ & $29,899,389$ & deletion & - & not rare \\
\hline 03889 chr6 & $31,358,955$ & $31,453,664$ & deletion & MICA & not rare \\
\hline 3889 chr6 & $32,508,741$ & $32,543,540$ & deletion & - & ot rare \\
\hline $03889 \mathrm{chr} 7$ & $141,766,850$ & $141,793,419$ & deletion & - & not rare \\
\hline 3889 chr7 & $148,956,486$ & $148,964,513$ & deletion & ZNF783 & not rare \\
\hline 3889 chr7 & $154,431,180$ & $154,459,073$ & deletion & - & rare \\
\hline 203889 chr8 & $39,232,581$ & $39,385,979$ & duplication & - & not rare \\
\hline $203889 \mathrm{chr} 9$ & $137,134,266$ & $137,140,972$ & deletion & - & not rare \\
\hline 03889 chr9 & $24,502,737$ & $24,518,795$ & deletion & - & not rare \\
\hline 203891 chr1 & $104,104,990$ & $104,211,046$ & duplication & \multicolumn{2}{|c|}{ AMY1C;AN not rare } \\
\hline 203891 chr1 & $187,717,153$ & $187,722,032$ & deletion & - & not rare \\
\hline 203891 chr1 & $25,598,276$ & $25,659,509$ & deletion & RHD & not rare \\
\hline 203891 chr11 & $49,715,154$ & $49,757,690$ & deletion & - & not rare \\
\hline 3891 chr12 & $33,300,995$ & $33,305,855$ & duplication & - & not rare \\
\hline 203891 chr14 & $86,457,952$ & $86,489,093$ & deletion & - & not rare \\
\hline 203891 chr15 & $24,055,488$ & $24,061,557$ & deletion & - & not rare \\
\hline 203891 chr15 & $34,717,697$ & $34,814,869$ & deletion & - & not rare \\
\hline 203891 chr15 & $56,789,979$ & $56,800,635$ & deletion & - & ot rare \\
\hline 203891 chr16 & $33,543,313$ & $33,579,417$ & deletion & - & not rare \\
\hline 203891 chr16 & $35,194,609$ & $35,197,323$ & deletion & - & not rare \\
\hline 203891 chr17 & $7,264,717$ & $7,265,681$ & deletion & - & not rare \\
\hline 203891 chr18 & $69,130,532$ & $69,132,262$ & deletion & & not rare \\
\hline 203891 chr19 & $20,601,006$ & $20,717,536$ & del & & not rare \\
\hline 203891 chr19 & $35,852,103$ & $35,861,695$ & deletion & & not rare \\
\hline 203891 chr19 & $41,362,173$ & $41,386,033$ & duplication & CYP2A7 & not rare \\
\hline 203891 chr2 & $158,973,416$ & $158,978,325$ & dele & & not rare \\
\hline 203891 chr2 & $180,414,715$ & $180,421,668$ & deletion & - & not rare \\
\hline 203891 chr2 & $208,351,315$ & $208,357,838$ & deletion & - & not rare \\
\hline 203891 chr2 & $4,212,725$ & $4,221,651$ & deletion & - & not rare \\
\hline 203891 chr21 & $10,724,858$ & $10,858,651$ & deletion & - & not rare \\
\hline 203891 chr3 & $131,711,896$ & $131,712,898$ & duplication & - & not rare \\
\hline 203891 chr3 & $139,698,588$ & $139,699,972$ & deletion & - & not rare \\
\hline $203891 \mathrm{chr} 3$ & $148,964,692$ & $148,969,220$ & deletion & - & not rare \\
\hline 203891 chr3 & $163,274,112$ & $163,525,723$ & duplication & - & rare \\
\hline 203891 chr3 & $89,402,409$ & $89,418,331$ & deletion & - & not rare \\
\hline $203891 \mathrm{chr} 4$ & $39,705,484$ & $39,713,134$ & deletion & - & not rare \\
\hline 203891 chr4 & $69,374,369$ & $69,489,473$ & deletion & UGT2B17 & not rare \\
\hline 203891 chr5 & 788,646 & 824,172 & deletion & ZDHHC11 & not rare \\
\hline 203891 chr6 & $29,920,769$ & $29,921,597$ & deletion & - & not rare \\
\hline 203891 chr6 & $29,921,700$ & $29,922,138$ & deletion & - & not rare \\
\hline 203891 chr6 & $69,231,883$ & $69,241,954$ & duplication & - & not rare \\
\hline 203891 chr7 & $29,688,760$ & $29,690,262$ & deletion & - & not rare \\
\hline 203891 chr7 & $38,291,851$ & $38,305,404$ & duplication & TARP & not rare \\
\hline 203891 chr7 & $62,149,006$ & $62,162,385$ & deletion & - & not rare \\
\hline 203891 chr8 & $24,972,808$ & $24,990,418$ & duplication & - & not rare \\
\hline 203891 chr8 & $51,031,221$ & $51,038,149$ & deletion & - & not rare \\
\hline
\end{tabular}




\begin{tabular}{|c|c|c|c|c|c|}
\hline $03891 \mathrm{chr} X$ & $98,423,975$ & $98,844,127$ & duplication & - & rare \\
\hline 03894 chr1 & $104,104,990$ & $104,211,046$ & duplication & \multicolumn{2}{|c|}{ AMY1C;AN not rare } \\
\hline 33894 chr1 & $111,380,720$ & $111,387,723$ & deletion & - & not rare \\
\hline $3894 \mathrm{chr} 1$ & $12,850,282$ & $12,944,989$ & deletion & \multicolumn{2}{|c|}{ HNRNPCL1 not rare } \\
\hline 03894 chr1 & $145,625,979$ & $145,831,160$ & duplication & \multicolumn{2}{|c|}{ CD160;RNF not rare } \\
\hline 03894 chr1 & $16,922,115$ & $16,971,106$ & duplication & - & not rare \\
\hline 03894 chr1 & $248,740,572$ & $248,795,110$ & deletion & \multicolumn{2}{|c|}{ OR2T10;OF not rare } \\
\hline 3894 chr1 & $25,598,276$ & $25,659,509$ & deletion & RHD & not rare \\
\hline 3894 chr10 & $20,850,624$ & $20,857,365$ & deletion & - & not rare \\
\hline 203894 chr10 & $47,541,708$ & $47,652,499$ & duplication & - & not rare \\
\hline 03894 chr10 & $58,739,433$ & $58,752,731$ & duplication & - & not rare \\
\hline 03894 chr11 & $132,824,262$ & $133,268,476$ & duplication & - & not rare \\
\hline 203894 chr11 & $49,715,154$ & $49,757,690$ & deletion & - & not rare \\
\hline 203894 chr11 & $83,829,316$ & $83,844,790$ & duplication & - & not rare \\
\hline 203894 chr12 & $11,511,132$ & $11,528,586$ & duplication & - & not rare \\
\hline 203894 chr13 & $62,621,651$ & $62,626,953$ & deletion & - & not rare \\
\hline 03894 chr13 & $64,343,579$ & $64,388,269$ & dupl & - & not rare \\
\hline 203894 chr14 & $106,062,675$ & $106,175,202$ & duplication & - & rare \\
\hline 203894 chr14 & $41,610,224$ & $41,661,685$ & deletion & - & not rare \\
\hline 203894 chr15 & $24,381,396$ & $24,438,156$ & dele & - & not rare \\
\hline 203894 chr15 & 24,5 & $24,779,134$ & dupl & - & rare \\
\hline 203894 chr15 & $43,916,071$ & $43,993,831$ & duplication & \multicolumn{2}{|c|}{ CKMT1A;C/not rare } \\
\hline 203894 chr16 & $70,016,280$ & $70,189,705$ & deletion & PDPR & not rare \\
\hline 203894 chr16 & $85,302,676$ & $85,304,326$ & dele & & not rare \\
\hline 203894 chr18 & $63,907,596$ & $63,910,708$ & dele & & not rare \\
\hline 203894 & $43,261,897$ & $43,352,823$ & dup & PSG8 & are \\
\hline 203894 chr19 & $54,730,202$ & $54,743,217$ & deletion & LILRA6 & not rare \\
\hline 203894 chr19 & $9,275,282$ & $9,282,176$ & deletion & - & not rare \\
\hline 203894 chr2 & $123,477,465$ & $123,482,301$ & dele & - & rare \\
\hline 203894 chr2 & $35,287,658$ & $35,551,622$ & dele & - & not rare \\
\hline 203894 chr2 & $52,759,890$ & $52,784,952$ & deletion & - & not rare \\
\hline 203894 chr2 & $89,616,271$ & $90,256,530$ & dele & - & not rare \\
\hline 203894 chr2 & $92,286,512$ & $92,304,211$ & duplication & - & not rare \\
\hline 203894 chr20 & $6,833,419$ & $6,835,158$ & duplication & - & not rare \\
\hline 203894 chr4 & $138,092,298$ & $138,098,601$ & delet & - & not rare \\
\hline 203894 chr4 & $152,790,580$ & $152,793,891$ & deletion & - & not rare \\
\hline 203894 chr4 & $152,991,094$ & $152,993,620$ & deletion & - & rare \\
\hline 203894 chr4 & $161,048,496$ & $161,072,119$ & deletion & - & not rare \\
\hline 203894 chr4 & $171,269,504$ & $171,273,360$ & deletion & - & not rare \\
\hline 203894 chr4 & $69,374,369$ & $69,489,473$ & deletion & UGT2B17 & not rare \\
\hline 203894 chr4 & $70,137,897$ & $70,229,260$ & deletion & UGT2B28 & not rare \\
\hline 203894 chr5 & $117,388,410$ & $117,393,058$ & deletion & - & not rare \\
\hline 203894 chr6 & $31,276,760$ & $31,278,472$ & deletion & - & not rare \\
\hline 203894 chr6 & $52,220,793$ & $52,230,542$ & duplication & - & not rare \\
\hline 203894 chr6 & $67,011,900$ & $67,048,885$ & deletion & - & not rare \\
\hline 203894 chr7 & $141,760,421$ & $141,784,299$ & duplication & $\mathrm{M}$ & not rare \\
\hline 203894 & $75,664,539$ & $75,667,743$ & deletion & - & not rare \\
\hline
\end{tabular}




\begin{tabular}{|c|c|c|c|c|c|}
\hline 203894 chr7 & $76,429,959$ & $76,638,975$ & duplication & - & not rare \\
\hline $203894 \mathrm{chr} 8$ & $16,260,166$ & $16,272,444$ & deletion & - & not rare \\
\hline 03894 chr9 & $20,082,242$ & $20,101,896$ & deletion & - & ot rare \\
\hline 03894 chr9 & $24,146,203$ & $24,150,034$ & deletion & - & not rare \\
\hline 203894 chr9 & $77,999,354$ & $78,011,616$ & deletion & - & not rare \\
\hline 203896 chr1 & $104,153,766$ & $104,161,227$ & deletion & AMY2A & not rare \\
\hline 03896 chr1 & $108,734,695$ & $108,737,586$ & deletion & SLC25A24 & not rare \\
\hline 203896 chr1 & $12,850,282$ & $12,919,928$ & deletion & HNRNPCL1 & not rare \\
\hline 203896 chr10 & $66,899,410$ & $66,911,038$ & duplication & - & not rare \\
\hline 203896 chr11 & $55,365,761$ & $55,443,269$ & deletion & OR4P4;ORL & $\angle$ not rare \\
\hline 203896 chr11 & $7,814,299$ & $7,827,879$ & deletion & OR5P2 & not rare \\
\hline 203896 chr12 & $68,654,215$ & $68,658,918$ & deletion & - & not rare \\
\hline 203896 chr12 & $70,679,864$ & $70,681,814$ & deletion & - & not rare \\
\hline 203896 chr13 & $34,134,809$ & $34,143,545$ & deletion & - & not rare \\
\hline 03896 chr13 & $69,247,022$ & $69,267,981$ & deletion & - & not rare \\
\hline 203896 chr14 & $41,610,224$ & $41,661,685$ & deletion & - & not rare \\
\hline 203896 chr15 & $34,717,697$ & $34,814,869$ & deletion & - & not rare \\
\hline 203896 chr15 & $60,086,249$ & $60,103,464$ & deletion & - & not rare \\
\hline 203896 chr16 & $19,945,540$ & $19,962,636$ & deletion & - & not rare \\
\hline 203896 chr16 & $32,542,736$ & $32,642,384$ & deletion & - & not rare \\
\hline 203896 chr16 & $33,380,180$ & $33,631,255$ & deletion & - & not rare \\
\hline 203896 chr17 & $10,891,369$ & $10,893,799$ & deletion & - & not rare \\
\hline 203896 chr17 & $16,576,609$ & $16,587,108$ & deletion & 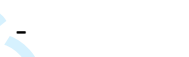 & not rare \\
\hline 203896 chr17 & $47,525,551$ & $47,571,886$ & dup & & rare \\
\hline 203896 chr2 & $34,697,895$ & $34,726,904$ & duplication & & not rare \\
\hline 203896 chr21 & $10,780,816$ & $10,858,651$ & duplication & - & not rare \\
\hline 203896 chr21 & $20,057,919$ & $20,078,801$ & duplication & - & not rare \\
\hline 203896 chr22 & $39,359,885$ & $39,385,663$ & deletion & C3B & not rare \\
\hline 203896 chr3 & $162,213,876$ & $162,234,970$ & deletion & - & not rare \\
\hline 203896 chr3 & $165,183,401$ & $165,187,567$ & deletion & - & not rare \\
\hline 203896 chr3 & $88,502,311$ & $88,691,895$ & duplication & - & rare \\
\hline 203896 chr3 & $89,402,409$ & $89,418,331$ & deletion & - & not rare \\
\hline $203896 \mathrm{chr} 4$ & $136,532,271$ & $136,601,944$ & deletion & - & not rare \\
\hline $203896 \mathrm{chr} 4$ & $190,903,688$ & $190,937,258$ & duplication & - & not rare \\
\hline 203896 chr4 & $34,785,955$ & $34,818,502$ & deletion & - & not rare \\
\hline $203896 \mathrm{chr} 4$ & $64,232,579$ & $64,241,398$ & deletion & - & not rare \\
\hline $203896 \mathrm{chr} 4$ & $92,889,426$ & $92,909,473$ & deletion & - & rare \\
\hline 203896 chr5 & $51,563,266$ & $51,569,099$ & deletion & - & not rare \\
\hline 203896 chr5 & $57,142,251$ & $57,153,542$ & deletion & - & not rare \\
\hline 203896 chr6 & $29,859,135$ & $29,899,389$ & deletion & - & not rare \\
\hline 203896 chr6 & $31,276,760$ & $31,278,472$ & deletion & - & not rare \\
\hline 203896 chr7 & $76,429,959$ & $76,637,441$ & duplication & - & not rare \\
\hline 203896 chr8 & $115,634,278$ & $115,642,459$ & deletion & - & not rare \\
\hline 203896 chr8 & $39,232,581$ & $39,385,979$ & duplication & - & not rare \\
\hline 203896 chr8 & $51,013,729$ & $51,038,149$ & deletion & - & not rare \\
\hline 203896 chr8 & $51,226,087$ & $51,227,777$ & deletion & - & not rare \\
\hline 203896 chr8 & $5,595,511$ & $5,605,706$ & deletion & - & not rare \\
\hline
\end{tabular}




\begin{tabular}{|c|c|c|c|c|c|}
\hline $203896 \mathrm{chr} 8$ & $85,261,157$ & $85,268,951$ & deletion & - & not rare \\
\hline 03896 chr9 & $104,715,329$ & $104,722,688$ & deletion & - & not rare \\
\hline 3896 chr9 & $23,363,526$ & $23,376,817$ & deletion & - & not rare \\
\hline 3896 chr9 & $6,664,282$ & $6,670,263$ & deletion & - & not rare \\
\hline $03896 \mathrm{chrX}$ & $54,715,118$ & $54,742,122$ & deletion & - & rare \\
\hline 203897 chr1 & $161,489,467$ & $161,619,741$ & duplication & \multicolumn{2}{|c|}{ FCGR3B;FC not rare } \\
\hline 203897 chr1 & $248,740,314$ & $248,856,024$ & duplication & \multicolumn{2}{|c|}{ OR2T10;OF not rare } \\
\hline 203897 chr10 & $20,850,624$ & $20,857,365$ & deletion & - & not rare \\
\hline 03897 chr10 & $71,282,981$ & $71,289,190$ & deletion & - & not rare \\
\hline 03897 chr11 & $49,715,154$ & $49,757,690$ & deletion & - & not rare \\
\hline 203897 chr11 & $55,365,761$ & $55,443,269$ & deletion & \multicolumn{2}{|c|}{$\mathrm{OR} 4 \mathrm{P} 4 ; \mathrm{OR} \angle$ not rare } \\
\hline 203897 chr12 & $11,511,132$ & $11,543,002$ & duplication & - & not rare \\
\hline 03897 chr12 & $33,300,995$ & $33,308,022$ & duplication & - & not rare \\
\hline 203897 chr13 & $34,134,809$ & $34,143,545$ & deletion & - & not rare \\
\hline 203897 chr13 & $69,247,022$ & $69,267,981$ & deletion & - & not rare \\
\hline 203897 chr14 & $41,610,224$ & $41,661,685$ & deletion & - & not rare \\
\hline 203897 chr15 & $34,717,697$ & $34,814,869$ & deletion & - & not rare \\
\hline 203897 chr16 & 205,035 & 212,649 & duplication & - & not rare \\
\hline 203897 chr16 & $60,556,223$ & $60,566,803$ & deletion & - & \\
\hline 203897 chr16 & $78,372,894$ & $78,383,509$ & deletion & - & not rare \\
\hline 203897 chr17 & $19,499,229$ & $19,537,470$ & deletion & - & not rare \\
\hline 203897 chr17 & $44,166,604$ & $44,344,749$ & duplication & KANSL1 & not rare \\
\hline 203897 chr17 & $7,264,717$ & $7,265,681$ & deletion & & not rare \\
\hline 203897 chr19 & 477,809 & 517,012 & duplication & \multicolumn{2}{|c|}{ TPGS1;MAI not rare } \\
\hline 203897 chr19 & $54,730,202$ & $54,743,217$ & duplication & LILRA6 & not rare \\
\hline 203897 chr19 & $9,275,282$ & $9,282,176$ & deletion & - & not rare \\
\hline 203897 chr2 & $123,477,465$ & $123,482,301$ & deletion & - & not rare \\
\hline 203897 chr2 & $14,704,369$ & $14,709,611$ & deletion & - & not rare \\
\hline 203897 chr2 & $51,926,599$ & $51,926,904$ & deletion & - & not rare \\
\hline 203897 chr2 & $52,759,890$ & $52,784,952$ & deletion & - & not rare \\
\hline 203897 chr2 & $97,738,917$ & $98,024,837$ & deletion & \multicolumn{2}{|c|}{ FAHD2B;Ar not rare } \\
\hline 203897 chr20 & $3,880,128$ & $3,885,100$ & deletion & - & not rare \\
\hline 203897 chr3 & $18,235,914$ & $18,244,588$ & deletion & - & not rare \\
\hline 203897 chr3 & $76,731,112$ & $76,735,823$ & deletion & - & not rare \\
\hline 203897 chr4 & $152,790,580$ & $152,793,891$ & deletion & - & not rare \\
\hline 203897 chr4 & $161,048,496$ & $161,072,119$ & deletion & - & not rare \\
\hline 203897 chr4 & $34,785,955$ & $34,818,502$ & deletion & - & not rare \\
\hline 203897 chr4 & $64,697,704$ & $64,709,753$ & deletion & - & not rare \\
\hline 203897 chr5 & $155,477,866$ & $155,488,917$ & deletion & - & not rare \\
\hline 203897 chr5 & $180,377,470$ & $180,424,820$ & deletion & \multicolumn{2}{|c|}{ BTNL8;BTN not rare } \\
\hline 203897 chr6 & $28,018,944$ & $28,021,775$ & deletion & - & not rare \\
\hline 203897 chr6 & $29,859,135$ & $29,899,389$ & deletion & - & not rare \\
\hline 203897 chr6 & $31,276,760$ & $31,278,472$ & deletion & - & not rare \\
\hline 203897 chr6 & $68,445,577$ & $68,453,376$ & deletion & - & not rare \\
\hline 203897 chr6 & $78,972,930$ & $79,035,739$ & deletion & - & not rare \\
\hline 203897 chr7 & $141,766,850$ & $141,793,419$ & deletion & - & not rare \\
\hline 203897 chr7 & $142,476,094$ & $142,487,836$ & deletion & - & not rare \\
\hline
\end{tabular}




\begin{tabular}{|c|c|c|c|c|c|}
\hline 203897 chr7 & $20,750,774$ & $20,753,987$ & deletion & - & not rare \\
\hline 203897 chr7 & $62,149,006$ & $62,162,385$ & deletion & - & not rare \\
\hline 203897 chr7 & $97,396,709$ & $97,401,677$ & deletion & - & not rare \\
\hline 203897 chr8 & $16,260,166$ & $16,272,444$ & deletion & - & not rare \\
\hline 203897 chr8 & $19,363,963$ & $19,434,698$ & deletion & - & rare \\
\hline 203897 chr8 & $2,887,395$ & $2,890,763$ & deletion & - & not rare \\
\hline 203897 chr8 & $3,786,543$ & $3,790,254$ & deletion & - & not rare \\
\hline 203897 chr8 & $39,232,581$ & $39,385,979$ & duplication & - & not rare \\
\hline 203897 chr8 & $40,184,872$ & $40,189,688$ & deletion & - & not rare \\
\hline 203897 chr8 & $51,013,729$ & $51,038,149$ & deletion & - & not rare \\
\hline 203897 chr9 & $107,799,020$ & $107,803,370$ & deletion & - & not rare \\
\hline 203897 chr9 & $135,949,324$ & $135,957,091$ & duplication & - & not rare \\
\hline 203897 chr9 & $23,363,526$ & $23,376,817$ & duplication & - & not rare \\
\hline 203897 chr9 & $78,004,378$ & $78,011,616$ & deletion & - & not rare \\
\hline 203897 chrX & $121,116,860$ & $121,188,308$ & deletion & - & rare \\
\hline 203898 chr1 & $104,153,766$ & $104,161,227$ & deletion & AMY2A & not rare \\
\hline 203898 chr1 & $158,532,198$ & $158,533,444$ & deletion & OR6P1 & not rare \\
\hline 203898 chr1 & $161,570,803$ & $161,619,741$ & deletion & FCGR3B & not rare \\
\hline 203898 chr1 & $62,113,597$ & $62,119,136$ & & - & \\
\hline 203898 chr10 & $83,883,951$ & $83,888,343$ & deletion & - & not rare \\
\hline 203898 chr11 & $55,365,761$ & $55,443,269$ & deletion & \multicolumn{2}{|c|}{$\mathrm{OR} 4 \mathrm{P} 4 ; \mathrm{OR} \angle$ not rare } \\
\hline 203898 chr12 & $43,021,431$ & $43,026,485$ & deletion & - & not rare \\
\hline 203898 chr13 & $27,976,955$ & $27,985,200$ & deletion & & not rare \\
\hline 203898 chr13 & $32,532,761$ & $32,537,768$ & & & not rare \\
\hline 203898 chr13 & $34,134,809$ & $34,143,545$ & deletion & & not rare \\
\hline 203898 chr13 & $58,589,893$ & $58,599,174$ & deletion & - & not rare \\
\hline 203898 chr14 & $106,103,358$ & $106,175,202$ & duplication & - & not \\
\hline 203898 chr14 & $106,784,840$ & $106,816,856$ & deletion & - & not rare \\
\hline 203898 chr15 & $24,705,252$ & $24,742,652$ & deletion & - & not rare \\
\hline 203898 chr15 & $97,815,182$ & $97,831,832$ & deletion & - & are \\
\hline 203898 chr16 & $19,945,540$ & $19,962,636$ & deletion & - & not rare \\
\hline 203898 chr16 & $33,519,862$ & $33,614,448$ & deletion & - & not rare \\
\hline 203898 chr16 & $33,882,506$ & $33,913,074$ & duplication & - & not rare \\
\hline 203898 chr17 & $10,891,369$ & $10,893,799$ & deletion & - & not rare \\
\hline 203898 chr17 & $14,997,297$ & $15,010,250$ & deletion & - & not rare \\
\hline 203898 chr17 & $15,044,804$ & $15,058,236$ & deletion & - & not rare \\
\hline 203898 chr17 & $44,166,604$ & $44,344,749$ & duplication & KANSL1 & not rare \\
\hline 203898 chr17 & $70,560,898$ & $70,565,828$ & deletion & - & not rare \\
\hline 203898 chr17 & $73,300,541$ & $73,305,159$ & deletion & - & not rare \\
\hline 203898 chr18 & $63,907,596$ & $63,910,708$ & deletion & - & not rare \\
\hline 203898 chr19 & $35,661,787$ & $35,665,515$ & deletion & - & not rare \\
\hline 203898 chr2 & $10,885,901$ & $10,890,881$ & duplication & - & not rare \\
\hline 203898 chr2 & $129,638,609$ & $129,645,321$ & deletion & - & not rare \\
\hline 203898 chr2 & $17,220,064$ & $17,239,502$ & deletion & - & not rare \\
\hline 203898 chr2 & $208,351,315$ & $208,357,838$ & deletion & - & not rare \\
\hline 203898 chr2 & $34,697,895$ & $34,726,904$ & duplication & - & not rare \\
\hline 203898 chr2 & $38,956,947$ & $38,971,623$ & duplication & GALM & not rare \\
\hline
\end{tabular}




\begin{tabular}{|c|c|c|c|c|c|}
\hline 203898 chr20 & $24,887,251$ & $24,893,947$ & deletion & - & not rare \\
\hline 203898 chr3 & $144,142,884$ & $144,186,265$ & deletion & - & rare \\
\hline 3898 chr3 & $61,479,175$ & $61,492,344$ & deletion & - & not rare \\
\hline 33898 chr4 & $108,064,023$ & $108,074,123$ & deletion & - & not rare \\
\hline $03898 \mathrm{chr} 4$ & $136,532,271$ & $136,601,944$ & deletion & - & not rare \\
\hline $203898 \mathrm{chr} 4$ & $190,903,688$ & $190,935,996$ & duplication & - & not rare \\
\hline $203898 \mathrm{chr} 4$ & $34,785,955$ & $34,818,502$ & duplication & - & not rare \\
\hline 203898 chr4 & $39,705,484$ & $39,713,134$ & deletion & - & not rare \\
\hline 203898 chr4 & $44,966,584$ & $45,004,987$ & duplication & - & not rare \\
\hline $03898 \mathrm{chr} 4$ & $60,325,242$ & $60,330,880$ & duplication & - & not rare \\
\hline 203898 chr4 & $69,374,369$ & $69,489,473$ & deletion & UGT2B17 & not rare \\
\hline 203898 chr4 & $88,839,476$ & $88,842,825$ & duplication & - & not rare \\
\hline $203898 \mathrm{chr} 4$ & $92,889,426$ & $92,909,473$ & deletion & - & rare \\
\hline 203898 chr5 & $117,388,410$ & $117,393,058$ & deletion & - & not rare \\
\hline 203898 chr5 & $133,395,820$ & $133,397,597$ & deletion & - & not rare \\
\hline 203898 chr5 & $17,600,053$ & $17,627,924$ & deletion & - & not rare \\
\hline 203898 chr5 & $180,377,470$ & $180,424,820$ & deletion & BTI & Inot rare \\
\hline 203898 chr5 & $51,563,266$ & $51,569,099$ & deletion & - & not rare \\
\hline 203898 chr5 & $57,142,251$ & $57,153,542$ & deletion & - & ot rare \\
\hline 203898 chr5 & $70,305,696$ & $70,308,251$ & duplication & NAIP & not rare \\
\hline 203898 chr6 & $30,994,114$ & $30,997,692$ & duplication & MUC22 & not rare \\
\hline 203898 chr6 & $32,498,447$ & $32,527,079$ & deletion & - & not rare \\
\hline 203898 chr6 & $67,011,900$ & $67,048,885$ & deletion & & not rare \\
\hline 203898 chr7 & $57,623,146$ & $57,670,249$ & duplication & 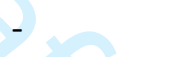 & not rare \\
\hline 203898 chr7 & $75,354,240$ & $75,370,368$ & duplication & HIP & not rare \\
\hline 203898 chr8 & $63,034,992$ & $63,039,751$ & deletion & - & not rare \\
\hline 203898 chr9 & $138,195,113$ & $138,195,756$ & deletion & - & not rare \\
\hline 203898 chr9 & $1,577,505$ & $1,580,979$ & deletion & - & not rare \\
\hline 203898 chr9 & $24,502,737$ & $24,518,795$ & deletion & - & not rare \\
\hline 203899 chr1 & $109,367,944$ & $109,371,874$ & deletion & AKI & not rare \\
\hline 203899 chr1 & $112,693,423$ & $112,704,581$ & deletion & - & not rare \\
\hline 203899 chr1 & $161,570,803$ & $161,619,741$ & deletion & FCGR3B & not rare \\
\hline 203899 chr1 & $62,113,597$ & $62,119,136$ & deletion & - & not rare \\
\hline 203899 chr10 & $58,516,470$ & $58,526,153$ & deletion & - & not rare \\
\hline 203899 chr10 & $71,282,981$ & $71,289,190$ & deletion & - & not rare \\
\hline 203899 chr10 & $73,292,293$ & $73,292,871$ & deletion & - & not rare \\
\hline 203899 chr11 & $125,075,575$ & $125,084,742$ & deletion & - & not rare \\
\hline 203899 chr11 & $55,365,761$ & $55,443,269$ & deletion & \multicolumn{2}{|c|}{$\mathrm{OR} 4 \mathrm{P} 4 ; \mathrm{OR} \angle$ not rare } \\
\hline 203899 chr11 & $67,501,626$ & $67,748,817$ & deletion & - & not rare \\
\hline 203899 chr11 & $90,193,807$ & $90,195,742$ & deletion & - & not rare \\
\hline 203899 chr12 & $126,994,506$ & $127,004,296$ & deletion & - & not rare \\
\hline 203899 chr12 & $30,237,351$ & $30,243,473$ & deletion & - & not rare \\
\hline 203899 chr12 & $32,052,422$ & $32,058,066$ & deletion & - & not rare \\
\hline 203899 chr12 & $43,021,431$ & $43,026,485$ & deletion & - & not rare \\
\hline 203899 chr12 & $6,241,446$ & $6,248,269$ & deletion & - & not rare \\
\hline 203899 chr13 & $20,458,701$ & $20,467,393$ & duplication & - & not rare \\
\hline 203899 chr13 & $80,681,247$ & $80,685,663$ & deletion & - & not rare \\
\hline
\end{tabular}




\begin{tabular}{|c|c|c|c|c|c|}
\hline 203899 chr13 & $83,788,742$ & $83,791,615$ & deletion & - & not rare \\
\hline 203899 chr14 & $20,197,311$ & $20,232,479$ & duplication & OR4Q3 & not rare \\
\hline 03899 chr14 & $20,363,759$ & $20,377,435$ & duplication & - & t rare \\
\hline 3899 chr16 & $33,543,313$ & $33,590,959$ & deletion & - & ot rare \\
\hline 03899 chr16 & $79,692,395$ & $79,696,939$ & deletion & - & not rare \\
\hline 3899 chr17 & $34,438,753$ & $34,480,718$ & duplication & - & not rare \\
\hline 3899 chr18 & $67,208,806$ & $67,217,271$ & deletion & - & not rare \\
\hline 203899 chr19 & $35,661,787$ & $35,665,515$ & deletion & - & not rare \\
\hline 203899 chr19 & $43,710,045$ & $43,759,382$ & deletion & PSG9 & not rare \\
\hline 03899 chr2 & $212,771,670$ & $212,773,893$ & deletion & - & not rare \\
\hline 203899 chr2 & $34,697,895$ & $34,726,904$ & deletion & - & rare \\
\hline 03899 chr2 & $51,926,599$ & $51,926,904$ & deletion & - & not rare \\
\hline 203899 chr21 & $44,822,871$ & $44,837,829$ & duplication & LOC102724 & not rare \\
\hline 203899 chr22 & $18,874,965$ & $21,464,479$ & duplication & RIMBP3;PI & rare \\
\hline 03899 chr22 & $39,359,885$ & $39,385,663$ & deletion & АРОВЕСЗВ & not rare \\
\hline $203899 \mathrm{chr} 3$ & $13,681,185$ & $13,682,137$ & deletion & - & ot rare \\
\hline $203899 \mathrm{chr} 3$ & $193,136,358$ & $193,140,348$ & deletion & - & not rare \\
\hline 203899 chr3 & $195,433,728$ & $195,462,414$ & duplication & MUC20 & not rare \\
\hline 203899 chr3 & $20,414,230$ & $20,416,003$ & deletion & - & \\
\hline 203899 chr3 & $76,731,112$ & $76,735,823$ & deletion & - & not rare \\
\hline $203899 \mathrm{chr} 4$ & $152,790,580$ & $152,793,891$ & deletion & - & not rare \\
\hline 203899 chr4 & $64,697,704$ & $64,709,753$ & deletion & - & not rare \\
\hline $203899 \mathrm{chr} 4$ & $69,374,369$ & $69,489,473$ & deletion & UGT2B17 & not rare \\
\hline $203899 \mathrm{chr} 4$ & $99,999,033$ & $100,000,541$ & & & not rare \\
\hline 203899 chr5 & 757,693 & 824,025 & duplication & ZDHHC11 & not rare \\
\hline 203899 chr6 & $29,857,565$ & $29,899,040$ & deletion & 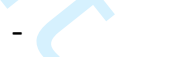 & not rare \\
\hline 203899 chr6 & $31,276,760$ & $31,278,472$ & & - & are \\
\hline 203899 chr6 & $81,284,205$ & $81,293,326$ & deletion & - & not rare \\
\hline 203899 chr7 & $142,098,342$ & $142,105,433$ & deletion & - & not rare \\
\hline 203899 chr7 & 151,829 & 159,207 & deletion & - & not rare \\
\hline 203899 chr7 & $155,882,711$ & $155,885,143$ & deletion & - & not rare \\
\hline 203899 chr7 & $26,114,836$ & $26,175,542$ & duplication & - & rare \\
\hline 203899 chr7 & $86,940,652$ & $86,948,364$ & deletion & - & not rare \\
\hline $203899 \mathrm{chr} 7$ & $91,033,074$ & $91,041,618$ & deletion & - & not rare \\
\hline 203899 chr9 & $104,715,329$ & $104,722,688$ & deletion & - & not rare \\
\hline 203899 chr9 & $2,148,213$ & $2,151,397$ & deletion & - & not rare \\
\hline $203900 \mathrm{chr} 1$ & $149,024,218$ & $149,237,009$ & deletion & - & not rare \\
\hline 203900 chr1 & $248,740,572$ & $248,795,110$ & deletion & \multicolumn{2}{|c|}{ OR2T10;OF not rare } \\
\hline 203900 chr10 & $38,792,512$ & $38,903,858$ & deletion & - & not rare \\
\hline 203900 chr11 & $55,365,761$ & $55,443,269$ & deletion & \multicolumn{2}{|c|}{ OR4P4;OR $\angle$ not rare } \\
\hline 203900 chr12 & $12,532,984$ & $12,541,869$ & deletion & - & not rare \\
\hline 203900 chr12 & $30,237,351$ & $30,243,473$ & deletion & - & not rare \\
\hline 203900 chr12 & $33,300,995$ & $33,305,855$ & duplication & - & not rare \\
\hline 203900 chr12 & $40,874,700$ & $40,875,963$ & deletion & MUC19 & not rare \\
\hline 203900 chr12 & $49,677,529$ & $49,691,250$ & duplication & PRPH & not rare \\
\hline 203900 chr12 & $6,255,862$ & $6,259,552$ & deletion & - & not rare \\
\hline 203900 chr13 & $34,134,809$ & $34,143,545$ & deletion & - & not rare \\
\hline
\end{tabular}




\begin{tabular}{|c|c|c|c|c|c|}
\hline 203900 chr13 & $58,589,893$ & $58,599,174$ & deletion & - & not rare \\
\hline 203900 chr13 & $69,247,022$ & $69,267,981$ & deletion & - & not rare \\
\hline 3900 chr14 & $41,610,224$ & $41,661,685$ & deletion & - & not rare \\
\hline 3900 chr16 & $63,141,937$ & $63,151,505$ & duplication & - & not rare \\
\hline $03900 \mathrm{chr} 17$ & $1,209,747$ & $1,209,989$ & deletion & - & not rare \\
\hline 203900 chr17 & $75,641,916$ & $75,650,825$ & deletion & - & ot rare \\
\hline 03900 chr18 & $55,931,179$ & $55,937,109$ & deletion & - & not rare \\
\hline 203900 chr19 & $24,564,592$ & $24,599,528$ & duplication & - & not rare \\
\hline 03900 chr19 & $51,331,915$ & $51,333,442$ & deletion & - & not rare \\
\hline 03900 chr2 & $38,956,947$ & $38,971,623$ & duplication & GA & not rare \\
\hline 203900 chr2 & $8,130,069$ & $8,136,251$ & deletion & - & not rare \\
\hline 203900 chr22 & $18,889,794$ & $19,010,149$ & duplication & PRODH;DG & not rare \\
\hline 203900 chr3 & $131,711,896$ & $131,712,898$ & deletion & - & not rare \\
\hline 203900 chr3 & $152,608,212$ & $152,612,537$ & deletion & - & not rare \\
\hline 203900 chr3 & $186,408,475$ & $186,419,335$ & deletion & - & not rare \\
\hline 203900 chr4 & $34,785,955$ & $34,818,502$ & duplication & - & not rare \\
\hline $203900 \mathrm{chr} 4$ & $42,697,553$ & $42,708,694$ & deletion & - & not rare \\
\hline $203900 \mathrm{chr} 4$ & $69,374,369$ & $69,489,473$ & deletion & UGT2B17 & not rare \\
\hline $203900 \mathrm{chr} 4$ & 69,461 & 125,751 & duplication & & rare \\
\hline 203900 chr5 & $151,581,680$ & $151,583,410$ & deletion & - & not rare \\
\hline 203900 chr5 & $52,670,932$ & $52,677,265$ & deletion & - & not rare \\
\hline 203900 chr5 & $58,762,723$ & $59,061,282$ & deletion & & rare \\
\hline 203900 chr6 & $69,231,883$ & $69,241,954$ & duplication & & not rare \\
\hline 203900 chr6 & $77,439,769$ & $77,451,301$ & deletion & & not rare \\
\hline 203900 chr6 & $78,972,930$ & $79,035,739$ & delet & & not rare \\
\hline 203900 chr7 & $62,049,484$ & $62,074,888$ & deletion & - & rare \\
\hline 203900 chr7 & $75,357,240$ & $75,370,368$ & duplication & HIP1 & not rare \\
\hline 203900 chr8 & $138,822,798$ & $138,824,695$ & deletion & - & not rare \\
\hline 203900 chr8 & $36,070,428$ & $36,076,949$ & deletion & - & not rare \\
\hline 203900 chr8 & $39,232,581$ & $39,385,979$ & duplication & - & not rare \\
\hline 203900 chr8 & $47,526,999$ & $47,539,088$ & duplication & - & not rare \\
\hline 203900 chr9 & $23,362,379$ & $23,376,817$ & duplication & - & not rare \\
\hline 203900 chr9 & $39,098,698$ & $39,155,885$ & duplication & AP3 & rare \\
\hline 203900 chr9 & $85,373,362$ & $85,383,934$ & deletion & - & not rare \\
\hline $203900 \mathrm{chrX}$ & $10,088,400$ & $10,328,162$ & duplication & \multicolumn{2}{|c|}{ WWC3;CLC rare } \\
\hline 203901 chr1 & $149,024,218$ & $149,237,009$ & deletion & - & not rare \\
\hline 203901 chr1 & $187,717,153$ & $187,722,032$ & deletion & - & not rare \\
\hline 203901 chr1 & $62,113,597$ & $62,119,136$ & deletion & - & not rare \\
\hline 203901 chr10 & 135,331 & 143,253 & deletion & - & not rare \\
\hline 203901 chr11 & $37,768,477$ & $37,839,784$ & duplication & - & not rare \\
\hline 203901 chr12 & $12,532,984$ & $12,541,869$ & deletion & - & not rare \\
\hline 203901 chr12 & $6,255,862$ & $6,259,552$ & deletion & - & not rare \\
\hline 203901 chr12 & $73,988,439$ & $73,995,487$ & deletion & - & not rare \\
\hline 203901 chr13 & $34,134,809$ & $34,143,545$ & deletion & - & not rare \\
\hline 203901 chr13 & $38,076,308$ & $38,085,537$ & deletion & - & not rare \\
\hline 203901 chr14 & $42,988,346$ & $42,992,844$ & deletion & - & not rare \\
\hline 203901 chr15 & $24,381,205$ & $24,436,527$ & duplication & - & not rare \\
\hline
\end{tabular}




\begin{tabular}{|c|c|c|c|c|c|}
\hline 03901 chr16 & 609 & $35,197,323$ & deletion & - & not rare \\
\hline 3901 chr17 & $75,641,916$ & $75,650,825$ & deletion & - & not rare \\
\hline 3901 chr19 & $51,331,915$ & $51,333,442$ & deletion & - & ot rare \\
\hline 3901 chr2 & $14,704,369$ & $14,709,611$ & deletion & - & not rare \\
\hline $3901 \mathrm{chr} 2$ & $48,851,541$ & $48,856,770$ & deletion & - & not rare \\
\hline 03901 chr2 & $8,130,069$ & $8,136,251$ & deletion & - & t rare \\
\hline 3901 chr2 & $87,392,157$ & $87,522,440$ & duplication & - & not rare \\
\hline 03901 chr2 & $87,768,332$ & $87,995,393$ & duplication & - & not rare \\
\hline 3901 chr22 & $18,889,794$ & $18,971,224$ & duplication & PRODH;DG & not rare \\
\hline 3901 chr22 & $18,977,743$ & $19,007,341$ & duplication & - & not rare \\
\hline 03901 chr22 & $39,359,885$ & $39,385,663$ & deletion & BEC $3 B$ & not rare \\
\hline 03901 chr3 & $186,408,475$ & $186,419,335$ & deletion & - & not rare \\
\hline 03901 chr3 & $197,052,973$ & $197,056,995$ & deletion & - & not rare \\
\hline 203901 chr3 & $197,844,320$ & $197,846,134$ & deletion & - & not rare \\
\hline 03901 chr3 & $32,945,865$ & $32,964,242$ & deletion & - & not rare \\
\hline 203901 chr3 & $4,644,551$ & $4,651,206$ & deletion & - & not rare \\
\hline 203901 chr3 & $96,308,055$ & $96,319,964$ & deletion & - & not rare \\
\hline 203901 chr4 & $116,168,158$ & $116,173,928$ & deletion & - & not rare \\
\hline 203901 chr4 & $122,285,263$ & $122,289,863$ & on & - & rare \\
\hline 203901 chr4 & $25,557,047$ & $25,576,330$ & duplication & - & not rare \\
\hline 203901 chr4 & $64,136,609$ & $64,154,477$ & deletion & - & not rare \\
\hline 203901 chr4 & $69,374,369$ & $69,489,473$ & deletion & UGT2B17 & not rare \\
\hline 203901 chr4 & 69,461 & 125,751 & duplication & ZNF595 & not rare \\
\hline 203901 chr5 & $113,327,484$ & $113,335,230$ & deletion & & not rare \\
\hline 203901 chr5 & $151,514,956$ & $151,518,615$ & dele & & not rare \\
\hline 203901 chr5 & $52,670,932$ & $52,677,265$ & deletion & . & not rare \\
\hline 203901 chr5 & 701,026 & 825,338 & ation & ZDHHC11 & no \\
\hline 203901 chr6 & $32,610,719$ & $32,614,917$ & duplication & - & not rare \\
\hline 203901 chr6 & $78,972,930$ & $79,035,739$ & deletion & - & not rare \\
\hline 203901 chr7 & $141,761,027$ & $141,787,327$ & duplication & $\mathrm{M}$ & not rare \\
\hline 203901 chr7 & $156,387,296$ & $156,394,307$ & deletion & - & not rare \\
\hline 203901 chr8 & $138,822,798$ & $138,824,695$ & deletion & - & not rare \\
\hline 203901 chr8 & $39,232,581$ & $39,385,979$ & duplication & - & not rare \\
\hline 203901 chr8 & $72,214,753$ & $72,217,690$ & deletion & - & not rare \\
\hline 203901 chr9 & $107,799,020$ & $107,803,370$ & deletion & - & not rare \\
\hline 203901 chr9 & $23,363,526$ & $23,376,817$ & duplication & - & not rare \\
\hline 203901 chr9 & $39,004,140$ & $39,151,736$ & duplication & CNTNAP3 & not rare \\
\hline 203901 chr9 & $85,373,362$ & $85,383,934$ & deletion & - & not rare \\
\hline 203901 chr9 & $94,396,059$ & $94,402,923$ & deletion & - & not rare \\
\hline 203901 chrX & $10,088,400$ & $10,328,162$ & duplication & \multicolumn{2}{|c|}{ WWC3;CLC rare } \\
\hline 204527 chr1 & $104,153,766$ & $104,211,046$ & deletion & \multicolumn{2}{|c|}{ AMY1A;AN not rare } \\
\hline 204527 chr1 & $109,367,944$ & $109,371,874$ & deletion & AKNAD1 & not rare \\
\hline 204527 chr1 & $142,603,938$ & $142,651,841$ & deletion & - & rare \\
\hline 204527 chr10 & $109,675,785$ & $109,679,542$ & deletion & - & not rare \\
\hline 204527 chr10 & $39,119,741$ & $39,149,666$ & duplication & - & not rare \\
\hline 204527 chr11 & $24,297,260$ & $24,311,034$ & deletion & - & not rare \\
\hline 204527 chr11 & $55,365,761$ & $55,443,269$ & deletion & \multicolumn{2}{|c|}{ OR4P4;OR $\angle$ not rare } \\
\hline
\end{tabular}




\begin{tabular}{|c|c|c|c|c|c|c|}
\hline 204527 & chr11 & 58, & $58,850,926$ & duplication & - & not rare \\
\hline 204527 & $\operatorname{chr} 12$ & $43,021,431$ & $43,026,485$ & deletion & - & not rare \\
\hline 04527 & chr12 & $70,679,864$ & $70,681,814$ & deletion & - & ot rare \\
\hline 4527 & chr13 & $32,532,761$ & $32,537,768$ & deletion & - & not rare \\
\hline 04527 & chr14 & $41,610,224$ & $41,661,685$ & deletion & - & not rare \\
\hline 04527 & chr17 & $10,891,369$ & $10,893,799$ & deletion & - & ot rare \\
\hline 04527 & chr17 & $14,997,297$ & $15,010,250$ & deletion & - & not rare \\
\hline 204527 & chr17 & $15,042,537$ & $15,058,236$ & deletion & - & not rare \\
\hline 204527 & chr18 & $63,907,596$ & $63,910,708$ & deletion & - & not rare \\
\hline 04527 & chr2 & $123,477,263$ & $123,482,301$ & deletion & - & not rare \\
\hline 204527 & chr2 & $240,562,883$ & $240,579,362$ & deletion & - & not rare \\
\hline 204527 & chr2 & $66,188,209$ & $66,196,726$ & deletion & - & not rare \\
\hline 204527 & $\operatorname{chr} 20$ & $14,573,513$ & $14,576,448$ & deletion & - & not rare \\
\hline 204527 & chr3 & $129,769,827$ & $129,798,600$ & deletion & - & not rare \\
\hline 204527 & chr3 & $193,136,358$ & $193,140,348$ & deletion & - & not rare \\
\hline 204527 & chr3 & $53,032,639$ & $53,042,099$ & deletion & - & not rare \\
\hline 204527 & chr4 & $138,092,298$ & $138,098,601$ & deletion & - & not rare \\
\hline 204527 & chr4 & $60,325,242$ & $60,330,880$ & duplication & - & not rare \\
\hline 204527 & chr4 & $70,137,897$ & $70,229,260$ & dele & 28 & rare \\
\hline 204527 & chr5 & $103,443,017$ & $103,452,929$ & deletion & - & not rare \\
\hline 204527 & chr5 & $140,225,908$ & $140,238,311$ & deletion & PCDHA10; & not rare \\
\hline 204527 & chr5 & $180,377,470$ & $180,424,820$ & deletion & BTNL8;E & Inot rare \\
\hline 204527 & chr6 & $29,921,001$ & $29,922,138$ & deletion & & not rare \\
\hline 204527 & chr6 & $38,741,576$ & $38,750,888$ & duplication & DNAH8 & not rare \\
\hline 204527 & chr6 & $66,074,421$ & $66,080,908$ & deletion & & not rare \\
\hline 204527 & chr7 & $141,766,850$ & $141,793,419$ & deletion & . & not rare \\
\hline 204527 & chr7 & $26,053,422$ & $26,190,652$ & ation & - & are \\
\hline 204527 & chr7 & 57,148 & 68,920 & duplication & - & not rare \\
\hline 204527 & chr8 & $115,634,278$ & $115,642,459$ & deletion & - & not rare \\
\hline 204527 & chr8 & $39,232,581$ & $39,385,979$ & duplication & - & not rare \\
\hline 204528 & chr1 & $196,823,300$ & $196,900,729$ & duplication & CFHR4 & not rare \\
\hline 204528 & chr12 & $6,255,862$ & $6,259,552$ & deletion & - & not rare \\
\hline 204528 & chr13 & $106,454,888$ & $106,464,877$ & duplication & - & not rare \\
\hline 204528 & chr13 & $69,247,022$ & $69,267,981$ & deletion & - & not rare \\
\hline 204528 & chr14 & $35,604,892$ & $35,613,500$ & deletion & - & not rare \\
\hline 204528 & chr15 & $92,577,334$ & $92,578,836$ & deletion & - & not rare \\
\hline 204528 & chr16 & $12,633,513$ & $12,638,489$ & deletion & - & not rare \\
\hline 204528 & chr18 & $2,193,994$ & $2,205,343$ & deletion & - & not rare \\
\hline 204528 & chr19 & $11,257,780$ & $11,343,795$ & duplication & \multicolumn{2}{|c|}{ DOCK6;KAI rare } \\
\hline 204528 & chr19 & $55,298,309$ & $55,354,873$ & deletion & \multicolumn{2}{|c|}{ KIR3DL1;KI not rare } \\
\hline 204528 & chr2 & $129,638,609$ & $129,645,321$ & deletion & - & not rare \\
\hline 204528 & $\operatorname{chr} 2$ & $184,085,551$ & $184,088,256$ & deletion & - & not rare \\
\hline 204528 & chr2 & $52,759,890$ & $52,784,952$ & deletion & - & not rare \\
\hline 204528 & chr20 & $61,381,140$ & $61,419,168$ & deletion & NTSR1 & rare \\
\hline 204528 & $\operatorname{chr} 21$ & $44,822,871$ & $44,837,829$ & duplication & \multicolumn{2}{|c|}{ LOC102724 not rare } \\
\hline 204528 & chr22 & $18,874,965$ & $19,010,149$ & deletion & \multicolumn{2}{|c|}{ PRODH;DG not rare } \\
\hline 204528 & chr22 & $25,663,994$ & $25,712,058$ & duplication & - & not rare \\
\hline
\end{tabular}




\begin{tabular}{|c|c|c|c|c|c|}
\hline 04528 chr22 & 25,7 & 25, & dup & LRP5L & not \\
\hline $04528 \mathrm{chr} 4$ & $152,790,580$ & $152,793,891$ & deletion & - & not rare \\
\hline $04528 \mathrm{chr} 4$ & $69,374,369$ & $69,489,473$ & deletion & UGT2B17 & not rare \\
\hline 4528 chr5 & $111,935,341$ & $111,944,746$ & deletion & - & not rare \\
\hline $4528 \mathrm{chr} 5$ & $140,225,908$ & $140,238,311$ & deletion & \multicolumn{2}{|c|}{ PCDHA10;F not rare } \\
\hline 04528 chr5 & $57,142,251$ & $57,153,542$ & deletion & - & not rare \\
\hline 4528 chr6 & $29,921,001$ & $29,922,138$ & deletion & - & not rare \\
\hline 04528 chr6 & $32,640,336$ & $32,657,373$ & deletion & - & not rare \\
\hline 04528 chr6 & $76,243,490$ & $76,265,724$ & deletion & - & not rare \\
\hline 4528 chr6 & $81,763,327$ & $81,818,875$ & deletion & - & rare \\
\hline 204528 chr7 & $141,766,850$ & $141,793,419$ & deletion & - & not rare \\
\hline 204528 chr7 & $21,548,379$ & $21,615,629$ & deletion & \multicolumn{2}{|c|}{ SP4;DNAH1 rare } \\
\hline 204528 chr7 & $62,018,789$ & $62,031,876$ & deletion & - & not rare \\
\hline 204528 chr8 & $39,232,581$ & $39,385,979$ & duplication & - & not rare \\
\hline 204528 chr9 & $135,935,394$ & $135,955,493$ & duplication & CEL & not rare \\
\hline 04528 chr9 & $138,195,113$ & $138,195,756$ & deletion & - & not rare \\
\hline 204528 chr9 & $23,363,526$ & $23,376,817$ & duplication & - & not rare \\
\hline 204528 chr9 & $44,779,633$ & $44,834,316$ & deletion & - & not rare \\
\hline 204530 chr1 & $149,022,110$ & $149,237,009$ & deletion & - & not rare \\
\hline 204530 chr1 & $187,717,153$ & $187,722,032$ & deletion & - & not rare \\
\hline $204530 \mathrm{chr} 1$ & $245,636,915$ & $245,647,809$ & deletion & - & not rare \\
\hline 204530 & $68,366,925$ & $68,414,024$ & deletion & - & rare \\
\hline 204530 chr10 & $120,897,479$ & $120,972,496$ & duplication & \multicolumn{2}{|c|}{ SFXN4;GRK rare } \\
\hline 204530 chr10 & $90,940,413$ & $90,945,998$ & deletion & & not rare \\
\hline 204530 chr11 & $24,446,609$ & $24,451,830$ & deletion & & not rare \\
\hline 204530 chr12 & $11,220,827$ & $11,244,117$ & deletion & TAS2R43 & not rare \\
\hline 204530 chr12 & $11,510,007$ & $11,543,002$ & deletion & - & not rare \\
\hline 204530 chr12 & $1,611,356$ & $1,614,080$ & deletion & - & not rare \\
\hline 204530 chr12 & $31,296,219$ & $31,407,303$ & duplication & - & not rare \\
\hline 204530 & $38,076,308$ & $38,085,537$ & dele & - & not rare \\
\hline 204530 chr14 & $20,331,117$ & $20,377,435$ & duplication & OR4K2 & not rare \\
\hline 204530 chr14 & $20,398,906$ & $20,422,799$ & duplication & OR4K1 & not rare \\
\hline 204530 chr14 & $73,527,253$ & $73,530,678$ & deletion & - & not rare \\
\hline 204530 chr15 & $24,705,252$ & $24,742,652$ & deletion & - & not rare \\
\hline 204530 chr16 & $78,372,894$ & $78,383,509$ & deletion & - & not rare \\
\hline 204530 chr19 & $20,601,006$ & $20,717,536$ & deletion & - & not rare \\
\hline 204530 chr2 & $133,924,454$ & $134,105,917$ & deletion & NCKAP5 & rare \\
\hline 204530 chr2 & $34,697,895$ & $34,726,904$ & duplication & - & not rare \\
\hline 204530 chr2 & $8,130,069$ & $8,136,251$ & deletion & - & not rare \\
\hline 204530 chr21 & $10,776,405$ & $10,858,651$ & duplication & - & not rare \\
\hline 204530 chr21 & $48,006,209$ & $48,095,982$ & duplication & \multicolumn{2}{|c|}{ PRMT2;S1C rare } \\
\hline 204530 chr22 & $25,663,994$ & $25,919,492$ & deletion & LRP5L & not rare \\
\hline 204530 chr22 & $42,957,288$ & $42,967,863$ & deletion & SERHL2 & not rare \\
\hline 204530 chr3 & $197,845,008$ & $197,846,927$ & deletion & - & not rare \\
\hline 204530 chr3 & $37,979,882$ & $37,986,734$ & deletion & - & not rare \\
\hline 204530 chr3 & $8,821,742$ & $8,855,880$ & duplication & - & not rare \\
\hline $204530 \mathrm{chr} 4$ & $42,697,553$ & $42,708,694$ & deletion & - & not rare \\
\hline
\end{tabular}




\begin{tabular}{|c|c|c|c|c|c|}
\hline $204530 \mathrm{chr} 4$ & $58,051,094$ & $58,100,571$ & duplication & - & not rare \\
\hline $204530 \mathrm{chr} 4$ & $70,137,897$ & $70,229,260$ & deletion & UGT2B28 & not rare \\
\hline 04530 chr5 & $98,090,590$ & $98,096,735$ & deletion & - & not rare \\
\hline 4530 chr6 & $16,568,792$ & $16,572,893$ & deletion & - & not rare \\
\hline 04530 chr6 & $3,640,561$ & $3,647,559$ & duplication & - & not rare \\
\hline 04530 chr6 & $78,972,930$ & $79,035,739$ & deletion & - & not rare \\
\hline 04530 chr7 & $14,051,536$ & $14,122,412$ & duplication & - & rare \\
\hline $204530 \mathrm{chr} 7$ & $141,766,850$ & $141,793,419$ & deletion & - & not rare \\
\hline 204530 chr7 & $142,098,342$ & $142,105,433$ & deletion & - & not rare \\
\hline 04530 chr7 & 41,421 & 68,920 & duplication & - & not rare \\
\hline 204530 chr7 & $43,416,186$ & $43,424,112$ & deletion & - & not rare \\
\hline $204530 \mathrm{chr} 7$ & $52,730,700$ & $52,744,420$ & duplication & - & not rare \\
\hline $204530 \mathrm{chr} 7$ & $67,198,842$ & $67,206,867$ & deletion & - & not rare \\
\hline 204530 chr7 & $79,961,091$ & $79,966,180$ & deletion & - & rare \\
\hline $204530 \mathrm{chr} 7$ & $86,940,652$ & $86,948,364$ & deletion & - & not rare \\
\hline 204530 chr8 & $15,778,007$ & $15,786,889$ & deletion & - & not rare \\
\hline 204530 chr8 & $39,232,581$ & $39,385,979$ & duplication & - & not rare \\
\hline 204531 chr1 & $60,445,753$ & $60,460,443$ & duplication & C1orf87 & not rare \\
\hline 204531 chr1 & $60,467,160$ & $60,824,982$ & dupl & & \\
\hline 204531 chr10 & $20,850,624$ & $20,857,365$ & deletion & - & not rare \\
\hline 204531 chr10 & $83,883,951$ & $83,888,343$ & deletion & - & not rare \\
\hline 204531 chr10 & $92,798,327$ & $92,811,789$ & dele & - & not rare \\
\hline 204531 chr11 & $3,239,988$ & $3,243,665$ & deletion & MRGPRG & not rare \\
\hline 204531 chr12 & $33,300,995$ & $33,305,855$ & duplication & - & not rare \\
\hline 204531 chr12 & $40,874,700$ & $40,875,963$ & deletion & MUC & not rare \\
\hline 204531 chr13 & $112,536,835$ & $112,539,078$ & deletion & - & not rare \\
\hline 204531 chr13 & $98,530,124$ & $98,532,757$ & dele & - & are \\
\hline 204531 chr15 & $32,509,090$ & $32,620,088$ & deletion & - & not rare \\
\hline 204531 chr16 & $78,372,894$ & $78,383,509$ & deletion & - & not rare \\
\hline 204531 chr19 & $38,551,881$ & $38,555,458$ & deletion & - & not rare \\
\hline 204531 chr19 & $51,331,915$ & $51,333,442$ & deletion & - & not rare \\
\hline 204531 chr19 & $55,298,309$ & $55,339,383$ & deletion & KIR3DL1;K & not rare \\
\hline 204531 chr19 & $9,275,282$ & $9,282,176$ & deletion & - & not rare \\
\hline 204531 chr2 & $227,345,365$ & $227,347,453$ & deletion & - & not rare \\
\hline 204531 chr21 & $10,787,677$ & $10,858,651$ & duplication & - & not rare \\
\hline 204531 chr3 & $128,382,258$ & $128,412,024$ & duplication & - & not rare \\
\hline 204531 chr3 & $13,681,185$ & $13,682,137$ & deletion & - & not rare \\
\hline 204531 chr3 & $191,989,642$ & $191,996,202$ & duplication & - & not rare \\
\hline 204531 chr3 & $195,458,723$ & $195,464,113$ & duplication & MUC20 & not rare \\
\hline 204531 chr3 & $21,869,649$ & $21,876,744$ & deletion & - & not rare \\
\hline 204531 chr3 & $53,032,639$ & $53,038,786$ & deletion & - & not rare \\
\hline 204531 chr3 & $8,821,742$ & $8,855,880$ & duplication & - & not rare \\
\hline 204531 chr4 & $138,092,298$ & $138,098,601$ & deletion & - & not rare \\
\hline 204531 chr4 & $34,785,955$ & $34,818,502$ & duplication & - & not rare \\
\hline 204531 chr4 & $69,374,369$ & $69,489,473$ & deletion & UGT2B17 & not rare \\
\hline 204531 chr5 & $180,377,470$ & $180,424,820$ & deletion & \multicolumn{2}{|c|}{ BTNL8;BTN not rare } \\
\hline 204531 chr5 & $24,778,523$ & $24,782,716$ & deletion & - & not rare \\
\hline
\end{tabular}




\begin{tabular}{|c|c|c|c|c|c|}
\hline 204531 chr6 & $12,832,840$ & $12,836,255$ & deletion & - & not rare \\
\hline 04531 chr6 & $16,391,928$ & $16,399,841$ & duplication & - & not rare \\
\hline 4531 chr6 & $67,011,900$ & $67,048,885$ & deletion & - & ot rare \\
\hline 4531 chr7 & $75,664,539$ & $75,667,743$ & deletion & - & not rare \\
\hline 04531 chr9 & $104,715,329$ & $104,722,688$ & deletion & - & not rare \\
\hline 04531 & $105,086,690$ & $105,273,648$ & deletion & - & rare \\
\hline 4531 chr9 & $135,939,032$ & $135,957,091$ & duplication & CEL & not rare \\
\hline 04531 chr9 & $66,820,824$ & $66,862,785$ & deletion & - & rare \\
\hline 04531 chr9 & $68,996,222$ & $69,156,191$ & deletion & - & rare \\
\hline 4531 chr9 & $69,808,877$ & $69,992,206$ & deletion & - & not rare \\
\hline 04533 chr1 & $104,153,766$ & $104,161,227$ & deletion & AMY2A & not rare \\
\hline 204533 chr1 & $22,913,051$ & $22,915,978$ & duplication & EPHA8 & not rare \\
\hline 04533 chr10 & $71,282,981$ & $71,289,190$ & deletion & - & not rare \\
\hline 204533 & $55,365,761$ & $55,451,045$ & deletion & 4;OR & are \\
\hline 204533 & $63,938,643$ & $63,953,114$ & duplication & - & not rare \\
\hline 204533 chr12 & $64,015,441$ & $64,129,108$ & duplication & DPY19L2 & not rare \\
\hline 204533 chr13 & $81,017,946$ & $81,022,929$ & deletion & - & not rare \\
\hline 04533 chr15 & $20,120,214$ & $20,144,522$ & deletion & - & not rare \\
\hline 04533 chr17 & $3,838,626$ & $3,852,881$ & deletion & ATP2A3 & not rare \\
\hline 204533 chr19 & $43,703,965$ & $43,759,382$ & deletion & PSG4;PSG9 & not rare \\
\hline 204533 chr19 & $53,518,747$ & $53,552,296$ & duplication & ERVV-1 & not rare \\
\hline 204533 chr2 & $195,677,877$ & $195,698,234$ & deletion & - & not rare \\
\hline 204533 chr20 & $24,887,251$ & $24,893,947$ & deletion & & not rare \\
\hline 204533 chr20 & $59,569,516$ & $59,590,280$ & du & & not rare \\
\hline 204533 chr21 & $10,797,565$ & $10,858,651$ & dup & & not rare \\
\hline 204533 chr3 & $173,256,552$ & $173,290,024$ & ation & . & not rare \\
\hline 204533 chr3 & $18,234,144$ & $18,244,588$ & dele & - & are \\
\hline 204533 chr3 & $29,835,768$ & $29,850,829$ & deletion & - & not rare \\
\hline 204533 chr3 & $37,979,882$ & $37,986,734$ & deletion & - & not rare \\
\hline 204533 chr3 & $49,690,199$ & $49,707,632$ & duplication & BSN & not rare \\
\hline 204533 chr3 & $63,578,618$ & $63,580,540$ & deletion & - & not rare \\
\hline 204533 chr4 & $161,048,496$ & $161,072,119$ & deletion & - & not rare \\
\hline 204533 chr4 & $60,325,242$ & $60,330,880$ & duplication & - & not rare \\
\hline 204533 chr4 & $69,374,369$ & $69,489,473$ & deletion & UGT2B17 & not rare \\
\hline 204533 chr5 & $103,443,017$ & $103,452,929$ & deletion & - & not rare \\
\hline 204533 chr5 & $144,679,911$ & $144,713,515$ & duplication & - & rare \\
\hline 204533 chr5 & $180,377,470$ & $180,424,820$ & deletion & \multicolumn{2}{|c|}{ BTNL8;BTN not rare } \\
\hline 204533 chr6 & $31,359,548$ & $31,453,781$ & deletion & MICA & not rare \\
\hline 204533 chr6 & 665,448 & 670,182 & deletion & - & not rare \\
\hline 204533 chr7 & $17,908,546$ & $18,069,638$ & duplication & \multicolumn{2}{|c|}{ PRPS1L1;SI rare } \\
\hline 204533 chr8 & $39,232,581$ & $39,385,979$ & duplication & - & not rare \\
\hline 204533 chr8 & $92,110,936$ & $92,185,168$ & deletion & LRRC69 & not rare \\
\hline 204533 chr9 & $1,446,522$ & $1,450,465$ & deletion & - & not rare \\
\hline 204533 chrX & $95,446,396$ & $95,480,629$ & deletion & - & not rare \\
\hline 204537 chr1 & $149,024,218$ & $149,237,009$ & deletion & - & not rare \\
\hline 204537 chr1 & $41,346,428$ & $41,380,543$ & duplication & - & not rare \\
\hline 204537 chr10 & $39,119,741$ & $39,154,535$ & duplication & - & not rare \\
\hline
\end{tabular}




\begin{tabular}{|c|c|c|c|c|c|c|}
\hline 04537 & chr11 & 70 & 2,221 & deletion & - & not rare \\
\hline 04537 & $\operatorname{chr} 11$ & $89,540,337$ & $89,692,656$ & deletion & \multicolumn{2}{|c|}{ TRIM64B;T not rare } \\
\hline 4537 & chr12 & $33,293,907$ & $33,305,855$ & duplication & - & not rare \\
\hline 4537 & $\operatorname{chr} 12$ & $43,021,431$ & $43,026,485$ & deletion & - & not rare \\
\hline 04537 & chr15 & $32,910,844$ & $33,016,154$ & duplication & \multicolumn{2}{|c|}{ SCG5;ARHC not rare } \\
\hline 04537 & chr15 & $33,028,724$ & $33,045,374$ & duplication & - & not rare \\
\hline 4537 & chr15 & $34,717,697$ & $34,814,869$ & deletion & - & not rare \\
\hline 04537 & chr16 & $54,408,688$ & $54,418,975$ & deletion & - & not rare \\
\hline 4537 & chr19 & $24,460,170$ & $24,462,326$ & deletion & - & not rare \\
\hline 4537 & chr19 & $43,393,926$ & $43,470,161$ & duplication & \multicolumn{2}{|c|}{ PSG6;PSG7 not rare } \\
\hline 204537 & chr19 & $43,703,965$ & $43,759,382$ & deletion & \multicolumn{2}{|c|}{ PSG4;PSG9 not rare } \\
\hline 204537 & chr2 & $223,865,393$ & $223,869,820$ & deletion & - & not rare \\
\hline 04537 & chr2 & $228,244,107$ & $228,258,288$ & duplication & - & not rare \\
\hline 204537 & chr2 & $41,324,723$ & $41,335,531$ & duplication & - & not rare \\
\hline 04537 & chr20 & $16,663,069$ & $16,666,053$ & deletion & - & not rare \\
\hline 204537 & $\operatorname{chr} 22$ & $24,516,291$ & $24,999,249$ & duplication & \multicolumn{2}{|c|}{ ADORA2A;: rare } \\
\hline 204537 & chr22 & $25,663,994$ & $25,919,492$ & duplication & LRP5L & not rare \\
\hline 204537 & chr3 & $128,382,258$ & $128,412,024$ & deletion & - & not rare \\
\hline 04537 & chr3 & 1,896 & $131,712,898$ & tion & - & \\
\hline 204537 & chr3 & $148,964,692$ & $148,969,220$ & deletion & - & not rare \\
\hline 204537 & chr3 & $6,318,899$ & $6,335,989$ & duplication & - & not rare \\
\hline 204537 & chr4 & $122,285,263$ & $122,289,863$ & deletion & - & not rare \\
\hline 204537 & chr4 & $69,374,369$ & $69,420,607$ & duplication & B17 & not rare \\
\hline 204537 & chr5 & $177,390,271$ & $177,400,704$ & deletion & & are \\
\hline 204537 & chr5 & $7,178,644$ & $7,191,074$ & dele & & not rare \\
\hline 204537 & chr6 & $132,114,944$ & $132,118,192$ & deletion & - & not rare \\
\hline 204537 & chr6 & 28,01 & $28,022,248$ & & - & \\
\hline 204537 & chr6 & $29,859,135$ & $29,899,389$ & deletion & - & not rare \\
\hline 204537 & chr6 & $31,276,760$ & $31,286,577$ & deletion & - & not rare \\
\hline 204537 & chr6 & $31,872,383$ & $31,878,433$ & deletion & - & not rare \\
\hline 204537 & chr6 & $67,011,900$ & $67,048,885$ & deletion & - & not rare \\
\hline 204537 & chr6 & $78,972,930$ & $79,035,739$ & deletion & - & not rare \\
\hline 204537 & chr7 & $3,721,572$ & $3,722,662$ & deletion & - & not rare \\
\hline 204537 & chr7 & $86,940,652$ & $86,948,364$ & deletion & - & not rare \\
\hline 204537 & chr8 & $24,972,808$ & $24,990,418$ & duplication & - & not rare \\
\hline 204537 & chr8 & $40,184,872$ & $40,189,688$ & deletion & - & not rare \\
\hline 204537 & chr8 & $51,155,162$ & $51,197,048$ & deletion & - & rare \\
\hline 204537 & chr8 & $60,996,982$ & $61,006,187$ & deletion & - & not rare \\
\hline 204537 & chr9 & $120,027,809$ & $120,041,792$ & duplication & - & not rare \\
\hline 204537 & chr9 & $23,363,526$ & $23,376,817$ & duplication & - & not rare \\
\hline 204537 & chr9 & $78,004,378$ & $78,011,616$ & deletion & - & not rare \\
\hline 204538 & chr1 & $108,734,695$ & $108,737,586$ & deletion & SLC25A24 & not rare \\
\hline 204538 & chr1 & $112,693,423$ & $112,704,581$ & deletion & - & not rare \\
\hline 204538 & chr1 & $187,717,153$ & $187,722,032$ & deletion & - & not rare \\
\hline 204538 & chr1 & $68,366,925$ & $68,414,024$ & deletion & - & rare \\
\hline 204538 & $\mathrm{nr} 10$ & $120,898,598$ & $120,976,743$ & duplication & \multicolumn{2}{|c|}{ SFXN4;GRK rare } \\
\hline 204538 & chr11 & $24,446,609$ & $24,451,830$ & deletion & - & not rare \\
\hline
\end{tabular}




\begin{tabular}{|c|c|c|c|c|c|}
\hline 204538 chr12 & 12,5 & $12,541,869$ & deletion & - & not rare \\
\hline 04538 chr12 & $1,611,356$ & $1,614,080$ & deletion & - & not rare \\
\hline 04538 chr12 & $33,300,995$ & $33,305,855$ & duplication & - & not rare \\
\hline 4538 chr12 & $43,021,431$ & $43,026,485$ & deletion & - & not rare \\
\hline 04538 chr12 & $63,839,168$ & $63,933,098$ & duplication & - & rare \\
\hline 04538 chr13 & $32,532,761$ & $32,537,768$ & deletion & - & not rare \\
\hline 04538 chr16 & $19,945,540$ & $19,962,636$ & deletion & - & not rare \\
\hline 204538 chr16 & $78,368,600$ & $78,383,509$ & deletion & - & not rare \\
\hline 204538 chr17 & $34,438,753$ & $34,480,718$ & duplication & - & not rare \\
\hline 04538 chr17 & $39,241,960$ & $39,252,167$ & duplication & - & not rare \\
\hline 204538 chr17 & $44,166,604$ & $44,344,749$ & duplication & KANSL1 & not rare \\
\hline 204538 chr19 & $20,601,006$ & $20,717,536$ & deletion & - & not rare \\
\hline 204538 chr19 & $24,460,170$ & $24,462,326$ & deletion & - & not rare \\
\hline 204538 chr2 & $133,924,454$ & $134,105,917$ & deletion & NC & rare \\
\hline 204538 chr2 & $38,963,211$ & $38,970,130$ & duplication & - & not rare \\
\hline 204538 chr2 & $8,130,069$ & $8,136,251$ & deletion & - & not rare \\
\hline 204538 chr3 & $131,711,896$ & $131,712,898$ & deletion & - & not rare \\
\hline 204538 chr3 & $162,130,691$ & $162,142,475$ & deletion & - & not rare \\
\hline 204538 chr3 & $186,408,475$ & $186,419,335$ & del & - & are \\
\hline 204538 chr3 & $37,979,882$ & $37,986,734$ & deletion & - & not rare \\
\hline 204538 chr3 & $8,821,742$ & $8,855,880$ & duplication & - & not rare \\
\hline 204538 chr4 & $42,697,553$ & $42,708,694$ & deletion & - & not rare \\
\hline 204538 chr5 & $113,327,484$ & $113,335,230$ & deletion & & not rare \\
\hline 204538 chr5 & $155,477,866$ & $155,488,917$ & de & & not rare \\
\hline 204538 chr5 & $180,176,005$ & $180,195,526$ & dup & & not rare \\
\hline 204538 chr6 & $78,972,930$ & $79,035,739$ & deletion & 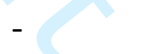 & not rare \\
\hline 204538 chr7 & $38,388,889$ & $38,402,517$ & deletion & - & not rare \\
\hline 204538 chr7 & $43,416,186$ & $43,424,112$ & deletion & - & not rare \\
\hline 204538 chr7 & 44,954 & 67,820 & duplication & - & not rare \\
\hline 204538 chr8 & $15,419,777$ & $15,432,653$ & deletion & - & not rare \\
\hline 204538 chr8 & $39,232,581$ & $39,385,979$ & duplication & - & not rare \\
\hline 204539 chr1 & $190,455,151$ & $190,472,727$ & deletion & - & not rare \\
\hline 204539 chr10 & $122,769,975$ & $122,784,282$ & deletion & - & not rare \\
\hline 204539 chr10 & $135,426,536$ & $135,446,327$ & deletion & FRG2B & not rare \\
\hline 204539 chr11 & $107,653,650$ & $107,670,228$ & duplication & SLC35F2 & not rare \\
\hline 204539 chr11 & $40,517,081$ & $40,537,540$ & deletion & - & rare \\
\hline 204539 chr11 & $50,691,976$ & $50,756,432$ & duplication & - & not rare \\
\hline 204539 chr11 & $5,876,086$ & $5,882,123$ & deletion & OR52E8 & not rare \\
\hline 204539 chr11 & $6,190,754$ & $6,192,944$ & deletion & OR52B2 & not rare \\
\hline 204539 chr11 & $7,814,299$ & $7,827,879$ & deletion & OR5P2 & not rare \\
\hline 204539 chr12 & $34,830,934$ & $34,853,011$ & duplication & - & not rare \\
\hline 204539 chr12 & $38,180,275$ & $38,190,103$ & deletion & - & not rare \\
\hline 204539 chr12 & $57,331,591$ & $57,385,834$ & deletion & RDH16 & not rare \\
\hline 204539 chr12 & $63,942,996$ & $64,129,108$ & duplication & DPY19L2 & not rare \\
\hline 204539 chr13 & $110,515,222$ & $110,526,176$ & deletion & - & not rare \\
\hline 204539 chr13 & $32,532,541$ & $32,537,768$ & deletion & - & not rare \\
\hline 204539 chr13 & $36,326,783$ & $36,334,301$ & duplication & - & not rare \\
\hline
\end{tabular}




\begin{tabular}{|c|c|c|c|c|c|}
\hline 204539 chr13 & 88,4 & $88,439,821$ & deletion & - & rare \\
\hline 04539 chr13 & $99,738,005$ & $99,740,450$ & deletion & DOCK9 & not rare \\
\hline 4539 chr14 & $107,157,561$ & $107,175,797$ & duplication & - & not rare \\
\hline 4539 chr14 & $61,964,069$ & $61,983,628$ & deletion & - & not rare \\
\hline 4539 chr14 & $94,547,478$ & $94,547,661$ & deletion & - & not rare \\
\hline 04539 chr14 & $96,967,955$ & $96,969,168$ & deletion & OLA & not rare \\
\hline 4539 chr15 & $32,509,090$ & $32,620,088$ & duplication & - & not rare \\
\hline 04539 chr15 & $56,789,979$ & $56,800,635$ & deletion & - & not rare \\
\hline 04539 chr15 & $97,815,182$ & $97,831,832$ & deletion & - & not rare \\
\hline 4539 chr16 & $28,609,775$ & $28,620,752$ & duplication & SULT1A1 & not rare \\
\hline 04539 chr16 & $34,471,298$ & $34,756,258$ & duplication & - & not rare \\
\hline 204539 chr16 & $78,372,894$ & $78,383,509$ & deletion & - & not rare \\
\hline 04539 chr17 & $34,438,753$ & $34,481,492$ & duplication & - & not rare \\
\hline 204539 chr17 & $73,429,056$ & $73,455,833$ & deletion & - & rare \\
\hline 204539 chr19 & $20,601,006$ & $20,717,536$ & deletion & - & not rare \\
\hline 204539 chr19 & $43,703,965$ & $43,759,382$ & deletion & PS & not rare \\
\hline 204539 chr19 & $51,256,120$ & $51,264,934$ & duplication & - & not rare \\
\hline 204539 chr19 & $53,518,747$ & $53,552,296$ & duplication & & not rare \\
\hline 204539 chr19 & $9,275,282$ & $9,282,176$ & & - & are \\
\hline 204539 chr2 & $130,550,135$ & $130,557,737$ & deletion & - & not rare \\
\hline 204539 chr2 & $185,099,836$ & $185,138,026$ & deletion & - & not rare \\
\hline 204539 chr20 & $16,566,568$ & $16,586,898$ & dele & - & not rare \\
\hline 204539 chr3 & $46,798,133$ & $46,845,618$ & del & & not rare \\
\hline $204539 \mathrm{chr} 4$ & $116,168,158$ & $116,173,928$ & & & not rare \\
\hline 204539 chr4 & $138,092,298$ & $138,098,601$ & del & & not rare \\
\hline 204539 chr4 & $29,034,685$ & $29,040,417$ & dele & . & not rare \\
\hline 204539 chr4 & $35,148,652$ & $35,151,237$ & & - & are \\
\hline 204539 chr4 & $38,456,729$ & $38,466,378$ & deletion & - & not rare \\
\hline $204539 \mathrm{chr} 4$ & $49,588,812$ & $49,628,667$ & duplication & - & rare \\
\hline $204539 \mathrm{chr} 4$ & $65,822,100$ & $65,840,580$ & deletion & - & not rare \\
\hline 204539 chr4 & $69,374,369$ & $69,489,473$ & deletion & UGT2B17 & not rare \\
\hline 204539 chr4 & $70,137,897$ & $70,229,260$ & deletion & UGT2B28 & not rare \\
\hline 204539 chr5 & $137,667,200$ & $137,677,862$ & deletion & CDC25C;FA & not rare \\
\hline 204539 chr5 & $140,720,954$ & $140,724,591$ & deletion & PCDHGA2; & Inot rare \\
\hline 204539 chr5 & $17,599,857$ & $17,642,516$ & deletion & - & not rare \\
\hline 204539 chr5 & $34,383,135$ & $34,410,258$ & duplication & - & rare \\
\hline 204539 chr5 & 722,205 & 840,717 & duplication & ZDHHC11 & not rare \\
\hline 204539 chr6 & $124,430,135$ & $124,471,820$ & duplication & NKAIN2 & not rare \\
\hline 204539 chr6 & $31,938,857$ & $31,940,315$ & deletion & DXO;STK19 & not rare \\
\hline 204539 chr6 & $32,811,530$ & $32,813,052$ & deletion & PSMB8 & not rare \\
\hline 204539 chr6 & $32,936,373$ & $32,940,815$ & deletion & BRD2 & not rare \\
\hline 204539 chr6 & $38,395,677$ & $38,404,140$ & deletion & - & not rare \\
\hline 204539 chr6 & $78,972,930$ & $79,035,739$ & deletion & - & not rare \\
\hline 204539 chr7 & $75,561,320$ & $75,576,189$ & deletion & - & not rare \\
\hline 204539 chr8 & $39,232,581$ & $39,385,979$ & duplication & - & not rare \\
\hline 204539 chr8 & $51,031,221$ & $51,038,149$ & deletion & - & not rare \\
\hline 204539 chr8 & $51,226,087$ & $51,227,777$ & deletion & - & not rare \\
\hline
\end{tabular}




\begin{tabular}{|c|c|c|c|c|c|}
\hline 204539 chr9 & $117,735,017$ & $117,744,829$ & deletion & - & not rare \\
\hline 04539 chr9 & $139,668,443$ & $139,676,734$ & deletion & - & not rare \\
\hline 04539 chr9 & $44,734,155$ & $44,841,930$ & duplication & - & not rare \\
\hline 04540 chr1 & $104,153,766$ & $104,161,227$ & deletion & $A M Y 2 A$ & not rare \\
\hline 4540 chr1 & $149,022,110$ & $149,237,009$ & deletion & - & not rare \\
\hline 04540 chr10 & $125,848,322$ & $125,856,308$ & deletion & - & not rare \\
\hline 4540 chr10 & $16,728,453$ & $16,732,150$ & deletion & - & not rare \\
\hline 204540 chr10 & $71,282,981$ & $71,289,190$ & deletion & - & not rare \\
\hline 4540 chr11 & $34,220,894$ & $34,223,636$ & deletion & - & not rare \\
\hline 4540 chr11 & $55,365,761$ & $55,451,045$ & deletion & \multicolumn{2}{|c|}{ OR4P4;OR $\angle$ not rare } \\
\hline 204540 chr11 & $7,814,299$ & $7,827,879$ & deletion & OR5P2 & not rare \\
\hline 204540 chr12 & $83,056,064$ & $83,229,883$ & duplication & TMTC2 & rare \\
\hline 04540 chr13 & $69,247,022$ & $69,267,981$ & deletion & - & not rare \\
\hline 204540 chr16 & $28,614,977$ & $28,620,752$ & duplication & SULT1A1 & not rare \\
\hline 204540 & $90,102,835$ & $90,170,495$ & duplication & \multicolumn{2}{|c|}{ GAS8;PRDA rare } \\
\hline 204540 chr17 & $10,891,369$ & $10,893,799$ & deletion & - & not rare \\
\hline 204540 chr19 & $53,518,747$ & $53,548,950$ & duplication & & not rare \\
\hline 204540 chr2 & $195,677,877$ & $195,698,234$ & deletion & - & not rare \\
\hline 204540 chr20 & $24,887,251$ & $24,893,947$ & deletion & - & not rare \\
\hline 204540 chr20 & $3,880,128$ & $3,885,100$ & deletion & - & not rare \\
\hline 204540 chr21 & $10,813,582$ & $10,858,651$ & duplication & - & not rare \\
\hline 204540 chr22 & $39,359,885$ & $39,385,663$ & & \multicolumn{2}{|c|}{ APOBEC3B not rare } \\
\hline 204540 chr3 & $131,711,896$ & $131,712,898$ & deletion & 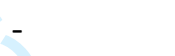 & not rare \\
\hline 204540 chr3 & $173,239,453$ & $173,290,024$ & cation & & not rare \\
\hline 204540 chr3 & 5,914 & 4,588 & dele & & \\
\hline 204540 chr3 & $29,835,768$ & $29,850,829$ & deletion & . & not rare \\
\hline 204540 chr3 & $37,979,882$ & $37,986,734$ & deletion & - & not rare \\
\hline 204540 chr3 & $63,578,618$ & $63,580,540$ & deletion & - & not rare \\
\hline $204540 \mathrm{chr} 4$ & $161,048,496$ & $161,072,119$ & deletion & - & not rare \\
\hline $204540 \mathrm{chr} 4$ & $31,166,614$ & $31,199,659$ & del & - & tale \\
\hline $204540 \mathrm{chr} 4$ & $60,325,242$ & $60,330,880$ & duplication & - & not rare \\
\hline $204540 \mathrm{chr} 4$ & $64,697,704$ & $64,709,753$ & deletion & - & not rare \\
\hline 204540 chr5 & $103,443,017$ & $103,452,929$ & deletion & - & not rare \\
\hline 204540 chr5 & $144,679,911$ & $144,713,515$ & duplication & - & rare \\
\hline 204540 chr5 & $46,266,196$ & $46,277,167$ & duplication & - & not rare \\
\hline 204540 chr5 & $70,305,696$ & $70,308,251$ & deletion & NAIP & not rare \\
\hline 204540 chr6 & $31,359,548$ & $31,453,781$ & deletion & MICA & not rare \\
\hline 204540 chr6 & 665,448 & 670,182 & deletion & - & not rare \\
\hline 204540 chr7 & $141,766,850$ & $141,793,419$ & deletion & - & not rare \\
\hline 204540 chr7 & $75,538,399$ & $75,653,545$ & deletion & \multicolumn{2}{|c|}{ STYXL1;POI rare } \\
\hline 204540 chr7 & $91,033,074$ & $91,041,618$ & deletion & - & not rare \\
\hline 204540 chr7 & $97,396,709$ & $97,401,677$ & deletion & - & not rare \\
\hline 204540 chr8 & $29,626,910$ & $29,631,546$ & deletion & - & not rare \\
\hline 204540 chr9 & $23,363,526$ & $23,376,817$ & duplication & - & not rare \\
\hline 204540 chr9 & $24,502,737$ & $24,518,795$ & deletion & - & not rare \\
\hline 204540 chrX & $95,446,396$ & $95,480,629$ & deletion & - & not rare \\
\hline 204541 chr1 & $104,153,766$ & $104,211,046$ & deletion & \multicolumn{2}{|c|}{ AMY1A;AN not rare } \\
\hline
\end{tabular}




\begin{tabular}{|c|c|c|c|c|c|}
\hline 204541 chr1 & $49,985,138$ & $50,093,073$ & duplication & - & rare \\
\hline 204541 chr1 & $62,113,597$ & $62,119,136$ & deletion & - & not rare \\
\hline 04541 chr1 & $9,847,290$ & $9,855,529$ & deletion & - & not rare \\
\hline 04541 chr11 & $22,842,737$ & $22,848,336$ & deletion & SVIP & not rare \\
\hline 04541 chr11 & $25,750,699$ & $25,753,430$ & deletion & - & ot rare \\
\hline 04541 chr11 & $55,365,761$ & $55,443,269$ & deletion & \multicolumn{2}{|c|}{ OR4P4;OR $\angle$ not rare } \\
\hline 204541 chr11 & $86,304,402$ & $86,306,401$ & deletion & - & not rare \\
\hline 04541 chr12 & $106,132,903$ & $106,136,330$ & deletion & - & not rare \\
\hline 4541 chr12 & $130,295,647$ & $130,297,819$ & deletion & - & not rare \\
\hline 204541 chr12 & $43,021,431$ & $43,026,485$ & deletion & - & not rare \\
\hline 204541 chr13 & $21,728,134$ & $21,732,193$ & duplication & SKA3 & not rare \\
\hline 04541 chr13 & $34,134,809$ & $34,143,545$ & deletion & - & not rare \\
\hline 204541 chr13 & $38,076,308$ & $38,085,537$ & deletion & - & ot rare \\
\hline 204541 chr14 & $41,610,224$ & $41,661,685$ & deletion & - & not rare \\
\hline 204541 chr14 & $42,988,346$ & $42,992,844$ & deletion & - & not rare \\
\hline 204541 chr14 & $44,504,048$ & $44,531,417$ & deletion & - & not rare \\
\hline 204541 chr15 & $26,751,068$ & $26,752,373$ & deletion & - & not rare \\
\hline 204541 chr15 & $55,010,971$ & $55,015,367$ & deletion & - & ot rare \\
\hline 204541 chr16 & $60,082,237$ & $60,098,544$ & deletion & - & not rare \\
\hline 204541 chr17 & $10,891,369$ & $10,893,799$ & deletion & - & not rare \\
\hline 204541 chr19 & $21,609,213$ & $21,614,203$ & & & rare \\
\hline 204541 chr19 & $35,661,787$ & $35,665,515$ & deletion & - & not rare \\
\hline 204541 chr2 & $195,979,780$ & $195,982,450$ & & & not rare \\
\hline 204541 chr2 & $212,771,670$ & $212,773,893$ & dele & & not rare \\
\hline 204541 chr2 & $214,319,016$ & $214,331,345$ & deletion & & not rare \\
\hline 204541 chr2 & $52,032,381$ & $52,077,000$ & del & & \\
\hline 204541 chr2 & $54,479,117$ & $54,481,636$ & deletion & - & not rare \\
\hline 204541 chr20 & $24,887,251$ & $24,893,947$ & deletion & - & not rare \\
\hline 204541 chr3 & $173,239,453$ & $173,290,024$ & cation & - & rare \\
\hline 204541 chr3 & $191,062,751$ & $191,070,300$ & duplication & - & not rare \\
\hline 204541 chr3 & $69,302,563$ & $69,319,503$ & duplication & - & not rare \\
\hline 204541 chr4 & $34,785,955$ & $34,818,502$ & duplication & - & not rare \\
\hline 204541 chr4 & $69,374,369$ & $69,489,473$ & deletion & UGT2B17 & not rare \\
\hline 204541 chr4 & $70,137,897$ & $70,229,260$ & deletion & 2B28 & not rare \\
\hline 204541 chr4 & $7,182,916$ & $7,187,397$ & deletion & - & not rare \\
\hline 204541 chr5 & $151,514,956$ & $151,518,615$ & deletion & - & not rare \\
\hline 204541 chr5 & $32,106,628$ & $32,164,826$ & duplication & \multicolumn{2}{|c|}{ GOLPH3;P[ not rare } \\
\hline 204541 chr6 & $29,854,392$ & $29,899,389$ & deletion & - & not rare \\
\hline 204541 chr6 & $31,276,760$ & $31,278,472$ & deletion & - & not rare \\
\hline 204541 chr6 & $31,785,453$ & $31,795,550$ & deletion & HSPA1A & not rare \\
\hline 204541 chr6 & $32,501,715$ & $32,520,907$ & deletion & - & not rare \\
\hline 204541 chr6 & $81,284,205$ & $81,293,326$ & deletion & - & not rare \\
\hline 204541 chr7 & $154,431,180$ & $154,459,674$ & deletion & - & rare \\
\hline 204541 chr7 & $38,388,889$ & $38,402,517$ & deletion & - & not rare \\
\hline 204541 chr8 & $115,634,278$ & $115,642,459$ & deletion & - & not rare \\
\hline 204541 & $85,261,157$ & $85,268,951$ & deletion & - & not rare \\
\hline 204541 & $21,193,860$ & $21,203,153$ & duplicatic & IF & not rare \\
\hline
\end{tabular}




\begin{tabular}{|c|c|c|c|c|}
\hline 184247 chr1 & $104,104,990$ & $104,211,046$ & duplication & AMY1C;AMY1A;AMY2A;AMY2B;AMY1B \\
\hline 184247 chr1 & $187,716,107$ & $187,722,032$ & deletion & - \\
\hline 184247 chr1 & $196,835,106$ & $196,846,344$ & deletion & - \\
\hline 184247 chr10 & $20,850,624$ & $20,857,365$ & deletion & - \\
\hline 184247 chr10 & $39,107,936$ & $39,149,666$ & duplication & - \\
\hline 184247 chr10 & $56,449,714$ & $56,468,486$ & duplication & - \\
\hline 184247 chr11 & $18,949,220$ & $18,961,743$ & duplication & MRGPRX1 \\
\hline 184247 chr11 & $5,487,596$ & $5,498,649$ & deletion & - \\
\hline 184247 chr11 & $86,304,402$ & $86,306,401$ & deletion & - \\
\hline 184247 chr11 & $93,683,680$ & $93,688,541$ & deletion & - \\
\hline 184247 chr11 & $96,204,555$ & $96,276,619$ & deletion & - \\
\hline 184247 chr12 & $33,300,995$ & $33,305,855$ & deletion & - \\
\hline 184247 chr12 & $6,241,446$ & $6,247,923$ & deletion & - \\
\hline 184247 chr13 & $38,076,308$ & $38,085,537$ & deletion & - \\
\hline 184247 chr14 & $106,891,524$ & $106,931,129$ & deletion & - \\
\hline 184247 chr14 & $20,337,127$ & $20,375,610$ & duplication & OR4K2 \\
\hline 184247 chr14 & $24,439,151$ & $24,484,060$ & deletion & DHRS4L2 \\
\hline 184247 chr15 & $24,055,488$ & $24,061,557$ & deletion & - \\
\hline 184247 chr15 & $86,511,657$ & $86,521,133$ & deletion & - \\
\hline 184247 chr16 & $55,798,095$ & $55,819,443$ & deletion & - \\
\hline 184247 chr16 & $71,356,102$ & $71,360,615$ & deletion & - \\
\hline 184247 chr17 & $44,166,604$ & $44,342,378$ & duplication & KANSL1 \\
\hline 184247 chr17 & $54,160,465$ & $54,172,668$ & deletion & - \\
\hline 184247 chr17 & $61,954,471$ & $61,963,199$ & deletion & $\mathrm{GH} 2$ \\
\hline 184247 chr17 & $67,268,689$ & $67,316,837$ & deletion & ABCA5 \\
\hline 184247 chr17 & $77,366,358$ & $77,392,062$ & duplication & - \\
\hline 184247 chr19 & $41,355,999$ & $41,387,154$ & duplication & CYP2A7;CYP2A6 \\
\hline 184247 chr19 & $43,298,958$ & $43,539,189$ & deletion & PSG6;PSG7;PSG11;PSG1 \\
\hline 184247 chr19 & $53,681,189$ & $53,708,639$ & duplication & ZNF665 \\
\hline 184247 chr19 & $55,298,309$ & $55,343,503$ & duplication & KIR3DL1;KIR2DL4 \\
\hline 184247 chr2 & $123,477,465$ & $123,482,301$ & deletion & - \\
\hline 184247 chr2 & $184,085,551$ & $184,088,256$ & deletion & - \\
\hline 184247 chr2 & $223,865,393$ & $223,869,820$ & deletion & - \\
\hline 184247 chr2 & $38,956,947$ & $38,971,623$ & duplication & GALM \\
\hline 184247 chr2 & $4,212,725$ & $4,222,144$ & deletion & - \\
\hline $184247 \mathrm{chr} 2$ & $52,759,890$ & $52,784,952$ & deletion & - \\
\hline 184247 chr20 & $29,428,644$ & $29,485,460$ & deletion & - \\
\hline 184247 chr3 & $131,711,896$ & $131,712,898$ & deletion & - \\
\hline $184247 \mathrm{chr} 3$ & $191,065,392$ & $191,070,300$ & deletion & - \\
\hline
\end{tabular}




\begin{tabular}{|c|c|c|c|c|}
\hline 184247 chr3 & $45,543,313$ & $45,552,027$ & deletion & - \\
\hline 184247 chr3 & $53,032,639$ & $53,038,786$ & deletion & - \\
\hline 184247 chr3 & $6,651,929$ & $6,654,060$ & deletion & - \\
\hline 184247 chr3 & $75,428,675$ & $75,643,261$ & deletion & - \\
\hline 184247 chr4 & $108,064,023$ & $108,074,123$ & deletion & - \\
\hline 184247 chr4 & $25,641,051$ & $25,681,579$ & duplication & SLC34A2 \\
\hline $184247 \mathrm{chr} 4$ & $69,374,369$ & $69,489,473$ & duplication & UGT2B17 \\
\hline 184247 chr4 & $70,137,897$ & $70,229,260$ & deletion & UGT2B28 \\
\hline 184247 chr5 & $111,937,859$ & $111,944,746$ & deletion & - \\
\hline 184247 chr5 & $12,687,617$ & $12,741,059$ & deletion & - \\
\hline 184247 chr5 & $155,477,866$ & $155,488,917$ & deletion & - \\
\hline 184247 chr5 & $27,607,894$ & $27,632,559$ & deletion & - \\
\hline 184247 chr5 & 788,646 & 825,338 & deletion & ZDHHC11 \\
\hline 184247 chr5 & $8,258,458$ & $8,260,630$ & deletion & - \\
\hline 184247 chr6 & $110,396,540$ & $110,414,749$ & deletion & - \\
\hline 184247 chr6 & $78,972,930$ & $79,035,739$ & deletion & - \\
\hline 184247 chr7 & $141,766,850$ & $141,793,521$ & deletion & - \\
\hline 184247 chr7 & $142,476,094$ & $142,484,042$ & deletion & - \\
\hline 184247 chr7 & $38,291,851$ & $38,305,404$ & duplication & TARP \\
\hline 184247 chr7 & $62,149,006$ & $62,162,385$ & deletion & - \\
\hline 184247 chr7 & $97,396,709$ & $97,401,677$ & deletion & - \\
\hline 184247 chr8 & $39,232,581$ & $39,382,903$ & deletion & - \\
\hline 184247 chr9 & $23,363,526$ & $23,376,817$ & deletion & - \\
\hline 184247 chr9 & $6,701,130$ & $6,707,794$ & deletion & - \\
\hline 184274 chr1 & $152,526,812$ & $152,552,461$ & deletion & LCE3D;LCE3E \\
\hline 184274 chr1 & $187,716,107$ & $187,722,032$ & deletion & - \\
\hline 184274 chr11 & $18,949,220$ & $18,961,743$ & duplication & MRGPRX1 \\
\hline 184274 chr11 & $24,083,684$ & $24,140,697$ & deletion & - \\
\hline 184274 chr11 & $55,365,761$ & $55,443,269$ & deletion & OR4P4;OR4C6;OR4C11;OR4S2 \\
\hline 184274 chr12 & $129,230,513$ & $129,233,013$ & deletion & - \\
\hline 184274 chr13 & $34,134,707$ & $34,143,545$ & deletion & - \\
\hline 184274 chr13 & $38,076,308$ & $38,085,537$ & deletion & - \\
\hline 184274 chr14 & $20,213,037$ & $20,398,906$ & duplication & OR4Q3;OR4N2;OR4K5;OR4M1;OR4K2 \\
\hline 184274 chr14 & $41,610,224$ & $41,661,685$ & deletion & - \\
\hline 184274 chr14 & $47,708,397$ & $47,715,702$ & deletion & - \\
\hline 184274 chr15 & $61,688,169$ & $61,698,179$ & deletion & - \\
\hline 184274 chr16 & $19,945,540$ & $19,962,636$ & deletion & - \\
\hline 184274 chr16 & $78,372,894$ & $78,383,509$ & deletion & - \\
\hline 184274 chr17 & $34,438,753$ & $34,483,342$ & duplication & - \\
\hline 184274 chr19 & $1,671,549$ & $1,677,696$ & deletion & - \\
\hline 184274 chr2 & $10,885,901$ & $10,890,881$ & duplication & - \\
\hline 184274 chr2 & $123,477,263$ & $123,482,301$ & deletion & - \\
\hline 184274 chr2 & $184,085,551$ & $184,088,256$ & deletion & - \\
\hline 184274 chr2 & $4,212,725$ & $4,222,144$ & deletion & - \\
\hline 184274 chr2 & $54,479,117$ & $54,481,636$ & deletion & - \\
\hline 184274 chr21 & $10,815,443$ & $10,863,251$ & duplication & - \\
\hline 184274 chr22 & $39,359,885$ & $39,385,663$ & deletion & АРОВЕСЗВ \\
\hline
\end{tabular}




\begin{tabular}{|c|c|c|c|c|}
\hline $184274 \mathrm{chr} 3$ & $114,288,641$ & $114,290,148$ & deletion & - \\
\hline $184274 \mathrm{chr} 3$ & $18,235,914$ & $18,244,588$ & deletion & - \\
\hline $184274 \mathrm{chr} 3$ & $191,065,392$ & $191,070,300$ & deletion & - \\
\hline $184274 \mathrm{chr} 3$ & $193,136,358$ & $193,140,348$ & deletion & - \\
\hline $184274 \mathrm{chr} 3$ & $195,457,853$ & $195,468,690$ & duplication & MUC20 \\
\hline 184274 chr3 & $29,835,031$ & $29,850,829$ & deletion & - \\
\hline 184274 chr3 & $53,032,639$ & $53,038,786$ & deletion & - \\
\hline $184274 \mathrm{chr} 3$ & $6,651,929$ & $6,654,060$ & deletion & - \\
\hline $184274 \mathrm{chr} 3$ & $68,793,418$ & $68,797,070$ & deletion & - \\
\hline 184274 chr4 & $58,256,666$ & $58,261,728$ & deletion & - \\
\hline $184274 \mathrm{chr} 4$ & $59,980,062$ & $59,988,095$ & duplication & - \\
\hline 184274 chr6 & $29,545,208$ & $29,551,861$ & deletion & - \\
\hline 184274 chr6 & $31,338,528$ & $31,341,357$ & deletion & - \\
\hline 184274 chr8 & $39,232,581$ & $39,385,979$ & duplication & - \\
\hline 184274 chr8 & $43,604,409$ & $43,625,127$ & deletion & - \\
\hline 184274 chr9 & $138,195,113$ & $138,195,756$ & deletion & - \\
\hline 184274 chr9 & $6,664,282$ & $6,670,263$ & deletion & - \\
\hline 184274 chr9 & $69,786,015$ & $69,992,206$ & deletion & - \\
\hline 184276 chr1 & $161,559,327$ & $161,619,741$ & duplication & FCGR2C;FCGR3B \\
\hline $184276 \mathrm{chr} 1$ & $187,716,107$ & $187,722,032$ & deletion & - \\
\hline 184276 chr1 & $245,636,900$ & $245,647,809$ & deletion & - \\
\hline 184276 chr10 & $39,127,750$ & $39,154,535$ & duplication & - \\
\hline 184276 chr11 & $18,949,220$ & $18,961,743$ & duplication & MRGPRX1 \\
\hline 184276 chr11 & $55,370,325$ & $55,422,275$ & deletion & OR4P4;OR4C11;OR4S2 \\
\hline 184276 chr12 & $11,220,827$ & $11,244,117$ & deletion & TAS2R43 \\
\hline 184276 chr12 & $132,131,087$ & $132,136,919$ & deletion & - \\
\hline 184276 chr12 & $31,907,259$ & $31,909,635$ & deletion & - \\
\hline 184276 chr13 & $64,223,907$ & $64,236,288$ & deletion & - \\
\hline 184276 chr13 & $84,161,129$ & $84,183,113$ & duplication & - \\
\hline 184276 chr14 & $41,610,224$ & $41,661,685$ & deletion & - \\
\hline 184276 chr15 & $20,196,661$ & $21,241,118$ & duplication & GOLGA6L6;POTEB2;POTEB;POTEB3 \\
\hline 184276 chr15 & $21,903,815$ & $22,753,733$ & duplication & GOLGA6L22;GOLGA6L1;OR4N4;POTEB3;OR4N \\
\hline $184276 \mathrm{chr} 16$ & $27,337,036$ & $27,350,687$ & deletion & - \\
\hline 184276 chr16 & $55,798,095$ & $55,819,443$ & deletion & - \\
\hline 184276 chr17 & $44,163,925$ & $44,224,272$ & duplication & KANSL1 \\
\hline 184276 chr18 & $55,931,179$ & $55,937,109$ & deletion & - \\
\hline 184276 chr18 & $66,746,208$ & $66,755,736$ & deletion & - \\
\hline 184276 chr19 & $41,355,999$ & $41,386,033$ & deletion & CYP2A7;CYP2A6 \\
\hline 184276 chr19 & $9,275,282$ & $9,282,176$ & deletion & - \\
\hline $184276 \mathrm{chr} 2$ & $123,477,465$ & $123,482,301$ & deletion & - \\
\hline $184276 \mathrm{chr} 2$ & $130,241,749$ & $130,250,978$ & deletion & - \\
\hline $184276 \mathrm{chr} 2$ & $38,956,947$ & $38,971,623$ & duplication & GALM \\
\hline $184276 \mathrm{chr} 20$ & $29,804,016$ & $29,982,948$ & duplication & DEFB116;DEFB115;DEFB118;DEFB119 \\
\hline $184276 \mathrm{chr} 3$ & $193,136,358$ & $193,140,348$ & deletion & - \\
\hline $184276 \mathrm{chr} 3$ & $195,457,853$ & $195,468,690$ & duplication & MUC20 \\
\hline $184276 \mathrm{chr} 3$ & $65,189,366$ & $65,214,685$ & deletion & - \\
\hline $184276 \mathrm{chr} 4$ & $108,064,023$ & $108,074,123$ & deletion & - \\
\hline
\end{tabular}




\begin{tabular}{|c|c|c|c|c|}
\hline $184276 \mathrm{chr} 4$ & $190,194,200$ & $190,199,993$ & deletion & - \\
\hline $184276 \mathrm{chr} 4$ & $34,785,955$ & $34,818,502$ & deletion & - \\
\hline $184276 \mathrm{chr} 4$ & $59,980,062$ & $59,988,095$ & duplication & - \\
\hline $184276 \mathrm{chr} 4$ & $64,136,609$ & $64,154,477$ & deletion & - \\
\hline $184276 \mathrm{chr} 4$ & $69,374,369$ & $69,489,473$ & deletion & UGT2B17 \\
\hline $184276 \mathrm{chr} 5$ & $180,176,005$ & $180,195,526$ & duplication & - \\
\hline 184276 chr5 & 762,435 & 825,338 & deletion & ZDHHC11 \\
\hline 184276 chr5 & $99,185,438$ & $99,231,572$ & duplication & - \\
\hline 184276 chr6 & $132,709,027$ & $132,711,200$ & deletion & - \\
\hline 184276 chr6 & $139,603,393$ & $139,606,731$ & deletion & - \\
\hline 184276 chr6 & $78,972,930$ & $79,035,739$ & deletion & - \\
\hline $184276 \mathrm{chr} 7$ & $17,557,177$ & $17,567,087$ & deletion & - \\
\hline 184276 chr7 & $62,149,006$ & $62,167,336$ & deletion & - \\
\hline 184276 chr7 & $67,198,842$ & $67,206,867$ & deletion & - \\
\hline 184276 chr8 & $39,232,581$ & $39,385,979$ & duplication & - \\
\hline 184276 chr9 & $32,257,426$ & $32,260,554$ & deletion & - \\
\hline 184276 chr9 & $4,045,236$ & $4,050,630$ & deletion & - \\
\hline $184281 \mathrm{chr} 1$ & $104,104,990$ & $104,211,046$ & duplication & AMY1C;AMY1A;AMY2A;AMY2B;AMY1B \\
\hline 184281 chr1 & $72,754,314$ & $72,763,324$ & deletion & - \\
\hline 184281 chr11 & $81,501,218$ & $81,517,261$ & deletion & - \\
\hline 184281 chr12 & $11,504,864$ & $11,547,551$ & deletion & PRB1;PRB2 \\
\hline 184281 chr12 & $129,230,513$ & $129,233,013$ & deletion & - \\
\hline 184281 chr12 & $43,021,431$ & $43,027,453$ & deletion & - \\
\hline 184281 chr12 & $6,241,446$ & $6,247,923$ & deletion & - \\
\hline 184281 chr13 & $34,134,809$ & $34,143,545$ & deletion & \\
\hline 184281 chr14 & $19,457,976$ & $19,470,537$ & deletion & - \\
\hline 184281 chr14 & $20,337,127$ & $20,404,736$ & duplication & OR4K5;OR4K1;OR4K2 \\
\hline 184281 chr14 & $79,160,530$ & $79,165,608$ & deletion & - \\
\hline 184281 chr15 & $34,717,697$ & $34,814,869$ & deletion & - \\
\hline 184281 chr15 & $56,789,979$ & $56,800,635$ & deletion & - \\
\hline 184281 chr16 & $78,372,894$ & $78,383,509$ & deletion & - \\
\hline 184281 chr19 & $43,647,130$ & $43,744,060$ & deletion & PSG4;PSG5 \\
\hline 184281 chr19 & $51,330,932$ & $51,333,442$ & deletion & KLK15 \\
\hline 184281 chr19 & $53,325,198$ & $53,358,889$ & duplication & ZNF468 \\
\hline 184281 chr19 & $55,293,760$ & $55,343,039$ & duplication & KIR3DL1;KIR2DL1;KIR2DL4 \\
\hline $184281 \mathrm{chr} 2$ & $195,979,780$ & $195,982,450$ & deletion & - \\
\hline $184281 \mathrm{chr} 2$ & $228,241,621$ & $228,258,288$ & duplication & TM4SF20 \\
\hline $184281 \mathrm{chr} 2$ & $233,211,861$ & $233,316,815$ & duplication & ALPP;ALPPL2 \\
\hline 184281 chr2 & $66,188,209$ & $66,196,726$ & deletion & - \\
\hline 184281 chr22 & $42,522,613$ & $42,536,917$ & deletion & CYP2D6 \\
\hline $184281 \mathrm{chr} 3$ & $125,438,148$ & $125,466,689$ & deletion & - \\
\hline $184281 \mathrm{chr} 4$ & $138,091,446$ & $138,098,601$ & deletion & - \\
\hline $184281 \mathrm{chr} 4$ & $190,194,200$ & $190,199,993$ & deletion & - \\
\hline $184281 \mathrm{chr} 4$ & $28,259,733$ & $28,270,157$ & duplication & - \\
\hline $184281 \mathrm{chr} 4$ & $34,897,243$ & $34,915,112$ & duplication & - \\
\hline $184281 \mathrm{chr} 4$ & $59,166,999$ & $59,180,928$ & deletion & - \\
\hline $184281 \mathrm{chr} 4$ & $69,374,369$ & $69,431,280$ & deletion & UGT2B17 \\
\hline
\end{tabular}




\begin{tabular}{|c|c|c|c|c|}
\hline $184281 \mathrm{chr} 5$ & $11,448,668$ & $11,499,250$ & deletion & - \\
\hline 184281 chr5 & $140,225,908$ & $140,238,311$ & deletion & PCDHA10;PCDHA9 \\
\hline 184281 chr5 & $155,477,866$ & $155,488,917$ & deletion & - \\
\hline $184281 \mathrm{chr} 5$ & $29,622,852$ & $29,634,027$ & duplication & - \\
\hline $184281 \mathrm{chr} 5$ & 760,502 & 836,608 & duplication & ZDHHC11 \\
\hline 184281 chr6 & $31,360,255$ & $31,453,640$ & duplication & MICA \\
\hline 184281 chr6 & $77,439,769$ & $77,451,301$ & deletion & - \\
\hline $184281 \mathrm{chr} 7$ & $159,118,443$ & $159,122,741$ & deletion & - \\
\hline $184281 \mathrm{chr} 8$ & $24,972,808$ & $24,990,418$ & duplication & - \\
\hline $184281 \mathrm{chr} 8$ & $3,786,543$ & $3,790,254$ & deletion & - \\
\hline 184281 chr8 & $39,236,582$ & $39,370,142$ & deletion & - \\
\hline 184281 chr8 & $51,013,729$ & $51,038,149$ & deletion & - \\
\hline 184281 chr9 & $44,068,410$ & $44,087,469$ & deletion & - \\
\hline 184281 chr9 & $78,004,378$ & $78,011,616$ & deletion & - \\
\hline 184281 chr9 & $85,373,362$ & $85,383,934$ & deletion & - \\
\hline 184282 chr1 & $104,104,990$ & $104,211,046$ & duplication & AMY1C;AMY1A;AMY2A;AMY2B;AMY1B \\
\hline $184282 \mathrm{chr} 1$ & $212,295,231$ & $212,322,447$ & deletion & - \\
\hline 184282 chr1 & $81,999,668$ & $82,019,285$ & deletion & - \\
\hline 184282 chr10 & $15,015,786$ & $15,036,242$ & deletion & - \\
\hline 184282 chr10 & $47,541,708$ & $47,756,065$ & duplication & ANTXRL;ANXA8L1 \\
\hline $184282 \mathrm{chr} 12$ & $11,511,132$ & $11,546,987$ & deletion & PRB2 \\
\hline 184282 chr12 & $59,935,926$ & $59,942,122$ & deletion & - \\
\hline $184282 \mathrm{chr} 12$ & $7,996,684$ & $8,123,306$ & duplication & SLC2A14;SLC2A3 \\
\hline $184282 \mathrm{chr} 13$ & $113,261,829$ & $113,341,464$ & duplication & ATP11AUN \\
\hline $184282 \mathrm{chr} 13$ & $34,134,809$ & $34,143,545$ & deletion & - \\
\hline $184282 \mathrm{chr} 13$ & $38,076,308$ & $38,085,537$ & deletion & - \\
\hline 184282 chr14 & $20,197,311$ & $20,425,051$ & duplication & OR4K5;OR4K1;OR4K2;OR4Q3;OR4N2;OR4M1 \\
\hline 184282 chr14 & $28,461,086$ & $28,519,804$ & deletion & - \\
\hline $184282 \mathrm{chr} 14$ & $66,054,583$ & $66,063,025$ & deletion & - \\
\hline $184282 \mathrm{chr} 15$ & $24,521,050$ & $24,660,014$ & duplication & - \\
\hline 184282 chr16 & $1,849,916$ & $1,855,743$ & deletion & - \\
\hline 184282 chr16 & $27,054,123$ & $27,087,947$ & duplication & C16orf82 \\
\hline $184282 \mathrm{chr} 16$ & $32,531,934$ & $32,833,893$ & deletion & TP53TG3B;TP53TG3;TP53TG3C \\
\hline $184282 \mathrm{chr} 16$ & $75,539,436$ & $75,578,459$ & duplication & TMEM231;CHST5 \\
\hline 184282 chr17 & $1,953,037$ & $1,964,441$ & deletion & HIC1 \\
\hline 184282 chr17 & $34,438,753$ & $34,534,918$ & duplication & CCL3L3;CCL3L1 \\
\hline 184282 chr19 & $53,516,663$ & $53,552,296$ & duplication & ERVV-1 \\
\hline 184282 chr2 & $161,446,028$ & $161,489,793$ & deletion & - \\
\hline 184282 chr2 & $4,212,725$ & $4,222,144$ & deletion & - \\
\hline 184282 chr2 & $52,759,890$ & $52,784,952$ & deletion & - \\
\hline 184282 chr2 & $77,977,262$ & $77,998,970$ & duplication & - \\
\hline 184282 chr20 & $16,663,069$ & $16,666,053$ & deletion & - \\
\hline 184282 chr20 & $36,895,860$ & $36,987,885$ & deletion & BPI;LBP \\
\hline 184282 chr20 & $37,008,769$ & $37,014,876$ & deletion & - \\
\hline 184282 chr21 & $23,655,900$ & $23,665,470$ & deletion & - \\
\hline $184282 \mathrm{chr} 3$ & $114,659,252$ & $114,667,896$ & deletion & - \\
\hline 184282 chr3 & $162,130,691$ & $162,142,475$ & deletion & - \\
\hline
\end{tabular}




\begin{tabular}{|c|c|c|c|c|}
\hline $184282 \mathrm{chr} 3$ & $197,844,320$ & $197,846,134$ & deletion & - \\
\hline $184282 \mathrm{chr} 3$ & $28,810,588$ & $28,817,583$ & deletion & - \\
\hline $184282 \mathrm{chr} 3$ & $89,402,409$ & $89,418,331$ & deletion & - \\
\hline $184282 \mathrm{chr} 4$ & $162,055,725$ & $162,062,505$ & deletion & - \\
\hline $184282 \mathrm{chr} 4$ & $162,221,384$ & $162,224,679$ & deletion & - \\
\hline $184282 \mathrm{chr} 4$ & $69,374,369$ & $69,467,200$ & deletion & UGT2B17 \\
\hline $184282 \mathrm{chr} 5$ & $117,388,410$ & $117,393,058$ & deletion & - \\
\hline 184282 chr5 & $151,514,956$ & $151,518,615$ & deletion & - \\
\hline 184282 chr5 & $45,858,842$ & $45,879,274$ & deletion & - \\
\hline 184282 chr6 & $29,856,693$ & $29,899,189$ & deletion & - \\
\hline 184282 chr6 & $31,281,682$ & $31,286,577$ & deletion & - \\
\hline 184282 chr6 & $56,915,058$ & $56,954,518$ & duplication & KIAA1586 \\
\hline 184282 chr6 & $67,011,900$ & $67,048,885$ & deletion & - \\
\hline 184282 chr6 & $77,436,935$ & $77,461,400$ & deletion & - \\
\hline 184282 chr6 & $78,326,093$ & $78,356,828$ & deletion & - \\
\hline $184282 \mathrm{chr} 7$ & 149,765 & 159,207 & deletion & - \\
\hline 184282 chr8 & $146,222,122$ & $146,238,334$ & deletion & - \\
\hline 184282 chr8 & $51,226,087$ & $51,227,777$ & deletion & - \\
\hline 184282 chr9 & $78,004,378$ & $78,011,616$ & deletion & - \\
\hline $184283 \mathrm{chr} 1$ & $149,022,110$ & $149,233,182$ & deletion & - \\
\hline 184283 chr11 & $81,501,218$ & $81,517,261$ & deletion & - \\
\hline 184283 chr11 & $92,025,597$ & $92,027,585$ & deletion & - \\
\hline 184283 chr12 & $59,935,926$ & $59,942,122$ & & . \\
\hline 184283 chr12 & $6,255,862$ & $6,259,552$ & deletion & - \\
\hline 184283 chr12 & $63,940,045$ & $64,129,108$ & duplication & 19L2 \\
\hline 184283 chr12 & $70,874,037$ & $70,879,131$ & deletion & - \\
\hline 184283 chr13 & $34,134,809$ & $34,143,545$ & deletion & - \\
\hline $184283 \mathrm{chr} 13$ & $38,076,308$ & $38,085,537$ & deletion & - \\
\hline 184283 chr13 & $69,247,022$ & $69,267,981$ & deletion & - \\
\hline 184283 chr14 & $106,891,524$ & $106,931,129$ & deletion & - \\
\hline 184283 chr14 & $41,610,224$ & $41,661,685$ & deletion & - \\
\hline 184283 chr15 & $25,420,978$ & $25,430,654$ & deletion & - \\
\hline 184283 chr15 & $61,688,169$ & $61,698,179$ & deletion & - \\
\hline 184283 chr16 & $19,945,540$ & $19,962,636$ & deletion & - \\
\hline 184283 chr17 & $34,438,753$ & $34,481,236$ & duplication & - \\
\hline 184283 chr19 & $54,728,767$ & $54,741,711$ & duplication & - \\
\hline $184283 \mathrm{chr} 2$ & $123,477,465$ & $123,482,301$ & deletion & - \\
\hline $184283 \mathrm{chr} 2$ & $13,203,874$ & $13,278,990$ & duplication & - \\
\hline $184283 \mathrm{chr} 2$ & $208,351,315$ & $208,357,838$ & deletion & - \\
\hline 184283 chr21 & $10,813,582$ & $10,858,651$ & duplication & - \\
\hline 184283 chr22 & $25,663,994$ & $25,911,071$ & duplication & LRP5L \\
\hline 184283 chr3 & $191,065,392$ & $191,070,300$ & deletion & - \\
\hline $184283 \mathrm{chr} 3$ & $53,032,639$ & $53,038,786$ & deletion & - \\
\hline $184283 \mathrm{chr} 4$ & $152,789,786$ & $152,793,891$ & deletion & - \\
\hline $184283 \mathrm{chr} 4$ & $185,753,007$ & $185,760,834$ & deletion & - \\
\hline $184283 \mathrm{chr} 4$ & $34,785,955$ & $34,818,502$ & deletion & - \\
\hline 184283 & $49,499,832$ & $49,522,346$ & deletion & - \\
\hline
\end{tabular}




\begin{tabular}{|c|c|c|c|c|}
\hline $184283 \mathrm{chr} 5$ & 701,026 & 807,499 & duplication & ZDHHC11 \\
\hline 184283 chr6 & $132,114,944$ & $132,118,192$ & deletion & - \\
\hline 184283 chr6 & $40,364,325$ & $40,368,860$ & deletion & - \\
\hline 184283 chr6 & $55,827,939$ & $55,847,710$ & deletion & - \\
\hline 184283 chr6 & $78,972,930$ & $79,035,739$ & deletion & - \\
\hline 184283 chr8 & $137,682,484$ & $137,862,080$ & deletion & - \\
\hline 184283 chr8 & $138,822,798$ & $138,824,695$ & deletion & - \\
\hline 184283 chr8 & $24,972,808$ & $24,990,418$ & duplication & - \\
\hline 184283 chr8 & $39,322,364$ & $39,345,720$ & deletion & - \\
\hline 184283 chr9 & $5,304,710$ & $5,337,760$ & deletion & RLN1 \\
\hline 184283 chr9 & $6,701,130$ & $6,707,794$ & deletion & - \\
\hline 184284 chr1 & $149,032,299$ & $149,233,182$ & deletion & - \\
\hline 184284 chr1 & $161,563,386$ & $161,619,741$ & duplication & FCGR2C;FCGR3B \\
\hline 184284 chr1 & $187,716,107$ & $187,722,032$ & deletion & - \\
\hline 184284 chr11 & $81,501,218$ & $81,517,261$ & deletion & - \\
\hline 184284 chr11 & $92,025,597$ & $92,027,585$ & deletion & - \\
\hline 184284 chr12 & $31,907,259$ & $31,909,635$ & deletion & - \\
\hline 184284 chr12 & $6,255,862$ & $6,259,552$ & deletion & - \\
\hline 184284 chr12 & $6,414,911$ & $6,420,513$ & deletion & - \\
\hline 184284 chr13 & $38,076,308$ & $38,085,537$ & deletion & - \\
\hline 184284 chr14 & $106,891,524$ & $106,931,129$ & deletion & - \\
\hline 184284 chr14 & $41,610,224$ & $41,661,685$ & deletion & - \\
\hline 184284 chr15 & $34,717,697$ & $34,814,869$ & deletion & - \\
\hline 184284 chr16 & $32,531,934$ & $32,833,893$ & deletion & TP53TG3B;TP53TG3;TP53TG3C \\
\hline 184284 chr16 & $33,551,243$ & $33,590,959$ & deletion & - \\
\hline 184284 chr17 & $34,438,753$ & $34,483,342$ & duplication & - \\
\hline 184284 chr17 & $70,562,063$ & $70,565,828$ & deletion & - \\
\hline $184284 \mathrm{chr} 19$ & $54,723,310$ & $54,743,217$ & duplication & LILRA6 \\
\hline 184284 chr2 & $123,477,465$ & $123,482,301$ & deletion & - \\
\hline 184284 chr2 & $129,638,609$ & $129,645,321$ & deletion & - \\
\hline $184284 \mathrm{chr} 2$ & $208,351,315$ & $208,357,838$ & deletion & - \\
\hline 184284 chr2 & $238,554,352$ & $238,558,478$ & deletion & - \\
\hline 184284 chr20 & $1,648,606$ & $1,670,319$ & duplication & - \\
\hline 184284 chr22 & $25,663,994$ & $25,911,071$ & duplication & LRP5L \\
\hline 184284 chr22 & $42,918,711$ & $42,950,033$ & duplication & SERHL2 \\
\hline $184284 \mathrm{chr} 3$ & $191,062,751$ & $191,070,300$ & deletion & - \\
\hline 184284 chr3 & $195,457,853$ & $195,468,690$ & duplication & MUC20 \\
\hline 184284 chr3 & $53,032,639$ & $53,042,099$ & deletion & - \\
\hline $184284 \mathrm{chr} 4$ & $190,194,200$ & $190,199,993$ & deletion & - \\
\hline 184284 chr4 & $34,785,955$ & $34,818,502$ & deletion & - \\
\hline $184284 \mathrm{chr} 4$ & $69,374,369$ & $69,395,903$ & deletion & - \\
\hline 184284 chr5 & $49,499,832$ & $49,522,346$ & deletion & - \\
\hline 184284 chr5 & $8,258,458$ & $8,260,630$ & deletion & - \\
\hline 184284 chr6 & $21,826,729$ & $21,834,059$ & deletion & - \\
\hline 184284 chr6 & $55,827,939$ & $55,847,710$ & deletion & - \\
\hline 184284 chr6 & $57,654,857$ & $58,071,881$ & duplication & - \\
\hline 184284 chr6 & $61,915,697$ & $62,124,535$ & duplication & - \\
\hline
\end{tabular}




\begin{tabular}{|c|c|c|c|c|c|}
\hline 184284 & chr7 & $29,676,097$ & $29,687,592$ & deletion & - \\
\hline 184284 & chr8 & $115,633,704$ & $115,642,459$ & deletion & - \\
\hline 184284 & chr8 & $16,262,222$ & $16,272,444$ & deletion & - \\
\hline 184284 & chr8 & $5,595,511$ & $5,605,706$ & deletion & - \\
\hline 184284 & chr9 & $23,363,526$ & $23,376,817$ & deletion & - \\
\hline 184284 & chr9 & $6,701,130$ & $6,707,794$ & deletion & - \\
\hline 184284 & chr9 & $73,906,136$ & $73,909,871$ & duplication & - \\
\hline 184285 & chr1 & $143,343,508$ & $143,544,285$ & duplication & - \\
\hline 184285 & chr1 & $17,233,799$ & $17,237,982$ & duplication & - \\
\hline 184285 & chr10 & $14,025,411$ & $14,036,601$ & deletion & - \\
\hline 184285 & chr10 & $46,949,421$ & $47,151,118$ & duplication & GPRIN2;CH17-360D5.1;NPY4R;SYT15 \\
\hline 184285 & chr11 & $90,192,538$ & $90,196,843$ & deletion & - \\
\hline 184285 & chr11 & $93,019,466$ & $93,028,940$ & deletion & - \\
\hline 184285 & chr12 & $12,532,984$ & $12,544,338$ & deletion & - \\
\hline 184285 & chr12 & $59,935,926$ & $59,942,122$ & deletion & - \\
\hline 184285 & chr14 & $106,023,250$ & $106,152,191$ & duplication & - \\
\hline 184285 & chr14 & $106,626,535$ & $106,774,755$ & duplication & - \\
\hline 184285 & chr14 & $107,157,561$ & $107,181,152$ & duplication & - \\
\hline 184285 & chr14 & $20,258,645$ & $20,420,338$ & duplication & OR4N2;OR4K5;OR4K1;OR4K2 \\
\hline 184285 & chr15 & $43,888,976$ & $43,939,642$ & duplication & STRC;CATSPER2;CKMT1B \\
\hline 184285 & chr16 & $32,469,035$ & $32,642,384$ & deletion & - \\
\hline 184285 & chr16 & $35,185,903$ & $35,197,323$ & deletion & - \\
\hline 184285 & chr16 & $78,372,894$ & $78,383,509$ & deletion & - \\
\hline 184285 & chr17 & $44,166,604$ & $44,347,946$ & duplication & KANSL1 \\
\hline 184285 & chr19 & $41,355,999$ & $41,384,578$ & deletion & CYP2A7;CYP2A6 \\
\hline 184285 & chr19 & $54,728,767$ & $54,743,217$ & duplication & LILRA6 \\
\hline 184285 & chr2 & $133,008,095$ & $133,044,318$ & duplication & - \\
\hline 184285 & chr2 & $208,351,315$ & $208,357,838$ & deletion & - \\
\hline 184285 & chr2 & $231,442,026$ & $231,445,696$ & deletion & - \\
\hline 184285 & chr2 & $241,390,516$ & $241,401,741$ & deletion & GPC1 \\
\hline 184285 & chr2 & $91,625,096$ & $91,974,299$ & duplication & - \\
\hline 184285 & chr20 & $29,454,201$ & $29,500,566$ & deletion & - \\
\hline 184285 & chr20 & $44,337,422$ & $44,378,173$ & deletion & SPINT4 \\
\hline 184285 & $\operatorname{chr} 21$ & $23,655,900$ & $23,666,129$ & deletion & - \\
\hline 184285 & chr3 & $148,964,692$ & $148,970,275$ & deletion & - \\
\hline 184285 & chr3 & $37,979,882$ & $37,990,299$ & deletion & CTDSPL \\
\hline 184285 & chr3 & $40,407,555$ & $40,447,632$ & deletion & ENTPD3 \\
\hline 184285 & chr3 & $75,428,675$ & $75,643,261$ & deletion & - \\
\hline 184285 & chr4 & $49,156,305$ & $49,580,447$ & duplication & - \\
\hline 184285 & chr4 & $64,136,609$ & $64,154,477$ & deletion & - \\
\hline 184285 & chr4 & 979,175 & 985,372 & deletion & SLC26A1;IDUA \\
\hline 184285 & chr6 & $21,826,729$ & $21,834,059$ & deletion & - \\
\hline 184285 & chr6 & $29,855,945$ & $29,899,189$ & deletion & - \\
\hline 184285 & chr6 & $67,011,900$ & $67,048,885$ & deletion & - \\
\hline 184285 & chr7 & $141,766,850$ & $141,793,521$ & deletion & - \\
\hline 184285 & chr7 & $17,177,492$ & $17,228,122$ & deletion & - \\
\hline 184285 & chr8 & $115,633,704$ & $115,642,459$ & deletion & - \\
\hline
\end{tabular}




\begin{tabular}{|c|c|c|c|c|}
\hline $184285 \mathrm{chr} 8$ & $144,976,572$ & $145,032,302$ & deletion & PLEC \\
\hline 184285 chr8 & $2,199,113$ & $2,259,412$ & duplication & - \\
\hline 184285 chr8 & $32,285,858$ & $32,290,601$ & deletion & - \\
\hline 184285 chr8 & $39,232,581$ & $39,385,979$ & deletion & - \\
\hline 184285 chr9 & $107,799,020$ & $107,803,370$ & deletion & - \\
\hline 184285 chr9 & $68,336,499$ & $68,440,324$ & duplication & - \\
\hline $184286 \mathrm{chr} 1$ & $25,598,276$ & $25,659,509$ & deletion & RHD \\
\hline 184286 chr10 & $39,107,936$ & $39,149,666$ & duplication & - \\
\hline 184286 chr11 & $107,653,650$ & $107,671,155$ & duplication & SLC35F2 \\
\hline 184286 chr11 & $18,949,220$ & $18,961,743$ & duplication & MRGPRX1 \\
\hline 184286 chr11 & $55,365,761$ & $55,427,700$ & deletion & OR4P4;OR4C11;OR4S2 \\
\hline 184286 chr11 & $7,814,299$ & $7,827,879$ & deletion & OR5P2 \\
\hline 184286 chr11 & $90,193,796$ & $90,196,843$ & deletion & - \\
\hline 184286 chr12 & $33,300,995$ & $33,305,855$ & deletion & - \\
\hline 184286 chr12 & $59,935,926$ & $59,942,122$ & deletion & - \\
\hline 184286 chr13 & $58,589,893$ & $58,599,174$ & deletion & - \\
\hline 184286 chr14 & $41,610,224$ & $41,661,685$ & deletion & - \\
\hline 184286 chr14 & $79,160,530$ & $79,165,608$ & deletion & - \\
\hline 184286 chr15 & $21,903,815$ & $22,753,733$ & duplication & GOLGA6L22;GOLGA6L1;OR4N4;POTEB3;OR4N \\
\hline 184286 chr15 & $24,381,205$ & $24,436,527$ & duplication & - \\
\hline $184286 \mathrm{chr} 16$ & $11,645,147$ & $11,739,455$ & duplication & LITAF \\
\hline 184286 chr16 & $55,798,095$ & $55,819,443$ & deletion & - \\
\hline 184286 chr17 & $34,438,753$ & $34,481,236$ & duplication & - \\
\hline $184286 \mathrm{chr} 17$ & $44,166,604$ & $44,292,896$ & duplication & KANSL1 \\
\hline 184286 chr18 & $22,055,650$ & $22,060,915$ & duplication & $\mathrm{HRH} 4$ \\
\hline 184286 chr19 & $41,355,999$ & $41,386,033$ & deletion & CYP2A7;CYP2A6 \\
\hline 184286 chr19 & $53,325,198$ & $53,358,889$ & deletion & ZNF468 \\
\hline 184286 chr19 & $53,518,747$ & $53,552,296$ & duplication & ERVV-1 \\
\hline 184286 chr19 & $9,275,282$ & $9,287,189$ & deletion & - \\
\hline $184286 \mathrm{chr} 2$ & $184,795,725$ & $184,803,456$ & deletion & - \\
\hline 184286 chr2 & $195,979,780$ & $195,982,450$ & deletion & - \\
\hline 184286 chr2 & $52,759,890$ & $52,784,952$ & deletion & - \\
\hline 184286 chr22 & $37,745,101$ & $37,749,946$ & deletion & - \\
\hline 184286 chr22 & $42,522,613$ & $42,536,917$ & deletion & CYP2D6 \\
\hline 184286 chr3 & $128,382,258$ & $128,412,024$ & duplication & - \\
\hline 184286 chr3 & $148,962,265$ & $148,970,275$ & deletion & - \\
\hline $184286 \mathrm{chr} 3$ & $189,364,424$ & $189,366,763$ & deletion & - \\
\hline $184286 \mathrm{chr} 3$ & $37,979,882$ & $37,986,734$ & deletion & - \\
\hline $184286 \mathrm{chr} 3$ & $46,798,133$ & $46,845,618$ & deletion & - \\
\hline 184286 chr3 & $75,428,675$ & $75,643,261$ & deletion & - \\
\hline $184286 \mathrm{chr} 4$ & $131,522,876$ & $131,569,148$ & deletion & - \\
\hline $184286 \mathrm{chr} 4$ & $188,856,430$ & $188,940,946$ & deletion & ZFP42 \\
\hline $184286 \mathrm{chr} 4$ & $190,194,200$ & $190,200,463$ & deletion & - \\
\hline $184286 \mathrm{chr} 4$ & $60,325,010$ & $60,330,880$ & duplication & - \\
\hline $184286 \mathrm{chr} 4$ & $69,374,369$ & $69,467,200$ & deletion & UGT2B17 \\
\hline 184286 chr5 & 717,675 & 840,717 & duplication & ZDHHC11 \\
\hline $184286 \mathrm{chr} 6$ & $11,634,722$ & $11,641,048$ & deletion & - \\
\hline
\end{tabular}




\begin{tabular}{|c|c|c|c|c|}
\hline 184286 chr6 & $95,372,264$ & $95,556,864$ & deletion & - \\
\hline 184286 chr7 & $15,013,768$ & $15,033,750$ & duplication & - \\
\hline 184286 chr8 & $137,682,484$ & $137,862,080$ & deletion & - \\
\hline 184286 chr8 & $138,821,460$ & $138,833,669$ & deletion & - \\
\hline 184286 chr9 & 107,153 & 290,670 & duplication & FOXD4;C9orf66;DOCK8;CBWD1 \\
\hline 184286 chr9 & $107,799,020$ & $107,803,370$ & deletion & - \\
\hline 184286 chr9 & $24,502,737$ & $24,520,633$ & deletion & - \\
\hline $184288 \mathrm{chr} 1$ & $149,032,299$ & $149,233,182$ & deletion & - \\
\hline $184288 \mathrm{chr} 1$ & $161,489,467$ & $161,619,741$ & duplication & FCGR3B;FCGR3A;HSPA6;FCGR2C \\
\hline 184288 chr1 & $16,363,925$ & $16,382,966$ & duplication & CLCNKB \\
\hline $184288 \mathrm{chr} 1$ & $248,740,314$ & $248,795,110$ & deletion & OR2T10;OR2T11 \\
\hline 184288 chr1 & $62,113,597$ & $62,119,136$ & deletion & - \\
\hline 184288 chr1 & $76,847,681$ & $76,853,011$ & deletion & - \\
\hline 184288 chr10 & $115,209,065$ & $115,216,378$ & deletion & - \\
\hline 184288 chr10 & $38,869,302$ & $38,903,858$ & deletion & - \\
\hline 184288 chr10 & $71,280,107$ & $71,289,190$ & deletion & - \\
\hline 184288 chr11 & $18,949,220$ & $18,961,743$ & duplication & MRGPRX1 \\
\hline 184288 chr11 & $49,715,154$ & $49,757,690$ & deletion & - \\
\hline 184288 chr12 & $2,245,636$ & $2,259,042$ & deletion & - \\
\hline 184288 chr12 & $59,935,926$ & $59,942,122$ & deletion & - \\
\hline 184288 chr13 & $21,728,134$ & $21,732,348$ & duplication & SKA3 \\
\hline 184288 chr13 & $38,076,308$ & $38,085,537$ & deletion & - \\
\hline 184288 chr14 & $106,891,524$ & $106,931,129$ & deletion & - \\
\hline 184288 chr14 & $20,197,311$ & $20,424,184$ & duplication & OR4K5;OR4K1;OR4K2;OR4Q3;OR4N2;OR4M1 \\
\hline $184288 \mathrm{chr} 15$ & $25,420,978$ & $25,430,654$ & deletion & - \\
\hline 184288 chr15 & $89,602,390$ & $89,609,950$ & deletion & - \\
\hline 184288 chr15 & $97,262,427$ & $97,268,718$ & deletion & - \\
\hline 184288 chr16 & $19,945,540$ & $19,962,636$ & deletion & - \\
\hline 184288 chr16 & $34,471,298$ & $34,756,258$ & duplication & - \\
\hline 184288 chr16 & $80,941,603$ & $80,952,656$ & deletion & - \\
\hline 184288 chr17 & $1,681,654$ & $1,703,156$ & deletion & SMYD4 \\
\hline 184288 chr17 & $44,166,604$ & $44,356,058$ & duplication & KANSL1 \\
\hline 184288 chr19 & $54,728,767$ & $54,743,217$ & duplication & LILRA6 \\
\hline 184288 chr2 & $123,477,263$ & $123,482,301$ & deletion & - \\
\hline 184288 chr2 & $227,339,461$ & $227,347,453$ & deletion & - \\
\hline $184288 \mathrm{chr} 2$ & $34,697,895$ & $34,726,904$ & deletion & - \\
\hline 184288 chr2 & $38,956,947$ & $38,971,623$ & duplication & GALM \\
\hline 184288 chr2 & $51,926,599$ & $51,926,904$ & deletion & - \\
\hline 184288 chr2 & $52,759,890$ & $52,784,952$ & deletion & - \\
\hline 184288 chr20 & $29,804,016$ & $29,814,437$ & duplication & - \\
\hline 184288 chr22 & $18,874,965$ & $18,942,570$ & duplication & PRODH;DGCR6 \\
\hline 184288 chr22 & $18,966,859$ & $19,016,663$ & duplication & - \\
\hline 184288 chr22 & $25,663,994$ & $25,919,492$ & duplication & LRP5L \\
\hline 184288 chr3 & $119,352,669$ & $119,353,893$ & deletion & - \\
\hline $184288 \mathrm{chr} 3$ & $6,651,929$ & $6,654,060$ & deletion & - \\
\hline 184288 chr3 & $85,336,559$ & $85,365,682$ & duplication & - \\
\hline $184288 \mathrm{chr} 3$ & $98,945,571$ & $98,947,547$ & deletion & - \\
\hline
\end{tabular}




\begin{tabular}{|c|c|c|c|c|}
\hline $184288 \mathrm{chr} 4$ & $190,194,200$ & $190,200,463$ & deletion & - \\
\hline $184288 \mathrm{chr} 4$ & $69,374,369$ & $69,489,473$ & deletion & UGT2B17 \\
\hline 184288 chr5 & $151,514,956$ & $151,518,615$ & deletion & - \\
\hline 184288 chr5 & $155,477,866$ & $155,488,917$ & deletion & - \\
\hline 184288 chr5 & $31,446,716$ & $31,894,881$ & duplication & C5orf22;DROSHA;PDZD2 \\
\hline $184288 \mathrm{chr} 5$ & $31,938,732$ & $32,275,467$ & duplication & GOLPH3;PDZD2;MTMR12 \\
\hline 184288 chr5 & 701,026 & 788,646 & duplication & - \\
\hline 184288 chr5 & $70,305,696$ & $70,308,251$ & deletion & NAIP \\
\hline 184288 chr6 & $140,463,013$ & $140,511,643$ & deletion & - \\
\hline 184288 chr6 & $77,439,769$ & $77,451,301$ & deletion & - \\
\hline 184288 chr8 & $138,822,798$ & $138,824,695$ & deletion & - \\
\hline 184288 chr8 & $24,972,808$ & $24,990,418$ & deletion & - \\
\hline 184288 chr8 & $39,232,581$ & $39,385,979$ & duplication & - \\
\hline 184288 chr9 & $10,315,193$ & $10,328,663$ & duplication & - \\
\hline 184288 chr9 & $135,947,165$ & $135,961,787$ & deletion & - \\
\hline 184288 chr9 & $2,148,213$ & $2,151,397$ & deletion & - \\
\hline 184288 chr9 & $78,004,378$ & $78,011,616$ & deletion & - \\
\hline $184288 \mathrm{chrX}$ & $66,639,351$ & $66,730,804$ & duplication & - \\
\hline 184291 chr1 & $104,153,766$ & $104,161,227$ & deletion & $A M Y 2 A$ \\
\hline 184291 chr1 & $149,032,299$ & $149,237,009$ & deletion & - \\
\hline 184291 chr1 & $187,716,107$ & $187,722,032$ & deletion & - \\
\hline $184291 \mathrm{chr} 1$ & $94,710,008$ & $94,916,649$ & deletion & $\mathrm{ABCD} 3$ \\
\hline 184291 chr10 & $104,051,165$ & $104,064,548$ & deletion & - \\
\hline 184291 chr14 & $34,741,677$ & $34,762,804$ & deletion & - \\
\hline 184291 chr14 & $86,285,493$ & $86,312,888$ & deletion & - \\
\hline 184291 chr15 & $22,299,434$ & $22,585,470$ & duplication & OR4M2;OR4N4 \\
\hline 184291 chr15 & $28,120,566$ & $28,146,440$ & deletion & - \\
\hline 184291 chr15 & $28,286,405$ & $28,303,203$ & deletion & - \\
\hline 184291 chr16 & $32,531,934$ & $32,833,893$ & deletion & TP53TG3B;TP53TG3;TP53TG3C \\
\hline 184291 chr16 & $35,185,903$ & $35,197,323$ & deletion & - \\
\hline $184291 \mathrm{chr} 16$ & $55,800,009$ & $55,819,443$ & deletion & - \\
\hline 184291 chr16 & $78,372,894$ & $78,383,509$ & deletion & - \\
\hline 184291 chr17 & $16,576,609$ & $16,587,108$ & deletion & - \\
\hline 184291 chr18 & $41,976,831$ & $41,988,970$ & deletion & - \\
\hline 184291 chr19 & $41,355,999$ & $41,386,033$ & deletion & CYP2A7;CYP2A6 \\
\hline $184291 \mathrm{chr} 2$ & $14,704,369$ & $14,709,611$ & deletion & - \\
\hline $184291 \mathrm{chr} 2$ & $34,697,895$ & $34,726,904$ & deletion & - \\
\hline 184291 chr3 & $195,433,728$ & $195,468,690$ & duplication & MUC20 \\
\hline 184291 chr3 & $3,052,153$ & $3,055,247$ & deletion & - \\
\hline $184291 \mathrm{chr} 4$ & $126,146,483$ & $126,159,605$ & duplication & - \\
\hline $184291 \mathrm{chr} 4$ & $190,194,200$ & $190,200,463$ & deletion & - \\
\hline $184291 \mathrm{chr} 4$ & $69,374,369$ & $69,447,592$ & duplication & UGT2B17 \\
\hline $184291 \mathrm{chr} 5$ & $111,937,859$ & $111,944,746$ & deletion & - \\
\hline 184291 chr5 & $17,345,282$ & $17,356,536$ & deletion & - \\
\hline 184291 chr5 & $32,106,628$ & $32,164,826$ & duplication & GOLPH3;PDZD2 \\
\hline 184291 chr5 & $97,933,671$ & $97,944,102$ & deletion & - \\
\hline 184291 chr7 & $3,721,572$ & $3,722,662$ & deletion & - \\
\hline
\end{tabular}




\begin{tabular}{|c|c|c|c|c|c|}
\hline 184291 & chr7 & $5,882,048$ & $5,886,060$ & deletion & - \\
\hline 184291 & chr8 & $39,232,581$ & $39,385,979$ & deletion & - \\
\hline 184291 & chr8 & $5,493,500$ & $5,536,072$ & deletion & - \\
\hline 184291 & chr9 & $107,799,020$ & $107,803,370$ & deletion & - \\
\hline 184291 & chr9 & $6,701,130$ & $6,707,794$ & deletion & - \\
\hline 184291 & chrX & $6,454,813$ & $6,591,870$ & duplication & - \\
\hline 184291 & chrX & $7,502,033$ & $8,135,053$ & duplication & VCX;PNPLA4 \\
\hline 184291 & chrX & $89,893,914$ & $89,912,332$ & deletion & - \\
\hline 184292 & chr1 & $104,153,766$ & $104,211,046$ & deletion & AMY1A;AMY2A;AMY1C;AMY1B \\
\hline 184292 & chr1 & $149,024,218$ & $149,432,892$ & deletion & - \\
\hline 184292 & chr1 & $174,794,144$ & $174,801,639$ & deletion & - \\
\hline 184292 & chr1 & $248,740,314$ & $248,795,110$ & deletion & OR2T10;OR2T11 \\
\hline 184292 & chr1 & $72,754,314$ & $72,763,324$ & deletion & - \\
\hline 184292 & chr10 & $6,655,306$ & $6,663,988$ & deletion & - \\
\hline 184292 & chr11 & $18,949,220$ & $18,961,743$ & deletion & MRGPRX1 \\
\hline 184292 & chr11 & $22,837,198$ & $22,881,341$ & deletion & SVIP;CCDC179 \\
\hline 184292 & chr11 & $55,365,761$ & $55,443,269$ & duplication & OR4P4;OR4C6;OR4C11;OR4S2 \\
\hline 184292 & chr11 & $81,501,218$ & $81,517,261$ & deletion & - \\
\hline 184292 & chr11 & $85,430,380$ & $85,470,893$ & deletion & SYTL2 \\
\hline 184292 & chr11 & $90,193,427$ & $90,196,843$ & deletion & - \\
\hline 184292 & chr13 & $19,342,790$ & $19,361,026$ & deletion & - \\
\hline 184292 & chr14 & $20,213,037$ & $20,232,479$ & duplication & OR4Q3 \\
\hline 184292 & chr14 & $20,363,759$ & $20,375,610$ & duplication & - \\
\hline 184292 & chr14 & $44,589,218$ & $44,592,123$ & deletion & - \\
\hline 184292 & chr14 & $86,285,493$ & $86,312,888$ & deletion & \\
\hline 184292 & chr15 & $20,196,661$ & $21,241,118$ & duplication & GOLGA6L6;POTEB2;POTEB;POTEB3 \\
\hline 184292 & chr15 & $21,903,815$ & $22,753,733$ & duplication & GOLGA6L22;GOLGA6L1;OR4N4;POTEB3;OR4N \\
\hline 184292 & chr15 & $28,120,566$ & $28,141,304$ & deletion & - \\
\hline 184292 & chr15 & $28,286,405$ & $28,303,203$ & deletion & - \\
\hline 184292 & chr16 & $19,945,540$ & $19,962,636$ & deletion & - \\
\hline 184292 & chr16 & 223,625 & 225,653 & deletion & - \\
\hline 184292 & chr16 & $35,185,903$ & $35,197,323$ & deletion & - \\
\hline 184292 & chr16 & $55,800,009$ & $55,819,443$ & deletion & - \\
\hline 184292 & chr17 & $8,704,176$ & $8,708,703$ & deletion & PIK3R6 \\
\hline 184292 & chr19 & $41,355,999$ & $41,386,033$ & deletion & CYP2A7;CYP2A6 \\
\hline 184292 & chr2 & $14,704,369$ & $14,709,611$ & deletion & - \\
\hline 184292 & chr2 & $208,351,315$ & $208,357,838$ & deletion & - \\
\hline 184292 & chr2 & $23,486,341$ & $23,494,356$ & deletion & - \\
\hline 184292 & chr2 & $35,581,797$ & $35,614,865$ & deletion & - \\
\hline 184292 & chr2 & $41,239,457$ & $41,248,468$ & deletion & - \\
\hline 184292 & chr2 & $58,567,018$ & $58,570,117$ & deletion & - \\
\hline 184292 & chr22 & $40,592,458$ & $40,599,631$ & deletion & - \\
\hline 184292 & chr3 & $141,914,586$ & $142,091,364$ & duplication & GK5;XRN1 \\
\hline 184292 & chr3 & $142,163,158$ & $142,188,392$ & duplication & ATR;XRN1 \\
\hline 184292 & chr3 & $191,061,214$ & $191,070,300$ & deletion & - \\
\hline 184292 & chr3 & $28,810,588$ & $28,817,583$ & deletion & - \\
\hline 84292 & chr3 & $53,032,639$ & $53,038,786$ & deletion & - \\
\hline
\end{tabular}




\begin{tabular}{|c|c|c|c|c|}
\hline $184292 \mathrm{chr} 4$ & $138,092,298$ & $138,098,601$ & deletion & - \\
\hline $184292 \mathrm{chr} 4$ & $190,194,200$ & $190,200,463$ & deletion & - \\
\hline $184292 \mathrm{chr} 4$ & $69,374,369$ & $69,489,473$ & duplication & UGT2B17 \\
\hline 184292 chr5 & $111,937,859$ & $111,944,746$ & deletion & - \\
\hline $184292 \mathrm{chr} 5$ & $140,555,135$ & $140,558,618$ & duplication & PCDHB8;PCDHB7 \\
\hline 184292 chr5 & $17,345,282$ & $17,356,536$ & deletion & - \\
\hline 184292 chr5 & $32,106,628$ & $32,164,826$ & duplication & GOLPH3;PDZD2 \\
\hline 184292 chr5 & $8,258,458$ & $8,260,630$ & deletion & - \\
\hline 184292 chr5 & $97,933,671$ & $97,939,616$ & deletion & - \\
\hline 184292 chr6 & $139,603,393$ & $139,606,731$ & deletion & - \\
\hline 184292 chr7 & $112,434,936$ & $112,474,811$ & duplication & C7orf60 \\
\hline $184292 \mathrm{chr} 7$ & $156,387,296$ & $156,394,307$ & deletion & - \\
\hline 184292 chr8 & $24,972,808$ & $24,990,418$ & duplication & - \\
\hline 184292 chr8 & $39,232,581$ & $39,385,979$ & deletion & - \\
\hline 184292 chr8 & $3,967,931$ & $3,969,537$ & deletion & - \\
\hline 184292 chr8 & $47,526,999$ & $47,539,088$ & duplication & - \\
\hline $184292 \mathrm{chr} 8$ & $51,029,371$ & $51,038,149$ & deletion & - \\
\hline 184292 chr8 & $73,596,566$ & $73,638,873$ & duplication & - \\
\hline 184292 chr9 & $135,947,165$ & $135,955,493$ & duplication & - \\
\hline 184292 chr9 & $23,363,526$ & $23,376,817$ & duplication & - \\
\hline $184293 \mathrm{chr} 1$ & $104,153,766$ & $104,211,046$ & deletion & AMY1A;AMY2A;AMY1C;AMY1B \\
\hline 184293 chr1 & $190,377,988$ & $190,410,679$ & deletion & - \\
\hline 184293 chr10 & $38,677,023$ & $38,972,666$ & duplication & - \\
\hline 184293 chr10 & $82,936,649$ & $82,945,572$ & deletion & \\
\hline 184293 chr12 & $2,245,636$ & $2,254,219$ & deletion & \\
\hline 184293 chr13 & $38,076,308$ & $38,085,537$ & deletion & - \\
\hline 184293 chr13 & $58,874,904$ & $58,928,118$ & duplication & - \\
\hline 184293 chr16 & $32,531,934$ & $32,833,893$ & deletion & TP53TG3B;TP53TG3;TP53TG3C \\
\hline 184293 chr17 & $6,458,427$ & $6,463,712$ & deletion & PITPNM3 \\
\hline 184293 chr19 & $54,728,767$ & $54,743,217$ & duplication & LILRA6 \\
\hline $184293 \mathrm{chr} 2$ & $14,704,369$ & $14,709,611$ & deletion & - \\
\hline 184293 chr20 & $59,568,187$ & $59,590,280$ & duplication & - \\
\hline 184293 chr21 & $21,308,651$ & $21,340,364$ & deletion & - \\
\hline 184293 chr22 & $37,745,101$ & $37,749,946$ & deletion & - \\
\hline 184293 chr3 & $6,318,899$ & $6,335,989$ & duplication & - \\
\hline $184293 \mathrm{chr} 4$ & $122,285,263$ & $122,289,863$ & deletion & - \\
\hline $184293 \mathrm{chr} 4$ & $69,374,369$ & $69,489,473$ & duplication & UGT2B17 \\
\hline $184293 \mathrm{chr} 4$ & $70,137,897$ & $70,229,260$ & deletion & UGT2B28 \\
\hline 184293 chr6 & $110,396,540$ & $110,412,756$ & deletion & - \\
\hline 184293 chr6 & $78,972,930$ & $79,035,739$ & deletion & - \\
\hline 184293 chr6 & $81,284,205$ & $81,293,326$ & deletion & - \\
\hline 184293 chr7 & $135,206,964$ & $135,215,397$ & deletion & - \\
\hline 184293 chr7 & $141,766,850$ & $141,793,521$ & deletion & - \\
\hline 184293 chr7 & $29,676,097$ & $29,687,592$ & deletion & - \\
\hline 184293 chr7 & $63,525,242$ & $63,789,687$ & deletion & ZNF736;ZNF679;ZNF727;ZNF735 \\
\hline $184293 \mathrm{chr} 8$ & $39,232,581$ & $39,385,979$ & duplication & - \\
\hline 184293 chr8 & $9,313,017$ & $9,315,807$ & deletion & - \\
\hline
\end{tabular}




\begin{tabular}{|c|c|c|c|c|}
\hline 184294 chr1 & $161,489,467$ & $161,619,741$ & duplication & FCGR3B;FCGR3A;HSPA6;FCGR2C \\
\hline 184294 chr1 & $196,823,300$ & $196,901,753$ & deletion & CFHR4 \\
\hline 184294 chr10 & $38,677,023$ & $38,972,666$ & duplication & - \\
\hline 184294 chr11 & $49,715,154$ & $49,757,690$ & deletion & - \\
\hline 184294 chr11 & $50,751,699$ & $50,780,629$ & deletion & - \\
\hline 184294 chr15 & $34,717,697$ & $34,814,869$ & deletion & - \\
\hline 184294 chr16 & $32,562,332$ & $32,649,736$ & deletion & - \\
\hline 184294 chr16 & $35,153,618$ & $35,285,609$ & duplication & - \\
\hline 184294 chr17 & $19,499,229$ & $19,537,470$ & deletion & - \\
\hline 184294 chr17 & $34,438,753$ & $34,483,342$ & duplication & - \\
\hline 184294 chr17 & $44,166,604$ & $44,225,181$ & duplication & KANSL1 \\
\hline $184294 \mathrm{chr} 2$ & $165,850,456$ & $165,864,123$ & deletion & - \\
\hline 184294 chr2 & $212,770,611$ & $212,776,546$ & deletion & - \\
\hline 184294 chr2 & $213,187,034$ & $213,191,389$ & deletion & - \\
\hline $184294 \mathrm{chr} 2$ & $228,241,621$ & $228,258,288$ & duplication & TM4SF20 \\
\hline 184294 chr20 & $29,454,201$ & $29,500,566$ & deletion & - \\
\hline $184294 \mathrm{chr} 4$ & $69,374,369$ & $69,489,473$ & duplication & UGT2B17 \\
\hline 184294 chr6 & $78,326,093$ & $78,356,828$ & deletion & - \\
\hline 184294 chr7 & 149,765 & 159,207 & deletion & - \\
\hline 184294 chr8 & $24,972,808$ & $24,990,418$ & deletion & - \\
\hline 184294 chr8 & $5,595,511$ & $5,605,706$ & deletion & - \\
\hline 184294 chr9 & $6,701,130$ & $6,707,794$ & deletion & - \\
\hline 184296 chr1 & $104,153,766$ & $104,211,046$ & deletion & AMY1A;AMY2A;AMY1C;AMY1B \\
\hline 184296 chr1 & $149,022,110$ & $149,237,009$ & deletion & - \\
\hline 184296 chr1 & $190,377,988$ & $190,410,679$ & deletion & - \\
\hline 184296 chr 1 & $22,316,110$ & $22,336,305$ & deletion & CELA3A \\
\hline 184296 chr11 & $3,239,077$ & $3,243,665$ & deletion & MRGPRG \\
\hline 184296 chr11 & $49,715,154$ & $49,757,690$ & deletion & - \\
\hline 184296 chr11 & $93,020,527$ & $93,028,940$ & deletion & - \\
\hline 184296 chr12 & $2,245,636$ & $2,259,042$ & deletion & - \\
\hline 184296 chr12 & $34,490,832$ & $34,550,058$ & deletion & - \\
\hline 184296 chr13 & $38,076,308$ & $38,085,537$ & deletion & - \\
\hline 184296 chr14 & $106,624,360$ & $106,816,856$ & duplication & - \\
\hline 184296 chr14 & $19,457,976$ & $20,424,184$ & deletion & OR4K5;OR4K1;OR4K2;OR4Q3;OR11H2;POTEN \\
\hline 184296 chr15 & $34,717,697$ & $34,859,791$ & deletion & GOLGA8B \\
\hline 184296 chr15 & $97,815,182$ & $97,831,832$ & deletion & - \\
\hline 184296 chr16 & $34,471,298$ & $34,756,258$ & duplication & - \\
\hline 184296 chr19 & $35,852,103$ & $35,861,695$ & deletion & - \\
\hline 184296 chr19 & $43,703,965$ & $43,759,382$ & deletion & PSG4;PSG9 \\
\hline 184296 chr19 & $51,330,932$ & $51,333,442$ & deletion & KLK15 \\
\hline 184296 chr19 & $54,728,767$ & $54,743,217$ & duplication & LILRA6 \\
\hline $184296 \mathrm{chr} 2$ & $14,704,369$ & $14,709,611$ & deletion & - \\
\hline $184296 \mathrm{chr} 2$ & $52,759,890$ & $52,784,952$ & deletion & - \\
\hline 184296 chr20 & $62,194,464$ & $62,200,576$ & deletion & HELZ2 \\
\hline 184296 chr21 & $47,387,112$ & $47,433,055$ & deletion & COL6A1 \\
\hline 184296 chr22 & $25,663,994$ & $25,919,492$ & duplication & LRP5L \\
\hline 184296 chr3 & $152,402,851$ & $152,627,946$ & duplication & P2RY1 \\
\hline
\end{tabular}




\begin{tabular}{|c|c|c|c|c|}
\hline $184296 \mathrm{chr} 3$ & $173,239,831$ & $173,290,024$ & duplication & - \\
\hline $184296 \mathrm{chr} 3$ & $6,318,899$ & $6,335,989$ & duplication & - \\
\hline $184296 \mathrm{chr} 4$ & $64,136,609$ & $64,154,477$ & deletion & - \\
\hline 184296 chr5 & $155,477,866$ & $155,488,917$ & deletion & - \\
\hline 184296 chr6 & $139,603,393$ & $139,606,731$ & deletion & - \\
\hline 184296 chr6 & $32,584,901$ & $32,586,024$ & deletion & - \\
\hline 184296 chr6 & $78,972,930$ & $79,035,739$ & deletion & - \\
\hline $184296 \mathrm{chr} 7$ & $141,766,850$ & $141,793,521$ & deletion & - \\
\hline 184296 chr7 & $142,476,094$ & $142,484,042$ & deletion & - \\
\hline 184296 chr7 & $29,676,097$ & $29,689,321$ & deletion & - \\
\hline 184296 chr8 & $39,232,581$ & $39,385,979$ & deletion & - \\
\hline 184296 chr9 & $1,446,149$ & $1,450,465$ & deletion & - \\
\hline 184296 chr9 & $24,502,737$ & $24,518,795$ & deletion & - \\
\hline 184297 chr1 & $189,617,072$ & $189,658,713$ & deletion & - \\
\hline 184297 chr10 & $58,516,470$ & $58,526,153$ & deletion & - \\
\hline 184297 chr11 & $49,715,154$ & $49,757,690$ & deletion & - \\
\hline 184297 chr12 & $11,220,827$ & $11,244,117$ & deletion & TAS2R43 \\
\hline 184297 chr12 & $43,021,431$ & $43,027,453$ & deletion & - \\
\hline 184297 chr12 & $7,996,684$ & $8,123,306$ & duplication & SLC2A14;SLC2A3 \\
\hline 184297 chr12 & $80,832,125$ & $80,854,787$ & duplication & PTPRQ \\
\hline 184297 chr13 & $64,223,907$ & $64,236,288$ & deletion & - \\
\hline 184297 chr14 & $20,197,311$ & $20,404,736$ & duplication & OR4K5;OR4K1;OR4K2;OR4Q3;OR4N2;OR4M1 \\
\hline 184297 chr15 & $23,624,148$ & $23,668,092$ & deletion & - \\
\hline 184297 chr16 & $34,482,158$ & $34,756,258$ & duplication & - \\
\hline 184297 chr17 & $33,681,335$ & $33,768,284$ & deletion & SLFN11;SLFN12;SLFN13 \\
\hline 184297 chr17 & $44,166,604$ & $44,285,531$ & duplication & KANSL1 \\
\hline 184297 chr18 & $67,208,806$ & $67,217,542$ & deletion & - \\
\hline 184297 chr19 & $43,243,403$ & $43,438,750$ & deletion & PSG6;PSG7;PSG3;PSG8;PSG1 \\
\hline 184297 chr19 & $43,703,965$ & $43,759,382$ & deletion & PSG4;PSG9 \\
\hline 184297 chr2 & $184,795,725$ & $184,803,456$ & deletion & - \\
\hline 184297 chr2 & $208,351,315$ & $208,357,838$ & deletion & - \\
\hline 184297 chr2 & $52,759,890$ & $52,784,952$ & deletion & - \\
\hline 184297 chr3 & $129,769,827$ & $129,806,304$ & deletion & ALG1L2 \\
\hline 184297 chr3 & $191,065,392$ & $191,070,300$ & deletion & - \\
\hline $184297 \mathrm{chr} 4$ & $112,238,706$ & $112,241,762$ & deletion & - \\
\hline 184297 chr4 & $188,924,979$ & $188,938,071$ & deletion & ZFP42 \\
\hline 184297 chr4 & $69,374,369$ & $69,467,200$ & deletion & UGT2B17 \\
\hline 184297 chr4 & $91,910,883$ & $91,913,329$ & deletion & - \\
\hline 184297 chr5 & $117,388,410$ & $117,393,058$ & deletion & - \\
\hline 184297 chr5 & 701,026 & 840,717 & duplication & ZDHHC11 \\
\hline 184297 chr6 & $29,855,945$ & $29,899,189$ & deletion & - \\
\hline 184297 chr6 & $81,284,205$ & $81,293,837$ & deletion & - \\
\hline 184297 chr7 & 41,421 & 73,060 & duplication & - \\
\hline 184297 chr7 & $52,730,700$ & $52,744,420$ & duplication & - \\
\hline 184297 chr8 & $24,972,808$ & $24,990,418$ & deletion & - \\
\hline 184297 chr8 & $39,232,581$ & $39,385,979$ & deletion & - \\
\hline 184297 chr9 & $107,799,020$ & $107,803,370$ & deletion & - \\
\hline
\end{tabular}




\begin{tabular}{|c|c|c|c|c|c|}
\hline 184297 & chr9 & $23,363,526$ & $23,376,817$ & deletion & - \\
\hline 184297 & chr9 & $24,146,203$ & $24,150,034$ & deletion & - \\
\hline 184297 & chr9 & $78,004,378$ & $78,012,382$ & deletion & - \\
\hline 184300 & chr1 & $104,153,766$ & $104,211,046$ & deletion & AMY1A;AMY2A;AMY1C;AMY1B \\
\hline 184300 & chr1 & $12,908,591$ & $12,919,928$ & deletion & PRAMEF2 \\
\hline 184300 & chr1 & $149,033,600$ & $149,233,182$ & deletion & - \\
\hline 184300 & chr1 & $245,636,900$ & $245,647,809$ & deletion & - \\
\hline 184300 & chr1 & $248,740,572$ & $248,795,110$ & deletion & OR2T10;OR2T11 \\
\hline 184300 & chr1 & $25,598,276$ & $25,659,509$ & deletion & RHD \\
\hline 184300 & chr11 & $134,602,333$ & $134,607,484$ & deletion & - \\
\hline 184300 & chr11 & $55,365,761$ & $55,427,700$ & deletion & OR4P4;OR4C11;OR4S2 \\
\hline 184300 & chr12 & $11,511,132$ & $11,546,987$ & deletion & PRB2 \\
\hline 184300 & chr13 & $38,076,308$ & $38,085,537$ & deletion & - \\
\hline 184300 & chr14 & $20,197,311$ & $20,424,184$ & duplication & OR4K5;OR4K1;OR4K2;OR4Q3;OR4N2;OR4M1 \\
\hline 184300 & chr14 & $41,610,224$ & $41,661,685$ & deletion & - \\
\hline 184300 & chr14 & $42,988,346$ & $42,992,844$ & deletion & - \\
\hline 184300 & chr14 & $50,721,266$ & $50,725,773$ & deletion & - \\
\hline 184300 & chr15 & $32,509,090$ & $32,515,849$ & deletion & - \\
\hline 184300 & chr15 & $43,916,071$ & $43,990,211$ & deletion & CKMT1A;CATSPER2 \\
\hline 184300 & chr15 & $97,262,427$ & $97,268,718$ & deletion & - \\
\hline 184300 & chr16 & $19,945,540$ & $19,962,636$ & deletion & - \\
\hline 184300 & chr16 & $34,471,298$ & $34,756,258$ & duplication & - \\
\hline 184300 & chr18 & $66,746,208$ & $66,755,736$ & deletion & - \\
\hline 184300 & chr18 & $69,428,556$ & $69,441,154$ & deletion & - \\
\hline 184300 & chr19 & $35,852,103$ & $35,861,695$ & deletion & \\
\hline 184300 & chr19 & $41,355,999$ & $41,386,033$ & deletion & CYP2A7;CYP2A6 \\
\hline 184300 & chr19 & $43,214,714$ & $43,378,265$ & duplication & PSG3;PSG8;PSG1 \\
\hline 184300 & chr19 & $51,330,932$ & $51,333,442$ & deletion & KLK15 \\
\hline 184300 & chr2 & $242,854,089$ & $243,034,519$ & deletion & - \\
\hline 184300 & chr2 & $34,697,895$ & $34,726,904$ & duplication & - \\
\hline 184300 & chr20 & $59,112,918$ & $59,122,693$ & deletion & - \\
\hline 184300 & chr21 & $10,802,502$ & $10,858,651$ & deletion & - \\
\hline 184300 & chr22 & $42,902,278$ & $42,950,033$ & duplication & SERHL2;RRP7A \\
\hline 184300 & chr3 & $162,130,691$ & $162,151,373$ & deletion & - \\
\hline 184300 & chr3 & $175,887,314$ & $175,909,890$ & deletion & - \\
\hline 184300 & chr3 & $191,062,751$ & $191,070,300$ & deletion & - \\
\hline 184300 & chr3 & $65,189,366$ & $65,214,685$ & deletion & - \\
\hline 184300 & chr4 & $126,146,483$ & $126,159,605$ & duplication & - \\
\hline 184300 & chr4 & $184,672,645$ & $184,799,985$ & duplication & - \\
\hline 184300 & chr4 & $34,785,955$ & $34,818,502$ & deletion & - \\
\hline 184300 & chr4 & $59,166,999$ & $59,180,928$ & deletion & - \\
\hline 184300 & chr5 & $140,225,908$ & $140,238,311$ & deletion & PCDHA10;PCDHA9 \\
\hline 184300 & chr6 & $151,507,355$ & $151,509,176$ & deletion & - \\
\hline 184300 & chr7 & $141,766,850$ & $141,793,521$ & deletion & - \\
\hline 184300 & chr7 & $155,882,711$ & $155,885,143$ & deletion & - \\
\hline 184300 & chr7 & $159,118,443$ & $159,122,741$ & deletion & - \\
\hline 184300 & chr7 & $38,388,889$ & $38,402,517$ & deletion & - \\
\hline
\end{tabular}




\begin{tabular}{|c|c|c|c|c|}
\hline 184300 chr7 & $75,664,539$ & $75,667,743$ & deletion & - \\
\hline $184300 \mathrm{chr} 8$ & $115,633,704$ & $115,642,459$ & deletion & - \\
\hline 184300 chr8 & $14,157,277$ & $14,161,700$ & deletion & - \\
\hline $184300 \mathrm{chr} 8$ & $24,972,808$ & $24,990,418$ & deletion & - \\
\hline $184300 \mathrm{chr} 8$ & $39,232,581$ & $39,385,979$ & duplication & - \\
\hline 184300 chr8 & $61,858,213$ & $61,869,649$ & deletion & - \\
\hline 184300 chr9 & $6,701,130$ & $6,707,794$ & deletion & - \\
\hline 184300 chr9 & $78,004,378$ & $78,011,616$ & deletion & - \\
\hline 184300 chr9 & $89,030,908$ & $89,038,427$ & duplication & - \\
\hline 184303 chr1 & $12,908,591$ & $12,919,928$ & deletion & PRAMEF2 \\
\hline 184303 chr1 & $149,032,299$ & $149,215,927$ & duplication & - \\
\hline 184303 chr1 & $152,526,812$ & $152,568,230$ & deletion & LCE3D;LCE3E \\
\hline 184303 chr1 & $152,648,950$ & $152,659,194$ & duplication & LCE2C \\
\hline 184303 chr1 & $196,823,300$ & $196,901,753$ & deletion & CFHR4 \\
\hline 184303 chr10 & $22,594,765$ & $22,621,933$ & deletion & COMMD3;COMMD3-BMI1;BMI1 \\
\hline 184303 chr10 & $35,123,178$ & $35,130,901$ & deletion & - \\
\hline 184303 chr10 & $71,278,805$ & $71,289,190$ & deletion & - \\
\hline 184303 chr11 & 346,381 & 436,461 & deletion & B4GALNT4;PKP3;ANO9;SIGIRR \\
\hline 184303 chr11 & $55,365,761$ & $55,427,700$ & deletion & OR4P4;OR4C11;OR4S2 \\
\hline 184303 chr13 & $100,619,146$ & $100,641,335$ & deletion & ZIC5;ZIC2 \\
\hline 184303 chr14 & $106,891,524$ & $106,931,129$ & deletion & - \\
\hline 184303 chr14 & $41,610,224$ & $41,661,685$ & deletion & - \\
\hline 184303 chr14 & $84,435,272$ & $84,440,535$ & deletion & - \\
\hline 184303 chr16 & 205,035 & 216,420 & deletion & HBM \\
\hline 184303 chr16 & $70,704,455$ & $70,742,141$ & deletion & MTSS1L;VAC14 \\
\hline 184303 chr17 & $6,105,070$ & $6,131,093$ & deletion & - \\
\hline 184303 chr17 & $6,294,175$ & $6,315,646$ & duplication & - \\
\hline 184303 chr19 & $54,728,767$ & $54,743,217$ & deletion & LILRA6 \\
\hline 184303 chr2 & $18,762,337$ & $18,811,756$ & deletion & NT5C1B-RDH14;NT5C1B \\
\hline 184303 chr2 & $34,697,895$ & $34,726,904$ & deletion & - \\
\hline 184303 chr2 & $89,540,620$ & $90,256,530$ & deletion & - \\
\hline 184303 chr20 & $52,647,631$ & $52,656,535$ & deletion & - \\
\hline 184303 chr22 & $37,745,101$ & $37,749,946$ & deletion & - \\
\hline 184303 chr22 & $39,359,885$ & $39,385,663$ & deletion & АРОВЕСЗВ \\
\hline 184303 chr3 & $129,317,585$ & $129,346,867$ & deletion & PLXND1 \\
\hline 184303 chr3 & $191,064,647$ & $191,070,300$ & deletion & - \\
\hline 184303 chr3 & $195,457,853$ & $195,468,690$ & duplication & MUC20 \\
\hline 184303 chr3 & $53,032,639$ & $53,038,786$ & deletion & - \\
\hline $184303 \mathrm{chr} 4$ & $69,374,369$ & $69,489,473$ & duplication & UGT2B17 \\
\hline 184303 chr5 & $21,852,537$ & $21,977,617$ & deletion & $\mathrm{CDH} 12$ \\
\hline 184303 chr5 & $97,048,466$ & $97,096,042$ & deletion & - \\
\hline 184303 chr5 & $97,933,671$ & $97,939,616$ & deletion & - \\
\hline 184303 chr6 & $66,074,421$ & $66,080,908$ & deletion & - \\
\hline 184303 chr7 & $140,195,001$ & $140,204,065$ & deletion & - \\
\hline 184303 chr7 & $1,572,616$ & $1,580,599$ & deletion & MAFK \\
\hline 184303 chr8 & $115,634,278$ & $115,642,459$ & deletion & - \\
\hline 184303 chr8 & $51,029,371$ & $51,038,149$ & deletion & - \\
\hline
\end{tabular}




\begin{tabular}{|c|c|c|c|c|}
\hline $184303 \mathrm{chr} 8$ & $98,193,889$ & $98,212,152$ & deletion & - \\
\hline 184303 chr9 & $24,502,737$ & $24,520,633$ & deletion & - \\
\hline 184303 chr9 & $44,739,805$ & $44,834,316$ & deletion & - \\
\hline 184303 chr9 & $78,004,378$ & $78,011,616$ & deletion & - \\
\hline $184303 \mathrm{chr} X$ & $51,741,943$ & $51,760,413$ & deletion & - \\
\hline $184306 \mathrm{chr} 1$ & $110,486,839$ & $110,494,874$ & deletion & - \\
\hline 184306 chr1 & $17,009,576$ & $17,288,389$ & duplication & FAM231A;FAM231C;MST1L;CROCC \\
\hline 184306 chr1 & $245,636,900$ & $245,647,809$ & deletion & - \\
\hline 184306 chr1 & $72,754,314$ & $72,763,324$ & deletion & - \\
\hline 184306 chr1 & $91,093,909$ & $91,099,903$ & deletion & - \\
\hline 184306 chr10 & $58,516,470$ & $58,526,153$ & deletion & - \\
\hline 184306 chr10 & $82,879,475$ & $82,891,283$ & deletion & - \\
\hline 184306 chr11 & $134,602,333$ & $134,607,484$ & deletion & - \\
\hline 184306 chr11 & $81,501,218$ & $81,517,261$ & deletion & - \\
\hline 184306 chr11 & $8,959,020$ & $8,964,938$ & deletion & ASCL3 \\
\hline 184306 chr11 & $90,193,796$ & $90,196,843$ & deletion & - \\
\hline 184306 chr12 & $43,021,431$ & $43,027,453$ & deletion & - \\
\hline 184306 chr15 & $25,420,978$ & $25,430,654$ & deletion & - \\
\hline 184306 chr15 & $34,717,697$ & $34,853,206$ & deletion & GOLGA8B \\
\hline 184306 chr15 & $56,789,979$ & $56,800,635$ & deletion & - \\
\hline 184306 chr16 & $34,471,298$ & $34,756,258$ & duplication & - \\
\hline 184306 chr17 & $44,166,604$ & $44,225,181$ & duplication & KANSL1 \\
\hline 184306 chr17 & $54,160,465$ & $54,172,668$ & deletion & - \\
\hline 184306 chr19 & $50,448,616$ & $50,458,071$ & deletion & SIGLEC11 \\
\hline 184306 chr2 & $123,477,263$ & $123,482,301$ & deletion & - \\
\hline 184306 chr2 & $208,351,315$ & $208,357,838$ & deletion & - \\
\hline 184306 chr20 & $3,729,899$ & $3,732,947$ & deletion & HSPA12B \\
\hline $184306 \mathrm{chr} 3$ & $192,777,710$ & $192,782,294$ & deletion & - \\
\hline 184306 chr3 & $37,979,882$ & $37,990,299$ & deletion & CTDSPL \\
\hline 184306 chr4 & $122,285,263$ & $122,289,863$ & deletion & - \\
\hline $184306 \mathrm{chr} 4$ & $161,032,995$ & $161,072,119$ & deletion & - \\
\hline 184306 chr4 & $34,785,955$ & $34,818,502$ & deletion & - \\
\hline $184306 \mathrm{chr} 4$ & $86,101,007$ & $86,118,526$ & deletion & - \\
\hline 184306 chr5 & $140,214,995$ & $140,228,164$ & deletion & PCDHA8;PCDHA9;PCDHA7 \\
\hline 184306 chr5 & $155,477,866$ & $155,488,917$ & deletion & - \\
\hline 184306 chr5 & $165,767,694$ & $165,779,913$ & deletion & - \\
\hline 184306 chr5 & $177,387,248$ & $177,400,704$ & deletion & - \\
\hline 184306 chr6 & $30,994,010$ & $30,994,787$ & deletion & MUC22 \\
\hline 184306 chr6 & $31,338,528$ & $31,341,357$ & deletion & - \\
\hline 184306 chr7 & $141,766,850$ & $141,793,521$ & deletion & - \\
\hline 184306 chr7 & $154,156,234$ & $154,169,390$ & deletion & - \\
\hline 184306 chr7 & $157,116,750$ & $157,122,691$ & deletion & - \\
\hline 184306 chr7 & $61,751,802$ & $61,793,162$ & deletion & - \\
\hline $184306 \mathrm{chr} 8$ & $39,232,581$ & $39,385,979$ & deletion & - \\
\hline 184306 chr8 & $39,575,853$ & $39,614,421$ & duplication & ADAM18;ADAM2 \\
\hline 184306 chr8 & $4,907,709$ & $4,914,546$ & deletion & - \\
\hline $184306 \mathrm{chr} 8$ & $72,214,753$ & $72,217,690$ & deletion & - \\
\hline
\end{tabular}




\begin{tabular}{|c|c|c|c|c|}
\hline 184306 chr8 & $87,177,402$ & $87,331,746$ & duplication & SLC7A13 \\
\hline 184315 chr1 & $104,103,057$ & $104,211,046$ & duplication & AMY1C;AMY1A;AMY2A;AMY2B;AMY1B \\
\hline $184315 \mathrm{chr} 1$ & $1,627,987$ & $1,668,298$ & deletion & SLC35E2;CDK11A;CDK11B \\
\hline 184315 chr1 & $210,602,708$ & $210,613,893$ & deletion & - \\
\hline 184315 chr1 & $72,754,314$ & $72,763,324$ & deletion & - \\
\hline 184315 chr11 & $18,949,220$ & $18,961,743$ & duplication & MRGPRX1 \\
\hline 184315 chr12 & $11,511,132$ & $11,546,987$ & deletion & PRB2 \\
\hline 184315 chr12 & $129,230,513$ & $129,233,013$ & deletion & - \\
\hline 184315 chr12 & $43,021,431$ & $43,027,453$ & deletion & - \\
\hline 184315 chr12 & $59,935,926$ & $59,942,122$ & deletion & - \\
\hline 184315 chr13 & $32,532,761$ & $32,537,768$ & deletion & - \\
\hline 184315 chr13 & $38,076,308$ & $38,085,537$ & deletion & - \\
\hline 184315 chr14 & $20,361,945$ & $20,404,736$ & duplication & OR4K5;OR4K1 \\
\hline 184315 chr14 & $41,610,224$ & $41,661,685$ & deletion & - \\
\hline 184315 chr14 & $47,708,397$ & $47,715,702$ & deletion & - \\
\hline 184315 chr15 & $56,789,979$ & $56,800,635$ & deletion & - \\
\hline 184315 chr16 & $32,531,934$ & $32,833,893$ & deletion & TP53TG3B;TP53TG3;TP53TG3C \\
\hline 184315 chr16 & $55,800,009$ & $55,819,443$ & deletion & - \\
\hline 184315 chr17 & $61,915,326$ & $61,984,208$ & deletion & $\mathrm{CSH} 2 ; \mathrm{SMARCD} 2 ; \mathrm{GH} 2 ; \mathrm{CSH} 1$ \\
\hline $184315 \mathrm{chr} 18$ & $41,976,831$ & $41,982,102$ & deletion & - \\
\hline 184315 chr19 & $35,852,103$ & $35,861,695$ & deletion & - \\
\hline $184315 \mathrm{chr} 2$ & $184,795,725$ & $184,803,456$ & deletion & - \\
\hline $184315 \mathrm{chr} 2$ & $213,187,034$ & $213,191,389$ & deletion & - \\
\hline 184315 chr2 & $38,956,947$ & $38,971,623$ & duplication & GALM \\
\hline 184315 chr20 & $52,647,631$ & $52,656,535$ & deletion & - \\
\hline 184315 chr22 & $42,902,872$ & $42,950,033$ & duplication & SERHL2;RRP7A \\
\hline 184315 chr3 & $195,418,291$ & $195,468,690$ & duplication & MUC20 \\
\hline $184315 \mathrm{chr} 4$ & $138,092,298$ & $138,098,601$ & deletion & - \\
\hline $184315 \mathrm{chr} 4$ & $152,789,786$ & $152,793,891$ & deletion & - \\
\hline $184315 \mathrm{chr} 4$ & $152,991,094$ & $152,993,620$ & deletion & - \\
\hline $184315 \mathrm{chr} 4$ & $171,267,562$ & $171,273,360$ & deletion & - \\
\hline $184315 \mathrm{chr} 4$ & $185,802,553$ & $185,812,040$ & duplication & - \\
\hline $184315 \mathrm{chr} 4$ & $34,785,955$ & $34,818,502$ & deletion & - \\
\hline 184315 chr4 & $64,697,704$ & $64,709,753$ & deletion & - \\
\hline 184315 chr5 & $140,225,908$ & $140,238,311$ & deletion & PCDHA10;PCDHA9 \\
\hline 184315 chr5 & $40,785,811$ & $40,858,699$ & duplication & RPL37;CARD6;PRKAA1 \\
\hline 184315 chr6 & $132,709,027$ & $132,711,200$ & deletion & - \\
\hline 184315 chr6 & $29,917,439$ & $29,924,440$ & deletion & - \\
\hline 184315 chr6 & $31,360,255$ & $31,453,640$ & deletion & MICA \\
\hline 184315 chr7 & $141,766,850$ & $141,793,521$ & deletion & - \\
\hline 184315 chr7 & $57,623,146$ & $57,670,249$ & duplication & - \\
\hline 184315 chr7 & $57,873,471$ & $57,886,113$ & duplication & - \\
\hline 184315 chr7 & $62,149,006$ & $62,162,385$ & deletion & - \\
\hline $184315 \mathrm{chr} 8$ & $117,293,884$ & $117,305,398$ & deletion & - \\
\hline 184315 chr8 & $24,972,808$ & $24,990,418$ & deletion & - \\
\hline 184315 chr8 & $47,526,999$ & $47,539,088$ & duplication & - \\
\hline 184315 chr9 & $78,004,378$ & $78,011,616$ & deletion & - \\
\hline
\end{tabular}




\begin{tabular}{|c|c|c|c|c|}
\hline 184316 chr1 & $104,104,990$ & $104,211,046$ & duplication & AMY1C;AMY1A;AMY2A;AMY2B;AMY1B \\
\hline $184316 \mathrm{chr} 1$ & $161,559,327$ & $161,619,741$ & deletion & FCGR2C;FCGR3B \\
\hline $184316 \mathrm{chr} 1$ & $210,602,708$ & $210,613,893$ & deletion & - \\
\hline 184316 chr1 & $248,740,314$ & $248,795,110$ & deletion & OR2T10;OR2T11 \\
\hline 184316 chr1 & $62,113,597$ & $62,119,136$ & deletion & - \\
\hline 184316 chr1 & $72,754,314$ & $72,763,324$ & deletion & - \\
\hline 184316 chr11 & $103,343,254$ & $103,381,461$ & duplication & DYNC2H1 \\
\hline 184316 chr11 & $55,370,325$ & $55,419,073$ & deletion & OR4P4;OR4C11;OR4S2 \\
\hline 184316 chr11 & $81,501,218$ & $81,517,261$ & deletion & - \\
\hline 184316 chr11 & $90,193,427$ & $90,195,742$ & deletion & - \\
\hline 184316 chr12 & $11,511,132$ & $11,543,084$ & duplication & - \\
\hline 184316 chr12 & $129,230,513$ & $129,233,013$ & deletion & - \\
\hline 184316 chr12 & $43,021,431$ & $43,027,453$ & deletion & - \\
\hline 184316 chr12 & $70,871,858$ & $70,879,131$ & deletion & - \\
\hline 184316 chr13 & $38,076,308$ & $38,085,537$ & deletion & - \\
\hline 184316 chr13 & $98,530,124$ & $98,532,757$ & deletion & - \\
\hline 184316 chr14 & $20,213,037$ & $20,422,799$ & duplication & OR4K5;OR4K1;OR4K2;OR4Q3;OR4N2;OR4M1 \\
\hline 184316 chr14 & $35,604,892$ & $35,613,500$ & deletion & - \\
\hline 184316 chr14 & $41,610,224$ & $41,661,685$ & deletion & - \\
\hline 184316 chr14 & $47,708,397$ & $47,715,702$ & deletion & - \\
\hline 184316 chr15 & $21,903,815$ & $22,753,733$ & duplication & GOLGA6L22;GOLGA6L1;OR4N4;POTEB3;OR4N \\
\hline 184316 chr16 & $32,531,934$ & $32,833,893$ & deletion & TP53TG3B;TP53TG3;TP53TG3C \\
\hline 184316 chr17 & $61,915,326$ & $61,984,208$ & deletion & $\mathrm{CSH} 2 ; \mathrm{SMARCD} 2 ; \mathrm{GH} 2 ; \mathrm{CSH} 1$ \\
\hline 184316 chr18 & $35,531,818$ & $35,536,374$ & deletion & 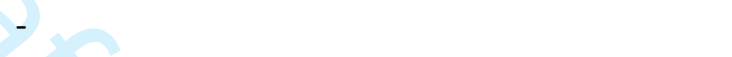 \\
\hline 184316 chr18 & $41,976,831$ & $41,982,102$ & deletion & \\
\hline 184316 chr19 & $35,852,103$ & $35,861,695$ & deletion & - \\
\hline 184316 chr19 & $43,261,897$ & $43,477,175$ & duplication & PSG6;PSG7;PSG8;PSG1 \\
\hline $184316 \mathrm{chr} 2$ & $121,137,382$ & $121,139,942$ & deletion & - \\
\hline $184316 \mathrm{chr} 2$ & $184,795,725$ & $184,803,456$ & deletion & - \\
\hline $184316 \mathrm{chr} 2$ & $38,956,947$ & $38,971,623$ & duplication & GALM \\
\hline $184316 \mathrm{chr} 2$ & $4,212,725$ & $4,222,144$ & deletion & - \\
\hline $184316 \mathrm{chr} 2$ & $51,926,599$ & $51,926,904$ & deletion & - \\
\hline 184316 chr2 & $97,738,917$ & $98,024,837$ & deletion & FAHD2B;ANKRD36 \\
\hline 184316 chr20 & $52,647,631$ & $52,656,535$ & deletion & - \\
\hline 184316 chr22 & $42,918,711$ & $42,950,033$ & duplication & SERHL2 \\
\hline $184316 \mathrm{chr} 3$ & $195,418,291$ & $195,475,799$ & duplication & MUC20;MUC4 \\
\hline $184316 \mathrm{chr} 4$ & $138,091,446$ & $138,098,601$ & deletion & - \\
\hline $184316 \mathrm{chr} 4$ & $152,790,580$ & $152,793,891$ & deletion & - \\
\hline $184316 \mathrm{chr} 4$ & $152,989,386$ & $152,993,620$ & deletion & - \\
\hline $184316 \mathrm{chr} 4$ & $34,785,955$ & $34,818,502$ & deletion & - \\
\hline $184316 \mathrm{chr} 5$ & $140,225,908$ & $140,238,311$ & deletion & PCDHA10;PCDHA9 \\
\hline 184316 chr6 & $31,276,513$ & $31,277,988$ & deletion & - \\
\hline 184316 chr6 & $31,360,255$ & $31,453,640$ & deletion & MICA \\
\hline $184316 \mathrm{chr} 6$ & $78,972,930$ & $79,035,739$ & deletion & - \\
\hline 184316 chr6 & $81,284,205$ & $81,293,837$ & deletion & - \\
\hline 184316 chr7 & $141,766,850$ & $141,793,521$ & deletion & - \\
\hline $184316 \mathrm{chr} 7$ & $85,634,656$ & $85,654,531$ & deletion & - \\
\hline
\end{tabular}




\begin{tabular}{|c|c|c|c|c|}
\hline 184316 chr8 & $117,293,884$ & $117,305,398$ & deletion & - \\
\hline $184316 \mathrm{chr} 8$ & $17,580,791$ & $17,581,700$ & deletion & MTUS1 \\
\hline 184316 chr8 & $39,236,582$ & $39,382,903$ & deletion & - \\
\hline 184321 chr1 & $104,104,990$ & $104,211,046$ & duplication & AMY1C;AMY1A;AMY2A;AMY2B;AMY1B \\
\hline 184321 chr1 & $187,716,107$ & $187,722,032$ & deletion & - \\
\hline 184321 chr11 & $106,398,020$ & $106,404,608$ & deletion & - \\
\hline 184321 chr11 & $18,949,220$ & $18,961,743$ & deletion & MRGPRX1 \\
\hline 184321 chr11 & $7,814,299$ & $7,827,879$ & deletion & OR5P2 \\
\hline 184321 chr11 & $90,193,796$ & $90,196,843$ & deletion & - \\
\hline 184321 chr12 & $19,469,320$ & $19,578,107$ & duplication & PLEKHA5 \\
\hline 184321 chr12 & $99,994,315$ & $100,008,773$ & deletion & - \\
\hline 184321 chr13 & $38,076,308$ & $38,085,537$ & deletion & - \\
\hline 184321 chr14 & $106,891,524$ & $106,931,129$ & deletion & - \\
\hline 184321 chr17 & $34,438,753$ & $34,534,918$ & duplication & CCL3L3;CCL3L1 \\
\hline 184321 chr19 & $35,852,103$ & $35,861,695$ & deletion & - \\
\hline 184321 chr19 & $53,518,747$ & $53,548,950$ & duplication & ERVV-1 \\
\hline 184321 chr19 & $54,728,767$ & $54,743,217$ & duplication & LILRA6 \\
\hline 184321 chr2 & $165,850,456$ & $165,864,123$ & deletion & - \\
\hline 184321 chr2 & $184,795,725$ & $184,803,456$ & deletion & - \\
\hline $184321 \mathrm{chr} 2$ & $238,554,352$ & $238,558,478$ & deletion & - \\
\hline 184321 chr2 & $51,926,599$ & $51,926,904$ & deletion & - \\
\hline 184321 chr2 & $8,130,069$ & $8,140,204$ & deletion & - \\
\hline 184321 chr3 & $162,130,691$ & $162,151,373$ & deletion & - \\
\hline 184321 chr3 & $195,457,853$ & $195,468,690$ & duplication & MUC20 \\
\hline $184321 \mathrm{chr} 3$ & $53,032,639$ & $53,042,099$ & deletion & - \\
\hline 184321 chr3 & $75,419,736$ & $75,643,261$ & deletion & - \\
\hline $184321 \mathrm{chr} 4$ & $122,285,263$ & $122,289,863$ & deletion & - \\
\hline $184321 \mathrm{chr} 4$ & $39,705,484$ & $39,713,134$ & deletion & - \\
\hline 184321 chr4 & $69,374,369$ & $69,489,473$ & deletion & UGT2B17 \\
\hline 184321 chr5 & $155,477,866$ & $155,500,953$ & deletion & - \\
\hline 184321 chr5 & $177,390,271$ & $177,400,704$ & deletion & - \\
\hline 184321 chr6 & $78,972,930$ & $79,035,739$ & deletion & - \\
\hline 184321 chr8 & $138,822,798$ & $138,833,669$ & deletion & - \\
\hline 184321 chr8 & $15,401,929$ & $15,411,867$ & deletion & - \\
\hline 184321 chr8 & $39,236,582$ & $39,372,087$ & deletion & - \\
\hline 184321 chr8 & $43,762,472$ & $43,772,232$ & deletion & - \\
\hline 184321 chr9 & $107,799,020$ & $107,803,370$ & deletion & - \\
\hline 184322 chr1 & $196,713,725$ & $196,901,753$ & deletion & CFHR3;CFHR1;CFHR4;CFH \\
\hline $184322 \mathrm{chr} 10$ & $107,984,226$ & $108,016,816$ & deletion & - \\
\hline 184322 chr11 & $18,949,220$ & $18,961,743$ & deletion & MRGPRX1 \\
\hline 184322 chr11 & $25,606,707$ & $25,625,889$ & deletion & - \\
\hline 184322 chr11 & $49,715,154$ & $49,757,690$ & deletion & - \\
\hline 184322 chr12 & $52,688,766$ & $52,782,141$ & duplication & KRT83;KRT84;KRT85;KRT86 \\
\hline 184322 chr12 & $59,935,926$ & $59,942,122$ & deletion & - \\
\hline 184322 chr13 & $38,076,308$ & $38,085,537$ & deletion & - \\
\hline 184322 chr13 & $58,589,893$ & $58,599,174$ & deletion & - \\
\hline 184322 chr14 & $20,197,311$ & $20,424,184$ & duplication & OR4K5;OR4K1;OR4K2;OR4Q3;OR4N2;O \\
\hline
\end{tabular}




\begin{tabular}{|c|c|c|c|c|}
\hline 184322 chr14 & $95,151,985$ & $95,154,930$ & duplication & - \\
\hline 184322 chr15 & $20,196,661$ & $21,241,118$ & deletion & GOLGA6L6;POTEB2;POTEB;POTEB3 \\
\hline 184322 chr15 & $34,717,697$ & $34,814,869$ & deletion & - \\
\hline 184322 chr15 & $97,815,182$ & $97,831,832$ & deletion & - \\
\hline 184322 chr16 & $20,012,297$ & $20,018,751$ & deletion & - \\
\hline $184322 \mathrm{chr} 16$ & 223,625 & 225,653 & deletion & - \\
\hline $184322 \mathrm{chr} 16$ & $85,302,707$ & $85,304,326$ & deletion & - \\
\hline 184322 chr17 & $15,044,804$ & $15,058,236$ & deletion & - \\
\hline 184322 chr17 & $76,624,853$ & $76,625,446$ & deletion & - \\
\hline 184322 chr18 & $63,907,596$ & $63,910,708$ & deletion & - \\
\hline 184322 chr19 & $20,601,006$ & $20,717,536$ & deletion & - \\
\hline $184322 \mathrm{chr} 19$ & $43,302,567$ & $43,744,060$ & deletion & PSG11;PSG6;PSG7;PSG4;PSG5;PSG2;PSG1 \\
\hline 184322 chr19 & $53,520,702$ & $53,552,296$ & duplication & - \\
\hline 184322 chr19 & $54,728,767$ & $54,743,217$ & duplication & LILRA6 \\
\hline 184322 chr19 & $9,275,282$ & $9,282,176$ & deletion & - \\
\hline 184322 chr2 & $114,155,972$ & $114,166,128$ & deletion & - \\
\hline $184322 \mathrm{chr} 2$ & $195,979,780$ & $195,982,450$ & deletion & - \\
\hline 184322 chr2 & $34,697,895$ & $34,726,904$ & duplication & - \\
\hline $184322 \mathrm{chr} 2$ & $87,768,332$ & $87,997,476$ & duplication & - \\
\hline $184322 \mathrm{chr} 20$ & $52,288,724$ & $52,290,223$ & deletion & - \\
\hline 184322 chr3 & $197,844,320$ & $197,846,134$ & deletion & - \\
\hline $184322 \mathrm{chr} 4$ & $34,785,955$ & $34,818,502$ & deletion & - \\
\hline $184322 \mathrm{chr} 4$ & $69,374,369$ & $69,489,473$ & duplication & UGT2B17 \\
\hline 184322 chr4 & $70,137,897$ & $70,229,260$ & deletion & UGT2B28 \\
\hline 184322 chr5 & $102,369,329$ & $102,392,398$ & deletion & - \\
\hline 184322 chr5 & $128,298,437$ & $128,303,353$ & deletion & SLC27A6 \\
\hline 184322 chr5 & $133,395,820$ & $133,399,148$ & deletion & - \\
\hline $184322 \mathrm{chr} 5$ & $151,514,956$ & $151,518,615$ & deletion & - \\
\hline 184322 chr5 & $155,477,866$ & $155,488,917$ & deletion & - \\
\hline 184322 chr5 & $37,911,022$ & $37,927,611$ & deletion & - \\
\hline 184322 chr6 & $162,795,058$ & $162,843,312$ & deletion & - \\
\hline 184322 chr6 & $31,281,682$ & $31,286,577$ & deletion & - \\
\hline 184322 chr7 & $111,727,046$ & $111,749,447$ & deletion & - \\
\hline 184322 chr7 & $141,766,850$ & $141,793,521$ & deletion & - \\
\hline $184322 \mathrm{chr} 7$ & $30,790,978$ & $30,795,841$ & deletion & INMT \\
\hline $184322 \mathrm{chr} 7$ & $30,810,505$ & $30,837,276$ & deletion & FAM188B \\
\hline 184322 chr7 & $62,149,006$ & $62,162,385$ & deletion & - \\
\hline $184322 \mathrm{chr} 7$ & $97,396,709$ & $97,401,677$ & deletion & - \\
\hline 184322 chr8 & $115,634,278$ & $115,642,459$ & deletion & - \\
\hline $184322 \mathrm{chr} 8$ & $24,972,808$ & $24,990,418$ & duplication & - \\
\hline 184322 chr8 & $5,595,511$ & $5,605,706$ & deletion & - \\
\hline 184322 chr8 & $6,977,269$ & $8,094,699$ & deletion & USP17L3;USP17L1;USP17L4;USP17L8;DEFB10 \\
\hline 184322 chr9 & $23,362,379$ & $23,376,817$ & duplication & - \\
\hline 184322 chr9 & $73,906,136$ & $73,909,871$ & duplication & - \\
\hline 184322 chr9 & $77,310,039$ & $77,318,073$ & deletion & - \\
\hline 184324 chr1 & $1,385,211$ & $1,430,707$ & deletion & ATAD3C;ATAD3B \\
\hline 184324 chr1 & $149,033,600$ & $149,233,182$ & deletion & - \\
\hline
\end{tabular}




\begin{tabular}{|c|c|c|c|c|}
\hline 184324 chr1 & $192,873,852$ & $192,895,587$ & deletion & - \\
\hline $184324 \mathrm{chr} 1$ & $213,002,088$ & $213,020,221$ & deletion & SPATA45 \\
\hline $184324 \mathrm{chr} 1$ & $245,636,900$ & $245,647,809$ & deletion & - \\
\hline 184324 chr1 & $62,766,001$ & $62,770,990$ & deletion & - \\
\hline 184324 chr10 & $13,056,587$ & $13,060,410$ & deletion & - \\
\hline 184324 chr10 & $60,090,140$ & $60,094,274$ & deletion & - \\
\hline 184324 chr10 & $60,261,697$ & $60,268,843$ & deletion & - \\
\hline 184324 chr11 & $18,949,220$ & $18,961,743$ & deletion & MRGPRX1 \\
\hline 184324 chr11 & $55,365,761$ & $55,443,269$ & duplication & OR4P4;OR4C6;OR4C11;OR4S2 \\
\hline 184324 chr12 & $11,511,132$ & $11,546,987$ & deletion & PRB2 \\
\hline 184324 chr12 & $80,155,600$ & $80,160,230$ & duplication & - \\
\hline 184324 chr13 & $23,545,717$ & $23,553,518$ & deletion & - \\
\hline 184324 chr13 & $38,076,308$ & $38,085,537$ & deletion & - \\
\hline 184324 chr14 & $20,197,311$ & $20,422,799$ & duplication & OR4K5;OR4K1;OR4K2;OR4Q3;OR4N2;OR4M1 \\
\hline 184324 chr14 & $41,610,224$ & $41,661,685$ & deletion & - \\
\hline 184324 chr14 & $42,988,346$ & $42,992,844$ & deletion & - \\
\hline 184324 chr14 & $73,527,253$ & $73,530,678$ & deletion & - \\
\hline 184324 chr16 & $19,945,540$ & $19,962,636$ & deletion & - \\
\hline 184324 chr17 & $44,234,944$ & $44,280,724$ & duplication & KANSL1 \\
\hline $184324 \mathrm{chr} 18$ & $63,200,484$ & $63,206,335$ & duplication & - \\
\hline 184324 chr19 & $43,298,958$ & $43,539,189$ & deletion & PSG6;PSG7;PSG11;PSG1 \\
\hline 184324 chr19 & $53,518,747$ & $53,552,296$ & duplication & ERVV-1 \\
\hline 184324 chr19 & $58,790,095$ & $58,790,807$ & deletion & ZNF8 \\
\hline 184324 chr19 & $9,275,282$ & $9,282,176$ & deletion & 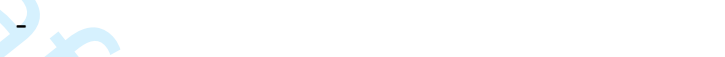 \\
\hline 184324 chr2 & $123,477,465$ & $123,482,301$ & deletion & \\
\hline 184324 chr2 & $17,220,064$ & $17,239,502$ & deletion & - \\
\hline $184324 \mathrm{chr} 2$ & $4,212,725$ & $4,222,144$ & deletion & - \\
\hline $184324 \mathrm{chr} 2$ & $51,926,599$ & $51,926,904$ & deletion & - \\
\hline $184324 \mathrm{chr} 2$ & $52,759,890$ & $52,784,952$ & deletion & - \\
\hline 184324 chr21 & $23,655,900$ & $23,665,470$ & deletion & - \\
\hline 184324 chr22 & $37,743,310$ & $37,749,946$ & deletion & - \\
\hline $184324 \mathrm{chr} 3$ & $191,064,647$ & $191,070,300$ & deletion & - \\
\hline 184324 chr3 & $37,979,882$ & $37,990,299$ & deletion & CTDSPL \\
\hline $184324 \mathrm{chr} 4$ & $171,269,504$ & $171,273,360$ & deletion & - \\
\hline $184324 \mathrm{chr} 4$ & $190,194,200$ & $190,200,463$ & deletion & - \\
\hline $184324 \mathrm{chr} 4$ & $34,785,955$ & $34,818,502$ & deletion & - \\
\hline 184324 chr5 & $140,225,908$ & $140,238,311$ & deletion & PCDHA10;PCDHA9 \\
\hline $184324 \mathrm{chr} 5$ & $151,514,956$ & $151,518,615$ & deletion & - \\
\hline 184324 chr5 & $155,477,866$ & $155,495,107$ & deletion & - \\
\hline 184324 chr6 & $19,041,346$ & $19,048,773$ & deletion & - \\
\hline 184324 chr6 & $78,972,930$ & $79,035,739$ & deletion & - \\
\hline 184324 chr7 & $141,766,850$ & $141,793,521$ & deletion & - \\
\hline 184324 chr8 & $1,305,163$ & $1,306,920$ & deletion & - \\
\hline $184324 \mathrm{chr} 8$ & $24,972,808$ & $24,990,418$ & deletion & - \\
\hline 184324 chr8 & $39,236,582$ & $39,382,903$ & deletion & - \\
\hline $184324 \mathrm{chr} 8$ & $79,180,414$ & $79,185,324$ & deletion & - \\
\hline 184324 chr8 & $9,055,379$ & $9,062,490$ & deletion & - \\
\hline
\end{tabular}




\begin{tabular}{|c|c|c|c|c|}
\hline 184324 chr9 & $23,363,526$ & $23,376,817$ & deletion & - \\
\hline 184324 chrX & $23,737,518$ & $23,742,979$ & deletion & АСОT9 \\
\hline $184328 \mathrm{chr} 1$ & $104,103,057$ & $104,211,046$ & duplication & AMY1C;AMY1A;AMY2A;AMY2B;AMY1B \\
\hline $184328 \mathrm{chr} 1$ & $143,343,508$ & $143,544,285$ & duplication & - \\
\hline 184328 chr1 & $149,032,299$ & $149,233,182$ & deletion & - \\
\hline $184328 \mathrm{chr} 1$ & $161,559,327$ & $161,619,741$ & duplication & FCGR2C;FCGR3B \\
\hline 184328 chr1 & $17,594,645$ & $17,620,391$ & duplication & PADI3 \\
\hline 184328 chr1 & $248,609,259$ & $248,645,623$ & duplication & OR2T2;OR2T3 \\
\hline 184328 chr1 & $248,740,572$ & $248,795,110$ & deletion & OR2T10;OR2T11 \\
\hline $184328 \mathrm{chr} 1$ & $62,113,597$ & $62,119,136$ & deletion & - \\
\hline 184328 chr1 & $72,754,314$ & $72,763,324$ & deletion & - \\
\hline 184328 chr10 & $132,635,031$ & $132,638,422$ & deletion & - \\
\hline 184328 chr10 & $53,204,654$ & $53,211,993$ & deletion & - \\
\hline 184328 chr11 & $90,193,796$ & $90,195,742$ & deletion & - \\
\hline 184328 chr12 & $11,504,864$ & $11,543,084$ & duplication & PRB1 \\
\hline 184328 chr12 & $132,323,294$ & $132,325,376$ & deletion & MMP17 \\
\hline 184328 chr12 & $31,266,287$ & $31,407,303$ & duplication & - \\
\hline 184328 chr12 & $33,300,995$ & $33,305,855$ & deletion & - \\
\hline 184328 chr12 & $38,315,433$ & $38,328,711$ & deletion & - \\
\hline 184328 chr12 & $59,935,026$ & $59,942,122$ & deletion & - \\
\hline 184328 chr12 & $6,241,446$ & $6,259,552$ & deletion & - \\
\hline 184328 chr13 & $21,728,134$ & $21,732,193$ & duplication & SKA3 \\
\hline 184328 chr15 & $20,196,661$ & $21,241,118$ & duplication & GOLGA6L6;POTEB2;POTEB;POTEB3 \\
\hline 184328 chr15 & $22,299,434$ & $22,652,330$ & duplication & OR4M2;OR4N4 \\
\hline $184328 \mathrm{chr} 16$ & $70,183,624$ & $70,196,139$ & duplication & PDPR \\
\hline 184328 chr18 & $63,907,596$ & $63,910,708$ & deletion & - \\
\hline 184328 chr19 & $20,601,006$ & $20,717,536$ & deletion & - \\
\hline $184328 \mathrm{chr} 19$ & $44,198,526$ & $44,210,219$ & duplication & - \\
\hline 184328 chr19 & $54,556,755$ & $54,560,078$ & deletion & - \\
\hline 184328 chr19 & $54,728,767$ & $54,743,217$ & duplication & LILRA6 \\
\hline $184328 \mathrm{chr} 2$ & $133,011,148$ & $133,031,030$ & duplication & - \\
\hline 184328 chr2 & $17,220,064$ & $17,239,502$ & deletion & - \\
\hline $184328 \mathrm{chr} 2$ & $184,795,725$ & $184,803,456$ & deletion & - \\
\hline $184328 \mathrm{chr} 2$ & $227,343,513$ & $227,347,453$ & deletion & - \\
\hline 184328 chr2 & $34,697,895$ & $34,726,904$ & deletion & - \\
\hline $184328 \mathrm{chr} 2$ & $41,239,457$ & $41,248,468$ & deletion & - \\
\hline 184328 chr2 & $48,851,541$ & $48,856,770$ & deletion & - \\
\hline 184328 chr2 & $52,759,890$ & $52,784,952$ & deletion & - \\
\hline 184328 chr20 & $62,194,128$ & $62,200,576$ & deletion & HELZ2 \\
\hline 184328 chr3 & $119,352,669$ & $119,353,893$ & deletion & - \\
\hline 184328 chr3 & $162,130,691$ & $162,142,475$ & deletion & - \\
\hline 184328 chr3 & $193,136,358$ & $193,140,348$ & deletion & - \\
\hline $184328 \mathrm{chr} 3$ & $195,457,853$ & $195,468,690$ & duplication & MUC20 \\
\hline $184328 \mathrm{chr} 3$ & $41,092,547$ & $41,144,695$ & deletion & - \\
\hline 184328 chr3 & $65,189,366$ & $65,214,685$ & deletion & - \\
\hline 184328 chr3 & $6,651,929$ & $6,654,060$ & deletion & - \\
\hline $184328 \mathrm{chr} 4$ & $10,396,298$ & $10,400,156$ & deletion & - \\
\hline
\end{tabular}




\begin{tabular}{|c|c|c|c|c|}
\hline $184328 \mathrm{chr} 4$ & $108,064,023$ & $108,074,123$ & deletion & - \\
\hline $184328 \mathrm{chr} 4$ & $49,094,981$ & $49,325,091$ & duplication & - \\
\hline $184328 \mathrm{chr} 4$ & $69,374,369$ & $69,431,280$ & deletion & UGT2B17 \\
\hline $184328 \mathrm{chr} 5$ & $151,514,956$ & $151,518,615$ & deletion & - \\
\hline 184328 chr6 & $19,040,857$ & $19,048,773$ & deletion & - \\
\hline 184328 chr6 & $32,486,060$ & $32,557,626$ & duplication & HLA-DRB1;HLA-DRB5 \\
\hline 184328 chr6 & $67,011,900$ & $67,048,885$ & deletion & - \\
\hline $184328 \mathrm{chr} 7$ & $142,476,094$ & $142,484,042$ & deletion & - \\
\hline 184328 chr7 & $156,387,296$ & $156,394,307$ & deletion & - \\
\hline 184328 chr7 & $20,750,774$ & $20,753,528$ & deletion & - \\
\hline 184328 chr8 & $15,401,929$ & $15,411,867$ & deletion & - \\
\hline $184328 \mathrm{chr} 8$ & $39,232,581$ & $39,385,979$ & duplication & - \\
\hline 184328 chr8 & $40,184,872$ & $40,189,688$ & deletion & - \\
\hline 184328 chr8 & $47,526,999$ & $47,539,088$ & duplication & - \\
\hline 184328 chr8 & $5,595,511$ & $5,605,706$ & deletion & - \\
\hline 184328 chr9 & $104,715,329$ & $104,722,688$ & deletion & - \\
\hline 184328 chr9 & $2,148,213$ & $2,151,397$ & deletion & - \\
\hline 184328 chr9 & $68,413,706$ & $68,502,538$ & duplication & - \\
\hline $184328 \mathrm{chr} X$ & $134,294,325$ & $134,324,449$ & duplication & СТ55 \\
\hline 184358 chr1 & $104,153,766$ & $104,161,227$ & duplication & AMY2A \\
\hline $184358 \mathrm{chr} 1$ & $153,674,704$ & $153,690,013$ & duplication & - \\
\hline 184358 chr1 & $196,712,596$ & $196,901,753$ & deletion & CFHR3;CFHR1;CFHR4;CFH \\
\hline $184358 \mathrm{chr} 1$ & $226,018,766$ & $226,045,056$ & duplication & TMEM63A;EPHX1 \\
\hline $184358 \mathrm{chr} 1$ & $248,740,572$ & $248,795,110$ & deletion & OR2T10;OR2T11 \\
\hline 184358 chr10 & $38,774,749$ & $38,877,428$ & duplication & \\
\hline 184358 chr10 & $83,883,951$ & $83,888,343$ & deletion & - \\
\hline 184358 chr11 & $134,602,333$ & $134,607,484$ & deletion & - \\
\hline 184358 chr11 & $55,365,761$ & $55,427,700$ & deletion & OR4P4;OR4C11;OR4S2 \\
\hline 184358 chr11 & $93,019,466$ & $93,028,940$ & deletion & - \\
\hline 184358 chr12 & $11,504,864$ & $11,546,987$ & deletion & PRB1;PRB2 \\
\hline 184358 chr12 & $12,532,984$ & $12,541,869$ & deletion & - \\
\hline 184358 chr12 & $43,021,431$ & $43,027,453$ & deletion & - \\
\hline 184358 chr12 & $59,935,926$ & $59,942,122$ & deletion & - \\
\hline 184358 chr13 & $111,587,429$ & $111,588,967$ & deletion & - \\
\hline 184358 chr13 & $114,050,622$ & $114,054,326$ & deletion & - \\
\hline 184358 chr13 & $19,342,790$ & $19,358,874$ & deletion & - \\
\hline 184358 chr15 & $20,870,571$ & $21,241,118$ & duplication & РОTЕВ2;РОТЕВ;РОТЕВ3 \\
\hline 184358 chr17 & $34,438,753$ & $34,534,918$ & duplication & CCL3L3;CCL3L1 \\
\hline 184358 chr17 & $54,160,465$ & $54,172,668$ & deletion & - \\
\hline 184358 chr18 & $14,549,799$ & $14,550,375$ & deletion & - \\
\hline 184358 chr19 & $35,852,103$ & $35,861,695$ & deletion & - \\
\hline $184358 \mathrm{chr} 2$ & $14,702,635$ & $14,709,611$ & deletion & - \\
\hline $184358 \mathrm{chr} 2$ & $17,220,064$ & $17,239,502$ & deletion & - \\
\hline 184358 chr2 & $184,795,725$ & $184,803,456$ & deletion & - \\
\hline 184358 chr2 & $195,979,780$ & $195,982,450$ & deletion & - \\
\hline 184358 chr21 & $31,136,207$ & $31,140,753$ & deletion & - \\
\hline 184358 chr22 & $22,313,128$ & $22,574,672$ & duplication & TOP3В \\
\hline
\end{tabular}




\begin{tabular}{|c|c|c|c|c|c|}
\hline 184358 & chr22 & $37,745,101$ & $37,749,946$ & deletion & - \\
\hline 184358 & chr3 & $11,411,823$ & $11,414,339$ & deletion & - \\
\hline 184358 & chr3 & $119,352,669$ & $119,353,893$ & deletion & - \\
\hline 184358 & chr3 & $173,239,453$ & $173,290,024$ & duplication & - \\
\hline 184358 & chr3 & $191,065,392$ & $191,070,300$ & deletion & - \\
\hline 184358 & chr3 & $6,651,929$ & $6,654,060$ & deletion & - \\
\hline 184358 & chr3 & $89,402,409$ & $89,418,331$ & deletion & - \\
\hline 184358 & chr3 & $98,945,571$ & $98,947,547$ & deletion & - \\
\hline 184358 & chr4 & $10,396,298$ & $10,400,156$ & deletion & - \\
\hline 184358 & chr4 & $116,168,158$ & $116,173,928$ & deletion & - \\
\hline 184358 & chr4 & $138,091,446$ & $138,098,601$ & deletion & - \\
\hline 184358 & chr4 & $34,785,955$ & $34,818,502$ & deletion & - \\
\hline 184358 & chr4 & $39,705,484$ & $39,713,134$ & deletion & - \\
\hline 184358 & chr4 & $69,374,369$ & $69,431,280$ & deletion & UGT2B17 \\
\hline 184358 & chr5 & $140,555,135$ & $140,558,618$ & duplication & PCDHB8;PCDHB7 \\
\hline 184358 & chr5 & $155,477,866$ & $155,488,917$ & deletion & - \\
\hline 184358 & chr5 & $9,267,171$ & $9,270,950$ & deletion & - \\
\hline 184358 & chr6 & $124,432,882$ & $124,470,796$ & duplication & NKAIN2 \\
\hline 184358 & chr6 & $19,041,346$ & $19,048,773$ & deletion & - \\
\hline 184358 & chr6 & $32,584,901$ & $32,586,024$ & deletion & - \\
\hline 184358 & chr6 & $78,972,930$ & $79,035,739$ & deletion & - \\
\hline 184358 & chr7 & $150,263,761$ & $150,268,098$ & deletion & GIMAP4 \\
\hline 184358 & chr7 & $38,291,851$ & $38,305,404$ & duplication & TARP \\
\hline 184358 & chr7 & $86,940,652$ & $86,948,364$ & deletion & - \\
\hline 184358 & chr7 & $97,430,156$ & $97,437,364$ & deletion & \\
\hline 184358 & chr8 & $17,580,791$ & $17,581,700$ & deletion & MTUS1 \\
\hline 184358 & chr8 & $39,232,581$ & $39,385,979$ & duplication & - \\
\hline 184358 & chr9 & $2,148,213$ & $2,151,397$ & deletion & - \\
\hline 184358 & chr9 & $6,701,130$ & $6,707,794$ & deletion & - \\
\hline 184358 & $\operatorname{chrX}$ & $6,019,681$ & $6,026,208$ & deletion & - \\
\hline 184359 & chr1 & $226,018,766$ & $226,045,805$ & duplication & TMEM63A;EPHX1 \\
\hline 184359 & chr1 & $248,740,572$ & $248,795,110$ & deletion & OR2T10;OR2T11 \\
\hline 184359 & chr10 & $127,071,929$ & $127,076,951$ & duplication & - \\
\hline 184359 & chr10 & $17,573,638$ & $17,697,879$ & deletion & HACD1;STAM \\
\hline 184359 & chr10 & $20,850,624$ & $20,857,365$ & deletion & - \\
\hline 184359 & chr10 & $38,774,749$ & $38,877,428$ & duplication & - \\
\hline 184359 & chr11 & $18,949,220$ & $18,961,743$ & duplication & MRGPRX1 \\
\hline 184359 & chr11 & $24,446,609$ & $24,451,830$ & deletion & - \\
\hline 184359 & chr11 & $55,365,761$ & $55,427,700$ & deletion & OR4P4;OR4C11;OR4S2 \\
\hline 184359 & chr11 & $93,020,527$ & $93,028,940$ & deletion & - \\
\hline 184359 & chr12 & $11,220,827$ & $11,244,117$ & deletion & TAS2R43 \\
\hline 184359 & chr12 & $11,504,864$ & $11,543,084$ & deletion & PRB1 \\
\hline 184359 & chr12 & $12,532,984$ & $12,544,338$ & deletion & - \\
\hline 184359 & chr12 & $33,300,995$ & $33,305,855$ & deletion & - \\
\hline 184359 & chr12 & $43,021,431$ & $43,027,453$ & deletion & - \\
\hline 184359 & chr13 & $111,587,429$ & $111,588,967$ & deletion & - \\
\hline 184359 & chr13 & $19,342,790$ & $19,358,874$ & deletion & - \\
\hline
\end{tabular}




\begin{tabular}{|c|c|c|c|c|}
\hline 184359 chr15 & $43,888,976$ & $43,939,642$ & deletion & STRC;CATSPER2;CKMT1B \\
\hline 184359 chr16 & $6,165,539$ & $6,190,035$ & deletion & - \\
\hline 184359 chr17 & $34,438,753$ & $34,483,342$ & duplication & - \\
\hline 184359 chr2 & $123,477,465$ & $123,482,301$ & deletion & - \\
\hline $184359 \mathrm{chr} 2$ & $184,795,725$ & $184,803,456$ & deletion & - \\
\hline 184359 chr2 & $34,697,895$ & $34,726,904$ & duplication & - \\
\hline $184359 \mathrm{chr} 2$ & $51,926,599$ & $51,926,904$ & deletion & - \\
\hline 184359 chr22 & $22,313,591$ & $22,574,672$ & duplication & TOP3B \\
\hline 184359 chr22 & $37,745,101$ & $37,749,946$ & deletion & - \\
\hline 184359 chr22 & $39,359,885$ & $39,385,663$ & deletion & АРОВЕСЗВ \\
\hline 184359 chr22 & $42,902,278$ & $42,950,033$ & duplication & SERHL2;RRP7A \\
\hline 184359 chr3 & $173,239,453$ & $173,292,699$ & duplication & - \\
\hline 184359 chr3 & $195,457,853$ & $195,468,690$ & duplication & MUC20 \\
\hline $184359 \mathrm{chr} 3$ & $6,651,929$ & $6,654,060$ & deletion & - \\
\hline $184359 \mathrm{chr} 4$ & $108,064,023$ & $108,074,123$ & deletion & - \\
\hline $184359 \mathrm{chr} 4$ & $116,168,158$ & $116,173,928$ & deletion & - \\
\hline $184359 \mathrm{chr} 4$ & $138,092,298$ & $138,098,601$ & deletion & - \\
\hline $184359 \mathrm{chr} 4$ & $186,397,915$ & $186,420,113$ & deletion & - \\
\hline $184359 \mathrm{chr} 4$ & $34,785,955$ & $34,818,502$ & deletion & - \\
\hline $184359 \mathrm{chr} 4$ & $9,370,814$ & $9,486,051$ & duplication & DEFB131 \\
\hline 184359 chr5 & $112,264,968$ & $112,277,452$ & deletion & - \\
\hline 184359 chr5 & $117,388,410$ & $117,393,058$ & deletion & - \\
\hline 184359 chr5 & $155,477,866$ & $155,488,917$ & deletion & - \\
\hline 184359 chr6 & $124,432,882$ & $124,475,211$ & duplication & NKAIN2 \\
\hline 184359 chr6 & $19,041,346$ & $19,048,773$ & deletion & - \\
\hline 184359 chr6 & $34,725,102$ & $34,887,804$ & duplication & SNRPC;TAF11;UHRF1BP1;ANKS1A \\
\hline 184359 chr6 & $78,972,930$ & $79,035,739$ & deletion & - \\
\hline 184359 chr7 & $150,263,761$ & $150,268,098$ & deletion & GIMAP4 \\
\hline 184359 chr7 & $38,291,851$ & $38,307,990$ & duplication & TARP \\
\hline 184359 chr8 & $17,580,791$ & $17,581,700$ & deletion & MTUS1 \\
\hline 184359 chr8 & $24,972,808$ & $24,990,418$ & duplication & - \\
\hline 184359 chr8 & $39,232,581$ & $39,385,979$ & deletion & - \\
\hline 184359 chr8 & $51,029,371$ & $51,038,149$ & deletion & - \\
\hline 184359 chr8 & $57,964,317$ & $57,967,489$ & deletion & - \\
\hline 184359 chr8 & $58,126,687$ & $58,130,784$ & deletion & - \\
\hline 184359 chr9 & $104,715,329$ & $104,722,688$ & deletion & - \\
\hline 184359 chr9 & $135,624,197$ & $135,626,396$ & deletion & - \\
\hline 184359 chr9 & $78,004,378$ & $78,011,616$ & deletion & - \\
\hline $184360 \mathrm{chr} 1$ & $104,153,766$ & $104,211,046$ & deletion & AMY1A;AMY2A;AMY1C;AMY1B \\
\hline $184360 \mathrm{chr} 1$ & $116,235,183$ & $116,287,357$ & duplication & CASQ2 \\
\hline 184360 chr10 & $89,068,877$ & $89,112,638$ & deletion & - \\
\hline 184360 chr11 & $7,814,299$ & $7,827,879$ & deletion & OR5P2 \\
\hline 184360 chr11 & $93,020,527$ & $93,028,940$ & deletion & - \\
\hline 184360 chr12 & $129,230,513$ & $129,233,013$ & deletion & - \\
\hline 184360 chr12 & $31,279,428$ & $31,407,303$ & duplication & - \\
\hline 184360 chr13 & $111,587,429$ & $111,588,967$ & deletion & - \\
\hline 184360 chr13 & $112,592,299$ & $112,597,912$ & deletion & - \\
\hline
\end{tabular}




\begin{tabular}{|c|c|c|c|c|}
\hline 184360 chr13 & $27,522,965$ & $27,531,466$ & deletion & - \\
\hline 184360 chr13 & $38,076,308$ & $38,085,537$ & deletion & - \\
\hline 184360 chr14 & $106,062,675$ & $106,178,945$ & duplication & - \\
\hline 184360 chr14 & $106,891,524$ & $106,931,129$ & deletion & - \\
\hline 184360 chr14 & $107,157,561$ & $107,181,703$ & duplication & - \\
\hline 184360 chr14 & $35,604,892$ & $35,613,500$ & deletion & - \\
\hline $184360 \mathrm{chr} 14$ & $42,988,346$ & $42,992,844$ & deletion & - \\
\hline 184360 chr14 & $79,160,530$ & $79,165,608$ & deletion & - \\
\hline 184360 chr16 & $32,542,736$ & $32,609,440$ & duplication & - \\
\hline $184360 \mathrm{chr} 16$ & $34,471,298$ & $34,756,258$ & duplication & - \\
\hline $184360 \mathrm{chr} 16$ & $55,800,009$ & $55,819,443$ & deletion & - \\
\hline $184360 \mathrm{chr} 16$ & $77,424,605$ & $77,434,657$ & duplication & - \\
\hline 184360 chr17 & $34,438,753$ & $34,534,918$ & duplication & CCL3L3;CCL3L1 \\
\hline 184360 chr18 & $55,931,179$ & $55,937,109$ & deletion & - \\
\hline 184360 chr19 & $35,852,103$ & $35,861,695$ & deletion & - \\
\hline $184360 \mathrm{chr} 2$ & $238,554,352$ & $238,558,478$ & deletion & - \\
\hline 184360 chr2 & $48,851,541$ & $48,856,770$ & deletion & - \\
\hline $184360 \mathrm{chr} 2$ & $51,926,599$ & $51,926,904$ & deletion & - \\
\hline $184360 \mathrm{chr} 2$ & $52,759,890$ & $52,784,952$ & deletion & - \\
\hline $184360 \mathrm{chr} 2$ & $89,540,620$ & $90,275,455$ & duplication & - \\
\hline 184360 chr22 & $22,313,128$ & $22,574,672$ & duplication & TOP3В \\
\hline 184360 chr22 & $37,745,101$ & $37,749,946$ & deletion & - \\
\hline $184360 \mathrm{chr} 3$ & $119,350,324$ & $119,353,893$ & deletion & - \\
\hline $184360 \mathrm{chr} 3$ & $173,239,453$ & $173,290,024$ & duplication & - \\
\hline 184360 chr3 & $175,887,314$ & $175,909,890$ & deletion & \\
\hline $184360 \mathrm{chr} 4$ & $116,168,158$ & $116,177,403$ & deletion & - \\
\hline $184360 \mathrm{chr} 4$ & $138,091,446$ & $138,098,601$ & deletion & - \\
\hline $184360 \mathrm{chr} 4$ & $171,269,504$ & $171,273,360$ & deletion & - \\
\hline $184360 \mathrm{chr} 4$ & $34,785,955$ & $34,818,502$ & deletion & - \\
\hline $184360 \mathrm{chr} 4$ & $64,136,609$ & $64,154,477$ & deletion & - \\
\hline $184360 \mathrm{chr} 4$ & $69,374,369$ & $69,429,037$ & deletion & UGT2B17 \\
\hline 184360 chr5 & $140,555,135$ & $140,558,618$ & duplication & PCDHB8;PCDHB7 \\
\hline 184360 chr6 & $124,432,882$ & $124,470,796$ & duplication & NKAIN2 \\
\hline 184360 chr6 & $19,041,346$ & $19,048,773$ & deletion & - \\
\hline $184360 \mathrm{chr} 6$ & $34,725,102$ & $34,887,804$ & duplication & SNRPC;TAF11;UHRF1BP1;ANKS1A \\
\hline $184360 \mathrm{chr} 6$ & $55,827,939$ & $55,847,710$ & deletion & - \\
\hline 184360 chr6 & $78,972,930$ & $79,035,739$ & deletion & - \\
\hline 184360 chr6 & $81,284,205$ & $81,293,326$ & deletion & - \\
\hline $184360 \mathrm{chr} 7$ & $38,291,851$ & $38,305,404$ & duplication & TARP \\
\hline $184360 \mathrm{chr} 7$ & $86,940,652$ & $86,948,364$ & deletion & - \\
\hline $184360 \mathrm{chr} 8$ & $125,868,822$ & $125,970,558$ & duplication & - \\
\hline 184360 chr8 & $16,733,791$ & $16,736,919$ & deletion & - \\
\hline $184360 \mathrm{chr} 8$ & $39,232,581$ & $39,382,903$ & deletion & - \\
\hline $184360 \mathrm{chr} 9$ & $2,148,213$ & $2,153,066$ & deletion & - \\
\hline 184360 chr9 & $23,363,526$ & $23,376,817$ & duplication & - \\
\hline 184360 chr9 & $44,739,805$ & $44,834,316$ & deletion & - \\
\hline 184360 chr9 & $6,701,130$ & $6,707,794$ & deletion & - \\
\hline
\end{tabular}




\begin{tabular}{|c|c|c|c|c|}
\hline 184362 chr1 & $104,153,766$ & $104,211,046$ & deletion & AMY1A;AMY2A;AMY1C;AMY1B \\
\hline $184362 \mathrm{chr} 1$ & $149,032,299$ & $149,432,892$ & deletion & - \\
\hline 184362 chr1 & $187,716,107$ & $187,722,032$ & deletion & - \\
\hline $184362 \mathrm{chr} 1$ & $62,280,304$ & $62,285,580$ & deletion & - \\
\hline $184362 \mathrm{chr} 10$ & $71,280,107$ & $71,289,190$ & deletion & - \\
\hline 184362 chr11 & $49,715,154$ & $49,757,690$ & deletion & - \\
\hline 184362 chr12 & $11,220,827$ & $11,244,117$ & deletion & TAS2R43 \\
\hline 184362 chr13 & $34,134,809$ & $34,143,545$ & deletion & - \\
\hline 184362 chr14 & $20,197,311$ & $20,424,184$ & duplication & OR4K5;OR4K1;OR4K2;OR4Q3;OR4N2;OR4M1 \\
\hline 184362 chr14 & $41,610,224$ & $41,661,685$ & deletion & - \\
\hline 184362 chr16 & $22,635,867$ & $22,703,741$ & deletion & - \\
\hline 184362 chr16 & $55,798,095$ & $55,819,443$ & deletion & - \\
\hline $184362 \mathrm{chr} 17$ & $44,166,604$ & $44,356,058$ & duplication & KANSL1 \\
\hline 184362 chr18 & $67,208,806$ & $67,217,271$ & deletion & - \\
\hline 184362 chr19 & $35,852,103$ & $35,861,695$ & deletion & - \\
\hline 184362 chr19 & $4,232,960$ & $4,237,067$ & deletion & $\mathrm{EBI3}$ \\
\hline 184362 chr19 & $43,647,130$ & $43,744,060$ & deletion & PSG4;PSG5 \\
\hline 184362 chr19 & $53,518,747$ & $53,552,296$ & duplication & ERVV-1 \\
\hline 184362 chr19 & $54,556,755$ & $54,560,078$ & deletion & - \\
\hline 184362 chr19 & $54,728,767$ & $54,743,217$ & duplication & LILRA6 \\
\hline $184362 \mathrm{chr} 2$ & $213,187,034$ & $213,191,389$ & deletion & - \\
\hline $184362 \mathrm{chr} 2$ & $242,854,089$ & $243,034,519$ & deletion & - \\
\hline $184362 \mathrm{chr} 2$ & $35,577,771$ & $35,683,261$ & deletion & - \\
\hline $184362 \mathrm{chr} 2$ & $48,851,541$ & $48,856,770$ & deletion & - \\
\hline 184362 chr21 & $10,818,766$ & $10,858,651$ & duplication & - \\
\hline 184362 chr3 & $129,769,827$ & $129,806,304$ & deletion & ALG1L2 \\
\hline $184362 \mathrm{chr} 3$ & $195,457,853$ & $195,468,690$ & duplication & MUC20 \\
\hline 184362 chr3 & $20,797,427$ & $20,815,352$ & deletion & - \\
\hline $184362 \mathrm{chr} 3$ & $53,032,639$ & $53,038,786$ & deletion & - \\
\hline $184362 \mathrm{chr} 3$ & $98,945,571$ & $98,947,547$ & deletion & - \\
\hline $184362 \mathrm{chr} 4$ & $108,064,023$ & $108,074,123$ & deletion & - \\
\hline $184362 \mathrm{chr} 4$ & $190,194,200$ & $190,200,463$ & deletion & - \\
\hline $184362 \mathrm{chr} 4$ & $34,785,955$ & $34,818,502$ & deletion & - \\
\hline $184362 \mathrm{chr} 5$ & $140,225,908$ & $140,238,311$ & deletion & PCDHA10;PCDHA9 \\
\hline 184362 chr5 & $141,290,904$ & $141,299,586$ & deletion & - \\
\hline 184362 chr6 & $132,709,027$ & $132,711,200$ & deletion & - \\
\hline $184362 \mathrm{chr} 6$ & $29,856,693$ & $29,899,189$ & deletion & - \\
\hline 184362 chr6 & $30,994,010$ & $30,994,787$ & deletion & MUC22 \\
\hline 184362 chr6 & $31,281,682$ & $31,299,163$ & deletion & - \\
\hline 184362 chr6 & $32,458,168$ & $32,538,693$ & deletion & HLA-DRB5 \\
\hline 184362 chr6 & $69,231,883$ & $69,241,954$ & duplication & - \\
\hline 184362 chr6 & $78,974,982$ & $79,012,428$ & duplication & - \\
\hline $184362 \mathrm{chr} 7$ & $142,476,094$ & $142,483,244$ & deletion & - \\
\hline $184362 \mathrm{chr} 7$ & $143,932,654$ & $143,969,480$ & deletion & CTAGE8;CTAGE4;OR2A7 \\
\hline $184362 \mathrm{chr} 7$ & $159,118,443$ & $159,122,741$ & deletion & - \\
\hline 184362 chr8 & $15,401,929$ & $15,411,867$ & deletion & - \\
\hline $184362 \mathrm{chr} 8$ & $39,313,590$ & $39,370,142$ & deletion & - \\
\hline
\end{tabular}




\begin{tabular}{|c|c|c|c|c|}
\hline $184362 \mathrm{chr} 8$ & $72,214,753$ & $72,217,690$ & deletion & - \\
\hline $184362 \mathrm{chr} 8$ & $87,188,572$ & $87,192,895$ & deletion & - \\
\hline 184362 chr9 & $23,363,526$ & $23,376,817$ & deletion & - \\
\hline 184362 chr9 & $24,146,203$ & $24,150,034$ & deletion & - \\
\hline 184362 chr9 & $66,533,728$ & $66,862,785$ & deletion & - \\
\hline 184362 chr9 & $69,786,015$ & $69,881,429$ & deletion & - \\
\hline $184364 \mathrm{chr} 1$ & $104,153,766$ & $104,161,227$ & deletion & AMY2A \\
\hline 184364 chr1 & $109,367,944$ & $109,371,874$ & deletion & AKNAD1 \\
\hline 184364 chr1 & $161,559,327$ & $161,619,741$ & deletion & FCGR2C;FCGR3B \\
\hline $184364 \mathrm{chr} 1$ & $187,716,107$ & $187,722,032$ & deletion & - \\
\hline 184364 chr1 & $72,754,314$ & $72,763,324$ & deletion & - \\
\hline 184364 chr10 & $132,635,459$ & $132,638,422$ & deletion & - \\
\hline 184364 chr10 & $71,279,367$ & $71,289,190$ & deletion & - \\
\hline 184364 chr11 & $18,949,220$ & $18,961,743$ & duplication & MRGPRX1 \\
\hline 184364 chr11 & $49,715,154$ & $49,757,690$ & deletion & - \\
\hline 184364 chr12 & $40,875,351$ & $40,875,963$ & deletion & MUC19 \\
\hline 184364 chr12 & $43,021,431$ & $43,027,453$ & deletion & - \\
\hline 184364 chr13 & $38,076,308$ & $38,085,537$ & deletion & - \\
\hline 184364 chr14 & $41,610,224$ & $41,661,685$ & deletion & - \\
\hline 184364 chr16 & $34,471,298$ & $34,756,258$ & duplication & - \\
\hline 184364 chr16 & $55,798,095$ & $55,819,443$ & deletion & - \\
\hline 184364 chr16 & $78,372,894$ & $78,383,509$ & deletion & - \\
\hline 184364 chr18 & $55,931,179$ & $55,937,109$ & deletion & - \\
\hline 184364 chr18 & $63,907,596$ & $63,910,708$ & deletion & \\
\hline 184364 chr18 & $66,746,208$ & $66,755,736$ & deletion & \\
\hline 184364 chr19 & $35,852,103$ & $35,861,695$ & deletion & - \\
\hline $184364 \mathrm{chr} 2$ & $14,704,369$ & $14,709,611$ & deletion & - \\
\hline 184364 chr2 & $184,085,551$ & $184,088,256$ & deletion & - \\
\hline $184364 \mathrm{chr} 2$ & $212,771,670$ & $212,773,893$ & deletion & - \\
\hline $184364 \mathrm{chr} 2$ & $38,301,735$ & $38,305,451$ & deletion & CYP1B1 \\
\hline 184364 chr21 & $25,296,221$ & $25,299,689$ & deletion & - \\
\hline 184364 chr21 & $28,242,508$ & $28,252,829$ & deletion & - \\
\hline 184364 chr3 & $191,061,214$ & $191,070,300$ & deletion & - \\
\hline $184364 \mathrm{chr} 4$ & $122,285,263$ & $122,289,863$ & deletion & - \\
\hline $184364 \mathrm{chr} 4$ & $64,136,609$ & $64,154,477$ & deletion & - \\
\hline $184364 \mathrm{chr} 4$ & $69,374,369$ & $69,431,280$ & deletion & UGT2B17 \\
\hline $184364 \mathrm{chr} 4$ & $70,137,897$ & $70,229,260$ & deletion & UGT2B28 \\
\hline $184364 \mathrm{chr} 5$ & $109,475,723$ & $109,487,887$ & duplication & - \\
\hline $184364 \mathrm{chr} 5$ & $120,424,662$ & $120,467,190$ & deletion & - \\
\hline 184364 chr5 & $151,514,956$ & $151,518,615$ & deletion & - \\
\hline 184364 chr5 & $155,477,866$ & $155,495,107$ & deletion & - \\
\hline $184364 \mathrm{chr} 5$ & 701,026 & 776,691 & duplication & - \\
\hline 184364 chr5 & $70,305,696$ & $70,308,251$ & deletion & NAIP \\
\hline 184364 chr6 & $40,364,085$ & $40,368,860$ & deletion & - \\
\hline $184364 \mathrm{chr} 7$ & $142,814,821$ & $142,890,868$ & deletion & PIP;TAS2R39 \\
\hline $184364 \mathrm{chr} 7$ & $62,149,006$ & $62,162,385$ & deletion & - \\
\hline $184364 \mathrm{chr} 8$ & $39,232,581$ & $39,385,979$ & duplication & - \\
\hline
\end{tabular}




\begin{tabular}{|c|c|c|c|c|}
\hline 184364 chr9 & $1,446,522$ & $1,450,465$ & deletion & - \\
\hline 184364 chr9 & $6,701,130$ & $6,707,794$ & deletion & - \\
\hline 184365 chr1 & $104,153,766$ & $104,161,227$ & deletion & AMY2A \\
\hline 184365 chr1 & $109,367,944$ & $109,371,874$ & deletion & AKNAD1 \\
\hline $184365 \mathrm{chr} 1$ & $1,590,681$ & $1,628,197$ & duplication & SLC35E2B \\
\hline 184365 chr1 & $161,559,327$ & $161,619,741$ & deletion & FCGR2C;FCGR3B \\
\hline 184365 chr1 & $248,740,572$ & $248,795,110$ & deletion & OR2T10;OR2T11 \\
\hline 184365 chr1 & $62,113,597$ & $62,119,136$ & deletion & - \\
\hline 184365 chr1 & $72,754,314$ & $72,763,324$ & deletion & - \\
\hline 184365 chr10 & $38,789,617$ & $38,877,428$ & duplication & - \\
\hline 184365 chr11 & $134,602,333$ & $134,607,484$ & deletion & - \\
\hline 184365 chr12 & $11,220,827$ & $11,244,117$ & deletion & TAS2R43 \\
\hline 184365 chr12 & $2,245,636$ & $2,259,042$ & deletion & - \\
\hline 184365 chr12 & $43,021,431$ & $43,027,453$ & deletion & - \\
\hline 184365 chr13 & $34,134,809$ & $34,143,545$ & deletion & - \\
\hline 184365 chr13 & $62,420,542$ & $62,499,471$ & duplication & - \\
\hline 184365 chr13 & $88,079,280$ & $88,088,427$ & deletion & - \\
\hline 184365 chr14 & $41,610,224$ & $41,661,685$ & deletion & - \\
\hline 184365 chr14 & $86,285,493$ & $86,312,888$ & deletion & - \\
\hline 184365 chr15 & $32,899,534$ & $33,105,403$ & duplication & SCG5;GREM1;FMN1;ARHGAP11A \\
\hline 184365 chr16 & $32,511,914$ & $32,642,384$ & duplication & - \\
\hline 184365 chr16 & $34,471,298$ & $34,756,258$ & duplication & - \\
\hline 184365 chr16 & $55,798,095$ & $55,819,443$ & deletion & - \\
\hline 184365 chr16 & $78,372,894$ & $78,383,509$ & deletion & \\
\hline 184365 chr16 & $85,302,707$ & $85,304,326$ & deletion & \\
\hline 184365 chr17 & $34,438,753$ & $34,483,342$ & duplication & - \\
\hline 184365 chr18 & $55,931,179$ & $55,937,109$ & deletion & - \\
\hline 184365 chr18 & $63,907,596$ & $63,910,708$ & deletion & - \\
\hline 184365 chr18 & $66,746,208$ & $66,755,736$ & deletion & - \\
\hline 184365 chr19 & $43,298,958$ & $43,539,189$ & deletion & PSG6;PSG7;PSG11;PSG1 \\
\hline 184365 chr19 & $9,275,282$ & $9,287,189$ & deletion & - \\
\hline 184365 chr2 & $14,704,369$ & $14,709,611$ & deletion & - \\
\hline $184365 \mathrm{chr} 2$ & $184,085,551$ & $184,088,256$ & deletion & - \\
\hline 184365 chr2 & $212,771,670$ & $212,773,893$ & deletion & - \\
\hline 184365 chr2 & $213,187,034$ & $213,191,389$ & deletion & - \\
\hline 184365 chr2 & $48,851,541$ & $48,856,770$ & deletion & - \\
\hline 184365 chr2 & $87,392,157$ & $87,997,476$ & duplication & - \\
\hline 184365 chr20 & $51,858,193$ & $51,865,183$ & deletion & - \\
\hline 184365 chr20 & $52,647,631$ & $52,656,535$ & deletion & - \\
\hline 184365 chr21 & $10,813,582$ & $10,858,651$ & duplication & - \\
\hline 184365 chr21 & $20,057,919$ & $20,081,051$ & duplication & - \\
\hline 184365 chr21 & $25,296,221$ & $25,299,689$ & deletion & - \\
\hline 184365 chr21 & $28,242,508$ & $28,252,829$ & deletion & - \\
\hline 184365 chr22 & $49,840,474$ & $49,907,281$ & deletion & - \\
\hline $184365 \mathrm{chr} 4$ & $64,136,609$ & $64,154,477$ & deletion & - \\
\hline 184365 chr4 & $70,137,897$ & $70,229,260$ & deletion & UGT2B28 \\
\hline 184365 chr5 & $117,385,681$ & $117,393,058$ & deletion & - \\
\hline
\end{tabular}




\begin{tabular}{|c|c|c|c|c|}
\hline 184365 chr5 & $140,225,908$ & $140,238,311$ & deletion & PCDHA10;PCDHA9 \\
\hline 184365 chr5 & $7,178,644$ & $7,191,074$ & deletion & - \\
\hline 184365 chr5 & $8,703,085$ & $8,748,627$ & deletion & - \\
\hline 184365 chr6 & $19,041,346$ & $19,048,773$ & deletion & - \\
\hline 184365 chr6 & $77,439,769$ & $77,451,301$ & deletion & - \\
\hline 184365 chr7 & $104,409,683$ & $104,479,443$ & duplication & - \\
\hline $184365 \mathrm{chr} 7$ & 41,421 & 73,060 & duplication & - \\
\hline 184365 chr7 & $70,757,913$ & $70,765,656$ & deletion & - \\
\hline 184365 chr7 & $97,396,709$ & $97,401,677$ & deletion & - \\
\hline 184365 chr8 & $72,214,753$ & $72,217,690$ & deletion & - \\
\hline 184365 chr9 & $44,739,805$ & $44,834,316$ & deletion & - \\
\hline 184365 chr9 & 718,835 & 721,782 & deletion & - \\
\hline 184365 chr9 & $78,004,378$ & $78,011,616$ & deletion & - \\
\hline 184366 chr1 & $104,153,766$ & $104,211,046$ & deletion & AMY1A;AMY2A;AMY1C;AMY1B \\
\hline $184366 \mathrm{chr} 1$ & $109,367,944$ & $109,371,874$ & deletion & AKNAD1 \\
\hline $184366 \mathrm{chr} 1$ & $62,113,597$ & $62,119,136$ & deletion & - \\
\hline 184366 chr1 & $72,754,314$ & $72,763,324$ & deletion & - \\
\hline 184366 chr10 & $132,635,459$ & $132,638,422$ & deletion & - \\
\hline 184366 chr10 & $38,677,023$ & $38,972,666$ & duplication & - \\
\hline 184366 chr10 & $39,058,671$ & $39,154,535$ & duplication & - \\
\hline 184366 chr10 & $42,791,910$ & $42,811,180$ & duplication & - \\
\hline 184366 chr10 & $71,282,981$ & $71,289,190$ & deletion & - \\
\hline 184366 chr11 & $18,949,220$ & $18,961,743$ & duplication & MRGPRX1 \\
\hline 184366 chr11 & $93,683,680$ & $93,688,541$ & deletion & - \\
\hline 184366 chr12 & $2,245,636$ & $2,254,219$ & deletion & \\
\hline 184366 chr12 & $40,875,351$ & $40,875,963$ & deletion & MUC19 \\
\hline 184366 chr12 & $43,021,431$ & $43,027,453$ & deletion & - \\
\hline 184366 chr13 & $111,587,429$ & $111,588,967$ & deletion & - \\
\hline 184366 chr13 & $21,728,134$ & $21,732,193$ & duplication & SKA3 \\
\hline 184366 chr13 & $62,420,542$ & $62,499,471$ & duplication & - \\
\hline 184366 chr14 & $106,891,524$ & $106,931,129$ & deletion & - \\
\hline 184366 chr14 & $41,610,224$ & $41,661,685$ & deletion & - \\
\hline 184366 chr15 & $32,899,534$ & $33,105,403$ & duplication & SCG5;GREM1;FMN1;ARHGAP11A \\
\hline $184366 \mathrm{chr} 16$ & $19,945,540$ & $19,962,636$ & deletion & - \\
\hline 184366 chr16 & $32,511,914$ & $32,609,440$ & duplication & - \\
\hline $184366 \mathrm{chr} 16$ & $34,471,298$ & $34,756,258$ & duplication & - \\
\hline 184366 chr16 & $35,153,618$ & $35,285,609$ & duplication & - \\
\hline 184366 chr16 & $60,556,223$ & $60,566,803$ & deletion & - \\
\hline 184366 chr19 & $35,852,103$ & $35,861,695$ & deletion & - \\
\hline $184366 \mathrm{chr} 2$ & $14,704,369$ & $14,709,611$ & deletion & - \\
\hline $184366 \mathrm{chr} 2$ & $212,771,670$ & $212,776,546$ & deletion & - \\
\hline $184366 \mathrm{chr} 2$ & $38,956,947$ & $38,971,623$ & duplication & GALM \\
\hline $184366 \mathrm{chr} 2$ & $52,759,890$ & $52,784,952$ & deletion & - \\
\hline 184366 chr20 & $29,804,016$ & $29,826,482$ & duplication & - \\
\hline 184366 chr20 & $51,858,193$ & $51,865,183$ & deletion & - \\
\hline 184366 chr20 & $52,647,631$ & $52,656,535$ & deletion & - \\
\hline 184366 chr21 & $10,815,443$ & $10,858,651$ & duplication & - \\
\hline
\end{tabular}




\begin{tabular}{|c|c|c|c|c|}
\hline 184366 chr21 & $28,242,508$ & $28,252,829$ & deletion & - \\
\hline $184366 \mathrm{chr} 4$ & $70,137,897$ & $70,229,260$ & deletion & UGT2B28 \\
\hline $184366 \mathrm{chr} 5$ & $109,475,723$ & $109,487,887$ & duplication & - \\
\hline $184366 \mathrm{chr} 5$ & $120,424,662$ & $120,467,190$ & deletion & - \\
\hline 184366 chr5 & $151,514,956$ & $151,518,615$ & deletion & - \\
\hline 184366 chr5 & $155,477,866$ & $155,488,917$ & deletion & - \\
\hline $184366 \mathrm{chr} 5$ & 701,026 & 776,691 & duplication & - \\
\hline 184366 chr5 & $70,305,696$ & $70,308,251$ & deletion & NAIP \\
\hline 184366 chr6 & $40,364,085$ & $40,368,860$ & deletion & - \\
\hline 184366 chr7 & $70,757,913$ & $70,765,656$ & deletion & - \\
\hline $184366 \mathrm{chr} 8$ & $87,188,572$ & $87,192,895$ & deletion & - \\
\hline $184366 \mathrm{chr} 9$ & $1,446,522$ & $1,450,465$ & deletion & - \\
\hline 184366 chr9 & $6,701,130$ & $6,707,794$ & deletion & - \\
\hline 184366 chr9 & $78,004,378$ & $78,011,616$ & deletion & - \\
\hline 184367 chr1 & $182,084,486$ & $182,087,284$ & deletion & - \\
\hline 184367 chr1 & $187,713,921$ & $187,722,032$ & deletion & - \\
\hline 184367 chr11 & $18,949,220$ & $18,961,743$ & duplication & MRGPRX1 \\
\hline 184367 chr12 & $11,220,827$ & $11,244,117$ & deletion & TAS2R43 \\
\hline 184367 chr12 & $27,648,411$ & $27,655,163$ & deletion & $\mathrm{SMCO} 2$ \\
\hline 184367 chr12 & $33,300,995$ & $33,305,855$ & deletion & - \\
\hline 184367 chr12 & $38,006,554$ & $38,053,317$ & duplication & - \\
\hline 184367 chr12 & $6,241,446$ & $6,247,923$ & deletion & - \\
\hline 184367 chr13 & $21,728,134$ & $21,732,193$ & duplication & SKA3 \\
\hline 184367 chr13 & $28,510,174$ & $28,524,904$ & duplication & - \\
\hline 184367 chr13 & $34,134,809$ & $34,143,545$ & deletion & \\
\hline 184367 chr14 & $35,604,892$ & $35,613,500$ & deletion & - \\
\hline 184367 chr15 & $20,870,571$ & $21,241,118$ & duplication & РОТЕВ2;РОТЕВ;РОТЕВ3 \\
\hline $184367 \mathrm{chr} 15$ & $21,903,815$ & $22,753,733$ & duplication & GOLGA6L22;GOLGA6L1;OR4N4;POTEB3;OR4N \\
\hline 184367 chr15 & $23,624,148$ & $23,668,092$ & deletion & - \\
\hline 184367 chr16 & $32,511,914$ & $32,603,033$ & duplication & - \\
\hline 184367 chr16 & $35,197,311$ & $35,223,008$ & duplication & - \\
\hline 184367 chr17 & $34,438,753$ & $34,483,342$ & duplication & - \\
\hline 184367 chr19 & $35,852,103$ & $35,861,695$ & deletion & - \\
\hline 184367 chr19 & $54,728,767$ & $54,743,217$ & deletion & LILRA6 \\
\hline $184367 \mathrm{chr} 2$ & $123,477,465$ & $123,482,301$ & deletion & - \\
\hline $184367 \mathrm{chr} 2$ & $195,979,780$ & $195,982,450$ & deletion & - \\
\hline $184367 \mathrm{chr} 2$ & $208,351,315$ & $208,357,838$ & deletion & - \\
\hline 184367 chr2 & $34,697,895$ & $34,726,904$ & deletion & - \\
\hline $184367 \mathrm{chr} 2$ & $41,239,457$ & $41,248,468$ & deletion & - \\
\hline 184367 chr2 & $51,926,599$ & $51,926,904$ & deletion & - \\
\hline 184367 chr20 & $29,428,644$ & $29,485,460$ & deletion & - \\
\hline 184367 chr21 & $44,822,871$ & $44,837,829$ & duplication & LOC102724428;SIK1 \\
\hline $184367 \mathrm{chr} 3$ & $131,711,896$ & $131,712,898$ & deletion & - \\
\hline $184367 \mathrm{chr} 3$ & $98,945,571$ & $98,947,547$ & deletion & - \\
\hline $184367 \mathrm{chr} 4$ & $10,396,298$ & $10,400,156$ & deletion & - \\
\hline $184367 \mathrm{chr} 4$ & $116,168,158$ & $116,173,928$ & deletion & - \\
\hline $184367 \mathrm{chr} 4$ & $138,092,298$ & $138,098,601$ & deletion & - \\
\hline
\end{tabular}




\begin{tabular}{|c|c|c|c|c|}
\hline $184367 \mathrm{chr} 4$ & $34,785,955$ & $34,815,308$ & duplication & - \\
\hline $184367 \mathrm{chr} 4$ & $69,374,369$ & $69,489,473$ & duplication & UGT2B17 \\
\hline $184367 \mathrm{chr} 5$ & $113,327,484$ & $113,335,230$ & deletion & - \\
\hline 184367 chr5 & $133,395,820$ & $133,397,597$ & deletion & - \\
\hline 184367 chr5 & $155,477,866$ & $155,488,917$ & deletion & - \\
\hline 184367 chr5 & $27,607,894$ & $27,632,559$ & deletion & - \\
\hline $184367 \mathrm{chr} 5$ & $5,694,586$ & $5,713,766$ & duplication & - \\
\hline 184367 chr5 & 701,026 & 825,338 & duplication & ZDHHC11 \\
\hline 184367 chr6 & $139,603,393$ & $139,606,731$ & deletion & - \\
\hline 184367 chr6 & $67,011,900$ & $67,048,885$ & deletion & - \\
\hline 184367 chr6 & $78,972,930$ & $79,035,739$ & deletion & - \\
\hline 184367 chr7 & $111,053,822$ & $111,199,771$ & deletion & IMMP2L \\
\hline 184367 chr7 & $38,291,851$ & $38,319,672$ & duplication & TARP \\
\hline 184367 chr8 & $51,029,371$ & $51,038,149$ & deletion & - \\
\hline $184367 \mathrm{chr} 8$ & $61,858,213$ & $61,869,649$ & deletion & - \\
\hline $184367 \mathrm{chr} 8$ & $9,055,379$ & $9,062,490$ & deletion & - \\
\hline 184367 chr9 & $107,799,020$ & $107,803,370$ & deletion & - \\
\hline 184367 chr9 & $23,363,526$ & $23,376,817$ & duplication & - \\
\hline $184368 \mathrm{chr} 1$ & $112,693,423$ & $112,704,581$ & deletion & - \\
\hline $184368 \mathrm{chr} 1$ & $149,039,031$ & $149,216,652$ & duplication & - \\
\hline 184368 chr1 & $16,491,406$ & $16,500,523$ & deletion & - \\
\hline $184368 \mathrm{chr} 1$ & $215,859,447$ & $215,861,792$ & deletion & - \\
\hline $184368 \mathrm{chr} 1$ & 809,700 & 823,155 & duplication & - \\
\hline 184368 chr1 & 849,998 & 856,352 & duplication & - \\
\hline $184368 \mathrm{chr} 10$ & $22,605,112$ & $22,616,244$ & deletion & COMMD3;COMMD3-BMI1;BMI1 \\
\hline 184368 chr10 & $38,774,749$ & $38,877,428$ & duplication & - \\
\hline 184368 chr11 & $23,420,932$ & $23,427,035$ & deletion & - \\
\hline $184368 \mathrm{chr} 11$ & $3,239,077$ & $3,243,435$ & deletion & MRGPRG \\
\hline 184368 chr11 & 346,381 & 436,461 & deletion & B4GALNT4;PKP3;ANO9;SIGIRR \\
\hline 184368 chr11 & $55,365,761$ & $55,443,269$ & duplication & OR4P4;OR4C6;OR4C11;OR4S2 \\
\hline 184368 chr12 & $11,220,827$ & $11,244,117$ & deletion & TAS2R43 \\
\hline 184368 chr12 & $11,511,132$ & $11,543,084$ & duplication & - \\
\hline 184368 chr12 & $127,308,735$ & $127,315,775$ & deletion & - \\
\hline 184368 chr13 & $100,628,807$ & $100,640,519$ & deletion & $\mathrm{ZIC2}$ \\
\hline 184368 chr13 & $79,172,756$ & $79,180,381$ & deletion & POU4F1 \\
\hline $184368 \mathrm{chr} 14$ & $33,400,709$ & $33,407,545$ & deletion & - \\
\hline 184368 chr14 & $74,240,176$ & $74,245,607$ & deletion & - \\
\hline $184368 \mathrm{chr} 15$ & $24,521,050$ & $24,660,014$ & duplication & - \\
\hline 184368 chr16 & $35,185,903$ & $35,197,323$ & deletion & - \\
\hline 184368 chr16 & $78,372,894$ & $78,383,509$ & deletion & - \\
\hline 184368 chr17 & $13,811,247$ & $13,818,217$ & deletion & - \\
\hline 184368 chr17 & $34,438,753$ & $34,481,236$ & duplication & - \\
\hline 184368 chr17 & $44,166,604$ & $44,292,896$ & duplication & KANSL1 \\
\hline $184368 \mathrm{chr} 18$ & $41,976,831$ & $41,982,102$ & deletion & - \\
\hline 184368 chr19 & $20,601,006$ & $20,717,536$ & deletion & - \\
\hline 184368 chr19 & $54,730,202$ & $54,743,217$ & duplication & LILRA6 \\
\hline $184368 \mathrm{chr} 2$ & $195,358,690$ & $195,368,541$ & deletion & - \\
\hline
\end{tabular}




\begin{tabular}{|c|c|c|c|c|}
\hline 184368 chr2 & $233,243,586$ & $233,272,162$ & deletion & ALPP;ALPPL2 \\
\hline $184368 \mathrm{chr} 2$ & $34,697,895$ & $34,726,904$ & duplication & - \\
\hline $184368 \mathrm{chr} 2$ & $77,977,262$ & $77,998,970$ & duplication & - \\
\hline 184368 chr22 & $42,522,613$ & $42,531,210$ & deletion & CYP2D6 \\
\hline 184368 chr3 & $189,364,424$ & $189,366,763$ & deletion & - \\
\hline $184368 \mathrm{chr} 3$ & $24,925,785$ & $24,942,213$ & deletion & - \\
\hline $184368 \mathrm{chr} 3$ & $3,052,153$ & $3,055,247$ & deletion & - \\
\hline $184368 \mathrm{chr} 3$ & $37,979,882$ & $37,986,734$ & deletion & - \\
\hline $184368 \mathrm{chr} 3$ & $75,428,675$ & $75,639,184$ & deletion & - \\
\hline $184368 \mathrm{chr} 4$ & $107,477,519$ & $107,489,998$ & duplication & - \\
\hline $184368 \mathrm{chr} 4$ & $138,092,298$ & $138,098,601$ & deletion & - \\
\hline $184368 \mathrm{chr} 4$ & $190,194,200$ & $190,200,463$ & deletion & - \\
\hline $184368 \mathrm{chr} 4$ & $3,767,394$ & $3,772,578$ & deletion & ADRA2C \\
\hline $184368 \mathrm{chr} 5$ & $70,305,696$ & $70,308,251$ & deletion & NAIP \\
\hline $184368 \mathrm{chr} 5$ & $72,741,402$ & $72,744,467$ & deletion & FOXD1 \\
\hline 184368 chr5 & $8,258,458$ & $8,260,630$ & deletion & - \\
\hline $184368 \mathrm{chr} 5$ & $84,087,297$ & $84,090,127$ & duplication & - \\
\hline 184368 chr6 & $31,276,513$ & $31,277,988$ & deletion & - \\
\hline $184368 \mathrm{chr} 6$ & $78,972,930$ & $79,035,739$ & deletion & - \\
\hline $184368 \mathrm{chr} 7$ & $111,199,771$ & $111,224,355$ & deletion & - \\
\hline 184368 chr7 & $141,766,850$ & $141,793,521$ & deletion & - \\
\hline $184368 \mathrm{chr} 7$ & $52,730,700$ & $52,744,420$ & duplication & - \\
\hline $184368 \mathrm{chr} 8$ & $16,262,222$ & $16,272,444$ & deletion & - \\
\hline $184368 \mathrm{chr} 8$ & $3,786,543$ & $3,790,254$ & deletion & . \\
\hline $184368 \mathrm{chr} 8$ & $39,232,581$ & $39,385,979$ & duplication & \\
\hline 184368 chr9 & $24,502,737$ & $24,518,795$ & deletion & - \\
\hline $184368 \mathrm{chrX}$ & $140,348,507$ & $140,779,598$ & duplication & LOC645188;SPANXA1;SPANXA2 \\
\hline $184369 \mathrm{chr} 1$ & $112,693,423$ & $112,704,581$ & deletion & - \\
\hline $184369 \mathrm{chr} 1$ & $149,032,299$ & $149,237,009$ & duplication & - \\
\hline $184369 \mathrm{chr} 1$ & $153,674,704$ & $153,690,013$ & duplication & - \\
\hline $184369 \mathrm{chr} 1$ & $161,559,327$ & $161,617,673$ & duplication & FCGR2C;FCGR3B \\
\hline $184369 \mathrm{chr} 1$ & $17,009,576$ & $17,093,414$ & duplication & FAM231A;FAM231C;MST1L \\
\hline $184369 \mathrm{chr} 1$ & $194,429,176$ & $194,456,489$ & deletion & - \\
\hline $184369 \mathrm{chr} 1$ & 797,820 & 863,812 & duplication & SAMD11 \\
\hline 184369 chr11 & $132,992,699$ & $133,006,277$ & deletion & - \\
\hline $184369 \mathrm{chr} 11$ & $3,239,077$ & $3,243,665$ & deletion & MRGPRG \\
\hline 184369 chr11 & $55,365,761$ & $55,443,269$ & duplication & OR4P4;OR4C6;OR4C11;OR4S2 \\
\hline 184369 chr12 & $11,504,864$ & $11,546,987$ & deletion & PRB1;PRB2 \\
\hline 184369 chr12 & $127,308,735$ & $127,315,775$ & deletion & - \\
\hline 184369 chr12 & $59,935,926$ & $59,942,122$ & deletion & - \\
\hline 184369 chr13 & $38,076,308$ & $38,085,537$ & deletion & - \\
\hline 184369 chr13 & $80,681,247$ & $80,685,663$ & deletion & - \\
\hline 184369 chr14 & $19,457,976$ & $19,470,537$ & deletion & - \\
\hline $184369 \mathrm{chr} 14$ & $20,341,645$ & $20,404,736$ & duplication & OR4K5;OR4K1;OR4K2 \\
\hline 184369 chr15 & $20,196,661$ & $21,241,118$ & duplication & GOLGA6L6;POTEB2;POTEB;POTEB3 \\
\hline 184369 chr16 & $35,185,903$ & $35,197,323$ & deletion & - \\
\hline 184369 chr16 & $79,260,765$ & $79,273,682$ & deletion & - \\
\hline
\end{tabular}




\begin{tabular}{|c|c|c|c|c|}
\hline 184369 chr16 & $87,040,145$ & $87,063,876$ & deletion & - \\
\hline $184369 \mathrm{chr} 17$ & $13,811,247$ & $13,818,217$ & deletion & - \\
\hline 184369 chr19 & $53,518,747$ & $53,552,296$ & duplication & ERVV-1 \\
\hline 184369 chr19 & $55,233,850$ & $55,339,383$ & duplication & KIR2DL3;KIR3DL1;KIR2DL1;KIR3DL3;KIR2DL4 \\
\hline $184369 \mathrm{chr} 2$ & $195,358,690$ & $195,368,541$ & deletion & - \\
\hline $184369 \mathrm{chr} 2$ & $233,243,586$ & $233,272,162$ & deletion & ALPP;ALPPL2 \\
\hline $184369 \mathrm{chr} 2$ & $52,759,890$ & $52,784,952$ & deletion & - \\
\hline 184369 chr20 & $29,428,644$ & $29,485,460$ & deletion & - \\
\hline $184369 \mathrm{chr} 22$ & $42,522,613$ & $42,531,210$ & deletion & CYP2D6 \\
\hline $184369 \mathrm{chr} 3$ & $37,979,882$ & $37,986,734$ & deletion & - \\
\hline $184369 \mathrm{chr} 3$ & $75,428,675$ & $75,639,184$ & deletion & - \\
\hline $184369 \mathrm{chr} 4$ & $107,486,721$ & $107,491,851$ & duplication & - \\
\hline $184369 \mathrm{chr} 4$ & $152,991,094$ & $152,993,620$ & deletion & - \\
\hline $184369 \mathrm{chr} 4$ & $190,194,200$ & $190,200,463$ & deletion & - \\
\hline $184369 \mathrm{chr} 4$ & $69,374,369$ & $69,431,280$ & deletion & UGT2B17 \\
\hline $184369 \mathrm{chr} 4$ & $93,314,859$ & $93,359,453$ & duplication & - \\
\hline 184369 chr5 & $140,225,908$ & $140,238,311$ & deletion & PCDHA10;PCDHA9 \\
\hline 184369 chr5 & $39,903,405$ & $39,913,978$ & deletion & - \\
\hline 184369 chr5 & $70,305,696$ & $70,308,251$ & deletion & NAIP \\
\hline 184369 chr6 & $77,439,769$ & $77,451,301$ & deletion & - \\
\hline $184369 \mathrm{chr} 7$ & $111,199,771$ & $111,224,355$ & deletion & - \\
\hline $184369 \mathrm{chr} 7$ & $141,766,850$ & $141,793,521$ & deletion & - \\
\hline $184369 \mathrm{chr} 7$ & $52,730,700$ & $52,744,420$ & duplication & - \\
\hline $184369 \mathrm{chr} 7$ & $97,396,709$ & $97,401,677$ & deletion & - \\
\hline $184369 \mathrm{chr} 8$ & $15,947,559$ & $16,023,673$ & deletion & MSR1 \\
\hline 184369 chr8 & $3,786,543$ & $3,790,254$ & deletion & - \\
\hline 184369 chr8 & $39,232,581$ & $39,385,979$ & duplication & - \\
\hline $184369 \mathrm{chr} 8$ & $51,029,371$ & $51,038,149$ & deletion & - \\
\hline 184369 chr9 & $23,363,526$ & $23,376,817$ & duplication & - \\
\hline $184369 \mathrm{chrX}$ & $40,444,431$ & $40,486,038$ & duplication & MPC1L;ATP6AP2 \\
\hline $184370 \mathrm{chr} 1$ & $1,385,211$ & $1,430,707$ & deletion & ATAD3C;ATAD3B \\
\hline 184370 chr1 & $22,316,110$ & $22,336,305$ & deletion & CELA3A \\
\hline 184370 chr10 & $123,882,245$ & $123,888,573$ & duplication & - \\
\hline 184370 chr10 & $42,523,752$ & $42,647,664$ & duplication & - \\
\hline $184370 \mathrm{chr} 10$ & $42,680,389$ & $42,825,180$ & duplication & - \\
\hline 184370 chr10 & $45,097,053$ & $45,126,974$ & deletion & - \\
\hline 184370 chr10 & $71,279,367$ & $71,289,190$ & deletion & - \\
\hline 184370 chr10 & $76,372,037$ & $76,374,526$ & deletion & - \\
\hline 184370 chr10 & $83,298,806$ & $83,307,529$ & deletion & - \\
\hline 184370 chr11 & $131,925,050$ & $131,929,618$ & duplication & - \\
\hline 184370 chr11 & $49,715,154$ & $49,757,690$ & deletion & - \\
\hline 184370 chr12 & $11,220,827$ & $11,258,011$ & deletion & TAS2R43 \\
\hline 184370 chr12 & $11,504,864$ & $11,546,987$ & deletion & PRB1;PRB2 \\
\hline 184370 chr12 & $132,131,087$ & $132,136,919$ & deletion & - \\
\hline 184370 chr12 & $53,823,307$ & $53,840,377$ & deletion & PRR13;AMHR2 \\
\hline 184370 chr15 & $21,903,815$ & $22,753,733$ & duplication & GOLGA6L22;GOLGA6L1;OR4N4;POTEB3;OR4N \\
\hline $184370 \mathrm{chr} 15$ & $82,370,777$ & $82,391,333$ & deletion & - \\
\hline
\end{tabular}




\begin{tabular}{|c|c|c|c|c|}
\hline $184370 \mathrm{chr} 16$ & $2,705,523$ & $2,737,071$ & duplication & KCTD5 \\
\hline 184370 chr16 & $32,511,914$ & $32,609,440$ & duplication & - \\
\hline 184370 chr16 & $78,372,894$ & $78,383,509$ & deletion & - \\
\hline 184370 chr17 & $34,438,753$ & $34,534,918$ & duplication & CCL3L3;CCL3L1 \\
\hline 184370 chr17 & $7,262,498$ & $7,265,681$ & deletion & - \\
\hline 184370 chr18 & $66,746,208$ & $66,755,736$ & deletion & - \\
\hline 184370 chr19 & $35,852,103$ & $35,861,695$ & deletion & - \\
\hline 184370 chr19 & $53,518,747$ & $53,548,950$ & duplication & ERVV-1 \\
\hline $184370 \mathrm{chr} 2$ & $223,865,393$ & $223,869,820$ & deletion & - \\
\hline $184370 \mathrm{chr} 2$ & $228,118,403$ & $228,246,702$ & duplication & COL4A3;TM4SF20;MFF \\
\hline $184370 \mathrm{chr} 2$ & $4,212,725$ & $4,222,144$ & deletion & - \\
\hline $184370 \mathrm{chr} 2$ & $66,188,209$ & $66,196,726$ & deletion & - \\
\hline $184370 \mathrm{chr} 20$ & $29,428,644$ & $29,485,460$ & deletion & - \\
\hline 184370 chr21 & $10,815,443$ & $10,858,651$ & duplication & - \\
\hline $184370 \mathrm{chr} 3$ & $162,130,691$ & $162,142,475$ & deletion & - \\
\hline $184370 \mathrm{chr} 3$ & $178,104,493$ & $178,112,910$ & deletion & - \\
\hline $184370 \mathrm{chr} 3$ & $195,457,853$ & $195,468,690$ & duplication & MUC20 \\
\hline $184370 \mathrm{chr} 4$ & $138,180,226$ & $138,194,992$ & deletion & - \\
\hline $184370 \mathrm{chr} 4$ & $152,789,786$ & $152,793,891$ & deletion & - \\
\hline $184370 \mathrm{chr} 4$ & $163,937,904$ & $163,952,337$ & duplication & - \\
\hline $184370 \mathrm{chr} 4$ & $69,374,369$ & $69,431,280$ & deletion & UGT2B17 \\
\hline $184370 \mathrm{chr} 4$ & $70,137,897$ & $70,229,260$ & deletion & UGT2B28 \\
\hline $184370 \mathrm{chr} 5$ & $111,937,859$ & $111,944,746$ & deletion & - \\
\hline 184370 chr5 & $112,264,968$ & $112,289,462$ & deletion & - \\
\hline $184370 \mathrm{chr} 5$ & 701,026 & 807,499 & duplication & ZDHHC11 \\
\hline $184370 \mathrm{chr} 5$ & $8,258,458$ & $8,260,630$ & deletion & - \\
\hline 184370 chr6 & $31,338,528$ & $31,341,357$ & deletion & - \\
\hline 184370 chr6 & $78,972,930$ & $79,035,739$ & deletion & - \\
\hline $184370 \mathrm{chr} 7$ & $8,827,959$ & $8,866,274$ & deletion & - \\
\hline $184370 \mathrm{chr} 7$ & $97,396,709$ & $97,401,677$ & deletion & - \\
\hline $184370 \mathrm{chr} 8$ & $17,580,791$ & $17,581,700$ & deletion & MTUS1 \\
\hline 184370 chr8 & $24,972,808$ & $24,990,418$ & deletion & - \\
\hline $184370 \mathrm{chr} 8$ & $5,595,511$ & $5,605,706$ & deletion & - \\
\hline 184370 chr9 & $2,148,213$ & $2,153,066$ & deletion & - \\
\hline 184370 chr9 & $23,363,526$ & $23,376,817$ & duplication & - \\
\hline 184371 chr1 & $104,104,990$ & $104,211,046$ & duplication & AMY1C;AMY1A;AMY2A;AMY2B;AMY1B \\
\hline $184371 \mathrm{chr} 1$ & $187,716,107$ & $187,722,032$ & deletion & - \\
\hline 184371 chr1 & $25,598,276$ & $25,659,509$ & deletion & RHD \\
\hline $184371 \mathrm{chr} 1$ & $49,936,419$ & $49,943,664$ & deletion & - \\
\hline 184371 chr1 & $62,113,597$ & $62,119,136$ & deletion & - \\
\hline $184371 \mathrm{chr} 1$ & $72,754,314$ & $72,763,324$ & deletion & - \\
\hline $184371 \mathrm{chr} 10$ & $132,635,459$ & $132,638,422$ & deletion & - \\
\hline 184371 chr10 & $16,931,255$ & $17,168,334$ & deletion & CUBN \\
\hline 184371 chr10 & $20,850,624$ & $20,857,365$ & deletion & - \\
\hline 184371 chr10 & $38,774,749$ & $38,871,868$ & duplication & - \\
\hline 184371 chr10 & $42,791,910$ & $42,811,180$ & duplication & - \\
\hline 184371 chr11 & $134,602,333$ & $134,607,484$ & deletion & - \\
\hline
\end{tabular}




\begin{tabular}{|c|c|c|c|c|}
\hline 184371 chr11 & $18,949,220$ & $18,961,743$ & duplication & MRGPRX1 \\
\hline 184371 chr11 & $90,193,796$ & $90,195,742$ & deletion & - \\
\hline 184371 chr12 & $11,511,132$ & $11,546,987$ & deletion & PRB2 \\
\hline 184371 chr12 & $30,237,351$ & $30,243,473$ & deletion & - \\
\hline 184371 chr12 & $31,279,428$ & $31,295,312$ & duplication & - \\
\hline 184371 chr14 & $25,610,677$ & $25,612,838$ & deletion & - \\
\hline 184371 chr14 & $47,708,397$ & $47,715,702$ & deletion & - \\
\hline 184371 chr15 & $63,267,778$ & $63,269,100$ & deletion & - \\
\hline 184371 chr16 & $32,528,023$ & $32,609,440$ & duplication & - \\
\hline 184371 chr16 & $35,194,609$ & $35,238,532$ & duplication & - \\
\hline 184371 chr17 & $44,215,394$ & $44,356,058$ & deletion & KANSL1 \\
\hline 184371 chr18 & $63,200,484$ & $63,206,335$ & duplication & - \\
\hline 184371 chr19 & $35,852,103$ & $35,861,695$ & deletion & - \\
\hline 184371 chr19 & $43,298,958$ & $43,539,189$ & deletion & PSG6;PSG7;PSG11;PSG1 \\
\hline 184371 chr19 & $54,728,767$ & $54,743,217$ & duplication & LILRA6 \\
\hline 184371 chr2 & $233,211,861$ & $233,291,701$ & duplication & ALPP;ALPPL2 \\
\hline 184371 chr2 & $4,212,725$ & $4,222,144$ & deletion & - \\
\hline 184371 chr2 & $48,851,541$ & $48,856,770$ & deletion & - \\
\hline 184371 chr2 & $52,759,890$ & $52,784,952$ & deletion & - \\
\hline 184371 chr2 & $87,392,157$ & $87,997,476$ & duplication & - \\
\hline 184371 chr20 & $54,750,577$ & $54,754,377$ & deletion & - \\
\hline 184371 chr21 & $10,813,582$ & $10,858,651$ & duplication & - \\
\hline 184371 chr22 & $39,359,885$ & $39,385,663$ & deletion & АРОВЕСЗВ \\
\hline 184371 chr3 & $110,793,050$ & $110,796,218$ & deletion & - \\
\hline 184371 chr3 & $131,711,896$ & $131,712,898$ & deletion & \\
\hline 184371 chr3 & $192,777,710$ & $192,782,294$ & deletion & - \\
\hline 184371 chr3 & $195,433,728$ & $195,475,799$ & duplication & MUC20;MUC4 \\
\hline $184371 \mathrm{chr} 4$ & $34,785,955$ & $34,818,502$ & deletion & - \\
\hline $184371 \mathrm{chr} 4$ & $64,136,609$ & $64,154,477$ & deletion & - \\
\hline 184371 chr4 & $64,697,704$ & $64,705,037$ & deletion & - \\
\hline $184371 \mathrm{chr} 4$ & $69,374,369$ & $69,431,280$ & deletion & UGT2B17 \\
\hline $184371 \mathrm{chr} 4$ & $70,137,897$ & $70,229,260$ & deletion & UGT2B28 \\
\hline 184371 chr5 & $101,953,047$ & $101,964,458$ & duplication & - \\
\hline 184371 chr5 & $17,599,857$ & $17,625,744$ & deletion & - \\
\hline 184371 chr5 & $32,106,628$ & $32,164,826$ & duplication & GOLPH3;PDZD2 \\
\hline 184371 chr6 & $13,534,503$ & $13,539,610$ & deletion & - \\
\hline 184371 chr6 & $32,486,060$ & $32,557,177$ & duplication & HLA-DRB1;HLA-DRB5 \\
\hline 184371 chr6 & $77,782,908$ & $77,792,085$ & deletion & - \\
\hline 184371 chr6 & $81,299,254$ & $81,416,489$ & deletion & - \\
\hline 184371 chr6 & $92,854,704$ & $92,865,417$ & deletion & - \\
\hline 184371 chr7 & $11,920,873$ & $11,922,312$ & deletion & - \\
\hline 184371 chr7 & $141,766,850$ & $141,793,521$ & deletion & - \\
\hline 184371 chr7 & $156,387,296$ & $156,394,307$ & deletion & - \\
\hline 184371 chr7 & $38,388,889$ & $38,402,517$ & deletion & - \\
\hline 184371 chr7 & $75,664,539$ & $75,667,743$ & deletion & - \\
\hline 184371 chr7 & $76,432,653$ & $76,631,856$ & duplication & - \\
\hline $184371 \mathrm{chr} 8$ & $138,822,798$ & $138,824,695$ & deletion & - \\
\hline
\end{tabular}




\begin{tabular}{|c|c|c|c|c|}
\hline 184371 chr8 & $39,232,581$ & $39,385,979$ & duplication & - \\
\hline 184371 chr8 & $51,029,371$ & $51,038,149$ & deletion & - \\
\hline 184371 chr8 & $72,214,753$ & $72,217,690$ & deletion & - \\
\hline 184371 chr9 & $104,715,329$ & $104,722,688$ & deletion & - \\
\hline $184371 \mathrm{chrX}$ & $144,331,003$ & $144,478,315$ & deletion & SPANXN1 \\
\hline 184372 chr1 & $104,104,990$ & $104,211,046$ & duplication & AMY1C;AMY1A;AMY2A;AMY2B;AMY1B \\
\hline 184372 chr1 & $25,598,276$ & $25,659,509$ & deletion & RHD \\
\hline 184372 chr1 & $49,936,419$ & $49,943,664$ & deletion & - \\
\hline $184372 \mathrm{chr} 1$ & $62,113,597$ & $62,119,136$ & deletion & - \\
\hline 184372 chr1 & $72,754,314$ & $72,763,324$ & deletion & - \\
\hline 184372 chr10 & $16,931,255$ & $17,168,334$ & deletion & CUBN \\
\hline 184372 chr10 & $20,850,624$ & $20,857,365$ & deletion & - \\
\hline 184372 chr10 & $38,774,749$ & $38,877,428$ & duplication & - \\
\hline 184372 chr11 & $134,602,333$ & $134,607,484$ & deletion & - \\
\hline 184372 chr11 & $61,793,535$ & $61,809,207$ & deletion & - \\
\hline 184372 chr12 & $11,511,132$ & $11,546,987$ & deletion & PRB2 \\
\hline $184372 \mathrm{chr} 12$ & $30,237,351$ & $30,243,473$ & deletion & - \\
\hline 184372 chr15 & $63,267,778$ & $63,269,100$ & deletion & - \\
\hline 184372 chr16 & $12,822,039$ & $12,882,093$ & deletion & CPPED1 \\
\hline 184372 chr16 & $15,288,094$ & $15,383,857$ & duplication & - \\
\hline 184372 chr16 & $28,614,734$ & $28,620,752$ & duplication & SULT1A1 \\
\hline 184372 chr16 & $34,471,298$ & $34,756,258$ & duplication & - \\
\hline $184372 \mathrm{chr} 16$ & $55,800,009$ & $55,819,443$ & deletion & - \\
\hline 184372 chr18 & $63,200,484$ & $63,206,335$ & duplication & - \\
\hline 184372 chr19 & $24,460,170$ & $24,462,326$ & deletion & \\
\hline 184372 chr19 & $35,852,103$ & $35,861,695$ & deletion & - \\
\hline 184372 chr19 & $43,298,958$ & $43,539,189$ & deletion & PSG6;PSG7;PSG11;PSG1 \\
\hline 184372 chr2 & $233,211,861$ & $233,316,179$ & duplication & ALPP;ALPPL2 \\
\hline 184372 chr2 & $34,697,895$ & $34,726,904$ & deletion & - \\
\hline 184372 chr20 & $54,750,577$ & $54,754,377$ & deletion & - \\
\hline 184372 chr21 & $10,818,766$ & $10,858,651$ & duplication & - \\
\hline 184372 chr22 & $39,359,885$ & $39,385,663$ & deletion & АРОВЕСЗВ \\
\hline 184372 chr3 & $110,793,050$ & $110,796,218$ & deletion & - \\
\hline 184372 chr3 & $131,711,896$ & $131,712,898$ & deletion & - \\
\hline 184372 chr3 & $195,457,853$ & $195,468,690$ & duplication & MUC20 \\
\hline 184372 chr3 & $37,979,882$ & $37,986,734$ & deletion & - \\
\hline 184372 chr3 & $53,032,639$ & $53,038,786$ & deletion & - \\
\hline 184372 chr4 & $34,785,955$ & $34,818,502$ & deletion & - \\
\hline 184372 chr4 & $39,705,484$ & $39,713,134$ & deletion & - \\
\hline $184372 \mathrm{chr} 4$ & $64,136,609$ & $64,154,477$ & deletion & - \\
\hline 184372 chr4 & $70,137,897$ & $70,229,260$ & deletion & UGT2B28 \\
\hline 184372 chr5 & $17,599,857$ & $17,627,924$ & deletion & - \\
\hline 184372 chr5 & $32,106,628$ & $32,164,826$ & duplication & GOLPH3;PDZD2 \\
\hline 184372 chr5 & 701,026 & 807,499 & duplication & ZDHHC11 \\
\hline 184372 chr5 & $8,258,458$ & $8,260,630$ & deletion & - \\
\hline 184372 chr6 & $139,603,393$ & $139,606,731$ & deletion & - \\
\hline 184372 chr6 & $151,507,355$ & $151,509,176$ & deletion & - \\
\hline
\end{tabular}




\begin{tabular}{|c|c|c|c|c|}
\hline 184372 chr6 & $69,231,883$ & $69,241,954$ & duplication & - \\
\hline 184372 chr6 & $77,315,788$ & $77,327,833$ & deletion & - \\
\hline 184372 chr6 & $78,985,226$ & $79,029,367$ & duplication & - \\
\hline 184372 chr6 & $89,155,213$ & $89,162,999$ & duplication & - \\
\hline 184372 chr7 & $141,766,850$ & $141,793,521$ & deletion & - \\
\hline $184372 \mathrm{chr} 7$ & $76,432,653$ & $76,631,856$ & duplication & - \\
\hline $184372 \mathrm{chr} 8$ & $138,822,798$ & $138,824,695$ & deletion & - \\
\hline 184372 chr8 & $39,232,581$ & $39,385,979$ & duplication & - \\
\hline 184372 chr8 & $72,214,753$ & $72,217,690$ & deletion & - \\
\hline 184372 chr9 & $104,715,329$ & $104,722,688$ & deletion & - \\
\hline $184372 \mathrm{chrX}$ & $144,331,003$ & $144,478,315$ & deletion & SPANXN1 \\
\hline 187235 chr1 & $161,559,327$ & $161,619,741$ & duplication & FCGR2C;FCGR3B \\
\hline 187235 chr1 & $62,113,597$ & $62,119,136$ & deletion & - \\
\hline 187235 chr10 & $33,441,324$ & $33,450,700$ & duplication & - \\
\hline 187235 chr11 & $55,365,761$ & $55,427,700$ & deletion & OR4P4;OR4C11;OR4S2 \\
\hline 187235 chr11 & $81,501,218$ & $81,517,261$ & deletion & - \\
\hline 187235 chr11 & $90,193,796$ & $90,196,843$ & deletion & - \\
\hline 187235 chr12 & $11,220,827$ & $11,244,117$ & deletion & TAS2R43 \\
\hline 187235 chr12 & $33,300,995$ & $33,305,855$ & deletion & - \\
\hline 187235 chr12 & $43,021,431$ & $43,027,453$ & deletion & - \\
\hline 187235 chr13 & $32,532,761$ & $32,537,768$ & deletion & - \\
\hline 187235 chr13 & $38,076,308$ & $38,085,537$ & deletion & - \\
\hline 187235 chr14 & $106,891,524$ & $106,931,129$ & deletion & - \\
\hline 187235 chr14 & $107,157,561$ & $107,181,152$ & duplication & . \\
\hline 187235 chr14 & $41,610,224$ & $41,661,685$ & deletion & \\
\hline 187235 chr15 & $63,267,778$ & $63,269,100$ & deletion & - \\
\hline 187235 chr16 & $32,528,023$ & $32,833,893$ & deletion & TP53TG3B;TP53TG3;TP53TG3C \\
\hline 187235 chr16 & $85,302,676$ & $85,304,326$ & deletion & - \\
\hline 187235 chr17 & $19,499,229$ & $19,537,470$ & deletion & - \\
\hline 187235 chr17 & $39,210,931$ & $39,220,285$ & deletion & KRTAP2-3;KRTAP2-2 \\
\hline 187235 chr18 & $66,746,208$ & $66,755,736$ & deletion & - \\
\hline 187235 chr19 & $43,214,714$ & $43,378,265$ & duplication & PSG3;PSG8;PSG1 \\
\hline 187235 chr19 & $43,703,965$ & $43,759,382$ & deletion & PSG4;PSG9 \\
\hline 187235 chr19 & $53,516,663$ & $53,548,950$ & duplication & ERVV-1 \\
\hline 187235 chr19 & $54,556,755$ & $54,560,078$ & deletion & - \\
\hline 187235 chr19 & $54,728,767$ & $54,743,217$ & duplication & LILRA6 \\
\hline 187235 chr2 & $4,212,725$ & $4,222,144$ & deletion & - \\
\hline 187235 chr20 & $59,112,918$ & $59,122,693$ & deletion & - \\
\hline 187235 chr21 & $44,822,871$ & $44,837,829$ & duplication & LOC102724428;SIK1 \\
\hline 187235 chr3 & $192,777,710$ & $192,782,294$ & deletion & - \\
\hline 187235 chr3 & $22,562,237$ & $22,608,487$ & deletion & - \\
\hline 187235 chr3 & $53,032,639$ & $53,038,786$ & deletion & - \\
\hline $187235 \mathrm{chr} 3$ & $6,651,929$ & $6,654,060$ & deletion & - \\
\hline $187235 \mathrm{chr} 3$ & $89,402,409$ & $89,418,331$ & deletion & - \\
\hline $187235 \mathrm{chr} 4$ & $69,374,369$ & $69,489,473$ & deletion & UGT2B17 \\
\hline $187235 \mathrm{chr} 4$ & $70,137,897$ & $70,229,260$ & deletion & UGT2B28 \\
\hline $187235 \mathrm{chr} 5$ & $155,477,866$ & $155,488,917$ & deletion & - \\
\hline
\end{tabular}




\begin{tabular}{|c|c|c|c|c|}
\hline 187235 chr5 & $180,377,470$ & $180,430,540$ & deletion & BTNL8;BTNL3 \\
\hline 187235 chr6 & $38,019,720$ & $38,447,949$ & deletion & BTBD9;ZFAND3 \\
\hline 187235 chr6 & $57,160,035$ & $57,165,948$ & deletion & - \\
\hline 187235 chr6 & $67,011,900$ & $67,048,885$ & deletion & - \\
\hline 187235 chr6 & $89,155,213$ & $89,162,999$ & duplication & - \\
\hline 187235 chr6 & $90,400,888$ & $90,491,285$ & deletion & MDN1 \\
\hline 187235 chr7 & $141,766,850$ & $141,793,521$ & deletion & - \\
\hline 187235 chr7 & $142,825,750$ & $142,890,868$ & deletion & PIP;TAS2R39 \\
\hline 187235 chr7 & $33,129,952$ & $33,187,279$ & duplication & RP9;BBS9 \\
\hline 187235 chr7 & $97,396,709$ & $97,401,677$ & deletion & - \\
\hline $187235 \mathrm{chr} 8$ & $115,633,704$ & $115,642,459$ & deletion & - \\
\hline 187235 chr8 & $15,401,929$ & $15,411,867$ & deletion & - \\
\hline 187235 chr8 & $39,232,581$ & $39,385,979$ & deletion & - \\
\hline 187235 chr9 & $107,799,020$ & $107,803,370$ & deletion & - \\
\hline $187236 \mathrm{chr} 1$ & $13,135,437$ & $13,182,303$ & duplication & - \\
\hline $187236 \mathrm{chr} 1$ & $149,032,299$ & $149,432,892$ & deletion & - \\
\hline 187236 chr1 & $62,113,597$ & $62,119,136$ & deletion & - \\
\hline 187236 chr10 & $39,058,671$ & $39,154,535$ & duplication & - \\
\hline 187236 chr11 & $100,279,379$ & $100,392,232$ & deletion & - \\
\hline 187236 chr11 & $48,755,477$ & $48,923,625$ & duplication & - \\
\hline 187236 chr11 & $55,365,761$ & $55,443,269$ & duplication & 4C11;OR4S2 \\
\hline 187236 chr11 & $8,959,020$ & $8,964,938$ & deletion & ASCL3 \\
\hline 187236 chr11 & $90,193,796$ & $90,196,843$ & deletion & 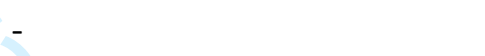 \\
\hline 187236 chr12 & $11,511,132$ & $11,543,084$ & duplication & - \\
\hline 187236 chr12 & $126,994,335$ & $127,004,296$ & deletion & \\
\hline 187236 chr12 & $2,245,636$ & $2,254,219$ & deletion & - \\
\hline 187236 chr12 & $30,237,351$ & $30,243,473$ & deletion & - \\
\hline 187236 chr12 & $31,907,259$ & $31,909,635$ & deletion & - \\
\hline 187236 chr13 & $32,532,761$ & $32,537,768$ & deletion & - \\
\hline 187236 chr16 & $22,635,867$ & $22,703,741$ & deletion & - \\
\hline 187236 chr16 & $55,798,095$ & $55,819,443$ & deletion & - \\
\hline 187236 chr16 & $76,143,934$ & $76,156,154$ & deletion & - \\
\hline 187236 chr18 & $76,157,694$ & $76,160,278$ & deletion & - \\
\hline 187236 chr19 & $35,852,103$ & $35,861,695$ & deletion & - \\
\hline 187236 chr19 & $43,703,965$ & $43,759,382$ & deletion & PSG4;PSG9 \\
\hline 187236 chr19 & $53,518,747$ & $53,552,296$ & duplication & ERVV-1 \\
\hline $187236 \mathrm{chr} 2$ & $34,697,895$ & $34,726,904$ & duplication & - \\
\hline $187236 \mathrm{chr} 2$ & $77,977,262$ & $77,998,970$ & duplication & - \\
\hline $187236 \mathrm{chr} 2$ & $87,392,157$ & $87,997,476$ & deletion & - \\
\hline 187236 chr21 & $23,655,900$ & $23,665,470$ & deletion & - \\
\hline 187236 chr22 & $42,912,029$ & $42,950,033$ & duplication & SERHL2;RRP7A \\
\hline $187236 \mathrm{chr} 3$ & $1,782,524$ & $1,787,581$ & deletion & - \\
\hline $187236 \mathrm{chr} 3$ & $8,825,626$ & $8,858,081$ & duplication & - \\
\hline $187236 \mathrm{chr} 3$ & $90,397,871$ & $90,409,592$ & duplication & - \\
\hline $187236 \mathrm{chr} 4$ & $171,269,504$ & $171,273,360$ & deletion & - \\
\hline $187236 \mathrm{chr} 4$ & $69,374,369$ & $69,467,200$ & deletion & UGT2B17 \\
\hline $187236 \mathrm{chr} 5$ & $41,224,284$ & $41,241,610$ & deletion & - \\
\hline
\end{tabular}




\begin{tabular}{|c|c|c|c|c|}
\hline 187236 chr6 & $139,601,136$ & $139,606,731$ & deletion & - \\
\hline $187236 \mathrm{chr} 6$ & $26,344,333$ & $26,351,349$ & deletion & - \\
\hline $187236 \mathrm{chr} 6$ & $78,972,930$ & $79,035,739$ & deletion & - \\
\hline 187236 chr7 & $3,394,468$ & $3,403,049$ & deletion & - \\
\hline 187236 chr8 & $15,419,777$ & $15,432,653$ & deletion & - \\
\hline $187236 \mathrm{chr} 8$ & $3,786,543$ & $3,790,254$ & deletion & - \\
\hline $187236 \mathrm{chr} 8$ & $39,236,582$ & $39,364,263$ & deletion & - \\
\hline $187236 \mathrm{chr} 8$ & $72,214,753$ & $72,217,690$ & deletion & - \\
\hline 187237 chr1 & $1,418,112$ & $1,430,707$ & deletion & ATAD3B \\
\hline 187237 chr1 & $148,937,908$ & $149,432,892$ & deletion & - \\
\hline 187237 chr10 & $135,320,016$ & $135,370,639$ & duplication & SYCE1;CYP2E1 \\
\hline 187237 chr10 & $38,774,749$ & $38,877,428$ & duplication & - \\
\hline 187237 chr11 & $100,286,219$ & $100,392,232$ & deletion & - \\
\hline 187237 chr11 & $18,949,220$ & $18,961,743$ & duplication & MRGPRX1 \\
\hline 187237 chr11 & $55,365,761$ & $55,443,269$ & duplication & OR4P4;OR4C6;OR4C11;OR4S2 \\
\hline 187237 chr11 & $86,304,402$ & $86,306,401$ & deletion & - \\
\hline 187237 chr11 & $90,193,796$ & $90,196,843$ & deletion & - \\
\hline 187237 chr12 & $11,511,132$ & $11,543,084$ & duplication & - \\
\hline 187237 chr12 & $126,994,506$ & $127,004,296$ & deletion & - \\
\hline 187237 chr12 & $2,245,636$ & $2,254,219$ & deletion & - \\
\hline 187237 chr12 & $29,943,923$ & $29,947,182$ & duplication & - \\
\hline 187237 chr12 & $30,237,351$ & $30,243,473$ & deletion & - \\
\hline 187237 chr12 & $31,907,259$ & $31,909,635$ & deletion & - \\
\hline 187237 chr14 & $41,610,224$ & $41,661,685$ & deletion & \\
\hline 187237 chr14 & $42,988,346$ & $42,992,844$ & deletion & t \\
\hline 187237 chr15 & $26,751,068$ & $26,752,373$ & deletion & - \\
\hline 187237 chr16 & 205,035 & 212,649 & duplication & - \\
\hline $187237 \mathrm{chr} 16$ & $76,143,934$ & $76,156,154$ & deletion & - \\
\hline 187237 chr19 & $43,703,965$ & $43,759,382$ & deletion & PSG4;PSG9 \\
\hline 187237 chr19 & $53,520,702$ & $53,552,296$ & duplication & - \\
\hline 187237 chr19 & $9,275,282$ & $9,282,176$ & deletion & - \\
\hline $187237 \mathrm{chr} 2$ & $209,033,557$ & $209,060,563$ & deletion & C2orf80 \\
\hline 187237 chr2 & $240,622,210$ & $240,627,052$ & deletion & - \\
\hline $187237 \mathrm{chr} 2$ & $77,977,262$ & $77,998,970$ & duplication & - \\
\hline $187237 \mathrm{chr} 2$ & $87,392,157$ & $87,997,476$ & deletion & - \\
\hline $187237 \mathrm{chr} 21$ & $23,655,900$ & $23,665,470$ & deletion & - \\
\hline 187237 chr22 & $42,902,278$ & $42,950,033$ & duplication & SERHL2;RRP7A \\
\hline $187237 \mathrm{chr} 3$ & $131,711,896$ & $131,712,898$ & deletion & - \\
\hline $187237 \mathrm{chr} 3$ & $1,782,524$ & $1,787,581$ & deletion & - \\
\hline 187237 chr3 & $45,799,871$ & $45,829,318$ & deletion & SLC6A20 \\
\hline $187237 \mathrm{chr} 3$ & $8,826,396$ & $8,850,723$ & duplication & - \\
\hline $187237 \mathrm{chr} 4$ & $34,785,955$ & $34,805,860$ & duplication & - \\
\hline 187237 chr5 & $146,305,665$ & $146,316,602$ & deletion & - \\
\hline $187237 \mathrm{chr} 5$ & $155,477,866$ & $155,488,917$ & deletion & - \\
\hline 187237 chr5 & 701,026 & 824,025 & duplication & ZDHHC11 \\
\hline 187237 chr6 & $122,159,344$ & $122,165,277$ & deletion & - \\
\hline 187237 chr6 & $139,603,393$ & $139,606,731$ & deletion & - \\
\hline
\end{tabular}




\begin{tabular}{|c|c|c|c|c|}
\hline 187237 chr6 & $30,253,011$ & $30,260,284$ & deletion & - \\
\hline 187237 chr6 & $31,281,682$ & $31,286,577$ & deletion & - \\
\hline 187237 chr6 & $78,972,930$ & $79,035,739$ & deletion & - \\
\hline 187237 chr7 & $3,394,468$ & $3,403,049$ & deletion & - \\
\hline 187237 chr7 & $91,033,074$ & $91,041,618$ & deletion & - \\
\hline 187237 chr8 & $112,714,749$ & $112,727,265$ & duplication & - \\
\hline 187237 chr8 & $3,786,543$ & $3,790,254$ & deletion & - \\
\hline 187241 chr1 & $161,559,327$ & $161,619,741$ & duplication & FCGR2C;FCGR3B \\
\hline 187241 chr1 & $187,716,107$ & $187,722,032$ & deletion & - \\
\hline 187241 chr1 & $245,636,900$ & $245,647,809$ & deletion & - \\
\hline 187241 chr10 & $38,792,512$ & $38,797,719$ & deletion & - \\
\hline 187241 chr10 & $38,869,302$ & $38,903,858$ & deletion & - \\
\hline 187241 chr10 & $68,629,972$ & $68,630,940$ & deletion & - \\
\hline 187241 chr10 & $82,879,475$ & $82,891,283$ & deletion & - \\
\hline 187241 chr11 & $93,683,680$ & $93,688,541$ & deletion & - \\
\hline 187241 chr12 & $19,469,320$ & $19,578,107$ & duplication & PLEKHA5 \\
\hline 187241 chr12 & $2,245,636$ & $2,254,219$ & deletion & - \\
\hline 187241 chr12 & $43,021,431$ & $43,027,453$ & deletion & - \\
\hline 187241 chr12 & $6,241,446$ & $6,247,923$ & deletion & - \\
\hline 187241 chr13 & $103,850,786$ & $103,870,753$ & deletion & - \\
\hline 187241 chr14 & $20,197,311$ & $20,424,184$ & deletion & OR4K5;OR4K1;OR4K2;OR4Q3;OR4N2;OR4M1 \\
\hline 187241 chr14 & $41,610,224$ & $41,661,685$ & deletion & - \\
\hline 187241 chr15 & $34,717,697$ & $34,814,869$ & deletion & - \\
\hline 187241 chr15 & $34,929,955$ & $34,982,488$ & deletion & - \\
\hline 187241 chr15 & $43,888,976$ & $43,939,642$ & duplication & STRC;CATSPER2;CKMT1B \\
\hline 187241 chr15 & $80,814,150$ & $80,826,489$ & deletion & - \\
\hline 187241 chr17 & $44,166,604$ & $44,356,058$ & duplication & KANSL1 \\
\hline 187241 chr17 & $70,815,357$ & $70,820,207$ & deletion & - \\
\hline 187241 chr18 & $14,054,744$ & $14,627,365$ & duplication & ZNF519;POTEC \\
\hline 187241 chr18 & $63,200,484$ & $63,206,335$ & duplication & - \\
\hline 187241 chr18 & $67,208,806$ & $67,217,271$ & deletion & - \\
\hline 187241 chr19 & $21,609,213$ & $21,614,203$ & deletion & ZNF493 \\
\hline 187241 chr19 & $54,556,755$ & $54,560,078$ & deletion & - \\
\hline 187241 chr19 & $54,728,767$ & $54,743,217$ & duplication & LILRA6 \\
\hline 187241 chr2 & $137,662,392$ & $137,707,025$ & deletion & - \\
\hline 187241 chr21 & $10,813,582$ & $10,858,651$ & duplication & - \\
\hline 187241 chr21 & $14,880,003$ & $15,119,875$ & duplication & POTED \\
\hline 187241 chr3 & $22,279,685$ & $22,286,262$ & deletion & - \\
\hline 187241 chr4 & $125,273,400$ & $125,325,858$ & deletion & - \\
\hline $187241 \mathrm{chr} 4$ & $138,092,298$ & $138,098,601$ & deletion & - \\
\hline $187241 \mathrm{chr} 4$ & $177,350,956$ & $177,356,320$ & deletion & - \\
\hline $187241 \mathrm{chr} 4$ & $64,697,704$ & $64,709,753$ & deletion & - \\
\hline 187241 chr5 & $121,254,774$ & $121,262,264$ & deletion & - \\
\hline 187241 chr5 & $123,191,125$ & $123,193,433$ & deletion & - \\
\hline 187241 chr5 & $17,639,913$ & $17,642,516$ & deletion & - \\
\hline 187241 chr5 & $8,258,458$ & $8,260,630$ & deletion & - \\
\hline 187241 chr6 & $30,994,010$ & $30,994,787$ & deletion & MUC22 \\
\hline
\end{tabular}




\begin{tabular}{|c|c|c|c|c|c|}
\hline 187241 & chr6 & $78,972,930$ & $79,035,739$ & deletion & - \\
\hline 187241 & chr7 & $142,476,094$ & $142,484,042$ & deletion & - \\
\hline 187241 & chr7 & $20,272,992$ & $20,277,232$ & deletion & - \\
\hline 187241 & chr7 & $20,750,774$ & $20,753,987$ & deletion & - \\
\hline 187241 & chr7 & $29,676,097$ & $29,687,592$ & deletion & - \\
\hline 187241 & chr7 & $62,137,274$ & $62,141,736$ & deletion & - \\
\hline 187241 & chr7 & $80,270,138$ & $80,282,734$ & deletion & CD36 \\
\hline 187241 & chr7 & $8,827,959$ & $8,866,383$ & deletion & - \\
\hline 187241 & chr8 & $115,634,278$ & $115,642,459$ & deletion & - \\
\hline 187241 & chr8 & $2,139,471$ & $2,153,305$ & duplication & - \\
\hline 187241 & chr8 & $39,232,581$ & $39,385,979$ & duplication & - \\
\hline 187241 & chr8 & $51,226,087$ & $51,227,777$ & deletion & - \\
\hline 187241 & chr9 & $78,004,378$ & $78,011,616$ & deletion & - \\
\hline 187244 & chr1 & $25,598,276$ & $25,659,509$ & deletion & RHD \\
\hline 187244 & chr1 & $62,113,597$ & $62,119,136$ & deletion & - \\
\hline 187244 & chr10 & $39,107,936$ & $39,149,666$ & duplication & - \\
\hline 187244 & chr10 & $81,794,192$ & $81,801,732$ & deletion & - \\
\hline 187244 & chr11 & $107,653,650$ & $107,671,155$ & duplication & SLC35F2 \\
\hline 187244 & chr11 & $18,949,220$ & $18,961,743$ & duplication & MRGPRX1 \\
\hline 187244 & chr11 & $55,365,761$ & $55,443,269$ & deletion & OR4P4;OR4C6;OR4C11;OR4S2 \\
\hline 187244 & chr11 & $96,439,090$ & $96,455,400$ & duplication & - \\
\hline 187244 & chr12 & $40,875,351$ & $40,875,963$ & deletion & MUC19 \\
\hline 187244 & chr12 & $6,255,862$ & $6,259,552$ & deletion & - \\
\hline 187244 & chr13 & $23,786,773$ & $23,818,456$ & deletion & SGCG \\
\hline 187244 & chr14 & $19,457,976$ & $19,470,537$ & deletion & - \\
\hline 187244 & chr14 & $20,197,311$ & $20,416,575$ & duplication & OR4K5;OR4K1;OR4K2;OR4Q3;OR4N2;OR4M1 \\
\hline 187244 & chr15 & $34,717,697$ & $34,814,869$ & deletion & - \\
\hline 187244 & chr15 & $97,815,182$ & $97,831,832$ & deletion & - \\
\hline 187244 & chr16 & $1,288,455$ & $1,297,145$ & duplication & TPSAB1 \\
\hline 187244 & chr16 & $55,798,095$ & $55,819,443$ & deletion & - \\
\hline 187244 & chr16 & $78,372,894$ & $78,383,509$ & deletion & - \\
\hline 187244 & chr17 & $44,166,604$ & $44,285,531$ & duplication & KANSL1 \\
\hline 187244 & chr19 & $34,365,706$ & $34,371,272$ & deletion & - \\
\hline 187244 & chr19 & $35,852,103$ & $35,861,695$ & deletion & - \\
\hline 187244 & chr19 & $54,728,767$ & $54,743,217$ & duplication & LILRA6 \\
\hline 187244 & chr2 & $195,979,780$ & $195,982,450$ & deletion & - \\
\hline 187244 & chr2 & $242,854,089$ & $243,034,519$ & deletion & - \\
\hline 187244 & chr20 & $14,916,657$ & $15,021,183$ & deletion & - \\
\hline 187244 & chr3 & $195,449,715$ & $195,468,690$ & duplication & MUC20 \\
\hline 187244 & chr3 & $89,120,471$ & $89,144,415$ & deletion & - \\
\hline 187244 & chr4 & $171,269,504$ & $171,273,360$ & deletion & - \\
\hline 187244 & chr4 & $69,374,369$ & $69,431,280$ & deletion & UGT2B17 \\
\hline 187244 & chr5 & $180,377,470$ & $180,430,540$ & deletion & BTNL8;BTNL3 \\
\hline 187244 & chr6 & $29,855,945$ & $29,899,189$ & deletion & - \\
\hline 187244 & chr6 & $55,827,939$ & $55,847,710$ & deletion & - \\
\hline 187244 & chr6 & $78,972,930$ & $79,035,739$ & deletion & - \\
\hline 187244 & chr7 & $157,116,750$ & $157,122,691$ & deletion & - \\
\hline
\end{tabular}




\begin{tabular}{|c|c|c|c|c|}
\hline 187244 chr7 & $38,388,889$ & $38,402,517$ & deletion & - \\
\hline 187244 chr8 & $115,634,278$ & $115,642,459$ & deletion & - \\
\hline 187244 chr8 & $39,236,582$ & $39,382,903$ & deletion & - \\
\hline 187244 chr8 & $3,967,931$ & $3,969,537$ & deletion & - \\
\hline 187244 chr8 & $5,595,511$ & $5,605,706$ & deletion & - \\
\hline 187244 chr9 & $133,030,684$ & $133,033,885$ & deletion & - \\
\hline 187244 chr9 & $24,502,737$ & $24,520,633$ & deletion & - \\
\hline 187244 chr9 & $6,701,130$ & $6,707,794$ & deletion & - \\
\hline 187244 chr9 & $94,396,059$ & $94,402,923$ & deletion & - \\
\hline 187248 chr1 & $12,852,748$ & $12,915,847$ & deletion & HNRNPCL1;HNRNPCL4;HNRNPCL3;PRAMEF1;F \\
\hline 187248 chr1 & $142,603,938$ & $143,978,807$ & duplication & FAM72D;FAM72C;PPIAL4G \\
\hline 187248 chr1 & $152,526,812$ & $152,568,230$ & deletion & LCE3D;LCE3E \\
\hline $187248 \mathrm{chr} 1$ & $196,835,106$ & $196,846,344$ & deletion & - \\
\hline 187248 chr1 & $248,740,572$ & $248,795,110$ & deletion & OR2T10;OR2T11 \\
\hline 187248 chr1 & $72,754,314$ & $72,763,324$ & deletion & - \\
\hline 187248 chr10 & $30,503,706$ & $30,550,587$ & deletion & - \\
\hline 187248 chr10 & $58,846,575$ & $58,849,853$ & deletion & - \\
\hline 187248 chr10 & $71,278,805$ & $71,289,190$ & deletion & - \\
\hline 187248 chr11 & $18,949,220$ & $18,961,743$ & duplication & MRGPRX1 \\
\hline 187248 chr11 & $36,892,734$ & $36,898,750$ & deletion & - \\
\hline 187248 chr11 & $86,304,402$ & $86,306,401$ & deletion & - \\
\hline 187248 chr12 & $43,021,431$ & $43,027,453$ & deletion & - \\
\hline 187248 chr12 & $99,994,315$ & $100,008,773$ & deletion & - \\
\hline 187248 chr13 & $31,327,623$ & $31,348,600$ & duplication & ALOX5AP \\
\hline 187248 chr14 & $41,610,224$ & $41,661,685$ & deletion & - \\
\hline 187248 chr16 & $19,945,540$ & $19,962,636$ & deletion & - \\
\hline 187248 chr16 & $32,528,023$ & $32,833,893$ & deletion & TP53TG3B;TP53TG3;TP53TG3C \\
\hline 187248 chr16 & $55,800,009$ & $55,819,443$ & deletion & - \\
\hline 187248 chr16 & $58,945,839$ & $58,948,100$ & deletion & - \\
\hline 187248 chr16 & $70,183,624$ & $70,196,139$ & duplication & PDPR \\
\hline 187248 chr16 & $78,372,894$ & $78,383,509$ & deletion & - \\
\hline 187248 chr16 & $8,861,083$ & $8,970,086$ & duplication & PMM2;TMEM186;ABAT;CARHSP1 \\
\hline 187248 chr18 & $41,976,831$ & $41,982,102$ & deletion & - \\
\hline 187248 chr18 & $67,208,806$ & $67,217,271$ & deletion & - \\
\hline 187248 chr19 & $51,330,932$ & $51,333,442$ & deletion & KLK15 \\
\hline 187248 chr19 & $53,518,747$ & $53,552,296$ & duplication & ERVV-1 \\
\hline 187248 chr19 & $54,556,755$ & $54,560,078$ & deletion & - \\
\hline 187248 chr2 & $14,704,369$ & $14,709,611$ & deletion & - \\
\hline 187248 chr2 & $184,085,551$ & $184,088,256$ & deletion & - \\
\hline 187248 chr2 & $38,301,735$ & $38,305,451$ & deletion & CYP1B1 \\
\hline 187248 chr2 & $48,851,541$ & $48,856,770$ & deletion & - \\
\hline 187248 chr20 & $59,112,918$ & $59,122,693$ & deletion & - \\
\hline 187248 chr4 & $18,932,707$ & $18,993,753$ & deletion & - \\
\hline 187248 chr4 & $34,785,955$ & $34,818,502$ & deletion & - \\
\hline $187248 \mathrm{chr} 4$ & $49,313,588$ & $49,651,131$ & duplication & - \\
\hline 187248 chr4 & $60,325,010$ & $60,330,880$ & duplication & - \\
\hline 187248 chr4 & $69,374,369$ & $69,489,473$ & deletion & UGT2B17 \\
\hline
\end{tabular}




\begin{tabular}{|c|c|c|c|c|}
\hline 187248 chr5 & $180,377,470$ & $180,430,540$ & deletion & BTNL8;BTNL3 \\
\hline $187248 \mathrm{chr} 5$ & $97,555,945$ & $97,563,781$ & deletion & - \\
\hline 187248 chr6 & $29,855,945$ & $29,899,189$ & deletion & - \\
\hline 187248 chr6 & $30,994,010$ & $30,994,787$ & deletion & MUC22 \\
\hline 187248 chr6 & $78,972,930$ & $79,035,739$ & deletion & - \\
\hline $187248 \mathrm{chr} 7$ & $156,387,296$ & $156,394,307$ & deletion & - \\
\hline 187248 chr7 & $159,118,443$ & $159,122,741$ & deletion & - \\
\hline 187248 chr7 & $20,750,774$ & $20,753,987$ & deletion & - \\
\hline 187248 chr7 & $7,097,615$ & $7,102,983$ & deletion & - \\
\hline 187248 chr8 & $39,232,581$ & $39,385,979$ & duplication & - \\
\hline 187248 chr9 & $68,421,637$ & $68,502,538$ & duplication & - \\
\hline 187248 chr9 & $94,396,059$ & $94,402,923$ & deletion & - \\
\hline 187249 chr1 & $142,603,938$ & $143,978,807$ & duplication & FAM72D;FAM72C;PPIAL4G \\
\hline $187249 \mathrm{chr} 1$ & $17,022,921$ & $17,288,389$ & duplication & FAM231A;FAM231C;MST1L;CROCC \\
\hline 187249 chr10 & $46,949,421$ & $47,151,118$ & duplication & GPRIN2;CH17-360D5.1;NPY4R;SYT15 \\
\hline 187249 chr11 & $21,671,750$ & $21,690,194$ & deletion & - \\
\hline 187249 chr11 & $24,446,609$ & $24,451,830$ & deletion & - \\
\hline 187249 chr11 & $90,193,427$ & $90,196,843$ & deletion & - \\
\hline 187249 chr12 & $11,511,132$ & $11,546,987$ & deletion & PRB2 \\
\hline 187249 chr12 & $12,532,984$ & $12,544,338$ & deletion & - \\
\hline 187249 chr12 & $43,021,431$ & $43,027,453$ & deletion & - \\
\hline 187249 chr14 & $106,891,524$ & $106,931,129$ & deletion & - \\
\hline 187249 chr14 & $20,197,311$ & $20,422,182$ & duplication & OR4K5;OR4K1;OR4K2;OR4Q3;OR4N2;OR4M1 \\
\hline 187249 chr14 & $47,708,397$ & $47,715,702$ & deletion & - \\
\hline $187249 \mathrm{chr} 14$ & $73,527,253$ & $73,530,678$ & deletion & - \\
\hline 187249 chr17 & $44,166,604$ & $44,356,058$ & duplication & KANSL1 \\
\hline 187249 chr17 & $54,160,465$ & $54,172,668$ & deletion & - \\
\hline $187249 \mathrm{chr} 17$ & $66,096,462$ & $66,178,186$ & deletion & - \\
\hline 187249 chr17 & $7,264,027$ & $7,265,681$ & deletion & - \\
\hline 187249 chr18 & $63,905,877$ & $63,910,708$ & deletion & - \\
\hline 187249 chr19 & $20,576,148$ & $20,717,536$ & deletion & - \\
\hline 187249 chr19 & $33,790,502$ & $33,812,241$ & deletion & CEBPA \\
\hline 187249 chr19 & $51,330,932$ & $51,333,442$ & deletion & KLK15 \\
\hline 187249 chr19 & $54,556,755$ & $54,560,078$ & deletion & - \\
\hline 187249 chr19 & $54,728,767$ & $54,743,217$ & duplication & LILRA6 \\
\hline 187249 chr19 & 566,738 & 568,189 & deletion & - \\
\hline $187249 \mathrm{chr} 2$ & $133,012,250$ & $133,023,841$ & duplication & - \\
\hline $187249 \mathrm{chr} 2$ & $18,319,713$ & $18,343,245$ & duplication & - \\
\hline 187249 chr2 & $184,085,551$ & $184,088,256$ & deletion & - \\
\hline $187249 \mathrm{chr} 2$ & $184,795,725$ & $184,803,456$ & deletion & - \\
\hline $187249 \mathrm{chr} 2$ & $34,697,895$ & $34,726,904$ & deletion & - \\
\hline 187249 chr2 & $8,170,162$ & $8,180,217$ & deletion & - \\
\hline 187249 chr20 & $29,454,201$ & $29,500,566$ & deletion & - \\
\hline $187249 \mathrm{chr} 20$ & $29,530,268$ & $29,839,864$ & duplication & - \\
\hline 187249 chr20 & $62,194,128$ & $62,204,897$ & deletion & HELZ2 \\
\hline 187249 chr21 & $10,699,687$ & $10,979,896$ & duplication & TPTE \\
\hline 187249 chr22 & $18,626,900$ & $18,629,320$ & deletion & - \\
\hline
\end{tabular}




\begin{tabular}{|c|c|c|c|c|}
\hline 187249 chr22 & $42,912,029$ & $42,961,860$ & duplication & SERHL2;RRP7A \\
\hline $187249 \mathrm{chr} 3$ & $128,382,258$ & $128,412,024$ & duplication & - \\
\hline $187249 \mathrm{chr} 3$ & $165,040,903$ & $165,074,650$ & deletion & - \\
\hline $187249 \mathrm{chr} 3$ & $189,364,424$ & $189,366,763$ & deletion & - \\
\hline 187249 chr3 & $191,061,214$ & $191,070,300$ & deletion & - \\
\hline $187249 \mathrm{chr} 3$ & $65,117,947$ & $65,118,762$ & deletion & - \\
\hline $187249 \mathrm{chr} 3$ & $6,651,929$ & $6,654,060$ & deletion & - \\
\hline $187249 \mathrm{chr} 4$ & $138,091,446$ & $138,098,601$ & deletion & - \\
\hline $187249 \mathrm{chr} 4$ & $34,785,955$ & $34,818,502$ & deletion & - \\
\hline $187249 \mathrm{chr} 4$ & $49,069,684$ & $49,651,131$ & duplication & - \\
\hline $187249 \mathrm{chr} 4$ & $70,137,897$ & $70,229,260$ & deletion & UGT2B28 \\
\hline 187249 chr5 & $155,477,866$ & $155,488,917$ & deletion & - \\
\hline 187249 chr5 & $8,703,085$ & $8,748,627$ & deletion & - \\
\hline 187249 chr6 & $31,785,453$ & $31,795,550$ & deletion & HSPA1A \\
\hline 187249 chr6 & $93,575,149$ & $93,578,359$ & deletion & - \\
\hline 187249 chr7 & $52,730,700$ & $52,744,420$ & duplication & - \\
\hline $187249 \mathrm{chr} 8$ & $115,633,704$ & $115,642,459$ & deletion & - \\
\hline 187249 chr8 & $144,976,572$ & $145,032,302$ & deletion & PLEC \\
\hline $187249 \mathrm{chr} 8$ & $15,400,574$ & $15,411,867$ & deletion & - \\
\hline $187249 \mathrm{chr} 8$ & $18,852,474$ & $18,865,181$ & deletion & - \\
\hline 187249 chr8 & $39,232,581$ & $39,385,979$ & duplication & - \\
\hline 187249 chr8 & $6,977,269$ & $8,094,699$ & duplication & USP17L3;USP17L1;USP17L4;USP17L8;DEFB10 \\
\hline 187249 chr9 & $44,734,155$ & $44,841,930$ & duplication & - \\
\hline 187249 chr9 & $68,307,449$ & $68,502,538$ & duplication & - \\
\hline 187249 chr9 & $69,838,649$ & $69,887,338$ & deletion & - \\
\hline $187250 \mathrm{chr} 1$ & $17,009,576$ & $17,288,389$ & duplication & FAM231A;FAM231C;MST1L;CROCC \\
\hline $187250 \mathrm{chr} 11$ & $21,673,210$ & $21,690,194$ & deletion & - \\
\hline $187250 \mathrm{chr} 11$ & $24,446,609$ & $24,451,830$ & deletion & - \\
\hline 187250 chr11 & $90,193,796$ & $90,196,843$ & deletion & - \\
\hline 187250 chr12 & $12,532,984$ & $12,544,338$ & deletion & - \\
\hline $187250 \mathrm{chr} 12$ & $33,300,995$ & $33,305,855$ & deletion & - \\
\hline 187250 chr12 & $43,021,431$ & $43,027,453$ & deletion & - \\
\hline $187250 \mathrm{chr} 13$ & $83,788,742$ & $83,791,615$ & deletion & - \\
\hline 187250 chr14 & $106,891,524$ & $106,931,129$ & deletion & - \\
\hline 187250 chr14 & $107,157,561$ & $107,181,152$ & duplication & - \\
\hline $187250 \mathrm{chr} 14$ & $20,258,645$ & $20,420,338$ & duplication & OR4N2;OR4K5;OR4K1;OR4K2 \\
\hline 187250 chr14 & $63,956,512$ & $63,965,522$ & deletion & - \\
\hline 187250 chr15 & $20,005,287$ & $20,173,102$ & duplication & - \\
\hline 187250 chr15 & $21,903,815$ & $22,753,733$ & duplication & GOLGA6L22;GOLGA6L1;OR4N4;POTEB3;OR4N \\
\hline $187250 \mathrm{chr} 15$ & $24,349,069$ & $24,710,031$ & duplication & - \\
\hline 187250 chr16 & $78,372,894$ & $78,383,509$ & deletion & - \\
\hline 187250 chr17 & $44,166,604$ & $44,259,615$ & duplication & KANSL1 \\
\hline 187250 chr17 & $4,692,559$ & $4,694,337$ & deletion & GLTPD2 \\
\hline 187250 chr17 & $54,160,465$ & $54,172,668$ & deletion & - \\
\hline 187250 chr17 & $66,096,462$ & $66,178,186$ & deletion & - \\
\hline 187250 chr19 & $5,452,363$ & $5,459,689$ & deletion & ZNRF4 \\
\hline 187250 chr19 & $54,728,767$ & $54,743,217$ & duplication & LILRA6 \\
\hline
\end{tabular}




\begin{tabular}{|c|c|c|c|c|}
\hline 187250 chr19 & $55,298,309$ & $55,338,833$ & deletion & KIR3DL1;KIR2DL4 \\
\hline $187250 \mathrm{chr} 2$ & $18,319,713$ & $18,343,245$ & duplication & - \\
\hline $187250 \mathrm{chr} 2$ & $184,085,551$ & $184,088,256$ & deletion & - \\
\hline $187250 \mathrm{chr} 2$ & $8,170,162$ & $8,180,217$ & deletion & - \\
\hline $187250 \mathrm{chr} 2$ & $87,392,157$ & $87,997,476$ & duplication & - \\
\hline 187250 chr20 & $52,284,831$ & $52,292,118$ & deletion & - \\
\hline 187250 chr20 & $62,194,128$ & $62,200,576$ & deletion & HELZ2 \\
\hline 187250 chr21 & $14,537,654$ & $15,087,262$ & deletion & POTED \\
\hline 187250 chr21 & $44,868,895$ & $44,891,194$ & deletion & - \\
\hline 187250 chr22 & $18,626,900$ & $18,629,849$ & deletion & - \\
\hline $187250 \mathrm{chr} 3$ & $165,040,903$ & $165,074,650$ & deletion & - \\
\hline $187250 \mathrm{chr} 3$ & $191,065,392$ & $191,070,300$ & deletion & - \\
\hline $187250 \mathrm{chr} 3$ & $4,088,770$ & $4,149,238$ & deletion & - \\
\hline $187250 \mathrm{chr} 3$ & $65,117,947$ & $65,118,762$ & deletion & - \\
\hline $187250 \mathrm{chr} 4$ & $44,966,584$ & $45,007,208$ & duplication & - \\
\hline $187250 \mathrm{chr} 4$ & $69,374,369$ & $69,417,886$ & deletion & UGT2B17 \\
\hline 187250 chr5 & $180,377,470$ & $180,430,540$ & deletion & BTNL8;BTNL3 \\
\hline 187250 chr6 & $31,275,174$ & $31,277,988$ & deletion & - \\
\hline 187250 chr6 & $31,785,453$ & $31,795,550$ & deletion & HSPA1A \\
\hline $187250 \mathrm{chr} 6$ & $32,448,904$ & $32,572,251$ & duplication & HLA-DRB1;HLA-DRB5 \\
\hline 187250 chr6 & $77,436,935$ & $77,451,301$ & deletion & - \\
\hline $187250 \mathrm{chr} 6$ & $78,972,930$ & $79,035,739$ & deletion & - \\
\hline $187250 \mathrm{chr} 7$ & $156,387,296$ & $156,394,307$ & deletion & - \\
\hline $187250 \mathrm{chr} 7$ & $52,730,700$ & $52,744,420$ & duplication & - \\
\hline $187250 \mathrm{chr} 8$ & $144,976,572$ & $145,032,302$ & deletion & PLEC \\
\hline $187250 \mathrm{chr} 8$ & $39,232,581$ & $39,385,979$ & duplication & - \\
\hline 187250 chr8 & $6,977,269$ & $8,094,699$ & duplication & USP17L3;USP17L1;USP17L4;USP17L8;DEFB10 \\
\hline $187250 \mathrm{chr} 9$ & $132,586,416$ & $132,597,621$ & deletion & C9orf78 \\
\hline 187250 chr9 & $44,734,155$ & $44,841,930$ & duplication & - \\
\hline 187252 chr11 & $55,370,325$ & $55,427,700$ & deletion & OR4P4;OR4C11;OR4S2 \\
\hline 187252 chr12 & $11,220,827$ & $11,244,117$ & duplication & TAS2R43 \\
\hline 187252 chr12 & $11,511,132$ & $11,546,987$ & deletion & PRB2 \\
\hline 187252 chr12 & $33,300,995$ & $33,305,855$ & deletion & - \\
\hline 187252 chr13 & $111,587,429$ & $111,588,967$ & deletion & - \\
\hline 187252 chr13 & $19,342,790$ & $19,358,874$ & deletion & - \\
\hline 187252 chr13 & $38,076,308$ & $38,085,537$ & deletion & - \\
\hline 187252 chr13 & $80,681,247$ & $80,685,663$ & deletion & - \\
\hline 187252 chr14 & $106,626,535$ & $106,774,755$ & deletion & - \\
\hline 187252 chr14 & $106,891,524$ & $106,931,129$ & deletion & - \\
\hline 187252 chr14 & $73,527,253$ & $73,530,678$ & deletion & - \\
\hline 187252 chr15 & $21,903,815$ & $22,753,733$ & duplication & GOLGA6L22;GOLGA6L1;OR4N4;POTEB3;OR4N \\
\hline 187252 chr16 & $76,717,061$ & $76,718,635$ & deletion & - \\
\hline 187252 chr16 & $88,788,715$ & $88,822,297$ & duplication & PIEZO1 \\
\hline 187252 chr17 & $44,169,808$ & $44,788,813$ & duplication & KANSL1;NSF;ARL17B;LRRC37A2;LRRC37A;ARL: \\
\hline 187252 chr18 & $70,941,877$ & $70,950,855$ & deletion & - \\
\hline $187252 \mathrm{chr} 2$ & $41,239,457$ & $41,248,468$ & deletion & - \\
\hline $187252 \mathrm{chr} 2$ & $52,759,890$ & $52,784,952$ & deletion & - \\
\hline
\end{tabular}




\begin{tabular}{|c|c|c|c|c|}
\hline 187252 chr2 & $66,188,209$ & $66,196,726$ & deletion & - \\
\hline 187252 chr21 & $20,057,919$ & $20,081,051$ & duplication & - \\
\hline 187252 chr21 & $44,822,871$ & $44,837,829$ & duplication & LOC102724428;SIK1 \\
\hline 187252 chr22 & $39,359,885$ & $39,385,663$ & deletion & АРОВЕСЗВ \\
\hline $187252 \mathrm{chr} 3$ & $191,065,392$ & $191,070,300$ & deletion & - \\
\hline $187252 \mathrm{chr} 3$ & $195,457,853$ & $195,468,690$ & duplication & MUC20 \\
\hline $187252 \mathrm{chr} 3$ & $53,032,639$ & $53,038,786$ & deletion & - \\
\hline $187252 \mathrm{chr} 3$ & $6,651,929$ & $6,654,060$ & deletion & - \\
\hline $187252 \mathrm{chr} 3$ & $75,419,736$ & $75,639,184$ & deletion & - \\
\hline $187252 \mathrm{chr} 3$ & $98,945,571$ & $98,947,547$ & deletion & - \\
\hline $187252 \mathrm{chr} 4$ & $1,212,670$ & $1,321,972$ & duplication & MAEA;CTBP1 \\
\hline $187252 \mathrm{chr} 4$ & $178,551,093$ & $179,144,866$ & duplication & - \\
\hline $187252 \mathrm{chr} 4$ & $34,785,955$ & $34,818,502$ & deletion & - \\
\hline $187252 \mathrm{chr} 4$ & $69,374,369$ & $69,489,473$ & duplication & UGT2B17 \\
\hline $187252 \mathrm{chr} 4$ & $70,137,897$ & $70,229,260$ & deletion & UGT2B28 \\
\hline 187252 chr5 & $113,327,484$ & $113,335,230$ & deletion & - \\
\hline 187252 chr5 & $117,388,410$ & $117,393,058$ & deletion & - \\
\hline 187252 chr5 & $17,609,458$ & $17,642,516$ & deletion & - \\
\hline 187252 chr5 & $180,377,470$ & $180,430,540$ & deletion & BTNL8;BTNL3 \\
\hline $187252 \mathrm{chr} 6$ & $30,994,010$ & $30,994,787$ & deletion & MUC22 \\
\hline 187252 chr6 & $78,979,398$ & $79,012,428$ & duplication & - \\
\hline $187252 \mathrm{chr} 7$ & $141,766,850$ & $141,793,521$ & deletion & - \\
\hline $187252 \mathrm{chr} 8$ & $24,972,808$ & $24,990,418$ & deletion & - \\
\hline 187252 chr8 & $39,232,581$ & $39,385,979$ & deletion & 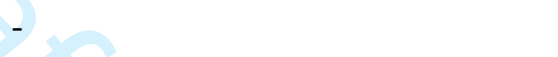 \\
\hline 187252 chr8 & $40,184,872$ & $40,189,688$ & deletion & \\
\hline 187252 chr8 & $43,762,472$ & $43,772,232$ & deletion & - \\
\hline 187252 chr8 & $5,595,511$ & $5,605,706$ & deletion & - \\
\hline 187252 chr9 & $23,363,526$ & $23,376,817$ & deletion & - \\
\hline 187252 chr9 & $6,701,130$ & $6,707,794$ & deletion & - \\
\hline 187252 chr9 & $73,766,471$ & $73,771,616$ & deletion & - \\
\hline 187252 chr9 & $78,004,378$ & $78,011,616$ & deletion & - \\
\hline $187252 \mathrm{chrX}$ & $103,179,170$ & $103,342,222$ & duplication & H2BFWT;TMSB15B;H2BFM \\
\hline 187252 chrX & $8,862,525$ & $8,996,401$ & duplication & FAM9B \\
\hline $187253 \mathrm{chr} 1$ & $17,009,576$ & $17,269,302$ & duplication & FAM231A;FAM231C;MST1L;CROCC \\
\hline $187253 \mathrm{chr} 1$ & $214,685,947$ & $214,690,845$ & deletion & - \\
\hline 187253 chr11 & $18,949,220$ & $18,961,743$ & deletion & MRGPRX1 \\
\hline 187253 chr11 & $24,446,609$ & $24,451,830$ & deletion & - \\
\hline 187253 chr11 & $55,365,761$ & $55,443,269$ & duplication & OR4P4;OR4C6;OR4C11;OR4S2 \\
\hline 187253 chr12 & $38,311,371$ & $38,328,711$ & deletion & - \\
\hline 187253 chr13 & $38,076,308$ & $38,085,537$ & deletion & - \\
\hline 187253 chr13 & $83,788,742$ & $83,791,615$ & deletion & - \\
\hline 187253 chr14 & $106,575,614$ & $106,774,755$ & deletion & - \\
\hline 187253 chr14 & $106,891,524$ & $106,931,129$ & deletion & - \\
\hline 187253 chr15 & $25,420,978$ & $25,430,654$ & deletion & - \\
\hline 187253 chr16 & $34,471,298$ & $34,756,258$ & duplication & - \\
\hline 187253 chr16 & $55,800,009$ & $55,819,443$ & deletion & - \\
\hline 187253 chr17 & $44,169,808$ & $44,347,946$ & duplication & L1 \\
\hline
\end{tabular}




\begin{tabular}{|c|c|c|c|c|}
\hline 187253 chr19 & $24,460,170$ & $24,462,326$ & deletion & - \\
\hline 187253 chr19 & $35,852,103$ & $35,865,496$ & deletion & - \\
\hline $187253 \mathrm{chr} 2$ & $129,638,609$ & $129,645,321$ & deletion & - \\
\hline 187253 chr2 & $34,697,895$ & $34,726,904$ & duplication & - \\
\hline $187253 \mathrm{chr} 2$ & $41,239,457$ & $41,248,468$ & deletion & - \\
\hline $187253 \mathrm{chr} 2$ & $48,851,541$ & $48,856,770$ & deletion & - \\
\hline 187253 chr21 & $10,815,443$ & $10,858,651$ & duplication & - \\
\hline 187253 chr22 & $39,359,885$ & $39,385,663$ & deletion & АРОВЕСЗВ \\
\hline 187253 chr3 & $192,777,710$ & $192,782,294$ & deletion & - \\
\hline 187253 chr3 & $193,136,358$ & $193,140,348$ & deletion & - \\
\hline $187253 \mathrm{chr} 3$ & $28,964,136$ & $29,000,920$ & deletion & - \\
\hline 187253 chr3 & $53,032,639$ & $53,042,099$ & deletion & - \\
\hline $187253 \mathrm{chr} 3$ & $75,419,736$ & $75,639,184$ & deletion & - \\
\hline 187253 chr3 & $98,945,571$ & $98,947,547$ & deletion & - \\
\hline $187253 \mathrm{chr} 4$ & $34,785,955$ & $34,818,502$ & deletion & - \\
\hline $187253 \mathrm{chr} 4$ & $69,374,369$ & $69,431,280$ & deletion & UGT2B17 \\
\hline 187253 chr5 & $113,327,484$ & $113,335,230$ & deletion & - \\
\hline 187253 chr5 & $155,477,866$ & $155,488,917$ & deletion & - \\
\hline 187253 chr5 & $17,345,282$ & $17,356,536$ & deletion & - \\
\hline 187253 chr5 & $17,609,458$ & $17,642,516$ & deletion & - \\
\hline $187253 \mathrm{chr} 5$ & $180,377,470$ & $180,430,540$ & deletion & BTNL8;BTNL3 \\
\hline 187253 chr5 & $70,305,696$ & $70,308,251$ & deletion & NAIP \\
\hline 187253 chr6 & $67,011,900$ & $67,048,885$ & deletion & - \\
\hline 187253 chr6 & $77,439,769$ & $77,451,301$ & deletion & - \\
\hline 187253 chr6 & $79,001,993$ & $79,029,367$ & duplication & \\
\hline 187253 chr7 & $141,766,850$ & $141,793,521$ & deletion & - \\
\hline $187253 \mathrm{chr} 8$ & $115,633,704$ & $115,642,459$ & deletion & - \\
\hline 187253 chr8 & $16,262,222$ & $16,272,444$ & deletion & - \\
\hline $187253 \mathrm{chr} 8$ & $39,232,581$ & $39,385,979$ & deletion & - \\
\hline 187253 chr8 & $40,184,872$ & $40,189,688$ & deletion & - \\
\hline $187253 \mathrm{chr} 8$ & $5,595,511$ & $5,605,706$ & deletion & - \\
\hline 187253 chr9 & $138,147,348$ & $138,308,172$ & duplication & C9orf62 \\
\hline 187253 chr9 & $6,701,130$ & $6,707,794$ & deletion & - \\
\hline 187253 chr9 & $73,766,471$ & $73,771,616$ & deletion & - \\
\hline 187253 chr9 & $78,004,378$ & $78,011,616$ & deletion & - \\
\hline 187254 chr1 & $223,627,639$ & $223,645,256$ & duplication & - \\
\hline $187254 \mathrm{chr} 1$ & $35,104,655$ & $35,111,147$ & deletion & - \\
\hline 187254 chr11 & $55,365,761$ & $55,427,700$ & deletion & OR4P4;OR4C11;OR4S2 \\
\hline 187254 chr12 & $40,874,700$ & $40,875,963$ & deletion & MUC19 \\
\hline 187254 chr12 & $63,927,412$ & $64,129,108$ & duplication & DPY19L2 \\
\hline 187254 chr12 & $7,996,684$ & $8,126,208$ & duplication & SLC2A14;SLC2A3 \\
\hline 187254 chr13 & $58,589,893$ & $58,599,174$ & deletion & - \\
\hline 187254 chr13 & $70,735,399$ & $70,773,605$ & deletion & - \\
\hline 187254 chr14 & $20,197,311$ & $20,402,122$ & duplication & OR4Q3;OR4N2;OR4K5;OR4M1;OR4K2 \\
\hline 187254 chr14 & $43,428,771$ & $43,436,560$ & deletion & - \\
\hline 187254 chr14 & $47,708,397$ & $47,715,702$ & deletion & - \\
\hline 187254 chr15 & $24,577,312$ & $24,779,134$ & deletion & - \\
\hline
\end{tabular}




\begin{tabular}{|c|c|c|c|c|}
\hline 187254 chr15 & $24,827,021$ & $25,190,843$ & duplication & NPAP1 \\
\hline 187254 chr15 & $56,789,979$ & $56,800,635$ & deletion & - \\
\hline 187254 chr16 & $19,945,540$ & $19,962,636$ & deletion & - \\
\hline 187254 chr16 & $6,924,927$ & $7,065,293$ & deletion & - \\
\hline 187254 chr18 & $63,200,484$ & $63,206,335$ & duplication & - \\
\hline 187254 chr18 & $69,428,556$ & $69,441,154$ & deletion & - \\
\hline 187254 chr19 & $20,601,006$ & $20,717,536$ & deletion & - \\
\hline 187254 chr19 & $27,735,604$ & $27,765,280$ & duplication & - \\
\hline 187254 chr19 & $35,852,103$ & $35,861,695$ & deletion & - \\
\hline 187254 chr2 & $120,046,497$ & $120,048,584$ & deletion & - \\
\hline $187254 \mathrm{chr} 2$ & $129,638,609$ & $129,645,321$ & deletion & - \\
\hline $187254 \mathrm{chr} 2$ & $38,298,139$ & $38,305,451$ & deletion & CYP1B1 \\
\hline $187254 \mathrm{chr} 2$ & $41,237,919$ & $41,248,468$ & deletion & - \\
\hline 187254 chr3 & $195,418,291$ & $195,468,690$ & duplication & MUC20 \\
\hline 187254 chr3 & $46,798,133$ & $46,845,618$ & deletion & - \\
\hline $187254 \mathrm{chr} 4$ & $64,136,609$ & $64,154,477$ & deletion & - \\
\hline $187254 \mathrm{chr} 4$ & $69,374,369$ & $69,489,473$ & deletion & UGT2B17 \\
\hline $187254 \mathrm{chr} 4$ & $9,370,814$ & $9,497,854$ & duplication & DEFB131 \\
\hline 187254 chr5 & 744,005 & 825,338 & deletion & ZDHHC11 \\
\hline $187254 \mathrm{chr} 6$ & $78,972,930$ & $79,035,739$ & deletion & - \\
\hline $187254 \mathrm{chr} 7$ & $142,476,094$ & $142,484,042$ & deletion & - \\
\hline $187254 \mathrm{chr} 8$ & $39,232,581$ & $39,385,979$ & duplication & - \\
\hline 187254 chr9 & $78,004,378$ & $78,011,616$ & deletion & - \\
\hline 187255 chr1 & $12,908,591$ & $12,944,989$ & deletion & PRAMEF4;PRAMEF2 \\
\hline $187255 \mathrm{chr} 1$ & $248,740,314$ & $248,813,829$ & deletion & OR2T10;OR2T11;OR2T27;OR2T35 \\
\hline 187255 chr1 & $25,598,276$ & $25,659,509$ & deletion & $\mathrm{RHD}$ \\
\hline $187255 \mathrm{chr} 1$ & $77,254,137$ & $77,259,478$ & deletion & - \\
\hline 187255 chr10 & $14,143,636$ & $14,148,035$ & deletion & - \\
\hline 187255 chr10 & $71,282,981$ & $71,289,190$ & deletion & - \\
\hline 187255 chr11 & $90,193,556$ & $90,196,843$ & deletion & - \\
\hline 187255 chr12 & $127,308,735$ & $127,315,775$ & deletion & - \\
\hline 187255 chr13 & $25,615,049$ & $25,623,454$ & deletion & - \\
\hline 187255 chr14 & $106,891,524$ & $106,931,129$ & deletion & - \\
\hline 187255 chr14 & $20,337,127$ & $20,375,610$ & duplication & OR4K2 \\
\hline 187255 chr17 & $34,438,753$ & $34,534,918$ & duplication & CCL3L3;CCL3L1 \\
\hline 187255 chr17 & $44,213,712$ & $44,788,813$ & duplication & KANSL1;NSF;ARL17B;LRRC37A2;LRRC37A;ARL: \\
\hline 187255 chr17 & $70,562,063$ & $70,565,828$ & deletion & - \\
\hline 187255 chr18 & 13,697 & 66,731 & duplication & - \\
\hline 187255 chr18 & $63,200,484$ & $63,206,335$ & duplication & - \\
\hline 187255 chr19 & $35,852,103$ & $35,861,695$ & deletion & - \\
\hline 187255 chr19 & $54,728,767$ & $54,743,217$ & duplication & LILRA6 \\
\hline 187255 chr19 & $55,248,107$ & $55,339,383$ & duplication & KIR2DL3;KIR3DL1;KIR2DL1;KIR2DL4 \\
\hline $187255 \mathrm{chr} 2$ & $188,059,145$ & $188,146,866$ & deletion & - \\
\hline $187255 \mathrm{chr} 2$ & $48,851,541$ & $48,856,770$ & deletion & - \\
\hline $187255 \mathrm{chr} 2$ & $52,759,890$ & $52,784,952$ & deletion & - \\
\hline 187255 chr2 & $57,398,769$ & $57,446,548$ & duplication & - \\
\hline 187255 chr20 & $29,804,016$ & $29,828,235$ & duplication & - \\
\hline
\end{tabular}




\begin{tabular}{|c|c|c|c|c|}
\hline 187255 chr20 & $52,288,724$ & $52,290,223$ & deletion & - \\
\hline 187255 chr21 & $23,655,900$ & $23,665,470$ & deletion & - \\
\hline 187255 chr22 & $18,626,900$ & $18,629,849$ & deletion & - \\
\hline 187255 chr3 & $193,136,358$ & $193,140,348$ & deletion & - \\
\hline 187255 chr3 & $4,087,818$ & $4,099,877$ & deletion & - \\
\hline $187255 \mathrm{chr} 3$ & $89,402,409$ & $89,418,331$ & deletion & - \\
\hline $187255 \mathrm{chr} 4$ & $39,705,484$ & $39,713,134$ & deletion & - \\
\hline $187255 \mathrm{chr} 4$ & $69,374,369$ & $69,431,280$ & deletion & UGT2B17 \\
\hline 187255 chr6 & $77,436,935$ & $77,451,301$ & deletion & - \\
\hline 187255 chr7 & $17,557,177$ & $17,567,087$ & deletion & - \\
\hline 187255 chr7 & $20,750,774$ & $20,753,528$ & deletion & - \\
\hline 187255 chr8 & $115,633,704$ & $115,642,459$ & deletion & - \\
\hline 187255 chr8 & $39,232,581$ & $39,385,979$ & deletion & - \\
\hline 187255 chr8 & $9,055,379$ & $9,062,490$ & deletion & - \\
\hline 187255 chr9 & $107,799,020$ & $107,803,370$ & deletion & - \\
\hline 187255 chr9 & $85,373,362$ & $85,383,934$ & deletion & - \\
\hline 187257 chr1 & $12,908,591$ & $12,920,067$ & deletion & PRAMEF2 \\
\hline 187257 chr1 & $142,603,938$ & $143,978,807$ & duplication & FAM72D;FAM72C;PPIAL4G \\
\hline $187257 \mathrm{chr} 1$ & $187,716,107$ & $187,722,032$ & deletion & - \\
\hline $187257 \mathrm{chr} 1$ & $248,621,384$ & $248,632,568$ & duplication & - \\
\hline 187257 chr1 & $248,740,572$ & $248,795,110$ & deletion & OR2T10;OR2T11 \\
\hline 187257 chr10 & $6,655,306$ & $6,663,988$ & deletion & - \\
\hline 187257 chr10 & $71,279,367$ & $71,289,190$ & deletion & - \\
\hline 187257 chr12 & $30,237,351$ & $30,243,631$ & deletion & - \\
\hline 187257 chr12 & $31,296,219$ & $31,407,303$ & duplication & - \\
\hline 187257 chr12 & $43,021,431$ & $43,027,453$ & deletion & - \\
\hline 187257 chr12 & $59,935,026$ & $59,942,122$ & deletion & - \\
\hline 187257 chr14 & $106,891,524$ & $106,931,129$ & deletion & - \\
\hline 187257 chr14 & $41,610,224$ & $41,661,685$ & deletion & - \\
\hline 187257 chr15 & $24,055,488$ & $24,061,557$ & deletion & - \\
\hline 187257 chr16 & 205,035 & 212,649 & deletion & - \\
\hline 187257 chr16 & $32,562,332$ & $32,609,440$ & duplication & - \\
\hline 187257 chr16 & $32,642,384$ & $33,827,904$ & duplication & TP53TG3B;TP53TG3;TP53TG3C \\
\hline 187257 chr16 & $70,183,624$ & $70,196,139$ & duplication & PDPR \\
\hline 187257 chr17 & $44,166,604$ & $44,356,058$ & duplication & KANSL1 \\
\hline 187257 chr17 & $70,562,063$ & $70,565,828$ & deletion & - \\
\hline 187257 chr17 & $7,262,226$ & $7,265,681$ & deletion & - \\
\hline 187257 chr19 & $35,852,103$ & $35,861,695$ & deletion & - \\
\hline 187257 chr19 & $54,556,755$ & $54,560,078$ & deletion & - \\
\hline 187257 chr19 & $54,728,767$ & $54,743,217$ & duplication & LILRA6 \\
\hline 187257 chr19 & $9,275,282$ & $9,282,176$ & deletion & - \\
\hline $187257 \mathrm{chr} 2$ & $123,477,263$ & $123,482,301$ & deletion & - \\
\hline $187257 \mathrm{chr} 2$ & $195,979,780$ & $195,982,450$ & deletion & - \\
\hline $187257 \mathrm{chr} 2$ & $34,697,895$ & $34,726,904$ & deletion & - \\
\hline 187257 chr2 & $52,759,890$ & $52,784,952$ & deletion & - \\
\hline 187257 chr20 & $58,296,967$ & $58,313,466$ & deletion & - \\
\hline 187257 chr22 & $42,902,278$ & $42,950,033$ & duplication & 2;RRP7A \\
\hline
\end{tabular}




\begin{tabular}{|c|c|c|c|c|}
\hline $187257 \mathrm{chr} 4$ & $134,919,721$ & $135,184,135$ & deletion & PABPC4L \\
\hline $187257 \mathrm{chr} 4$ & $70,137,897$ & $70,229,260$ & deletion & UGT2B28 \\
\hline $187257 \mathrm{chr} 5$ & $151,514,956$ & $151,518,615$ & deletion & - \\
\hline 187257 chr5 & $155,477,866$ & $155,488,917$ & deletion & - \\
\hline 187257 chr5 & $180,377,470$ & $180,430,540$ & deletion & BTNL8;BTNL3 \\
\hline 187257 chr6 & $124,432,882$ & $124,470,796$ & duplication & NKAIN2 \\
\hline 187257 chr6 & $151,507,355$ & $151,509,176$ & deletion & - \\
\hline 187257 chr6 & $19,041,346$ & $19,048,773$ & deletion & - \\
\hline 187257 chr6 & $77,439,769$ & $77,451,301$ & deletion & - \\
\hline 187257 chr7 & $142,476,094$ & $142,484,042$ & deletion & - \\
\hline 187257 chr7 & $156,387,296$ & $156,394,307$ & deletion & - \\
\hline 187257 chr7 & $159,118,443$ & $159,122,741$ & deletion & - \\
\hline 187257 chr8 & $15,401,929$ & $15,411,867$ & deletion & - \\
\hline 187257 chr8 & $39,232,581$ & $39,385,979$ & deletion & - \\
\hline 187257 chr9 & $68,413,706$ & $68,502,538$ & duplication & - \\
\hline 187258 chr10 & $87,953,705$ & $87,959,563$ & deletion & - \\
\hline 187258 chr11 & $49,715,154$ & $49,757,690$ & deletion & - \\
\hline 187258 chr12 & $11,220,827$ & $11,244,117$ & deletion & TAS2R43 \\
\hline 187258 chr12 & $3,052,731$ & $3,062,407$ & duplication & - \\
\hline 187258 chr12 & $31,283,913$ & $31,407,303$ & duplication & - \\
\hline 187258 chr13 & $32,532,541$ & $32,537,768$ & deletion & - \\
\hline 187258 chr14 & $20,197,311$ & $20,422,799$ & duplication & OR4K5;OR4K1;OR4K2;OR4Q3;OR4N2;OR4M1 \\
\hline $187258 \mathrm{chr} 14$ & $35,604,892$ & $35,613,500$ & deletion & - \\
\hline 187258 chr14 & $99,178,143$ & $99,193,627$ & deletion & C14orf177 \\
\hline $187258 \mathrm{chr} 15$ & $24,521,050$ & $24,714,849$ & duplication & - \\
\hline 187258 chr15 & $46,149,594$ & $46,289,459$ & deletion & - \\
\hline $187258 \mathrm{chr} 16$ & $35,185,903$ & $35,197,323$ & deletion & - \\
\hline $187258 \mathrm{chr} 19$ & $51,330,932$ & $51,333,442$ & deletion & KLK15 \\
\hline 187258 chr19 & $53,518,747$ & $53,548,950$ & duplication & ERVV-1 \\
\hline $187258 \mathrm{chr} 2$ & $158,973,416$ & $158,978,325$ & deletion & UPP2 \\
\hline $187258 \mathrm{chr} 2$ & $184,085,170$ & $184,088,256$ & deletion & - \\
\hline $187258 \mathrm{chr} 2$ & $207,861,511$ & $207,864,911$ & deletion & - \\
\hline $187258 \mathrm{chr} 2$ & $242,854,089$ & $243,034,519$ & deletion & - \\
\hline $187258 \mathrm{chr} 2$ & $38,956,947$ & $38,971,623$ & duplication & GALM \\
\hline 187258 chr20 & $44,350,423$ & $44,378,173$ & deletion & SPINT4 \\
\hline $187258 \mathrm{chr} 3$ & $129,769,827$ & $129,806,304$ & deletion & ALG1L2 \\
\hline $187258 \mathrm{chr} 3$ & $13,681,185$ & $13,682,747$ & deletion & - \\
\hline $187258 \mathrm{chr} 3$ & $148,964,692$ & $148,969,220$ & deletion & - \\
\hline $187258 \mathrm{chr} 3$ & $46,798,133$ & $46,845,618$ & deletion & - \\
\hline $187258 \mathrm{chr} 4$ & $69,374,369$ & $69,467,200$ & deletion & UGT2B17 \\
\hline $187258 \mathrm{chr} 4$ & $70,137,897$ & $70,229,260$ & deletion & UGT2B28 \\
\hline 187258 chr5 & $109,475,723$ & $109,484,586$ & duplication & - \\
\hline 187258 chr5 & $155,477,866$ & $155,495,107$ & deletion & - \\
\hline $187258 \mathrm{chr} 5$ & $26,221,764$ & $26,238,194$ & deletion & - \\
\hline 187258 chr5 & $97,048,466$ & $97,096,042$ & deletion & - \\
\hline 187258 chr6 & $31,281,682$ & $31,286,577$ & deletion & - \\
\hline 187258 chr6 & $67,011,900$ & $67,048,885$ & deletion & - \\
\hline
\end{tabular}




\begin{tabular}{|c|c|c|c|c|c|}
\hline 187258 & chr6 & $77,436,935$ & $77,451,301$ & deletion & - \\
\hline 187258 & chr7 & $29,676,097$ & $29,687,592$ & deletion & - \\
\hline 187258 & chr9 & $24,146,203$ & $24,150,034$ & deletion & - \\
\hline 187259 & chr1 & $25,598,276$ & $25,659,509$ & deletion & RHD \\
\hline 187259 & chr10 & $135,242,873$ & $135,381,927$ & duplication & SYCE1;CYP2E1 \\
\hline 187259 & chr10 & $38,789,617$ & $38,871,868$ & duplication & - \\
\hline 187259 & chr10 & $56,449,714$ & $56,468,486$ & duplication & - \\
\hline 187259 & chr11 & $24,446,609$ & $24,451,830$ & deletion & - \\
\hline 187259 & chr11 & $90,193,796$ & $90,195,742$ & deletion & - \\
\hline 187259 & chr12 & $11,511,132$ & $11,546,987$ & deletion & PRB2 \\
\hline 187259 & chr12 & $31,296,219$ & $31,407,303$ & duplication & - \\
\hline 187259 & chr13 & $34,134,809$ & $34,143,545$ & deletion & - \\
\hline 187259 & chr13 & $38,076,308$ & $38,085,537$ & deletion & - \\
\hline 187259 & chr14 & $20,197,311$ & $20,424,184$ & duplication & OR4K5;OR4K1;OR4K2;OR4Q3;OR4N2;OR4M1 \\
\hline 187259 & chr14 & $41,610,224$ & $41,661,685$ & deletion & - \\
\hline 187259 & chr15 & $34,717,697$ & $34,814,869$ & deletion & - \\
\hline 187259 & chr16 & $19,945,540$ & $19,962,636$ & deletion & - \\
\hline 187259 & chr16 & $28,609,775$ & $28,620,752$ & deletion & SULT1A1 \\
\hline 187259 & chr16 & $78,372,894$ & $78,383,509$ & deletion & - \\
\hline 187259 & chr17 & $44,166,604$ & $44,285,531$ & duplication & KANSL1 \\
\hline 187259 & chr19 & $20,601,006$ & $20,717,536$ & deletion & - \\
\hline 187259 & chr19 & $41,355,999$ & $41,386,033$ & deletion & CYP2A7;CYP2A6 \\
\hline 187259 & chr2 & $195,979,780$ & $195,982,450$ & deletion & - \\
\hline 187259 & chr2 & 33,012 & 35,070 & deletion & - \\
\hline 187259 & chr2 & $41,239,457$ & $41,248,468$ & deletion & \\
\hline 187259 & chr2 & $4,212,725$ & $4,222,144$ & deletion & - \\
\hline 187259 & chr21 & $23,655,900$ & $23,665,470$ & deletion & - \\
\hline 187259 & chr22 & $37,745,101$ & $37,749,946$ & deletion & - \\
\hline 187259 & chr22 & $42,902,278$ & $42,950,033$ & deletion & SERHL2;RRP7A \\
\hline 187259 & chr22 & $45,134,981$ & $45,136,558$ & deletion & - \\
\hline 187259 & chr3 & $172,587,737$ & $172,592,311$ & deletion & - \\
\hline 187259 & chr3 & $191,989,376$ & $191,996,202$ & duplication & - \\
\hline 187259 & chr3 & $193,136,358$ & $193,140,348$ & deletion & - \\
\hline 187259 & chr3 & $195,457,853$ & $195,468,690$ & duplication & MUC20 \\
\hline 187259 & chr4 & $10,396,298$ & $10,400,156$ & deletion & - \\
\hline 187259 & chr4 & $108,064,023$ & $108,074,123$ & deletion & - \\
\hline 187259 & chr4 & $69,374,369$ & $69,489,473$ & duplication & UGT2B17 \\
\hline 187259 & chr4 & $70,137,897$ & $70,229,260$ & deletion & UGT2B28 \\
\hline 187259 & chr5 & $133,395,820$ & $133,397,597$ & deletion & - \\
\hline 187259 & chr5 & $37,197,429$ & $37,251,893$ & deletion & C5orf42 \\
\hline 187259 & chr5 & 717,675 & 807,499 & duplication & ZDHHC11 \\
\hline 187259 & chr6 & $151,507,355$ & $151,509,176$ & deletion & - \\
\hline 187259 & chr6 & $19,041,346$ & $19,048,773$ & deletion & - \\
\hline 187259 & chr6 & $30,994,010$ & $30,994,787$ & deletion & MUC22 \\
\hline 187259 & chr7 & $11,920,873$ & $11,922,312$ & deletion & - \\
\hline 187259 & chr8 & $146,020,871$ & $146,032,842$ & deletion & ZNF517 \\
\hline 187259 & chr8 & $18,852,474$ & $18,865,181$ & deletion & - \\
\hline
\end{tabular}




\begin{tabular}{|c|c|c|c|c|}
\hline 187259 chr8 & $39,232,581$ & $39,385,979$ & deletion & - \\
\hline 187259 chr8 & $40,184,872$ & $40,189,688$ & deletion & - \\
\hline 187259 chr9 & $107,799,020$ & $107,803,370$ & deletion & - \\
\hline 187259 chr9 & $23,363,526$ & $23,376,817$ & duplication & - \\
\hline $187259 \mathrm{chrX}$ & $134,756,517$ & $134,917,010$ & duplication & СТ45A9;CT45A8;CT45A1;CT45A3;CT45A2;CT4! \\
\hline $187260 \mathrm{chr} 1$ & $104,103,057$ & $104,132,315$ & duplication & AMY2B \\
\hline $187260 \mathrm{chr} 1$ & $174,797,867$ & $174,801,639$ & deletion & - \\
\hline $187260 \mathrm{chr} 1$ & $72,754,314$ & $72,763,324$ & deletion & - \\
\hline 187260 chr10 & $135,242,873$ & $135,381,927$ & duplication & SYCE1;CYP2E1 \\
\hline $187260 \mathrm{chr} 10$ & $56,449,714$ & $56,468,486$ & duplication & - \\
\hline $187260 \mathrm{chr} 10$ & $71,282,981$ & $71,289,190$ & deletion & - \\
\hline 187260 chr12 & $11,220,827$ & $11,244,117$ & deletion & TAS2R43 \\
\hline 187260 chr12 & $11,504,864$ & $11,546,987$ & deletion & PRB1;PRB2 \\
\hline $187260 \mathrm{chr} 12$ & $73,988,439$ & $73,995,487$ & deletion & - \\
\hline $187260 \mathrm{chr} 13$ & $19,342,790$ & $19,358,874$ & deletion & - \\
\hline 187260 chr13 & $34,134,809$ & $34,143,545$ & deletion & - \\
\hline 187260 chr13 & $38,076,308$ & $38,085,537$ & deletion & - \\
\hline $187260 \mathrm{chr} 14$ & $41,610,224$ & $41,661,685$ & deletion & - \\
\hline $187260 \mathrm{chr} 15$ & $20,870,571$ & $21,241,118$ & duplication & РОТЕВ2;РОТЕВ;РОТЕВ3 \\
\hline $187260 \mathrm{chr} 15$ & $21,903,815$ & $22,753,733$ & duplication & GOLGA6L22;GOLGA6L1;OR4N4;POTEB3;OR4N \\
\hline 187260 chr15 & $34,717,697$ & $34,814,869$ & deletion & - \\
\hline $187260 \mathrm{chr} 16$ & $61,673,570$ & $61,682,736$ & deletion & - \\
\hline $187260 \mathrm{chr} 16$ & $78,372,894$ & $78,383,509$ & deletion & - \\
\hline 187260 chr17 & $34,438,753$ & $34,481,236$ & duplication & - \\
\hline 187260 chr17 & $44,166,604$ & $44,356,058$ & duplication & KANSL1 \\
\hline $187260 \mathrm{chr} 18$ & $41,976,831$ & $41,982,102$ & deletion & - \\
\hline $187260 \mathrm{chr} 19$ & $20,601,006$ & $20,717,536$ & deletion & - \\
\hline $187260 \mathrm{chr} 19$ & $51,330,932$ & $51,333,442$ & deletion & KLK15 \\
\hline $187260 \mathrm{chr} 2$ & $123,477,465$ & $123,482,301$ & deletion & - \\
\hline $187260 \mathrm{chr} 2$ & $129,638,609$ & $129,645,321$ & deletion & - \\
\hline $187260 \mathrm{chr} 2$ & $195,979,780$ & $195,982,450$ & deletion & - \\
\hline $187260 \mathrm{chr} 2$ & $41,239,457$ & $41,248,468$ & deletion & - \\
\hline $187260 \mathrm{chr} 2$ & $4,212,725$ & $4,222,144$ & deletion & - \\
\hline 187260 chr2 & $51,926,599$ & $51,926,904$ & deletion & - \\
\hline $187260 \mathrm{chr} 2$ & $79,332,083$ & $79,343,124$ & duplication & - \\
\hline $187260 \mathrm{chr} 21$ & $23,655,900$ & $23,665,470$ & deletion & - \\
\hline $187260 \mathrm{chr} 3$ & $172,587,737$ & $172,592,311$ & deletion & - \\
\hline 187260 chr3 & $191,065,392$ & $191,070,300$ & deletion & - \\
\hline $187260 \mathrm{chr} 3$ & $191,989,376$ & $191,996,202$ & duplication & - \\
\hline 187260 chr3 & $193,136,358$ & $193,140,348$ & deletion & - \\
\hline $187260 \mathrm{chr} 3$ & $197,844,320$ & $197,846,134$ & deletion & - \\
\hline $187260 \mathrm{chr} 3$ & $65,117,947$ & $65,118,762$ & deletion & - \\
\hline $187260 \mathrm{chr} 4$ & $167,162,342$ & $167,186,484$ & deletion & - \\
\hline $187260 \mathrm{chr} 4$ & $190,194,200$ & $190,200,463$ & deletion & - \\
\hline $187260 \mathrm{chr} 4$ & $34,785,955$ & $34,814,472$ & duplication & - \\
\hline 187260 chr5 & $133,395,820$ & $133,397,597$ & deletion & - \\
\hline 187260 chr5 & $17,599,857$ & $17,625,744$ & deletion & - \\
\hline
\end{tabular}




\begin{tabular}{|c|c|c|c|c|}
\hline 187260 chr5 & $37,197,160$ & $37,251,893$ & deletion & C5orf42 \\
\hline 187260 chr5 & $3,958,271$ & $3,970,470$ & deletion & - \\
\hline $187260 \mathrm{chr} 6$ & $19,041,346$ & $19,048,773$ & deletion & - \\
\hline 187260 chr6 & $30,994,010$ & $30,994,787$ & deletion & MUC22 \\
\hline 187260 chr6 & $78,979,398$ & $79,009,742$ & duplication & - \\
\hline $187260 \mathrm{chr} 7$ & $11,920,873$ & $11,922,312$ & deletion & - \\
\hline $187260 \mathrm{chr} 7$ & $142,476,094$ & $142,484,042$ & deletion & - \\
\hline 187260 chr7 & 151,829 & 159,207 & deletion & - \\
\hline 187260 chr7 & $156,387,296$ & $156,394,307$ & deletion & - \\
\hline 187260 chr7 & $97,396,709$ & $97,401,677$ & deletion & - \\
\hline $187260 \mathrm{chr} 8$ & $146,020,871$ & $146,032,842$ & deletion & ZNF517 \\
\hline $187260 \mathrm{chr} 8$ & $18,852,474$ & $18,865,181$ & deletion & - \\
\hline 187260 chr8 & $39,232,581$ & $39,385,979$ & deletion & - \\
\hline $187260 \mathrm{chr} 8$ & $40,184,872$ & $40,189,688$ & deletion & - \\
\hline $187260 \mathrm{chr} 8$ & $51,029,371$ & $51,038,149$ & deletion & - \\
\hline 187260 chr9 & $107,799,020$ & $107,809,131$ & deletion & - \\
\hline 187260 chr9 & $23,363,526$ & $23,376,817$ & duplication & - \\
\hline 187261 chr1 & $12,908,591$ & $12,919,928$ & deletion & PRAMEF2 \\
\hline $187261 \mathrm{chr} 1$ & $185,098,927$ & $185,128,607$ & duplication & TRMT1L \\
\hline 187261 chr 1 & $62,113,597$ & $62,119,136$ & deletion & - \\
\hline 187261 chr1 & $9,847,290$ & $9,856,826$ & deletion & - \\
\hline 187261 chr10 & $71,282,981$ & $71,289,190$ & deletion & - \\
\hline 187261 chr10 & $90,941,441$ & $90,949,605$ & dele & 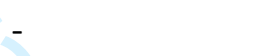 \\
\hline 187261 chr11 & $107,653,650$ & $107,671,155$ & duplication & SLC35F2 \\
\hline 187261 chr11 & $134,602,333$ & $134,607,484$ & deletion & - \\
\hline 187261 chr11 & $55,370,325$ & $55,408,494$ & deletion & OR4P4;O \\
\hline 187261 chr11 & $86,304,402$ & $86,306,401$ & deletion & - \\
\hline 187261 chr13 & $34,134,809$ & $34,143,545$ & deletion & - \\
\hline 187261 chr13 & $38,076,308$ & $38,085,537$ & deletion & - \\
\hline 187261 chr14 & $41,610,224$ & $41,661,685$ & deletion & - \\
\hline 187261 chr14 & $47,708,397$ & $47,715,702$ & deletion & - \\
\hline 187261 chr15 & $97,815,182$ & $97,831,832$ & deletion & - \\
\hline 187261 chr16 & $19,945,540$ & $19,962,636$ & deletion & - \\
\hline 187261 chr16 & $55,800,009$ & $55,819,443$ & deletion & - \\
\hline 187261 chr16 & $78,372,894$ & $78,383,509$ & deletion & - \\
\hline 187261 chr17 & $6,300,290$ & $6,312,899$ & duplication & - \\
\hline 187261 chr19 & $54,728,767$ & $54,743,217$ & duplication & LILRA6 \\
\hline 187261 chr2 & $15,467,179$ & $15,540,714$ & deletion & NBAS \\
\hline 187261 chr2 & $184,085,551$ & $184,088,256$ & deletion & - \\
\hline $187261 \mathrm{chr} 2$ & $19,386,794$ & $19,414,509$ & deletion & - \\
\hline $187261 \mathrm{chr} 2$ & $57,398,769$ & $57,446,548$ & duplication & - \\
\hline $187261 \mathrm{chr} 3$ & $195,457,853$ & $195,468,690$ & duplication & MUC20 \\
\hline $187261 \mathrm{chr} 3$ & $63,751,834$ & $63,756,093$ & deletion & - \\
\hline $187261 \mathrm{chr} 4$ & $16,920,838$ & $16,929,643$ & deletion & - \\
\hline $187261 \mathrm{chr} 4$ & $66,754,450$ & $66,812,771$ & deletion & - \\
\hline 187261 chr5 & $70,305,696$ & $70,308,251$ & deletion & NAIP \\
\hline 187261 & 717,675 & 807,499 & duplication & ZDHHC11 \\
\hline
\end{tabular}




\begin{tabular}{|c|c|c|c|c|}
\hline 187261 chr6 & $132,709,027$ & $132,711,200$ & deletion & - \\
\hline 187261 chr6 & $30,994,010$ & $30,994,787$ & deletion & MUC22 \\
\hline 187261 chr6 & $31,281,682$ & $31,286,577$ & deletion & - \\
\hline 187261 chr7 & $142,476,094$ & $142,484,042$ & deletion & - \\
\hline $187261 \mathrm{chr} 7$ & $38,292,745$ & $38,305,404$ & duplication & TARP \\
\hline 187261 chr7 & $6,669,926$ & $6,671,907$ & deletion & - \\
\hline 187261 chr7 & $97,396,709$ & $97,401,677$ & deletion & - \\
\hline 187261 chr8 & $39,232,581$ & $39,385,979$ & duplication & - \\
\hline 187261 chr9 & $104,715,329$ & $104,722,688$ & deletion & - \\
\hline 187261 chr9 & $23,363,526$ & $23,376,817$ & deletion & - \\
\hline 187261 chr9 & $24,502,737$ & $24,518,795$ & deletion & - \\
\hline 187261 chr9 & $32,081,117$ & $32,087,232$ & duplication & - \\
\hline 187262 chr1 & $149,022,110$ & $149,237,009$ & deletion & - \\
\hline 187262 chr1 & $161,492,587$ & $161,563,386$ & duplication & FCGR3A;HSPA6;FCGR2C \\
\hline 187262 chr1 & $1,627,987$ & $1,675,041$ & deletion & SLC35E2;CDK11A;CDK11B \\
\hline 187262 chr1 & $187,716,107$ & $187,722,032$ & deletion & - \\
\hline $187262 \mathrm{chr} 1$ & $19,595,194$ & $19,609,204$ & deletion & - \\
\hline $187262 \mathrm{chr} 1$ & $196,823,300$ & $196,901,753$ & duplication & CFHR4 \\
\hline $187262 \mathrm{chr} 1$ & $245,636,059$ & $245,647,809$ & deletion & - \\
\hline 187262 chr11 & $18,949,220$ & $18,961,743$ & deletion & MRGPRX1 \\
\hline 187262 chr11 & $85,263,724$ & $85,271,049$ & deletion & - \\
\hline 187262 chr12 & $11,220,827$ & $11,244,117$ & deletion & TAS2R43 \\
\hline 187262 chr12 & $132,131,087$ & $132,136,919$ & deletion & - \\
\hline 187262 chr12 & $40,875,351$ & $40,875,963$ & deletion & MUC19 \\
\hline 187262 chr12 & $6,241,446$ & $6,247,923$ & deletion & - \\
\hline 187262 chr12 & $83,167,766$ & $83,212,731$ & deletion & - \\
\hline 187262 chr13 & $32,532,761$ & $32,537,768$ & deletion & - \\
\hline 187262 chr13 & $38,076,308$ & $38,085,537$ & deletion & - \\
\hline 187262 chr14 & $20,197,311$ & $20,404,736$ & duplication & OR4K5;OR4K1;OR4K2;OR4Q3;OR4N2;OR4M1 \\
\hline 187262 chr15 & $25,420,978$ & $25,430,654$ & deletion & - \\
\hline 187262 chr15 & $61,688,169$ & $61,698,179$ & deletion & - \\
\hline 187262 chr15 & $97,815,182$ & $97,831,832$ & deletion & - \\
\hline 187262 chr16 & $34,471,298$ & $34,756,258$ & duplication & - \\
\hline 187262 chr16 & $54,408,688$ & $54,418,975$ & deletion & - \\
\hline 187262 chr16 & $75,533,714$ & $75,572,495$ & deletion & CHST5 \\
\hline 187262 chr19 & $20,601,006$ & $20,717,536$ & deletion & - \\
\hline 187262 chr19 & $55,298,309$ & $55,378,621$ & deletion & KIR3DL1;KIR2DS4;KIR3DL2;KIR2DL4 \\
\hline 187262 chr19 & $6,986,214$ & $6,994,310$ & deletion & - \\
\hline 187262 chr21 & $44,827,959$ & $44,837,829$ & duplication & LOC102724428;SIK1 \\
\hline 187262 chr22 & $39,359,885$ & $39,385,663$ & deletion & АРОВЕСЗВ \\
\hline 187262 chr3 & $46,794,496$ & $46,845,618$ & deletion & - \\
\hline $187262 \mathrm{chr} 3$ & $75,428,675$ & $75,643,261$ & deletion & - \\
\hline $187262 \mathrm{chr} 4$ & $138,091,446$ & $138,098,601$ & deletion & - \\
\hline $187262 \mathrm{chr} 4$ & $69,374,369$ & $69,467,200$ & deletion & UGT2B17 \\
\hline $187262 \mathrm{chr} 4$ & $92,280,583$ & $92,284,935$ & deletion & - \\
\hline 187262 chr5 & $151,514,956$ & $151,518,615$ & deletion & - \\
\hline 187262 chr5 & $17,609,458$ & $17,642,516$ & deletion & - \\
\hline
\end{tabular}




\begin{tabular}{|c|c|c|c|c|}
\hline 187262 chr6 & $169,271,809$ & $169,273,635$ & deletion & - \\
\hline 187262 chr6 & $29,855,945$ & $29,899,189$ & deletion & - \\
\hline 187262 chr6 & $31,338,528$ & $31,341,357$ & deletion & - \\
\hline 187262 chr6 & $67,011,900$ & $67,048,885$ & deletion & - \\
\hline 187262 chr6 & $77,436,935$ & $77,451,301$ & deletion & - \\
\hline 187262 chr6 & $78,972,930$ & $79,035,739$ & deletion & - \\
\hline 187262 chr7 & 151,829 & 159,207 & deletion & - \\
\hline 187262 chr7 & $38,291,851$ & $38,306,366$ & duplication & TARP \\
\hline 187262 chr7 & $8,827,959$ & $8,866,274$ & deletion & - \\
\hline 187262 chr8 & $24,972,808$ & $24,990,418$ & deletion & - \\
\hline 187262 chr9 & $135,947,165$ & $135,957,452$ & duplication & - \\
\hline 187262 chr9 & $78,004,378$ & $78,011,616$ & deletion & - \\
\hline $187262 \mathrm{chrX}$ & $115,941,080$ & $116,083,902$ & duplication & - \\
\hline 187263 chr1 & $12,900,831$ & $12,919,928$ & deletion & HNRNPCL1;HNRNPCL3;HNRNPCL4;PRAMEF2 \\
\hline $187263 \mathrm{chr} 1$ & $248,740,314$ & $248,795,110$ & deletion & OR2T10;OR2T11 \\
\hline 187263 chr1 & $5,113,911$ & $5,174,078$ & deletion & - \\
\hline 187263 chr10 & $135,242,873$ & $135,381,927$ & duplication & SYCE1;CYP2E1 \\
\hline 187263 chr10 & $71,280,107$ & $71,289,190$ & deletion & - \\
\hline 187263 chr11 & $134,347,693$ & $134,721,543$ & duplication & - \\
\hline 187263 chr11 & $55,365,761$ & $55,443,269$ & deletion & OR4P4;OR4C6;OR4C11;OR4S2 \\
\hline 187263 chr12 & $6,255,862$ & $6,259,552$ & deletion & - \\
\hline 187263 chr13 & $19,948,553$ & $19,976,389$ & deletion & - \\
\hline 187263 chr13 & $69,247,022$ & $69,267,981$ & deletion & - \\
\hline 187263 chr14 & $106,575,614$ & $106,809,871$ & deletion & - \\
\hline 187263 chr14 & $41,610,224$ & $41,661,685$ & deletion & - \\
\hline 187263 chr15 & $22,299,434$ & $22,477,128$ & duplication & OR4M2;OR4N4 \\
\hline 187263 chr15 & $56,789,979$ & $56,800,635$ & deletion & - \\
\hline 187263 chr15 & $97,815,182$ & $97,831,832$ & deletion & - \\
\hline 187263 chr16 & $28,609,775$ & $28,620,752$ & deletion & SULT1A1 \\
\hline 187263 chr16 & $32,511,914$ & $32,833,893$ & deletion & TP53TG3B;TP53TG3;TP53TG3C \\
\hline 187263 chr17 & $34,438,753$ & $34,483,342$ & duplication & - \\
\hline 187263 chr19 & $43,703,965$ & $43,759,382$ & deletion & PSG4;PSG9 \\
\hline 187263 chr19 & $53,518,747$ & $53,552,296$ & duplication & ERVV-1 \\
\hline 187263 chr19 & $54,728,767$ & $54,743,217$ & duplication & LILRA6 \\
\hline $187263 \mathrm{chr} 2$ & $123,477,263$ & $123,482,301$ & deletion & - \\
\hline 187263 chr2 & $228,244,107$ & $228,258,288$ & duplication & - \\
\hline 187263 chr2 & $78,703,558$ & $78,723,039$ & deletion & - \\
\hline $187263 \mathrm{chr} 3$ & $128,382,258$ & $128,417,293$ & duplication & - \\
\hline 187263 chr3 & $191,061,214$ & $191,070,300$ & deletion & - \\
\hline $187263 \mathrm{chr} 3$ & $193,136,358$ & $193,140,348$ & deletion & - \\
\hline 187263 chr3 & $36,264,033$ & $36,288,652$ & duplication & - \\
\hline $187263 \mathrm{chr} 3$ & $37,979,882$ & $37,990,299$ & deletion & CTDSPL \\
\hline $187263 \mathrm{chr} 4$ & $156,959,568$ & $156,973,205$ & deletion & - \\
\hline $187263 \mathrm{chr} 4$ & $29,927,916$ & $29,938,283$ & deletion & - \\
\hline $187263 \mathrm{chr} 4$ & $69,374,369$ & $69,393,356$ & deletion & - \\
\hline 187263 chr5 & $155,477,866$ & $155,488,917$ & deletion & - \\
\hline 187263 chr5 & $8,258,458$ & $8,260,630$ & deletion & - \\
\hline
\end{tabular}




\begin{tabular}{|c|c|c|c|c|}
\hline 187263 chr6 & $146,870,307$ & $146,874,547$ & deletion & RAB32 \\
\hline 187263 chr7 & $52,730,700$ & $52,744,420$ & duplication & - \\
\hline 187263 chr8 & $16,262,222$ & $16,272,444$ & deletion & - \\
\hline 187263 chr8 & $18,852,474$ & $18,865,181$ & deletion & - \\
\hline 187263 chr8 & $2,256,926$ & $2,263,719$ & deletion & - \\
\hline 187263 chr9 & $6,701,130$ & $6,707,794$ & deletion & - \\
\hline 187264 chr1 & $12,908,591$ & $12,919,928$ & deletion & PRAMEF2 \\
\hline 187264 chr1 & $248,740,572$ & $248,795,110$ & deletion & OR2T10;OR2T11 \\
\hline 187264 chr1 & $25,598,276$ & $25,659,509$ & deletion & RHD \\
\hline 187264 chr1 & $5,113,911$ & $5,174,078$ & deletion & - \\
\hline 187264 chr10 & $27,227,105$ & $27,227,458$ & duplication & - \\
\hline 187264 chr10 & $98,838,488$ & $98,997,986$ & duplication & SLIT1;ARHGAP19 \\
\hline 187264 chr11 & $55,365,761$ & $55,443,269$ & deletion & OR4P4;OR4C6;OR4C11;OR4S2 \\
\hline $187264 \mathrm{chr} 12$ & $11,494,021$ & $11,568,822$ & deletion & PRB1;PRB2 \\
\hline $187264 \mathrm{chr} 12$ & $132,131,087$ & $132,136,919$ & deletion & - \\
\hline 187264 chr12 & $24,746,899$ & $24,751,003$ & deletion & - \\
\hline $187264 \mathrm{chr} 12$ & $59,935,026$ & $59,942,122$ & deletion & - \\
\hline 187264 chr12 & $6,255,862$ & $6,259,552$ & deletion & - \\
\hline $187264 \mathrm{chr} 13$ & $69,247,022$ & $69,267,981$ & deletion & - \\
\hline 187264 chr13 & $91,024,205$ & $91,027,128$ & deletion & - \\
\hline 187264 chr14 & $106,575,614$ & $106,774,755$ & deletion & - \\
\hline 187264 chr14 & $20,197,311$ & $20,404,736$ & duplication & OR4K5;OR4K1;OR4K2;OR4Q3;OR4N2;OR4M1 \\
\hline 187264 chr14 & $41,610,224$ & $41,661,685$ & deletion & - \\
\hline 187264 chr16 & $28,603,889$ & $28,643,773$ & deletion & SULT1A1;SULT1A2 \\
\hline $187264 \mathrm{chr} 16$ & $32,528,023$ & $32,833,893$ & deletion & TP53TG3B;TP53TG3;TP53TG3C \\
\hline 187264 chr16 & $68,568,132$ & $68,573,347$ & deletion & - \\
\hline 187264 chr17 & $34,438,753$ & $34,483,342$ & duplication & - \\
\hline $187264 \mathrm{chr} 19$ & $43,703,965$ & $43,759,382$ & deletion & PSG4;PSG9 \\
\hline 187264 chr19 & $53,518,747$ & $53,548,950$ & duplication & ERVV-1 \\
\hline 187264 chr19 & $54,728,767$ & $54,743,217$ & duplication & LILRA6 \\
\hline 187264 chr19 & $9,275,282$ & $9,282,176$ & deletion & - \\
\hline 187264 chr2 & $115,532,886$ & $115,542,382$ & deletion & - \\
\hline $187264 \mathrm{chr} 2$ & $38,956,947$ & $38,971,623$ & duplication & GALM \\
\hline 187264 chr2 & $49,534,329$ & $49,538,143$ & deletion & - \\
\hline 187264 chr2 & $78,703,558$ & $78,723,039$ & deletion & - \\
\hline $187264 \mathrm{chr} 3$ & $128,382,258$ & $128,412,024$ & duplication & - \\
\hline 187264 chr3 & $191,062,751$ & $191,070,300$ & deletion & - \\
\hline $187264 \mathrm{chr} 3$ & $193,136,358$ & $193,140,348$ & deletion & - \\
\hline 187264 chr3 & $37,979,882$ & $37,986,734$ & deletion & - \\
\hline 187264 chr3 & $6,318,899$ & $6,335,989$ & duplication & - \\
\hline $187264 \mathrm{chr} 4$ & $138,092,298$ & $138,098,601$ & deletion & - \\
\hline $187264 \mathrm{chr} 4$ & $165,569,844$ & $165,587,340$ & deletion & - \\
\hline $187264 \mathrm{chr} 4$ & $34,785,955$ & $34,818,502$ & deletion & - \\
\hline $187264 \mathrm{chr} 4$ & $60,325,010$ & $60,330,880$ & duplication & - \\
\hline $187264 \mathrm{chr} 4$ & $69,374,369$ & $69,467,200$ & deletion & UGT2B17 \\
\hline $187264 \mathrm{chr} 4$ & $7,182,916$ & $7,187,397$ & deletion & - \\
\hline 187264 chr5 & $117,388,410$ & $117,393,058$ & deletion & - \\
\hline
\end{tabular}




\begin{tabular}{|c|c|c|c|c|}
\hline 187264 chr6 & $146,870,307$ & $146,874,547$ & deletion & RAB32 \\
\hline 187264 chr6 & $30,995,187$ & $30,996,363$ & deletion & MUC22 \\
\hline 187264 chr7 & $156,387,296$ & $156,394,307$ & deletion & - \\
\hline 187264 chr7 & $39,548,060$ & $39,562,295$ & deletion & - \\
\hline 187264 chr7 & $97,396,709$ & $97,401,677$ & deletion & - \\
\hline 187264 chr8 & $137,682,484$ & $137,862,080$ & deletion & - \\
\hline 187264 chr8 & $16,262,222$ & $16,272,444$ & deletion & - \\
\hline 187264 chr8 & $18,852,474$ & $18,865,181$ & deletion & - \\
\hline 187264 chr8 & $2,256,926$ & $2,263,719$ & deletion & - \\
\hline $187264 \mathrm{chr} 8$ & $39,236,582$ & $39,382,903$ & deletion & - \\
\hline 187265 chr1 & $12,900,831$ & $12,919,928$ & deletion & HNRNPCL1;HNRNPCL3;HNRNPCL4;PRAMEF2 \\
\hline 187265 chr1 & $248,740,314$ & $248,795,110$ & deletion & OR2T10;OR2T11 \\
\hline 187265 chr1 & $25,598,276$ & $25,659,509$ & deletion & RHD \\
\hline $187265 \mathrm{chr} 1$ & $5,113,911$ & $5,174,078$ & deletion & - \\
\hline 187265 chr10 & $98,835,644$ & $98,997,986$ & duplication & SLIT1;ARHGAP19 \\
\hline 187265 chr11 & $55,365,761$ & $55,443,269$ & deletion & OR4P4;OR4C6;OR4C11;OR4S2 \\
\hline 187265 chr12 & $11,494,021$ & $11,568,822$ & deletion & PRB1;PRB2 \\
\hline 187265 chr12 & $132,131,087$ & $132,136,919$ & deletion & - \\
\hline 187265 chr12 & $24,746,899$ & $24,751,003$ & deletion & - \\
\hline 187265 chr12 & $59,935,926$ & $59,942,122$ & deletion & - \\
\hline 187265 chr12 & $6,255,862$ & $6,261,600$ & deletion & - \\
\hline 187265 chr13 & $69,247,022$ & $69,267,981$ & deletion & - \\
\hline 187265 chr14 & $106,575,614$ & $106,774,755$ & deletion & - \\
\hline 187265 chr14 & $20,213,037$ & $20,422,799$ & duplication & OR4K5;OR4K1;OR4K2;OR4Q3;OR4N2;OR4M1 \\
\hline 187265 chr14 & $41,610,224$ & $41,661,685$ & deletion & - \\
\hline 187265 chr16 & $28,603,889$ & $28,643,773$ & deletion & SULT1A1;SULT1A2 \\
\hline 187265 chr17 & $34,438,753$ & $34,483,342$ & duplication & - \\
\hline 187265 chr19 & $43,703,965$ & $43,759,382$ & deletion & PSG4;PSG9 \\
\hline 187265 chr19 & $53,518,747$ & $53,553,992$ & duplication & ERVV-1;ERVV-2 \\
\hline 187265 chr19 & $54,728,767$ & $54,743,217$ & duplication & LILRA6 \\
\hline 187265 chr19 & $9,275,282$ & $9,282,176$ & deletion & - \\
\hline 187265 chr2 & $38,956,947$ & $38,971,623$ & duplication & GALM \\
\hline 187265 chr2 & $49,534,329$ & $49,538,143$ & deletion & - \\
\hline $187265 \mathrm{chr} 2$ & $78,703,558$ & $78,723,039$ & deletion & - \\
\hline 187265 chr3 & $128,382,258$ & $128,417,293$ & duplication & - \\
\hline 187265 chr3 & $193,136,358$ & $193,140,348$ & deletion & - \\
\hline 187265 chr3 & $37,979,882$ & $37,990,299$ & deletion & CTDSPL \\
\hline 187265 chr3 & $6,318,899$ & $6,335,989$ & duplication & - \\
\hline $187265 \mathrm{chr} 4$ & $138,092,298$ & $138,098,601$ & deletion & - \\
\hline $187265 \mathrm{chr} 4$ & $165,569,844$ & $165,587,340$ & deletion & - \\
\hline $187265 \mathrm{chr} 4$ & $60,325,010$ & $60,330,880$ & duplication & - \\
\hline $187265 \mathrm{chr} 5$ & $117,388,410$ & $117,393,058$ & deletion & - \\
\hline 187265 chr5 & $180,176,005$ & $180,195,526$ & duplication & - \\
\hline 187265 chr5 & $8,258,458$ & $8,260,630$ & deletion & - \\
\hline 187265 chr6 & $146,870,307$ & $146,874,547$ & deletion & RAB32 \\
\hline 187265 chr7 & $156,387,296$ & $156,394,307$ & deletion & - \\
\hline 187265 chr7 & $39,546,038$ & $39,562,295$ & deletion & - \\
\hline
\end{tabular}




\begin{tabular}{|c|c|c|c|c|}
\hline 187265 chr7 & $97,396,709$ & $97,401,677$ & deletion & - \\
\hline 187265 chr8 & $137,682,484$ & $137,862,080$ & deletion & - \\
\hline 187265 chr8 & $16,262,222$ & $16,272,444$ & deletion & - \\
\hline 187265 chr8 & $18,852,474$ & $18,865,181$ & deletion & - \\
\hline 187265 chr8 & $2,256,926$ & $2,263,719$ & deletion & - \\
\hline 187266 chr1 & $62,113,597$ & $62,119,136$ & deletion & - \\
\hline 187266 chr11 & $44,729,754$ & $44,733,505$ & deletion & - \\
\hline 187266 chr11 & $83,209,945$ & $83,215,072$ & deletion & - \\
\hline 187266 chr11 & $90,193,427$ & $90,196,843$ & deletion & - \\
\hline 187266 chr12 & $43,021,431$ & $43,027,453$ & deletion & - \\
\hline 187266 chr14 & $50,714,985$ & $50,725,773$ & deletion & - \\
\hline 187266 chr15 & $56,083,103$ & $56,089,103$ & deletion & - \\
\hline 187266 chr15 & $87,825,010$ & $87,870,024$ & deletion & - \\
\hline 187266 chr16 & $32,528,023$ & $32,833,893$ & deletion & TP53TG3B;TP53TG3;TP53TG3C \\
\hline $187266 \mathrm{chr} 16$ & $52,445,515$ & $52,451,224$ & deletion & - \\
\hline 187266 chr17 & $33,548,928$ & $33,601,223$ & duplication & SLFN5 \\
\hline 187266 chr17 & $34,438,753$ & $34,483,342$ & duplication & - \\
\hline $187266 \mathrm{chr} 18$ & $67,208,806$ & $67,217,271$ & deletion & - \\
\hline 187266 chr19 & $54,728,767$ & $54,743,217$ & duplication & LILRA6 \\
\hline $187266 \mathrm{chr} 2$ & $123,477,263$ & $123,482,301$ & deletion & - \\
\hline 187266 chr2 & $41,239,457$ & $41,248,468$ & deletion & - \\
\hline 187266 chr2 & $48,851,541$ & $48,856,770$ & deletion & - \\
\hline $187266 \mathrm{chr} 20$ & $59,112,918$ & $59,122,693$ & deletion & - \\
\hline 187266 chr3 & $129,769,827$ & $129,806,304$ & deletion & ALG1L2 \\
\hline 187266 chr3 & $193,136,358$ & $193,140,348$ & deletion & - \\
\hline 187266 chr3 & $195,457,853$ & $195,468,690$ & duplication & MUC20 \\
\hline $187266 \mathrm{chr} 3$ & $4,175,624$ & $4,313,485$ & deletion & - \\
\hline $187266 \mathrm{chr} 3$ & $65,189,366$ & $65,214,685$ & deletion & - \\
\hline 187266 chr3 & $75,428,675$ & $75,639,184$ & deletion & - \\
\hline 187266 chr4 & $126,688,743$ & $126,696,308$ & deletion & - \\
\hline $187266 \mathrm{chr} 4$ & $138,092,298$ & $138,098,601$ & deletion & - \\
\hline 187266 chr4 & $152,790,580$ & $152,793,891$ & deletion & - \\
\hline $187266 \mathrm{chr} 4$ & $152,991,094$ & $152,993,620$ & deletion & - \\
\hline $187266 \mathrm{chr} 4$ & $34,785,955$ & $34,818,502$ & deletion & - \\
\hline $187266 \mathrm{chr} 4$ & $64,136,609$ & $64,154,477$ & deletion & - \\
\hline 187266 chr5 & $155,477,866$ & $155,488,917$ & deletion & - \\
\hline 187266 chr6 & $31,281,682$ & $31,286,577$ & deletion & - \\
\hline 187266 chr6 & $32,486,060$ & $32,527,045$ & deletion & HLA-DRB5 \\
\hline 187266 chr6 & $78,972,930$ & $79,035,739$ & deletion & - \\
\hline 187266 chr7 & $156,387,296$ & $156,394,307$ & deletion & - \\
\hline 187266 chr7 & $62,149,006$ & $62,162,385$ & deletion & - \\
\hline 187266 chr8 & $24,972,808$ & $24,990,418$ & deletion & - \\
\hline 187266 chr8 & $3,786,543$ & $3,790,254$ & deletion & - \\
\hline 187266 chr9 & $115,793,311$ & $115,821,485$ & deletion & ZFP37 \\
\hline 187266 chr9 & $2,148,213$ & $2,151,397$ & deletion & - \\
\hline 187266 chr9 & $7,226,519$ & $7,234,019$ & deletion & - \\
\hline 187267 chr1 & $76,976,522$ & $77,357,556$ & duplication & ST6GALNAC5;ST6GALNAC3 \\
\hline
\end{tabular}




\begin{tabular}{|c|c|c|c|c|c|}
\hline 187267 & chr10 & $35,956,671$ & $35,985,781$ & deletion & - \\
\hline 187267 & $\operatorname{chr} 11$ & $3,235,343$ & $3,247,976$ & deletion & MRGPRG \\
\hline 187267 & $\operatorname{chr} 11$ & $55,365,761$ & $55,451,045$ & deletion & OR4P4;OR4C6;OR4C11;OR4S2 \\
\hline 187267 & chr11 & $81,501,218$ & $81,517,261$ & deletion & - \\
\hline 187267 & chr11 & $86,304,402$ & $86,306,401$ & deletion & - \\
\hline 187267 & $\operatorname{chr} 12$ & $7,996,684$ & $8,126,208$ & duplication & SLC2A14;SLC2A3 \\
\hline 187267 & chr14 & $106,062,675$ & $106,178,945$ & deletion & - \\
\hline 187267 & chr14 & $20,326,628$ & $20,422,799$ & duplication & OR4K5;OR4K1;OR4K2 \\
\hline 187267 & chr14 & $41,610,224$ & $41,661,685$ & deletion & - \\
\hline 187267 & chr15 & $97,806,730$ & $97,831,832$ & deletion & - \\
\hline 187267 & chr16 & $78,372,894$ & $78,383,509$ & deletion & - \\
\hline 187267 & chr19 & $56,332,199$ & $56,355,061$ & duplication & - \\
\hline 187267 & chr2 & $213,187,034$ & $213,196,099$ & deletion & - \\
\hline 187267 & chr2 & $228,244,107$ & $228,258,288$ & duplication & - \\
\hline 187267 & chr2 & $38,951,666$ & $38,971,623$ & duplication & GALM \\
\hline 187267 & chr2 & $89,540,620$ & $90,256,530$ & deletion & - \\
\hline 187267 & chr21 & $23,655,900$ & $23,665,470$ & deletion & - \\
\hline 187267 & $\operatorname{chr} 22$ & $42,902,872$ & $42,950,033$ & duplication & SERHL2;RRP7A \\
\hline 187267 & chr3 & $89,187,123$ & $89,248,378$ & duplication & - \\
\hline 187267 & chr3 & $89,402,409$ & $89,418,331$ & deletion & - \\
\hline 187267 & chr4 & $108,064,023$ & $108,076,199$ & deletion & - \\
\hline 187267 & chr4 & $67,078,994$ & $67,114,908$ & duplication & - \\
\hline 187267 & chr6 & $29,855,945$ & $29,899,189$ & deletion & - \\
\hline 187267 & chr6 & $78,972,930$ & $79,035,739$ & deletion & - \\
\hline 187267 & chr7 & $142,825,750$ & $142,890,868$ & deletion & PIP;TAS2R39 \\
\hline 187267 & chr7 & $156,387,296$ & $156,394,307$ & deletion & - \\
\hline 187267 & chr8 & $51,029,371$ & $51,038,149$ & deletion & - \\
\hline 187267 & chr9 & $23,363,526$ & $23,376,817$ & deletion & - \\
\hline 187268 & chr1 & $223,627,639$ & $223,643,527$ & duplication & - \\
\hline 187268 & chr10 & $17,095,299$ & $17,102,123$ & deletion & - \\
\hline 187268 & chr11 & $55,370,325$ & $55,419,073$ & deletion & OR4P4;OR4C11;OR4S2 \\
\hline 187268 & chr12 & $7,996,684$ & $8,125,745$ & duplication & SLC2A14;SLC2A3 \\
\hline 187268 & chr14 & $43,428,771$ & $43,436,560$ & deletion & - \\
\hline 187268 & chr14 & $47,708,397$ & $47,715,702$ & deletion & - \\
\hline 187268 & chr15 & $56,789,979$ & $56,800,635$ & deletion & - \\
\hline 187268 & chr16 & $28,614,734$ & $28,620,752$ & deletion & SULT1A1 \\
\hline 187268 & chr16 & $31,965,625$ & $32,649,736$ & duplication & TP53TG3D \\
\hline 187268 & chr16 & $33,533,297$ & $33,631,255$ & duplication & - \\
\hline 187268 & chr16 & $34,471,298$ & $34,756,258$ & duplication & - \\
\hline 187268 & chr16 & $6,924,927$ & $7,065,293$ & deletion & - \\
\hline 187268 & chr17 & $34,438,753$ & $34,483,342$ & duplication & - \\
\hline 187268 & chr17 & $54,160,465$ & $54,172,668$ & deletion & - \\
\hline 187268 & $\operatorname{chr} 18$ & $63,200,484$ & $63,206,335$ & duplication & - \\
\hline 187268 & $\operatorname{chr} 18$ & $69,428,556$ & $69,441,154$ & deletion & - \\
\hline 187268 & chr18 & $76,157,694$ & $76,160,278$ & deletion & - \\
\hline 187268 & chr19 & $20,601,006$ & $20,717,536$ & deletion & - \\
\hline 187268 & chr19 & $35,852,103$ & $35,861,695$ & deletion & - \\
\hline
\end{tabular}




\begin{tabular}{|c|c|c|c|c|}
\hline 187268 chr2 & $184,085,551$ & $184,088,256$ & deletion & - \\
\hline $187268 \mathrm{chr} 2$ & $51,926,599$ & $51,926,904$ & deletion & - \\
\hline $187268 \mathrm{chr} 2$ & $52,759,890$ & $52,784,952$ & deletion & - \\
\hline $187268 \mathrm{chr} 2$ & $87,392,157$ & $87,997,476$ & duplication & - \\
\hline $187268 \mathrm{chr} 3$ & $193,136,358$ & $193,140,348$ & deletion & - \\
\hline $187268 \mathrm{chr} 3$ & $46,798,133$ & $46,845,618$ & deletion & - \\
\hline $187268 \mathrm{chr} 4$ & $190,194,200$ & $190,200,463$ & deletion & - \\
\hline $187268 \mathrm{chr} 4$ & $69,374,369$ & $69,489,473$ & deletion & UGT2B17 \\
\hline $187268 \mathrm{chr} 4$ & $70,137,897$ & $70,229,260$ & deletion & UGT2B28 \\
\hline 187268 chr6 & $78,972,930$ & $79,035,739$ & deletion & - \\
\hline 187268 chr7 & $142,476,094$ & $142,484,042$ & deletion & - \\
\hline $187268 \mathrm{chr} 8$ & $137,682,484$ & $137,862,080$ & deletion & - \\
\hline $187268 \mathrm{chr} 8$ & $39,322,364$ & $39,385,979$ & deletion & - \\
\hline $187268 \mathrm{chr} 8$ & $61,858,213$ & $61,869,649$ & deletion & - \\
\hline 187268 chr9 & $78,004,378$ & $78,011,616$ & deletion & - \\
\hline $187269 \mathrm{chr} 1$ & $104,104,990$ & $104,211,046$ & duplication & AMY1C;AMY1A;AMY2A;AMY2B;AMY1B \\
\hline $187269 \mathrm{chr} 1$ & $161,490,896$ & $161,510,021$ & duplication & HSPA6 \\
\hline $187269 \mathrm{chr} 1$ & $187,716,107$ & $187,722,032$ & deletion & - \\
\hline $187269 \mathrm{chr} 1$ & $231,711,489$ & $231,810,657$ & duplication & DISC1 \\
\hline $187269 \mathrm{chr} 1$ & $248,740,572$ & $248,795,110$ & deletion & OR2T10;OR2T11 \\
\hline 187269 chr10 & $33,855,128$ & $33,865,876$ & duplication & - \\
\hline 187269 chr11 & $18,949,220$ & $18,961,743$ & duplication & MRGPRX1 \\
\hline 187269 chr11 & $55,365,761$ & $55,427,700$ & deletion & OR4P4;OR4C11;OR4S2 \\
\hline 187269 chr11 & $90,193,796$ & $90,196,843$ & deletion & - \\
\hline 187269 chr12 & $11,220,827$ & $11,244,117$ & deletion & TAS2R43 \\
\hline 187269 chr12 & $29,206,071$ & $29,226,080$ & duplication & - \\
\hline 187269 chr13 & $19,342,790$ & $19,358,874$ & deletion & - \\
\hline 187269 chr14 & $106,891,524$ & $106,931,129$ & deletion & - \\
\hline 187269 chr14 & $41,610,224$ & $41,661,685$ & deletion & - \\
\hline 187269 chr15 & $24,381,396$ & $24,439,268$ & deletion & - \\
\hline 187269 chr15 & $24,577,312$ & $24,779,134$ & duplication & - \\
\hline 187269 chr15 & $61,688,169$ & $61,699,175$ & deletion & - \\
\hline 187269 chr18 & $69,428,556$ & $69,441,154$ & deletion & - \\
\hline $187269 \mathrm{chr} 2$ & $178,551,924$ & $178,573,842$ & duplication & PDE11A \\
\hline $187269 \mathrm{chr} 2$ & $208,351,315$ & $208,357,838$ & deletion & - \\
\hline 187269 chr21 & $44,822,871$ & $44,837,829$ & duplication & LOC102724428;SIK1 \\
\hline 187269 chr22 & $25,758,275$ & $25,849,589$ & deletion & - \\
\hline 187269 chr22 & $44,562,756$ & $44,567,854$ & deletion & PARVB \\
\hline $187269 \mathrm{chr} 3$ & $195,457,853$ & $195,468,690$ & duplication & MUC20 \\
\hline $187269 \mathrm{chr} 3$ & $27,448,619$ & $27,553,889$ & duplication & SLC4A7 \\
\hline $187269 \mathrm{chr} 3$ & $28,810,588$ & $28,817,583$ & deletion & - \\
\hline $187269 \mathrm{chr} 3$ & $37,979,882$ & $37,990,299$ & deletion & CTDSPL \\
\hline $187269 \mathrm{chr} 3$ & $53,032,639$ & $53,042,099$ & deletion & - \\
\hline $187269 \mathrm{chr} 3$ & $80,117,870$ & $80,124,685$ & deletion & - \\
\hline 187269 chr4 & $162,055,725$ & $162,062,505$ & deletion & - \\
\hline $187269 \mathrm{chr} 4$ & $64,136,609$ & $64,154,477$ & deletion & - \\
\hline $187269 \mathrm{chr} 4$ & $69,374,369$ & $69,489,473$ & deletion & UGT2B17 \\
\hline
\end{tabular}




\begin{tabular}{|c|c|c|c|c|}
\hline 187269 chr5 & $140,225,908$ & $140,238,311$ & deletion & PCDHA10;PCDHA9 \\
\hline $187269 \mathrm{chr} 5$ & 788,646 & 825,338 & deletion & ZDHHC11 \\
\hline $187269 \mathrm{chr} 6$ & $30,994,010$ & $30,994,787$ & deletion & MUC22 \\
\hline 187269 chr6 & $78,972,930$ & $79,035,739$ & deletion & - \\
\hline 187269 chr8 & $143,806,896$ & $143,809,811$ & deletion & THEM6 \\
\hline $187269 \mathrm{chrX}$ & $120,819,108$ & $120,920,493$ & deletion & - \\
\hline $187269 \mathrm{chrX}$ & $149,169,688$ & $149,233,585$ & deletion & - \\
\hline $187270 \mathrm{chr} 1$ & $161,489,467$ & $161,563,386$ & duplication & FCGR3A;HSPA6;FCGR2C \\
\hline $187270 \mathrm{chr} 1$ & $72,754,314$ & $72,763,324$ & deletion & - \\
\hline $187270 \mathrm{chr} 1$ & $92,240,685$ & $92,248,312$ & deletion & - \\
\hline 187270 chr10 & $38,792,512$ & $38,903,858$ & deletion & - \\
\hline 187270 chr10 & $56,449,714$ & $56,468,486$ & duplication & - \\
\hline 187270 chr11 & $55,370,325$ & $55,424,207$ & deletion & OR4P4;OR4C11;OR4S2 \\
\hline 187270 chr12 & $129,230,513$ & $129,233,013$ & deletion & - \\
\hline 187270 chr12 & $30,237,351$ & $30,243,473$ & deletion & - \\
\hline 187270 chr12 & $6,241,446$ & $6,247,923$ & deletion & - \\
\hline 187270 chr13 & $58,589,893$ & $58,599,174$ & deletion & - \\
\hline 187270 chr13 & $64,225,427$ & $64,236,288$ & deletion & - \\
\hline 187270 chr13 & $83,786,517$ & $83,791,615$ & deletion & - \\
\hline 187270 chr14 & $41,610,224$ & $41,661,685$ & deletion & - \\
\hline 187270 chr16 & $55,798,095$ & $55,819,443$ & deletion & - \\
\hline 187270 chr18 & $67,208,806$ & $67,217,271$ & deletion & - \\
\hline $187270 \mathrm{chr} 2$ & $184,085,551$ & $184,088,256$ & deletion & - \\
\hline $187270 \mathrm{chr} 2$ & $208,351,315$ & $208,357,838$ & deletion & - \\
\hline 187270 chr2 & $38,956,947$ & $38,971,623$ & duplication & GALM \\
\hline $187270 \mathrm{chr} 2$ & $48,851,541$ & $48,856,770$ & deletion & - \\
\hline $187270 \mathrm{chr} 2$ & $73,859,139$ & $73,918,312$ & deletion & NAT8 \\
\hline 187270 chr21 & $20,057,919$ & $20,081,051$ & duplication & - \\
\hline 187270 chr21 & $23,655,900$ & $23,665,470$ & deletion & - \\
\hline 187270 chr21 & $44,822,871$ & $44,837,829$ & duplication & LOC102724428;SIK1 \\
\hline 187270 chr22 & $25,663,994$ & $25,919,492$ & duplication & LRP5L \\
\hline 187270 chr22 & $42,902,278$ & $42,950,033$ & duplication & SERHL2;RRP7A \\
\hline $187270 \mathrm{chr} 3$ & $100,340,145$ & $100,441,716$ & duplication & ADGRG7;TFG \\
\hline $187270 \mathrm{chr} 3$ & $128,382,258$ & $128,412,024$ & duplication & - \\
\hline $187270 \mathrm{chr} 3$ & $129,769,827$ & $129,806,304$ & deletion & ALG1L2 \\
\hline $187270 \mathrm{chr} 3$ & $131,711,896$ & $131,712,898$ & deletion & - \\
\hline 187270 chr3 & $2,572,993$ & $2,574,706$ & duplication & - \\
\hline $187270 \mathrm{chr} 4$ & $190,194,200$ & $190,199,993$ & deletion & - \\
\hline $187270 \mathrm{chr} 4$ & $69,374,369$ & $69,489,473$ & duplication & UGT2B17 \\
\hline $187270 \mathrm{chr} 4$ & $70,137,897$ & $70,229,260$ & deletion & UGT2B28 \\
\hline 187270 chr5 & $8,436,209$ & $8,445,657$ & deletion & - \\
\hline 187270 chr5 & $8,703,085$ & $8,748,627$ & deletion & - \\
\hline 187270 chr8 & $24,972,808$ & $24,990,418$ & duplication & - \\
\hline $187270 \mathrm{chr} 8$ & $39,232,581$ & $39,385,979$ & duplication & - \\
\hline 187270 chr9 & $6,701,130$ & $6,707,794$ & deletion & - \\
\hline 187271 chr1 & $112,693,423$ & $112,704,581$ & deletion & - \\
\hline 187271 chr1 & $161,559,327$ & $161,619,741$ & duplication & 2C;FCGR3B \\
\hline
\end{tabular}




\begin{tabular}{|c|c|c|c|c|}
\hline 187271 chr1 & $25,601,221$ & $25,655,538$ & deletion & RHD \\
\hline 187271 chr10 & $20,850,624$ & $20,857,365$ & deletion & - \\
\hline 187271 chr10 & $39,058,671$ & $39,154,535$ & duplication & - \\
\hline 187271 chr10 & $47,141,927$ & $47,703,946$ & duplication & FAM25C;ANXA8;ANTXRL;FAM25G;AGAP9 \\
\hline 187271 chr10 & $56,449,714$ & $56,468,486$ & duplication & - \\
\hline 187271 chr11 & $107,653,650$ & $107,671,155$ & duplication & SLC35F2 \\
\hline 187271 chr11 & $125,076,981$ & $125,084,742$ & deletion & - \\
\hline 187271 chr11 & $90,193,796$ & $90,196,843$ & deletion & - \\
\hline 187271 chr12 & $11,220,827$ & $11,244,117$ & deletion & TAS2R43 \\
\hline 187271 chr12 & $2,245,636$ & $2,259,042$ & deletion & - \\
\hline 187271 chr13 & $32,532,761$ & $32,537,768$ & deletion & - \\
\hline 187271 chr14 & $106,891,524$ & $106,931,129$ & deletion & - \\
\hline 187271 chr14 & $20,197,311$ & $20,422,799$ & duplication & OR4K5;OR4K1;OR4K2;OR4Q3;OR4N2;OR4M1 \\
\hline 187271 chr16 & $34,471,298$ & $34,756,258$ & duplication & - \\
\hline 187271 chr16 & $55,798,095$ & $55,819,443$ & deletion & - \\
\hline 187271 chr17 & $39,212,718$ & $39,227,984$ & deletion & KRTAP2-3;KRTAP2-4 \\
\hline 187271 chr18 & $3,304,629$ & $3,312,834$ & duplication & - \\
\hline 187271 chr18 & $63,907,596$ & $63,910,708$ & deletion & - \\
\hline 187271 chr19 & $43,703,965$ & $43,766,659$ & deletion & PSG4;PSG9 \\
\hline 187271 chr19 & $53,518,747$ & $53,548,950$ & duplication & ERVV-1 \\
\hline 187271 chr19 & $54,728,767$ & $54,743,217$ & duplication & LILRA6 \\
\hline $187271 \mathrm{chr} 2$ & $140,711,341$ & $140,780,343$ & deletion & - \\
\hline $187271 \mathrm{chr} 2$ & $17,220,064$ & $17,239,502$ & deletion & - \\
\hline 187271 chr2 & $48,851,541$ & $48,856,770$ & deletion & - \\
\hline 187271 chr22 & $39,359,885$ & $39,385,663$ & deletion & АРОВЕСЗВ \\
\hline 187271 chr3 & $195,457,853$ & $195,468,690$ & duplication & MUC20 \\
\hline $187271 \mathrm{chr} 3$ & $6,651,929$ & $6,654,060$ & deletion & - \\
\hline $187271 \mathrm{chr} 4$ & $156,959,568$ & $156,973,205$ & deletion & - \\
\hline $187271 \mathrm{chr} 4$ & $171,269,504$ & $171,273,360$ & deletion & - \\
\hline 187271 chr6 & $161,815,583$ & $161,859,655$ & deletion & - \\
\hline 187271 chr6 & $29,855,945$ & $29,899,189$ & deletion & - \\
\hline 187271 chr6 & $5,199,979$ & $5,412,543$ & duplication & FARS2;LYRM4 \\
\hline 187271 chr6 & $78,972,930$ & $79,035,739$ & deletion & - \\
\hline $187271 \mathrm{chr} 7$ & $142,479,577$ & $142,484,042$ & deletion & - \\
\hline $187271 \mathrm{chr} 7$ & $75,664,539$ & $75,667,743$ & deletion & - \\
\hline $187271 \mathrm{chr} 8$ & $15,778,007$ & $15,786,889$ & deletion & - \\
\hline 187271 chr8 & $39,279,418$ & $39,382,903$ & deletion & - \\
\hline $187271 \mathrm{chr} 8$ & $51,029,371$ & $51,038,149$ & deletion & - \\
\hline 187271 chr8 & $5,595,511$ & $5,605,706$ & deletion & - \\
\hline 187273 chr1 & $185,098,927$ & $185,128,607$ & duplication & TRMT1L \\
\hline 187273 chr1 & $62,113,597$ & $62,119,136$ & deletion & - \\
\hline 187273 chr10 & $132,635,459$ & $132,638,422$ & deletion & - \\
\hline 187273 chr10 & $71,282,981$ & $71,289,190$ & deletion & - \\
\hline 187273 chr11 & $107,653,650$ & $107,671,037$ & duplication & SLC35F2 \\
\hline 187273 chr11 & $134,602,333$ & $134,607,484$ & deletion & - \\
\hline 187273 chr11 & $55,367,889$ & $55,427,700$ & deletion & OR4P4;OR4C11;OR4S2 \\
\hline 187273 chr11 & $86,304,402$ & $86,306,401$ & deletion & - \\
\hline
\end{tabular}




\begin{tabular}{|c|c|c|c|c|}
\hline 187273 chr12 & $43,021,431$ & $43,027,453$ & deletion & - \\
\hline 187273 chr13 & $34,134,809$ & $34,143,545$ & deletion & - \\
\hline 187273 chr13 & $38,076,308$ & $38,085,537$ & deletion & - \\
\hline 187273 chr14 & $20,197,311$ & $20,424,184$ & duplication & OR4K5;OR4K1;OR4K2;OR4Q3;OR4N2;OR4M1 \\
\hline 187273 chr14 & $24,429,849$ & $24,484,060$ & duplication & DHRS4;DHRS4L2 \\
\hline 187273 chr14 & $41,610,224$ & $41,661,685$ & deletion & - \\
\hline 187273 chr16 & $19,945,540$ & $19,962,636$ & deletion & - \\
\hline 187273 chr16 & $4,135,350$ & $4,140,459$ & deletion & - \\
\hline 187273 chr16 & $78,372,894$ & $78,383,509$ & deletion & - \\
\hline 187273 chr17 & $44,166,604$ & $44,249,838$ & duplication & KANSL1 \\
\hline 187273 chr17 & $6,300,290$ & $6,312,899$ & duplication & - \\
\hline 187273 chr19 & $35,852,103$ & $35,861,695$ & deletion & - \\
\hline 187273 chr19 & $54,728,767$ & $54,743,217$ & duplication & LILRA6 \\
\hline 187273 chr2 & $129,638,609$ & $129,645,321$ & deletion & - \\
\hline 187273 chr2 & $184,085,551$ & $184,088,256$ & deletion & - \\
\hline 187273 chr2 & $19,386,794$ & $19,414,509$ & deletion & - \\
\hline 187273 chr2 & $34,697,895$ & $34,726,904$ & duplication & - \\
\hline 187273 chr2 & $38,301,735$ & $38,305,451$ & deletion & CYP1B1 \\
\hline 187273 chr2 & $52,759,890$ & $52,784,952$ & deletion & - \\
\hline 187273 chr20 & $29,454,201$ & $29,500,566$ & deletion & - \\
\hline 187273 chr20 & $52,647,631$ & $52,656,535$ & deletion & - \\
\hline 187273 chr21 & $47,552,130$ & $47,552,449$ & deletion & COL6A2 \\
\hline 187273 chr3 & $195,433,728$ & $195,468,690$ & duplication & MUC20 \\
\hline 187273 chr3 & $65,189,366$ & $65,214,685$ & deletion & - \\
\hline 187273 chr3 & $75,428,675$ & $75,639,184$ & deletion & \\
\hline $187273 \mathrm{chr} 4$ & $16,920,838$ & $16,929,643$ & deletion & - \\
\hline 187273 chr4 & $190,194,200$ & $190,200,463$ & deletion & - \\
\hline 187273 chr4 & $34,785,955$ & $34,818,502$ & deletion & - \\
\hline $187273 \mathrm{chr} 4$ & $92,076,288$ & $92,079,569$ & deletion & - \\
\hline 187273 chr5 & $117,388,410$ & $117,393,058$ & deletion & - \\
\hline 187273 chr5 & 701,026 & 807,499 & duplication & ZDHHC11 \\
\hline 187273 chr6 & $132,709,027$ & $132,711,200$ & deletion & - \\
\hline 187273 chr6 & $139,603,393$ & $139,606,731$ & deletion & - \\
\hline 187273 chr6 & $169,271,809$ & $169,273,635$ & deletion & - \\
\hline 187273 chr6 & $30,994,010$ & $30,994,787$ & deletion & MUC22 \\
\hline 187273 chr6 & $31,281,682$ & $31,286,577$ & deletion & - \\
\hline 187273 chr6 & $77,439,769$ & $77,451,301$ & deletion & - \\
\hline 187273 chr7 & $11,920,873$ & $11,922,312$ & deletion & - \\
\hline 187273 chr7 & $142,476,094$ & $142,484,042$ & deletion & - \\
\hline 187273 chr7 & $38,291,851$ & $38,305,404$ & duplication & TARP \\
\hline 187273 chr7 & $97,396,709$ & $97,401,677$ & deletion & - \\
\hline 187273 chr8 & $39,232,581$ & $39,382,903$ & deletion & - \\
\hline 187273 chr8 & $6,827,299$ & $6,829,137$ & deletion & - \\
\hline 187273 chr9 & $23,363,526$ & $23,376,817$ & deletion & - \\
\hline 187273 chr9 & $24,502,737$ & $24,520,633$ & deletion & - \\
\hline $190680 \mathrm{chr} 1$ & $143,481,684$ & $143,504,049$ & deletion & - \\
\hline $190680 \mathrm{chr} 1$ & $16,368,977$ & $16,386,395$ & duplication & FAM131C;CLCNKB \\
\hline
\end{tabular}




\begin{tabular}{|c|c|c|c|c|}
\hline $190680 \mathrm{chr} 10$ & $39,119,741$ & $39,154,535$ & duplication & - \\
\hline $190680 \mathrm{chr} 10$ & $42,653,286$ & $42,669,575$ & deletion & - \\
\hline $190680 \mathrm{chr} 11$ & $133,453,021$ & $133,458,545$ & deletion & - \\
\hline 190680 chr11 & $7,814,299$ & $7,827,879$ & deletion & OR5P2 \\
\hline $190680 \mathrm{chr} 12$ & $31,907,259$ & $31,909,635$ & deletion & - \\
\hline $190680 \mathrm{chr} 12$ & $33,300,995$ & $33,305,855$ & duplication & - \\
\hline 190680 chr12 & $99,994,315$ & $100,008,773$ & deletion & - \\
\hline 190680 chr14 & $41,610,224$ & $41,661,685$ & deletion & - \\
\hline 190680 chr15 & $20,120,214$ & $20,144,522$ & deletion & - \\
\hline $190680 \mathrm{chr} 15$ & $20,878,391$ & $21,200,107$ & deletion & РОTEB2;POTEB;POTEB3 \\
\hline 190680 chr15 & $22,512,203$ & $22,516,945$ & deletion & - \\
\hline $190680 \mathrm{chr} 15$ & $92,577,334$ & $92,578,836$ & deletion & - \\
\hline $190680 \mathrm{chr} 16$ & $19,945,540$ & $19,962,636$ & deletion & - \\
\hline $190680 \mathrm{chr} 16$ & $46,471,024$ & $46,492,062$ & deletion & - \\
\hline $190680 \mathrm{chr} 17$ & $25,268,096$ & $25,276,889$ & deletion & - \\
\hline 190680 chr17 & $34,438,753$ & $34,480,718$ & duplication & - \\
\hline $190680 \mathrm{chr} 18$ & 13,697 & 60,360 & deletion & - \\
\hline $190680 \mathrm{chr} 18$ & $68,942,222$ & $68,959,727$ & duplication & - \\
\hline 190680 chr19 & $51,256,120$ & $51,264,336$ & duplication & - \\
\hline 190680 chr2 & $132,781,784$ & $132,834,032$ & deletion & - \\
\hline $190680 \mathrm{chr} 2$ & $223,865,393$ & $223,869,820$ & deletion & - \\
\hline $190680 \mathrm{chr} 2$ & $242,854,089$ & $243,034,519$ & deletion & - \\
\hline $190680 \mathrm{chr} 2$ & $34,697,895$ & $34,726,904$ & deletion & . \\
\hline 190680 chr2 & $52,243,162$ & $52,248,140$ & deletion & 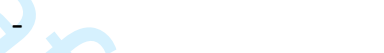 \\
\hline 190680 chr20 & $25,890,697$ & $25,910,523$ & deletion & \\
\hline 190680 chr20 & $29,450,698$ & $29,483,606$ & deletion & - \\
\hline 190680 chr20 & $29,566,903$ & $29,585,801$ & deletion & - \\
\hline 190680 chr21 & $44,396,144$ & $44,422,296$ & deletion & - \\
\hline 190680 chr22 & $18,626,900$ & $18,629,320$ & deletion & - \\
\hline 190680 chr22 & $37,738,621$ & $37,749,946$ & deletion & - \\
\hline $190680 \mathrm{chr} 4$ & $109,962,007$ & $109,964,916$ & deletion & - \\
\hline $190680 \mathrm{chr} 4$ & $122,285,263$ & $122,289,863$ & deletion & - \\
\hline $190680 \mathrm{chr} 4$ & $190,196,067$ & $190,200,455$ & deletion & - \\
\hline $190680 \mathrm{chr} 4$ & $34,785,955$ & $34,818,502$ & duplication & - \\
\hline $190680 \mathrm{chr} 4$ & $69,374,369$ & $69,489,473$ & deletion & UGT2B17 \\
\hline 190680 chr5 & $140,225,908$ & $140,238,311$ & deletion & PCDHA10;PCDHA9 \\
\hline 190680 chr5 & 682,606 & 776,691 & duplication & - \\
\hline 190680 chr5 & $8,591,633$ & $8,597,846$ & deletion & - \\
\hline 190680 chr6 & $67,011,900$ & $67,048,885$ & deletion & - \\
\hline 190680 chr6 & $78,972,930$ & $79,035,739$ & deletion & - \\
\hline 190680 chr7 & $156,387,296$ & $156,394,307$ & deletion & - \\
\hline 190680 chr7 & $61,761,653$ & $61,774,925$ & deletion & - \\
\hline 190680 chr7 & $61,796,377$ & $61,820,891$ & deletion & - \\
\hline 190680 chr7 & $62,089,067$ & $62,090,042$ & deletion & - \\
\hline 190680 chr7 & $75,357,240$ & $75,370,368$ & duplication & HIP1 \\
\hline $190680 \mathrm{chr} 8$ & $24,972,808$ & $24,990,418$ & duplication & - \\
\hline 190680 chr8 & $39,232,581$ & $39,385,979$ & duplication & - \\
\hline
\end{tabular}




\begin{tabular}{|c|c|c|c|c|}
\hline $190680 \mathrm{chr} 8$ & $51,031,221$ & $51,038,149$ & deletion & - \\
\hline 190680 chr9 & $24,502,737$ & $24,518,795$ & deletion & - \\
\hline 190680 chr9 & $44,057,312$ & $44,100,710$ & deletion & - \\
\hline 190680 chr9 & $5,304,710$ & $5,337,760$ & deletion & RLN1 \\
\hline $190680 \mathrm{chrX}$ & $33,895,605$ & $34,024,193$ & deletion & - \\
\hline 190681 chr1 & $143,134,057$ & $143,284,605$ & deletion & - \\
\hline 190681 chr1 & $143,481,684$ & $143,503,159$ & deletion & - \\
\hline 190681 chr1 & $143,513,594$ & $143,531,627$ & deletion & - \\
\hline 190681 chr11 & $24,446,609$ & $24,451,830$ & deletion & - \\
\hline 190681 chr12 & $11,220,827$ & $11,244,117$ & deletion & TAS2R43 \\
\hline 190681 chr12 & $118,944,329$ & $118,949,436$ & deletion & - \\
\hline 190681 chr12 & $32,052,422$ & $32,058,066$ & deletion & - \\
\hline 190681 chr13 & $38,076,308$ & $38,085,537$ & deletion & - \\
\hline 190681 chr15 & $20,120,214$ & $20,144,522$ & deletion & - \\
\hline 190681 chr15 & $20,878,391$ & $21,157,642$ & deletion & РОТЕВ2;РОТЕВ;РОТЕВЗ \\
\hline 190681 chr15 & $22,342,453$ & $22,516,945$ & deletion & OR4M2;OR4N4 \\
\hline 190681 chr15 & $24,624,598$ & $24,742,652$ & duplication & - \\
\hline 190681 chr16 & $34,471,298$ & $34,756,258$ & duplication & - \\
\hline 190681 chr16 & $46,471,024$ & $46,492,062$ & deletion & - \\
\hline 190681 chr17 & $25,268,096$ & $25,276,983$ & deletion & - \\
\hline 190681 chr17 & $34,438,753$ & $34,480,718$ & duplication & - \\
\hline 190681 chr19 & $20,601,006$ & $20,717,536$ & deletion & - \\
\hline 190681 chr19 & $30,139,462$ & $30,149,581$ & duplication & - \\
\hline 190681 chr19 & $54,723,029$ & $54,743,217$ & duplication & LILRA6;LILRB3 \\
\hline 190681 chr19 & $5,832,209$ & $5,842,169$ & duplication & FUT6 \\
\hline 190681 chr2 & $132,781,784$ & $132,834,032$ & deletion & - \\
\hline 190681 chr2 & $14,704,369$ & $14,709,611$ & deletion & - \\
\hline 190681 chr2 & $213,187,034$ & $213,191,389$ & deletion & - \\
\hline 190681 chr2 & $38,955,129$ & $38,971,623$ & duplication & GALM \\
\hline 190681 chr2 & $41,239,457$ & $41,248,468$ & deletion & - \\
\hline 190681 chr20 & $25,890,697$ & $25,896,760$ & deletion & - \\
\hline 190681 chr20 & $29,566,903$ & $29,585,801$ & deletion & - \\
\hline 190681 chr20 & $44,350,423$ & $44,380,052$ & deletion & SPINT4 \\
\hline 190681 chr21 & $11,055,527$ & $11,063,273$ & deletion & BAGE2;BAGE3 \\
\hline 190681 chr22 & $37,745,101$ & $37,749,946$ & deletion & - \\
\hline 190681 chr22 & $39,359,885$ & $39,385,663$ & deletion & AРОВЕСЗВ \\
\hline 190681 chr3 & $37,979,882$ & $37,986,734$ & deletion & - \\
\hline 190681 chr3 & $6,651,929$ & $6,654,060$ & deletion & - \\
\hline 190681 chr3 & $73,388,366$ & $73,424,330$ & deletion & - \\
\hline $190681 \mathrm{chr} 4$ & $190,194,200$ & $190,200,455$ & deletion & - \\
\hline $190681 \mathrm{chr} 4$ & $64,697,704$ & $64,709,753$ & duplication & - \\
\hline 190681 chr5 & $111,935,341$ & $111,944,746$ & deletion & - \\
\hline 190681 chr5 & $114,013,380$ & $114,015,705$ & deletion & - \\
\hline 190681 chr5 & $154,912,236$ & $154,924,820$ & duplication & - \\
\hline 190681 chr5 & $155,477,866$ & $155,488,917$ & deletion & - \\
\hline 190681 chr5 & $17,345,282$ & $17,356,536$ & deletion & - \\
\hline 190681 chr6 & $132,709,027$ & $132,711,200$ & deletion & - \\
\hline
\end{tabular}




\begin{tabular}{|c|c|c|c|c|}
\hline 190681 chr6 & $29,921,001$ & $29,921,927$ & deletion & - \\
\hline 190681 chr6 & $40,365,601$ & $40,368,860$ & deletion & - \\
\hline 190681 chr6 & $69,231,883$ & $69,241,954$ & duplication & - \\
\hline 190681 chr6 & $8,926,695$ & $8,932,296$ & deletion & - \\
\hline 190681 chr7 & 151,829 & 159,207 & deletion & - \\
\hline 190681 chr7 & $38,291,851$ & $38,305,404$ & duplication & TARP \\
\hline 190681 chr7 & 41,421 & 67,820 & duplication & - \\
\hline 190681 chr7 & $57,991,743$ & $58,010,754$ & deletion & - \\
\hline 190681 chr7 & $61,761,653$ & $61,774,925$ & deletion & - \\
\hline 190681 chr7 & $61,796,377$ & $61,820,891$ & deletion & - \\
\hline 190681 chr8 & $115,634,278$ & $115,642,459$ & deletion & - \\
\hline 190681 chr8 & $6,129,892$ & $6,303,409$ & duplication & $\mathrm{MCPH} 1$ \\
\hline 190681 chr9 & $44,057,312$ & $44,100,710$ & deletion & - \\
\hline 190681 chr9 & $78,004,378$ & $78,011,616$ & deletion & - \\
\hline 190682 chr1 & $111,380,720$ & $111,387,723$ & deletion & - \\
\hline 190682 chr1 & $112,693,423$ & $112,704,581$ & deletion & - \\
\hline 190682 chr1 & $143,513,594$ & $143,531,627$ & deletion & - \\
\hline 190682 chr11 & $24,446,609$ & $24,451,830$ & deletion & - \\
\hline 190682 chr11 & $81,501,218$ & $81,517,261$ & deletion & - \\
\hline 190682 chr12 & $12,532,984$ & $12,541,869$ & deletion & - \\
\hline 190682 chr12 & $6,255,862$ & $6,259,552$ & deletion & - \\
\hline 190682 chr15 & $20,120,214$ & $20,144,522$ & deletion & - \\
\hline 190682 chr15 & $22,383,292$ & $22,447,130$ & deletion & N4 \\
\hline 190682 chr15 & $94,136,730$ & $94,166,312$ & deletion & 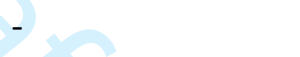 \\
\hline 190682 chr16 & $22,552,780$ & $22,693,375$ & deletion & \\
\hline 190682 chr16 & $46,471,024$ & $46,492,062$ & deletion & - \\
\hline 190682 chr17 & $25,268,096$ & $25,278,249$ & deletion & - \\
\hline 190682 chr17 & $34,438,753$ & $34,480,718$ & duplication & - \\
\hline 190682 chr17 & $70,815,357$ & $70,820,207$ & deletion & - \\
\hline 190682 chr19 & $20,601,006$ & $20,717,536$ & deletion & - \\
\hline 190682 chr19 & $30,139,462$ & $30,149,581$ & duplication & - \\
\hline 190682 chr19 & $35,661,787$ & $35,665,515$ & deletion & - \\
\hline 190682 chr19 & $54,730,202$ & $54,743,217$ & duplication & LILRA6 \\
\hline 190682 chr19 & $5,832,209$ & $5,842,169$ & duplication & FUT6 \\
\hline 190682 chr2 & $132,781,784$ & $132,869,329$ & deletion & - \\
\hline 190682 chr2 & $223,865,393$ & $223,869,820$ & deletion & - \\
\hline 190682 chr20 & $25,890,697$ & $25,896,760$ & deletion & - \\
\hline 190682 chr20 & $29,627,973$ & $29,629,342$ & deletion & - \\
\hline 190682 chr20 & $44,350,423$ & $44,380,052$ & deletion & SPINT4 \\
\hline 190682 chr21 & $11,055,462$ & $11,063,273$ & deletion & BAGE2;BAGE3 \\
\hline 190682 chr22 & $18,626,900$ & $18,629,320$ & deletion & - \\
\hline 190682 chr22 & $23,050,265$ & $23,079,635$ & deletion & - \\
\hline 190682 chr22 & $37,745,101$ & $37,749,946$ & deletion & - \\
\hline 190682 chr22 & $39,359,885$ & $39,385,663$ & deletion & АРОВЕСЗВ \\
\hline 190682 chr3 & $13,681,185$ & $13,682,137$ & deletion & - \\
\hline 190682 chr3 & $73,388,366$ & $73,424,330$ & deletion & - \\
\hline 190682 chr5 & $140,225,908$ & $140,238,311$ & deletion & PCDHA10;PCDHA9 \\
\hline
\end{tabular}




\begin{tabular}{|c|c|c|c|c|}
\hline 190682 chr5 & $52,670,932$ & $52,677,265$ & deletion & - \\
\hline 190682 chr5 & $7,178,644$ & $7,191,074$ & deletion & - \\
\hline $190682 \mathrm{chr} 5$ & 730,567 & 825,338 & deletion & ZDHHC11 \\
\hline 190682 chr6 & $132,709,027$ & $132,711,200$ & deletion & - \\
\hline 190682 chr6 & $29,857,565$ & $29,899,389$ & deletion & - \\
\hline $190682 \mathrm{chr} 7$ & $141,766,850$ & $141,793,419$ & deletion & - \\
\hline $190682 \mathrm{chr} 7$ & 41,421 & 68,920 & duplication & - \\
\hline 190682 chr7 & $52,730,700$ & $52,744,420$ & duplication & - \\
\hline 190682 chr7 & $57,991,743$ & $58,010,754$ & deletion & - \\
\hline 190682 chr7 & $61,761,653$ & $61,774,925$ & deletion & - \\
\hline $190682 \mathrm{chr} 7$ & $61,794,652$ & $61,820,891$ & deletion & - \\
\hline $190682 \mathrm{chr} 8$ & $115,634,278$ & $115,642,459$ & deletion & - \\
\hline 190682 chr8 & $39,232,581$ & $39,385,979$ & duplication & - \\
\hline $190682 \mathrm{chr} 8$ & $6,130,415$ & $6,162,349$ & duplication & - \\
\hline 190682 chr8 & $6,171,324$ & $6,180,270$ & duplication & - \\
\hline 190682 chr8 & $6,189,821$ & $6,303,409$ & duplication & МСРH1 \\
\hline 190682 chr9 & $104,715,329$ & $104,722,688$ & deletion & - \\
\hline 190682 chr9 & $23,363,526$ & $23,376,817$ & duplication & - \\
\hline $190682 \mathrm{chrX}$ & $34,045,676$ & $34,069,410$ & deletion & - \\
\hline $190683 \mathrm{chr} 1$ & $111,380,720$ & $111,387,723$ & deletion & - \\
\hline 190683 chr1 & $1,651,355$ & $1,668,298$ & deletion & SLC35E2;CDK11A;CDK11B \\
\hline $190683 \mathrm{chr} 1$ & $245,636,915$ & $245,647,809$ & deletion & - \\
\hline 190683 chr10 & $27,226,851$ & $27,227,458$ & deletion & - \\
\hline 190683 chr11 & $42,968,875$ & $42,973,253$ & duplication & - \\
\hline 190683 chr12 & $116,983,786$ & $117,040,415$ & deletion & MAP1LC3B2 \\
\hline 190683 chr12 & $2,245,636$ & $2,254,219$ & deletion & - \\
\hline 190683 chr12 & 574,811 & 616,214 & deletion & - \\
\hline $190683 \mathrm{chr} 14$ & $22,811,629$ & $22,828,889$ & duplication & - \\
\hline 190683 chr15 & $20,120,214$ & $20,144,522$ & deletion & - \\
\hline 190683 chr15 & $20,878,391$ & $21,200,107$ & deletion & РОTEB2;РОTEB;РОTEB3 \\
\hline 190683 chr15 & $22,652,330$ & $23,228,712$ & deletion & CYFIP1;GOLGA6L22;NIPA1;GOLGA6L1;NIPA2; 1 \\
\hline 190683 chr15 & $92,577,334$ & $92,578,836$ & deletion & - \\
\hline 190683 chr16 & $46,416,238$ & $46,455,400$ & deletion & - \\
\hline 190683 chr16 & $78,372,894$ & $78,383,509$ & deletion & - \\
\hline 190683 chr17 & $25,268,096$ & $25,307,180$ & deletion & - \\
\hline $190683 \mathrm{chr} 17$ & $44,166,604$ & $44,347,946$ & duplication & KANSL1 \\
\hline 190683 chr17 & $70,815,357$ & $70,820,207$ & deletion & - \\
\hline 190683 chr19 & $53,518,747$ & $53,552,296$ & duplication & ERVV-1 \\
\hline $190683 \mathrm{chr} 2$ & $132,781,784$ & $132,797,583$ & deletion & - \\
\hline $190683 \mathrm{chr} 2$ & $184,795,725$ & $184,803,456$ & deletion & - \\
\hline 190683 chr20 & $25,890,697$ & $25,914,745$ & deletion & - \\
\hline 190683 chr20 & $29,450,698$ & $29,483,606$ & deletion & - \\
\hline 190683 chr20 & $29,566,903$ & $29,585,801$ & deletion & - \\
\hline 190683 chr20 & $29,619,497$ & $29,629,342$ & deletion & - \\
\hline 190683 chr20 & $49,749,265$ & $49,757,672$ & deletion & - \\
\hline 190683 chr20 & $52,288,724$ & $52,290,223$ & deletion & - \\
\hline 190683 chr21 & $10,791,037$ & $10,858,651$ & duplication & - \\
\hline
\end{tabular}




\begin{tabular}{|c|c|c|c|c|}
\hline $190683 \mathrm{chr} 21$ & $11,055,527$ & $11,063,273$ & deletion & 2;BAGE3 \\
\hline $190683 \mathrm{chr} 4$ & $179,725,423$ & $179,729,425$ & deletion & - \\
\hline $190683 \mathrm{chr} 4$ & $92,280,583$ & $92,284,935$ & deletion & - \\
\hline $190683 \mathrm{chr} 5$ & $140,225,908$ & $140,238,311$ & deletion & PCDHA10;PCDHA9 \\
\hline $190683 \mathrm{chr} 5$ & $155,477,866$ & $155,488,917$ & deletion & - \\
\hline 190683 chr5 & $32,120,414$ & $32,164,826$ & duplication & GOLPH3 \\
\hline 190683 chr5 & $8,258,458$ & $8,260,630$ & deletion & - \\
\hline 190683 chr6 & $32,458,168$ & $32,543,540$ & deletion & HLA-DRB5 \\
\hline $190683 \mathrm{chr} 6$ & $78,972,930$ & $79,035,739$ & deletion & - \\
\hline $190683 \mathrm{chr} 7$ & $142,825,750$ & $142,890,868$ & deletion & PIP;TAS2R39 \\
\hline 190683 chr7 & $61,760,702$ & $61,774,925$ & deletion & - \\
\hline 190683 chr8 & $24,972,808$ & $24,990,418$ & deletion & - \\
\hline 190683 chr9 & $10,660,982$ & $10,688,528$ & deletion & - \\
\hline 190683 chr9 & $135,949,324$ & $135,956,640$ & deletion & - \\
\hline 190683 chr9 & $23,363,526$ & $23,376,817$ & deletion & - \\
\hline $190684 \mathrm{chr} 1$ & $112,693,423$ & $112,704,581$ & deletion & - \\
\hline $190684 \mathrm{chr} 1$ & $143,134,057$ & $143,284,605$ & deletion & - \\
\hline $190684 \mathrm{chr} 1$ & $143,481,684$ & $143,531,627$ & deletion & - \\
\hline 190684 chr10 & $42,626,276$ & $42,669,575$ & deletion & - \\
\hline 190684 chr11 & $81,501,218$ & $81,517,261$ & deletion & - \\
\hline 190684 chr12 & $12,532,984$ & $12,541,869$ & deletion & - \\
\hline 190684 chr12 & $27,648,411$ & $27,654,998$ & deletion & SMCO2 \\
\hline 190684 chr12 & $32,052,422$ & $32,058,066$ & deletion & . \\
\hline 190684 chr12 & $32,331,195$ & $32,334,813$ & deletion & \\
\hline 190684 chr13 & $38,076,308$ & $38,085,537$ & deletion & \\
\hline 190684 chr15 & $20,120,214$ & $20,144,522$ & deletion & - \\
\hline 190684 chr15 & $25,420,978$ & $25,430,654$ & deletion & - \\
\hline 190684 chr17 & $25,268,096$ & $25,286,503$ & deletion & - \\
\hline 190684 chr17 & $34,438,753$ & $34,480,718$ & duplication & - \\
\hline 190684 chr19 & $20,601,006$ & $20,717,536$ & deletion & - \\
\hline 190684 chr19 & $30,139,462$ & $30,149,581$ & duplication & - \\
\hline 190684 chr19 & $35,661,787$ & $35,665,515$ & deletion & - \\
\hline $190684 \mathrm{chr} 2$ & $132,781,784$ & $132,797,583$ & deletion & - \\
\hline $190684 \mathrm{chr} 2$ & $184,795,725$ & $184,803,456$ & deletion & - \\
\hline 190684 chr2 & $195,364,534$ & $195,366,958$ & deletion & - \\
\hline 190684 chr2 & $213,187,034$ & $213,191,389$ & deletion & - \\
\hline $190684 \mathrm{chr} 2$ & $38,955,129$ & $38,971,623$ & duplication & GALM \\
\hline $190684 \mathrm{chr} 2$ & $41,239,457$ & $41,248,468$ & deletion & - \\
\hline 190684 chr20 & $25,890,697$ & $25,896,760$ & deletion & - \\
\hline 190684 chr21 & $11,054,573$ & $11,063,273$ & deletion & BAGE2;BAGE3 \\
\hline 190684 chr22 & $37,745,101$ & $37,749,946$ & deletion & - \\
\hline $190684 \mathrm{chr} 3$ & $13,681,185$ & $13,682,137$ & deletion & - \\
\hline 190684 chr3 & $75,735,726$ & $75,743,254$ & deletion & - \\
\hline $190684 \mathrm{chr} 4$ & $190,196,246$ & $190,200,455$ & deletion & - \\
\hline $190684 \mathrm{chr} 4$ & $87,446,936$ & $87,506,685$ & deletion & - \\
\hline 190684 chr5 & $111,935,341$ & $111,944,746$ & deletion & - \\
\hline 190684 chr5 & $114,013,380$ & $114,015,705$ & deletion & - \\
\hline
\end{tabular}




\begin{tabular}{|c|c|c|c|c|}
\hline 190684 chr5 & $154,912,236$ & $154,924,820$ & duplication & - \\
\hline $190684 \mathrm{chr} 5$ & $155,477,866$ & $155,488,917$ & deletion & - \\
\hline 190684 chr5 & $7,178,644$ & $7,191,074$ & deletion & - \\
\hline 190684 chr5 & 749,946 & 836,608 & deletion & ZDHHC11 \\
\hline 190684 chr6 & $40,365,601$ & $40,368,860$ & deletion & - \\
\hline 190684 chr6 & $55,827,939$ & $55,846,527$ & deletion & - \\
\hline 190684 chr6 & $69,231,883$ & $69,241,954$ & duplication & - \\
\hline 190684 chr6 & $8,926,695$ & $8,932,296$ & deletion & - \\
\hline $190684 \mathrm{chr} 7$ & $20,750,774$ & $20,753,528$ & deletion & - \\
\hline 190684 chr7 & $61,796,377$ & $61,820,891$ & deletion & - \\
\hline $190684 \mathrm{chr} 8$ & $39,232,581$ & $39,385,979$ & duplication & - \\
\hline 190684 chr9 & $44,057,312$ & $44,100,710$ & deletion & - \\
\hline 190685 chr1 & $104,103,057$ & $104,211,046$ & duplication & AMY1C;AMY1A;AMY2A;AMY2B;AMY1B \\
\hline 190685 chr1 & $243,074,818$ & $243,081,905$ & duplication & - \\
\hline 190685 chr10 & $20,850,624$ & $20,857,365$ & deletion & - \\
\hline 190685 chr10 & $38,869,302$ & $38,903,858$ & deletion & - \\
\hline 190685 chr12 & $8,000,912$ & $8,118,205$ & duplication & SLC2A14;SLC2A3 \\
\hline 190685 chr13 & $69,247,022$ & $69,267,981$ & deletion & - \\
\hline 190685 chr13 & $98,530,124$ & $98,532,757$ & deletion & - \\
\hline 190685 chr14 & $74,026,686$ & $74,051,642$ & deletion & ACOT2 \\
\hline 190685 chr15 & $20,120,214$ & $20,145,193$ & deletion & - \\
\hline 190685 chr15 & $20,878,391$ & $21,157,642$ & deletion & РОТЕВ2;РОТЕВ;РОТЕВ3 \\
\hline $190685 \mathrm{chr} 16$ & $46,471,024$ & $46,492,062$ & deletion & - \\
\hline 190685 chr17 & $25,268,096$ & $25,278,249$ & deletion & 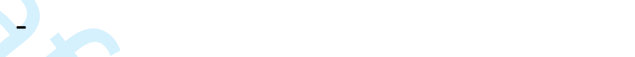 \\
\hline 190685 chr19 & $20,601,006$ & $20,717,536$ & deletion & \\
\hline 190685 chr2 & $49,534,329$ & $49,538,143$ & deletion & - \\
\hline $190685 \mathrm{chr} 2$ & $92,293,128$ & $92,304,211$ & deletion & - \\
\hline 190685 chr20 & $25,890,697$ & $25,896,760$ & deletion & - \\
\hline 190685 chr21 & $10,780,816$ & $10,858,651$ & duplication & - \\
\hline 190685 chr21 & $11,055,462$ & $11,063,273$ & deletion & BAGE2;BAGE3 \\
\hline $190685 \mathrm{chr} 3$ & $128,382,258$ & $128,412,024$ & duplication & - \\
\hline 190685 chr3 & $13,681,185$ & $13,682,137$ & deletion & - \\
\hline 190685 chr3 & $37,979,882$ & $37,986,734$ & deletion & - \\
\hline 190685 chr3 & $89,402,409$ & $89,418,331$ & deletion & - \\
\hline $190685 \mathrm{chr} 4$ & $104,205,273$ & $104,247,262$ & deletion & - \\
\hline $190685 \mathrm{chr} 4$ & $190,189,043$ & $190,200,455$ & deletion & - \\
\hline 190685 chr4 & $69,374,369$ & $69,489,473$ & deletion & UGT2B17 \\
\hline 190685 chr6 & $70,214,844$ & $70,224,911$ & duplication & - \\
\hline 190685 chr6 & $81,284,205$ & $81,293,326$ & deletion & - \\
\hline 190685 chr7 & $20,750,774$ & $20,753,528$ & deletion & - \\
\hline 190685 chr7 & $62,149,006$ & $62,159,926$ & deletion & - \\
\hline 190685 chr8 & $57,307,609$ & $57,321,536$ & deletion & - \\
\hline 190685 chr9 & $23,363,526$ & $23,376,817$ & deletion & - \\
\hline 190685 chr9 & $44,057,312$ & $44,100,710$ & deletion & - \\
\hline 190686 chr1 & $43,447,091$ & $43,451,901$ & deletion & - \\
\hline 190686 chr11 & $18,949,220$ & $18,961,743$ & deletion & MRGPRX1 \\
\hline 190686 chr11 & $24,446,609$ & $24,451,830$ & deletion & - \\
\hline
\end{tabular}




\begin{tabular}{|c|c|c|c|c|}
\hline 190686 chr11 & $3,239,988$ & $3,243,665$ & deletion & MRGPRG \\
\hline 190686 chr11 & $49,715,154$ & $49,757,690$ & deletion & - \\
\hline 190686 chr12 & $127,489,891$ & $127,493,679$ & deletion & - \\
\hline 190686 chr12 & $21,555,582$ & $21,571,978$ & duplication & - \\
\hline 190686 chr13 & $21,728,134$ & $21,732,193$ & duplication & SKA3 \\
\hline 190686 chr14 & $35,604,892$ & $35,613,500$ & deletion & - \\
\hline 190686 chr14 & $41,610,224$ & $41,661,685$ & deletion & - \\
\hline 190686 chr14 & $56,460,374$ & $56,480,275$ & duplication & - \\
\hline 190686 chr15 & $20,120,214$ & $20,144,522$ & deletion & - \\
\hline 190686 chr16 & $19,945,540$ & $19,962,636$ & deletion & - \\
\hline 190686 chr16 & $46,471,024$ & $46,492,062$ & deletion & - \\
\hline 190686 chr16 & $7,212,889$ & $7,239,880$ & deletion & - \\
\hline 190686 chr18 & $66,746,208$ & $66,755,736$ & deletion & - \\
\hline 190686 chr19 & $54,730,202$ & $54,743,217$ & duplication & LILRA6 \\
\hline 190686 chr19 & $9,275,282$ & $9,282,176$ & deletion & - \\
\hline 190686 chr2 & $228,241,621$ & $228,258,288$ & duplication & TM4SF20 \\
\hline $190686 \mathrm{chr} 2$ & $76,937,446$ & $76,949,101$ & deletion & - \\
\hline 190686 chr20 & $25,890,697$ & $25,910,523$ & deletion & - \\
\hline 190686 chr22 & $25,663,994$ & $25,919,492$ & duplication & LRP5L \\
\hline 190686 chr3 & $191,065,392$ & $191,070,300$ & deletion & - \\
\hline 190686 chr3 & $49,385,099$ & $49,393,607$ & deletion & - \\
\hline 190686 chr3 & $57,938,822$ & $57,979,767$ & duplication & - \\
\hline 190686 chr3 & $65,117,947$ & $65,118,762$ & deletion & - \\
\hline $190686 \mathrm{chr} 4$ & $190,194,200$ & $190,199,993$ & deletion & - \\
\hline $190686 \mathrm{chr} 4$ & $49,588,812$ & $49,620,898$ & deletion & - \\
\hline $190686 \mathrm{chr} 4$ & $60,325,242$ & $60,330,880$ & duplication & - \\
\hline 190686 chr5 & $111,935,341$ & $111,944,746$ & deletion & - \\
\hline 190686 chr5 & $133,415,061$ & $133,416,500$ & deletion & - \\
\hline 190686 chr6 & $132,114,944$ & $132,118,192$ & deletion & - \\
\hline 190686 chr6 & $27,634,640$ & $27,659,192$ & duplication & - \\
\hline 190686 chr6 & $78,972,930$ & $79,035,739$ & deletion & - \\
\hline 190686 chr7 & $75,357,240$ & $75,369,103$ & duplication & HIP1 \\
\hline 190686 chr8 & $137,682,484$ & $137,862,080$ & deletion & - \\
\hline 190686 chr8 & $15,401,929$ & $15,411,867$ & deletion & - \\
\hline 190686 chr8 & $24,972,808$ & $24,990,418$ & deletion & - \\
\hline 190686 chr8 & $51,013,729$ & $51,038,149$ & deletion & - \\
\hline 190686 chr9 & $107,799,020$ & $107,803,370$ & deletion & - \\
\hline 190686 chr9 & $31,396,656$ & $31,399,459$ & deletion & - \\
\hline 190686 chr9 & $44,069,106$ & $44,100,710$ & deletion & - \\
\hline 190687 chr1 & $104,153,766$ & $104,211,046$ & deletion & AMY1A;AMY2A;AMY1C;AMY1B \\
\hline 190687 chr1 & $12,850,282$ & $12,919,928$ & deletion & HNRNPCL1;HNRNPCL3;HNRNPCL4;PRAMEF11 \\
\hline 190687 chr1 & $149,024,218$ & $149,237,009$ & deletion & - \\
\hline 190687 chr1 & $161,570,803$ & $161,619,741$ & deletion & FCGR3B \\
\hline 190687 chr1 & $189,337,997$ & $189,542,729$ & deletion & - \\
\hline 190687 chr1 & $225,533,111$ & $225,534,348$ & deletion & DNAH14 \\
\hline $190687 \mathrm{chr} 1$ & $248,740,572$ & $248,795,110$ & deletion & OR2T10;OR2T11 \\
\hline 190687 chr1 & $9,847,290$ & $9,855,529$ & deletion & - \\
\hline
\end{tabular}




\begin{tabular}{|c|c|c|c|c|}
\hline 190687 chr10 & $132,692,121$ & $132,693,122$ & deletion & \\
\hline 190687 chr10 & $20,850,624$ & $20,857,365$ & deletion & - \\
\hline 190687 chr10 & $46,989,839$ & $47,122,413$ & duplication & GPRIN2;CH17-360D5.1;NPY4R \\
\hline 190687 chr10 & $47,541,708$ & $47,703,946$ & duplication & ANTXRL \\
\hline 190687 chr11 & $132,452,659$ & $132,458,871$ & deletion & - \\
\hline 90687 chr11 & $134,601,963$ & $134,606,394$ & deletion & - \\
\hline 190687 chr11 & $25,585,180$ & $25,751,372$ & deletion & - \\
\hline 90687 chr11 & $48,876,899$ & $48,901,553$ & duplication & - \\
\hline 190687 chr11 & $49,715,154$ & $49,757,690$ & deletion & - \\
\hline $190687 \mathrm{chr} 11$ & $93,698,060$ & $93,702,069$ & deletion & - \\
\hline 190687 chr12 & $130,295,647$ & $130,297,819$ & deletion & - \\
\hline 190687 chr12 & $30,237,351$ & $30,243,473$ & deletion & - \\
\hline 190687 chr12 & $31,945,622$ & $31,951,237$ & deletion & - \\
\hline 190687 chr12 & $33,300,995$ & $33,305,855$ & duplication & - \\
\hline 190687 chr15 & $26,751,068$ & $26,752,373$ & deletion & - \\
\hline 190687 chr15 & $63,267,778$ & $63,268,686$ & deletion & - \\
\hline 190687 chr16 & $25,420,840$ & $25,445,588$ & duplication & - \\
\hline 190687 chr16 & $32,542,736$ & $32,642,384$ & deletion & - \\
\hline 190687 chr16 & $46,471,024$ & $46,492,062$ & deletion & - \\
\hline 190687 chr16 & $55,800,009$ & $55,819,443$ & deletion & - \\
\hline 190687 chr16 & $60,556,223$ & $60,566,803$ & deletion & - \\
\hline 190687 chr17 & $42,659,043$ & $42,662,966$ & deletion & - \\
\hline 190687 chr19 & $43,298,958$ & $43,539,189$ & deletion & PSG6;PSG7;PSG11;PSG1 \\
\hline 190687 chr19 & $43,710,045$ & $43,759,382$ & deletion & PSG9 \\
\hline 190687 chr19 & $51,331,915$ & $51,333,442$ & deletion & - \\
\hline 190687 chr19 & $56,710,941$ & $56,739,605$ & duplication & ZSCAN5A \\
\hline $190687 \mathrm{chr} 2$ & $123,477,465$ & $123,482,301$ & deletion & - \\
\hline $190687 \mathrm{chr} 2$ & $29,577,405$ & $29,596,963$ & deletion & - \\
\hline $190687 \mathrm{chr} 2$ & $40,927,466$ & $40,949,168$ & deletion & - \\
\hline 190687 chr20 & $25,890,697$ & $25,896,449$ & deletion & - \\
\hline 190687 chr20 & $29,566,903$ & $29,585,801$ & deletion & - \\
\hline 190687 chr22 & $18,889,794$ & $19,010,149$ & duplication & PRODH;DGCR6 \\
\hline 190687 chr22 & $39,359,885$ & $39,385,663$ & deletion & АРОВЕСЗВ \\
\hline 190687 chr22 & $42,526,710$ & $42,531,210$ & duplication & CYP2D6 \\
\hline $190687 \mathrm{chr} 3$ & $131,711,896$ & $131,712,898$ & duplication & - \\
\hline $190687 \mathrm{chr} 4$ & $122,285,263$ & $122,289,863$ & deletion & - \\
\hline $190687 \mathrm{chr} 4$ & $171,269,504$ & $171,273,360$ & deletion & - \\
\hline $190687 \mathrm{chr} 4$ & $34,785,955$ & $34,818,502$ & duplication & - \\
\hline $190687 \mathrm{chr} 4$ & $64,697,704$ & $64,709,753$ & duplication & - \\
\hline $190687 \mathrm{chr} 4$ & $69,374,369$ & $69,489,473$ & deletion & UGT2B17 \\
\hline $190687 \mathrm{chr} 5$ & $161,140,039$ & $161,142,633$ & deletion & - \\
\hline 190687 chr6 & $29,853,641$ & $29,899,389$ & deletion & - \\
\hline 190687 chr6 & $31,281,682$ & $31,286,577$ & deletion & - \\
\hline 190687 chr6 & $81,293,837$ & $81,787,190$ & duplication & - \\
\hline 190687 chr6 & $81,988,694$ & $82,195,906$ & duplication & - \\
\hline 190687 chr7 & $111,228,173$ & $111,334,573$ & deletion & - \\
\hline $190687 \mathrm{chr} 7$ & $141,766,850$ & $141,793,419$ & deletion & - \\
\hline
\end{tabular}




\begin{tabular}{|c|c|c|c|c|}
\hline 190687 chr7 & $142,476,094$ & $142,487,836$ & deletion & - \\
\hline 190687 chr7 & $156,387,296$ & $156,394,307$ & deletion & - \\
\hline 190687 chr7 & $20,750,774$ & $20,753,528$ & deletion & - \\
\hline 190687 chr7 & 41,421 & 68,920 & duplication & - \\
\hline $190687 \mathrm{chr} 7$ & $62,688,981$ & $62,740,018$ & deletion & - \\
\hline 190687 chr8 & $115,634,278$ & $115,642,459$ & deletion & - \\
\hline 190687 chr8 & $18,852,474$ & $18,865,181$ & deletion & - \\
\hline 190687 chr9 & $44,070,629$ & $44,100,710$ & deletion & - \\
\hline 190687 chr9 & $6,701,130$ & $6,707,794$ & deletion & - \\
\hline 190687 chr9 & $69,494,085$ & $69,808,877$ & deletion & - \\
\hline 190687 chr9 & $69,824,191$ & $69,881,429$ & deletion & - \\
\hline 190687 chrX & $134,772,011$ & $134,947,796$ & duplication & СТ45A9;CT45A8;CT45A1;CT45A3;CT45A2;CT4! \\
\hline 198122 chr1 & $112,983,544$ & $112,987,487$ & deletion & - \\
\hline 198122 chr1 & $149,024,218$ & $149,237,009$ & deletion & - \\
\hline 198122 chr1 & $1,651,355$ & $1,668,298$ & deletion & SLC35E2;CDK11A;CDK11B \\
\hline 198122 chr10 & $58,901,976$ & $58,936,939$ & deletion & - \\
\hline $198122 \mathrm{chr} 11$ & $55,365,761$ & $55,443,269$ & deletion & OR4P4;OR4C6;OR4C11;OR4S2 \\
\hline 198122 chr11 & $58,820,988$ & $58,836,622$ & duplication & - \\
\hline 198122 chr12 & $11,511,132$ & $11,546,987$ & deletion & PRB2 \\
\hline 198122 chr13 & $22,929,489$ & $22,948,820$ & deletion & - \\
\hline $198122 \mathrm{chr} 13$ & $32,532,761$ & $32,537,768$ & deletion & - \\
\hline 198122 chr13 & $43,598,844$ & $43,607,143$ & duplication & - \\
\hline 198122 chr14 & $106,784,840$ & $106,816,856$ & deletion & - \\
\hline 198122 chr14 & $41,610,224$ & $41,661,685$ & deletion & - \\
\hline 198122 chr15 & $20,878,391$ & $21,200,107$ & duplication & РОTEB2;РОTEB;РОТЕВ3 \\
\hline 198122 chr15 & $26,751,068$ & $26,752,373$ & deletion & - \\
\hline 198122 chr15 & $32,509,090$ & $32,620,088$ & duplication & - \\
\hline 198122 chr16 & $33,863,814$ & $33,882,506$ & deletion & - \\
\hline 198122 chr16 & $84,304,110$ & $84,310,740$ & duplication & - \\
\hline 198122 chr17 & $10,891,369$ & $10,893,799$ & deletion & - \\
\hline 198122 chr17 & $15,044,804$ & $15,058,236$ & deletion & - \\
\hline 198122 chr17 & $15,641,109$ & $15,674,245$ & deletion & TBC1D26 \\
\hline $198122 \mathrm{chr} 18$ & $44,534,144$ & $44,588,009$ & duplication & TCEB3CL2;TCEB3B;TCEB3C;KATNAL2;TCEB3CL \\
\hline $198122 \mathrm{chr} 18$ & $66,746,208$ & $66,755,736$ & deletion & - \\
\hline 198122 chr2 & $10,885,901$ & $10,890,881$ & duplication & - \\
\hline 198122 chr2 & $123,477,465$ & $123,482,301$ & deletion & - \\
\hline $198122 \mathrm{chr} 2$ & $212,771,670$ & $212,773,893$ & deletion & - \\
\hline 198122 chr2 & $34,697,895$ & $34,726,904$ & duplication & - \\
\hline 198122 chr2 & $35,676,218$ & $35,682,937$ & deletion & - \\
\hline 198122 chr2 & $49,533,498$ & $49,538,143$ & deletion & - \\
\hline 198122 chr2 & $79,332,083$ & $79,343,124$ & duplication & - \\
\hline 198122 chr21 & $14,338,959$ & $14,348,261$ & deletion & - \\
\hline 198122 chr21 & $23,655,900$ & $23,667,066$ & deletion & - \\
\hline 198122 chr3 & $162,213,876$ & $162,234,970$ & deletion & - \\
\hline 198122 chr3 & $193,136,358$ & $193,140,348$ & deletion & - \\
\hline $198122 \mathrm{chr} 4$ & $161,014,288$ & $161,019,327$ & deletion & - \\
\hline 198122 chr4 & $161,861,640$ & $161,931,797$ & duplication & - \\
\hline
\end{tabular}




\begin{tabular}{|c|c|c|c|c|}
\hline $198122 \mathrm{chr} 4$ & $34,785,955$ & $34,818,502$ & duplication & - \\
\hline $198122 \mathrm{chr} 4$ & $69,374,369$ & $69,489,473$ & deletion & UGT2B17 \\
\hline $198122 \mathrm{chr} 5$ & $17,639,913$ & $17,651,582$ & deletion & - \\
\hline 198122 chr6 & $120,987,090$ & $121,016,276$ & deletion & - \\
\hline 198122 chr6 & $140,387,753$ & $140,391,106$ & deletion & - \\
\hline 198122 chr6 & $169,506,837$ & $169,520,560$ & deletion & - \\
\hline 198122 chr6 & $29,859,135$ & $29,899,389$ & deletion & - \\
\hline 198122 chr6 & $32,550,802$ & $32,555,728$ & deletion & HLA-DRB1 \\
\hline 198122 chr6 & $32,600,317$ & $32,629,207$ & deletion & HLA-DQB1;HLA-DQA1 \\
\hline 198122 chr6 & $37,963,308$ & $37,981,179$ & duplication & - \\
\hline $198122 \mathrm{chr} 7$ & 151,829 & 159,207 & deletion & - \\
\hline 198122 chr7 & $61,840,856$ & $61,853,795$ & duplication & - \\
\hline 198122 chr7 & $62,688,981$ & $62,740,018$ & deletion & - \\
\hline $198122 \mathrm{chr} 7$ & $91,033,074$ & $91,041,618$ & deletion & - \\
\hline 198122 chr8 & $2,150,949$ & $2,159,660$ & deletion & - \\
\hline 198122 chr8 & $39,232,581$ & $39,385,979$ & deletion & - \\
\hline 198122 chr8 & $6,120,770$ & $6,124,908$ & deletion & - \\
\hline 198122 chr9 & $13,798,727$ & $13,800,109$ & deletion & - \\
\hline 198122 chr9 & $23,363,526$ & $23,376,817$ & duplication & - \\
\hline $198127 \mathrm{chr} 1$ & $104,153,766$ & $104,161,227$ & deletion & AMY2A \\
\hline 198127 chr1 & $17,202,073$ & $17,269,302$ & deletion & CROCC \\
\hline $198127 \mathrm{chr} 1$ & $84,126,005$ & $84,127,067$ & deletion & - \\
\hline 198127 chr12 & $119,988,733$ & $119,991,777$ & deletion & - \\
\hline 198127 chr12 & $30,237,351$ & $30,243,473$ & deletion & \\
\hline 198127 chr12 & $32,004,170$ & $32,061,988$ & duplication & - \\
\hline 198127 chr13 & $38,076,308$ & $38,085,537$ & deletion & - \\
\hline 198127 chr14 & $20,365,265$ & $20,422,799$ & duplication & OR4K5;OR4K1 \\
\hline 198127 chr14 & $47,708,397$ & $47,715,702$ & deletion & - \\
\hline 198127 chr15 & $32,509,090$ & $32,620,088$ & deletion & - \\
\hline 198127 chr15 & $43,888,976$ & $43,939,642$ & duplication & STRC;CATSPER2;CKMT1B \\
\hline 198127 chr16 & $21,530,772$ & $21,839,340$ & deletion & METTL9;IGSF6;OTOA \\
\hline 198127 chr16 & $78,368,600$ & $78,383,509$ & deletion & - \\
\hline 198127 chr16 & $78,427,766$ & $78,430,875$ & duplication & - \\
\hline 198127 chr16 & $83,463,871$ & $83,467,391$ & deletion & - \\
\hline 198127 chr18 & $37,065,326$ & $37,200,183$ & deletion & - \\
\hline 198127 chr19 & $53,518,747$ & $53,552,296$ & duplication & ERVV-1 \\
\hline 198127 chr2 & $195,677,877$ & $195,698,234$ & deletion & - \\
\hline 198127 chr2 & $208,351,315$ & $208,357,838$ & deletion & - \\
\hline 198127 chr2 & $38,956,947$ & $38,971,623$ & duplication & GALM \\
\hline $198127 \mathrm{chr} 2$ & $49,534,329$ & $49,538,143$ & deletion & - \\
\hline 198127 chr2 & $52,759,890$ & $52,784,952$ & deletion & - \\
\hline 198127 chr20 & $52,647,631$ & $52,656,535$ & deletion & - \\
\hline 198127 chr3 & $128,382,258$ & $128,412,024$ & duplication & - \\
\hline $198127 \mathrm{chr} 3$ & $131,711,896$ & $131,712,898$ & deletion & - \\
\hline 198127 chr3 & $1,782,524$ & $1,787,581$ & deletion & - \\
\hline 198127 chr3 & $197,844,320$ & $197,846,134$ & deletion & - \\
\hline 198127 chr3 & $8,821,742$ & $8,855,880$ & duplication & - \\
\hline
\end{tabular}




\begin{tabular}{|c|c|c|c|c|}
\hline 198127 chr3 & $98,945,571$ & $98,947,547$ & deletion & - \\
\hline $198127 \mathrm{chr} 4$ & $116,168,158$ & $116,173,928$ & deletion & - \\
\hline $198127 \mathrm{chr} 4$ & $69,374,369$ & $69,489,473$ & deletion & UGT2B17 \\
\hline 198127 chr5 & $140,225,908$ & $140,238,311$ & deletion & PCDHA10;PCDHA9 \\
\hline 198127 chr5 & $70,305,696$ & $70,308,251$ & duplication & NAIP \\
\hline 198127 chr5 & 788,646 & 825,338 & deletion & ZDHHC11 \\
\hline 198127 chr5 & $99,507,450$ & $99,510,375$ & deletion & - \\
\hline 198127 chr6 & $139,603,393$ & $139,606,731$ & deletion & - \\
\hline 198127 chr6 & $31,281,682$ & $31,286,577$ & deletion & - \\
\hline 198127 chr6 & $77,439,769$ & $77,451,301$ & deletion & - \\
\hline 198127 chr7 & $142,476,094$ & $142,487,836$ & deletion & - \\
\hline 198127 chr7 & $145,148,265$ & $145,151,681$ & deletion & - \\
\hline 198127 chr7 & $156,387,296$ & $156,394,307$ & deletion & - \\
\hline 198127 chr7 & $75,664,539$ & $75,667,743$ & deletion & - \\
\hline 198127 chr8 & $39,232,581$ & $39,385,979$ & duplication & - \\
\hline 198127 chr8 & $40,106,630$ & $40,108,527$ & deletion & - \\
\hline 198127 chr8 & $85,261,157$ & $85,268,951$ & deletion & - \\
\hline 203892 chr1 & $143,279,808$ & $143,544,285$ & duplication & - \\
\hline 203892 chr1 & $175,161,599$ & $175,162,805$ & deletion & - \\
\hline 203892 chr1 & $175,430,855$ & $175,762,061$ & duplication & - \\
\hline 203892 chr1 & $187,717,153$ & $187,722,032$ & deletion & - \\
\hline 203892 chr1 & $231,710,453$ & $231,813,134$ & duplication & DISC1 \\
\hline 203892 chr1 & $248,740,572$ & $248,795,110$ & deletion & OR2T10;OR2T11 \\
\hline 203892 chr1 & 534,247 & 761,764 & duplication & OR4F29;OR4F3;OR4F16 \\
\hline 203892 chr1 & $59,247,993$ & $59,251,097$ & deletion & JUN \\
\hline 203892 chr1 & $72,754,314$ & $72,763,324$ & deletion & - \\
\hline 203892 chr10 & $33,853,394$ & $33,865,876$ & duplication & - \\
\hline 203892 chr11 & $134,601,963$ & $134,606,394$ & deletion & - \\
\hline 203892 chr11 & $7,814,299$ & $7,827,879$ & deletion & OR5P2 \\
\hline 203892 chr11 & $86,304,402$ & $86,306,401$ & deletion & - \\
\hline 203892 chr11 & $92,025,597$ & $92,027,585$ & deletion & - \\
\hline 203892 chr11 & $93,902,945$ & $93,910,895$ & deletion & - \\
\hline 203892 chr12 & $11,511,132$ & $11,546,987$ & deletion & PRB2 \\
\hline 203892 chr14 & $106,062,675$ & $106,175,202$ & duplication & - \\
\hline 203892 chr14 & $106,891,524$ & $106,917,103$ & deletion & - \\
\hline 203892 chr14 & $94,547,478$ & $94,547,661$ & deletion & - \\
\hline 203892 chr15 & $24,578,496$ & $24,741,382$ & duplication & - \\
\hline 203892 chr16 & $32,542,736$ & $32,642,384$ & deletion & - \\
\hline 203892 chr16 & $35,194,609$ & $35,197,323$ & deletion & - \\
\hline 203892 chr17 & $44,215,394$ & $44,347,946$ & duplication & KANSL1 \\
\hline 203892 chr17 & $48,636,534$ & $48,639,367$ & deletion & CACNA1G \\
\hline 203892 chr18 & $47,814,249$ & $47,814,821$ & deletion & - \\
\hline 203892 chr18 & $69,428,556$ & $69,441,154$ & deletion & - \\
\hline 203892 chr19 & $43,555,291$ & $43,848,797$ & deletion & PSG4;PSG5;PSG2;PSG9 \\
\hline 203892 chr19 & $53,518,747$ & $53,552,296$ & duplication & ERVV-1 \\
\hline $203892 \mathrm{chr} 2$ & $177,374,056$ & $177,377,367$ & deletion & - \\
\hline 203892 chr2 & $208,351,315$ & $208,357,838$ & deletion & - \\
\hline
\end{tabular}




\begin{tabular}{|c|c|c|c|c|}
\hline 203892 chr2 & $34,697,895$ & $34,726,904$ & deletion & - \\
\hline 203892 chr21 & $10,699,687$ & $10,979,896$ & duplication & TPTE \\
\hline 203892 chr21 & $44,824,695$ & $44,837,829$ & duplication & LOC102724428;SIK1 \\
\hline 203892 chr22 & $17,564,478$ & $17,565,932$ & deletion & - \\
\hline 203892 chr22 & $44,564,297$ & $44,567,854$ & deletion & PARVB \\
\hline $203892 \mathrm{chr} 3$ & $162,130,691$ & $162,142,475$ & deletion & - \\
\hline $203892 \mathrm{chr} 3$ & $197,810,178$ & $197,891,568$ & duplication & FAM157A \\
\hline 203892 chr3 & $27,445,270$ & $27,554,292$ & duplication & SLC4A7 \\
\hline 203892 chr3 & $28,810,588$ & $28,817,583$ & deletion & - \\
\hline 203892 chr3 & $37,979,882$ & $37,986,734$ & deletion & - \\
\hline 203892 chr3 & $75,427,141$ & $75,643,261$ & deletion & - \\
\hline 203892 chr4 & $42,697,553$ & $42,708,694$ & deletion & - \\
\hline 203892 chr4 & $43,341,411$ & $43,344,992$ & deletion & - \\
\hline 203892 chr4 & $49,164,409$ & $49,325,091$ & duplication & - \\
\hline $203892 \mathrm{chr} 4$ & $69,374,369$ & $69,489,473$ & deletion & UGT2B17 \\
\hline 203892 chr5 & $155,477,866$ & $155,488,917$ & deletion & - \\
\hline 203892 chr5 & $180,377,470$ & $180,424,820$ & deletion & BTNL8;BTNL3 \\
\hline 203892 chr5 & 749,946 & 776,691 & deletion & - \\
\hline 203892 chr5 & $8,258,458$ & $8,260,630$ & deletion & - \\
\hline 203892 chr6 & $124,430,135$ & $124,471,820$ & duplication & NKAIN2 \\
\hline 203892 chr6 & $30,180,922$ & $30,182,395$ & deletion & - \\
\hline 203892 chr6 & $30,312,962$ & $30,313,340$ & deletion & RPP21;TRIM39-RPP21 \\
\hline 203892 chr6 & $32,610,719$ & $32,610,994$ & duplication & 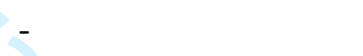 \\
\hline 203892 chr6 & $36,646,788$ & $36,648,464$ & deletion & \\
\hline 203892 chr6 & $67,011,900$ & $67,048,885$ & deletion & \\
\hline 203892 chr6 & $78,972,930$ & $79,035,739$ & deletion & . \\
\hline 203892 chr6 & $81,284,205$ & $81,293,326$ & deletion & - \\
\hline 203892 chr7 & $156,387,296$ & $156,394,307$ & deletion & - \\
\hline 203892 chr8 & $17,580,791$ & $17,581,700$ & deletion & MTUS1 \\
\hline 203892 chr8 & $24,972,808$ & $24,990,418$ & deletion & - \\
\hline $203892 \mathrm{chrX}$ & $120,819,108$ & $120,920,493$ & deletion & - \\
\hline 203892 chrX & $149,168,778$ & $149,233,585$ & deletion & - \\
\hline 203895 chr1 & $111,380,720$ & $111,387,723$ & deletion & - \\
\hline 203895 chr1 & $143,272,542$ & $143,284,605$ & deletion & - \\
\hline 203895 chr1 & $187,717,153$ & $187,722,032$ & deletion & - \\
\hline 203895 chr10 & $90,940,413$ & $90,945,998$ & deletion & - \\
\hline 203895 chr11 & $18,949,220$ & $18,961,743$ & deletion & MRGPRX1 \\
\hline 203895 chr11 & $49,715,154$ & $49,757,690$ & deletion & - \\
\hline 203895 chr12 & $12,532,984$ & $12,541,869$ & deletion & - \\
\hline 203895 chr13 & $58,589,893$ & $58,599,174$ & deletion & - \\
\hline 203895 chr14 & $41,610,224$ & $41,661,685$ & deletion & - \\
\hline 203895 chr15 & $20,120,214$ & $20,144,522$ & deletion & - \\
\hline 203895 chr16 & $19,945,540$ & $19,962,636$ & deletion & - \\
\hline 203895 chr16 & $28,609,775$ & $28,620,752$ & deletion & SULT1A1 \\
\hline 203895 chr18 & $63,201,694$ & $63,206,335$ & duplication & - \\
\hline 203895 chr19 & $54,730,202$ & $54,743,217$ & duplication & LILRA6 \\
\hline 203895 chr19 & $9,275,282$ & $9,282,176$ & deletion & - \\
\hline
\end{tabular}




\begin{tabular}{|c|c|c|c|c|}
\hline 203895 chr2 & $184,085,551$ & $184,088,256$ & deletion & - \\
\hline 203895 chr2 & $184,795,725$ & $184,803,456$ & deletion & - \\
\hline 203895 chr2 & $195,364,534$ & $195,366,958$ & deletion & - \\
\hline 203895 chr2 & $208,351,315$ & $208,357,838$ & deletion & - \\
\hline 203895 chr2 & $242,854,089$ & $243,034,519$ & deletion & - \\
\hline 203895 chr2 & $87,559,968$ & $87,972,393$ & duplication & - \\
\hline 203895 chr21 & $10,847,940$ & $10,858,651$ & duplication & - \\
\hline 203895 chr21 & $20,057,919$ & $20,078,801$ & duplication & - \\
\hline 203895 chr3 & $129,769,827$ & $129,798,600$ & deletion & - \\
\hline 203895 chr3 & $13,681,185$ & $13,682,137$ & deletion & - \\
\hline 203895 chr3 & $148,964,692$ & $148,969,220$ & deletion & - \\
\hline 203895 chr3 & $195,458,723$ & $195,462,414$ & duplication & MUC20 \\
\hline $203895 \mathrm{chr} 3$ & $53,032,639$ & $53,038,786$ & deletion & - \\
\hline 203895 chr3 & $5,452,140$ & $5,471,296$ & deletion & - \\
\hline 203895 chr3 & $65,189,366$ & $65,214,685$ & deletion & - \\
\hline 203895 chr3 & $98,945,571$ & $98,947,547$ & deletion & - \\
\hline 203895 chr4 & $10,396,298$ & $10,400,156$ & deletion & - \\
\hline 203895 chr4 & $138,092,298$ & $138,098,601$ & deletion & - \\
\hline 203895 chr5 & $155,477,866$ & $155,488,917$ & deletion & - \\
\hline 203895 chr5 & $173,113,526$ & $173,121,339$ & deletion & - \\
\hline 203895 chr5 & $180,377,470$ & $180,424,820$ & deletion & BTNL8;BTNL3 \\
\hline 203895 chr6 & $110,396,540$ & $110,411,065$ & deletion & - \\
\hline 203895 chr6 & $29,915,469$ & $29,928,649$ & deletion & - \\
\hline 203895 chr7 & $111,943,602$ & $111,972,365$ & deletion & ZNF277 \\
\hline 203895 chr7 & $142,476,094$ & $142,487,836$ & deletion & - \\
\hline 203895 chr8 & $10,628,947$ & $10,635,788$ & deletion & - \\
\hline 203895 chr8 & $115,634,278$ & $115,642,459$ & deletion & - \\
\hline 203895 chr8 & $39,232,581$ & $39,385,979$ & deletion & - \\
\hline 203895 chr8 & $7,254,609$ & $7,371,590$ & deletion & DEFB106A;DEFB106B;DEFB104B;DEFB4B;DEFE \\
\hline 203895 chr9 & $23,363,526$ & $23,376,817$ & deletion & - \\
\hline 203895 chr9 & $77,999,354$ & $78,013,319$ & deletion & - \\
\hline 204524 chr1 & $112,693,423$ & $112,704,581$ & deletion & - \\
\hline 204524 chr1 & $149,024,218$ & $149,237,009$ & deletion & - \\
\hline 204524 chr1 & $25,598,276$ & $25,669,057$ & deletion & RHD;TMEM50A \\
\hline 204524 chr11 & $7,814,299$ & $7,827,879$ & deletion & OR5P2 \\
\hline 204524 chr12 & $11,232,402$ & $11,252,845$ & duplication & TAS2R43 \\
\hline 204524 chr12 & $12,532,984$ & $12,541,869$ & deletion & - \\
\hline 204524 chr12 & $126,994,506$ & $127,004,296$ & deletion & - \\
\hline 204524 chr12 & $131,727,571$ & $131,825,359$ & deletion & - \\
\hline 204524 chr12 & $70,875,808$ & $70,877,258$ & deletion & - \\
\hline 204524 chr13 & $38,076,308$ & $38,085,537$ & deletion & - \\
\hline 204524 chr13 & $92,608,088$ & $92,622,045$ & deletion & - \\
\hline 204524 chr15 & $24,578,496$ & $24,741,382$ & duplication & - \\
\hline 204524 chr16 & $32,257,633$ & $32,649,736$ & duplication & TP53TG3D \\
\hline 204524 chr16 & $33,380,180$ & $33,631,255$ & duplication & - \\
\hline 204524 chr16 & $34,471,298$ & $34,756,258$ & duplication & - \\
\hline 204524 chr17 & $34,438,753$ & $34,481,492$ & duplication & - \\
\hline
\end{tabular}




\begin{tabular}{|c|c|c|c|c|}
\hline 204524 chr19 & $43,710,045$ & $43,759,382$ & deletion & PSG9 \\
\hline 204524 chr2 & $110,852,875$ & $110,982,530$ & deletion & MALL;NPHP1 \\
\hline 204524 chr2 & $19,576,591$ & $19,582,415$ & deletion & - \\
\hline 204524 chr2 & $213,187,034$ & $213,191,389$ & deletion & - \\
\hline 204524 chr2 & $48,851,541$ & $48,856,770$ & deletion & - \\
\hline 204524 chr2 & $51,926,599$ & $51,926,904$ & deletion & - \\
\hline 204524 chr21 & $10,813,582$ & $10,837,083$ & deletion & - \\
\hline 204524 chr21 & $11,055,462$ & $11,060,553$ & deletion & BAGE2;BAGE3 \\
\hline 204524 chr22 & $25,663,994$ & $25,919,492$ & deletion & LRP5L \\
\hline 204524 chr3 & $1,782,524$ & $1,787,581$ & deletion & - \\
\hline 204524 chr3 & $22,279,685$ & $22,286,262$ & deletion & - \\
\hline 204524 chr3 & $75,428,675$ & $75,703,113$ & deletion & - \\
\hline 204524 chr4 & $161,048,496$ & $161,072,119$ & deletion & - \\
\hline 204524 chr4 & $42,697,553$ & $42,708,694$ & deletion & - \\
\hline 204524 chr5 & $150,585,169$ & $150,591,079$ & deletion & - \\
\hline 204524 chr5 & $155,477,866$ & $155,488,917$ & deletion & - \\
\hline 204524 chr5 & $17,600,053$ & $17,627,924$ & deletion & - \\
\hline 204524 chr5 & $50,231,548$ & $50,269,870$ & duplication & - \\
\hline 204524 chr6 & $132,114,944$ & $132,118,192$ & deletion & - \\
\hline 204524 chr6 & 266,035 & 379,638 & duplication & DUSP22 \\
\hline 204524 chr6 & $32,610,719$ & $32,614,917$ & duplication & - \\
\hline 204524 chr6 & $77,439,769$ & $77,451,301$ & deletion & - \\
\hline 204524 chr7 & $75,664,539$ & $75,667,743$ & deletion & - \\
\hline 204524 chr7 & $97,396,709$ & $97,401,677$ & deletion & - \\
\hline 204524 chr8 & $24,972,808$ & $24,990,418$ & duplication & 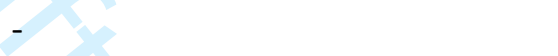 \\
\hline 204524 chr8 & $47,526,999$ & $47,539,088$ & duplication & - \\
\hline 204524 chr9 & $6,701,130$ & $6,707,794$ & deletion & - \\
\hline 204524 chr9 & $85,373,362$ & $85,383,934$ & deletion & - \\
\hline 204525 chr1 & $104,153,766$ & $104,211,046$ & deletion & AMY1A;AMY2A;AMY1C;AMY1B \\
\hline 204525 chr1 & $149,024,218$ & $149,237,009$ & deletion & - \\
\hline 204525 chr10 & $81,788,104$ & $81,791,683$ & deletion & - \\
\hline 204525 chr11 & $49,715,154$ & $49,757,690$ & deletion & - \\
\hline 204525 chr11 & $81,501,218$ & $81,517,261$ & deletion & - \\
\hline 204525 chr11 & $83,829,316$ & $83,844,790$ & duplication & - \\
\hline 204525 chr12 & $11,220,827$ & $11,244,117$ & deletion & TAS2R43 \\
\hline 204525 chr12 & $6,255,862$ & $6,259,552$ & deletion & - \\
\hline 204525 chr13 & $80,681,247$ & $80,685,663$ & deletion & - \\
\hline 204525 chr14 & $106,067,375$ & $106,072,316$ & duplication & - \\
\hline 204525 chr15 & $20,444,796$ & $21,200,107$ & duplication & GOLGA6L6;POTEB2;POTEB;POTEB3 \\
\hline 204525 chr15 & $22,383,292$ & $22,528,723$ & duplication & OR4N4 \\
\hline 204525 chr16 & $60,556,223$ & $60,566,803$ & deletion & - \\
\hline 204525 chr17 & $34,438,753$ & $34,480,718$ & duplication & - \\
\hline 204525 chr19 & $20,601,006$ & $20,717,536$ & deletion & - \\
\hline 204525 chr19 & $53,518,747$ & $53,548,950$ & duplication & ERVV-1 \\
\hline 204525 chr2 & $106,347,912$ & $106,353,107$ & deletion & - \\
\hline 204525 chr2 & $114,155,972$ & $114,166,128$ & deletion & - \\
\hline 204525 chr2 & $177,374,056$ & $177,377,367$ & deletion & - \\
\hline
\end{tabular}




\begin{tabular}{|c|c|c|c|c|}
\hline $204525 \mathrm{chr} 2$ & $18,171,812$ & $18,193,277$ & deletion & - \\
\hline $204525 \mathrm{chr} 2$ & $208,351,315$ & $208,357,838$ & deletion & - \\
\hline 204525 chr2 & $228,241,621$ & $228,258,288$ & duplication & TM4SF20 \\
\hline 204525 chr2 & $34,697,895$ & $34,726,904$ & duplication & - \\
\hline $204525 \mathrm{chr} 2$ & $38,956,947$ & $38,971,623$ & duplication & GALM \\
\hline 204525 chr2 & $48,851,541$ & $48,856,770$ & deletion & - \\
\hline 204525 chr20 & $14,936,935$ & $15,276,852$ & deletion & MACROD2 \\
\hline 204525 chr20 & $44,350,423$ & $44,380,052$ & deletion & SPINT4 \\
\hline 204525 chr21 & $10,766,457$ & $10,858,651$ & duplication & - \\
\hline 204525 chr3 & $13,681,185$ & $13,682,137$ & deletion & - \\
\hline 204525 chr3 & $195,458,723$ & $195,462,414$ & duplication & MUC20 \\
\hline 204525 chr3 & $37,979,882$ & $37,986,734$ & deletion & - \\
\hline $204525 \mathrm{chr} 3$ & $75,428,675$ & $75,703,113$ & deletion & - \\
\hline 204525 chr3 & $98,945,571$ & $98,947,547$ & deletion & - \\
\hline 204525 chr4 & $152,991,094$ & $152,993,620$ & deletion & - \\
\hline $204525 \mathrm{chr} 4$ & $156,959,568$ & $156,974,697$ & deletion & - \\
\hline $204525 \mathrm{chr} 4$ & $160,244,212$ & $160,379,451$ & duplication & RAPGEF2 \\
\hline 204525 chr4 & $25,557,047$ & $25,576,330$ & duplication & - \\
\hline 204525 chr5 & $140,225,908$ & $140,238,311$ & deletion & PCDHA10;PCDHA9 \\
\hline 204525 chr5 & $155,477,866$ & $155,488,917$ & deletion & - \\
\hline 204525 chr5 & $18,996,966$ & $19,015,104$ & duplication & - \\
\hline 204525 chr5 & $19,285,249$ & $19,309,903$ & duplication & - \\
\hline 204525 chr5 & 788,646 & 836,608 & deletion & ZDHHC11 \\
\hline 204525 chr6 & $78,972,930$ & $79,035,739$ & deletion & . \\
\hline 204525 chr7 & $20,750,774$ & $20,753,528$ & deletion & \\
\hline 204525 chr8 & $18,852,474$ & $18,865,181$ & deletion & - \\
\hline 204525 chr8 & $24,972,808$ & $24,990,418$ & duplication & - \\
\hline 204525 chr8 & $29,173,434$ & $29,176,919$ & deletion & - \\
\hline 204525 chr8 & $72,214,753$ & $72,217,690$ & deletion & - \\
\hline 204525 chr9 & $23,363,526$ & $23,376,817$ & duplication & - \\
\hline 204525 chr9 & $24,502,737$ & $24,518,795$ & deletion & - \\
\hline 204525 chrX & $74,494,796$ & $74,653,212$ & duplication & ZDHHC15;UPRT \\
\hline 204526 chr1 & $108,734,695$ & $108,737,586$ & deletion & SLC25A24 \\
\hline 204526 chr1 & $112,693,423$ & $112,704,581$ & deletion & - \\
\hline 204526 chr1 & $187,717,153$ & $187,722,032$ & deletion & - \\
\hline 204526 chr1 & $240,711,075$ & $240,748,758$ & deletion & - \\
\hline $204526 \mathrm{chr} 1$ & $248,740,572$ & $248,795,110$ & deletion & OR2T10;OR2T11 \\
\hline 204526 chr1 & $25,598,276$ & $25,659,509$ & deletion & RHD \\
\hline 204526 chr11 & $131,925,581$ & $131,931,753$ & duplication & - \\
\hline 204526 chr11 & $134,601,963$ & $134,606,394$ & deletion & - \\
\hline 204526 chr11 & $55,365,761$ & $55,451,045$ & deletion & OR4P4;OR4C6;OR4C11;OR4 \\
\hline 204526 chr11 & $81,501,218$ & $81,517,261$ & deletion & - \\
\hline 204526 chr12 & $30,237,351$ & $30,243,473$ & deletion & - \\
\hline 204526 chr14 & $42,988,346$ & $42,992,844$ & deletion & - \\
\hline 204526 chr15 & $102,004,318$ & $102,010,213$ & deletion & - \\
\hline 204526 chr15 & $91,983,376$ & $91,989,276$ & deletion & - \\
\hline 204526 chr17 & $71,655,923$ & $71,667,181$ & deletion & - \\
\hline
\end{tabular}




\begin{tabular}{|c|c|c|c|c|}
\hline 204526 chr18 & $57,454,578$ & $57,456,108$ & deletion & - \\
\hline 204526 chr19 & $43,298,958$ & $43,539,189$ & deletion & PSG6;PSG7;PSG11;PSG1 \\
\hline 204526 chr2 & $120,046,497$ & $120,048,584$ & deletion & - \\
\hline 204526 chr2 & $34,697,895$ & $34,726,904$ & deletion & - \\
\hline 204526 chr2 & $9,324,513$ & $9,357,400$ & duplication & ASAP2 \\
\hline 204526 chr21 & $25,499,807$ & $25,500,458$ & deletion & - \\
\hline 204526 chr22 & $17,985,994$ & $17,994,585$ & deletion & CECR2 \\
\hline 204526 chr3 & $164,813,376$ & $164,815,463$ & deletion & - \\
\hline 204526 chr3 & $197,845,008$ & $197,846,927$ & deletion & - \\
\hline 204526 chr3 & $37,979,882$ & $37,986,734$ & deletion & - \\
\hline $204526 \mathrm{chr} 3$ & $65,189,366$ & $65,214,685$ & deletion & - \\
\hline 204526 chr4 & $138,092,298$ & $138,098,601$ & deletion & - \\
\hline 204526 chr4 & $172,772,609$ & $172,782,420$ & deletion & - \\
\hline $204526 \mathrm{chr} 4$ & $18,124,909$ & $18,131,245$ & deletion & - \\
\hline $204526 \mathrm{chr} 4$ & $70,137,897$ & $70,229,260$ & deletion & UGT2B28 \\
\hline 204526 chr5 & $63,850,027$ & $63,851,332$ & deletion & - \\
\hline 204526 chr6 & $30,994,114$ & $30,994,787$ & deletion & MUC22 \\
\hline 204526 chr6 & $31,337,850$ & $31,343,632$ & deletion & - \\
\hline 204526 chr6 & $31,785,453$ & $31,795,550$ & deletion & HSPA1A \\
\hline 204526 chr6 & $78,972,930$ & $79,035,739$ & deletion & - \\
\hline 204526 chr8 & $3,786,543$ & $3,790,254$ & deletion & - \\
\hline 204526 chr8 & $39,232,581$ & $39,385,979$ & deletion & - \\
\hline $204526 \mathrm{chr} 8$ & $51,226,087$ & $51,227,777$ & deletion & - \\
\hline 204526 chr9 & $23,363,526$ & $23,376,817$ & deletion & - \\
\hline 204529 chr1 & $104,153,766$ & $104,161,227$ & deletion & AMY2A \\
\hline 204529 chr1 & $158,493,726$ & $158,497,751$ & deletion & - \\
\hline $204529 \mathrm{chr} 1$ & $187,717,153$ & $187,722,032$ & deletion & - \\
\hline $204529 \mathrm{chr} 1$ & $25,598,276$ & $25,669,057$ & deletion & RHD;TMEM50A \\
\hline 204529 chr10 & $135,351,137$ & $135,434,303$ & duplication & SYCE1;CYP2E1 \\
\hline 204529 chr11 & $55,365,761$ & $55,443,269$ & deletion & OR4P4;OR4C6;OR4C11;OR4S2 \\
\hline 204529 chr11 & $81,501,218$ & $81,517,261$ & deletion & - \\
\hline 204529 chr12 & $12,532,984$ & $12,541,869$ & deletion & - \\
\hline 204529 chr15 & $20,120,214$ & $20,144,522$ & deletion & - \\
\hline 204529 chr16 & $60,556,223$ & $60,566,803$ & deletion & - \\
\hline 204529 chr17 & $34,438,753$ & $34,480,718$ & duplication & - \\
\hline 204529 chr19 & $20,601,006$ & $20,717,536$ & deletion & - \\
\hline 204529 chr19 & $53,518,747$ & $53,548,950$ & duplication & ERVV-1 \\
\hline 204529 chr2 & $114,155,972$ & $114,166,128$ & deletion & - \\
\hline 204529 chr2 & $129,638,609$ & $129,645,321$ & deletion & - \\
\hline 204529 chr2 & $180,414,715$ & $180,421,668$ & deletion & - \\
\hline 204529 chr2 & $223,865,393$ & $223,869,820$ & deletion & - \\
\hline 204529 chr2 & $38,956,947$ & $38,971,623$ & duplication & GALM \\
\hline 204529 chr2 & $51,926,599$ & $51,926,904$ & deletion & - \\
\hline $204529 \mathrm{chr} 2$ & $66,188,209$ & $66,196,726$ & deletion & - \\
\hline 204529 chr20 & $44,350,423$ & $44,380,052$ & deletion & SPINT4 \\
\hline 204529 chr3 & $195,433,728$ & $195,462,414$ & duplication & MUC20 \\
\hline 204529 chr3 & $37,979,882$ & $37,986,734$ & deletion & - \\
\hline
\end{tabular}




\begin{tabular}{|c|c|c|c|c|}
\hline $204529 \mathrm{chr} 3$ & $75,419,736$ & $75,703,113$ & deletion & - \\
\hline $204529 \mathrm{chr} 4$ & $152,991,094$ & $152,993,620$ & deletion & - \\
\hline 204529 chr4 & $156,959,568$ & $156,974,697$ & deletion & - \\
\hline $204529 \mathrm{chr} 4$ & $160,261,725$ & $160,377,484$ & duplication & RAPGEF2 \\
\hline $204529 \mathrm{chr} 4$ & $25,557,047$ & $25,577,184$ & duplication & - \\
\hline 204529 chr4 & $34,785,955$ & $34,818,502$ & duplication & - \\
\hline 204529 chr5 & $140,225,908$ & $140,238,311$ & deletion & PCDHA10;PCDHA9 \\
\hline 204529 chr5 & $155,477,866$ & $155,488,917$ & deletion & - \\
\hline 204529 chr5 & $19,285,249$ & $19,309,903$ & duplication & - \\
\hline 204529 chr5 & 788,646 & 836,608 & deletion & ZDHHC11 \\
\hline 204529 chr6 & $29,859,135$ & $29,899,389$ & deletion & - \\
\hline 204529 chr7 & $156,387,296$ & $156,394,307$ & deletion & - \\
\hline $204529 \mathrm{chr} 8$ & $18,852,474$ & $18,865,181$ & deletion & - \\
\hline 204529 chr8 & $39,232,581$ & $39,385,979$ & duplication & - \\
\hline 204529 chr8 & $51,031,221$ & $51,038,149$ & deletion & - \\
\hline 204529 chr8 & $72,214,753$ & $72,217,690$ & deletion & - \\
\hline 204529 chr8 & $9,055,379$ & $9,062,490$ & deletion & - \\
\hline 204529 chr9 & $118,073,084$ & $118,082,546$ & deletion & - \\
\hline 204529 chr9 & $6,701,130$ & $6,707,794$ & deletion & - \\
\hline $204529 \mathrm{chrX}$ & $74,494,796$ & $74,653,212$ & duplication & ZDHHC15;UPRT \\
\hline 204532 chr1 & $104,153,766$ & $104,211,046$ & duplication & AMY1A;AMY2A;AMY1C;AMY1B \\
\hline 204532 chr1 & $149,022,110$ & $149,237,009$ & deletion & - \\
\hline 204532 chr1 & $17,202,073$ & $17,269,302$ & deletion & CROCC \\
\hline 204532 chr1 & $187,717,153$ & $187,722,032$ & deletion & - \\
\hline 204532 chr1 & $196,823,300$ & $196,901,753$ & duplication & CFHR4 \\
\hline 204532 chr1 & $87,028,669$ & $87,039,309$ & deletion & CLCA4 \\
\hline 204532 chr10 & $27,226,851$ & $27,227,458$ & deletion & - \\
\hline 204532 chr10 & $28,649,384$ & $28,652,892$ & deletion & - \\
\hline 204532 chr10 & $39,119,741$ & $39,149,666$ & duplication & - \\
\hline 204532 chr11 & $70,075,666$ & $70,082,221$ & deletion & - \\
\hline 204532 chr12 & $30,237,351$ & $30,243,473$ & deletion & - \\
\hline 204532 chr15 & $20,120,214$ & $20,144,522$ & deletion & - \\
\hline 204532 chr15 & $20,878,391$ & $21,157,642$ & deletion & РОTЕВ2;РОТЕВ;РОТЕВ3 \\
\hline 204532 chr15 & $22,299,434$ & $22,516,945$ & deletion & OR4M2;OR4N4 \\
\hline 204532 chr15 & $34,717,697$ & $34,814,869$ & deletion & - \\
\hline 204532 chr15 & $56,789,979$ & $56,800,635$ & deletion & - \\
\hline 204532 chr16 & $34,471,298$ & $34,756,258$ & duplication & - \\
\hline 204532 chr16 & $70,016,280$ & $70,141,115$ & deletion & - \\
\hline 204532 chr19 & $35,852,103$ & $35,861,695$ & deletion & - \\
\hline 204532 chr19 & $53,518,747$ & $53,548,950$ & duplication & ERVV-1 \\
\hline 204532 chr2 & $184,795,725$ & $184,803,456$ & deletion & - \\
\hline $204532 \mathrm{chr} 2$ & $208,351,315$ & $208,357,838$ & deletion & - \\
\hline 204532 chr2 & $79,752,148$ & $79,761,222$ & deletion & - \\
\hline 204532 chr3 & $128,382,258$ & $128,412,024$ & duplication & - \\
\hline 204532 chr3 & $197,844,320$ & $197,846,927$ & deletion & - \\
\hline 204532 chr3 & $46,798,133$ & $46,845,618$ & deletion & - \\
\hline 204532 chr3 & $75,428,675$ & $75,703,113$ & deletion & - \\
\hline
\end{tabular}




\begin{tabular}{|c|c|c|c|c|}
\hline 204532 chr4 & $69,374,369$ & $69,489,473$ & deletion & UGT2B17 \\
\hline 204532 chr6 & $32,508,741$ & $32,543,540$ & deletion & - \\
\hline 204532 chr6 & $67,011,900$ & $67,048,885$ & deletion & - \\
\hline 204532 chr6 & $69,231,883$ & $69,241,954$ & duplication & - \\
\hline 204532 chr8 & $3,786,543$ & $3,790,254$ & deletion & - \\
\hline 204532 chr8 & $39,232,581$ & $39,385,979$ & deletion & - \\
\hline 204532 chr9 & $23,363,526$ & $23,376,817$ & deletion & - \\
\hline 204532 chr9 & $77,999,354$ & $78,011,616$ & deletion & - \\
\hline 204534 chr1 & $16,368,481$ & $16,386,395$ & duplication & FAM131C;CLCNKB \\
\hline 204534 chr1 & $1,651,355$ & $1,668,298$ & deletion & SLC35E2;CDK11A;CDK11B \\
\hline 204534 chr1 & $205,438,040$ & $205,456,128$ & duplication & - \\
\hline 204534 chr10 & $12,558,701$ & $12,576,005$ & deletion & - \\
\hline 204534 chr10 & $135,256,575$ & $135,349,801$ & duplication & CYP2E1 \\
\hline 204534 chr10 & $135,354,068$ & $135,378,802$ & duplication & SYCE1 \\
\hline 204534 chr10 & $20,850,624$ & $20,857,365$ & deletion & - \\
\hline 204534 chr10 & $39,119,741$ & $39,149,666$ & duplication & - \\
\hline 204534 chr11 & $3,239,988$ & $3,243,665$ & deletion & MRGPRG \\
\hline 204534 chr11 & $90,193,427$ & $90,196,250$ & duplication & - \\
\hline 204534 chr12 & $119,988,733$ & $119,991,380$ & deletion & - \\
\hline 204534 chr12 & $131,794,405$ & $131,816,213$ & deletion & - \\
\hline 204534 chr13 & $111,587,429$ & $111,588,967$ & deletion & - \\
\hline 204534 chr13 & $34,134,809$ & $34,143,545$ & deletion & - \\
\hline 204534 chr13 & $64,225,427$ & $64,236,288$ & deletion & - \\
\hline 204534 chr16 & $22,166,976$ & $22,178,256$ & duplication & VWA3A \\
\hline 204534 chr16 & $65,710,913$ & $65,723,074$ & duplication & - \\
\hline 204534 chr17 & $33,681,335$ & $33,768,284$ & deletion & SLFN11;SLFN12;SLFN13 \\
\hline 204534 chr19 & $43,298,958$ & $43,539,189$ & deletion & PSG6;PSG7;PSG11;PSG1 \\
\hline 204534 chr19 & $53,518,747$ & $53,552,296$ & duplication & ERVV-1 \\
\hline 204534 chr2 & $95,529,090$ & $95,550,262$ & duplication & TEKT4 \\
\hline 204534 chr20 & $14,455,497$ & $14,467,744$ & deletion & - \\
\hline 204534 chr21 & $11,055,462$ & $11,063,273$ & deletion & BAGE2;BAGE3 \\
\hline $204534 \mathrm{chr} 3$ & $13,619,303$ & $13,714,683$ & duplication & FBLN2 \\
\hline 204534 chr3 & $197,845,008$ & $197,846,927$ & deletion & - \\
\hline 204534 chr4 & $35,148,652$ & $35,151,237$ & deletion & - \\
\hline 204534 chr5 & $155,477,866$ & $155,488,917$ & deletion & - \\
\hline 204534 chr6 & $125,222,272$ & $125,230,575$ & deletion & - \\
\hline 204534 chr6 & $78,972,930$ & $79,035,739$ & deletion & - \\
\hline 204534 chr7 & $159,118,443$ & $159,123,700$ & deletion & - \\
\hline 204534 chr8 & $137,682,484$ & $137,862,080$ & deletion & - \\
\hline $204534 \mathrm{chr} 8$ & $3,443,992$ & $3,450,317$ & deletion & - \\
\hline 204534 chr8 & $9,055,379$ & $9,062,490$ & deletion & - \\
\hline 204535 chr1 & $174,797,867$ & $174,801,639$ & deletion & - \\
\hline 204535 chr10 & $43,206,700$ & $43,247,120$ & duplication & - \\
\hline 204535 chr10 & $58,516,470$ & $58,526,153$ & deletion & - \\
\hline 204535 chr11 & $7,814,299$ & $7,827,879$ & deletion & OR5P2 \\
\hline 204535 chr12 & $12,532,984$ & $12,541,869$ & deletion & - \\
\hline 204535 chr12 & $126,994,335$ & $127,004,296$ & deletion & - \\
\hline
\end{tabular}




\begin{tabular}{|c|c|c|c|c|c|}
\hline 204535 & $\operatorname{chr} 12$ & $31,907,259$ & $31,909,635$ & deletion & - \\
\hline 204535 & chr12 & $32,331,195$ & $32,334,813$ & deletion & - \\
\hline 204535 & chr12 & $60,874,409$ & $60,877,239$ & deletion & - \\
\hline 204535 & chr13 & $98,530,124$ & $98,532,757$ & deletion & - \\
\hline 204535 & chr14 & $106,891,524$ & $106,917,103$ & deletion & - \\
\hline 204535 & chr15 & $24,578,496$ & $24,741,382$ & duplication & - \\
\hline 204535 & chr16 & $22,552,780$ & $22,703,741$ & deletion & - \\
\hline 204535 & chr16 & $78,372,894$ & $78,383,509$ & deletion & - \\
\hline 204535 & chr16 & $85,300,519$ & $85,304,486$ & deletion & - \\
\hline 204535 & chr17 & $34,438,753$ & $34,480,718$ & duplication & - \\
\hline 204535 & chr17 & $54,160,465$ & $54,172,668$ & deletion & - \\
\hline 204535 & chr17 & $70,815,357$ & $70,820,207$ & deletion & - \\
\hline 204535 & chr17 & $7,264,717$ & $7,265,681$ & deletion & - \\
\hline 204535 & chr19 & $43,703,965$ & $43,759,382$ & deletion & PSG4;PSG9 \\
\hline 204535 & chr2 & $213,187,034$ & $213,191,389$ & deletion & - \\
\hline 204535 & chr2 & $34,697,895$ & $34,726,904$ & duplication & - \\
\hline 204535 & chr2 & $51,926,599$ & $51,926,904$ & deletion & - \\
\hline 204535 & chr22 & $25,663,994$ & $25,919,492$ & deletion & LRP5L \\
\hline 204535 & chr22 & $33,862,494$ & $33,933,970$ & duplication & - \\
\hline 204535 & chr22 & $39,359,885$ & $39,385,663$ & deletion & АРОВЕСЗВ \\
\hline 204535 & chr3 & $131,711,896$ & $131,712,898$ & duplication & - \\
\hline 204535 & chr3 & $85,593,984$ & $85,613,258$ & duplication & - \\
\hline 204535 & chr3 & $98,945,571$ & $98,947,547$ & deletion & - \\
\hline 204535 & chr4 & $165,559,842$ & $166,180,003$ & duplication & APELA;TMEM192;KLHL2;TRIM60;TRIM61;FAN \\
\hline 204535 & chr4 & $34,785,955$ & $34,818,502$ & duplication & \\
\hline 204535 & chr4 & $42,697,553$ & $42,708,694$ & deletion & - \\
\hline 204535 & chr4 & $69,374,369$ & $69,489,473$ & deletion & UGT2B17 \\
\hline 204535 & chr4 & $70,137,897$ & $70,229,260$ & deletion & UGT2B28 \\
\hline 204535 & chr4 & $92,280,583$ & $92,284,935$ & deletion & - \\
\hline 204535 & chr5 & $150,585,169$ & $150,591,079$ & deletion & - \\
\hline 204535 & chr5 & $155,477,866$ & $155,488,917$ & deletion & - \\
\hline 204535 & chr6 & $132,114,944$ & $132,118,192$ & deletion & - \\
\hline 204535 & chr6 & $69,231,883$ & $69,241,954$ & duplication & - \\
\hline 204535 & chr6 & $77,439,769$ & $77,451,301$ & deletion & - \\
\hline 204535 & chr6 & $78,972,930$ & $79,035,739$ & deletion & - \\
\hline 204535 & chr7 & $23,544,373$ & $23,582,792$ & deletion & TRA2A \\
\hline 204535 & chr7 & $30,404,788$ & $30,407,445$ & deletion & - \\
\hline 204535 & chr7 & $75,664,539$ & $75,667,743$ & deletion & - \\
\hline 204535 & chr8 & $3,786,543$ & $3,790,254$ & deletion & - \\
\hline 204535 & chr8 & $63,034,992$ & $63,039,751$ & deletion & - \\
\hline 204535 & chr9 & $85,373,362$ & $85,383,934$ & deletion & - \\
\hline 204536 & chr1 & $104,153,766$ & $104,161,227$ & deletion & $A M Y 2 A$ \\
\hline 204536 & chr1 & $149,022,110$ & $149,237,009$ & deletion & - \\
\hline 204536 & chr1 & $213,003,428$ & $213,011,812$ & deletion & SPATA45 \\
\hline 204536 & chr1 & $25,598,276$ & $25,669,057$ & deletion & RHD;TMEM50A \\
\hline 204536 & chr10 & $81,788,104$ & $81,791,683$ & deletion & - \\
\hline 204536 & chr11 & $55,365,761$ & $55,443,269$ & deletion & OR4P4;OR4C6;OR4C11;OR4S2 \\
\hline
\end{tabular}




\begin{tabular}{|c|c|c|c|c|}
\hline 204536 chr12 & $33,300,995$ & $33,305,855$ & deletion & - \\
\hline 204536 chr12 & $61,879,699$ & $61,886,284$ & deletion & - \\
\hline 204536 chr12 & $6,255,862$ & $6,259,552$ & deletion & - \\
\hline 204536 chr13 & $21,728,134$ & $21,732,193$ & duplication & SKA3 \\
\hline 204536 chr13 & $80,681,247$ & $80,685,663$ & deletion & - \\
\hline 204536 chr14 & $106,103,358$ & $106,175,202$ & duplication & - \\
\hline 204536 chr14 & $23,047,799$ & $23,053,564$ & deletion & - \\
\hline 204536 chr16 & $60,556,223$ & $60,566,803$ & deletion & - \\
\hline 204536 chr16 & $85,302,676$ & $85,304,326$ & deletion & - \\
\hline 204536 chr17 & $34,438,753$ & $34,480,718$ & duplication & - \\
\hline 204536 chr17 & $7,264,717$ & $7,265,681$ & deletion & - \\
\hline 204536 chr19 & $20,601,006$ & $20,717,536$ & deletion & - \\
\hline 204536 chr19 & 293,913 & 296,902 & deletion & - \\
\hline 204536 chr19 & $51,331,915$ & $51,333,442$ & deletion & - \\
\hline 204536 chr19 & $53,518,747$ & $53,548,950$ & duplication & ERVV-1 \\
\hline 204536 chr19 & $54,730,202$ & $54,743,217$ & deletion & LILRA6 \\
\hline 204536 chr2 & $106,347,912$ & $106,353,107$ & deletion & - \\
\hline 204536 chr2 & $114,155,972$ & $114,166,128$ & deletion & - \\
\hline 204536 chr2 & $14,704,369$ & $14,709,611$ & deletion & - \\
\hline 204536 chr2 & $180,414,715$ & $180,421,668$ & deletion & - \\
\hline 204536 chr2 & $18,171,812$ & $18,193,277$ & deletion & - \\
\hline 204536 chr2 & $223,865,393$ & $223,869,820$ & deletion & - \\
\hline 204536 chr2 & $38,956,947$ & $38,971,623$ & duplication & GALM \\
\hline 204536 chr2 & $51,926,599$ & $51,926,904$ & deletion & - \\
\hline 204536 chr20 & $44,350,423$ & $44,380,052$ & deletion & SPINT4 \\
\hline 204536 chr21 & $44,822,871$ & $44,837,829$ & duplication & LOC102724428;SIK1 \\
\hline 204536 chr3 & $195,458,723$ & $195,462,414$ & duplication & MUC20 \\
\hline 204536 chr3 & $37,979,882$ & $37,986,734$ & deletion & - \\
\hline 204536 chr3 & $75,419,736$ & $75,703,113$ & deletion & - \\
\hline 204536 chr3 & $96,308,055$ & $96,319,964$ & deletion & - \\
\hline 204536 chr4 & $116,168,158$ & $116,173,928$ & deletion & - \\
\hline $204536 \mathrm{chr} 4$ & $34,785,955$ & $34,818,502$ & duplication & - \\
\hline 204536 chr5 & $140,225,908$ & $140,238,311$ & deletion & PCDHA10;PCDHA9 \\
\hline 204536 chr5 & $155,477,866$ & $155,488,917$ & deletion & - \\
\hline 204536 chr5 & $19,285,249$ & $19,309,903$ & duplication & - \\
\hline 204536 chr5 & 788,646 & 824,172 & deletion & ZDHHC11 \\
\hline 204536 chr7 & $142,476,094$ & $142,487,836$ & deletion & - \\
\hline 204536 chr8 & $24,972,808$ & $24,990,418$ & duplication & - \\
\hline 204536 chr9 & $23,363,526$ & $23,376,817$ & duplication & - \\
\hline 204536 chr9 & $6,701,130$ & $6,707,794$ & deletion & - \\
\hline 204542 chr1 & $1,651,355$ & $1,668,298$ & deletion & SLC35E2;CDK11A;CDK11B \\
\hline 204542 chr1 & $205,438,040$ & $205,454,349$ & duplication & - \\
\hline 204542 chr1 & $213,003,428$ & $213,011,812$ & deletion & SPATA45 \\
\hline 204542 chr1 & $248,740,314$ & $248,795,110$ & deletion & OR2T10;OR2T11 \\
\hline 204542 chr10 & $71,282,981$ & $71,289,190$ & deletion & - \\
\hline 204542 chr10 & $98,968,967$ & $98,971,025$ & deletion & - \\
\hline 204542 chr11 & $3,239,988$ & $3,243,665$ & deletion & MRGPRG \\
\hline
\end{tabular}




$\begin{array}{llllll}204542 & \text { chr12 } & 12,532,984 & 12,541,869 & \text { deletion } & - \\ 204542 & \text { chr12 } & 131,794,405 & 131,816,213 & \text { deletion } & - \\ 204542 & \text { chr12 } & 132,115,667 & 132,136,919 & \text { duplication } & - \\ 204542 & \text { chr14 } & 44,166,914 & 44,202,432 & \text { deletion } & - \\ 204542 & \text { chr15 } & 34,717,697 & 34,814,869 & \text { deletion } & - \\ 204542 & \text { chr16 } & 22,166,976 & 22,178,256 & \text { duplication } & \text { VWA3A } \\ 204542 & \text { chr16 } & 2,653,599 & 2,725,256 & \text { duplication } & - \\ 204542 & \text { chr16 } & 65,710,913 & 65,723,074 & \text { duplication } & - \\ 204542 & \text { chr17 } & 33,681,335 & 33,768,284 & \text { deletion } & \text { SLFN11;SLFN12;SLFN13 } \\ 204542 & \text { chr18 } & 63,201,694 & 63,206,335 & \text { duplication } & - \\ 204542 & \text { chr19 } & 43,298,958 & 43,539,189 & \text { deletion } & \text { PSG6;PSG7;PSG11;PSG1 } \\ 204542 & \text { chr19 } & 53,518,747 & 53,552,296 & \text { duplication } & \text { ERVV-1 } \\ 204542 & \text { chr2 } & 177,374,056 & 177,377,367 & \text { deletion } & - \\ 204542 & \text { chr20 } & 37,008,769 & 37,014,876 & \text { deletion } & - \\ 204542 & \text { chr3 } & 13,619,303 & 13,714,683 & \text { duplication } & \text { FBLN2 } \\ 204542 & \text { chr3 } & 197,844,320 & 197,846,927 & \text { deletion } & - \\ 204542 & \text { chr3 } & 29,559,526 & 29,568,381 & \text { deletion } & - \\ 204542 & \text { chr4 } & 35,148,652 & 35,151,237 & \text { deletion } & - \\ 204542 & \text { chr4 } & 64,136,609 & 64,154,477 & \text { deletion } & - \\ 204542 & \text { chr5 } & 155,477,866 & 155,488,917 & \text { deletion } & - \\ 204542 & \text { chr5 } & 757,693 & 825,338 & \text { duplication } & \text { ZDHHC11 } \\ 204542 & \text { chr6 } & 32,044,172 & 32,047,436 & \text { duplication } & \text { TNXB } \\ 204542 & \text { chr6 } & 78,972,930 & 79,035,739 & \text { deletion } & - \\ 204542 & \text { chr7 } & 142,476,094 & 142,487,836 & \text { deletion } & - \\ 204542 & \text { chr8 } & 137,682,484 & 137,862,080 & \text { deletion } & - \\ 204542 & \text { chr8 } & 3,443,992 & 3,450,317 & \text { deletion } & - \\ 204542 & \text { chr9 } & 78,004,378 & 78,011,616 & \text { deletion } & - \\ & & & & & \end{array}$


12;POTEB2;POTEB 
12;POTEB2;POTEB

https://mc06.manuscriptcentral.com/bcb-pubs 
12;РOTEB2;POTEB 
l;OR4M1;POTEG;OR4N2 
12;POTEB2;POTEB 
12;POTEB2;POTEB 
12;РОTEB2;РOTEB 
'RAMEF11

https://mc06.manuscriptcentral.com/bcb-pubs 
$17 \mathrm{~A}$

https://mc06.manuscriptcentral.com/bcb-pubs 
5A5;CT45A4;CT45A7;CT45A6

12;РOTEB2;РOTEB 
;PRAMEF1;PRAMEF2

https://mc06.manuscriptcentral.com/bcb-pubs 
5A5;CT45A4;CT45A7;CT45A6;CT45A10 
3104A;SPAG11B;DEFB107A;DEFB107B;DEFB105A;DEFB105B;DEFB103B;DEFB103A 
1218A 
JEFB107B;DEFB107A;DEFB105B;PRR23D2;PRR23D1;DEFB103B;DEFB103A 
JEFB107B;DEFB107A;DEFB105B;PRR23D2;PRR23D1;DEFB103B;DEFB103A 
JEFB107B;DEFB107A;DEFB105B;PRR23D2;PRR23D1;DEFB103B;DEFB103A 


\begin{tabular}{|c|c|c|c|}
\hline sample & gender & status & relatedness.PLINK \\
\hline 184245 & $\mathrm{~F}$ & case & - \\
\hline 184246 & $M$ & case & - \\
\hline 184247 & $\mathrm{~F}$ & control & - \\
\hline 184248 & $\mathrm{~F}$ & case & - \\
\hline 184250 & $M$ & case & - \\
\hline 184251 & $M$ & case & - \\
\hline 184252 & $\mathrm{~F}$ & case & 184254-sibling \\
\hline 184254 & $\mathrm{~F}$ & case & 184252-sibling \\
\hline 184255 & $\mathrm{~F}$ & case & - \\
\hline 184256 & $\mathrm{~F}$ & case & - \\
\hline 184257 & $\mathrm{~F}$ & case & 184258-related \\
\hline 184258 & $\mathrm{~F}$ & case & 184257-related \\
\hline 184260 & $M$ & case & - \\
\hline 184261 & $M$ & case & 184263-sibling;184262-sibling \\
\hline 184262 & $M$ & case & 184263-sibling;184261-sibling \\
\hline 184263 & $\mathrm{~F}$ & case & 184262-sibling;184261-sibling \\
\hline 184265 & $M$ & case & 184274-related \\
\hline 184266 & $M$ & case & - \\
\hline 184267 & $M$ & case & - \\
\hline 184269 & $\mathrm{~F}$ & case & - \\
\hline 184270 & $M$ & case & 184271-related \\
\hline 184271 & $\mathrm{~F}$ & case & 184270-related \\
\hline 184273 & $\mathrm{~F}$ & case & - \\
\hline 184274 & $M$ & control & 184265-related \\
\hline 184275 & $\mathrm{M}$ & case & - \\
\hline 184276 & $M$ & control & - \\
\hline 184277 & $M$ & case & 184279-related \\
\hline 184279 & $\mathrm{~F}$ & case & 184277-related \\
\hline 184281 & $\mathrm{~F}$ & control & - \\
\hline 184282 & $\mathrm{~F}$ & control & - \\
\hline 184283 & $M$ & control & 184284-sibling \\
\hline 184284 & $\mathrm{~F}$ & control & 184283-sibling \\
\hline 184285 & $M$ & control & - \\
\hline 184286 & $\mathrm{~F}$ & control & - \\
\hline 184288 & $M$ & control & - \\
\hline 184291 & $\mathrm{~F}$ & control & 184292-sibling \\
\hline 184292 & $\mathrm{~F}$ & control & 184291-sibling \\
\hline 184293 & $M$ & control & 184296-sibling \\
\hline 184294 & $M$ & control & - \\
\hline 184296 & $M$ & control & 184293-sibling \\
\hline 184297 & $\mathrm{~F}$ & control & - \\
\hline 184298 & $M$ & case & - \\
\hline 184300 & $\mathrm{~F}$ & control & - \\
\hline 184301 & $\mathrm{~F}$ & case & 184302-related \\
\hline 184302 & $\mathrm{~F}$ & case & 184301-related \\
\hline 184303 & $M$ & control & - \\
\hline
\end{tabular}




\begin{tabular}{|c|c|c|}
\hline $184304 \mathrm{M}$ & case & - \\
\hline $184306 \mathrm{M}$ & control & - \\
\hline $184307 \mathrm{M}$ & case & - \\
\hline $184308 \mathrm{~F}$ & case & - \\
\hline $184315 \mathrm{M}$ & control & 184316-sibling \\
\hline $184316 \mathrm{~F}$ & control & 184315-sibling \\
\hline $184317 \mathrm{~F}$ & case & - \\
\hline $184320 \mathrm{~F}$ & case & - \\
\hline $184321 \mathrm{~F}$ & control & - \\
\hline $184322 \mathrm{M}$ & control & - \\
\hline $184324 \mathrm{~F}$ & control & - \\
\hline $184325 \mathrm{M}$ & case & - \\
\hline $184327 \mathrm{~F}$ & case & - \\
\hline $184328 \mathrm{M}$ & control & - \\
\hline $184329 \mathrm{~F}$ & case & - \\
\hline $184332 \mathrm{M}$ & case & - \\
\hline $184334 \mathrm{~F}$ & case & 184341-sibling;184340-related \\
\hline $184335 \mathrm{M}$ & case & - \\
\hline $184336 \mathrm{~F}$ & case & 184337-sibling \\
\hline $184337 \mathrm{~F}$ & case & 184336-sibling \\
\hline $184338 \mathrm{~F}$ & case & - \\
\hline $184339 \mathrm{~F}$ & case & - \\
\hline $184340 \mathrm{~F}$ & case & 184341-related;184334-related \\
\hline $184341 \mathrm{~F}$ & case & 184334-sibling;184340-related \\
\hline $184342 \mathrm{~F}$ & case & - \\
\hline $184343 \mathrm{M}$ & case & - \\
\hline $184344 \mathrm{M}$ & case & - \\
\hline $184345 \mathrm{M}$ & case & 184346-related \\
\hline $184346 \mathrm{~F}$ & case & 184345-related \\
\hline $184347 \mathrm{M}$ & case & - \\
\hline $184349 \mathrm{M}$ & case & - \\
\hline $184350 \mathrm{M}$ & case & 184351-sibling \\
\hline $184351 \mathrm{M}$ & case & 184350-sibling \\
\hline $184353 \mathrm{~F}$ & case & 184354-sibling \\
\hline $184354 \mathrm{M}$ & case & 184353-sibling \\
\hline $184356 \mathrm{~F}$ & case & 184357-sibling \\
\hline $184357 \mathrm{M}$ & case & 184356-sibling \\
\hline $184358 \mathrm{~F}$ & control & 184359-sibling;184360-related \\
\hline $184359 \mathrm{~F}$ & control & 184358-sibling;184360-related \\
\hline $184360 \mathrm{M}$ & control & 184359-related;184358-related \\
\hline $184362 \mathrm{M}$ & control & - \\
\hline $184364 \mathrm{M}$ & control & 184366-sibling;184365-sibling \\
\hline $184365 \mathrm{M}$ & control & 184366-sibling;184364-sibling \\
\hline $184366 \mathrm{M}$ & control & 184364-sibling;184365-sibling \\
\hline $184367 \mathrm{M}$ & control & - \\
\hline $184368 \mathrm{~F}$ & control & 184369-sibling \\
\hline $184369 \mathrm{~F}$ & control & 184368-sibling \\
\hline
\end{tabular}




\begin{tabular}{|c|c|c|}
\hline $184370 \mathrm{M}$ & control & - \\
\hline $184371 \mathrm{~F}$ & control & 184372-sibling \\
\hline $184372 \mathrm{M}$ & control & 184371-sibling \\
\hline $187232 \mathrm{~F}$ & case & 187233-sibling \\
\hline $187233 \mathrm{M}$ & case & 187232-sibling \\
\hline $187235 \mathrm{~F}$ & control & - \\
\hline $187236 \mathrm{M}$ & control & 187237-sibling \\
\hline $187237 \mathrm{~F}$ & control & 187236-sibling \\
\hline $187238 \mathrm{~F}$ & case & - \\
\hline $187239 \mathrm{M}$ & case & - \\
\hline $187240 \mathrm{~F}$ & case & - \\
\hline $187241 \mathrm{M}$ & control & - \\
\hline $187242 \mathrm{~F}$ & case & 187243-sibling \\
\hline $187243 \mathrm{~F}$ & case & 187242-sibling \\
\hline $187244 \mathrm{M}$ & control & - \\
\hline $187248 \mathrm{~F}$ & control & - \\
\hline $187249 \mathrm{M}$ & control & 187250-sibling \\
\hline $187250 \mathrm{M}$ & control & 187249-sibling \\
\hline $187251 \mathrm{~F}$ & case & - \\
\hline $187252 \mathrm{~F}$ & control & 187253-sibling \\
\hline $187253 \mathrm{~F}$ & control & 187252-sibling \\
\hline $187254 \mathrm{~F}$ & control & 187268-sibling \\
\hline $187255 \mathrm{M}$ & control & - \\
\hline $187257 \mathrm{M}$ & control & - \\
\hline $187258 \mathrm{M}$ & control & - \\
\hline $187259 \mathrm{M}$ & control & 187260-sibling \\
\hline $187260 \mathrm{M}$ & control & 187259-sibling \\
\hline $187261 \mathrm{~F}$ & control & 187273-sibling \\
\hline $187262 \mathrm{~F}$ & control & - \\
\hline $187263 \mathrm{~F}$ & control & 187264-sibling;187265-sibling \\
\hline $187264 \mathrm{M}$ & control & 187263-sibling;187265-twin_or_replicate \\
\hline $187265 \mathrm{M}$ & control & 187263-sibling;187264-twin_or_replicate \\
\hline $187266 \mathrm{M}$ & control & - \\
\hline $187267 \mathrm{~F}$ & control & - \\
\hline $187268 \mathrm{~F}$ & control & 187254-sibling \\
\hline $187269 \mathrm{~F}$ & control & 203892-sibling \\
\hline $187270 \mathrm{~F}$ & control & - \\
\hline $187271 \mathrm{~F}$ & control & - \\
\hline $187273 \mathrm{~F}$ & control & 187261-sibling \\
\hline $190680 \mathrm{~F}$ & control & - \\
\hline $190681 \mathrm{~F}$ & control & 190684-sibling;190682-sibling \\
\hline $190682 \mathrm{~F}$ & control & 190684-sibling;190681-sibling \\
\hline $190683 \mathrm{M}$ & control & - \\
\hline $190684 \mathrm{~F}$ & control & 190681-sibling;190682-sibling \\
\hline $190685 \mathrm{M}$ & control & - \\
\hline $190686 \mathrm{M}$ & control & - \\
\hline $190687 \mathrm{~F}$ & control & - \\
\hline
\end{tabular}




\begin{tabular}{|c|c|c|}
\hline $198116 \mathrm{M}$ & case & 198117-related \\
\hline $198117 \mathrm{~F}$ & case & 198116-related \\
\hline $198118 \mathrm{~F}$ & case & 198119-related;198120-related \\
\hline $198119 \mathrm{~F}$ & case & 198120-related;198118-related \\
\hline $198120 \mathrm{M}$ & case & 198119-related;198118-related \\
\hline $198121 \mathrm{~F}$ & case & - \\
\hline $198122 \mathrm{M}$ & control & - \\
\hline $198125 \mathrm{~F}$ & case & - \\
\hline $198126 \mathrm{~F}$ & case & - \\
\hline $198127 \mathrm{~F}$ & control & - \\
\hline $198128 \mathrm{~F}$ & case & - \\
\hline $203888 F$ & case & - \\
\hline $203889 M$ & case & - \\
\hline $203891 \mathrm{~F}$ & case & - \\
\hline $203892 \mathrm{~F}$ & control & 187269-sibling \\
\hline $203894 F$ & case & - \\
\hline $203895 \mathrm{~F}$ & control & - \\
\hline 203896 M & case & 203898-related \\
\hline $203897 M$ & case & - \\
\hline $203898 M$ & case & 203896-related \\
\hline $203899 \mathrm{~F}$ & case & - \\
\hline $203900 \mathrm{~F}$ & case & 203901-sibling \\
\hline $203901 \mathrm{~F}$ & case & 203900-sibling \\
\hline $204524 F$ & control & 204535-sibling \\
\hline $204525 \mathrm{~F}$ & control & 204536-sibling;204529-sibling \\
\hline $204526 \mathrm{~F}$ & control & - \\
\hline $204527 M$ & case & - \\
\hline $204528 M$ & case & - \\
\hline $204529 \mathrm{~F}$ & control & 204536-sibling;204525-sibling \\
\hline $204530 \mathrm{~F}$ & case & 204538-sibling \\
\hline $204531 M$ & case & - \\
\hline $204532 F$ & control & - \\
\hline $204533 \mathrm{~F}$ & case & 204540-sibling \\
\hline $204534 \mathrm{~F}$ & control & 204542-sibling \\
\hline $204535 M$ & control & 204524-sibling \\
\hline $204536 M$ & control & 204525-sibling;204529-sibling \\
\hline $204537 M$ & case & - \\
\hline $204538 \mathrm{~F}$ & case & 204530-sibling \\
\hline $204539 \mathrm{~F}$ & case & - \\
\hline $204540 \mathrm{~F}$ & case & 204533-sibling \\
\hline $204541 M$ & case & - \\
\hline $204542 F$ & control & 204534-sibling \\
\hline
\end{tabular}




\begin{tabular}{|l|l|l|}
\hline GeneSet ID (Suppl DataSets) & Figure Label & GeneSet FullName \\
\hline BspanVH_Ig2rpkm4.74 & Brain exp. V. high & Brain very high expr \\
\hline BspanHM_Ig2rpkm3.21 & Brain exp. High/med & Brain high/medium expr \\
\hline BspanML_Ig2rpkm0.93 & Brain exp. Med/low & Brain medium/low expr \\
\hline BspanLA_Ig2rpkm.MIN & Brain exp. Low/absent & Brain low/absent expr \\
\hline
\end{tabular}




\begin{tabular}{|l|l|}
\hline GeneSet Group & \#Genes in Set \\
\hline BrainExpression (BrainSpan) & 4600 \\
\hline BrainExpression (BrainSpan) & 4605 \\
\hline BrainExpression (BrainSpan) & 4596 \\
\hline BrainExpression (BrainSpan) & 4601 \\
\hline
\end{tabular}




\section{GeneSet Definition}

Genes with at least 5 BrainSpan data points $\log 2(\mathrm{rpkm})>=4.74$

Genes with at least 5 BrainSpan data points $\log 2(\mathrm{rpkm})>=3.21$, but not in BspanVH_lg2rpkm4.74 Genes with at least 5 BrainSpan data points $\log 2(\mathrm{rpkm})>=0.93$, but not in BspanVH_lg2rpkm4.74 or BspanHM_lg.

Genes with at least 5 BrainSpan data points $\log 2(\mathrm{rpkm})>=\mathrm{MIN}$, but not in BspanVH_lg2rpkm4.74 or BspanHM_lg: 
2rpkm3.21

2rpkm3.21 or BspanML_Ig2rpkm0.93 